Philopatrie versus Emigration:

Analysen zur Fitnessmaximierung adulter Söhne und Töchter einer semifreilebenden Weißbüschelaffen-Sozietät

(Callithrix jacchus)

\author{
Dissertation \\ zur Erlangung des Doktorgrades \\ der Mathematisch-Naturwissenschaftlichen Fakultäten \\ der Georg-August-Universität zu Göttingen
}

vorgelegt von

Ariane Thieß

aus Hamburg

Göttingen 2004 
D7

Referent: Prof. Dr. Hartmut Rothe

Korreferent: Prof. Dr. Ulrich Ehlers

Tag der mündlichen Prüfung: 01.07.2004 
„Love the animals for themselves first, then strain for general explanations, and, with good fortune, discoveries will follow. If they don't, the love and the pleasure will have been enough. " (Wilson 1994) 


\section{ABSTRACT}

During a two and a half year study, the mechanisms leading to dispersal and expulsions of nonreproductive offspring within large semi-free living families of common marmosets (Callithrix jacchus) were analysed. A model was developed on factors internal and external to the family, which were examined for their effect on the decision for philopatry or dispersal. The readiness for dispersal should become clear with peripheralisations.

The study was performed at the ethological field station Sennickerode from the Institute of Zoology and Anthropology of the University of Goettingen, Germany. During the whole study period the focal family consisted of 7-11 members living in a 1.5 ha home range within a 6.5 ha semi-free area. The group was exposed to all weather conditions and there was predatory risk due to the presence of native predators, especially raptors. The marmosets could seek shelter at any time in wooden huts, small wooden nest boxes which were installed all over their home range and a variety of trees and bushes.

The behaviour of each animal was recorded with instantaneous sampling together with the number of family members in close proximity to the focal animal. To record special or rare events ad libitum sampling was used as well. Instantaneous records were allocated to one of six categories with time budgets describing the proportion of each category. The whereabouts of the family members were recorded by an electronic identification system. Lure marmosets were exposed during experiments to the focal family in an experimental area separated from the family's home range by a 30m wide grassland belt after a control without and a control with a neighbouring family. The presence of lure females or lure males should provoke the nonreproductive adult offspring to peripheralize or leave their natal family.

Probabilities for expulsions dependent on the group size, age of the current offspring and sex and age of the expelled individuals were calculated from data collected in the C. jacchus colony of the Institute of Anthropology during 1972-2003, and compared with the results of this study. Probabilities for expulsions were also calculated in dependence of some activities. Expulsion probabilities where greater at group sizes of 11, 13 and 17 individuals within the first 20 days after birth and for males at the age of 15-25 months and females at the age of 15-30 months respectively. The sex of the evicted individuals, the presence of lure marmosets and activities like infant-carrying, scanning, non-tactile interactions with unknown marmosets and tactile interactions with family members had no influence on expulsion probabilities. The body temperature of some focal animals, which had implanted temperature buttons, was measured to detect dynamic processes before they became obvious in the behaviour of the animals. Due to 
technical and methodological problems it was not possible to record temperature accelerations that were triggered off by distress.

During the study no offspring left the natal family voluntarily, however, there were eight expulsions. Usually individuals were expelled by same sex siblings. When the parents took part in aggressive behaviour leading to evictions, they only harassed offspring of their own sex which was concluded to be a result of sexual conflict. In all adult offspring the presence of certain lure marmosets led to non-tactile interactions with unknown marmosets. Male offspring reduced their affiliative interactions with each other when lure females were presented, probably due to sexual conflict. Their spatial association, however, did not change. Analyses of the family structure showed trends towards brother-conflicts for social partners, too. In the presence of two lure males reductions of affiliative interactions and spatial associations among most of the siblings were observed. During this experiment the expulsion of a son occurred and at the same time a pair of offspring was born, therefore clear conclusions about the influence of the two lure males on sibling rivalry cannot be made. However, possible causalities between group size, number of helpers and sibling rivalry are discussed with a model.

A reduced spatial association to the family was observed in all adult offspring of both sexes, but no voluntary dispersal occurred. In accordance with this, peripheralisations could not be explained as readiness for dispersal. Sudden reduction of the spatial association of an offspring to the family, accompanied by reduced frequencies of having a near neighbour and reduced affiliative interactions could be valued as an indicator of forthcoming expulsions.

It became apparent that the offspring and the parents participate in dynamic processes, though it is not completely understood to what extent individuals can promote or delay their expulsion from the family with their behaviour. 


\section{INHALTSVERZEICHNIS}

1.1 Soziale Organisation und Reproduktionssystem von Callithrix jacchus ...........1

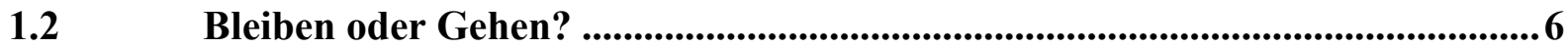

1.2.1 Einflussfaktoren auf die Entscheidung über Philopatrie und Emigration .............. 6

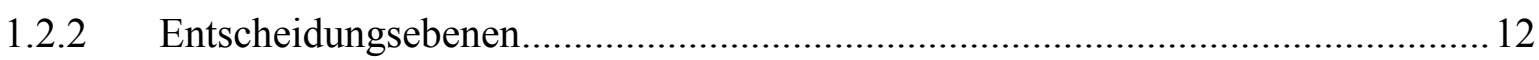

1.3 Spieltheoretische Betrachtung der Analyse von Entscheidungen .................... 14

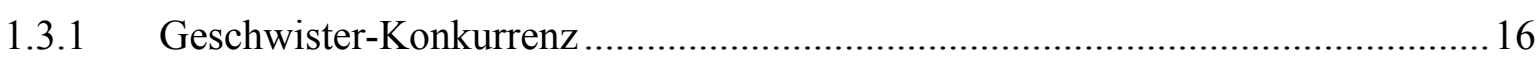

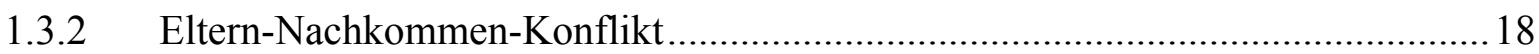

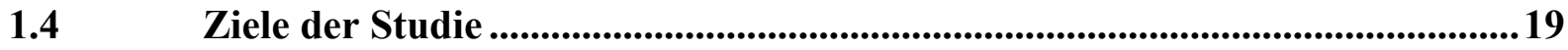

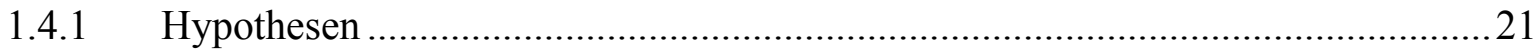

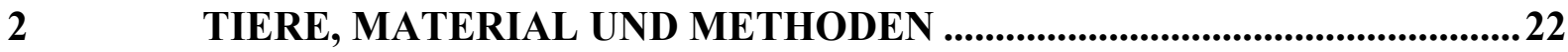

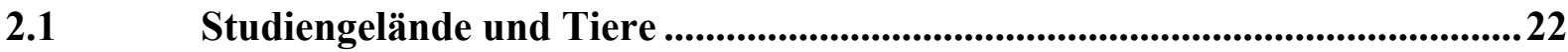

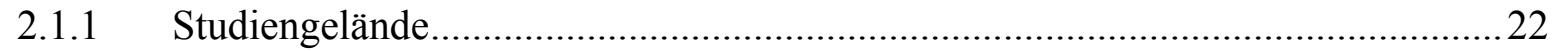

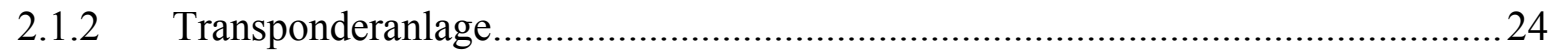

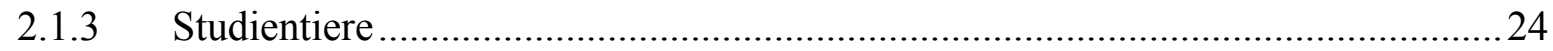

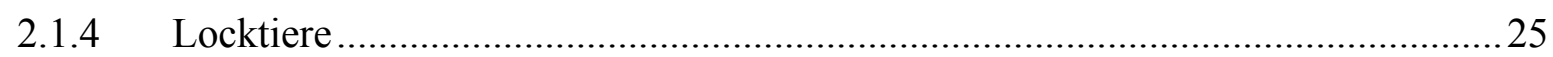

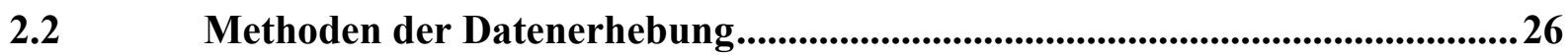

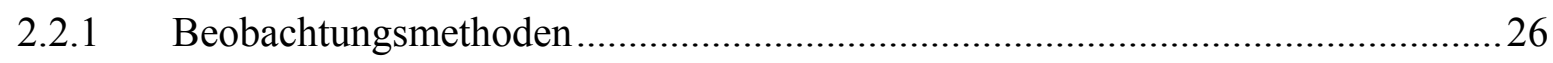

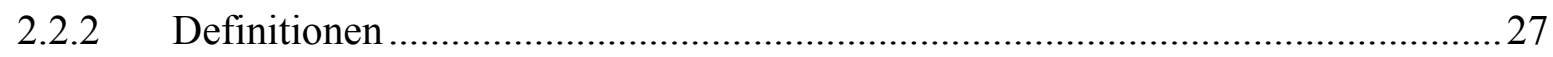

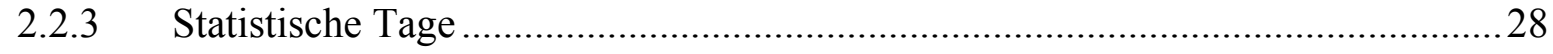

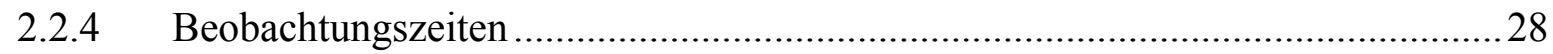

2.2.5 Beobachtungsperiode im ersten Beobachtungsjahr (2000) .................................29

2.2.6 Beobachtungsperiode im zweiten Beobachtungsjahr (2001) ...............................29

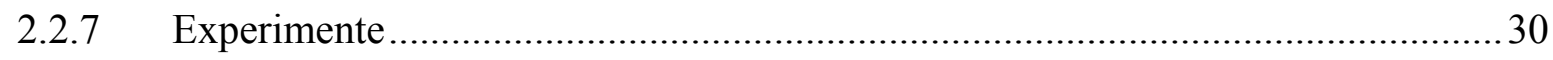

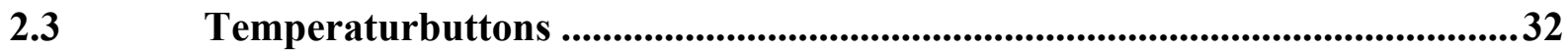

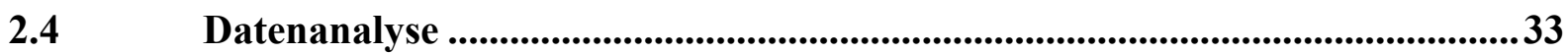


2.4.1 Zuordnung der Verhaltenseinheiten in Verhaltensbereiche ..................................33

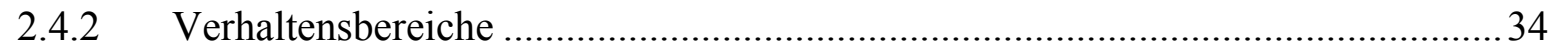

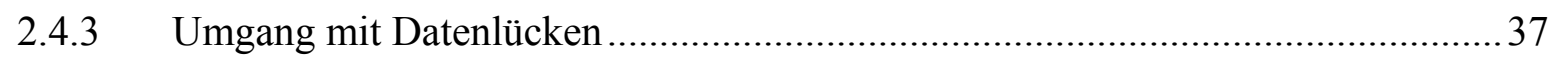

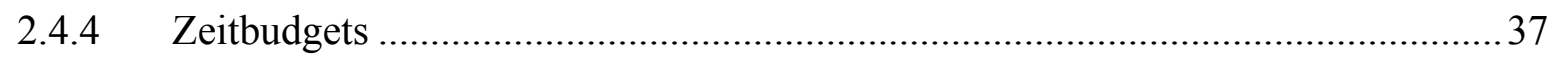

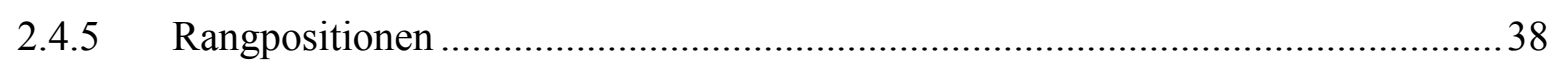

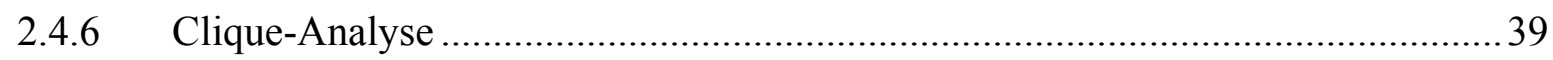

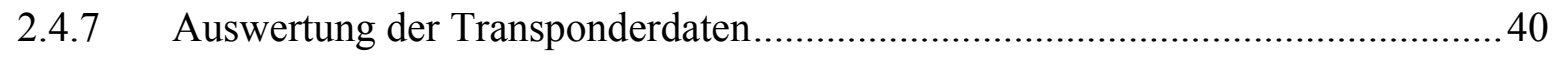

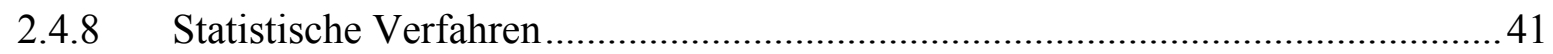

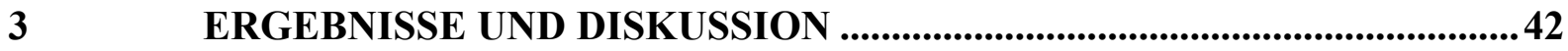

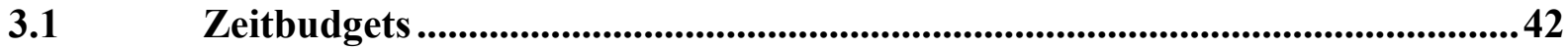

3.1.1 Zeitbudget der Familie im ersten Beobachtungsjahr ........................................ 42

3.1.2 Zeitbudget der Familie im zweiten Beobachtungsjahr ..................................... 43

3.1.3 Vergleich der Zeitbudgets der Familienmitglieder im ersten Beobachtungsjahr .. 45

3.1.4 Vergleich der Zeitbudgets der Familienmitglieder im zweiten Beobachtungsjahr48

3.1.5 Zeitbudgets der Familienmitglieder während der Experimente im ersten

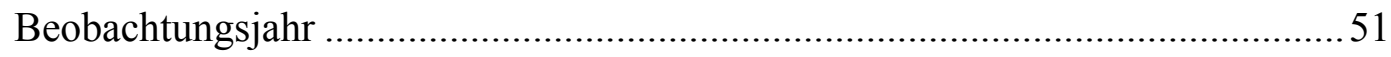

3.1.6 Zeitbudgets der Familienmitglieder während der Experimente im zweiten

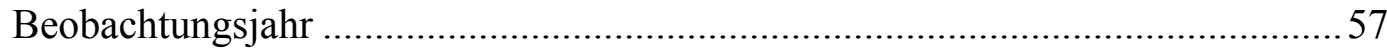

3.1.7 Qualitative Verhaltensunterschiede zwischen den Familienmitgliedern im ersten Beobachtungsjahr

3.1.8 Qualitative Verhaltensunterschiede zwischen den Familienmitgliedern im zweiten

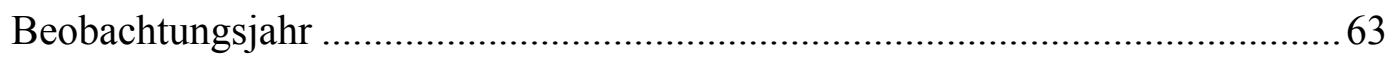

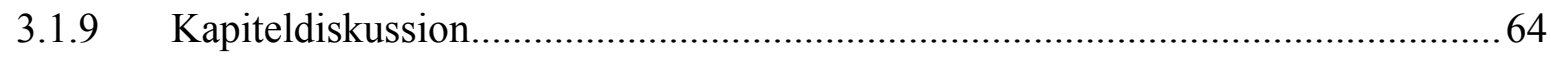

Emigrationen und Rauswürfe ..........................................................................66

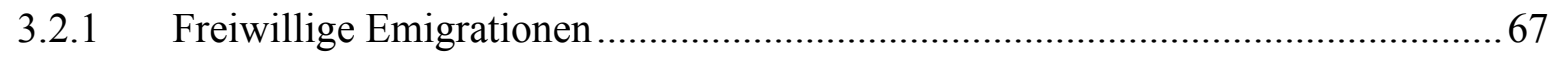

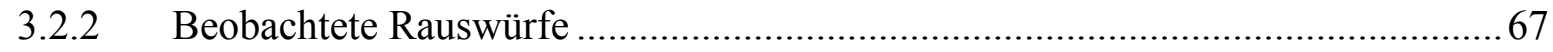

3.2.3 Rauswürfe in Abhängigkeit von individuen-externen Faktoren..........................69

3.2.4 Rauswürfe in Abhängigkeit von individuellen Faktoren.................................... 76

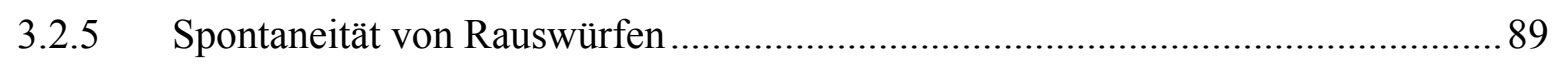

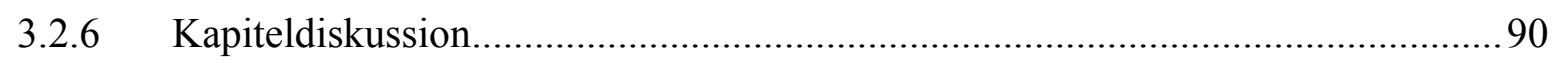


3.3 Untersuchungen zur Geschwister-Konkurrenz und zum Eltern-Nachkommen-

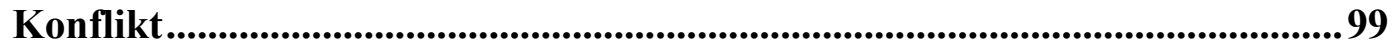

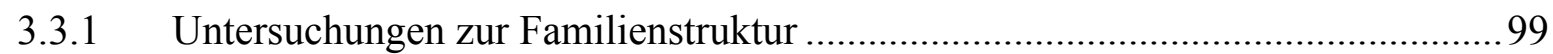

3.3.2 Einfluss der Locktiere auf die Beziehungen der Geschwister ............................. 113

3.3.3 Einfluss der Eltern auf die gruppendynamischen Prozesse ............................... 145

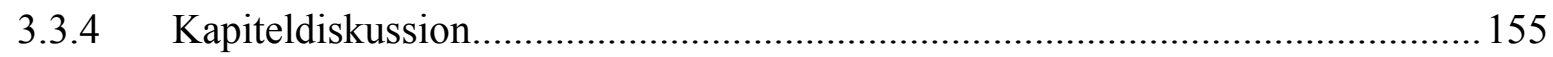

3.4 Untersuchungen zur Emigrationsneigung anhand der Aufenthaltsorte und räumlichen Assoziation der Familienmitglieder .................................................. 163

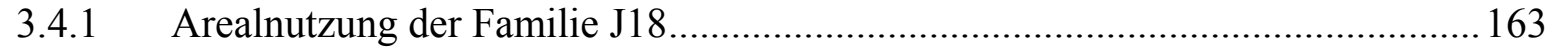

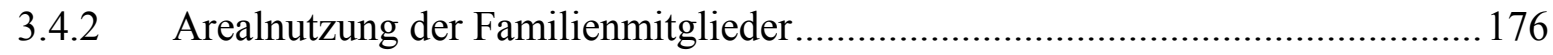

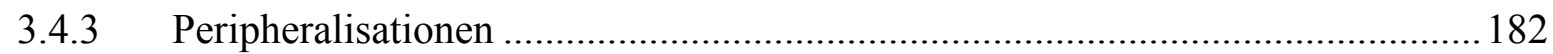

3.4.4 Zusammenhänge zwischen sozialen Faktoren und Peripheralisationen..............205

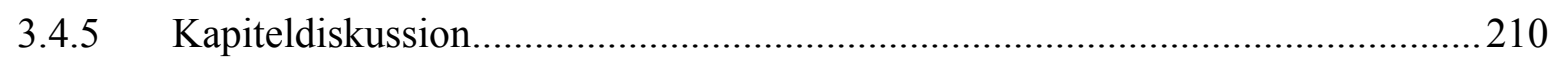

3.5 Überprüfung einzelner Modellkomponenten...................................................216

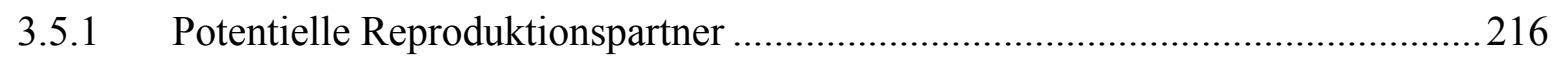

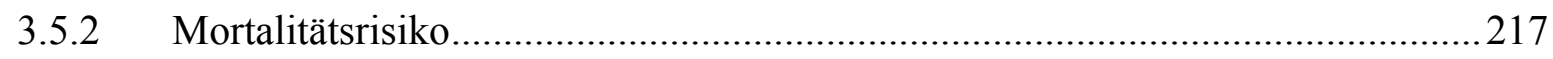

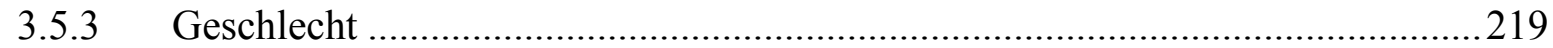

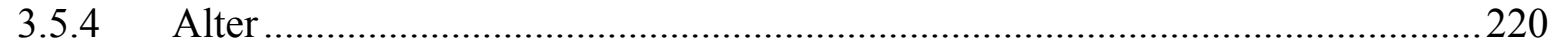

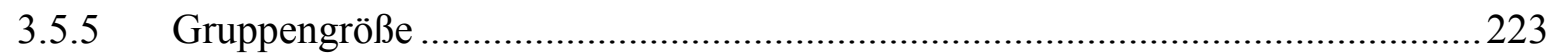

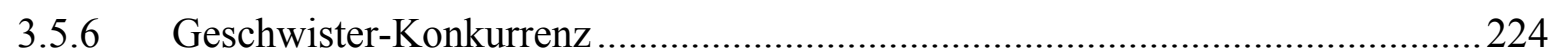

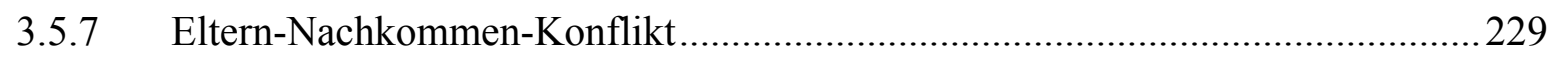

4 SCHLUSSFOLGERUNG UND AUSBLICK.............................................2232

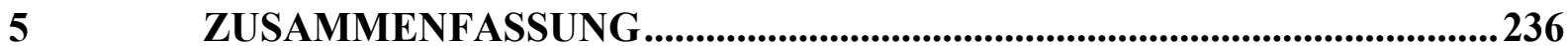

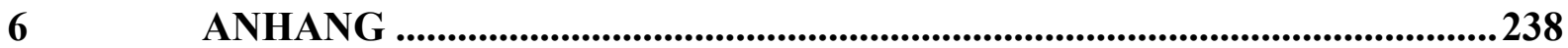

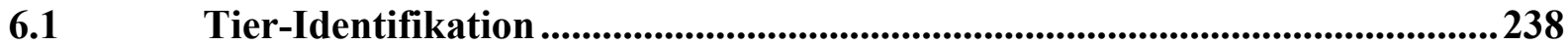

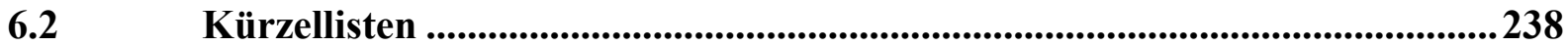

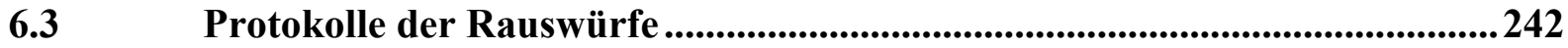

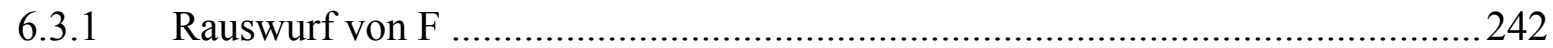

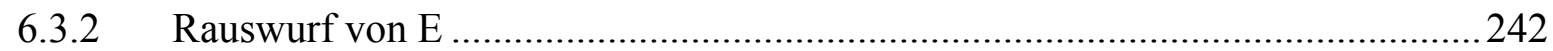




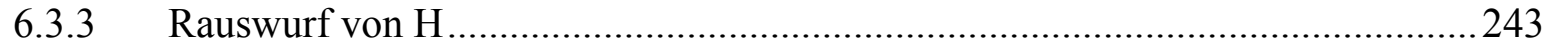

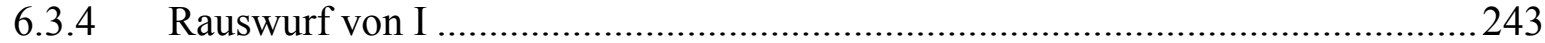

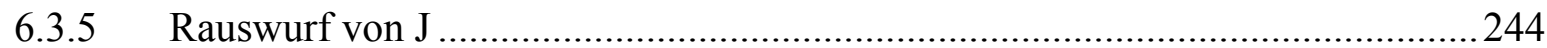

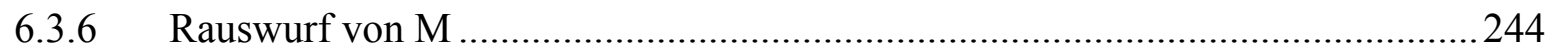

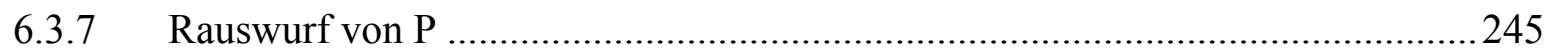

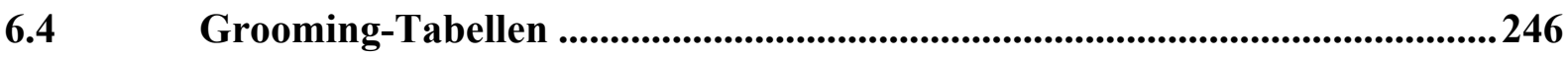

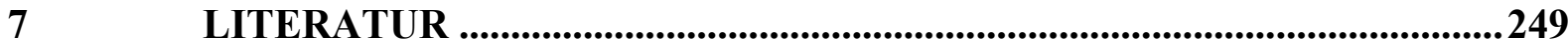

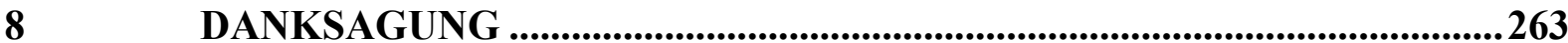

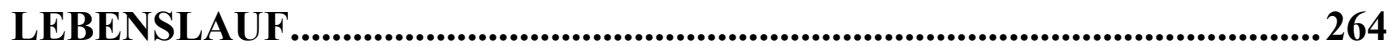




\section{EINLEITUNG}

\subsection{Soziale Organisation und Reproduktionssystem von Callithrix jacchus}

Weißbüschelaffen (Callithrix jacchus), die zur Familie der Krallenaffen (Callitrichidae) gehören und im nordöstlichen Brasilien beheimatet sind, leben in großen Familienverbänden (extended families sensu McGrew 1986), welche aus einem monogamen Elternpaar und ihren Nachkommen bestehen. Adulte Nachkommen verbleiben häufig mehrere Jahre in der Natalfamilie (Geburtsfamilie) und beteiligen sich als nicht-reproduktive Helfer an der Jungtieraufzucht. Das Alpha-Weibchen inhibiert in der Regel Ovulationen ihrer geschlechtsreifen Töchter (Abbott 1984, 1986, Hearn 1983), so dass sich meist nur das Alpha-Weibchen reproduziert. Das reproduktive Alpha-Männchen sichert seine Fortpflanzung vor den fertilen Söhnen durch aktives mate-guarding (Partnerbewachung) während des Östrus des AlphaWeibchens (Anzenberger 1983, Darms 1987, Jünemann 1990, Radespiel 1990, Rothe und Darms 1993, Soini 1987 für Cebuella). Die reproduktive Unterdrückung der geschlechtsreifen Nachkommen innerhalb der Natalfamilie wird als Strategie zur Vermeidung von Inzucht angesehen (z.B. Ralls et al. 1986). Inzwischen wird hypothetisiert, dass sexuelle und reproduktive Repression auch eine wichtige Funktion bei der Vermeidung geschwisterlicher Aggression übernimmt (Schaffner und Caine 2000).

Die vorwiegend aus Laborstudien gewonnenen Erkenntnisse über die Reproduktionssysteme der Marmosetten und Tamarine (Callitrichidae), gingen zunächst von einer strikten, reproduktiven Monogamie aus. Freilandstudien berichten jedoch von Callithrix-Gruppen, bei denen nicht nur die Alpha-Tiere an Kopulationen beteiligt waren (Ferrari 1988b, Ferrari and Lopes Ferrari 1989, Rylands 1982, 1986) oder in denen mehr als ein reproduktives Weibchen beobachtet werden konnten (Digby 1995a, 1999, Hubrecht 1989, Mendes Pontes und Monteiro da Cruz 1995, Scanlon et al. 1988). Price und McGrew (1991) veröffentlichten einen Überblick über die Reproduktionssysteme von Tamarinen (Saguinus oedipus) und Rothe und König (1991) stellten eine entsprechende Übersicht für die Reproduktionssysteme von Marmosetten (Callithrix jacchus) zusammen. Polyandrie, Polygynie, als auch Polygynandrie waren bei beiden Spezies, allerdings mit unterschiedlicher Häufigkeit, vertreten. Bei Abweichungen von der Monogamie entstanden bei Saguinus oedipus häufiger spontan polyandrische Systeme, während sich bei Callithrix jacchus öfter polygyne Gruppen fanden (z.B. Rothe und König 1987). In polyandrischen Systemen leben zwei oder mehr Männchen mit einem Weibchen zusammen, wobei jedes Männchen mit dem Weibchen kopuliert. 
Beteiligen sich die reproduktiven Männchen an der Jungtieraufzucht, wird von kooperativer Polyandrie gesprochen. Wenn Männchen mit einem oder mehreren Brüdern polyandrisch leben (fraternale kooperative Polyandrie sensu McGrew 1986) ist ihr genetischer Gewinn höher als in einer polyandrischen Gemeinschaft mit nicht-verwandten Männchen. Bei einer Gruppenneugründung von C. jacchus konnte fraternale kooperative Polyandrie in der Semifreilandstation Sennickerode beobachtet werden (Kretzer 1996). Nachdem das polyandrische System mehrere Monate stabil war, schloss das dominante Männchen die Brüder aus, und das nun monogame System des reproduktiven Paares erwies sich lange in dieser Form stabil. Die fraternale kooperative Polyandrie trat somit nur transitional auf (Rothe und Thieß 2001b).

Ferrari und Digby (1996) und Digby (1995a,b) erbrachten Nachweise für polygyne Reproduktionssysteme bei Marmosetten im Freiland. In mehreren C. jacchus-Gruppen waren zwei Weibchen reproduktiv, von denen sehr wahrscheinlich ein Weibchen die adulte Tochter des anderen Weibchens war (Ferrari und Digby 1996). Die beobachteten promisken Paarungen waren meistens inzestuös und daher nicht adaptiv (Ferrari und Lopes Ferrari 1989). Bei der Gattung Callithrix treten Abweichungen von monogamen Paar-Bindungen nur selten und damit weniger variabel als bei Tamarinen auf (Rothe und Darms 1993, Rothe und König 1991, Ferrari und Lopes Ferrari 1989). Nach rein soziographischen Gesichtspunkten leben Krallenaffensozietäten grundsätzlich polygynandrisch (Sussmann und Garber 1987, Stevenson und Rylands 1988, Ferrari und Lopes Ferrari 1989, Rothe und Darms 1993), da fast immer nicht-reproduktive Tiere beider Geschlechter in einer Gruppe anwesend sind.

Verglichen mit anderen Primatengattungen haben reproduktive Callithrix-Weibchen eine höhere energetische Belastung (z.B. Tardif 1994), da sie zwei Jungtiere mit hohem relativen Geburtsgewicht (z.B. Leutenegger 1980) und hoher postnataler Wachstumsrate (Wright 1984) tragen und säugen. Aufgrund eines Postpartum-Östrus wenige Tage nach der Geburt, können die laktierenden Weibchen erneut trächtig werden, so dass bei einer Trächtigkeitsphase von ca. 144 Tagen zwei Jungtierwürfe im Jahr aufgezogen werden können. Indirekte Belastung erfährt das reproduktive Weibchen durch eine anstrengendere Nahrungssuche (Oliveira et al. 1999, Rothe et al. 2003), eine erhöhte Immobilität (Clarke 1987, Terborgh \& Goldizen 1985, Goldizen 1987, Snowdon \& Soini 1988, Tardif \& Harrison 1990, Price 1992, Digby \& Barreto 1996, Sanchez et al. 1999), ein größeres Prädationsrisiko (z.B. Tardif 1994, Caine 1997), verringerte Wachsamkeit (Price 1992), als auch eine verkürzte Zeit für die Nahrungssuche (Terborgh \& Goldizen 1985, Goldizen 1987, Price 1992 für Saguinus; Lopes \& Ferrari 1994, 
Tardif 1994, Digby \& Barreto 1996 für C. jacchus). Infolge des zunehmenden Alters der Jungtiere kommt es z.T. zur Gewichtsreduktion des Alpha-Weibchens (Garber und Teaford 1986 für Saguinus, Goldizen et al. 1988 für S. fuscicollis, Sanchez et al. 1999 für S. oedipus).

Neben dem reproduktiven Männchen unterstützen die philopatrischen Nachkommen als nichtreproduktive Helfer die Mutter bei der Aufzucht der Geschwisterjungtiere (Garber et al. 1984, Price und Evans 1991, Rothe et al. 2003, Sussman \& Garber 1987, Terborgh \& Goldizen 1985). Ein solches Helfersystem (communal rearing system sensu Brown 1987, siehe auch Price und Evans 1991), das bei einigen anderen Säugetierarten wie z.B. Afrikanischen Wildhunden (Lycaon pictus: Malcolm und Marten 1982), Erdmännnchen (Suricatta suricatta: Clutton-Brock et al. 1999), Wölfen (Canis lupus: Packard et al. 1985) und Zwergmangusten (Helogale parvula: Rasa 1989) vorkommt, bildet unter den Mammalia jedoch eher eine Ausnahme. Etwas häufiger sind "Helfer-am-Nest-Syteme" bei Vögeln verbreitet, z.B. bei Blaubuschhähern (Aphalocoma caerulescens: Woolfenden und Fitzpatrick 1984) oder Graufischern (Ceryle rudis: Reyer 1980).

Bei Weißbüschelaffen, wie auch bei anderen Callitrichiden, beteiligen sich adulte Nachkommen neben der expliziten Jungtierfürsorge auch an aktiver Raubfeindvermeidung, der Sicherung des Streifgebietes und an der gemeinsamen Nahrungssuche. Für die reproduktiven Tiere liegt der Vorteil eines Helfersystems darin, dass (bis zu einem Grenzwert) mit zunehmender Anzahl der Helfer der Aufzuchtserfolg ansteigt (König und Rothe 1991, Rothe und Darms 1993, Schradin und Anzenberger 2001). Nachteile wären gegeben, wenn adulte Nachkommen mit den Eltern um die reproduktive Position konkurrieren oder wenn sie bei schlechten Habitatbedingungen zu Nahrungskonkurrenten werden (z.B. Rothe und König 1987, 1991, Ferrari und Diego 1992 für C. flaviceps). Die Gründe, weshalb es sich für die Nachkommen lohnt, in der elterlichen Familie zu bleiben, auf die eigene Fortpflanzung zu verzichten bzw. sie zu verschieben und sich als nicht-reproduktive Helfer nützlich zu machen, werden kontrovers diskutiert. Die „pay-to-stay“-Hypothese von Gaston (1978) besagt zum Beispiel, dass sich adulte Nachkommen als Konkurrenten der Eltern durch gruppendienliches Verhalten (Helfen) ihr Aufenthaltsrecht in der Natalfamilie erkaufen müssten. Da die Bereitschaft, Jungtiere zu tragen, bei nicht-reproduktiven Helfern allerdings auch zu erheblichen energetischen Kosten führt (Sanchez et al. 1996) und den Aufzuchtserfolg der reproduktiven Tiere steigern kann (Lucas et al. 1997), ist fraglich, ob von einem einseitig ausgerichteten Vorteil die Rede sein kann. Wenn die reproduktiven Tiere einen größeren Nutzen aus der Hilfeleistung ihrer adulten Nachkommen ziehen als Letztere, 
so müssten die Eltern den Nachkommen spätestens dann einen Anreiz geben, zu bleiben, wenn diese vorteilhafte Umweltbedingungen vorfinden, die ihnen die erfolgreiche Aufzucht eigener Nachkommen ermöglichen (Emlen 1982b, Keller und Reeve 1994).

Es wurde argumentiert, dass andere Vorteile der Philopatrie den Nachkommen den eigentlichen Nutzen bringen würden, während die Hilfeleistung bei der Jungtieraufzucht nur ein Nebeneffekt der Philopatrie sei (Emlen 1982a, Koenig und Pitelka 1981). So wäre Philopatrie vorteilhaft, wenn die adulten Nachkommen aufgrund einer hohen Populationsdichte keine freien Streifgebiete (dispersal sinks sensu Lidicker 1975) oder keine Reproduktionspartner finden würden. Ein hohes Mortalitätsrisiko migrierender Individuen durch Prädation oder Verhungern (Caine 1993, Cheney und Seyfarth 1983, Gaines und McClenaghan 1980, Goldizen und Terborgh 1989; Smith 1987, Waser 1988, Voland 2000) können außerdem ausschlaggebend sein für die Entstehung der philopatrischen Lebensweise. Individuen, welche die Abwanderung aus dem Natalgebiet aufschieben, wären im heimatlichen Gruppenverband und durch die Kenntnis von Versteck-, Schlaf- und Nahrungsplätzen einem geringeren Mortalitätsrisiko ausgesetzt als Emigranten. Nach der „benefits-of-philopatry“-Hypothese ${ }^{1}$ von Stacey und Ligon (1991) kann die Sättigung des Habitats durch Artgenossen allerdings keine Begründung für Philopatrie sein, da Arten, die nicht-kooperativ leben auch gesättigte Habitate vorfinden und trotzdem abwandern, um vorerst als Floater ${ }^{2}$ zu leben. Wäre das Mortalitätsrisiko von Emigranten allerdings derart hoch, dass die Überlebenswahrscheinlichkeit philopatrischer Nachkommen jene der Emigranten übersteigt, so könnte das Helferverhalten in der Tat eine Alternative zur eigenen Reproduktion darstellen, mit der in die indirekte Fitness investiert würde (,,making the best of a bad job“-Hypothese; Komdeur 1992, Überblick in Rothe und Darms 1993).

Mit Fitness wird die Fähigkeit eines Individuums beschrieben, sich in einer gegebenen Umwelt erfolgreich fortzupflanzen und damit die eigenen Gene im Genpool der Population dauerhaft zu etablieren. Die Gesamtfitness oder inklusive Fitness setzt sich aus der direkten Fitness (Anzahl eigener Nachkommen) und der indirekten Fitness (Verwandtenunterstützung) zusammen (Hamilton 1964). Besteht also für ein Individuum keine Möglichkeit durch eigene Reproduktion in die direkte Fitness zu investieren, kann es durch die Unterstützung von

\footnotetext{
${ }^{1}$ „benefits-of-philopatry“-Hypothese: Philopatrie ist eine Anpassung an variable Umweltbedingungen kooperativ lebender Arten, so dass der Fitnessgewinn von Nachkommen im Natalterritorium trotz Einbuße der eigenen Reproduktion höher ist, als wenn sie abwandern würden.

${ }^{2}$ Floater: Begriff für migrierende Individuen, die kein eigenes Streifgebiet haben und (noch) nicht in eine neue Sozietät integriert sind.
} 
Verwandten seine Gesamtfitness maximieren. Stellt man eine Kosten-Nutzen-Bilanz für die Fitnesserträge eines nicht-reproduktiven Helfers auf, so wird deutlich, dass mit der Aufzucht eines Geschwisters ein ebenso großer Anteil seiner Gene, nämlich 0,5, an die folgende Generation weitergegeben wird, wie mit der Aufzucht eines eigenen Nachkommen (Alexander 1974). Maynard-Smith (1964) prägte dafür den Begriff der Verwandtenselektion. Altruismus gegenüber Verwandten, ist deshalb als gen-egoistisches Verhalten anzusehen (Voland 2000). Tardif (1997) argumentierte, dass Individuen mit Helfererfahrung einen höheren Aufzuchtserfolg in der eigenen Jungtierfürsorge aufwiesen als solche ohne entsprechende Erfahrung. Rothe und Darms (1993) konnten derartige Zusammenhänge nicht bestätigen, fanden aber, dass das Erlernen sozialer Kompetenz (etwa in peer-groups) im juvenilen Alter maßgeblich für die erfolgreiche Jungtieraufzucht war.

Nachkommen des Elternpaares, die sich an der Aufzucht ihrer jüngeren Geschwister beteiligen, werden als primäre Helfer bezeichnet. Tiere, die nicht mit dem Elternpaar verwandt sind, aber bei der Jungtieraufzucht helfen, gelten als sekundäre Helfer und können mit der Hilfeleistung ihre indirekte Fitness nicht steigern, da sie mit den Jungtieren nicht verwandt sind (Krebs und Davies 1996). Reyer (1984) berechnete für männliche Graufischer (Ceryle rudis), die im ersten Lebensjahr primäre Helfer, sekundäre Helfer oder Aufschieber ${ }^{3}$ waren, die Gesamtfitness nach ihrer ersten eigenen Brut. Es stellte sich heraus, dass der Reproduktionserfolg der sekundären Helfer viermal höher war, als bei den Aufschiebern. Die Existenz sekundärer Helfer konnte in freilebenden Tamaringruppen nachgewiesen werden (Garber et al. 1984 und Goldizen 1987). Beobachtungen von Box (1977) und Ferrari (1988b) liefern Hinweise, dass hin und wieder auch in Callithrix-Sozietäten nicht-verwandte Tiere dem Alpha-Weibchen bei der Aufzucht ihrer Nachkommen helfen.

Beruht ein Sozialsystem auf engen Verwandtschaftsverhältnissen und altruistischem Verhalten, dann könnte sich die Einwanderung eines einzigen nicht-verwandten Individuums für alle beteiligten Sozietätsmitglieder nachteilig auswirken, da der Eindringling, abgesehen vom Erwerb der Helfererfahrung, kein Interesse an der Unterstützung nicht-verwandter Tiere hätte und beim Durchsetzen seiner Eigeninteressen potentiellen Konkurrenten schaden könnte (Darms 1987). Im Falle der Integration eines nicht-verwandten Artgenossen in die Familie, wird nicht mehr von einer Familie, sondern von einer Gruppe gesprochen (sensu McGrew 1986). Den reproduktiven Tieren gegenüber wäre ein nicht-verwandter gleichgeschlechtlicher

\footnotetext{
${ }^{3}$ Aufschieber: Tiere, die in ihrem ersten Lebensjahr nicht helfen und sich auch nicht reproduzierten.
} 
Artgenosse ein sexueller Konkurrent, während der Austausch eines der reproduktiven Tiere für nicht-reproduktive Nachkommen zu einem geringeren Verwandtschaftsgrad zu den neuen Nachkommengenerationen führen würde, wodurch somit ihre indirekte Fitness minimiert würde. Befunde aus Konfrontationsexperimenten über Reaktionen von C. jacchus auf familienfremde einzelne Artgenossen, machten Unterschiede zwischen verpaarten Männchen und Weibchen deutlich (Anzenberger 1983). Alpha-Weibchen sind ihrem Paarungspartner „treu“, während Alpha-Männchen sexuelle Kontakte mit fremden Weibchen eingingen. Die weitgehend geschlossene Familie ist also unter bestimmten Umständen durchlässig, zumindest für extra-group-copulations (siehe Digby 1999). Die Versuche, einzelne Individuen in bereits bestehende Familien $\mathrm{zu}$ integrieren, scheiterten meist an den dissoziativen Handlungen der Nachkommen gegenüber dem Eindringling (Darms 1987), was die Annahme der Verwandtenselektion bestärkt.

\subsection{Bleiben oder Gehen?}

Sobald ein Weißbüschelaffe innerhalb seiner Natalfamilie soziale Kompetenz erlernt und die Geschlechtsreife erreicht hat, sind zwei alternative Taktiken für ihn denkbar. Eine Taktik besteht darin, in der Natalfamilie zu bleiben, und die eigene Reproduktion aufzuschieben (Dunbar 1988, S. 27f). Die andere Taktik wäre die Emigration aus der Natalfamilie (nataldispersal) mit dem Ziel, selber Nachkommen zu produzieren (vgl. Emlen 1982b, Lucas et al. 1997). Die Entscheidung für eine der beiden Taktiken, wird von zahlreichen Faktoren beeinflusst (Abb. 1.1).

Der Begriff Entscheidung wird in der vorliegenden Arbeit als „...handelnder Vollzug selbst aufgefasst...“, welcher ,...sich in der Wahl eines bestimmten...Reaktionsmusters in einer mehrdeutigen Situation“ manifestiert (Fröhlich 2000, S.154). Die Entscheidung ist damit kein bewusst geplanter, gedanklicher Vorgang, sondern ist selbst die eine ausgeführte Handlung von mehreren Alternativen.

\subsubsection{Einflussfaktoren auf die Entscheidung über Philopatrie und Emigration}

- In dem monogamen Reproduktionssystem der Weißbüschelaffen werden die reproduktiven Aktivitäten aller Nachkommen von den Alpha-Tieren unterdrückt. Daraus ergibt sich für einen Nachkommen die Notwendigkeit, aus der Familie zu emigrieren, um in die direkte Fitness zu investieren. Dies hat somit einen Einfluss auf die Entscheidung über Philopatrie oder Emigration eines Individuums. 
- Die hohe energetische Belastung des Alpha-Weibchens wird durch die Entstehung eines Helfersystems reduziert, indem nicht-reproduktive Nachkommen die Aufzucht von Geschwistern unterstützen und damit (indirekte) Fitnessgewinne erzielen. Ob einem Nachkommen eine Helferposition zur Verfügung steht, hängt unter anderem von der Gruppengröße ab. Mit jedem erfolgreich aufgezogenen Jungtier nimmt die Zahl der nichtreproduktiven Helfer in einer Gruppe zu, was zu Konkurrenz unter den Geschwistern um Helferpositionen führen kann (König und Rothe 1991).

- Bei Callitrichiden beobachteten Hubrecht (1984), Rylands (1986) und Stevenson und Rylands (1988) maximale Gruppengrößen von 9 bis 13 Tieren. Ferrari und Digby (1996) berichten von Gruppen mit 9 bis 15 Individuen. Die optimale Gruppengröße entspricht, laut Rothe et al. (1993a) 8 bis 10 Mitgliedern (Jungtiere nicht mitgezählt) und damit 2 bis 4 adulten Helfern, denn sie fanden bei einer Anzahl von 10 bis 11 Gruppenmitgliedern den größten Aufzuchtserfolg. Eine Erhöhung der Gruppengröße über 10 bis 12 Mitglieder würde sich nach Ferrari und Digby (1996), Tardif et al. (1984) und Goldizen (1987) auf alle Gruppenmitglieder nachteilig auswirken. Chase (1980) vertrat die Ansicht, dass mit steigender Gruppengröße das Investment des Einzeltieres in die Jungtierfürsorge abnimmt. Dieser Zusammenhang konnte aber von König und Rothe (1991) nicht bestätigt werden, da in ihrer Studie die gesamte Trageleistung von 3 bis 6 Tieren bewältigt wurde. Demnach würde die Gruppengröße von den zu vergebenden Helferpositionen abhängen. Die Wahrscheinlichkeit, dass Tiere die Gruppe verlassen (müssen) steigt danach ab einer Gruppengröße von 8 bis 10 Tieren an und wird ab 13 Gruppenmitgliedern maximal. In der Anzahl der Helfer ausgedrückt, sind die Wahrscheinlichkeiten für Emigrationen also minimal, wenn weniger als zwei nicht-reproduktive Helfer und maximal, wenn mehr als vier nichtreproduktive Helfer in der Sozietät anwesend sind. Abhängig von den jeweiligen Umweltbedingungen muss eine gewisse Variabilität eingeräumt werden, denn Soini (1982 für Cebuella pygmaea) zeigte, dass mit abnehmender Ressourcenmenge und -qualität die Größe der vom Autor beobachteten Gruppen abnahmen. Eine Form der Größenregulation besteht im Ausschluss einzelner Tiere aus dem Gruppenverband (Rothe et al. 1986, Stevenson und Rylands 1988 und Darms 1989). Da mit jeder Geburt die Gruppengröße bei C. jacchus um ein bis zwei Mitglieder anwächst, ist zu erwarten, dass ab einer kritischen Gruppengröße um den Zeitpunkt einer Geburt Nachkommen hinausgedrängt werden. Die Gruppengröße bleibt damit stabil. 
- Die Gruppengröße steht zu dem Mortalitätsrisiko jedes einzelnen Familienmitgliedes im heimatlichen Streifgebiet in wechselseitiger Abhängigkeit. Die Mortalitätsrate von S. oedipus geoffroyi beträgt im Freiland z.B. 50\% (Dawson 1978) und angesichts der vielen Raubfeinde von Callitrichiden, wie Tayras (Eira barbara), Ozelote (Felis pardalis), Adler (Spizaetus ornatus), Falken (Micrastur ruficollis) und Schlangen (Eunectes murinus) (Dawson 1976, Heymann 1987, 1990, Vasquez und Heymann 2001, Shahuano Tello et al. 2002) wird von einem hohen Prädationsrisiko ausgegangen. Doch nicht nur direkte Beobachtungen der Prädation auf Callitrichiden, sondern auch das „Scannen“ (Def. im Anhang S. 241) als Ausdruck eines effektiven Wächtersystems lassen einen hohen Raubfeinddruck vermuten (Caine 1984, 1986, 1993, Bartecki und Heymann 1987, Buchanan-Smith 1990, Ferrari und Lopes Ferrai 1990, König 1992, vgl. Sherman 1985 für Belding-Ziesel und Rasa 1984 für Zwergmangusten). Mit steigender Gruppengröße kann durch das Wächtersystem das Mortalitätsrisiko verringert werden (Clutton-Brock et al. 1999 für Suricatta suricatta). Ferrari und Lopes Ferrari (1990) fanden bei C. flaviceps-Gruppen zum Beispiel, dass trotz eines hohen Prädationsrisikos keine Attacke eines Beutegreifers erfolgreich war und führen dies auf die große Anzahl der Gruppenmitglieder bei Marmosetten zurück (siehe auch Ferrari und Diego 1992, Rylands 1982 für C. humeralifer).

- Das Mortalitätsrisiko im heimatlichen Streifgebiet hat direkte Auswirkungen auf die Emigrationsentscheidung, da mit steigendem Mortalitätsrisiko die Vorteile, sich im heimatlichen Streifgebiet aufzuhalten, vermindert werden.

- Das Mortalitätsrisiko während einer Migration und in einem neuen Streifgebiet hat Auswirkungen auf die Entscheidung über Philopatrie oder Emigration. Ebenso beeinflusst die Wahrscheinlichkeit ein Streifgebiet und/oder einen Reproduktionspartner $\mathrm{zu}$ finden die Entscheidung. In der Literatur finden sich zumeist Angaben über die Häufigkeiten von Emigrationen, während die Lebensläufe der Emigranten nur selten weiter untersucht wurden (Digby und Ferrari 1994, Ferrari und Digby 1996, Goldizen und Terborgh 1989, Nievergelt et al. 2000, Savage et al. 1996, Scanlon et al. 1988). Digby und Barreto (1993) beobachteten jeweils mehrere Wochen lang drei emigrierende Weibchen, denen es nicht gelang, in eine Gruppe zu immigrieren bzw. eine neue Gruppe zu gründen. Allgemein gilt für Primaten, dass Immigranten in neuen Streifgebieten ca. sechs Monate lang einem größeren Mortalitätsrisiko unterliegen als philopatrische Tiere, da sie die Kenntnis von Futter-, Schlaf- und Versteckplätzen erst erwerben und den Aggressionen fremder Artgenossen widerstehen müssen (Isbell 1994). 


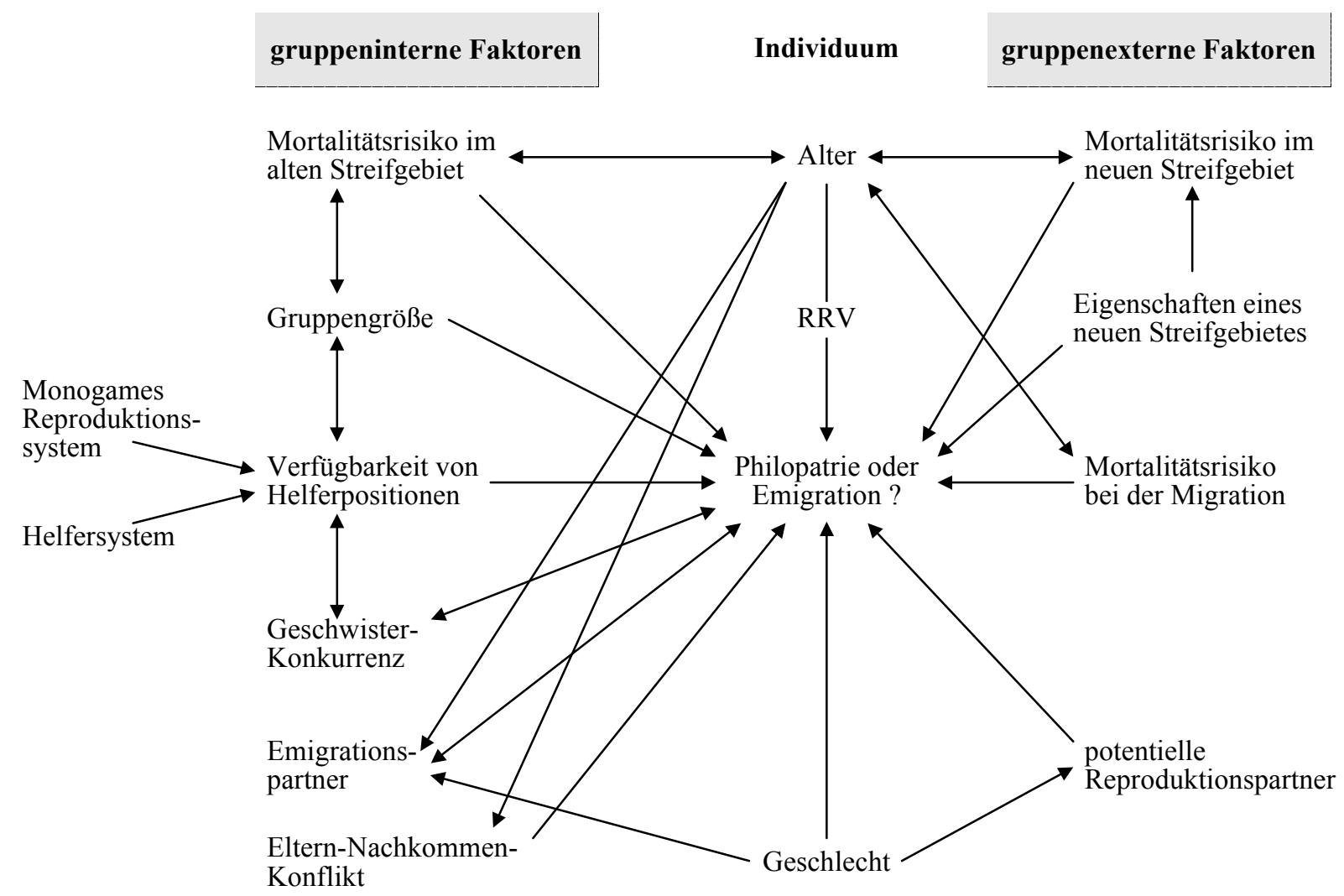

Abb. 1.1 Flussdiagramm über die Einflussfaktoren auf die Entscheidung zwischen Philopatrie und Emigration. Im Zentrum der Grafik befindet sich die Entscheidungsfrage: Philopatrie oder Emigration? Die auf sie zuführenden Pfeile stehen für die Auswirkungen der Faktoren auf die Entscheidung eines Individuums. Pfeile, die von der Frage wegführen, stehen für die Beeinflussung der Entscheidung eines Individuums auf die angeführten Faktoren. Über und unter der Entscheidungsfrage befinden sich die Life-History-Parameter des Individuums, sein Alter und sein Geschlecht. Sie haben z.T. Auswirkungen auf die Faktoren und damit indirekt auf die Entscheidungsfrage. Links sind gruppeninterne Faktoren aufgelistet, d.h. Größen, die auf das Individuum einwirken, solange es sich in der Natalfamilie aufhält und rechts gruppenexterne Faktoren, d.h. Größen, welche auf das Individuum einwirken werden, wenn es die Natalfamilie verlassen hat und damit Einfluss auf die Entscheidung nehmen. Das Diagramm ist folgendermaßen zu lesen: Das monogame Reproduktionssystem und die damit einhergehende reproduktive Unterdrückung der adulten Nachkommen ist eine Voraussetzung für die Notwendigkeit einer Emigration aus der Natalfamilie, wenn in direkte Fitness investiert werden soll. Die energetische Belastung des Alpha-Weibchens bei der Jungtieraufzucht (durch Zwillinge, hohes relatives Geburtsgewicht, Laktation, Postpartum-Östrus) macht ein Helfersystem notwendig und hat damit auch einen Einfluss auf die Verfügbarkeit von Helferpositionen. Letztere ist zudem von der Gruppengröße abhängig, da mit steigender Gruppengröße die Konkurrenz um Helferpositionen zunehmen und zur Geschwister-Konkurrenz führen kann. Die Gruppengröße steht in wechselseitiger Beeinflussung zum Mortalitätsrisiko im Streifgebiet der Natalfamilie und hängt von der Anzahl der Tiere ab, die aus der Familie abwandern, bzw. bleiben. Das Mortalitätsrisiko hat ebenfalls Auswirkungen auf die Entscheidung über Philopatrie und Emigration, denn je größer das Risiko ist, zu sterben, desto größer ist die Wahrscheinlichkeit, dass ein Individuum die Familie verlässt. Das Mortalitätsrisiko hängt außerdem mit dem Alter des Individuums zusammen; sehr junge und alte Tiere sind einem größeren Risiko ausgesetzt. Ein hohes Sterblichkeitsrisiko mindert wiederum die Wahrscheinlichkeit, älter zu werden. Das Alter beeinflusst den Eltern-Nachkommen-Konflikt und bestimmt den Restreproduktionswert (RRV), der einen Einfluss auf den Zeitpunkt der Entscheidung hat. Das Alter hat außerdem Auswirkungen auf das Mortalitätsrisiko von migrierenden Tieren, als auch von Individuen, welche erfolgreich in ein neues Streifgebiet immigriert sind. Je nach Höhe des jeweiligen Mortalitätsrisikos wird auch die Entscheidung über Philopatrie und Emigration beeinflusst. Das Mortalitätsrisiko wird von den ökologischen Bedingungen des Habitats, in dem das Streifgebiet liegt, mit bestimmt. Die Auswirkungen des Geschlechts auf die Entscheidung sollte unter anderem von der Anwesenheit potentieller Reproduktionspartner abhängen. Die Wahrscheinlichkeit, einen Emigrationspartner zu finden, hängt vom Alter und Geschlecht des Individuums ab. 
- Eine Strategie zur Minimierung des Prädationsrisikos kann die gemeinsame Emigration eines Individuums mit einem Emigrationspartner sein (Baker 1991, Garber 1994, Kretzer 1996, Pusey und Packer 1987, Sugiyama 1976). Brüder können nach einer Co-Emigration in fraternaler Polyandrie leben. Die von Rothe und Thieß (2001b) aus dem Semifreiland beobachtete temporäre fraternale Polyandrie resultierte jedoch darin, dass das dominante Männchen seine beiden Brüder nacheinander verjagte und den Nachwuchs in einem monogamen Paarsystem aufzog. Die Rekrutierung eines Emigrationspartners kann also für das Überleben und die Neubildung einer Gruppe nützlich, für subordinierte Tiere aber auch risikoreich sein. Denn der Anschluss an ein ranghöheres und damit meist älteres Geschwister würde zwar bedeuten, von dessen Erfahrungen zu profitieren, aber auch, selber im Falle einer Gruppenneubildung nur in die indirekte Fitness investieren zu können oder gar vertrieben zu werden (siehe auch Terborgh und Goldizen 1985; Siess 1988). Wie groß die Wahrscheinlichkeit für ein jüngeres Geschwister ist, sich an der Reproduktion zu beteiligen oder in die Alpha-Position aufzusteigen, muss noch geklärt werden. Erfolgversprechender erscheint die Gewinnung eines jüngeren Geschwisters, da so der Vorteil einer gemeinsamen Emigration und außerdem die Sicherung der Alpha-Position bei einer Gruppenneugründung wahrscheinlicher wären (Siess 1988; Rothe und Thieß 2001b).

- Generell haben auch die Eigenschaften des Habitats, in dem sich das Streifgebiet befindet, Auswirkungen auf das Mortalitätsrisiko eines Tieres. Abhängig von den Nahrungsbedingungen, Versteck- und Schlafplätzen, die das Streifgebiet bietet, sinkt oder steigt das Überlebensrisiko eines Individuums. Die Nahrungsbedingungen haben auch einen direkten Einfluss auf die Streifgebietsgröße. Denn je weniger Nahrungsressourcen in dem Lebensraum vorhanden sind, umso größer muss das Streifgebiet ausfallen, damit das Individuum genug Nahrung finden kann. Da Vertreter des Genus Callithrix an die Nutzung von Pflanzensäften angepasst sind, sind sie in der Lage, in relativ kleinen Streifgebieten zu leben (Rylands 1996, Ferrari und Lopes Ferrari 1989, Maier et al. 1982, siehe Soini 1982, 1988 für Cebuella).

- In der Lebensspanne eines Tieres ist das Alter ein wesentlicher Faktor, wenn es um die Fragen geht, wann sich ein Tier zum ersten Mal fortpflanzt, wie viele Junge es zur Welt bringt und wie viel Energie es in die Aufzucht der Nachkommen investiert (Ross und Jones 1999, Barash 1980). Eine häufig genutzte Größe zur Charakterisierung der Life-History ist der Reproduktionswert, mit dem der Beitrag von Individuen verschiedener Altersklassen zum Populationswachstum gewichtet wird (Goodman 1982). Da die Reproduktion für Individuen auch Kosten verursacht, unterschied Williams (1966) zwischen der aktuellen und der zu 
erwartenden zukünftigen Reproduktion einer bestimmten Altersklasse (Restreproduktionswert $=\mathrm{RRV})$. Der Restreproduktionswert kann damit Auswirkungen auf den Zeitpunkt einer Emigration haben (mehr Details in 3.5.4). Von dem Alter eines Tieres hängt sein jeweiliges Mortalitätsrisiko ab, da die Sterblichkeit von jungen und alten Individuen generell höher ist als von Tieren mittleren Alters (Ross und Jones 1999, Stearns 1992).

- In vielen Primatenarten verlassen nur männliche oder nur weibliche Nachkommen das Geburtsgebiet, um sich mit nichtverwandten Artgenossen fortzupflanzen (Pusey \& Packer 1987). Beispielsweise emigrieren bei Makaken, Languren und Meerkatzen nur männliche Nachkommen (Colvin 1983, Hrdy 1977, Henzi \& Lucas 1980). Bei Krallenaffen-Arten sind Migrationen beider Geschlechter zu beobachten (Ferrari und Digby 1996, Mendes Pontes und Monteiro da Cruz 1995, Ferrari 1988b für Callithrix; Dawson 1978, Neyman 1978 für Saguinus). Das Mortalitätsrisiko während Migrationen sollte bei Krallenaffen für beide Geschlechter gleich groß sein, da kein signifikanter Sexualdimorphismus besteht und die physiologischen Ansprüche (Nahrungsbedarf, Umgebungstemperatur etc.) beider Geschlechter mit Ausnahme des reproduktiven Weibchens einander ähneln (Dawson 1976 für S. oedipus). Obwohl Weibchen in der Regel etwas größer und schwerer als Männchen sind, fand Dawson (1976) bei S. oedipus keine Unterschiede im Prädationsrisiko. Bisher konnte auch bei Marmosetten keine unterschiedlich hohe Sterblichkeit zwischen den Geschlechtern nachgewiesen werden (Hubrecht 1984, 1985, Stevenson und Rylands 1988, Scanlon et al. 1988, Rothe et al. 1992 für C. jacchus in Laborhaltung, Soini 1982 für Cebuella). Das Geschlecht des Individuums beeinflusst die Entscheidung über Philopatrie und Emigration indirekt, indem das Geschlechterverhältnis für den Aufzuchtserfolg einer Gruppe von Bedeutung sein kann (König 1995, Garber 1984 für S. mystax). Das Geschlecht hat außerdem Auswirkungen darauf, ob Geschwister zu Emigrationspartnern rekrutiert und Reproduktionspartner gefunden werden können.

- Das Geschlechterverhältnis hat möglicherweise Auswirkungen darauf, welches Individuum sich für Philopatrie und welches sich für eine Emigration entscheidet, da mit steigender Anzahl der Männchen innerhalb der Gruppe der Aufzuchtserfolg steigt (König 1995). Durch Abwanderungen kann sich das Geschlechterverhältnis ändern.

- Der Eltern-Nachkommen-Konflikt und die Geschwister-Konkurrenz sind Größen, die durch Interaktionen zwischen den Familienmitgliedern deutlich werden. Beide können auf die Emigrationsentscheidung Einfluss nehmen. In Kapitel 1.3 wird auf diese Aspekte näher eingegangen. 


\subsubsection{Entscheidungsebenen}

Bei Callithrix-Gruppen wurde unter Laborbedingungen häufig beobachtet, dass Individuen durch dissoziative Handlungen einzelner oder aller Gruppenmitglieder aus der Gruppe ausgeschlossen wurden (Darms 1989, König und Rothe 1991, Rothe et al. 1986, Rothe und König 1987, eigene Beobachtungen für C. jacchus, McGrew und McLuckie 1986 und McGrew 1997 für S. oedipus). Die Entscheidung über Philopatrie und Emigration ${ }^{4}$ eines Individuums kann also durchaus auch von der Gruppe oder einzelnen Gruppenmitgliedern getroffen werden und findet daher auf zwei Ebenen statt, nämlich auf der Individuen-Ebene und auf der GruppenEbene (Abb. 1.2).

Das Individuum kann zwischen Philopatrie und der freiwilligen Emigration wählen. Fällt die Entscheidung auf Philopatrie, so trifft die Gruppe zeitgleich eine Entscheidung, ob das Individuum toleriert wird oder nicht. Wird es toleriert, kann das Individuum in der Natalgruppe verbleiben. Entscheidet sich die Gruppe, das Individuum nicht mehr als Mitglied zu akzeptieren, kann es zu einem Rauswurf kommen. Die zu Rauswürfen hinführenden Prozesse und initialen Auslöser sind bisher jedoch weitgehend ungeklärt (Darms 1987, Digby 1995b, Ferrari 1988b, Rothe und Darms 1993; Stevenson und Rylands 1988, Baker 1987 für L. rosalia, McGrew 1997 für S. oedipus). Epple (1967) und Kleiman (1981) sehen die Verantwortlichkeit für Rauswürfe bei den Eltern, während Rothe (1978) den Geschwistern des betroffenen Individuums eine besondere Rolle bei gruppendynamischen Prozessen einräumt. Anzenberger (1983) und Spichiger-Carlsson (1982) vermuten einen kombinierten Mechanismus, indem ein Elter den Rauswurf eines gleichgeschlechtlichen Nachkommen initiiert und der Rauswurf selber von einem Geschwister durchgeführt wird.

Das „Interesse“ der Sozietät ist die Aufrechterhaltung eines funktionierenden Helfersystems. Nach Darms (1987) sind die Sozietätsmitglieder keinem starren Verhaltensprogramm unterworfen und ,..die Aufrechterhaltung des Fließgleichgewichtes -Sozietät- kann hinsichtlich der Gruppengröße, Altersstruktur und Geschlechterrelation innerhalb gewisser Toleranzgrenzen erfolgen“. Möglicherweise lässt sich eine Emigration nicht nur als eine rein freiwillige oder eine durch Gruppenmitglieder hervorgerufene Entscheidung auffassen, denn die Interessen des Einzelnen stehen in Abhängigkeit zu denen der Gruppe, so dass der Emigrationsprozess beschleunigt oder verlangsamt werden kann. Trifft das Individuum seine

\footnotetext{
${ }^{4}$ Unter Laborbedingungen können keine Aussagen zur Spontaneität von Emigrationen gemacht werden, da die Individuen sich nicht freiwillig aus dem Streifgebiet der Natalfamilie entfernen können.
} 
Entscheidung für die freiwillige Emigration, so könnten Gruppenmitglieder durch Rückhaltemechanismen versuchen, auf die Entscheidung des Individuums Einfluss zu nehmen. Entscheidungen unterliegen daher immer einer hierarchischen Ordnung, denn der Entscheidung, zu helfen, muss die Entscheidung, philopatrisch zu leben, als auch die Entscheidung der Gruppenmitglieder, ein Individuum in der Gruppe zu tolerieren, vorausgehen.
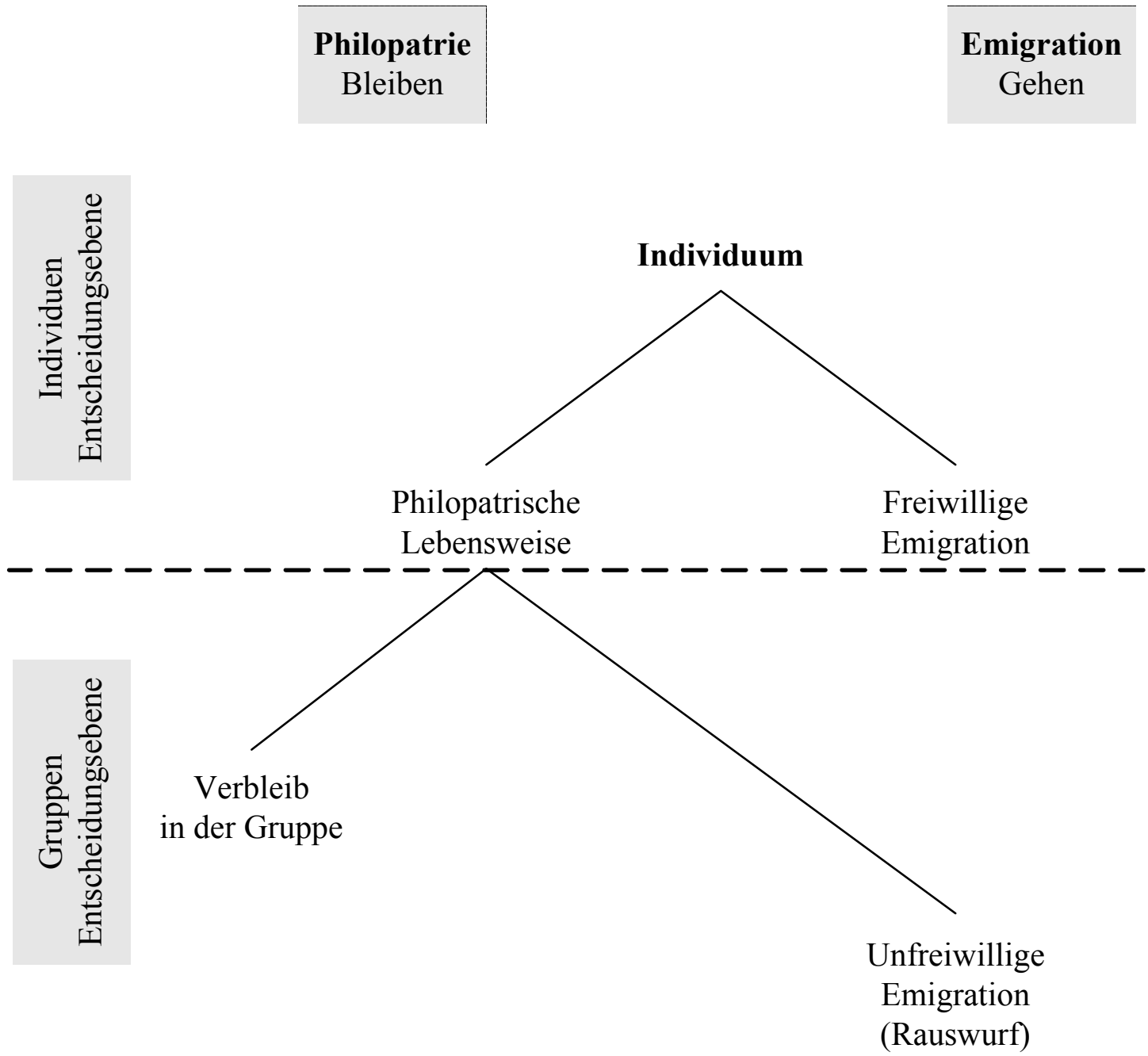

Abb. 1.2 Individuen-Entscheidungsebene und Gruppen-Entscheidungsebene bei der Entscheidung über Philopatrie und Emigration bei $C$. jacchus. Das Individuum (nicht-reproduktiver Nachkomme) trifft eine Entscheidung für eine philopatrische Lebensweise (links) oder für eine freiwillige Emigration (rechts) aus der Natalgruppe heraus. Seine Entscheidung wird von der Gruppe beeinflusst, indem auf der Gruppen-Entscheidungsebene entschieden wird, ob das Individuum verbleiben darf (links) oder rausgeworfen wird (rechts). Die Entscheidung auf der Gruppenebene geschieht jedoch nicht nach, sondern wird zeitgleich zur Entscheidung auf der Individuenebene gefällt.

Unter Laborbedingungen kann ein Tier vor den dissoziativen Handlungen der Gruppenmitglieder nicht fliehen und ist ihnen hilflos ausgeliefert, so dass es unter Umständen von der Gruppe entfernt werden muss. Außerdem macht die Käfighaltung eine freiwillige Emigration 
unmöglich. Für die Interpretation von Emigrationen oder Rauswürfen im Freiland fehlt jedoch häufig die Kenntnis der Verwandtschaftsverhältnisse der Gruppenmitglieder (Digby und Barreto 1993). Der große Vorteil der Semifreilandhaltung für diese Fragestellung zeigt sich nun darin, dass ein Gruppenmitglied die Möglichkeit hat, vor seinen Angreifern zu fliehen, als auch freiwillig das Streifgebiet der Sozietät $\mathrm{zu}$ verlassen, so dass beide extremen Ausprägungsformen des natal-dispersal den Nachkommen damit ermöglicht werden. Ein weiterer Vorteil besteht in der genauen Kenntnis der verwandtschaftlichen Verhältnisse und Altersstrukturen der Gruppen, als auch in der Möglichkeit, experimentell Variablen zu kontrollieren (vgl. Gaines und McClenaghan 1980).

Rauswürfe, die für den Beobachter meist spontan geschehen, können lange vor dem eigentlichen Ausschluss des Tieres verdeckt, d.h. für den Beobachter nicht wahrnehmbar, vorbereitet werden, so dass häufig erst nach einem Rauswurf retrospektiv die proximaten Ursachen (kausalen Mechanismen) für einen Rauswurf rekonstruiert werden müssen. Sind die Auslöser eines Rauswurfes erkannt und geklärt, kann letztendlich seine ultimate Funktion, also sein Überlebenswert diskutiert werden (Krebs und Davies 1996).

\subsection{Spieltheoretische Betrachtung der Analyse von Entscheidungen}

Die Entscheidungsalternativen des Flussdiagramms in Abb.1.1, nämlich Philopatrie und Emigration, konnten beide in Weißbüschelaffensozietäten beobachtet werden. Warum bei $C$. jacchus ein Nachkomme mehrere Jahre innerhalb der Natalgruppe bleibt, während ein anderer emigriert oder rausgeworfen wird, konnte bisher nicht ausreichend geklärt werden. Eine Möglichkeit zur Analyse von strategischen Entscheidungssituationen bietet die Spieltheorie. Ihre Lösungskonzepte können angewendet werden, wenn die Entscheidung eines Individuums von der Entscheidung anderer Individuen abhängig ist und sich alle Entscheidungsträger dieser wechselseitigen Abhängigkeit (Interdependenz) bewusst sind. Von Neumann und Morgenstern (1944) entwickelten die Spieltheorie ursprünglich für Entscheidungsfragen der Ökonomie ${ }^{5}$. Eine Entscheidungssituation kann nach dieser Theorie in der Form eines Spieles beschrieben werden, in denen die Spieler (Entscheidungsträger) bestimmten Spielregeln unterworfen sind. Es gibt eine festgelegte Menge an möglichen Strategiekombinationen und die dazugehörigen Auszahlungen für die jeweiligen Spieler. In Lösungskonzepten kann

\footnotetext{
${ }^{5}$ Die Spieltheorie wird auch als ökonomische Entscheidungstheorie bezeichnet (Amann 1999)
} 
erarbeitet werden, welche Strategie ein Spieler wählen sollte, um eine möglichst hohe Auszahlung zu erhalten (Holler und Illing 1999).

Die evolutionäre Spieltheorie, erstmals zusammenfassend dargestellt von Maynard Smith (1982), unterscheidet sich von der klassischen Spieltheorie nach von Neumann und Morgenstern (1944) darin, dass keine bewussten strategischen Entscheidungen von den Spielern erwartet werden. Weil vorausgesetzt wird, dass der Spieler keine bewusste Entscheidung trifft, kann er auch mit der Strategie, die er verkörpert, gleichgesetzt werden. Der Erfolg einer Strategie (s) drückt sich in der Fitness aller Spieler aus, welche diese Strategie (s) verfolgen. Indem retrospektiv das Wachstum der Population von Spielern mit der Strategie (s) untersucht wird, kann der Erfolg der Strategie (s) beurteilt werden (Holler und Illing 1999).

Ein Lösungskonzept, das von Maynard Smith und Price (1973) entwickelt wurde, ist die evolutionsstabile Strategie (ESS). Eine ESS herrscht z.B. dann, wenn alle Mitglieder einer Population dieselbe Strategie verfolgen und sich keine neu hinzukommende Strategie (z.B. durch Mutation) in der Population ausbreiten kann. Es gibt jedoch auch Strategien, die nur dann erfolgreich sein können, wenn sie sich im Umfeld anderer Strategien befinden. Dieser Zusammenhang soll kurz am Beispiel der Produzenten und Schnorrer erläutert werden. Es wird vorausgesetzt, dass Produzenten sich selber ihre Nahrung suchen und niemals Ressourcen anderer Individuen nutzen, während Schnorrer nie selber Nahrung suchen und immer die Ressourcen der Produzenten ausbeuten. Eine reine Schnorrerpopulation könnte nicht existieren, da es keine Produzenten zum ausbeuten gäbe. Befinden sich in einer Population nur Produzenten, so ist sie jedoch immer anfällig für die Ausbreitung von Schnorrern, so dass eine reine Produzentenpopulation in der Natur nur selten vorkommt. Die beiden Strategien existieren daher in einem Gleichgewicht (Giraldeau und Livoreil 1998). Gibt es mindestens zwei alternative Strategien innerhalb einer Population, ist die Population polymorph. Manifestieren sich die Strategien im Verhalten von Organismen, wird von einem ethologischen Polymorphismus gesprochen (Voland 2000). Der Erfolg einer Strategie hängt dabei von ihrer Häufigkeit innerhalb der Population ab. Je mehr Schnorrer sich in einer Population befinden, umso geringer wird die Ausbeute der Schnorrer sein, weil der Anteil an Produzenten immer weniger wird. An diesem Punkt wird der Unterschied der evolutionären Spieltheorie zu einfachen Optimalitätsmodellen (z.B. optimal foraging theory: Charnov 1976, Pulliam 1974) deutlich, da letztere frequenzunabhängig sind (Dugatkin und Reeve 1998).

Ein umfangreiches Modell zur Entscheidung über Philopatrie und Emigration stellen Lucas et al. (1997) für Helogale parvula vor, welches jedoch für C. jacchus in der bestehenden Form 
nicht anwendbar ist, da die Möglichkeit durch andere Gruppenmitglieder aus der Gruppe geworfen zu werden, in diesem Modell nicht berücksichtigt wird. Der Einfluss der Taktiken der anderen Gruppenmitglieder auf die optimale Entscheidung wird in dem Modell 1.2 dieser Arbeit miteinbezogen und im Folgenden anhand der Geschwister-Konkurrenz und des ElternNachkommen-Konfliktes ${ }^{6}$ dargestellt.

\subsubsection{Geschwister-Konkurrenz}

Viele Modelle sehen die Ursache der Philopatrie in ungünstigen Umweltbedingungen (z.B. Habitatsättigung, Migrationsrisiken etc.) und vernachlässigen häufig den Einfluss sozialer Faktoren innerhalb der Sozietät eines Individuums auf die Entscheidung über Philopatrie und Emigration, wie z.B. die Geschwister-Konkurrenz. Rauswürfe in Krallenaffensozietäten werden häufig von gleichgeschlechtlichen Geschwistern initiiert und häufig auch durchgeführt (Rothe 1975, Snowdon und Pickhard 1999, Rothe, pers. Mtlg.). Mehrere Studien belegen jedoch auch, dass junge Gruppenmitglieder von ihren Eltern ausgestoßen wurden (Baker 1987 für Leontopithecus rosalia, McGrew und McLuckie 1986 bei Saguinus oedipus, Rothe pers. Mtlg. für C. jacchus, Soini 1988 für Cebuella pygmaea). Dissoziative Interaktionen von Eltern und Nachkommen werden im nächsten Abschnitt noch ausführlich behandelt. Generell sind bei Krallenaffen die Dominanzverhältnisse infolge der Seniorität bereits geklärt (Sutcliffe und Poole 1984). Ein älteres Gruppenmitglied dominiert ein jüngeres. Zwillinge (twins) haben dieselbe Seniorität, so dass die Dominanzverhältnisse im Alter von 5-10 Monaten häufig in einem twin-fight ausgetragen werden. Es gibt Hinweise darauf, dass später häufig jene Tiere rausgeworfen wurden, die den twin-fight verloren hatten (Sutcliffe und Poole 1984, Rothe und Darms 1993). Dies stützt die Vermutung, dass dominante Gruppenmitglieder subordinierte aus der Gruppe werfen, also die Rauswürfe hierarchisch abwärts geschehen (McGrew und McLuckie 1984, Snowdon und Pickhard 1999 für S. oedipus). Bei C. jacchus treten allerdings auch Rauswürfe hierarchisch aufwärts auf (Rothe pers. Mtlg., eigene Beobachtungen).

\footnotetext{
6 Die beiden Begriffe Konkurrenz und Konflikt werden in der Literatur oft nur unzureichend bzw. recht weitreichend definiert. Konkurrenz und Konflikte tatsächlich nachzuweisen ist problematisch, da ihr Nachweis auf der Interpretation bestimmter Verhaltensweisen (z.B. dissoziativen Handlungen) aufbaut, und somit das Wissen um die Handlungsmotivation der Individuen vorausgesetzt wird, welches nach dem bisherigen Stand der Forschung nicht vollständig vorliegt. Die Analysen in dieser Arbeit können daher nur Hinweise auf mögliche Konkurrenz oder Konflikte innerhalb der untersuchten Krallenaffensozietät liefern. Im Folgenden werden diese Begriffe benutzt, da sie am ehesten die unterschiedlichen Interessen der Familienmitglieder beschreiben, die für diese Studie relevant sind.
} 
Wenn Rauswürfe durch Geschwister ein Ausdruck von Konkurrenz um den Verbleib im heimatlichen Streifgebiet sind, kann gefolgert werden, dass Philopatrie anstelle einer Emigration bevorzugt wird (siehe auch Ekman et al. 2002, Stacey und Ligon 1991). Es wurde deshalb erwartet, dass der Verbleib in der Natalfamilie adulten Nachkommen Vorteile bietet. Verspätete Emigrationen könnten demnach eine Taktik zur Optimierung des zukünftigen Reproduktionserfolges sein (Kokko und Johnstone 1999). Dominante Tiere können von diesen Vorteilen profitieren, wenn sie subordinierte Gruppenmitglieder verdrängen.

Price (1991) wies nach, dass Nachkommen in Tamarinfamilien ( $S$. oedipus) darum konkurrieren, die Jungtiere zu tragen. Dabei übernahmen die Geschwister in größeren Gruppen häufiger Jungtiere, lehnten die Jungtierübernahme seltener $a b$ und wiesen Geschwister, welche Jungtiere übernehmen wollten häufiger ab als in kleineren Gruppen. Der erste Zugang zum neugeborenen Nachwuchs wird zunächst von der Mutter bzw. den Eltern kontrolliert, indem diese Einfluss darauf nehmen, welcher Nachkomme die Neugeborenen übernehmen darf (eigene Beobachtungen bei C. jacchus). Der jungtiertragende Nachkomme kann (von den Eltern kontrolliert) die Entscheidung über die Weitergabe der Jungtiere beeinflussen, indem es bestimmte Geschwister abwehrt und anderen die Jungtiere übergibt. Mock et al. (1998) beschreiben eine Hierarchie in der Weitergabe von Nahrungsstücken entsprechend der Seniorität bei Vogelnestlingen. Eine Hierarchie entsprechend der Seniorität in der Jungtiertragehäufigkeit konnte auch in einigen C. jacchus-Gruppen beobachtet werden (Rothe et al. 1993).

Eine weitere Ressource, um die Geschwister möglicherweise miteinander konkurrieren, sind Sozialpartner, insbesondere die Alpha-Tiere (Seyfarth 1983). Dies ist auch für Krallenaffen plausibel, da enge Beziehungen (geäußert in regelmäßigen, häufigen affiliativen Interaktionen) zu den ranghöchsten Gruppenmitgliedern (Eltern) die Akzeptanz im elterlichen Streifgebiet steigern könnten. Affiliative Interaktionen wären damit eine Währung für die pay-to-stay-Hypothese.

Eine Erklärung für Konkurrenz zwischen Geschwistern könnte ein Modell von Creel (1990) und Lucas et al. (1996) darstellen. Bei der Berechnung der inklusiven Fitness von nichtreproduktiven Helfern müssen die Auswirkungen von Gruppenmitgliedern auf ein Individuum berücksichtigt werden (Creel 1990). Der von Hamilton (1964) formulierte durchschnittliche Effekt eines Helfers auf den Reproduktionserfolg des reproduktiven Tieres multipliziert mit dem Anteil der Helfer in einer Gruppe ergibt Hamilton's Sozialfaktor $e^{o}$ (Creel 1990). Da $e^{o}$ von der indirekten Fitness des Helfers subtrahiert werden muss, kann es 
zu negativen inklusiven Fitnesswerten der Helfer kommen. Der Fitnessertrag sinkt damit für den einzelnen Helfer mit einer zunehmenden Anzahl an Helfern (Lucas et al. 1996a). Somit kann angenommen werden, dass eine wachsende Anzahl an philopatrischen Geschwistern, die sich an der Jungtieraufzucht beteiligen, die inklusive Fitness aller nicht-reproduktiven Helfer verringert. Rauswürfe zwischen Geschwistern könnten damit ein Ausdruck der Fitnessmaximierung aller Familienmitglieder sein, wenn die Fitness der nicht-reproduktiven Helfer durch die Anwesenheit eines weiteren Geschwisters negativ beeinflusst würde.

\subsubsection{Eltern-Nachkommen-Konflikt}

Der klassische Eltern-Nachkommen-Konflikt bezieht sich auf das Ringen um die Dauer und Intensität der Investition elterlicher Leistungen in die Aufzucht der Nachkommen (ElternInvestment nach Trivers 1972). Eltern sollten demnach versuchen, den Aufwand der Fürsorge so gering wie möglich zu halten, da er zulasten der Investition in die zukünftige Reproduktion geschieht. Da die Überlebenswahrscheinlichkeit der Nachkommen und ihr zukünftiger Reproduktionserfolg von der Investition der Eltern abhängen, muss diese wiederum groß genug ausfallen, damit die Fitness maximal wird. Die Nachkommen werden versuchen, ihre „gen-egoistischen Ziele...durchzusetzen“ (Voland 2000) und das für sie maximale Investment von den Eltern einzufordern, was zu Konflikten zwischen beiden Parteien führt, z.B. Entwöhnungskonflikt (Trivers 1985). Wichtige Faktoren, die das Verhalten der Eltern und Nachkommen beeinflussen und Handlungsvariationen ermöglichen, sind das jeweilige Alter und Geschlecht der Eltern und Nachkommen. Bleiben Nachkommen nach ihrer Geschlechtsreife in der Natalfamilie, so zögern sie das elterliche Investment hinaus, indem sie die Ressourcen des heimatlichen Streifgebiets nutzen (Emlen 1982b). Werden die Nachkommen zu Nahrungskonkurrenten oder sexuellen Rivalen, sollten die Eltern versuchen, die Nachkommen aus ihrem Streifgebiet zu vertreiben (Ekman und Rosander 1992). In Weißbüschelaffenfamilien profitieren die Eltern jedoch von der Hilfeleistung der adulten Nachkommen, so dass für sie abzuwägen ist, durch welches Verhalten gegenüber ihren Nachkommen der größtmögliche Nutzen resultiert. In Krallenaffengruppen konnte sowohl beobachtet werden, wie nicht-reproduktive Töchter aggressiv von ihren Müttern aus der Gruppe vertrieben und Nachkommen von beiden Elternteilen sozial isoliert wurden (Epple 1975a für Callithrix und Saguinus, Baker 1987 für Leontopithecus, Soini 1988 für Cebuella), als auch von einer hohen Toleranz der Eltern gegenüber adulten Nachkommen. Abbott (1984), Hubrecht (1989), Lazaro-Perea et al. (1997) und Rothe (in Vorbereitung) berichten zum Beispiel über $C$. jacchus, dass Töchter innerhalb der Natalfamilie ovulierten und zwischen Mutter und ältester 
Tochter keine aggressiven Interaktionen auftraten. Häufig wird der ältesten Tochter in C. jacchus-Familien nach Geburten von Gruppenmitgliedern aggressiv begegnet, jedoch selten von ihren Müttern (Saltzman et al. 1997a). Damit bleibt fraglich, ob Aggressionen von Weibchen gegenüber ihren Töchtern aufgrund von sexueller Konkurrenz entstehen (Snowdon et al. 1993).

Fitnesserfolge der Nachkommen zahlen sich auch für die Eltern aus. Wenn das Überleben und die Chancen auf bestmögliche Reproduktion mit der längerfristigen Nutzung des elterlichen Streifgebiets gewährleistet wären, könnte Philopatrie der Nachkommen auch für die Eltern die optimale Option darstellen. Brown und Brown (1984) wendeten dieses Modell bei kooperativ brütenden Vögeln (Graubrusthäher, Aphelocoma ultramarina) an, wiesen jedoch darauf hin, dass sich ihr Konzept ebenso auf soziale Insekten und andere hochsoziale Tiere anwenden ließe. Sie machten deutlich, dass sich der höchste Reproduktionserfolg dann einstellte, wenn drei Nachkommen aufgezogen wurden, von denen zwei bleiben durften und einer das natale Territorium verließ. Der ethologische Polymorphismus könnte aus Selektionsdrücken resultieren, indem länger Bleibende, als auch frühe Abwanderer Vor- und Nachteile haben (Bekoff 1977). Wenn man das Modell auf Callithrix jacchus anwendet, könnte die optimale Strategie darin bestehen, dass ein bestimmter Teil der Nachkommen langfristig benötigt bzw. geduldet und ein anderer Teil aus der Gruppe herausgedrängt wird (vgl. GeschwisterKonkurrenz).

Garber (1997) vermutet, dass Tamarine kooperatives Verhalten nutzen, um die Gruppenkohäsion zu stärken, soziale und sexuelle Bindungen aufzubauen und die Anwesenheit von mehreren adulten Helfern zu sichern, da Helfer wesentlich für das Überleben der jüngsten Nachkommen sind. Es ist daher vorstellbar, dass Eltern versuchen, nicht-reproduktive Helfer von einer Emigration abzuhalten, falls adulte Nachkommen die Absicht erkennen lassen, die Natalfamilie zu verlassen, etwa durch Verringerung der räumlichen Assoziation zur Gruppe. So können sich Rückhalteversuche seitens der Eltern durch Kontrolle der Helfer, affiliatives Verhalten (Lazaro-Perea et al. 2004) oder gar durch kurzfristige Aufhebung der sexuellen Unterdrückung äußern (Emlen und Wrege 1992, Kokko und Johnstone 1999, Rylands1996).

\subsection{Ziele der Studie}

Das Ziel dieser Studie ist die Analyse gruppendynamischer Prozesse semifreilebender Weißbüschelaffenfamilien (Callithrix jacchus) anhand der hypothetisierten Regulationsmechanismen Emigration und/oder Rauswurf. Dabei wird analysiert, welche Ursachen die 
alternativen Taktiken (Philopatrie oder Emigration) zur Fitnessmaximierung adulter Nachkommen haben.

Welche sozialen und ökologischen Bedingungen erfüllt sein müssen, damit Nachkommen sich für einen Wechsel von der philopatrischen Lebensweise zur Emigration (und damit zur eigenen Reproduktion) entscheiden bzw. unter welchen Voraussetzungen einzelne Familienmitglieder von der Familie ausgestoßen werden, war Gegenstand der Untersuchung. Von besonderem Interesse ist dabei, ob die Dynamik der sozialen Integration auf den Einfluss der Eltern, der betrachteten Individuen selbst oder deren Geschwister zurückzuführen ist. Ein wichtiger Aspekt dieser Arbeit ist deshalb, im Rückblick nach beobachteten Emigrationen bzw. Rauswürfen Hinweise auf die Auslöser dieser gruppengrößenregulierenden Prozesse zu finden und festzustellen, unter welchen Bedingungen welches Gruppenmitglied emigrierte bzw. rausgeworfen wurde. Dabei werden die Auswirkungen der Gruppengröße, des Alters und Geschlechts eines Individuums, der Anwesenheit von potentiellen Reproduktionspartnern, als auch familiärer Strukturen und Beziehungen auf die Entscheidung zwischen Philopatrie und Emigration berücksichtigt. Ein weiteres Augenmerk gilt Peripheralisationen einzelner Mitglieder von der Gruppe, welche bisher als Emigrationsneigungen interpretiert wurden (Geyer 1993, Rothe und Darms 1993). Es wird untersucht, inwiefern beobachtete Peripheralisierungen von Mitgliedern der Fokusfamilie mit Emigrationen bzw. Rauswürfen in Zusammenhang gebracht werden können.

Angesichts des Facettenreichtums des Modells (Abb. 1.1) wird im Rahmen dieser Studie den oben genannten Einflussfaktoren nachgegangen. Mortalitätsrisiken, die auf ein emigrierendes Individuum außerhalb seiner Natalgruppe einwirken und Eigenschaften eines potentiellen neuen Streifgebietes konnten aufgrund der Semifreiland-Haltungsbedingungen nicht untersucht werden. Auf die Verfügbarkeit von Helferpositionen (möglicherweise Rollen) wurde indirekt über die Gruppengröße und die Geschwister-Konkurrenz eingegangen.

Die der Arbeit zugrunde liegenden Hypothesen (siehe 1.4.1) orientieren sich an dem dargestellten Modell aus 1.2 und testen gezielte Einflussfaktoren (z.T. mit experimentellem Versuchsaufbau) auf Zusammenhänge mit den beobachteten Entscheidungen der Gruppenmitglieder. 


\subsubsection{Hypothesen}

\section{Emigrationen und Rauswürfe}

Hypothese 1: Die Wahrscheinlichkeit, dass ein Rauswurf geschieht, ist von individuenexternen Faktoren unabhängig.

Hypothese 2: Die Wahrscheinlichkeit, rausgeworfen zu werden, ist für alle Nachkommen gleich groß.

Hypothese 3: Rauswürfe deuten sich nicht durch einen Anstieg der Körperkerntemperatur des betroffenen Tieres an.

\section{Untersuchungen zur Geschwister-Konkurrenz und zum EItern-Nachkommen- Konflikt}

Hypothese 4: Rauswürfe ereignen sich unabhängig von Geschwister-Konkurrenz.

Hypothese 5: Die Beziehungen der Geschwister (räumliche Assoziation und affiliative Interaktionen) bleiben von wechselnden Experimentalbedingungen unbeeinflusst.

Hypothese 6: Rauswürfe ereignen sich unabhängig von Eltern-Nachkommen-Konflikten.

III. Untersuchungen zur Emigrationsneigung anhand der Aufenthaltsorte und räumlichen Assoziation der Familienmitglieder

Hypothese 7: Die Arealnutzung ist gleichmäßig und weder von den Experimenten, noch von den Familienmitgliedern abhängig.

Hypothese 8: Peripheralisationen sind von individuenexternen Faktoren unabhängig. 


\section{TIERE, MATERIAL UND METHODEN}

\subsection{Studiengelände und Tiere}

\subsubsection{Studiengelände}

Die Untersuchungen wurden in der Semifreianlage der Ethologischen Station Sennickerode (Gleichen) der Universität Göttingen durchgeführt. In dem ca. 6,5ha großen Gelände wurden mehrere Callithrix jacchus-Familien gehalten, deren Streifgebiete (engl. home-ranges) durch mindestens $30 \mathrm{~m}$ breite und 1,50m hohe, dichte Grasflächen voneinander getrennt waren. Die einzelnen Streifgebiete betrugen ca. 1,5ha und waren mit Klettergerüsten, Laufleisten, mehreren Schlafkästen, Futterbrettern und drei beheizbaren Schutzhütten ausgestattet. Entlang der Kletter- und Laufeinrichtungen waren seit 1995 unterschiedliche Baum- und Straucharten (Ahorn, Erle, Linde, Pappel, Robinie) kontinuierlich gepflanzt worden, so dass sich die Studientiere während des Untersuchungszeitraumes (2000-2002) zum Teil in kleinen Wäldchen bewegen und ihr gesamtes Streifgebiet erreichen konnten, ohne den Boden zu betreten (Abb. 2.1).

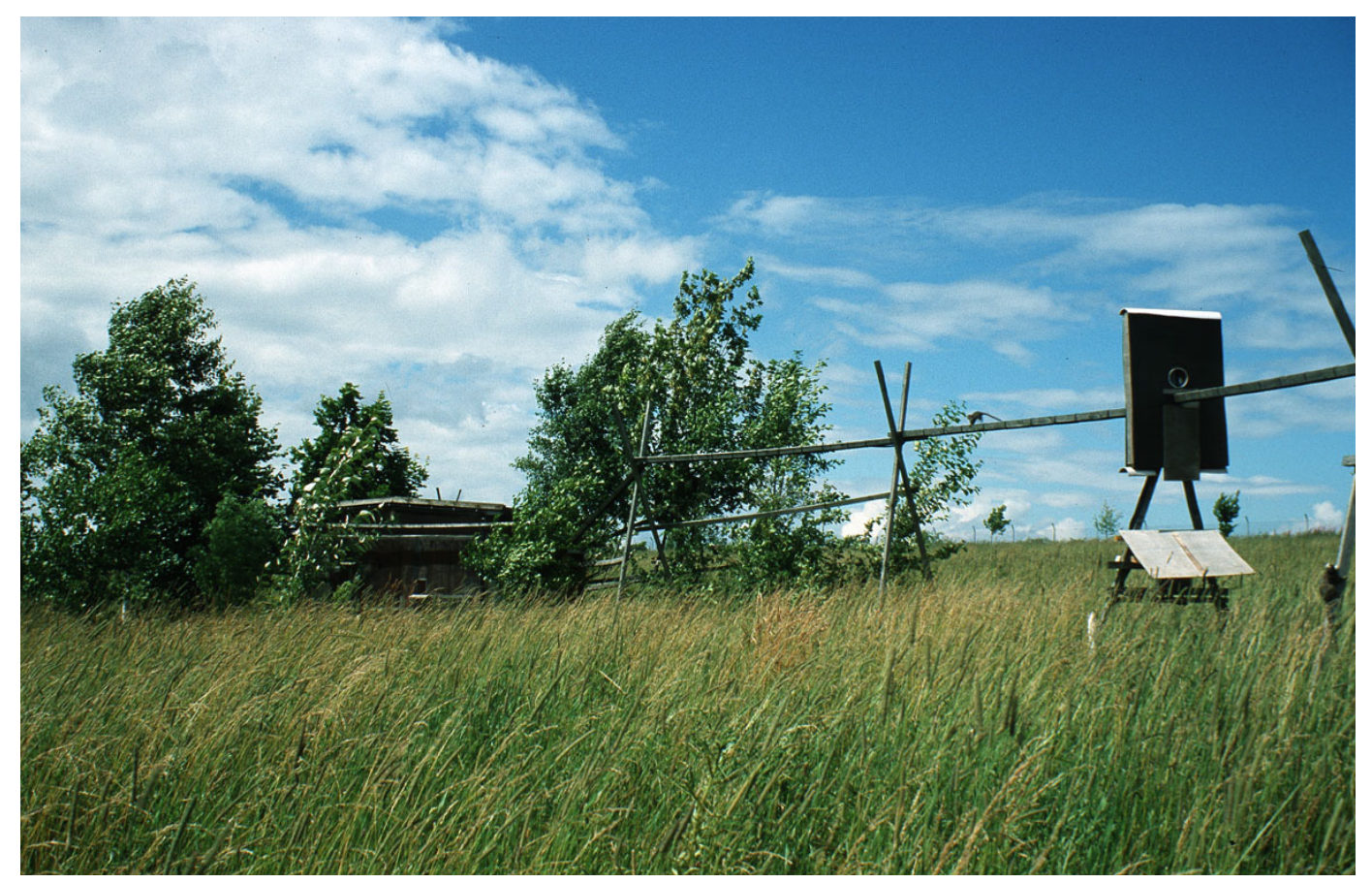

Abb. 2.1 Semifreianlage der Ethologischen Station Sennickerode (Ausschnitt). Rechts im Bild ist eine Transponderschleuse (vgl. 2.1.2) zu erkennen. 


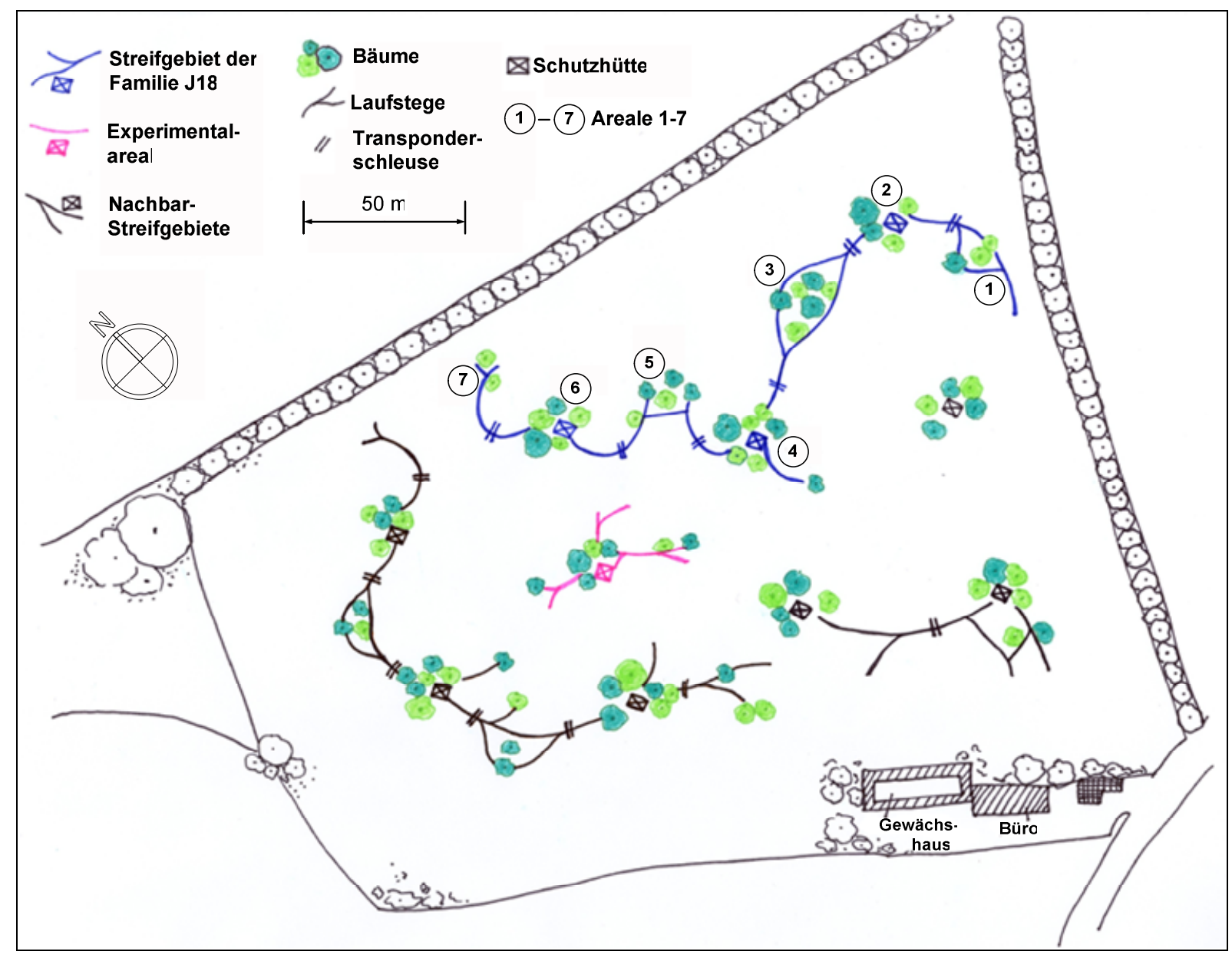

Abb. 2.2 Übersichtsskizze der Semifreianlage der Ethologischen Station Sennickerode (Anlage von oben).

Eine besonders dichte Vegetation war in den Arealen 3 und 5 vorzufinden. Ein deutlich kleineres Streifgebiet, das Experimentalareal, welches im Zentrum des gesamten Geländes angesiedelt war, wurde nur während bestimmter Experimente mit Locktieren besetzt (Abb. 2.2). Eine Ausführliche Beschreibung des Geländes findet sich bei Rothe et al. (1997).

Die Studientiere hatten während der Vegetationsperiode (Mai bis November) Zugang zum Semifreigelände und waren daher Wetterbedingungen, saisonalen Tageslicht- und Temperaturschwankungen sowie dem Raubfeindrisiko durch Greifvögel, Füchse, Katzen und Marder ausgesetzt. Der Grasgürtel zwischen den Streifgebieten diente als natürliche „Weglaufsperre“ für die arborikolen Tiere, welche nur selten überwunden wurde (z.B. wenn ein Tier von der Gruppe ausgeschlossen wurde und es aus dem Streifgebiet abwanderte). Während der Wintermonate in der Zeit von Dezember bis April war der Aktionsraum der Tiere auf eine Schutzhütte (2,7 x 2,7 x 2,4m) mit Veranda und drei Außenkäfigen (jeweils 1,3 x $1,3 \times 2,6 \mathrm{~m}$ ) beschränkt. 


\subsubsection{Transponderanlage}

Mit Hilfe einer elektronischen Identifizierungsanlage (Transponderanlage) war es möglich, die Aufenthaltsorte der Affen auf dem Gelände auch ohne Anwesenheit eines Beobachters während der gesamten Sommerperioden zu verfolgen und elektronisch zu speichern. Das Streifgebiet der Fokusfamilie wurde durch Schleusen (siehe Abb. 2.1) in sieben Areale unterteilt. Diese konnten nur von einem Tier pro Zeiteinheit passiert werden. Befand sich das Tier im Durchgang, wurde sein trovan ${ }^{\circledR}$ Transponder mit individueller Erkennungsnummer, der jedem Tier in den Schulterbereich injiziert worden war, elektronisch ausgelesen.

Die Auswertung der Daten über die Aufenthaltsorte der Affen wird in 2.4.7 dargestellt. Gelegentlich kam es zu Ausfällen der gesamten Anlage oder einzelner Schleusen (etwa durch Gewitter oder starke Erwärmung aufgrund von Sonneneinstrahlung), so dass die Aufzeichnung an einigen Tagen Lücken aufweist.

\subsubsection{Studientiere}

Für die Studie wurde die Familie J18 ausgewählt, eine Weißbüschelaffenfamilie, die 1995 im Semifreigelände angesiedelt worden war. Die Familie bestand im ersten Beobachtungsjahr aus einem Elternpaar (A und B), zwei adulten Söhnen (C und E), einer adulten Tochter (D), einem subadulten Sohn $(F)$, einem juvenilen Sohn $(\mathrm{G})$, einer juvenilen Tochter $(\mathrm{H})$ und zwei männlichen Jungtieren (I und J), also insgesamt 10 Tieren. Die Altersklassen wurden nach der Definition von Stevenson und Rylands (1988) folgendermaßen festgelegt: infantil: 0-3 Monate; juvenil: 4-5 Monate; adoleszent: 6-10 Monate; subadult: 11-15 Monate; adult: älter als 15 Monate. In Tabelle 2.1. sind die wichtigsten demographischen Angaben über die Familienmitglieder zusammengefasst.

Durch Ausschlüsse einiger Familienmitglieder (E, F und H) und die Geburten von K, M, P und R veränderte sich die Zusammensetzung der Familie im zweiten Beobachtungsjahr. G war am Anfang des zweiten Jahres subadult, die Zwillingsschwestern K und M juvenil und die Zwillingsbrüder P und $\mathrm{R}$ infantil. Die Gruppengröße schwankte während des zweiten Beobachtungsjahres zwischen 9 und 11 Mitgliedern. Im dritten Jahr der Studie wurden zwei neue Jungtiere geboren, die Nachkommen J, M, P und R aus der Gruppe geworfen und das Alpha-Weibchen starb im Sommer 2002, so dass die Gruppengröße sich auf 7 Mitglieder verkleinerte. 
Tabelle 2.1 Demographische Angaben zu den Mitgliedern der Familie J18. Die Angaben der Altersklassen beziehen sich auf die Beobachtungszeiträume in den jeweiligen Jahren.

\begin{tabular}{|c|c|c|c|c|c|}
\hline $\begin{array}{c}\text { Tier- } \\
\text { bezeichnung }\end{array}$ & Geschlecht & $\begin{array}{c}\text { Geburts- } \\
\text { datum }\end{array}$ & $\begin{array}{l}\text { Altersklasse } \\
2000\end{array}$ & $\begin{array}{l}\text { Altersklasse } \\
2001\end{array}$ & Bemerkungen \\
\hline A & $0^{\pi}$ & 01.03.1992 & adult & adult & \\
\hline B & q & 30.10 .1991 & adult & adult & \\
\hline C & $0^{\pi}$ & 10.06 .1995 & adult & adult & \\
\hline D & q & 01.06 .1997 & adult & adult & \\
\hline$E$ & $\hat{0}$ & 29.03 .1998 & adult & - & Rauswurf am 27.03.2001 \\
\hline $\mathbf{F}$ & $0^{\pi}$ & 29.01 .1999 & subadult & - & Rauswurf am 06.07.2000 \\
\hline G & $\pi$ & 09.10 .1999 & adoleszent & adult & \\
\hline $\mathrm{H}$ & q & 09.10 .1999 & adoleszent & adult & Rauswurf am 21.05.2001 \\
\hline I & $\hat{0}$ & 05.04 .2000 & infantil/juvenil & subadult & Rauswurf am 29.06.2001 \\
\hline$J$ & $\widehat{0}$ & 05.04 .2000 & infantil/juvenil & subadult & Rauswurf am 21.10.2001 \\
\hline K & q & 13.12 .2000 & - & adoleszent & \\
\hline M & q & 13.12 .2000 & - & adoleszent & Rauswurf am 24.03.2002 \\
\hline $\mathbf{P}$ & $\pi$ & 10.06 .2001 & - & infantil/juvenil & Rauswurf am 11.06.2002 \\
\hline $\mathbf{R}$ & $0^{\pi}$ & 10.06 .2001 & - & infantil/juvenil & Rauswurf am 30.07.2002 \\
\hline
\end{tabular}

\subsubsection{Locktiere}

Für die Durchführung der Experimente wurden Locktiere (siehe Tabelle 2.2) in das Experimentalareal gebracht. Eine detaillierte Beschreibung der Experimente erfolgt in Kapitel 2.2.7. In den Experimenten $\mathrm{C} 1$ und $\mathrm{C} 2$ des ersten Beobachtungsjahres dienten einzelne adulte Weibchen als Locktiere. Im zweiten Beobachtungsjahr befand sich in Experiment E1 eine vierköpfige Familie mit einem subadulten Sohn und einer subadulten Tochter im Experimentalareal. Für die darauffolgenden Experimente E2 und E3 wurden die beiden weiblichen Tiere aus dem Experimentalareal heraus genommen, so dass nur noch Vater und Sohn als Lockmännchen dienten. Während des letzten Experiments E4 befand sich ein adultes Lockmännchen im Experimentalareal. 
Tabelle 2.2 Demographische Angaben zu den Individuen, die im ersten und zweiten Beobachtungsjahr dieser Studie als Locktiere verwendet wurden.

\begin{tabular}{|c|c|c|c|}
\hline Experiment & Geschlecht & Geburtsdatum & Altersklasse \\
\hline C1/2000 & q & 14.05 .1998 & adult \\
\hline C2/2000 & q & 01.08 .1998 & adult \\
\hline \multirow[t]{4}{*}{ E1/2001 } & $\hat{0}$ & 29.10 .1997 & adult \\
\hline & 우 & 09.05 .1997 & adult \\
\hline & $\widehat{0}$ & 01.05 .2000 & subadult \\
\hline & 우 & 01.05 .2000 & subadult \\
\hline \multirow[t]{2}{*}{ E2 und E3/2001 } & $\widehat{0}$ & 29.10 .1997 & adult \\
\hline & $0^{\lambda}$ & 01.05 .2000 & subadult \\
\hline E4/2001 & $0^{\lambda}$ & 03.08.1999 & adult \\
\hline
\end{tabular}

\subsection{Methoden der Datenerhebung}

\subsubsection{Beobachtungsmethoden}

Zur quantitativen Datenerhebung wurden interaktive und nicht-interaktiven Verhaltenseinheiten von jedem Tier der Fokusfamilie mit instantaneous sampling aufgenommen (focal animal sampling nach Martin und Bateson 1986). Dazu wurden zum Zeitpunkt des instants die Verhaltenseinheit, gegebenenfalls Rezipienten des Verhaltens und der Aufenthaltsort des Fokustiers festgehalten. Ein Protokoll wurde beendet, wenn alle Familienmitglieder einmal beobachtet worden waren, wobei sich die gesamte Beobachtung eines Fokustieres aus zehn Intervallen von jeweils 60 Sekunden Länge zusammen setzte. Die Reihenfolge der Fokustiere wurde vor der ersten Beobachtung festgelegt, wobei jedes Protokoll mit einem anderen Fokustier startete. Damit wurde verhindert, dass ein und dasselbe Tier zur gleichen Zeit beobachtet wurde. Instantaneous sampling erfasst Wahrscheinlichkeiten, keine realen Häufigkeiten (Martin und Bateson 1986).

Die Eintragung der Daten erfolgte in ein transportables elektronisches Datenaufnahmegerät (Newton Message Pad 120, Apple; siehe Westermann und Rothe 1996). Parallel dazu wurden ad libitum auffällige Aktivitäten (z.B. Keckern) und besonderen Ereignisse (z.B. Kopulationen) notiert, die mit instantaneous sampling nicht erhoben werden konnten, weil sie zu selten auftraten (Altmann 1974). Mit instantaneous sampling war es z.B. möglich zu beschreiben, wie häufig oder wie lange zwei Individuen sich im Nahbereich zueinander befanden. Welches Tier eine Nahbereichskonstellation initiierte oder beendete wurde hiermit 
jedoch nur selten oder gar nicht ermittelt. Zur qualitativen Feinanalyse einer Beziehung zweier Individuen war deshalb das ad libitum - Protokoll eine wichtige Ergänzung.

\subsubsection{Definitionen}

Für eine unmissverständliche Einordnung wurden die Verhaltensweisen vor der Datenaufnahme definiert. Falls nicht anders erwähnt, wurden die Begriffe und Definitionen von Rothe (1979) übernommen. Die Liste der beobachteten Verhaltenseinheiten und ihrer zugehörigen Kürzel befindet sich im Anhang auf Seite 238ff. Einige wichtige Begriffe wurden unter folgender Bedeutung verwendet:

affiliatives Verhalten: unter affiliativem Verhalten wurden alle Verhaltenseinheiten (VE) zusammengefasst, bei denen ein Tier in soziopositive Interaktionen mit einem Familienmitglied eingebunden war (z.B.: Grooming, Handauflegen, Kuscheln, Sozialspiel).

dissoziatives Verhalten: unter dissoziativem Verhalten finden sich all jene Verhaltenseinheiten, bei denen ein Tier in sozionegative Interaktionen mit einem Familienmitglied eingebunden war (z.B. Ausweichen, Abwenden, Fellsträuben, Genitalpräsentieren, Katzbuckellaufen, Keckern).

Emigration: irreversible Abwanderung eines Familienmitgliedes aus seiner Sozietät.

Familie und Gruppe: die Beobachtungen dieser Arbeit beschränken sich auf eine Callithrix jacchus - Familie, in der alle Tiere (mit Ausnahme der Eltern) miteinander verwandt sind. Nach rein soziographischen Gesichtspunkten handelt es sich daher um keine Gruppe (Rothe und Darms 1993). Da die Verwandtschaftsverhältnisse der Mitglieder freilebender Sozietäten oft unbekannt sind, wird in diesen Fällen der Begriff Gruppe (sensu McGrew 1986) verwendet.

Auch im Zusammenhang mit der Fokusfamilie dieser Studie werden im Sinne einer Sozietät die Begriffe Gruppengröße, Gruppendynamik etc. verwendet.

gruppendienliches Verhalten: Verhalten, von dem Gruppenmitglieder profitieren, wie z.B. Jungtierfürsorge, Scanning und Territorialverteidigung (Patrouillieren an Streifgebietsgrenzen, Bißmarken anlegen, Markieren).

Initiierung affiliativer Kontakte: Verhalten, bei dem ein Tier aktiv die freundliche Interaktion mit einem Familienmitglied beginnt (z.B. Aufforderung zum Grooming, freundliches Annähern, Kontaktaufnahme). 
Nahbereich: Der Nahbereich eines Tieres wurde mit einem Radius von ca. $50 \mathrm{~cm}$ (doppelte Armlänge) um das Tier herum definiert, so dass alle Familienmitglieder, die sich innerhalb dieses Radius aufhielten, im Nahbereich des betreffenden Tieres waren.

Peripheralisation: Verminderte räumliche Assoziation zu den übrigen Familienmitgliedern. Ein verringerter Anteil an affiliativen Interaktionen mit Familienmitgliedern als auch der Nahbereichshäufigkeiten kann damit einhergehen.

Rauswurf: Unfreiwilliger Ausschluss eines Familienmitgliedes aus der Familie, der durch aggressives Verhalten von Familienmitgliedern hervorgerufen wird. Damit einhergehend wird das Tier von der Nutzung jeglicher Ressourcen der Sozietät ausgeschlossen.

\subsubsection{Statistische Tage}

Zur statistischen Analyse der gesamten Tagesaktivität wurden statistische Tage eingeführt. An vier aufeinander folgenden Beobachtungstagen wurden dafür die Tiere jeweils zu einem Viertel ihrer täglichen Aktivitätsperiode beobachtet, so dass nach vier Tagen die gesamte Aktivitätsperiode abgedeckt und ein statistischer Tag beendet werden konnte. Die Anzahl der Stunden, die diesem Viertel entsprachen, hing von der Sonnenscheindauer entsprechend der Jahreszeit ab. Suchi (1996) berechnete die Länge eines Tagesviertels in Stunden und fand, dass im Sommer (Ende Juli) täglich vier Stunden und im Herbst (November) zwei Stunden dem Viertel der Tagesaktivität der Affen in Sennickerode entsprachen. Die tägliche Beobachtungszeit wurde der jeweiligen Aktivtätsdauer angepasst.

\subsubsection{Beobachtungszeiten}

Vom 22.-24.05.2000 wurden Voruntersuchungen zur tageszeitlichen Aktivität der Tiere durchgeführt, um abschätzen zu können, in welchem Zeitraum des Tages Beobachtungen aussagekräftig waren, da Ruhe- und Schlafphasen für die Analyse der Gruppendynamik von geringerer Bedeutung sind. Suchi (1996) beobachtete Schlaf- oder Ruhephasen vorwiegend im Zeitraum zwischen 9:00 und 11:00 Uhr. Die Voruntersuchungen zeigten aber, dass die genauen Zeiträume der Schlafphasen nicht vorhergesehen werden konnten, so dass gemäß der Lichtperiode zunächst von einem $16 \mathrm{~h}$ Tag ausgegangen werden musste. Die Voruntersuchungen ergaben eine kürzere Aktivitätszeit der Affen, die zwischen zehn und dreizehn Stunden täglich lag, was sich mit Beobachtungen von Ahlborn (1996), Suchi (1996) und Maier et al. (1982) weitestgehend deckt. Daher wurde zunächst in einem Zeitraum zwischen 
6:00 und 19:20 Uhr beobachtet. Nach Rothe (pers. Mtlg) hielten sich die Affen in Sennickerode seit 1995 auch während der Sommermonate mit längerer Tageslänge nur noch selten nach 19:00 Uhr außerhalb ihrer Schutzhütten auf, was in den ersten drei statistischen Tagen der ersten Kontrollphase der vorliegenden Studie bestätigt werden konnte. Demnach wurden die Beobachtungszeiten um jeweils 20 Minuten vorgezogen und in der zweiten Kontrollphase entgültig auf einen Zeitraum von 6:00 bis 18:00 Uhr festgelegt.

\subsubsection{Beobachtungsperiode im ersten Beobachtungsjahr (2000)}

Die Beobachtungsperiode dauerte vom 25.05.2000 bis zum 07.10.2000. Die Anzahl der Beobachtungstage, die Beobachtungsdauer pro Tier und für die gesamte Familie, als auch die Anzahl der beobachteten Tiere sind in Tabelle 2.3 für jedes Experiment aufgeführt.

Im Sommer 2000 wurde die Familie J18 insgesamt 286,66 Stunden beobachtet. Davon entfallen auf jedes Familienmitglied 30,66 Stunden Beobachtungszeit. Die angeführten Beobachtungsstunden beziehen sich ausschließlich auf die Datenaufnahme mit instantaneous sampling.

Tabelle 2.3 Beobachtungsstunden im ersten Beobachtungsjahr 2000

\begin{tabular}{c|c|c|c|c|c}
\hline Phase & Datum & $\begin{array}{c}\text { Anzahl der } \\
\text { Tage }\end{array}$ & $\begin{array}{c}\text { Beobachtungsdauer } \\
\text { pro Tier (in Min.) }\end{array}$ & $\begin{array}{c}\text { Anzahl der } \\
\text { Tiere }\end{array}$ & $\begin{array}{c}\text { Beobachtungsdauer } \\
\text { insgesamt (in Min.) }\end{array}$ \\
\hline Hüttenphase 1(A1) & $25.05 .00-05.06 .00$ & 12 & 240 & 10 & 2400 \\
Kontrollphase 1(B1) & $12.06 .00-01.07 .00$ & 20 & 400 & 10 & 4000 \\
Kontrollphase 2 (B2) & $10.07 .00-29.07 .00$ & 20 & 400 & 9 & 3600 \\
Experiment 1 (C1) & $11.08 .00-01.09 .00$ & 20 & 400 & 9 & 3600 \\
Experiment 2 (C2) & $16.09 .00-07.10 .00$ & 20 & 400 & 9600 \\
\hline
\end{tabular}

\subsubsection{Beobachtungsperiode im zweiten Beobachtungsjahr (2001)}

Die zweite Beobachtungsperiode dauerte vom 30.04.2001 bis zum 26.09.2001. Details werden in Tabelle 2.4 dargestellt. Im Sommer 2001 wurde die Familie J18 insgesamt 225,5 Stunden beobachtet. Davon entfallen auf jedes Familienmitglied 26,17 Stunden Beobachtungszeit. Die angeführten Beobachtungsstunden beziehen sich ebenfalls ausschließlich auf die Datenaufnahme instantaneous sampling. 
Tabelle 2.4 Beobachtungsstunden im zweiten Beobachtungsjahr 2001

\begin{tabular}{c|c|c|c|c|c}
\hline Phase & Datum & $\begin{array}{c}\text { Anzahl der } \\
\text { Tage }\end{array}$ & $\begin{array}{c}\text { Beobachtungsdauer } \\
\text { pro Tier (in Min.) }\end{array}$ & $\begin{array}{c}\text { Anzahl der } \\
\text { Tiere }\end{array}$ & $\begin{array}{c}\text { Beobachtungsdauer } \\
\text { insgesamt (in Min.) }\end{array}$ \\
\hline Hüttenphase 2 (D1) & $30.04 .01-19.05 .01$ & 20 & 200 & 10 & 2000 \\
Experiment 1 (E1) & $28.05 .01-18.06 .01$ & 20 & 400 & 9 & 3600 \\
Experiment 2 (E2) & $21.06 .01-29.06 .01$ & 9 & 170 & 9 & 1530 \\
Experiment 3 (E3) & $30.06 .01-21.07 .01$ & 20 & 400 & 8 & 3200 \\
Experiment 4 (E4) & $05.09 .01-26.09 .01$ & 20 & 400 & 8 & 3200 \\
\hline
\end{tabular}

\subsubsection{Experimente}

Während der Sommermonate 2000 und 2001 wurden mit der Fokusfamilie unterschiedliche Experimente durchgeführt. Die Umweltbedingungen der Gruppe wurden derart manipuliert, dass potentiellen Emigranten verschiedene dispersal sinks (Streifgebiete zum Immigrieren) angeboten wurden. In das Experimentalareal, welches räumlich durch einen mindestens $30 \mathrm{~m}$ breiten Grasgürtel von dem Areal der Fokusfamilie entfernt lag und mit einer Schutzhütte, Futterbrettern, einigen Bäumen und Laufleisten ausgestattet war, wurden für die Familie unbekannte Artgenossen in unterschiedlichen Konstellationen gebracht, womit getestet werden sollte, welcher Anreiz die Nachkommen der Fokusfamilie zu welcher Entscheidung über Philopatrie und Emigration provoziert. Es wurde erwartet, dass männliche Nachkommen durch weibliche Locktiere und weibliche Nachkommen durch männliche Locktiere dazu verleitet werden, aus der Natalfamilie zu emigrieren.

\subsubsection{Voruntersuchungen zu den Experimenten}

Bevor die Affen aus den Blockhütten, die ihnen als Winterquartier dienten, in das Semifreigelände entlassen wurden, wurden im ersten Jahr zweimal täglich in einer zwölftägigen Beobachtungsperiode (Hüttenphase 1) und im folgenden Jahr einmal täglich in einer zwanzigtägigen Beobachtungsperiode (Hüttephase 2) Daten mit instantaneous sampling erhoben, um die Auswirkungen der Haltungsänderung von einem vergleichsweise räumlich beengten Hütten-Käfig-Komplex zu einer weitläufigen Freilandanlage auf die Fokustiere zu protokollieren. Mit dem Tag der Freilassung begannen die Kontrollphasen bzw. die Experimente. Nach einer ersten Kontrollphase (B1), in der nur die Fokusfamilie J18 zur Semifreianlage Zugang hatte, wurde in einer zweiten Kontrollphase (B2) eine Familie mit sechs Familienmitgliedern (J27) in das Nachbarareal gesetzt. Die Kontrollphasen dauerten 
jeweils 20 Tage und sollten Aufschluss über die Nutzung des Streifgebietes und die Beziehungen innerhalb der Studienfamilie geben.

\subsubsection{Experimente im Sommer 2000}

In der ersten Kontrollphase (B1) gab es freie Streifgebiete, in der zweiten Kontrollphase (B2) eine Nachbarfamilie, und ein freies Streifgebiet. Für das darauf folgende erste Experiment wurde ein Lockweibchen in das Experimentalareal gebracht, so dass eine Reproduktionsposition für ein Männchen frei war und männlichen Nachkommen der Fokusfamilie ein Anreiz zur Emigration gegeben wurde. Die Affen der Familie J18 konnten sowohl Sicht-, als auch akustischen Kontakt zum Lockweibchen aufnehmen. Am Ende des Experiments (nach 20 Tagen) wurde das Lockweibchen aus dem Areal heraus genommen. Das zweite Experiment wurde nach einer Pause von 14 Tagen mit einem anderen Lockweibchen unter gleichen Bedingungen durchgeführt. Das Lockweibchen des zweiten Experiments (C2) gab weniger Kontaktlaute (Distanz-Phi und Twitter) als das erste Lockweibchen von sich und zeigte sich der Fokusfamilie seltener.

\subsubsection{Experimente im Sommer 2001}

Im Sommer 2001 wurden Bedingungen getestet, welche vor allem den Töchtern der Fokusfamilie einen Emigrationsanreiz geben sollten. Die Familie im Nachbarareal wurde aus tierpflegerischen Gründen durch eine andere ersetzt (J29).

Im ersten Experiment des zweiten Beobachtungsjahres sollte getestet werden, ob eine Kleinfamilie (bestehend aus den Eltern und einem männlichen und einem weiblichen subadulten Nachkommen), deren Alpha-Weibchen einen verletzten Fuß hatte und sich in schlechter Kondition befand, für die Töchter der Fokusfamilie einen Emigrationsanreiz darstellt. Aus Freilandstudien ist bekannt, dass die reproduktive Position des AlphaWeibchens von anderen, meist jüngeren Weibchen übernommen werden kann (Ferrari und Lopes Ferrari 1989, Rylands 1982 für C. humeralifer) und Rothe und König (1987) beobachteten, dass Töchter mit ihrem Vater sexuell interagierten, wenn ihre Mutter aus Krankheitsgründen geschwächt war. Anzenberger (1983) und Radespiel (1990) berichten außerdem, dass reproduktive Männchen in Laborhaltung bei der Konfrontation mit fremden Weibchen Sexualverhalten zeigten bzw. mit den Weibchen sexuell interagierten (siehe auch Hubrecht 1985 und Digby 1999). Aufgrund dieser Beobachtungen wurde hypothetisiert, dass eine Tochter der Fokusfamilie entweder emigriert und die reproduktive Position des kranken 
Alpha-Weibchens übernimmt oder aber zumindest mit dem fremden Alpha-Männchen kopuliert. Für das fremde Alpha-Männchen wäre dies eine Option gewesen, sich mit einer Tochter der Fokusfamilie zu reproduzieren, anstatt bei einer temporär nicht-reproduktiven Partnerin zu bleiben.

Für das zweite Experiment wurden die beiden weiblichen Tiere aus der Kleinfamilie entfernt, so dass nur noch zwei Männchen (Vater und Sohn) der Fokusfamilie präsentiert wurden. Mit der Anwesenheit zweier Männchen wurde den Töchtern die Möglichkeit zur polyandrischen Reproduktion und den männlichen Nachkommen der Fokusfamilie eine peer-group (,Junggesellengruppe“/Freundes-Gruppe) geboten. Am neunten Tag des zweiten Experiments wurde ein männlicher Nachkomme aus der Fokusfamilie ausgestoßen, so dass damit das zweite Experiment beendet war. Am darauffolgenden Tag begann ein weiteres Experiment mit denselben Locktieren (Experiment 3), welches wieder regulär 20 Tage lang andauern sollte. Am Ende wurden die beiden Männchen aus dem Experimentalareal entfernt. Nach einer längeren Pause von 45 Tagen begann das vierte und letzte zwanzigtägige Experiment, in dem ein einzelnes, adultes Männchen in das Experimentalareal gebracht wurde.

\subsection{Temperaturbuttons}

Die Entscheidung, die Natalfamilie zu verlassen, wird meistens erst durch die Emigration bzw. den Rauswurf ersichtlich. Um den Beginn des Entscheidungsprozesses erkennen zu können, sollten physiologische Parameter ermittelt werden. Es wurde deshalb eine Pilotstudie zur Messung von Körperkerntemperaturen der Affen durchgeführt (Thieß und Rothe 2002).

Die regelmäßige Messung der Körpertemperaturen sollte Aufschluss darüber liefern, ob Erhöhungen der Körpertemperaturen als Indikator für sozialen Stress mit dem beobachteten Verhalten korreliert werden können. Ebenso wurde nach Zusammenhängen zwischen Ovulationen und den Temperaturen der weiblichen Tiere gesucht (vgl. Maier 1988, Petry und Maier 1990, Schnell 1993, 1997). Für die Messung der Körperkerntemperaturen der Affen dienten Temperaturbuttons der Firma ADS (Agricultural Data Systems). Die Vorteile dieser Buttons waren (1) die Möglichkeit bei semifreilebenden Tieren Körpertemperaturen zu messen, wobei die Tiere völlig ungestört blieben, (2) die geringe Größe der Buttons (17,35mm Durchmesser, 5,89mm Höhe, 3,3g), so dass das Wohlbefinden der Tiere mit implantierten Buttons gewährleistet war, (3) dass das Material und die Verarbeitung der Buttons keine chemischen Reaktionen mit tierischem Gewebe hervorruft, (4) abhängig von der Messhäufigkeit die Buttons mehrere Monate durchgehend einsetzbar sind, (5) die Buttons 
leicht $\mathrm{zu}$ implantieren sind und (6) der finanzielle und zeitliche Aufwand gering gehalten wird.

Vor Beginn der Datenerhebung 2001 wurden fünf adulten Tieren der Familie J18 (A, C, D, G und H) Temperaturbuttons implantiert ${ }^{7}$. Dem trächtigen Alpha-Weibchen wurde kein Button eingesetzt, um die Gesundheit der Föten nicht zu gefährden. Die Buttons waren so konfiguriert, dass sie alle drei Stunden (23:00, 2:00, 5:00, 8:00, 11:00, 14:00, 17:00 und 20:00 Uhr) die Körperkerntemperatur des jeweiligen Tieres maßen und speicherten. Wichtig erschienen während der Planung vor allem die Nachtzeiten, weil davon ausgegangen werden konnte, dass die Tiere nachts schliefen und eine gesteigerte Aktivität als Auslöser möglicher Temperaturerhöhungen ausgeschlossen werden konnten. Nach zehn Monaten wurden die Buttons explantiert und mit der zugehörigen Software ausgewertet. Die von der Herstellerfirma angegebene Messgenauigkeit der Temperatur-Buttons erfüllte nicht die Erwartungen der Pilotstudie, da die Temperatur zwar genau gemessen $\left(0,1^{\circ} \mathrm{C}\right.$-Schritte), aber aufgrund der begrenzten Kapazität der Batterie auf $0,5^{\circ} \mathrm{C}$-Schritte gerundet innerhalb des Buttons gespeichert wurde. Die Information über die begrenzte Batteriekapazität und der Rundung in $0,5^{\circ} \mathrm{C}$-Schritten wurde von der Herstellerfirma erst nach langem Nachfragen geliefert, nachdem die Ergebnisse bereits vorlagen. Die exakte Temperatur konnte deshalb nicht mehr nachvollzogen werden.

\subsection{Datenanalyse}

\subsubsection{Zuordnung der Verhaltenseinheiten in Verhaltensbereiche}

Die mit instantaneous sampling gewonnenen Verhaltenseinheiten wurden sechs Verhaltensbereichen zugeordnet:

- taktile Interaktion mit Familienmitgliedern (TIF)

- nicht-taktile Interaktion mit Familienmitgliedern (NTF)

- nicht-taktile Interaktion mit fremden Artgenossen (NTA)

- Erwerb und Sichern von Ressourcen und Raubfeindvermeidung (RR)

- stoffwechselbedingtes Verhalten und Körperpflege (SK)

- Körperhaltung und Lokomotion (KL) 
Durch die Zusammenfassung der Verhaltenseinheiten zu Verhaltensbereichen wurde die Erstellung von Zeitbudgets möglich. Die Bereiche mussten sich qualitativ unterscheiden und weitestgehend voneinander abgrenzbar sein. Waren bestimmte Verhaltenseinheiten bereichsübergreifend, musste abgewogen werden, welche Bedeutung sie für die Beantwortungen der Fragestellungen hatten. Im Zweifelsfall wurde meist ihrer Wirkung auf die Fokusfamilie Vorrang gegeben, da die Analyse der Gruppendynamik im Vordergrund der Arbeit steht. Am Beispiel der Verhaltenseinheit Scannen (Definition S. 247) soll diese Vorgehensweise kurz verdeutlicht werden.

Während der Datenaufnahme wurde Scannen synonym gebraucht für „Wachsamkeitsverhalten“, “Ausschau halten“ und „schweifende Blicke“ (König 1992) was der Raubfeindvermeidung, der Ressourcensicherung und der Kommunikation mit fremden Artgenossen dienen konnte. Für die Interpretation des Verhaltens war es notwendig, dass Raubfeinde oder Artgenossen im Sichtbereich des Fokustieres auch für den Beobachter sichtbar waren. Da eine Verhaltenseinheit nicht mehreren Bereichen zugeordnet werden konnte, mussten Festlegungen getroffen werden. Befanden sich Artgenossen im Sichtfeld des Fokustieres, wurde das Scannen der nicht-taktilen Interaktion mit fremden Artgenossen zugeordnet. Konnte kein Artgenosse gesichtet werden oder zeigte sich ein Greifvogel, erfolgte die Einordnung in den Bereich Erwerb und Sichern von Ressourcen und Raubfeindvermeidung (RR).

Schwierig ist die Zuordnung multifunktionaler Verhaltenseinheiten, wie z.B. Katzbuckeln. Es hat einerseits eine starke Wirkung auf die Familienmitglieder, andererseits unter Umständen auch auf fremde Artgenossen in Nachbararealen und drittens ist es eine spezielle Form der Lokomotion. Eine Zuordnung zu mehreren Verhaltensbereichen ist damit möglich. Um die Auswirkungen des Katzbuckelns auf die Familie auswerten zu können, wurde diese Verhaltenseinheit dem Bereich nicht-taktile Interaktion mit Familienmitgliedern zugeordnet.

\subsubsection{Verhaltensbereiche}

\subsubsection{1 taktile Interaktion innerhalb der Familie (TIF)}

Unter dem Verhaltensbereich TIF werden alle Verhaltenseinheiten zusammengefasst, bei denen eine körperliche Berührung mit mindestens einem Familienmitglied zustande kommt $(\rightarrow$ taktil). Mit der Herstellung des Körperkontaktes wird der persönliche Raum (personal space) des beteiligten Tieres unterschritten und dies lässt Aussagen über die Qualität der

\footnotetext{
${ }^{7}$ von der Bezirksregierung Braunschweig unter Aktenzeichen 509.42502/01-21.01 genehmigt
} 
Interaktion zwischen zwei Tieren zu. Da alle übrigen Verhaltensbereiche physischen Kontakt ausschließen, ist die Zuordnung eindeutig.

Affiliative VE: Grooming, Auffordern zum Grooming, Beschnuppern, Handauflegen, Kontaktaufnahme, Körperkontakt, Kuscheln, Spielaufforderung, Soziales Liegen, Soziales Ruhen, Soziales Schlafen, Sozialspiel, Umarmen

Dissoziative VE: Angreifen, Beißen, Schlagen, Schupsen, Unterkriechen, Wegdrücken Jungtierfürsorge: Hüten, Jungtierabsteigen, Jungtierabstreifen, Jungtieraufnahme, Jungtieraufsteigen, Jungtiertragen, Jungtierübernahme, Tragtierwechsel, Zitzenkontakt

Sexualverhalten: Folgen, Genitalkontrollieren, Kopulation, Urinlecken

Die Jungtierfürsorge nimmt hier eine Sonderstellung ein, weil Jungtiere auch als schützenswerte Ressourcen begriffen werden können. Es ist daher eine Zuordnung in den Bereich Erwerb und Sichern von Ressourcen und Raubfeindvermeidung denkbar. Da der Zugang zu den Jungtieren wesentlich von der Duldung der Eltern abhängt, so dass die Jungtierfürsorge eine spezielle Form der taktilen Interaktion mit Familienmitgliedern darstellt, wird sie in diesem Bereich belassen.

\subsubsection{2 nicht-taktile Interaktion innerhalb der Familie (NTF)}

Im Verhaltensbereich NTF werden jene Verhaltenseinheiten zusammengefasst, die eine Signalwirkung auf die Familienmitglieder ausüben, ohne in physischen Kontakt zu treten. Dazu gehören außer visueller und akustischer Kommunikation auch lokomotorisches Verhalten, welches gezielt auf ein Familienmitglied zu- oder von ihm wegführt und damit das Fokustier mit dem Familienmitglied in Beziehung setzt.

akustische Interaktion: Futtertriller, Keckern, Lippenschmatzen, Mobbing-Laute, Phi (leise), Quärren, Triller, Twitter, Whisper

visuelle Interaktion: Abwenden, Anblicken, Annähern, Ausweichen, Beobachten von Familienmitgliedern, Blickabwenden, Büschelanlegen, Büschelheben, Büschelklappen, Büschelstellen, Fellsträuben, Fliehen, Genitalpräsentieren, Katzbuckellaufen/-stehen ${ }^{8}$, Langen, Nachlaufen,

\footnotetext{
${ }^{8}$ Katzbuckellaufen wird unter visuelle Interaktion eingeordnet, da es eine optische Wirkung auf die Gruppenmitglieder hat. Im engeren Sinne stellt es keine visuelle Interaktion dar, weil sich das katzbuckelnde Tier nicht direkt mit Familienmitgliedern in Beziehung setzt.
} 
Schlitzaugen machen, Sich nähern, Spiel beenden, Über die Schulter blicken, Weggehen, Wegjagen

Distanzphi- und Doppelphi-Laute können Signalwirkungen sowohl auf die eigene Familie, als auch auf fremde Artgenossen haben. Aus methodischen Gründen mussten diese Verhaltenseinheiten trotz Überschneidungen einem Verhaltensbereich zugeordnet werden. Da sie vorwiegend in Kontexten auftreten, die eine Interaktion mit fremden Artgenossen beinhalten, findet die Zuordnung in den Bereich nicht-taktile Interaktion mit fremden Artgenossen statt.

\subsubsection{3 nicht-taktile Interaktion mit fremden Artgenossen (NTA)}

In diesem Bereich finden sich Verhaltenseinheiten, die vor allem der akustischen und visuellen Kommunikation mit fremden Artgenossen dienen und die über weite Entfernungen reichen. Da während der Experimente insbesondere Reaktionen der Familienmitglieder auf fremde Artgenossen untersucht wurden, finden Distanz- und Doppel-Phi ihre Einordnung in diesem Bereich, auch wenn in diesem Fall ihre kommunikative Funktion innerhalb der Familie vernachlässigt wird.

akustische Interaktion: Distanz-Phi, Doppel-Phi ${ }^{9}$, Twitter zu fremden Artgenossen visuelle Interaktion: Beobachten von fremden Artgenossen, Scannen $\mathrm{zu}$ fremden Artgenossen

\subsubsection{Erwerb und Sichern von Ressourcen und Raubfeindvermeidung (RR)}

Die Verhaltenseinheiten dieses Bereichs dienen der Erkundung, des Erwerbs und der Sicherung eigener Ressourcen. Ressourcen können Beutetiere und andere Nahrung, das gemeinsame Streifgebiet, die Familie ${ }^{10}$, als auch die Gesundheit, bzw. das eigene Leben sein $(\rightarrow$ Raubfeindvermeidung). Die olfaktorische Kommunikationskomponente der Verhaltenseinheiten Substratmarkieren, Bissmarke anlegen und Markenkontrollen zwischen Familienmitgliedern wird hier vernachlässigt.

Nahrungserwerb: Anblicken und Beobachten von Gegenständen und Nahrungsressourcen, Benagen, Erkunden, Foraging, Futterbetteln, Futterstehlen, Futtertransport, Kauen, Lecken, Manipulieren

\footnotetext{
${ }^{9}$ Distanz-Phi und Doppel-Phi können auch der Kommunikation innerhalb der Gruppe dienen.

${ }^{10}$ Jungtierfürsorge wird aufgrund der taktilen Komponente und sozialen Sonderstellung der Jungtiere dem Verhaltensbereich taktile Interaktion mit Familienmitgliedern zugeordnet.
} 
Sicherung des Streifgebietes ${ }^{11}$ : Bissmarke anlegen, Markenkontrolle, Nagen, Scannen, Substratmarkieren

Raubfeindvermeidung: $\quad$ Greifvogel-Scannen, Umherblicken, Verstecken, Warnlaut

\subsubsection{Stoffwechselbedingtes Verhalten und Körperpflege (SK)}

$\mathrm{Zu}$ stoffwechselbedingtem Verhalten und Körperpflege zählen Verhaltenseinheiten, die aufgrund von physischen Bedürfnissen auftreten.

stoffwechselbedingte VE: Defäkieren, Fressen, Gähnen, Räkeln, Ruhen, Schlafen, Trinken, Urinieren

Körperpflege: $\quad$ Autogrooming, Kratzen, Lippenlecken, Sich schütteln

\subsubsection{Körperhaltung und Lokomotion (KL)}

Hier werden verschiedene Körperhaltungen und Positionen, als auch Verhaltensweisen zusammengefasst, die in unterschiedlichen Kontexten $\mathrm{zu}$ beobachten sind und deren Bedeutung häufig erst durch den Kontext ersichtlich werden ( $\rightarrow$ Lokomotion):

Aufblicken, Aufrichten, Beobachtet Beobachter, Ducken, Hängen, Klammern, Kopfdrehen, Liegen, Lokomotion, Schleichen, Sit-and-look, Solitärspiel, Umwenden, Verharren

\subsubsection{Umgang mit Datenlücken}

Wenn ein Fokustier während eines Intervalls im instantaneous sampling nicht beobachtet werden konnte, blieb das Intervall leer und ging nicht in die Auswertung der Daten mit ein. Konnten beispielsweise von 100 Intervallen 10 nicht eingetragen werden, so waren 90 Intervalle die Grundlage für die Analyse und wurden mit 100\% gleichgesetzt.

\subsubsection{Zeitbudgets}

Für die Familie und jedes Fokustier wurden Zeitbudgets (engl. time-budgets) für beide Beobachtungsjahre erstellt, so dass ermittelt werden konnte, zu welchen Anteilen an der Gesamtaktivität die Familie bzw. die Familienmitglieder Verhaltenseinheiten der einzelnen Verhaltenbereiche zeigten. Die mit instantaneous sampling erhobenen Daten geben nicht die

\footnotetext{
${ }^{11}$ Dreschler (2002) weist darauf hin, dass C. jacchus-Familien im Semifreiland ein Gebiet, möglicherweise Territorium, innerhalb ihres Streifgebietes bevorzugt markieren (Substratmarkieren).
} 
gesamte Aktivität, sondern nur Ausschnitte der Aktivität der Tiere in einer bestimmten Beobachtungsperiode wieder. Zur Vereinfachung der Darstellung der Ergebnisse wird jedoch der Begriff Gesamtaktivität verwendet. Da die Versuchsbedingungen von einem zum nächsten Experiment variierten, wurde für jede Phase pro Tier ein eigenes Zeitbudget angefertigt, dem jeweils die Daten fünf aufeinander folgender statistischer Tage $(=40$ Beobachtungen pro Tier) zugrunde liegen. Die Gesamtbeobachtungsdauer eines Experiments wurde mit $100 \%$ der Aktivität gleichgesetzt, so dass die Anzahl der Intervallhäufigkeiten ${ }^{12}$ der Verhaltensbereiche in Prozent angegeben werden konnten.

\subsubsection{Rangpositionen}

Rangpositionen, in der Literatur oft als „status“ bezeichnet, können z.B. über die Analyse von Dominanzbeziehungen der Mitglieder einer Sozietät ermittelt werden (Hinde 1983). Durch die Ermittlung der Rangposition kann auf die Bedeutung des Affen für die Familie geschlossen werden (Sade 1972, für Macaca mulatta), womit möglicherweise Hinweise auf Konkurrenz um Sozialpartner gefunden werden können.

Sade (1972) schlägt als beste Verhaltenseinheit für die Analyse und Beschreibung eines solchen status von Altweltaffen das Grooming-Verhalten (Fellpflege mit sozialer Bedeutung) vor. Die Bedeutung eines Affen für seine Sozietät wird dabei durch die Anzahl der Tiere, die ihn groomen, angegeben. Je größer die Anzahl der Gruppenmitglieder ist, die ein bestimmtes Tier groomen, umso höher ist seine Grooming-Rangposition innerhalb der Gruppe. Da bei Altweltaffen hochrangige Tiere häufiger gegroomt werden als niederrangige Tiere, haben hochrangige Tiere auch eine höhere Grooming-Rangposition (Sade 1972).

Eine Grooming-Rangposition kann z.B. über 1-Schritt-Verbindungen (1-step-linkages) ermittelt werden (siehe Abb. 2.3). Über die Analyse von 1-Schritt-Verbindungen haben Tier a und Tier c die gleiche Grooming-Rangposition, da sie beide von einem Tier gegroomt werden. Wird ein Tier von mehreren Tieren gegroomt (Tier d) hat es eine höhere GroomingRangposition.

\footnotetext{
${ }^{12}$ Als Intervallhäufigkeit wird die Anzahl der Einträge einer Verhaltenseinheit im instantaneous samplingProtokoll bezeichnet. Sie ist keine reale Häufigkeit, sondern ein Maß für die Wahrscheinlichkeit des Auftretens dieser Verhaltenseinheit.
} 


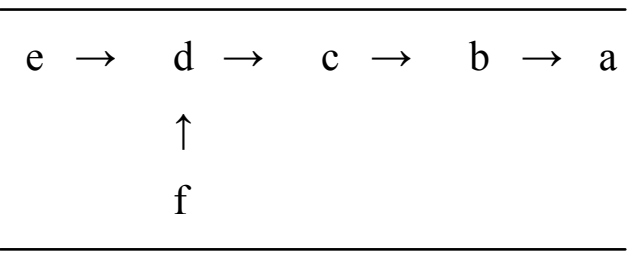

Abb. 2.3 Darstellung eines Grooming-Netzwerks nach Sade (1972); Pfeile: Richtung der Grooming-Episode; a f: hypothetische Affen. Tier a wird gegroomt von Tier b, Tier b wird gegroomt von Tier c, Tier c wird gegroomt von Tier $\mathrm{d}$ und Tier d wird gegroomt von Tier e und Tier f. Über die Analyse von 1-Schritt-Verbindungen haben Tier a und Tier c die gleiche Grooming-Rangposition, da sie beide von einem Affen gegroomt werden. Tier $d$ hat eine höhere Grooming-Rangposition, da es von zwei Tieren gegroomt wird.

Da bei Callitrichiden keine lineare Hierarchie nachgewiesen werden konnte (vgl. Epple 1975 und Rothe 1978) werden in dieser Studie für die Analyse der Gruppendynamik relevante Rangpositionen verschiedener affiliativer Verhaltenseinheiten anhand von 1-step-linkages berechnet und anhand von dissoziativen Interaktionen an den Futterplätzen ermittelt.

\subsubsection{Clique-Analyse}

Mit Hilfe der Clique-Analyse nach Sade (1972) können Subgruppen innerhalb größerer Sozietäten ermittelt werden. Die Datengrundlage der Clique-Analyse sind Interaktionen (z.B. Grooming) der Gruppenmitglieder. Dabei können mehrere Cliquen nebeneinander existieren und einzelne Individuen können Mitglieder verschiedener Cliquen sein. 1-Cliquen, die auch als extreme Cliquen bezeichnet werden, beschreiben eine Gemeinschaft von mindestens drei Affen, die jeweils einander als Interaktionspartner gewählt haben (Abb. 2.4.).

Mitglieder von 2-Cliquen sind Tiere, die nur über einen gemeinsamen Interaktionspartner miteinander verbunden sind. Der Berechnung von Cliquen werden Interaktions-Matrizen ${ }^{13}$ zugrunde gelegt, die in binäre Matrizen ${ }^{14}$ umgewandelt und mehrfach mit sich selbst multipliziert werden. Die einzelnen Schritte der Vorgehensweise sind im Appendix II bei Sade (1972) dargestellt.

Bisher wurde dem Grooming bei Neuweltaffen nicht die soziale Bedeutung wie bei den Affen der Alten Welt zugeordnet (Dunbar 1991). Da jüngste Untersuchungen bei verschiedenen Platyrrhini-Arten jedoch im Gegensatz zu Dunbar's Annahme von einer wichtigen sozialen

\footnotetext{
${ }^{13}$ Interaktions-Matrizen: Die Anzahl der Interaktionen zwischen Gruppenmitgliedern werden in der Form einer Kreuztabelle (Matrize) dargestellt. Die Aktoren stehen in der ersten Spalte und die Rezipienten in der ersten Zeile der Kreuztabelle. Die Einträge sind die Häufigkeiten der Interaktionen (Beispiel im Anhang S. 246).

${ }^{14}$ binäre Matrize: Binäre Matrizen erhält man, indem die Einträge $\neq 0$ einer Interaktions-Matrize $=1$ gesetzt werden und Einträge $=0$ nicht verändert werden.
} 
Bedeutung dieses Verhaltens ausgehen (z.B. Lazaro-Perea et al. 2004 für Callithrix jacchus, Manson et al. 1999 für Cebus capucinus, Sanchez-Viagra et al. 1998 für Alouatta seniculus), wird für das Grooming neben anderen affiliativen Interaktionen (z.B. Kuscheln, Handauflegen und soziales Spielen) eine Clique-Analyse durchgeführt.

(a)

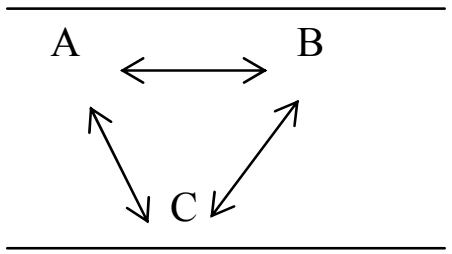

(b)

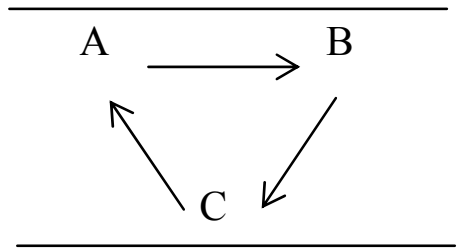

Abb. 2.4 (a) Darstellung einer 1-Clique: mindestens drei Affen (z.B. A, B und C) interagieren wechselseitig (bidirektional) miteinander. (b) Darstellung einer 2-Clique: Interaktionen können unidirektional verlaufen. Pfeile: Richtung der Interaktion, z.B. Grooming.

\subsubsection{Auswertung der Transponderdaten}

Die Auswertung der Transponderdaten wurde mit einem von der Firma Dataquest entwickelten Computerprogramm basierend auf MS Access 2.0 durchgeführt.

Aufenthaltswahrscheinlichkeiten der Familienmitglieder in bestimmten Arealen, Betritthäufigkeiten der Familienmitglieder von bestimmten Arealen und die räumliche Assoziation eines Familienmitgliedes $\mathrm{zu}$ allen anderen Familienmitgliedern (kurz Restfamilie oder Familie) wurden mit dem Programm berechnet.

Fiel eine Schleuse in der Mitte der Anlage aus, so wurde ein Ortswechsel der Affen in das betreffende Areal solange nicht registriert, bis der Affe durch eine andere funktionsfähige Schleuse gelaufen war. Das Programm berücksichtigte dabei, dass ein Areal übersprungen wurde und gab demnach an, dass der Affe eine unbestimmte Zeit in dem nichtregistrierten Areal gewesen sein musste. Die genaue Dauer konnte nicht nachvollzogen werden, genauso wenig wie die Aufenthaltsdauer in dem Areal, in dem der Affe registriert wurde. Aufgrund der Registrierung des Tieres bei Betreten und Verlassen eines der Areale, ging jedoch die Angabe über die maximale Aufenthaltsdauer in die Auswertung mit ein. Bei Ausfällen der Transponderschleusen 10 und 15, also der Schleusen, welche die Randareale anbinden (vgl. Abb. 2.2, S.23), konnte nachträglich nicht erfasst werden, ob sich ein Tier im Randareal oder im benachbarten Areal aufgehalten hatte. Das Programm war jedoch so konzipiert, dass die Wahrscheinlichkeit über die Verlässlichkeit der Daten mit in die Auswertung einbezogen wurde. 


\subsubsection{Statistische Verfahren}

Die Konfigurations-Frequenz-Analyse (KFA) nach Bortz et al. (1990) wurde für den Vergleich von Häufigkeiten angewendet. Dabei wurden Beobachtungswerte mit Erwartungswerten verglichen.

$\mathrm{EWB}=e \pm(u \times \sqrt{ } e(1-p))$

$e=N / n=$ Erwartungswert

$u=$ Abzissenwert der Standardnormalverteilung für $\alpha^{*}$

$p=1 / n$

$n=$ Anzahl der Klassen (Tiere)

$\mathrm{N}=$ Gesamtheit der beobachteten Werte

Der $\alpha$-Wert muss der jeweiligen Anzahl der Klassen ( $n$ ) angepasst werden.

Die Abhängigkeit einer Variablen von ein oder zwei Faktoren wurde mit einer Varianzanalyse (ANOVA) überprüft, sofern keine Abweichung von der Normalverteilung auftrat. Ergaben die erhobenen Daten keine Normalverteilung, wurden die Varianzen auf Homogenität geprüft und die Korrelation von Varianzen und Mittelwerten getestet. Trat eine negative oder keine Korrelation auf, war eine ANOVA zulässig. War eine positive Korrelation zu verzeichnen, musste auf die Ein-Wege-Rangvarianzanalyse nach Kruskal und Wallis ausgewichen werden. Bei einer Stichprobe von $n>200$ war in jedem Fall eine ANOVA zulässig. Als post-hoc Test einer ANOVA diente entweder der Student Newman Keuls-Test oder der Least Significant Difference-Test (Bortz et al. 1990).

Verhaltenunterschiede zwischen rausgeworfenen und verbliebenen Nachkommen der Fokusfamilie wurden mit dem Mann-Whitney-U-Test auf Zufälligkeit überprüft (Siegel 1987).

Für Korrelationsanalysen wurde die Spearman-Rang-Korrelation verwendet, da die Daten mindestens auf Ordinalniveau erhoben wurden, was als Voraussetzung für diesen Test gilt (Siegel 1987). Korrelationsanalysen wurden nur dann durchgeführt, wenn die Stichprobe mindestens sieben Untersuchungseinheiten (Intervalle, Tage oder Tiere) betrug.

Für alle statistischen Verfahren wurde eine Signifikanzgrenze von $\alpha=0,05$ festgelegt.

Die Varianz- und Korrelationsanalysen wurden mit Hilfe des Computerprogramms Statistica für Windows 97 (Version 5) der Firma Statsoft erstellt. 


\section{ERGEBNISSE UND DISKUSSION}

\subsection{Zeitbudgets}

„A decision can be thought of as a commitment of time to some particular course of action when an animal has more than one alternative behaviour available to it. "(Lucas et al. 1996b, S.501)

Das Zitat von Lucas et al. (1996) erklärt deutlich, warum für eine Analyse von Entscheidungen eines Tieres (oder einer Gruppe) die Kenntnis seines Zeitbudgets von großer Bedeutung ist. Mit jeder Entscheidung, die ein Individuum trifft, widmet es einen Abschnitt seiner ihm zur Verfügung stehenden Zeit einer bestimmten Aktivität. Während dieses Zeitabschnitts kann es gleichzeitig nicht anders handeln, so dass jede Entscheidung ein Kompromiss der Aufteilung der ihm zur Verfügung stehenden Zeit für bestimmte Aktivitäten ist.

Für die Überprüfung der Hypothesen 2, 5, 6 und 8 war es wichtig, eine Analyse der Zeitbudgets der gesamten Familie, als auch der einzelnen Familienmitglieder durchzuführen. Die Ergebnisse über die Zeitbudgets werden daher in diesem Kapitel zusammengefasst, um Wiederholungen der Ergebnisse zu vermeiden.

\subsubsection{Zeitbudget der Familie im ersten Beobachtungsjahr}

Die Anteile der sechs Verhaltensbereiche an der Gesamtaktivität der Familie waren im ersten Beobachtungsjahr unterschiedlich groß (Kruskal-Wallis-Test, $\mathrm{N}=24, \mathrm{H}=20,98, \mathrm{p}<0,001$ ). Die Familienmitglieder verbrachten im Durchschnitt 25,2\% der beobachteten Zeit mit taktiler Interaktion mit Familienmitgliedern und 36,8\% mit dem Erwerb und der Sicherung von Ressourcen und der Raubfeindvermeidung. 7,4\% entfielen auf stoffwechselbedingte Aktivitäten und 6,6\% auf nicht-taktile Interaktion mit Familienmitgliedern. Um 0,3\% lag der Anteil nicht-takiler Interaktion mit fremden Artgenossen (Nachbarn und Locktiere). Körperhaltung und Lokomotion, die im Zusammenhang mit diversen Kontexten stehen konnten, füllten 23,2\% der Gesamtaktivität (Abb. 3.1).

Zwischen den vier Experimentalphasen (B1 bis $\mathrm{C} 2$ ) bestanden keine Unterschiede im Zeitbudget der Familie (Kruskal-Wallis-Test, N=24, H=0,113, n. s.). 


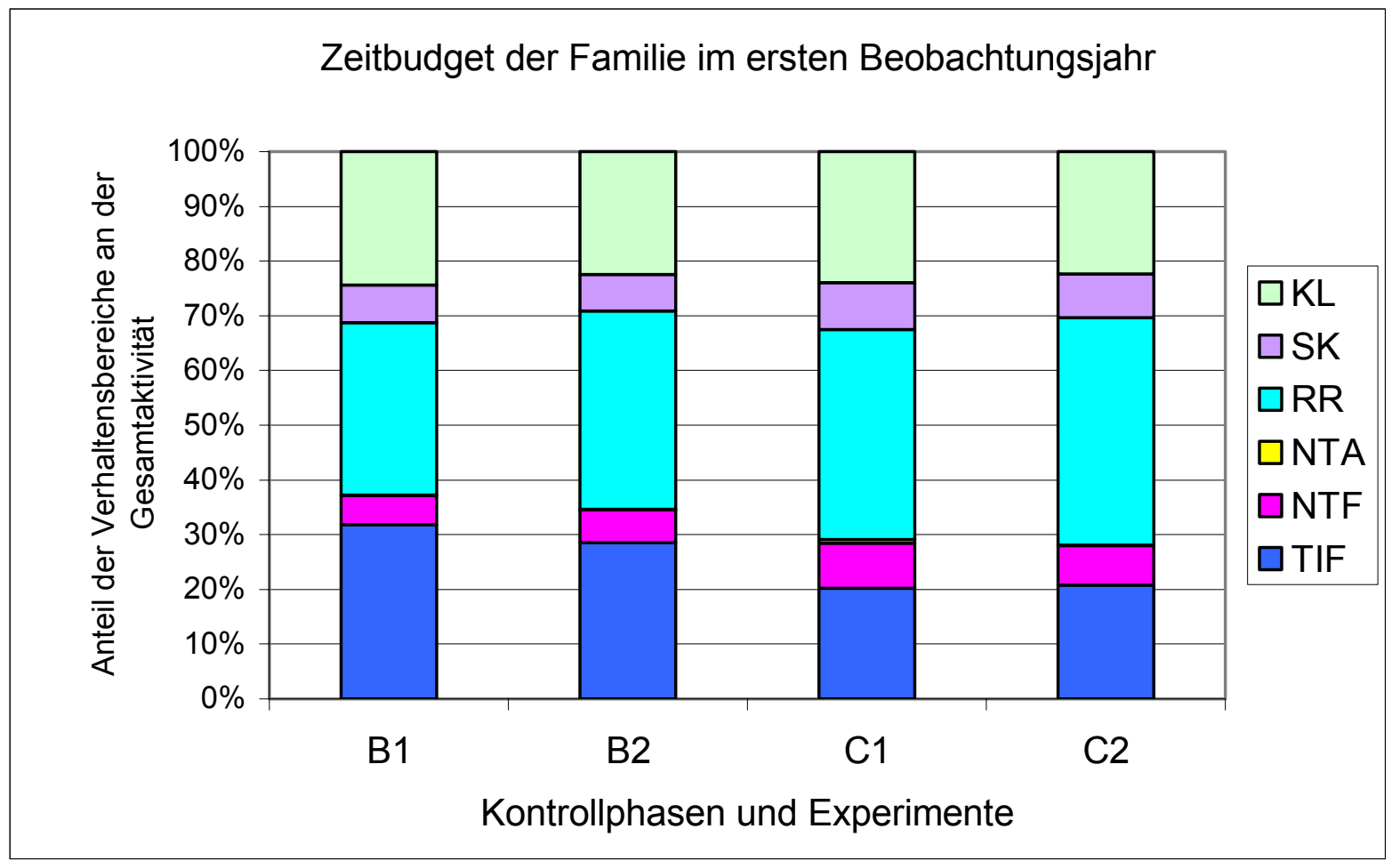

Abb. 3.1 Zeitbudget der Familie J18 im ersten Beobachtungsjahr 2000. In der Graphik ist der prozentuale Anteil der Verhaltensbereiche (TIF=taktile Interaktion mit Familienmitgliedern, NTF=nicht-taktile Interaktion mit Familienmitgliedern, NTA=nicht-taktile Interaktion mit fremden Artgenossen, RR=Ressourcensicherung und Raubfeindvermeidung, SK=stoffwechselbedingtes Verhalten und Körperpflege, $\mathrm{KL}=\mathrm{Körperhaltung}$ und Lokomotion) an der Gesamtaktivität (100\%) für jedes Experiment (B1-C2) eingetragen.

\subsubsection{Zeitbudget der Familie im zweiten Beobachtungsjahr}

Auch im zweiten Beobachtungsjahr unterschieden sich die prozentualen Anteile der Verhaltensbereiche am Zeitbudget voneinander (Kruskal-Wallis-Test, $\mathrm{N}=24, \mathrm{H}=22,01$, $\mathrm{p}<0,001)$. Der durchschnittliche Anteil an taktiler Interaktion mit Familienmitgliedern lag bei 35,5\% und war damit ca. 10\% höher als im ersten Jahr. Erwerb und Sichern von Ressourcen und Raubfeindvermeidung lag mit 27,3\% um ca. 9\% niedriger als im Vorjahr. Die nichttaktile Interaktion mit Familienmitgliedern mit 7,8\% und stoffwechselbedingte Verhaltensweisen mit 6,9\% zeigten keine auffälligen Veränderungen. Die nicht-taktile Interaktion mit fremden Artgenossen stieg im Vergleich zum ersten Jahr um mehr als das vierfache auf 1,4\% der Beobachtungszeit an, während der Bereich Körperhaltung und Lokomotion auf 19,4\% sank (Abb. 3.2). Auch im zweiten Jahr gab es zwischen den vier Experimentalphasen (E1 bis E4) wieder keine Unterschiede im Zeitbudget der Familie (Kruskal-Wallis-Test, N=24, $\mathrm{H}=0,287$, n.s.). Wurden die Zeitbudgets der beiden Beobachtungsjahre miteinander verglichen, zeigte sich keine Veränderung. In Abhängigkeit von den Beobachtungsjahren gab es allerdings Veränderungen in den Anteilen der einzelnen Verhaltensbereiche am Zeitbudget der Familie (Abb. 3.3). Während der Anteil an taktiler Interaktion im zweiten Beobachtungs- 
jahr deutlich anstieg, zeigte sich bei den Verhaltensbereichen RR und KL eine Verringerung im zweiten Beobachtungsjahr. Die Verhaltensbereiche NTF, NTA und SK änderten sich nicht.

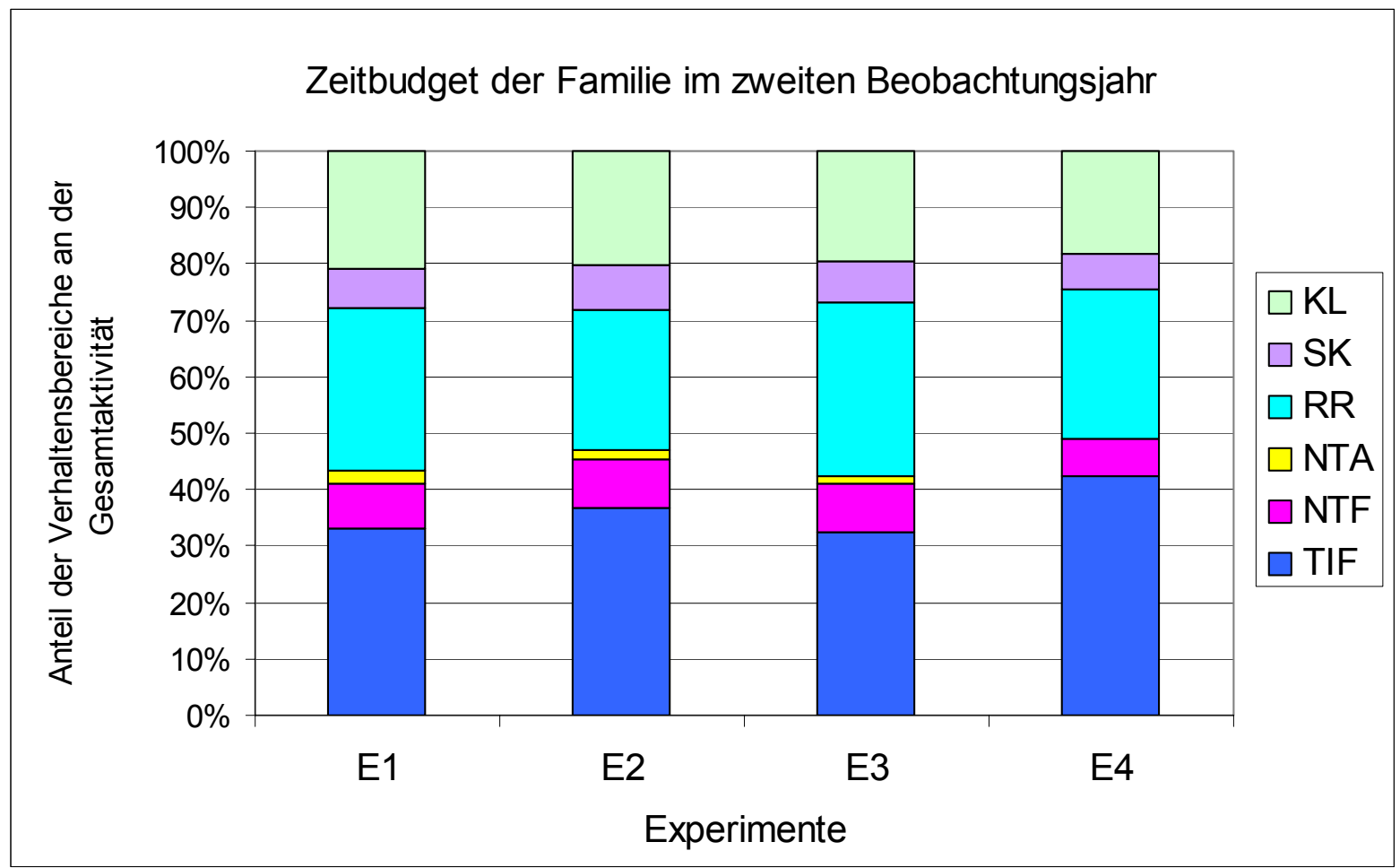

Abb. 3.2 Zeitbudget der Familie J18 im zweiten Beobachtungsjahr. In der Graphik ist der prozentuale Anteil der Verhaltensbereiche an der Gesamtaktivität (100\%) für jedes Experiment (E1-E4) eingetragen. Abkürzungen wie Abb. 3.1.

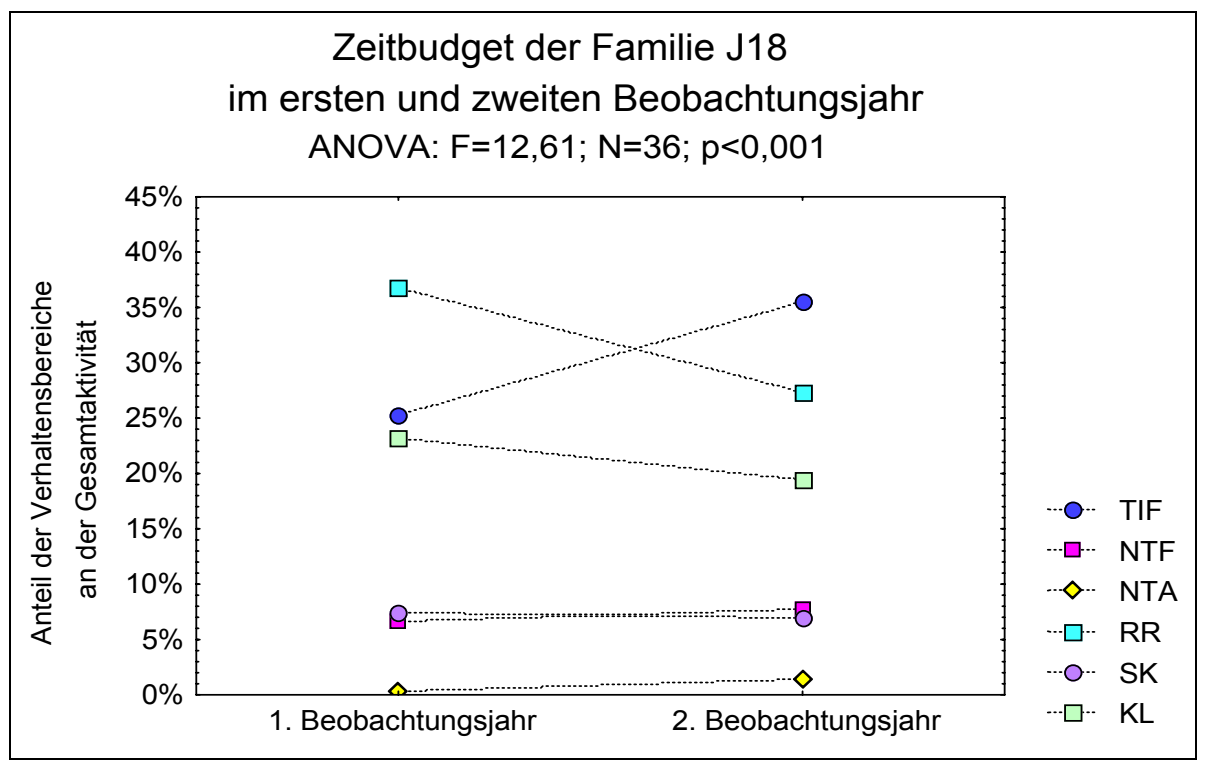

Abb. 3.3 Zeitbudget der Familie J18 im ersten und zweiten Beobachtungsjahr. Es gab keine Unterschiede im Zeitbudget der Familie zwischen den Beobachtungsjahren (ANOVA, $\mathrm{F}=0,06 ; \mathrm{N}=36 ; \mathrm{p}<0,80$ ). In Abhängigkeit von den Beobachtungsjahren veränderten sich jedoch die Anteile der einzelnen Verhaltensbereiche am Zeitbudget der Familie (ANOVA, F=12,61; N=36; $<<0,001$ ). Abkürzungen wie in Abb. 3.1. 


\subsubsection{Vergleich der Zeitbudgets der Familienmitglieder im ersten Beobachtungsjahr}

Bei einem Vergleich der Tiere in der ersten Kontrollphase (Abb. 3.4) auf der Ebene der Verhaltensbereiche zeigte sich, dass die Nachkommen D, E und F seltener als die anderen taktile Interaktionen mit Familienmitgliedern hatten. Der Vater und die Juvenilen I und J interagierten häufiger als erwartet taktil mit Familienmitgliedern (KFA, EW=31,63 n=10). Relativ hohe Anteile an nicht-taktiler Interaktion mit Familienmitgliedern hatten A, E und F, wobei $\mathrm{F}$ signifikant vom Erwartungswert abwich (KFA, EW=5,29 n=10). Die Familienmitglieder zeigten keinen Unterschied im Bereich nicht-taktile Interaktion mit fremden Artgenossen (KFA, EW=0,13 n=10). Die ältesten Nachkommen C, D und E zeigten häufiger als erwartet Ressourcensicherung und Raubfeindvermeidung (KFA, EW=38,66 n=10). F wies einen erhöhten Anteil an stoffwechselbedingtem Verhalten und Körperpflege auf, während H in diesem Verhaltensbereich seltener beobachtet wurde (KFA, EW=6,8 $n=10)$. Im Bereich Körperhaltung und Lokomotion gab es keine Unterschiede zwischen den Familienmitgliedern $(\mathrm{KFA}, \mathrm{EW}=24,34 \mathrm{n}=10)$.

Die zweite Kontrollphase (Abb. 3.5) zeichnete sich dadurch aus, dass D und E seltener als andere taktile Interaktionen hatten, dafür aber häufiger, wie auch $\mathrm{C}$, Ressourcen sicherten. A interagierte häufiger als andere nicht-taktil mit Familienmitgliedern, während E häufiger akustische und visuelle Kontakte zu fremden Artgenossen suchte (KFA, EW=0,20, n=9).

Im ersten Experiment (Abb. 3.6) waren alle drei adulten Nachkommen (C, D und E) seltener an taktiler Interaktion beteiligt und sicherten weiterhin überdurchschnittlich häufig Ressourcen, was A seltener als andere Familienmitglieder tat. Er investierte nach wie vor mehr Zeit in nicht-taktile Interaktion zu Familienmitgliedern. D und E kommunizierten häufiger mit fremden Artgenossen als die anderen (KFA, EW=0,76, n=9).

Im zweiten Experiment (Abb. 3.7) nahm D, wie auch in den Phasen vorher, seltener an taktiler Interaktion teil (KFA, $E W=20,03, n=9)$ und A kommunizierte mehr mit Familienmitgliedern (KFA, EW=7,06, n=9). Der einzige Verhaltensbereich, in den nur einige Familienmitglieder einen Anteil ihrer Zeit investierten, war die nicht-taktile Interaktion mit fremden Artgenossen. Nur das Alpha-Männchen und die adulten Nachkommen C, D und E zeigten im ersten Beobachtungsjahr Aktivitäten aus diesem Verhaltensbereich. 


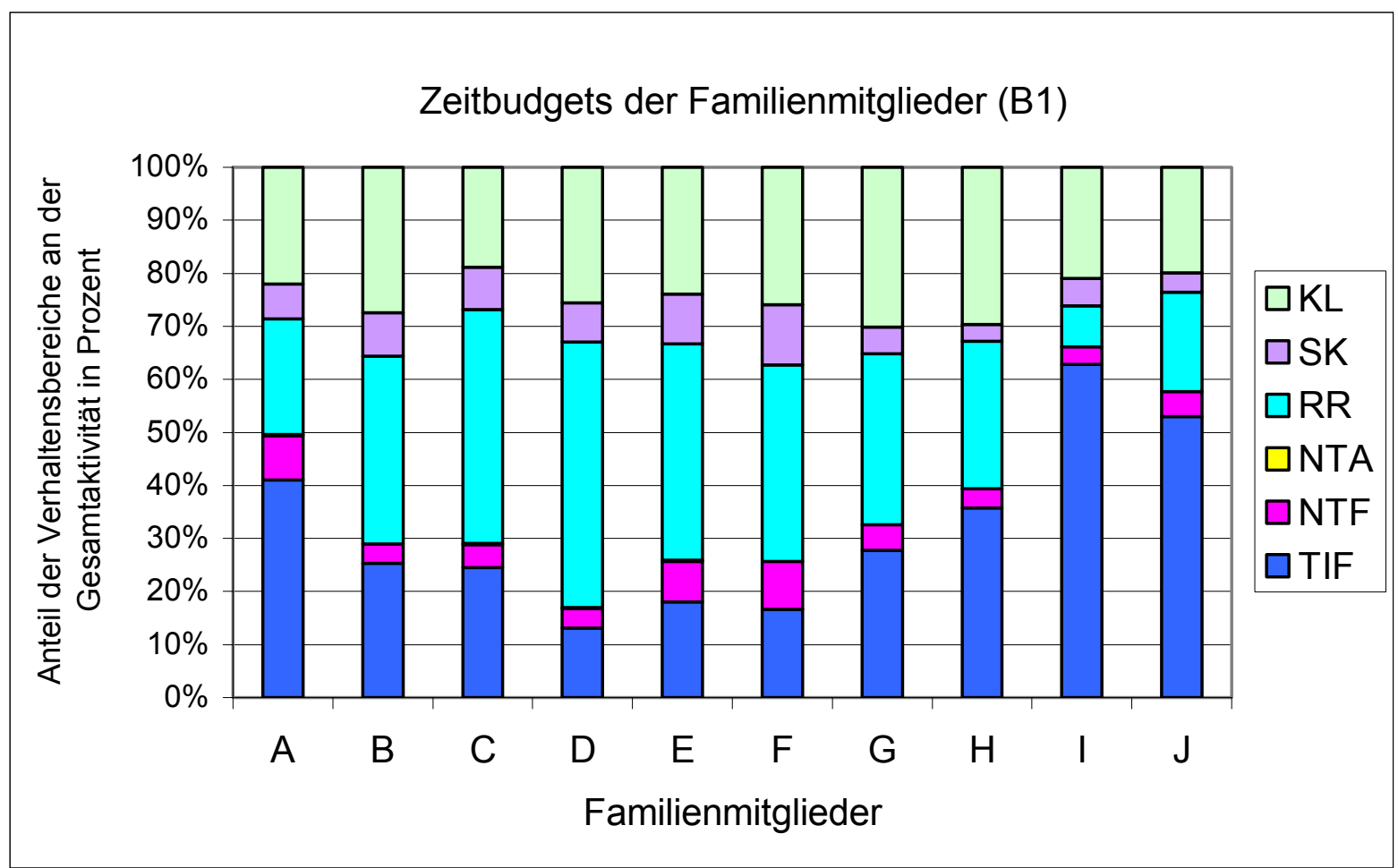

Abb. 3.4 Zeitbudgets der Familienmitglieder in der ersten Kontrollphase (B1) im ersten Beobachtungsjahr. In der Graphik ist der prozentuale Anteil der Verhaltensbereiche an der Gesamtaktivität (100\%) eingetragen. Abkürzungen wie in Abb. 3.1.

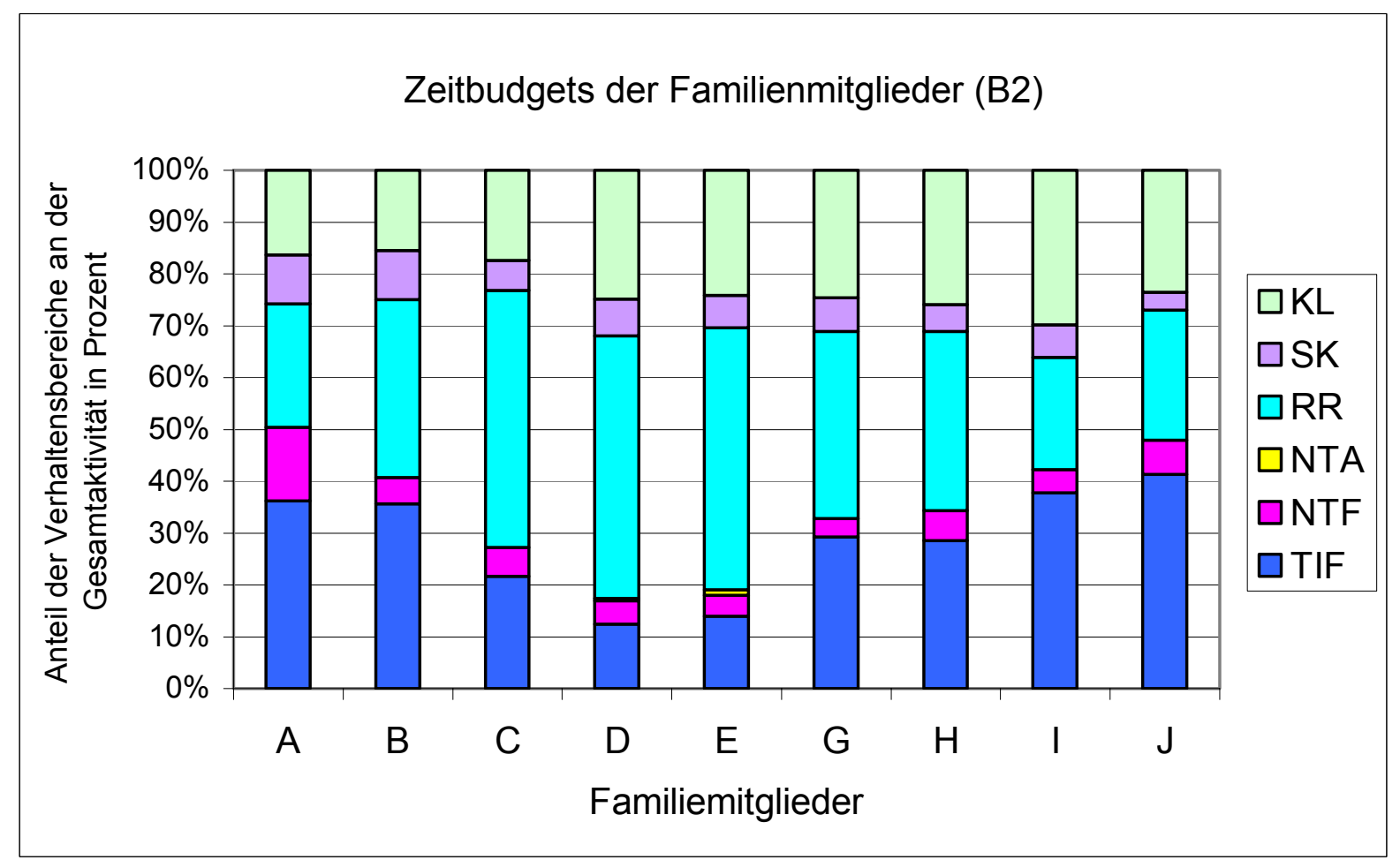

Abb. 3.5 Zeitbudgets der Familienmitglieder in der zweiten Kontrollphase (B2) im ersten Beobachtungsjahr. In der Graphik ist der prozentuale Anteil der Verhaltensbereiche an der Gesamtaktivität (100\%) eingetragen. Abkürzungen wie in Abb. 3.1. 


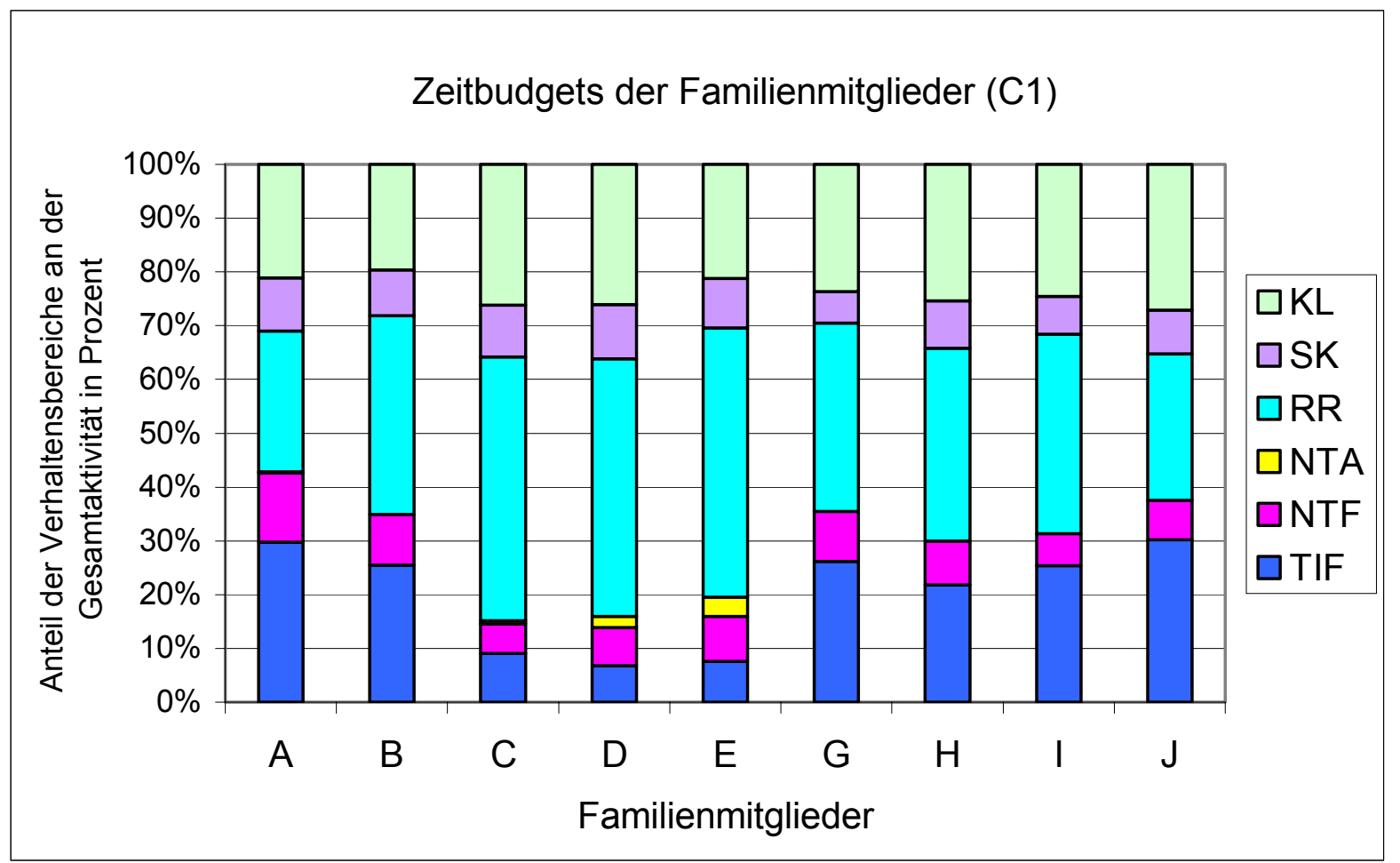

Abb. 3.6 Zeitbudgets der Familienmitglieder im ersten Experiment (C1) im ersten Beobachtungsjahr. In der Graphik ist der prozentuale Anteil der Verhaltensbereiche an der Gesamtaktivität (100\%) eingetragen. Abkürzungen wie in Abb. 3.1.

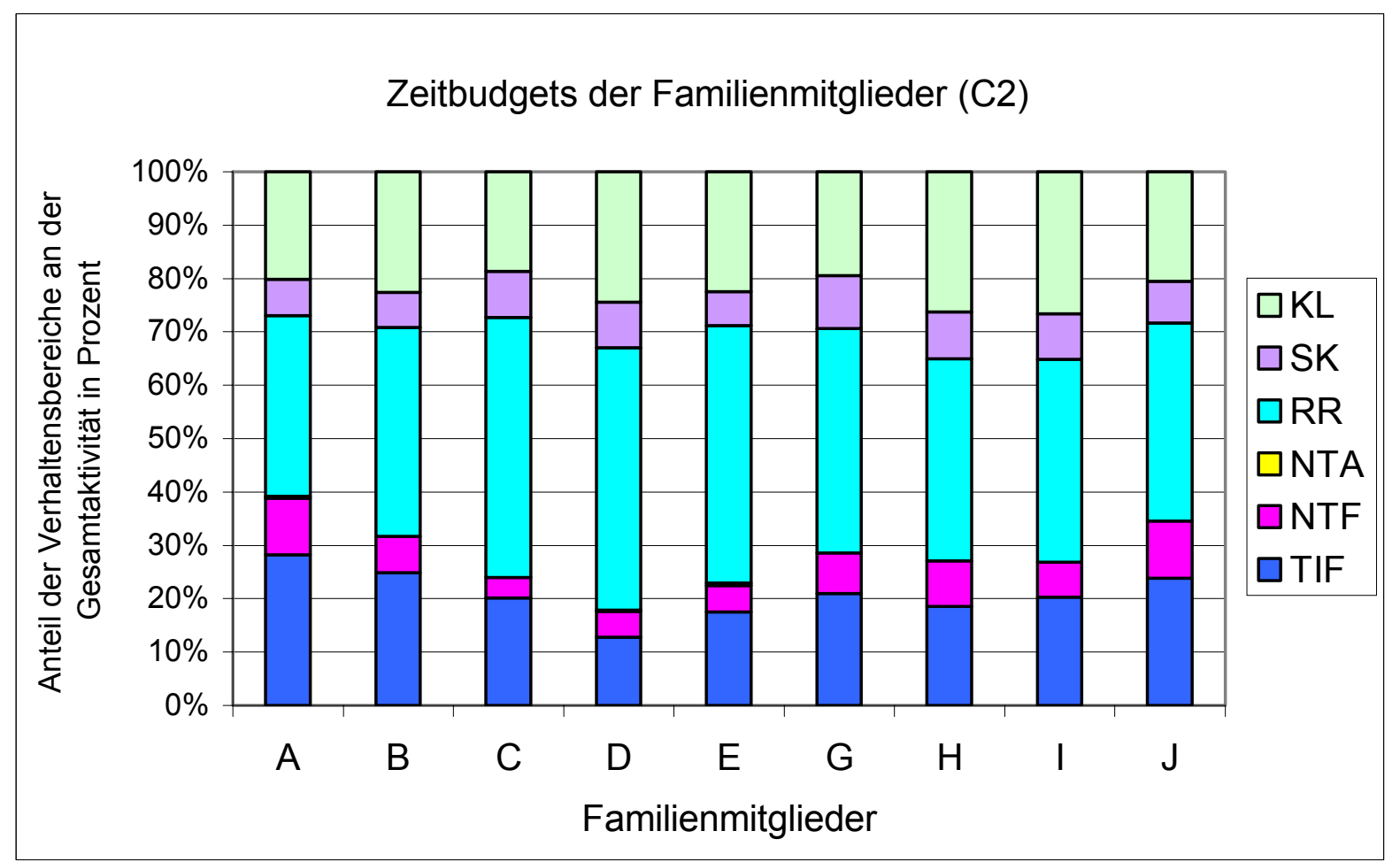

Abb. 3.7 Zeitbudgets der Familienmitglieder im zweiten Experiment (C2) im ersten Beobachtungsjahr. In der Graphik ist der prozentuale Anteil der Verhaltensbereiche an der Gesamtaktivität (100\%) eingetragen. Abkürzungen wie in Abb. 3.1. 


\subsubsection{Vergleich der Zeitbudgets der Familienmitglieder im zweiten Beobachtungsjahr}

Während der Hüttenphase D1 verbrachte A mehr Zeit als andere mit nicht-taktiler Interaktion mit Familienmitgliedern, was D nur unterdurchschnittlich zeigte (KFA, EW=12,05 n=9). J sicherte häufiger Ressourcen (KFA, EW=27,07 $\mathrm{n}=9$ ) und wurde seltener als andere im Bereich Körperhaltung und Lokomotion (KFA, EW=35,01 n=9) beobachtet.

In der ersten Experimentalphase (Abb. 3.8) investierten die ältesten Nachkommen (C, D und G) mehr Zeit in die nicht-taktile Interaktion mit fremden Artgenossen als andere Familienmitglieder (KFA, EW=1,71 n=9), während die taktile Interaktion mit Familienmitgliedern bei D, G und I seltener als erwartet vorkam (KFA, EW=34,46 n=9). Die AlphaTiere hatten häufiger als andere taktile Interaktionen und sicherten seltener Ressourcen (KFA, $E W=28,76 \mathrm{n}=9$ ). J sicherte ebenfalls seltener Ressourcen. C zeigte überdurchschnittlich häufig stoffwechselbedingtes Verhalten und Körperpflege (KFA, EW=6,94 n=9).

Im zweiten Experiment (Abb. 3.9) interagierten D und I seltener als andere taktil mit Familienmitgliedern, während die Alpha-Tiere hier häufiger vertreten waren (KFA, $\mathrm{EW}=36,59 \mathrm{n}=9$ ). I war dafür häufiger als andere mit Ressourcensicherung beschäftigt (KFA, $\mathrm{EW}=24,23 \mathrm{n}=9$ ) und $\mathrm{G}$ kommunizierte häufiger als andere mit fremden Artgenossen (KFA, $\mathrm{EW}=2,03 \mathrm{n}=9$ ). $\mathrm{D}$ investierte im zweiten, wie auch im dritten Experiment, mehr Zeit in stoffwechselbedingte Aktivitäten und Körperpflege (KFA, EW=7,47 n=9).

Im dritten Experiment (Abb. 3.10) zeigte sich $\mathrm{G}$ weniger in taktile Interaktionen (KFA, $\mathrm{EW}=33,01 \mathrm{n}=8$ ) involviert, dafür aber gemeinsam mit $\mathrm{C}$ vermehrt in nicht-taktiler Interaktion mit fremden Artgenossen (KFA, EW=1,39 n=8). Ebenso verbrachten D und G mehr Zeit mit Ressourcensicherung als andere Familienmitglieder (KFA, EW=30,85 n=8). Während A überdurchschnittlich häufig mit Familienmitgliedern nicht-taktil kommunizierte (KFA, $\mathrm{EW}=7,56 \mathrm{n}=8$ ), zeigte er weniger Ressourcensicherung (s.o.), stoffwechselbedingtes Verhalten (KFA, EW=7,22 n=8) und Lokomotion (KFA, EW=19,56 n=8). C trat seltener als andere in nicht-taktile Interaktionen mit Familienmitgliedern (s.o.).

Im vierten Experiment (Abb. 3.11) hatten $\mathrm{C}$ und $\mathrm{D}$ seltener taktile Interaktionen (KFA, $E W=43,47 n=8$ ), während sie überdurchschnittlich häufig Zeit in die Ressourcensicherung investierten (KFA, EW=26,4 n=8). A und B interagierten dafür häufiger taktil mit Familienmitgliedern und A und G zeigten weniger Ressourcensicherung als der Rest der Familie (s.o.). 
$\mathrm{K}$ verbrachte mehr Zeit mit stoffwechselbedingten Aktivitäten als andere Familienmitglieder $(\mathrm{KFA}, \mathrm{EW}=5,9 \mathrm{n}=8)$.

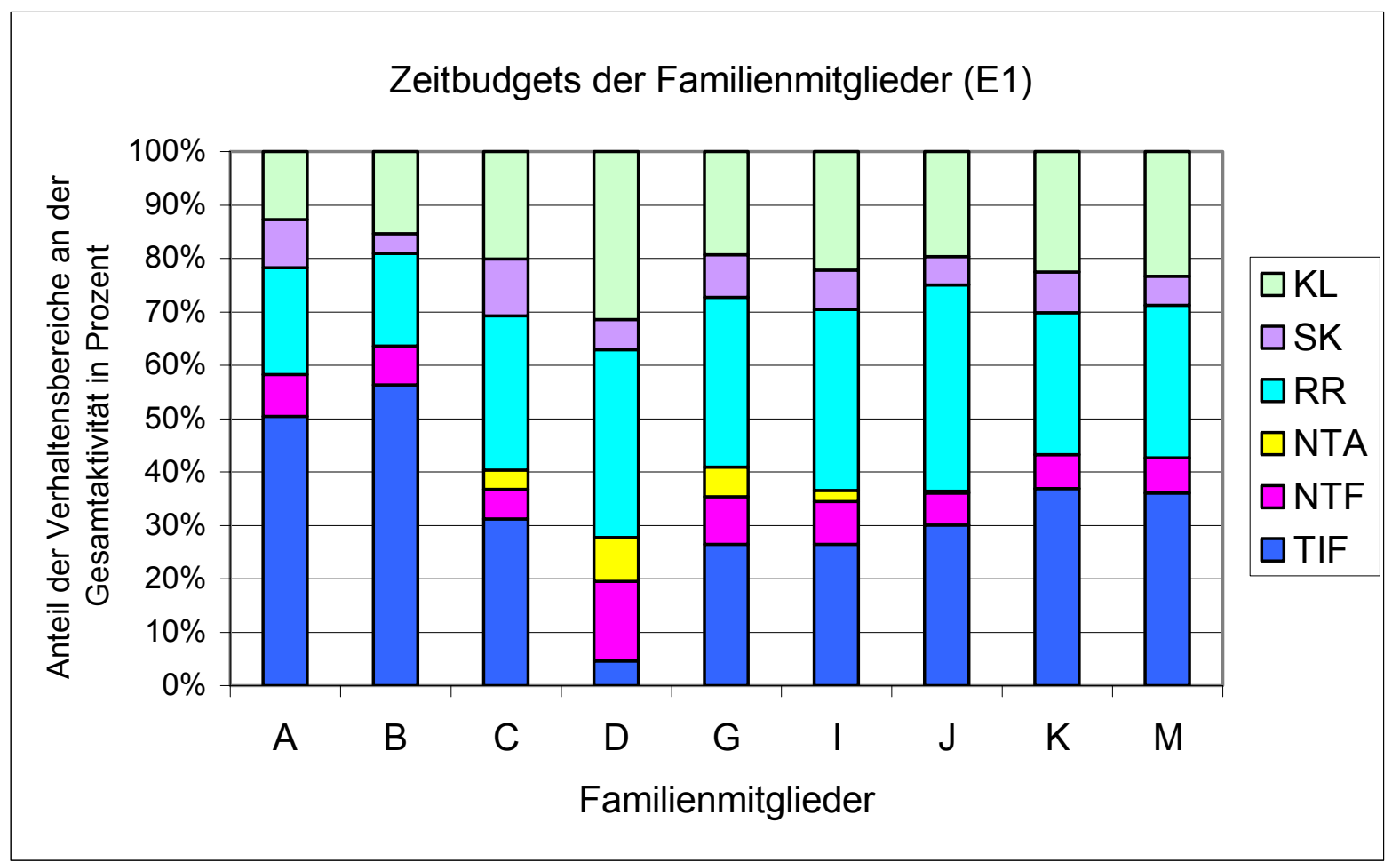

Abb. 3.8 Zeitbudgets der Familienmitglieder im ersten Experiment (E1) im zweiten Beobachtungsjahr. In der Graphik ist der prozentuale Anteil der Verhaltensbereiche an der Gesamtaktivität (100\%) eingetragen. Abkürzungen wie in Abb. 3.1.

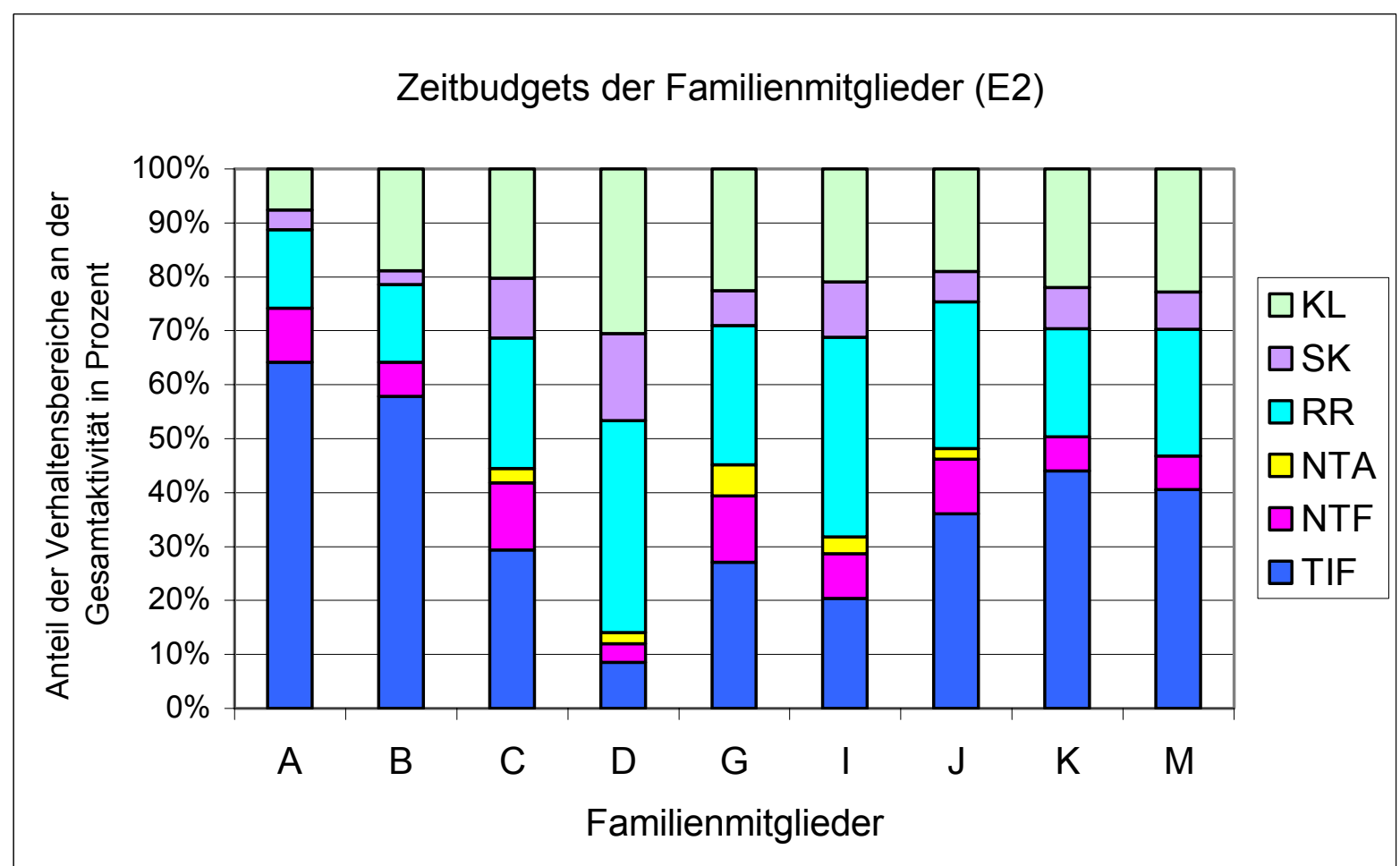

Abb. 3.9 Zeitbudgets der Familienmitglieder im zweiten Experiment (E2) im zweiten Beobachtungsjahr. In der Graphik ist der prozentuale Anteil der Verhaltensbereiche an der Gesamtaktivität (100\%) eingetragen. Abkürzungen wie in Abb. 3.1. 


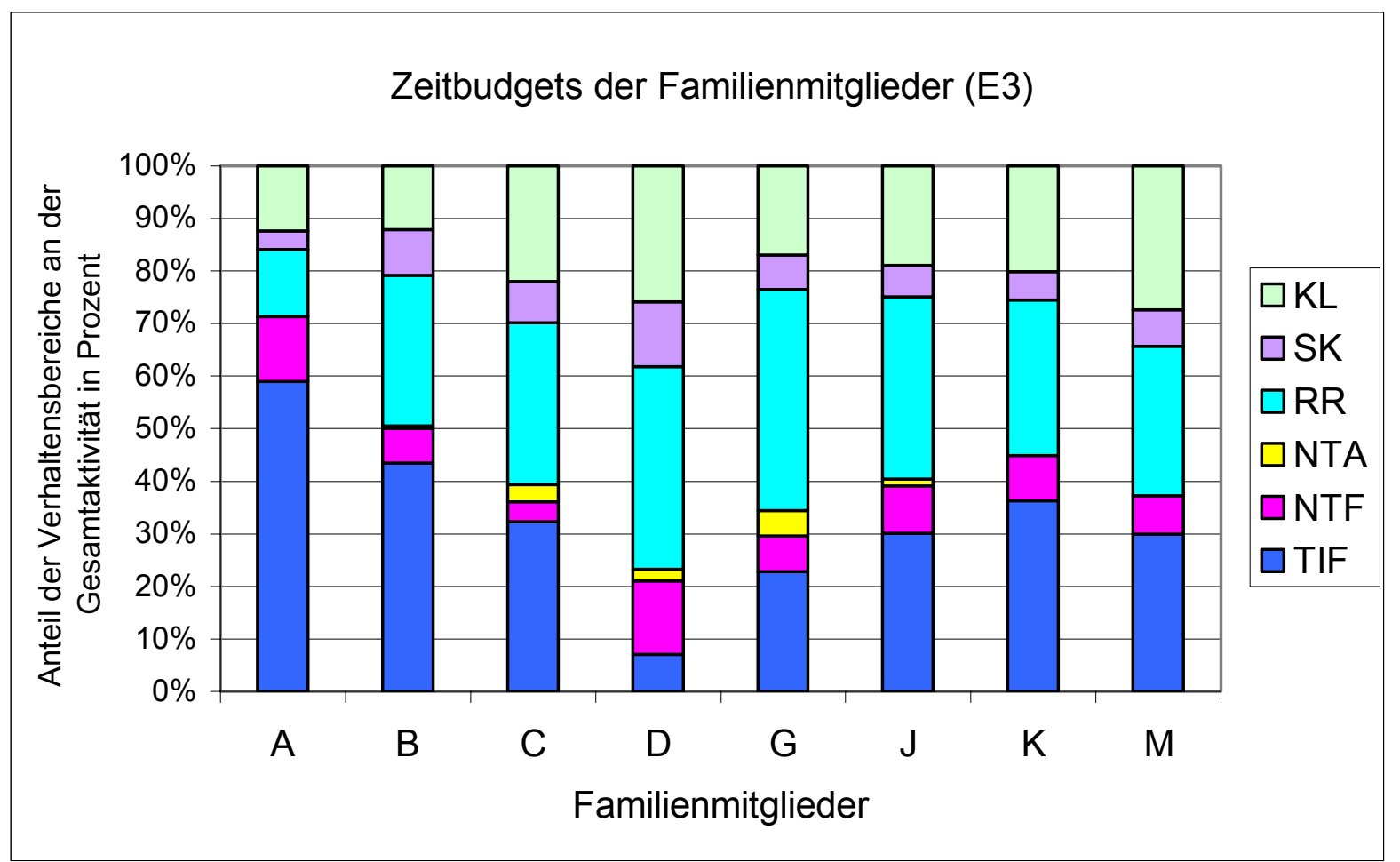

Abb. 3.10 Zeitbudgets der Familienmitglieder im dritten Experiment (E3) im zweiten Beobachtungsjahr. In der Graphik ist der prozentuale Anteil der Verhaltensbereiche an der Gesamtaktivität (100\%) eingetragen. Abkürzungen wie in Abb. 3.1.

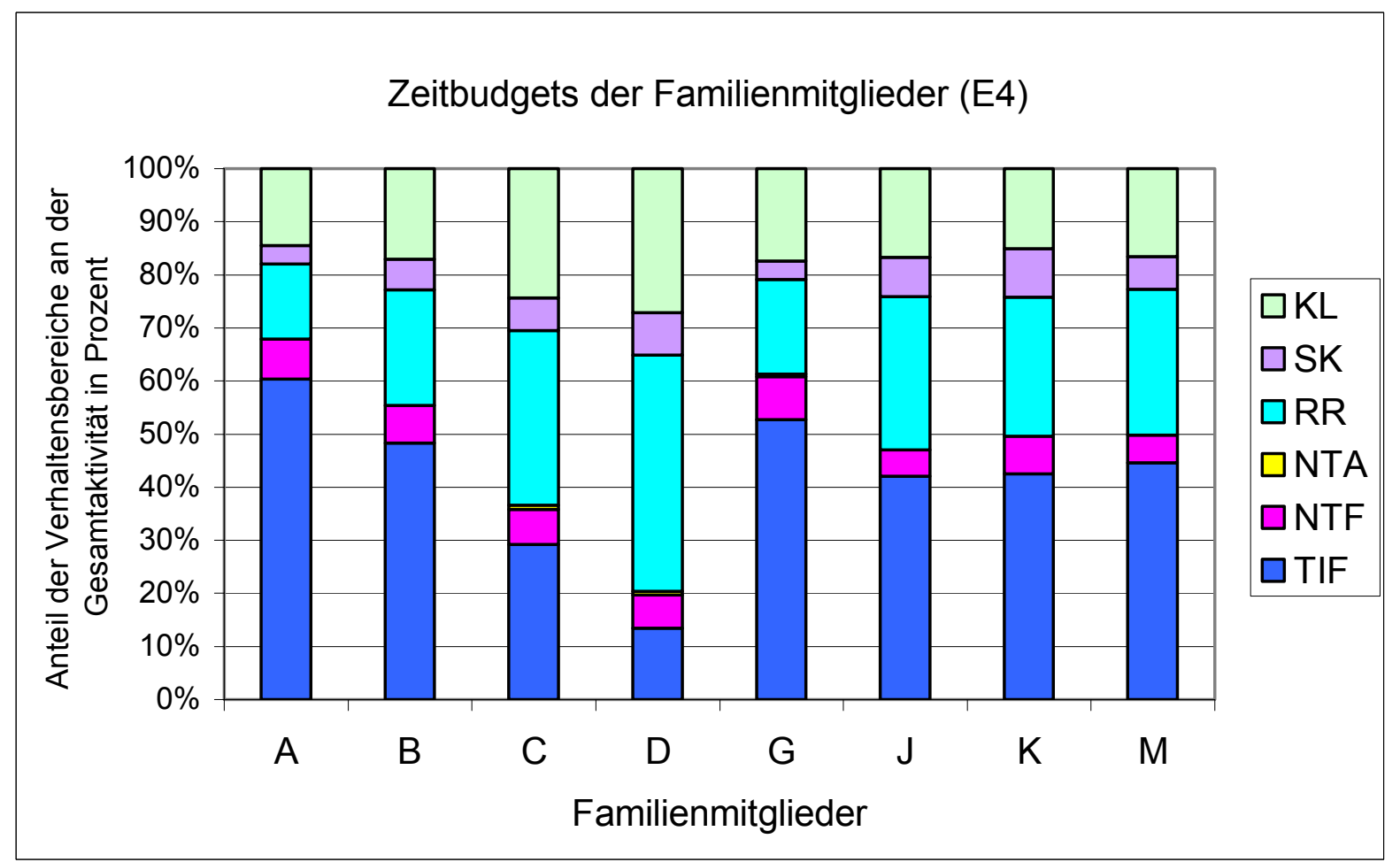

Abb. 3.11 Zeitbudgets der Familienmitglieder im vierten Experiment (E4) im zweiten Beobachtungsjahr. In der Graphik ist der prozentuale Anteil der Verhaltensbereiche an der Gesamtaktivität (100\%) eingetragen. Abkürzungen wie in Abb. 3.1. 
Auch im zweiten Beobachtungsjahr beteiligten sich nicht alle Familienmitglieder an der nichttaktilen Interaktion mit fremden Artgenossen. Alle adulten und subadulten Nachkommen kommunizierten mit fremden Artgenossen. Das Alpha-Weibchen zeigte im dritten Experiment ebenfalls dieses Verhalten.

\subsubsection{Zeitbudgets der Familienmitglieder während der Experimente im ersten Beobachtungsjahr}

Das Alpha-Männchen (Abb. 3.12) hatte während der ersten Kontrollphase häufiger als erwartet taktile Interaktionen mit Familienmitgliedern, in der zweiten Kontrolle und während der Experimente jedoch seltener als erwartet (KFA, EW=33,67 n=4). Während des zweiten Experiments war sein Anteil an Ressourcensicherung und Raubfeindvermeidung höher als in den vorherigen Phasen (KFA, EW=26,29 n=4). Das Alpha-Weibchen (Abb. 3.13) zeigte in der ersten Kontrollphase häufiger als erwartet Verhaltenseinheiten aus dem Bereich Körperhaltung und Lokomotion (KFA, EW=21,2 n=4). In der zweiten Kontrollphase sank dieser Verhaltensbereich unter den Erwartungswert, während sie häufiger als erwartet taktil mit Familienmitgliedern interagierte (KFA, EW=27,65 n=4).

Im ersten Experiment $\mathrm{C} 1$ verringerte sich bei allen adulten Nachkommen $(\mathrm{C}, \mathrm{D}$ und $\mathrm{E}$ : $\mathrm{Abb}$. 3.14 - 3.16) der Anteil an taktiler Interaktion mit Familienmitgliedern (KFA, C: EW=18,71; D: $E W=11,24 ; E: E W=14,27$ n=4). Bei D und $E$ war dafür der Anteil an nicht-taktiler Interaktion mit fremden Artgenossen höher als erwartet (KFA, D: EW=0,76; E: EW=1,36 $\mathrm{n}=4$ ), während bei $\mathrm{C}$ der Anteil am Verhaltensbereich Körperhaltung und Lokomotion zunahm (KFA, EW=20,24 n=4). Der adoleszente Sohn G (Abb. 3.17) investierte im ersten Experiment mehr Zeit als erwartet in Verhaltenseinheiten des Bereiches Körperhaltung und Lokomotion (KFA, EW=24,22 n=4), während sein Anteil an nicht-taktiler Interaktion mit Familienmitgliedern im ersten Experiment (KFA, EW=9,14 n=4) und der Anteil an stoffwechselbedingtem Verhalten und Körperpflege im zweiten Experiment höher als erwartet war (KFA, EW=6,84 n=4). Seine Zwillingsschwester H (Abb. 3.18) zeigte Verhaltenseinheiten dieses Verhaltensbereichs in der ersten Kontrollphase seltener als erwartet (KFA; $\mathrm{EW}=6,47 \mathrm{n}=4$ ), wobei sie häufiger an taktiler Interaktion mit Familienmitgliedern beteiligt war $(\mathrm{KFA}, \mathrm{EW}=25,8 \mathrm{n}=4)$. H hatte im Laufe des Jahres immer weniger taktile Interaktionen mit Familienmitgliedern, bis sie im zweiten Experiment seltener als erwartet taktil mit Familienmitgliedern interagierte. 
Bei den juvenilen I und J (Abb. 3.19 und 3.20) konnte im Verhaltensbereich taktile Interaktion mit Familienmitgliedern ein kontinuierlicher Rückgang im Jahresverlauf beobachtet werden (KFA, I: $E W=36,34 ; \mathrm{J}: \mathrm{EW}=36,78 \mathrm{n}=4$ ), währenddessen ihr Anteil an Ressourcensicherung und Raubfeindvermeidung stetig zunahm (KFA, I: EW=26,1; J: $\mathrm{EW}=26,83 \mathrm{n}=4$ ). Bei J war außerdem eine Steigerung der nicht-taktilen Interaktionen mit Familienmitgliedern im zweiten Experiment zu verzeichnen (KFA, EW=7,25 n=4).

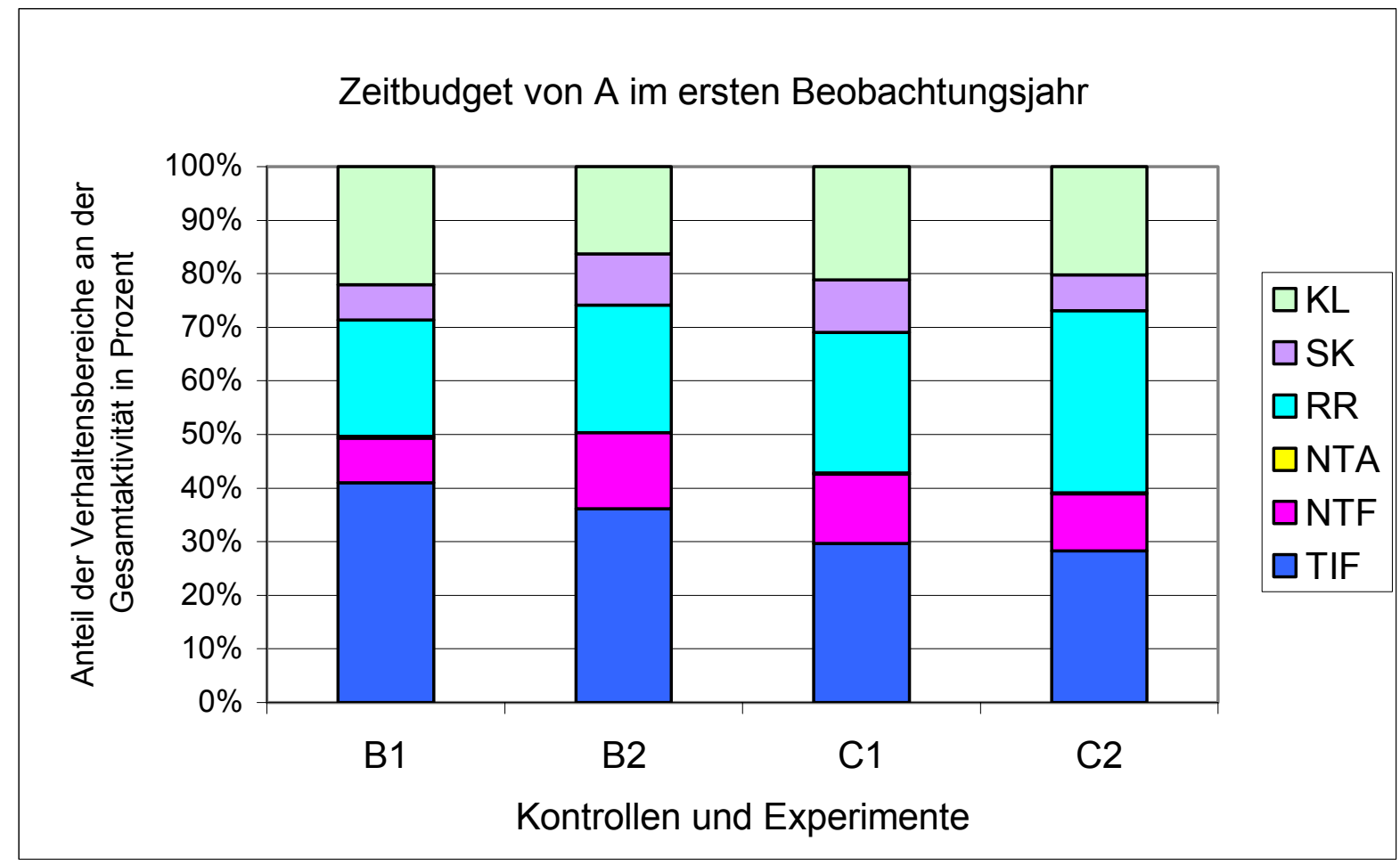

Abb. 3.12 Zeitbudget von A (Alpha-Männchen) im ersten Beobachtungsjahr. In der Graphik ist der prozentuale Anteil der Verhaltensbereiche an der Gesamtaktivität (100\%) für die Kontrollphasen (B1 und B2) und Experimente ( $\mathrm{C} 1$ und $\mathrm{C} 2$ ) eingetragen. 


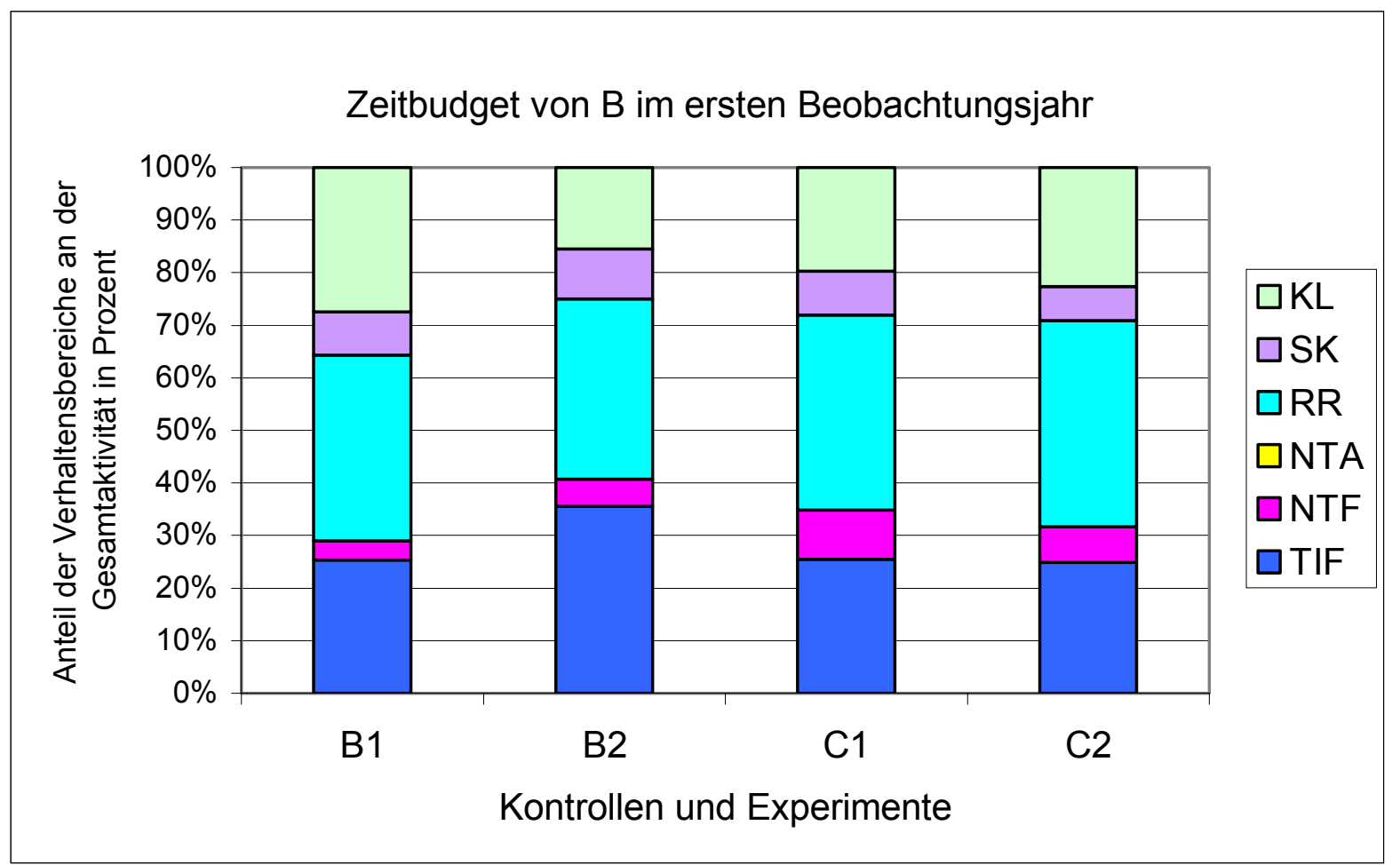

Abb. 3.13 Zeitbudget von B (Alpha-Weibchen) im ersten Beobachtungsjahr. In der Graphik ist der prozentuale Anteil der Verhaltensbereiche an der Gesamtaktivität (100\%) für die Kontrollphasen (B1 und B2) und Experimente ( $\mathrm{C} 1$ und $\mathrm{C} 2$ ) eingetragen.

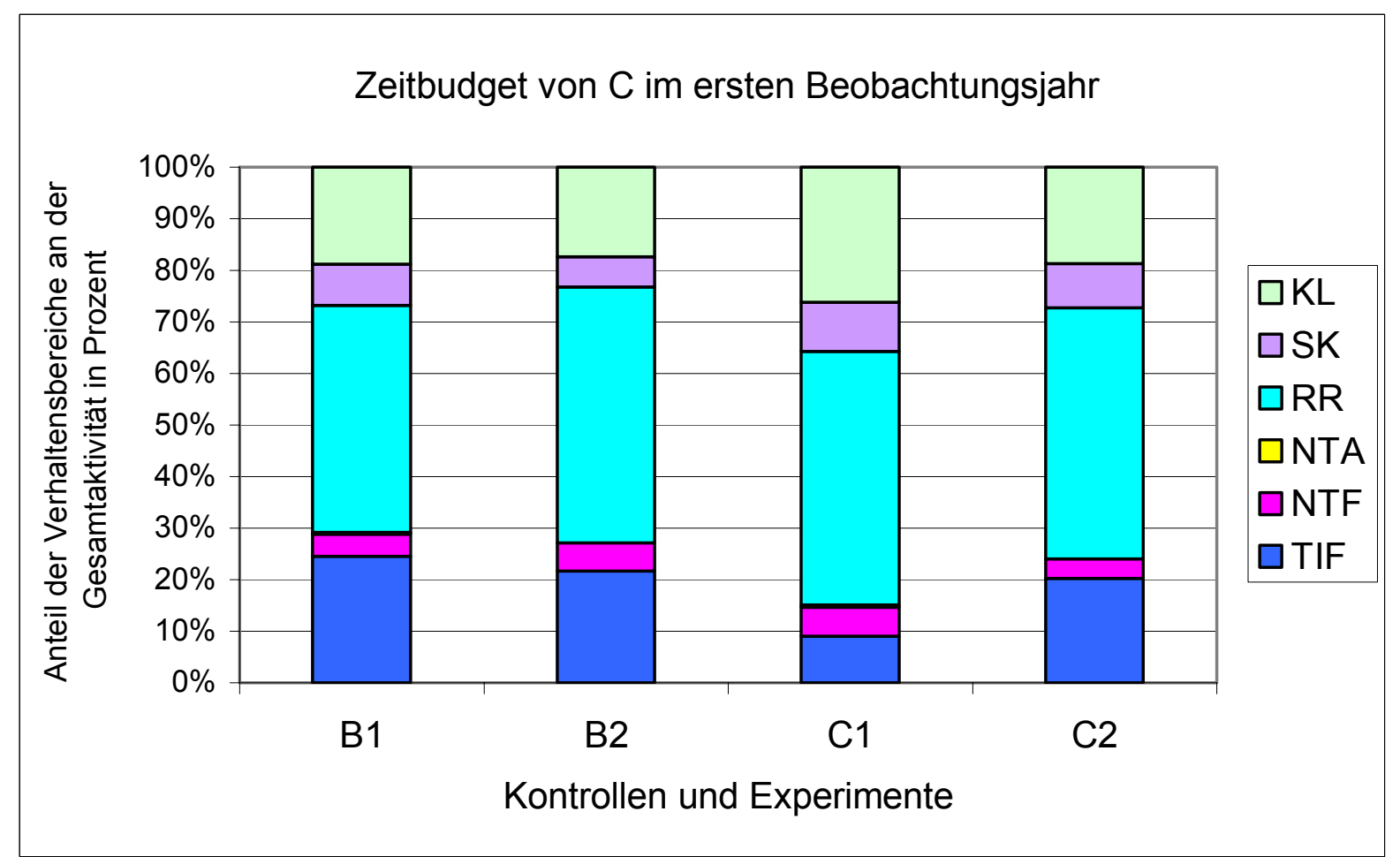

Abb. 3.14 Zeitbudget von C (männlich) im ersten Beobachtungsjahr. In der Graphik ist der prozentuale Anteil der Verhaltensbereiche an der Gesamtaktivität (100\%) für die Kontrollphasen (B1 und B2) und Experimente (C1 und $\mathrm{C} 2$ ) eingetragen. 


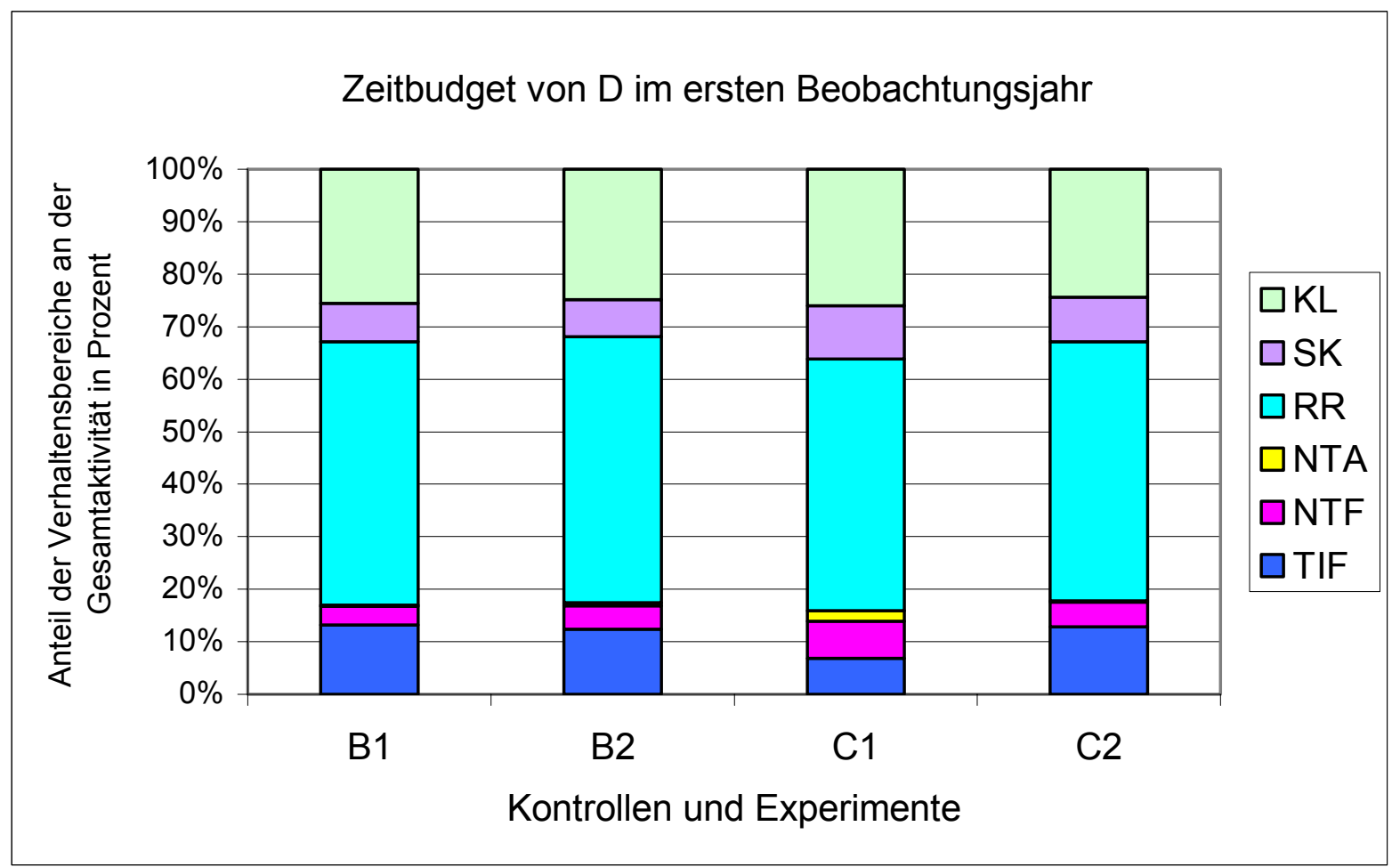

Abb. 3.15 Zeitbudget von D (weiblich) im ersten Beobachtungsjahr. In der Graphik ist der prozentuale Anteil der Verhaltensbereiche an der Gesamtaktivität (100\%) für die Kontrollphasen (B1 und B2) und Experimente (C1 und $\mathrm{C} 2$ ) eingetragen.

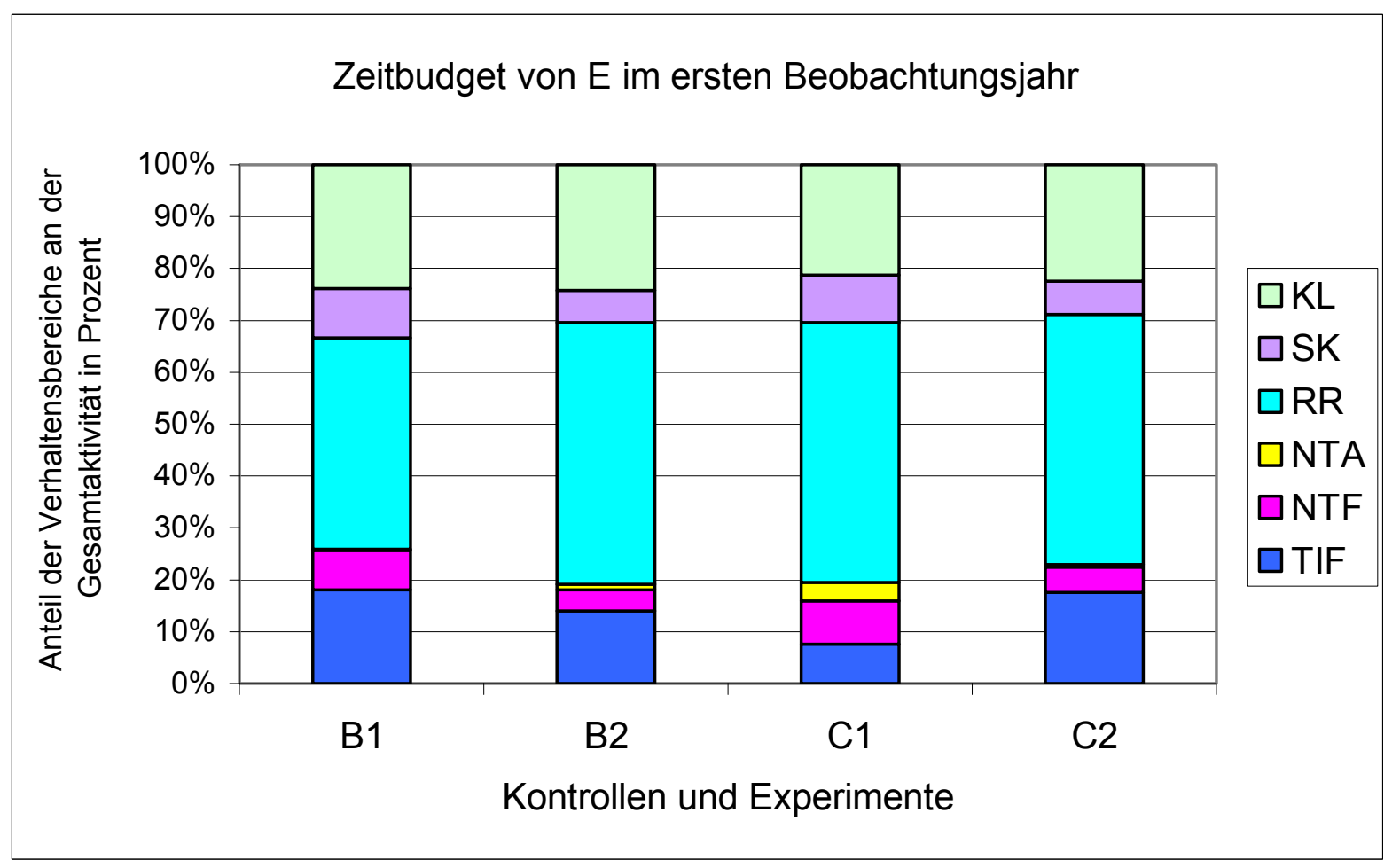

Abb. 3.16 Zeitbudget von E (männlich) im ersten Beobachtungsjahr. In der Graphik ist der prozentuale Anteil der Verhaltensbereiche an der Gesamtaktivität (100\%) für die Kontrollphasen (B1 und B2) und Experimente (C1 und $\mathrm{C} 2$ ) eingetragen. 


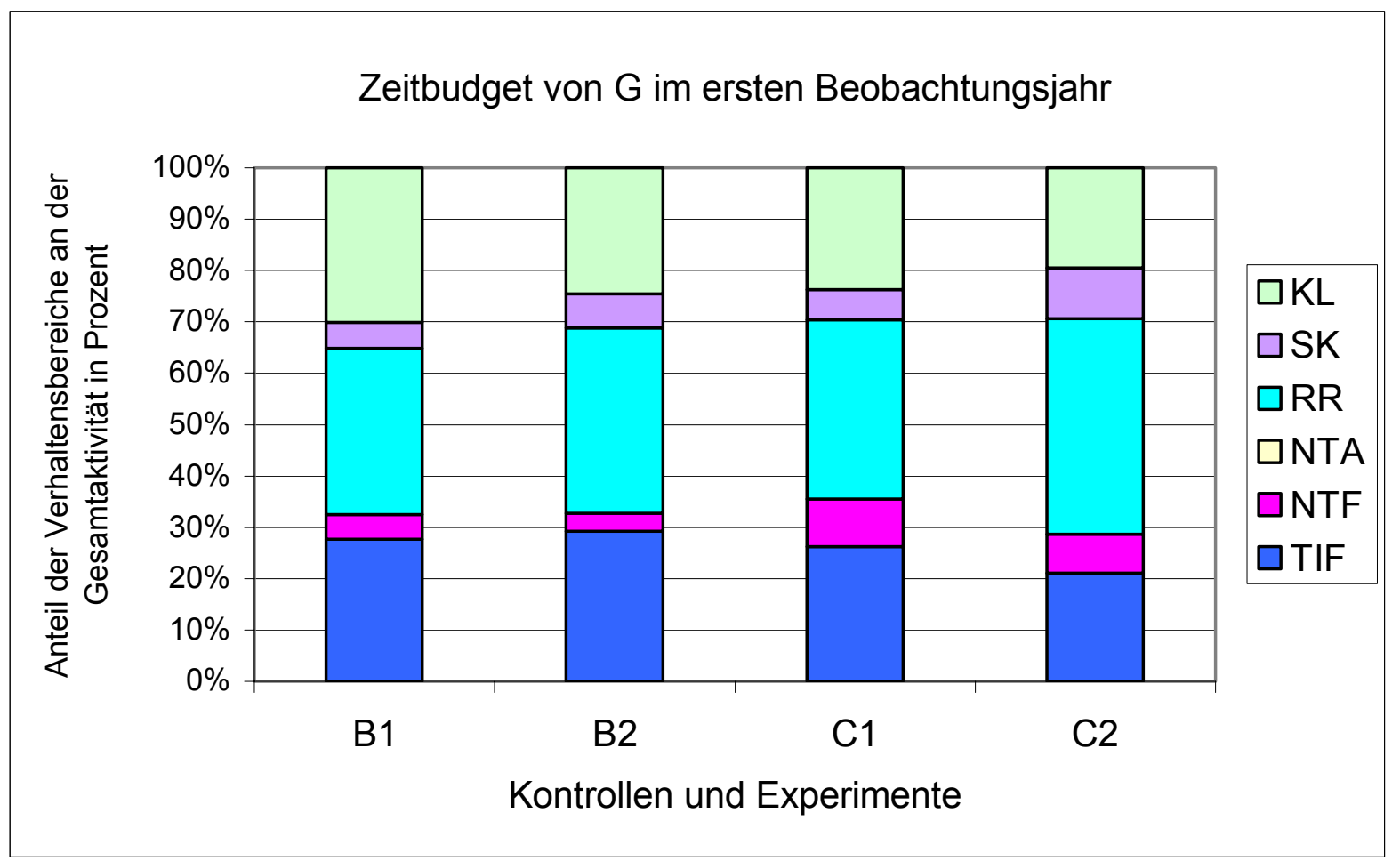

Abb. 3.17 Zeitbudget von G (männlich) im ersten Beobachtungsjahr. In der Graphik ist der prozentuale Anteil der Verhaltensbereiche an der Gesamtaktivität (100\%) für die Kontrollphasen (B1 und B2) und Experimente (C1 und $\mathrm{C} 2$ ) eingetragen.

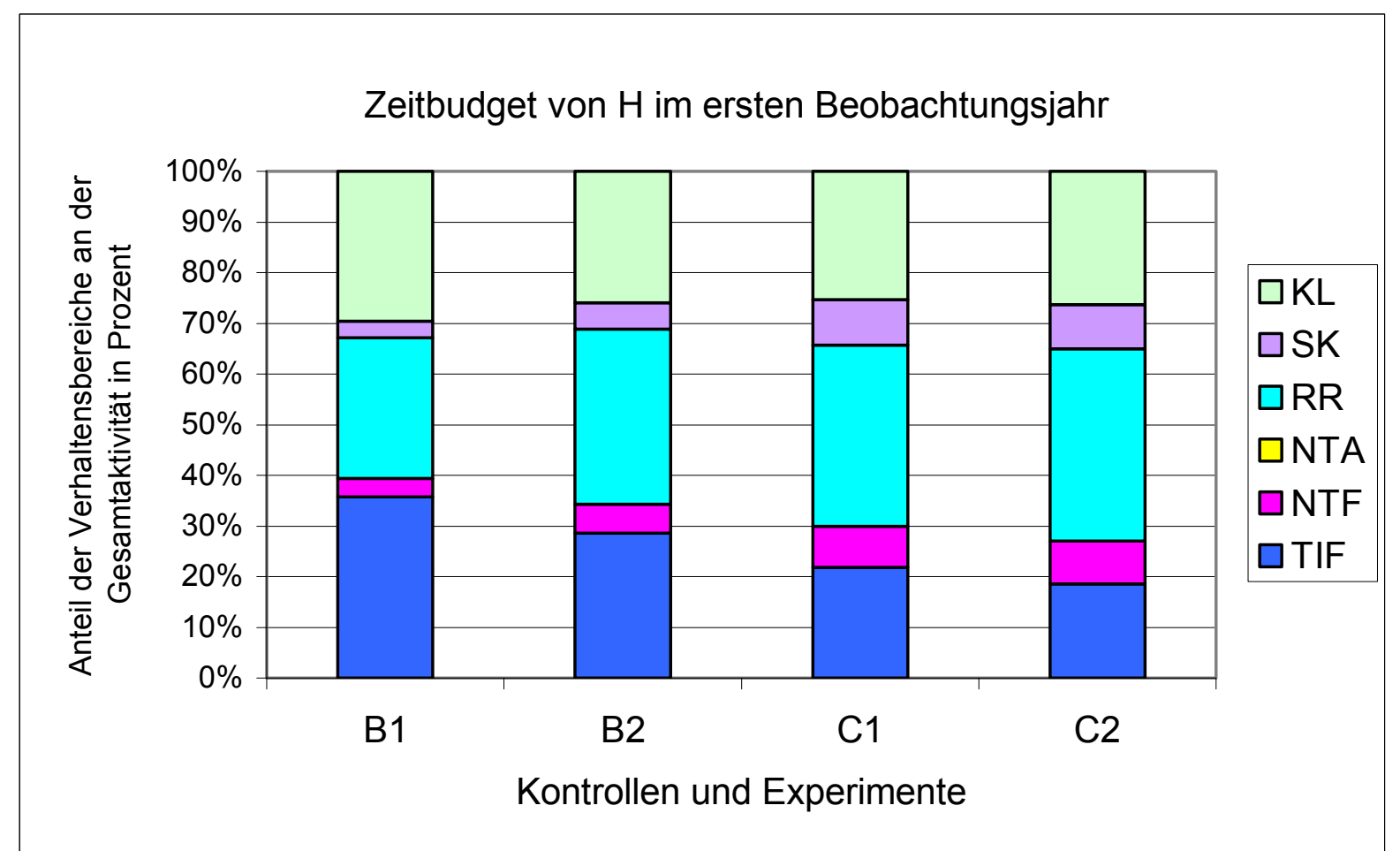

Abb. 3.18 Zeitbudget von H (weiblich) im ersten Beobachtungsjahr. In der Graphik ist der prozentuale Anteil der Verhaltensbereiche an der Gesamtaktivität (100\%) für die Kontrollphasen (B1 und B2) und Experimente (C1 und $\mathrm{C} 2$ ) eingetragen. 


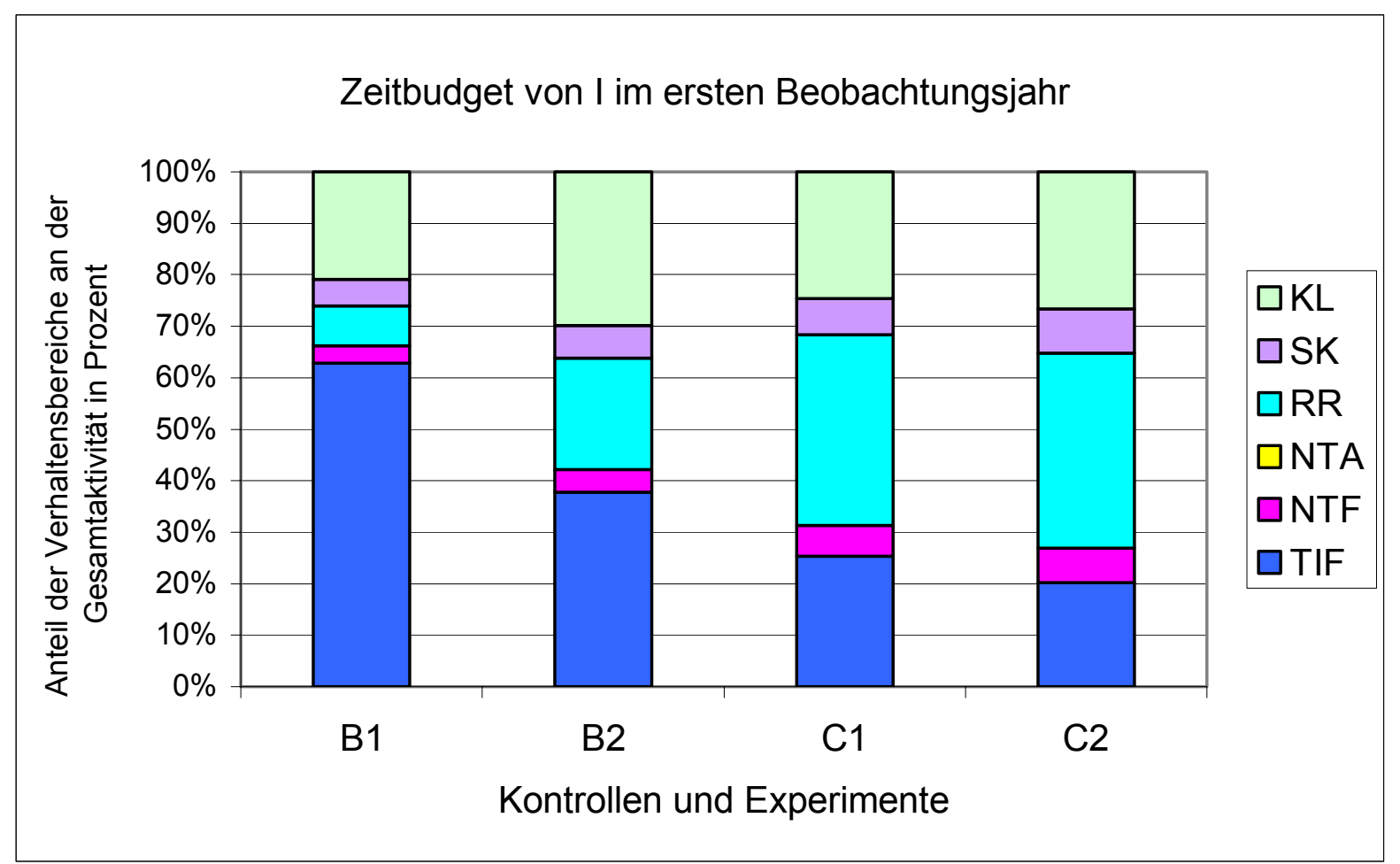

Abb. 3.19 Zeitbudget von I (männlich) im ersten Beobachtungsjahr. In der Graphik ist der prozentuale Anteil der Verhaltensbereiche an der Gesamtaktivität (100\%) für die Kontrollphasen (B1 und B2) und Experimente (C1 und $\mathrm{C} 2$ ) eingetragen.

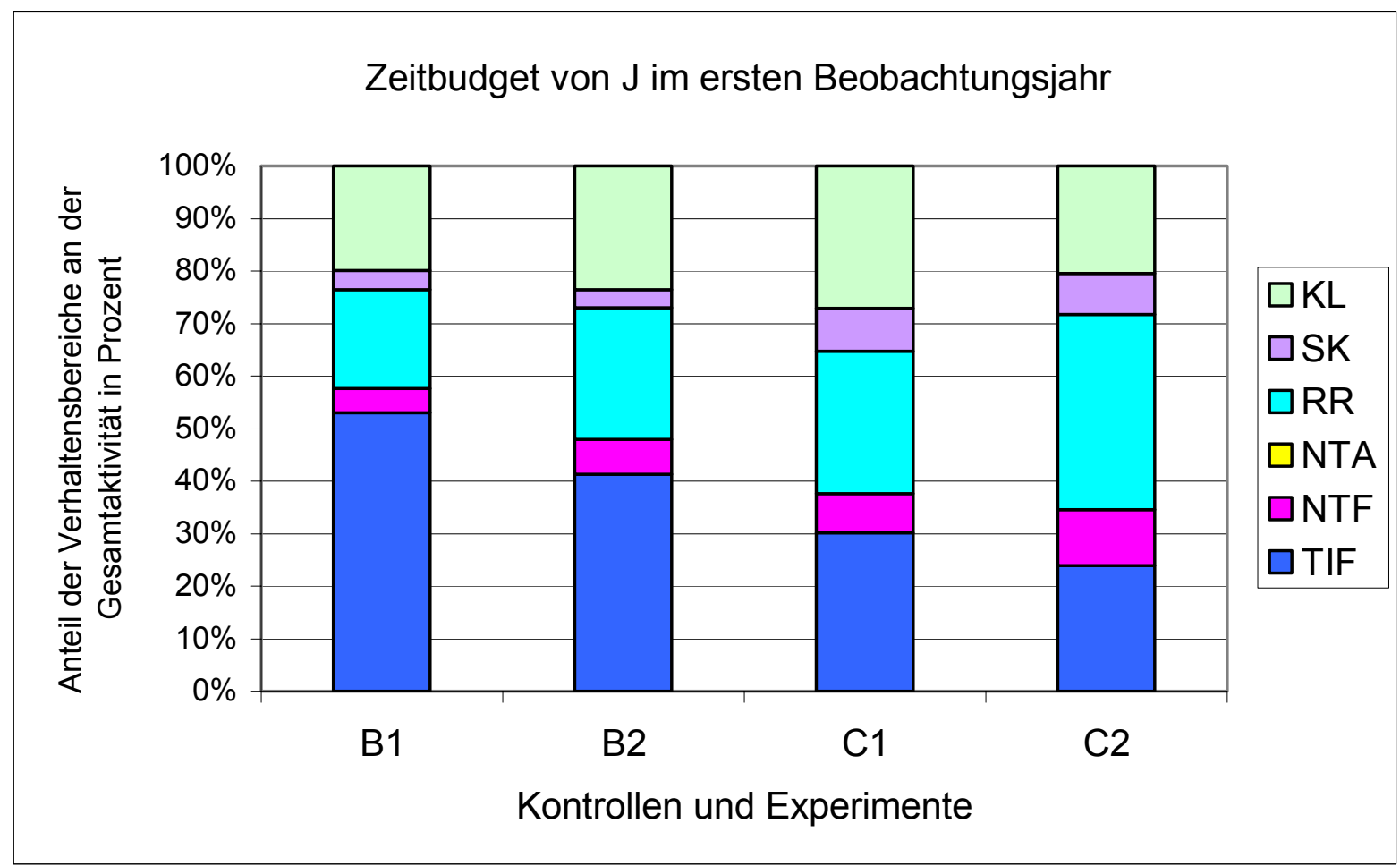

Abb. 3.20 Zeitbudget von J (männlich) im ersten Beobachtungsjahr. In der Graphik ist der prozentuale Anteil der Verhaltensbereiche an der Gesamtaktivität (100\%) für die Kontrollphasen (B1 und B2) und Experimente (C1 und $\mathrm{C} 2$ ) eingetragen. 


\subsubsection{Zeitbudgets der Familienmitglieder während der Experimente im zweiten Beobachtungsjahr}

Im Zeitbudget des Alpha-Männchens war nur der Verhaltensbereich stoffwechselbedingtes Verhalten und Körperpflege im ersten Experiment höher als während des restlichen Jahres (KFA, EW=5,1 n=4) und das Alpha-Weibchen hatte im dritten Experiment mehr Zeit als erwartet in Ressourcensicherung und Raubfeindvermeidung investiert (KFA, EW=21,1 n=4). Alle anderen Verhaltenbereiche blieben bei den Alpha-Tieren im Laufe der Experiment gleich (Abb. 3.21 und 3.22).

Während des zweiten Experiments im zweiten Beobachtungsjahr war bei $\mathrm{C}$ der Anteil an nicht-taktiler Interaktion mit Familienmitgliedern im Zeitbudget erhöht (KFA, EW=6,08 $\mathrm{n}=4$ ). Obwohl sich die Experimentalbedingungen im dritten Experiment nicht geändert hatten, sank der Anteil seiner nicht-taktilen Interaktionen mit Familienmitgliedern wieder in den Erwartungsbereich (Abb. 3.23).

D zeigte im ersten Experiment häufiger als in den darauffolgenden Phasen nicht-taktile Interaktion sowohl mit Familienmitgliedern (KFA, EW=7,37 $\mathrm{n}=4$ ), als auch mit fremden Artgenossen (KFA, EW=1,95 n=4). Im zweiten Experiment war ihr Anteil an nicht-taktiler Interaktion mit Familienmitgliedern unter den Erwartungsbereich gesunken und überstieg im dritten Experiment wieder den Erwartungsbereich (Abb. 3.24). Ebenso war auch ihr Anteil an stoffwechselbedingtem Verhalten und Körperpflege in den Experimenten 2 und 3 erhöht $(\mathrm{KFA}, \mathrm{EW}=8,97 \mathrm{n}=4)$.

Während des dritten Experiments war bei G der Anteil an taktiler Interaktion verringert und während des zweiten Experiments höher als erwartet (KFA, EW=32,94 n=4). Ressourcensicherung und Raubfeindvermeidung zeigte $\mathrm{G}$ im dritten Experiment häufiger und im vierten Experiment seltener als erwartet (KFA, EW=29,69 n=4). Nicht-taktile Interaktionen mit fremden Artgenossen waren bei $\mathrm{G}$ im vierten Experiment ebenfalls seltener als erwartet $(\mathrm{KFA}, \mathrm{EW}=3,84 \mathrm{n}=4)$. Alle adulten Nachkommen interagierten im vierten Experiment seltener nicht-taktil mit fremden Artgenossen (Abb. 3.25).

Bei allen nicht-adulten Familienmitgliedern gab es keine Veränderungen des Zeitbudgets in Abhängigkeit von den Experimenten (Abb. 3.26 bis 3.29). 


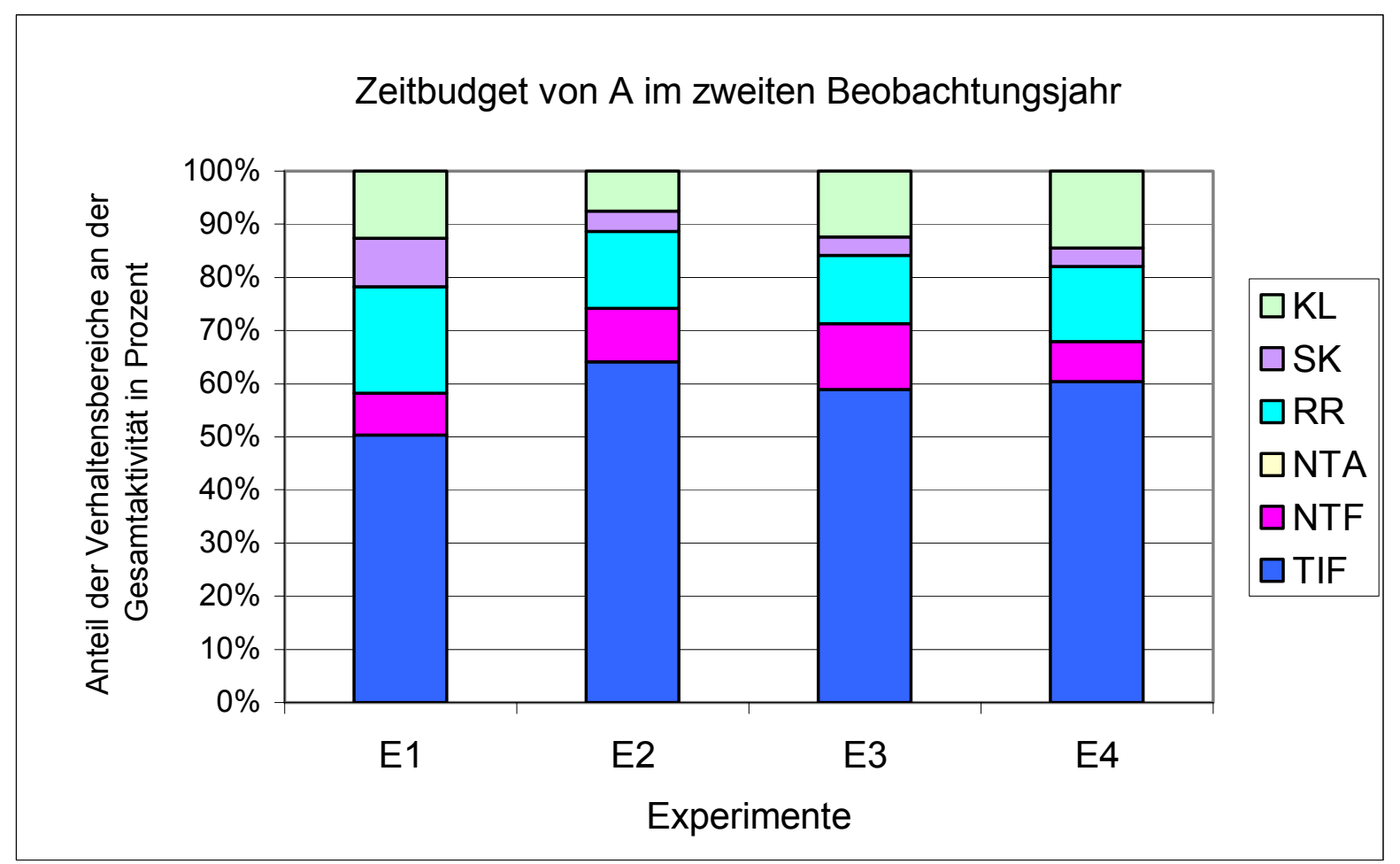

Abb. 3.21 Zeitbudget von A (Alpha-Männchen) im zweiten Beobachtungsjahr. In der Graphik ist der prozentuale Anteil der Verhaltensbereiche an der Gesamtaktivität (100\%) für die Experimente (E1 bis E4) eingetragen. Abkürzungen wie Abb. 3.1.

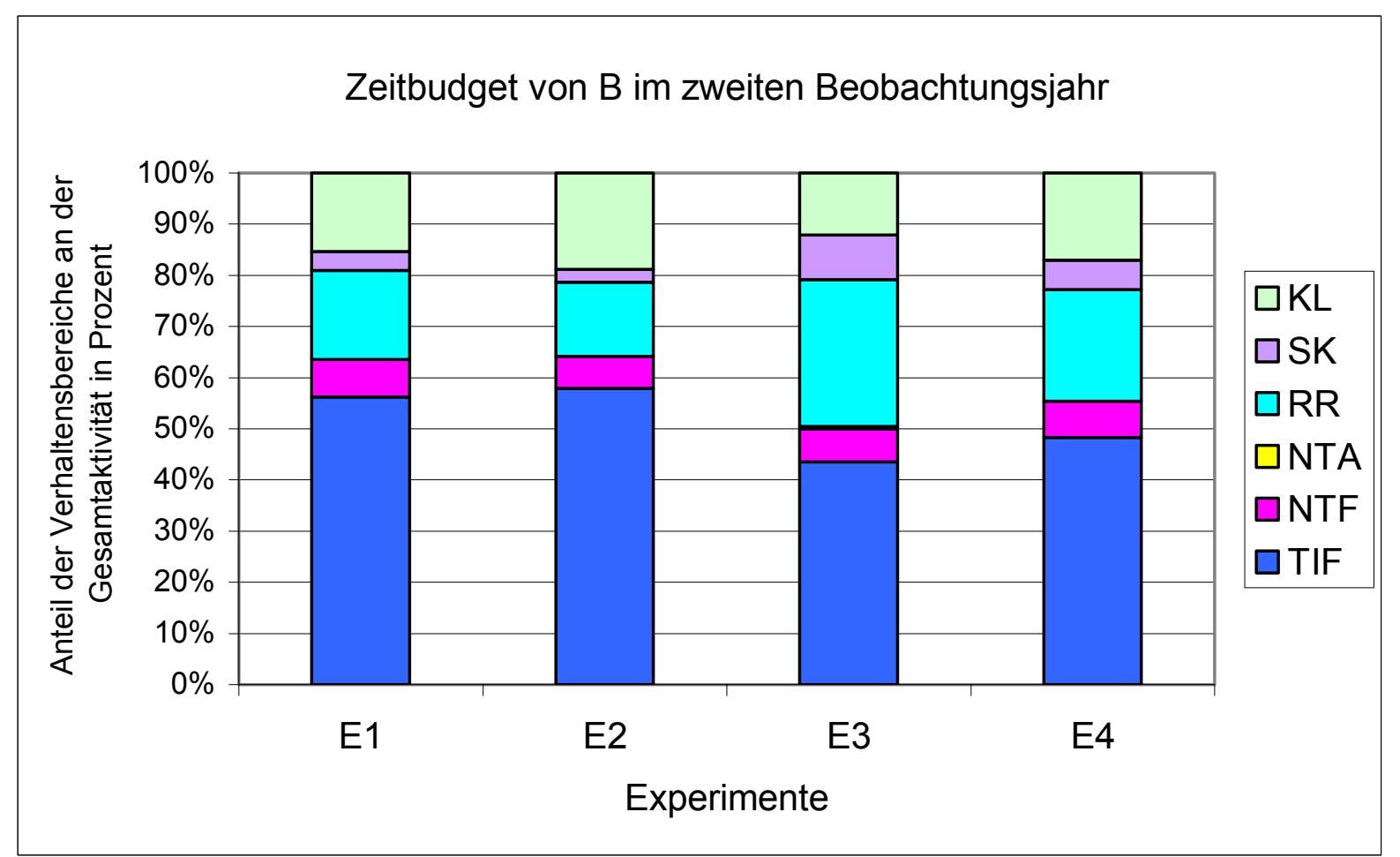

Abb. 3.22 Zeitbudget von B (Alpha-Weibchen) im zweiten Beobachtungsjahr. In der Graphik ist der prozentuale Anteil der an der Gesamtaktivität (100\%) für die Experimente (E1 bis E4) eingetragen. Abkürzungen wie Abb. 3.1 . 


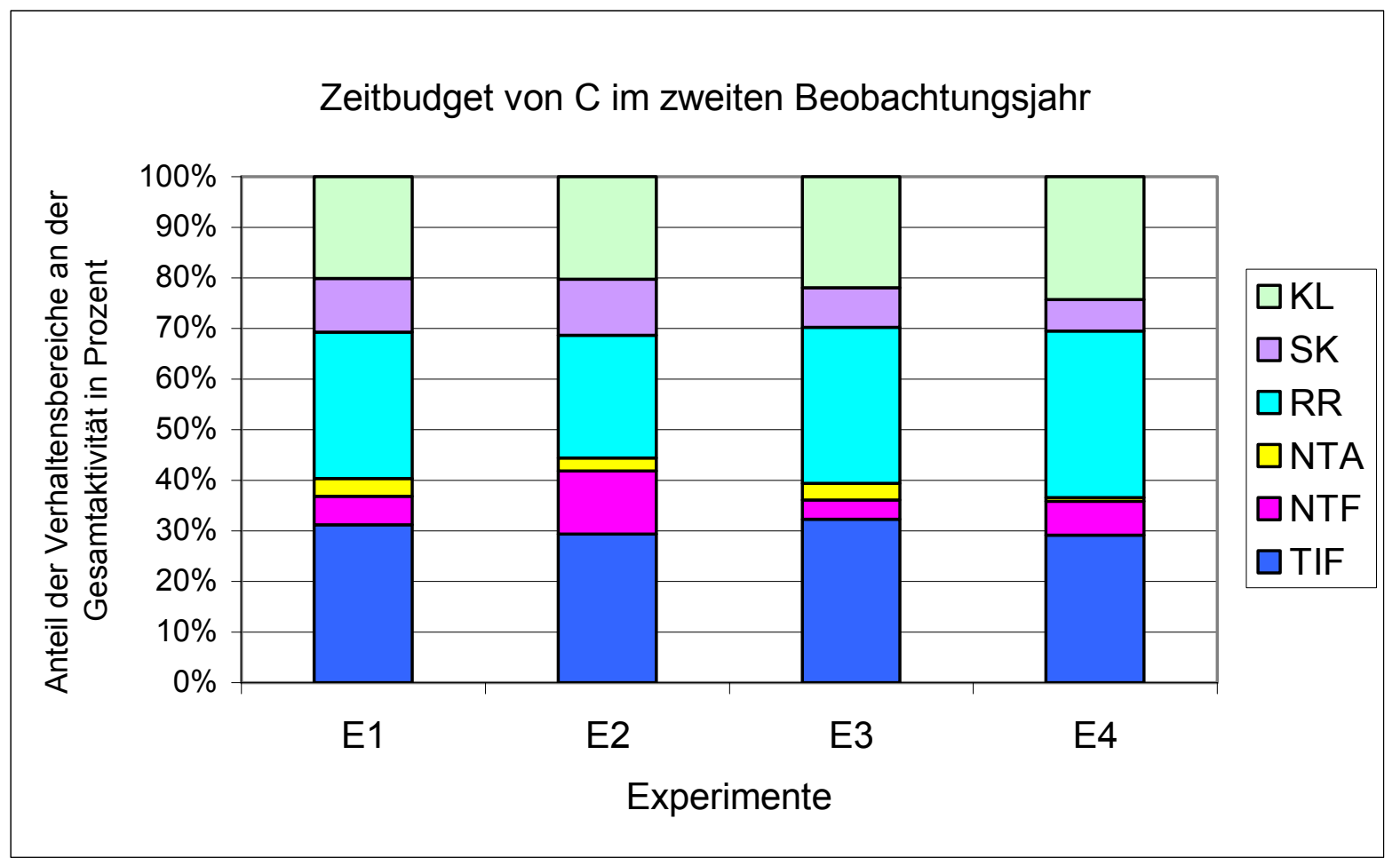

Abb. 3.23 Zeitbudget von C (Männchen) im zweiten Beobachtungsjahr. In der Graphik ist der prozentuale Anteil der Verhaltensbereiche an der Gesamtaktivität (100\%) für die Experimente (E1 bis E4) eingetragen. Abkürzungen wie Abb. 3.1.

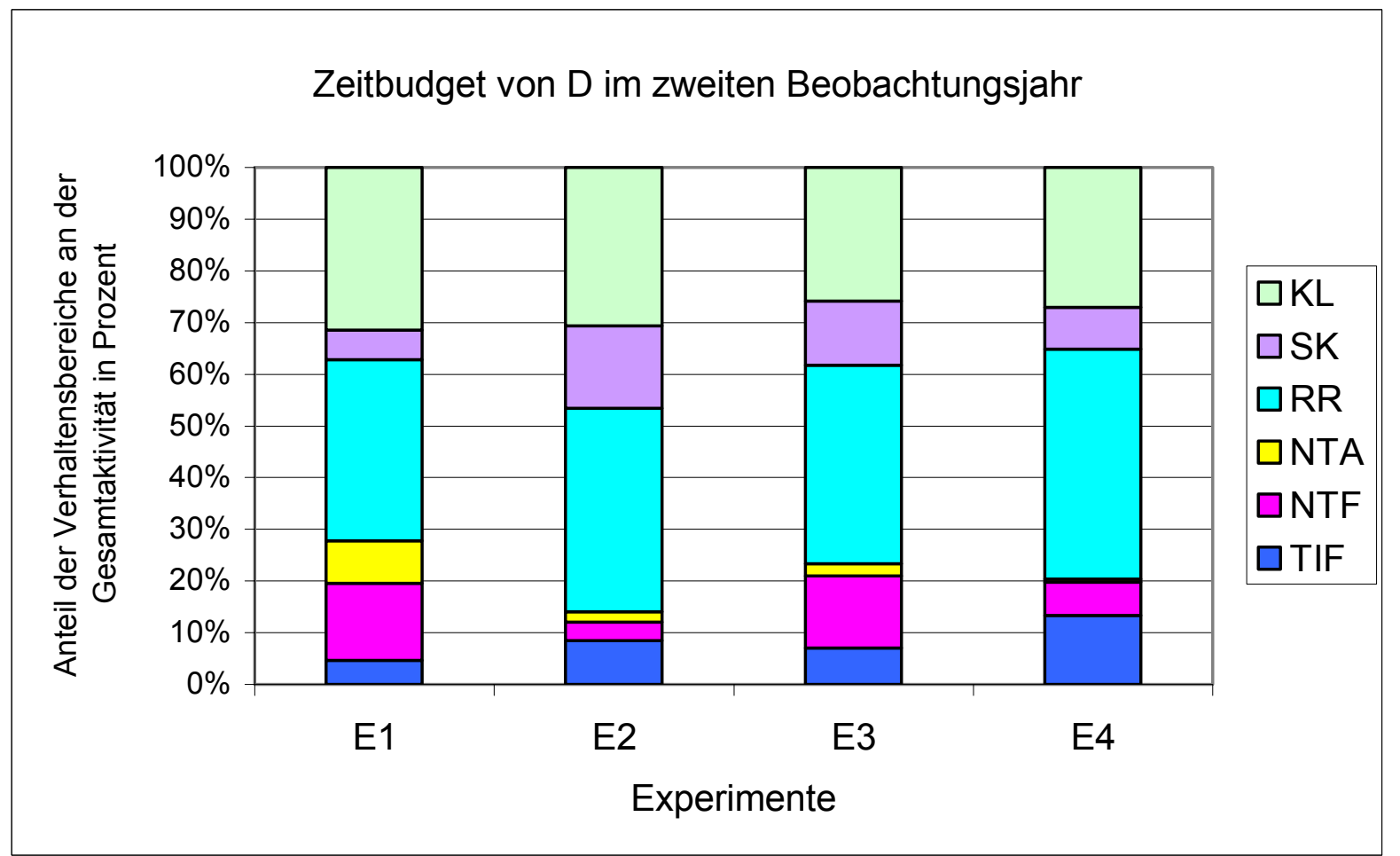

Abb. 3.24 Zeitbudget von D (Weibchen) im zweiten Beobachtungsjahr. In der Graphik ist der prozentuale Anteil der Verhaltensbereiche an der Gesamtaktivität (100\%) für die Experimente (E1 bis E4) eingetragen. Abkürzungen wie Abb. 3.1. 


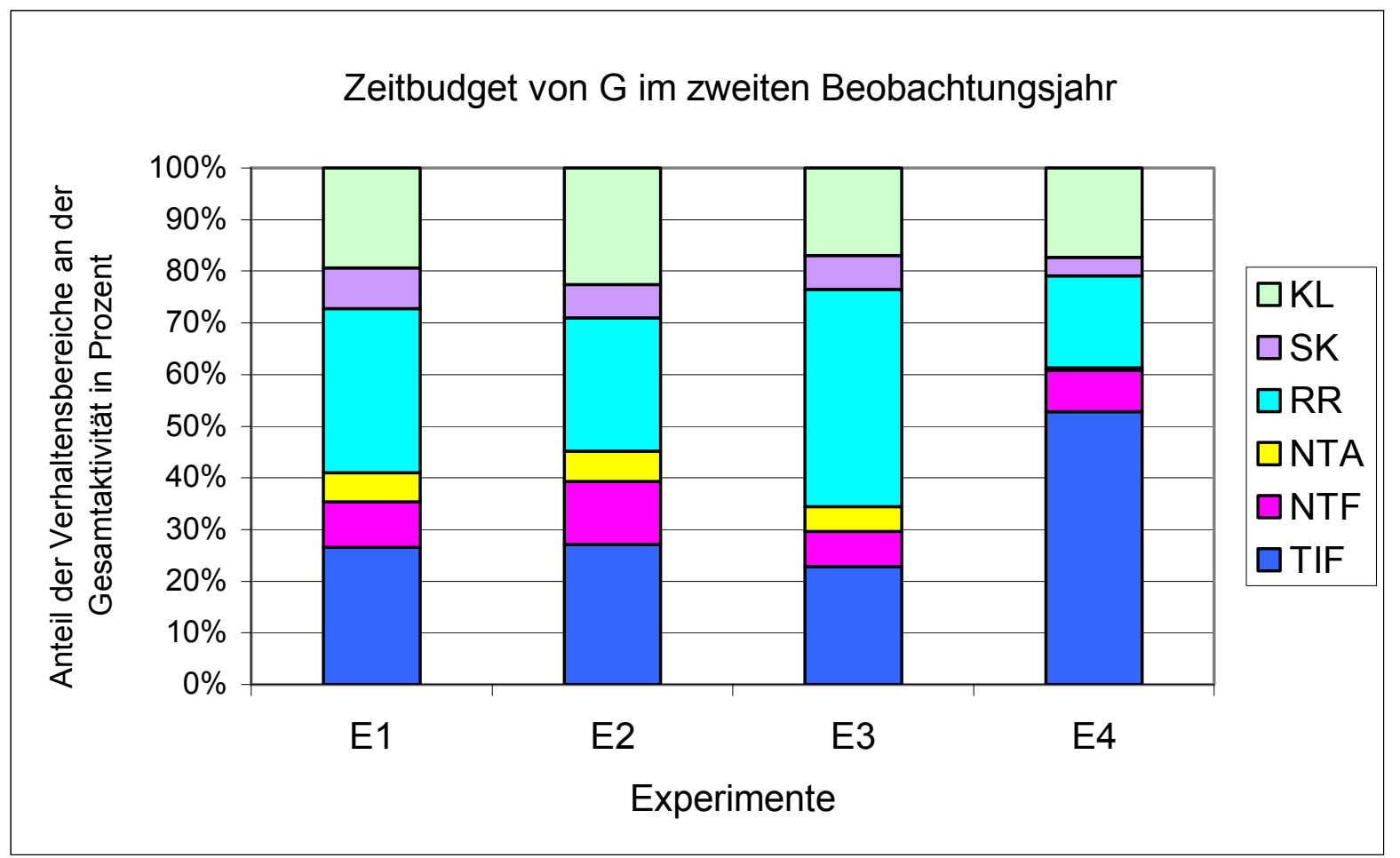

Abb. 3.25 Zeitbudget von G (Männchen) im zweiten Beobachtungsjahr. In der Graphik ist der prozentuale Anteil der Verhaltensbereiche an der Gesamtaktivität (100\%) für die Experimente (E1 bis E4) eingetragen. Abkürzungen wie Abb. 3.1.

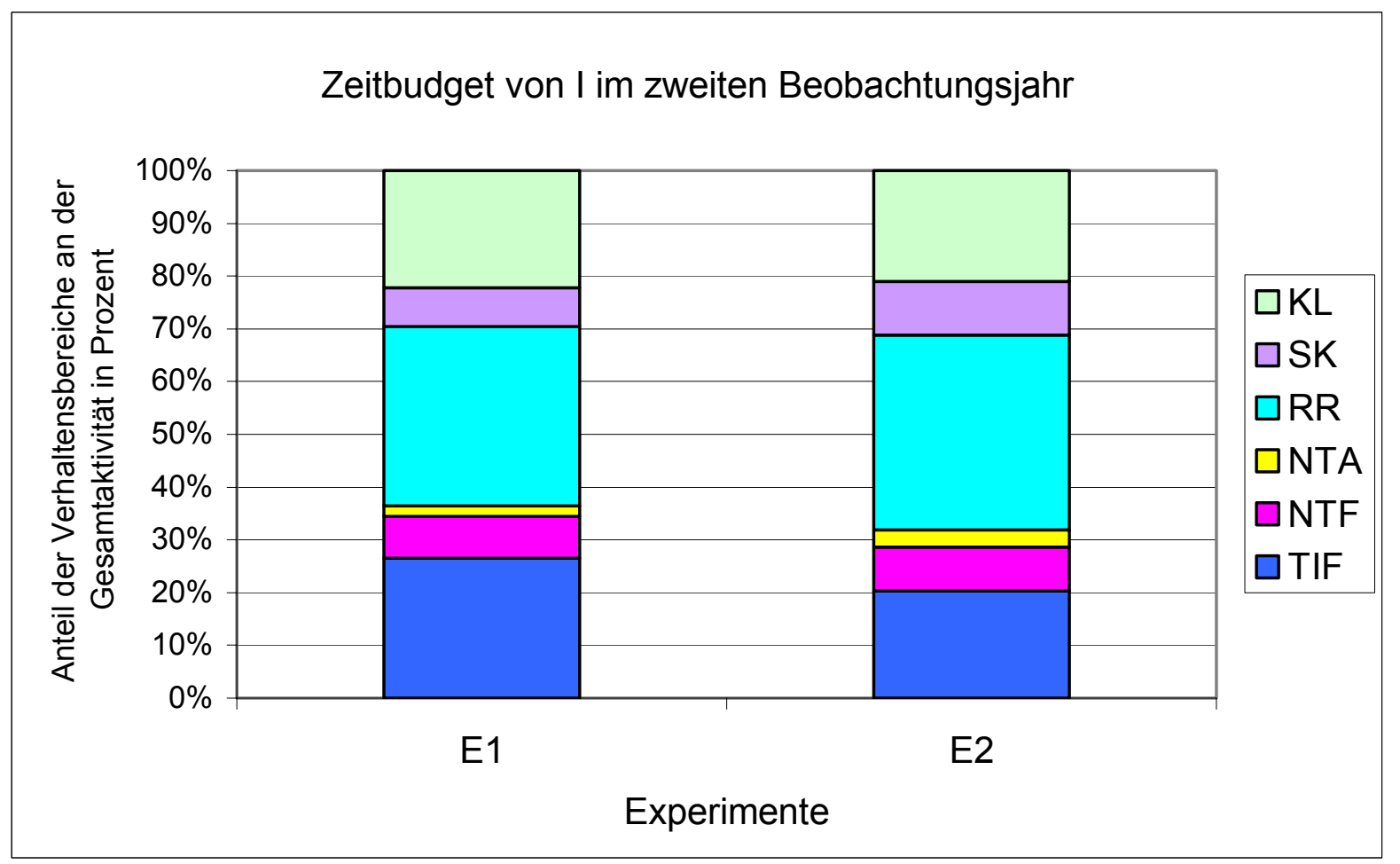

Abb. 3.26 Zeitbudget von I (Männchen) im zweiten Beobachtungsjahr. In der Graphik ist der prozentuale Anteil der Verhaltensbereiche an der Gesamtaktivität (100\%) für die Experimente (E1 und E2) eingetragen. Abkürzungen wie Abb. 3.1. 


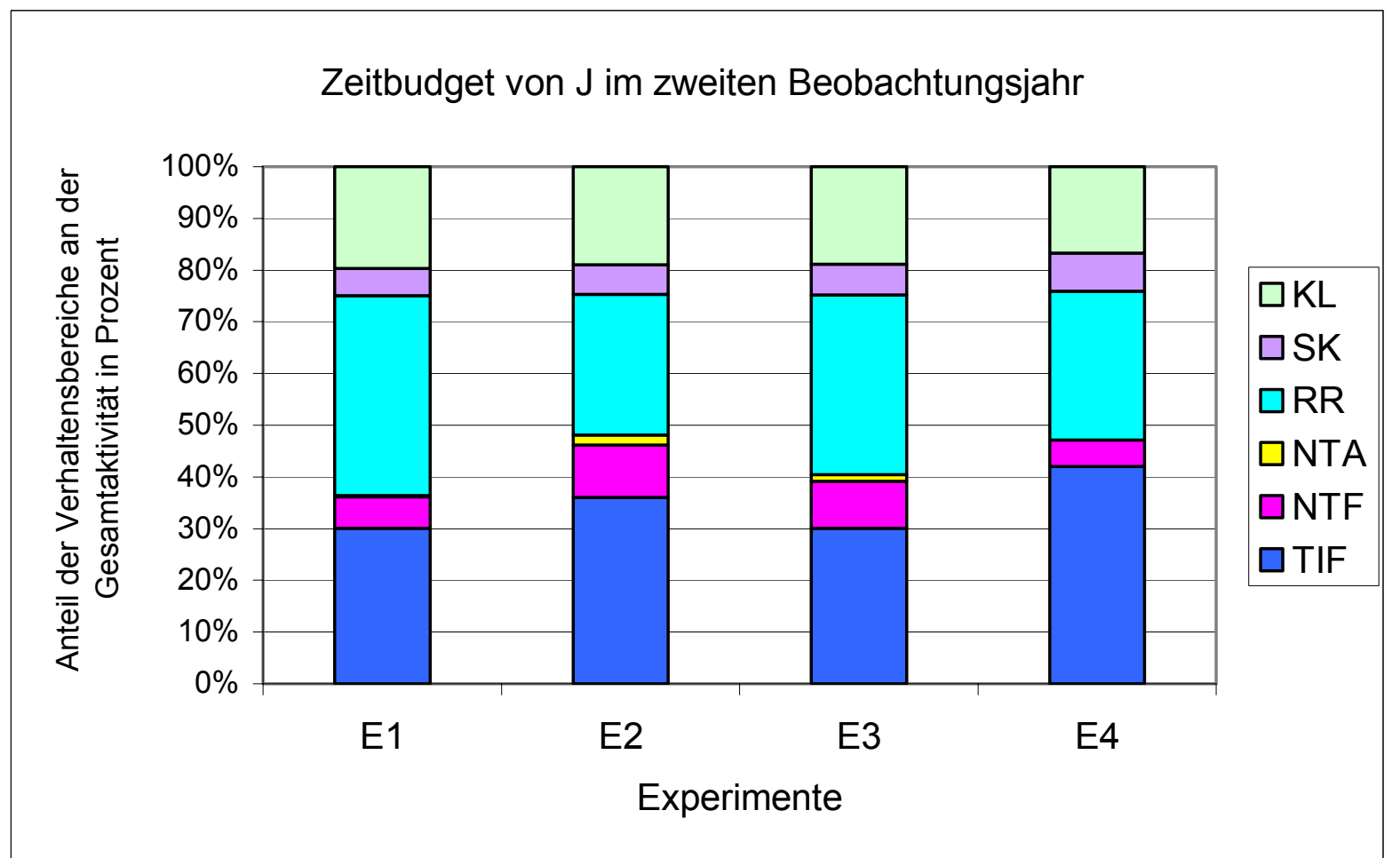

Abb. 3.27 Zeitbudget von J (Männchen) im zweiten Beobachtungsjahr. In der Graphik ist der prozentuale Anteil der Verhaltensbereiche an der Gesamtaktivität (100\%) für die Experimente (E1 bis E4) eingetragen. Abkürzungen wie Abb. 3.1.

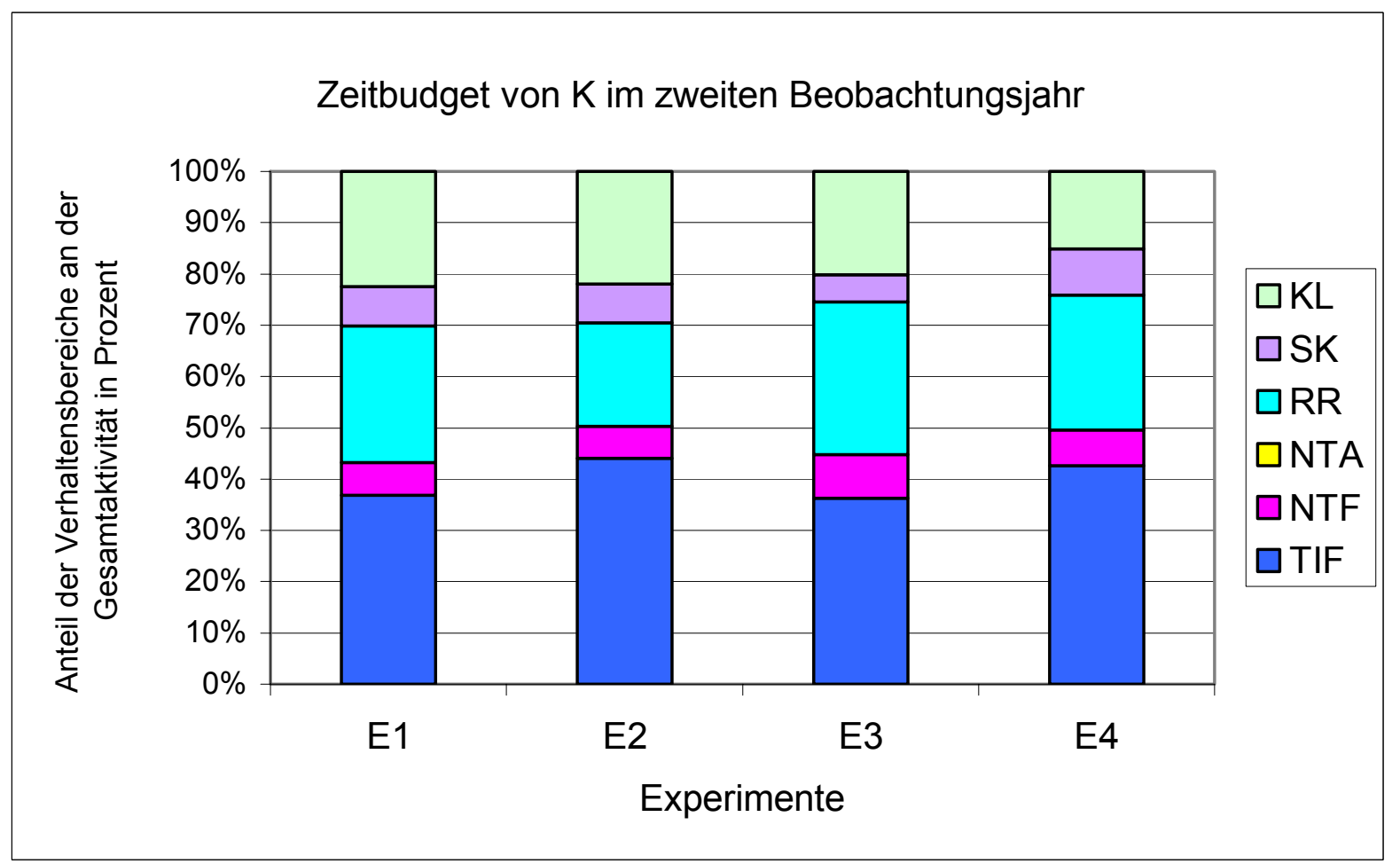

Abb. 3.28 Zeitbudget von K (Weibchen) im zweiten Beobachtungsjahr. In der Graphik ist der prozentuale Anteil der Verhaltensbereiche an der Gesamtaktivität (100\%) für die Experimente (E1 bis E4) eingetragen. Abkürzungen wie Abb. 3.1. 


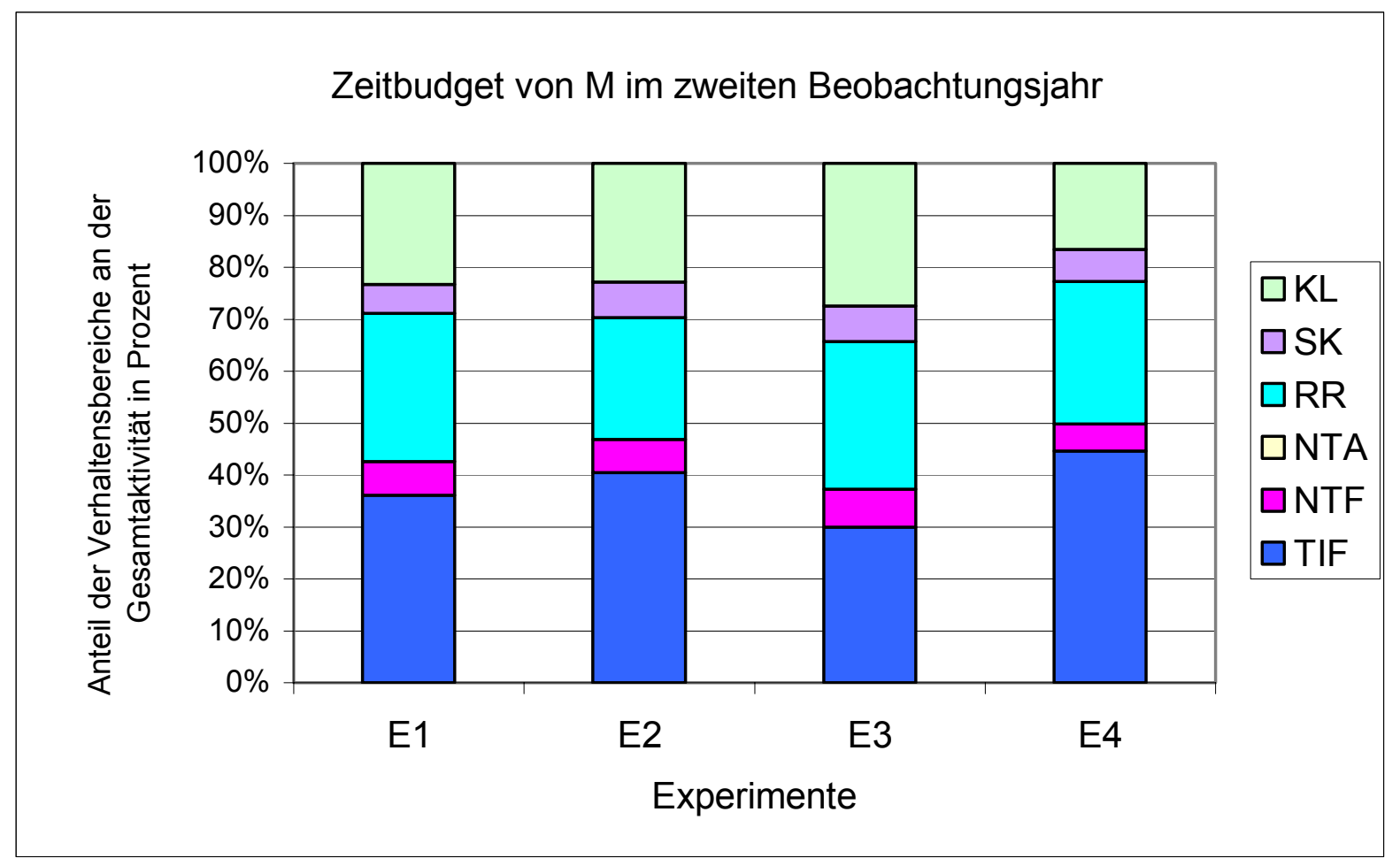

Abb. 3.29 Zeitbudget von M (Weibchen) im zweiten Beobachtungsjahr. In der Graphik ist der prozentuale Anteil der Verhaltensbereiche an der Gesamtaktivität (100\%) für die Experimente (E1 bis E4) eingetragen. Abkürzungen wie Abb. 3.1.

\subsubsection{Qualitative Verhaltensunterschiede zwischen den Familienmitgliedern im ersten Beobachtungsjahr}

Einige Verhaltenseinheiten wurde nur bei bestimmten Familienmitgliedern beobachtet. In den folgenden beiden Abschnitten werden diese Unterschiede beschrieben.

Während des instantaneous sampling wurden Angriffe und Keckern nur bei den Alpha-Tieren (A, B) beobachtet (siehe Tab. 3.1). Ausweichen, Fellsträuben, Fliehen, Katzbuckellaufen, Scannen und Twitter zu fremden Artgenossen, Twitter zu Familienmitgliedern, Warnlaute und Wegjagen konnte nur bei adoleszenten bis adulten Tieren (A-H) beobachtet werden, während Futterbetteln und Schlagen nur von Infantilen/Juvenilen (I, J) gezeigt wurde.

Verhalten, welches ausschließlich bei adulten und subadulten nicht-reproduktiven Nachkommen $(\mathrm{C}-\mathrm{H})$ vorkam, waren Fliehen, Twitter zu fremden Artgenossen, Warnlaute und Wegjagen. Markenkontrollen, Mobbing-Laute, Distanz-Phi-Laute und Über-die-Schulterblicken wurden von Individuen aller Altersklassen gezeigt. 
Tabelle 3.1 Verhaltenseinheiten, die während des instantaneous sampling im ersten Beobachtungsjahr nur bei bestimmten Familienmitgliedern beobachtet wurden. Die Familienmitglieder sind mit Großbuchstaben A - J gekennzeichnet. $\mathrm{x}=$ das betreffende Tier zeigte das Verhalten, welches links in der jeweiligen Zeile eingetragen ist. Die Tabelle gibt nur Angaben über die Qualität, nicht über die Häufigkeiten der Verhaltenseinheiten. Die grau unterlegte Fläche kennzeichnet die nicht-reproduktiven adulten und subadulten Nachkommen.

\begin{tabular}{|c|c|c|c|c|c|c|c|c|c|c|}
\hline Verhaltenseinheit & $A$ & B & C & $\mathrm{D}$ & $E$ & $\mathrm{~F}$ & G & $\mathrm{H}$ & I & $\mathrm{J}$ \\
\hline Angreifen & & $x$ & & & & & & & & \\
\hline Ausweichen & & & & & & & & $x$ & & \\
\hline Fellsträuben & $x$ & $x$ & $x$ & $x$ & $x$ & $x$ & & $x$ & & \\
\hline Fliehen & & & & $x$ & & $x$ & & & & \\
\hline Futterbetteln & & & & & & & & & $x$ & $x$ \\
\hline Genitalpräsentieren & $x$ & & & & $x$ & & & & & $\mathrm{x}$ \\
\hline Katzbuckellaufen & $x$ & $x$ & & $x$ & $x$ & & & $x$ & & \\
\hline Keckern & $x$ & $x$ & & & & & & & & \\
\hline Markenkontrolle & $x$ & $x$ & $x$ & $x$ & $x$ & & $x$ & $x$ & $x$ & $\mathrm{x}$ \\
\hline Mobbing-Laute & $x$ & $x$ & & $x$ & & $x$ & $x$ & $x$ & $x$ & $x$ \\
\hline Distanz-Phi-Laut & $x$ & & $x$ & $x$ & $x$ & & $x$ & $x$ & $x$ & \\
\hline Quärren & & & & $x$ & & $x$ & $x$ & $x$ & $x$ & $\mathrm{x}$ \\
\hline $\begin{array}{l}\text { Scannen zu fremden } \\
\text { Artgenossen }\end{array}$ & $x$ & & $x$ & $x$ & $x$ & & $x$ & & & \\
\hline Schlagen & & & & & & & & & $x$ & \\
\hline $\begin{array}{l}\text { Twitter zu fremden } \\
\text { Artgenossen }\end{array}$ & & & & $x$ & $x$ & & & & & \\
\hline $\begin{array}{l}\text { Twitter zu } \\
\text { Familienmitgliedern }\end{array}$ & $x$ & $x$ & $x$ & $x$ & $x$ & & & $x$ & & \\
\hline $\begin{array}{l}\text { Über-die-Schulter- } \\
\text { blicken }\end{array}$ & & $x$ & $x$ & & $x$ & & & $x$ & & $\mathrm{x}$ \\
\hline Warnlaut & & & & $x$ & $x$ & & $x$ & & & \\
\hline Wegjagen & & & $x$ & & $x$ & & & & & \\
\hline
\end{tabular}

\subsubsection{Qualitative Verhaltensunterschiede zwischen den Familienmitgliedern im zweiten Beobachtungsjahr}

Im zweiten Beobachtungsjahr wurde Erkunden, Kopulieren und Schlitzaugen-machen nur bei den Alpha-Tieren beobachtet. Büschelklappen und Genitalpräsentieren wurde bei den AlphaTieren und den juvenilen Familienmitgliedern ( $\mathrm{K}, \mathrm{M})$ beobachtet, während Angreifen, Futterbetteln, Distanz-Phi-Laute und Wegjagen nur bei adulten und subadulten Nachkommen (C-J) beobachtet werden konnte. Ausweichen, Fliehen, Mobbing-Laute und Quärren waren Verhaltenseinheiten, die nur von den Nachkommen (C-M) gezeigt wurden. Folgen, Genitalkontrollieren, Katzbuckellaufen, Schlagen, Schupsen/Wegdrücken, Twitter zu fremden 
Artgenossen und Twitter zu Familienmitgliedern wurden nur bei adulten und subadulten Familienmitgliedern beobachtet (siehe Tab. 3.2).

Tabelle 3.2 Verhaltenseinheiten, die während des instantaneous sampling im zweiten Beobachtungsjahr nur bei bestimmten Familienmitgliedern beobachtet wurden. Die Familienmitglieder sind mit Großbuchstaben A - M gekennzeichnet. $\mathrm{x}=$ das betreffende Tier zeigte das Verhalten, welches links in der jeweiligen Zeile eingetragen ist. Die Tabelle gibt nur Angaben über die Qualität, nicht über die Häufigkeiten der Verhaltenseinheiten. Die grau unterlegte Fläche kennzeichnet die nicht-reproduktiven adulten und subadulten Nachkommen.

\begin{tabular}{|c|c|c|c|c|c|c|c|c|c|}
\hline Verhaltenseinheit & A & B & C & D & G & 1 & $\mathrm{~J}$ & $\mathrm{~K}$ & $\mathrm{M}$ \\
\hline Angreifen & & & $x$ & & & & & & \\
\hline Ausweichen & & & & & & $x$ & $x$ & $x$ & \\
\hline Büschelklappen & $x$ & & & & & & & $x$ & $\mathrm{x}$ \\
\hline Erkunden & & $\mathrm{x}$ & & & & & & & \\
\hline Fliehen & & & & $x$ & & $x$ & & $x$ & \\
\hline Folgen & $x$ & & & & $x$ & & $x$ & & \\
\hline Futterbetteln & & & & & & & $x$ & & \\
\hline Genitalkontrollieren & $x$ & & & & $x$ & & & & \\
\hline Genitalpräsentieren & & $x$ & & & & & & & $\mathrm{x}$ \\
\hline Katzbuckellaufen & $x$ & $x$ & $x$ & $x$ & $x$ & $x$ & $x$ & & \\
\hline Keckern & & $x$ & & $x$ & & & $x$ & & $x$ \\
\hline Kopulieren & $x$ & $x$ & & & & & & & \\
\hline Mobbing-Laute & & & $x$ & $\mathrm{x}$ & & $x$ & $x$ & $x$ & $x$ \\
\hline Distanz-Phi-Laut & & & $x$ & $\mathrm{x}$ & $x$ & $\mathrm{x}$ & $x$ & & \\
\hline Quärren & & & & $x$ & & & $x$ & $x$ & $\mathrm{x}$ \\
\hline Schlagen & & $\mathrm{x}$ & & & & & $x$ & & \\
\hline Schlitzaugen & $x$ & & & & & & & & \\
\hline $\begin{array}{l}\text { Schupsen/ } \\
\text { Wegdrücken }\end{array}$ & $x$ & $x$ & & & & & $x$ & & \\
\hline $\begin{array}{l}\text { Twitter zu fremden } \\
\text { Artgenossen }\end{array}$ & & $x$ & & $x$ & $x$ & $x$ & & & \\
\hline $\begin{array}{l}\text { Twitter zu } \\
\text { Familienmitgliedern }\end{array}$ & $x$ & $x$ & $x$ & $\mathrm{x}$ & $x$ & $x$ & $x$ & & \\
\hline Wegjagen & & & & & $x$ & & & & \\
\hline
\end{tabular}

\subsubsection{Kapiteldiskussion}

Da es innerhalb beider Beobachtungsjahre keine Veränderungen des Zeitbudgets der Familie gab und sich im Vergleich der Beobachtungsjahre keine Änderungen ergaben, kann davon ausgegangen werden, dass das Zeitbudget der Familie langfristig stabil war und die Anwesenheit der Locktiere auf dem Semifreigelände keinen Einfluss auf das Zeitbudget der Familie nahm. Einzelne Verhaltensbereiche hatten in den beiden Beobachtungsjahren aber unterschiedlich große Anteile an der Gesamtaktivität. Der höhere Anteil an taktiler Interaktion 
im zweiten Beobachtungsjahr mag darin begründet liegen, dass Jungtiere geboren worden waren, mit denen die Familienmitglieder häufig taktil interagierten.

Da während beider Beobachtungsjahre nur die adulten und subadulten Nachkommen und im ersten Beobachtungsjahr auch das Alpha-Männchen mit fremden Artgenossen nicht-taktil interagierten, kann angenommen werden, dass diese die Funktion der Verteidigung des Streifgebietes übernahmen (Radespiel 1990, König und Rothe 1991, Darms 1987), was ihnen andererseits die Möglichkeit bot, Kontakte mit potentiellen zukünftigen Paarungspartnern herzustellen. Hubrecht (1985) beschreibt, dass der Aufenthalt von Familienmitgliedern an den Grenzen des Streifgebietes der Verteidigung des Streifgebietes diente und dass es an den Grenzen außerdem zu „extra-group-copulations“ eines nicht-reproduktiven Weibchens (ca.1015 Monate alt) mit Männchen fremder Gruppen kam. Ebenso berichtet Digby (1999) von einer C. jacchus-Population, in der $24 \%$ aller beobachteten Kopulationsversuche zwischen Mitgliedern verschiedener Gruppen auftraten. Die Beobachtungen der vorliegenden Studie ergaben, dass das reproduktive Weibchen, als auch die adoleszenten und juvenilen Nachkommen sich nicht quantitativ messbar an der Kommunikation mit fremden Artgenossen beteiligten, was dafür spricht, dass die nicht-taktile Interaktion eine der oben genannten Funktionen ausübte. Dies gilt allerdings nur für die in der Studie gewählten Verhaltenseinheiten und olfaktorische Signale können nicht ausgeschlossen werden.

Da bei den Nachkommen D und E im ersten Beobachtungsjahr der Anteil an nicht-taktiler Interaktion mit fremden Artgenossen im ersten Experiment höher war als in den anderen Phasen, verdeutlicht sich eine starke Reaktion von D und E auf die Anwesenheit des Lockweibchens. Einhergehend mit der Präsenz des Lockweibchens verminderten die adulten Nachkommen zudem ihren Zeitanteil an taktiler Interaktion mit Familienmitgliedern, was eine Orientierung der Nachkommen von der Familie weg zum Lockweibchen aufzeigt. Während des zweiten Beobachtungsjahres reagierten die adulten Nachkommen C, D und G auf die Präsentation des einzelnen Lockmännchens anders als auf die Anwesenheit des einzelnen Lockweibchens im Jahr vorher. Der verringerte Anteil an nicht-taktiler Interaktion mit fremden Artgenossen von G kann durch seine Krankheit während des vierten Experiments erklärt werden, in dem er gehäuft an taktiler Interaktion mit Familienmitgliedern beteiligt war. Mögliche Ursachen für das geringe Interesse der ältesten Tochter D an dem Lockmännchen können z.B. die hormonelle Unterdrückung, die Präsenz der Brüder bzw. der Familie oder persönliche Abneigung (vgl. Geyer 1993) sein. War die bevorzugte Strategie der Tochter der 
Verbleib im Natalgebiet, um später die reproduktive Position zu erben, dann wäre das Interesse an fremden Artgenossen von untergeordneter Bedeutung.

Am Ende des zweiten Experiments war I von G aus der Familie vertrieben worden. Nach dem Rauswurf von I markierte G häufiger und legte mehr Bissmarken an, wodurch der vergrößerte Anteil an Ressourcensicherung und Raubfeindvermeidung im dritten Experiment erklärt werden kann. Markierverhalten wird in vielen Freilandstudien in Zusammenhang mit aggressivem Intra- und Intergruppenverhalten gebracht (Rylands 1990). Aggressives Verhalten und ein hoher sozialer Rang gehen mit häufigem Markierverhalten einher und es wird vermutet, dass das aggressiv motivierte Tier subordinierte Individuen und fremde Artgenossen mit diesen olfaktorischen Signalen kontrolliert bzw. abschreckt (Epple et al. 1993). Das Markierverhalten von G nach dem Rauswurf seines jüngeren Bruders I weist auf eine solche Demonstration seines hohen Ranges hin.

Die älteste Tochter hatte während der Präsentation der Kleinfamilie im zweiten Beobachtungsjahr häufiger als während anderer Phasen nicht-taktil mit Familienmitgliedern und fremden Artgenossen interagiert. Da in dieser Phase die Jungtiere geboren worden waren, bleibt jedoch unklar, ob und auf welche Bedingungen ihr Verhalten zurückzuführen ist (Locktiere oder Geburt).

Die kontinuierliche Verringerung der taktilen Interaktion mit Familienmitgliedern und die Zunahme der Ressourcensicherung und Raubfeindvermeidung bei den juvenilen I und J spiegelt deren Alterszuwachs und die damit einhergehende Verselbständigung im Laufe des ersten Beobachtungsjahres wieder (z.B. Yamamoto 1993).

\subsection{Emigrationen und Rauswürfe}

In diesem Kapitel wird das Resultat der in der Einleitung gestellten Entscheidungsfrage über Philopatrie und Emigration für die adulten und subadulten Nachkommen der Fokusfamilie J18 beschrieben. Darauffolgend wird nach Abhängigkeiten der Wahrscheinlichkeit, aus der Familie geworfen zu werden, von verschiedenen Faktoren gesucht.

Eine freiwillige Emigration wird im Folgenden als irreversible Abwanderung aus der Familie definiert, welcher keine aggressiven Handlungen von Familienmitgliedern gegenüber dem emigrierenden Individuum vorausgingen. Unter Rauswurf werden all jene Dezimierungen der Gruppengröße verstanden, welche durch aggressives Verhalten der Familienmitglieder gegenüber einem Individuum hervorgerufen wurden, woraufhin das Individuum entweder selber die Familie verließ oder aus der Familie entfernt werden musste (in Käfighaltung). 
Inwiefern Individuen „absichtlich“ durch ein bestimmtes Verhalten einen Rauswurf aus der Familie provozierten und damit eine gewisse Freiwilligkeit impliziert werden kann, muss vorerst offen bleiben (vgl. Darms 1987).

\subsubsection{Freiwillige Emigrationen}

Freiwillige Emigrationen von Familienmitgliedern konnten in der Fokusfamilie J18 während des Beobachtungszeitraums von 2000-2002 nicht beobachtet werden. Die Versuchsbedingungen, in denen potentiellen Emigranten freie Streifgebiete (dispersal sinks) und abwechselnd geschlechtsreife Einzeltiere, ein Männchenpaar und eine Kleinfamilie mit eingeschränkt reproduktivem Alpha-Weibchen zugänglich waren, führten zu keiner Abwanderung, ohne vorhergehende aggressive Handlungen von Familienmitgliedern.

\subsubsection{Beobachtete Rauswürfe}

Während der Studie kam es zu acht Rauswürfen: sechs Männchen (75\%) und zwei Weibchen (25\%). Der Verlauf der Rauswürfe ist im Anhang auf S. 242ff. dargestellt. Die Tiere wurden von gleichgeschlechtlichen Geschwistern rausgeworfen. In zwei Fällen war auch je ein Elter an den Rauswürfen gleichgeschlechtlicher Nachkommen beteiligt. Das Alter der rausgeworfenen Tiere betrug 12 bis 36 Monate und lag im Durchschnitt bei 18,4 Monaten, d.h. alle rausgeworfenen Tiere waren subadult, pubertär oder älter (Abbott und Hearn 1978). Die Rauswerfer waren im Mittel 26,7 (15,3 - 47,7) Monate alt. Die Gruppengröße betrug durchschnittlich 10,1 (8-11), bevor die Tiere ausgestoßen wurden und 9,1 (7-10) nach dem Rauswurf. Tabelle 3.3 bietet eine Übersicht über die Rauswürfe .

Der Rauswurf von R ereignete sich außerhalb der Beobachtungsperioden und kann deshalb nur marginal behandelt werden. Ein anderer Rauswurf trat infolge schwerer Krankheit des Familienmitgliedes $\mathrm{J}$ auf, nachdem das Tier eine Woche lang von der Familie entfernt werden musste. Eine Eingliederung war nach dieser Zeit aufgrund von dissoziativen Handlungen durch den älteren Bruder $\mathrm{G}$ nicht mehr möglich. Wegen der besonderen Umstände muss die Analyse dieses Rauswurfes ebenfalls mit Vorsicht erfolgen. Der Rauswurf von P ereignete sich, nachdem das Alpha-Weibchen gestorben war. Da im Labor gehaltene Familien nach dem Tod eines Alpha-Tieres häufig instabil werden, könnte dieser Rauswurf mit der besonderen Situation der Familie in Zusammenhang gebracht werden (König et al. 1988, Rothe et al. 1986). 
Tabelle 3.3 Rauswürfe aus Familie J18 im Zeitraum 2000-2002. Für jedes rausgeworfene Tier wurde das Geschlecht, das Alter beim Rauswurf und der Rauswerfer (mit Geschlechts- und Altersangabe) angegeben. Das Datum des Rauswurfs, die Anwesenheit von Locktieren, das Alter der Jungtiere in der Fokusfamilie, die Veränderung der Gruppengröße durch den Rauswurf und das Geschlechterverhältnis vor dem Rauswurf sind ebenfalls abgebildet.

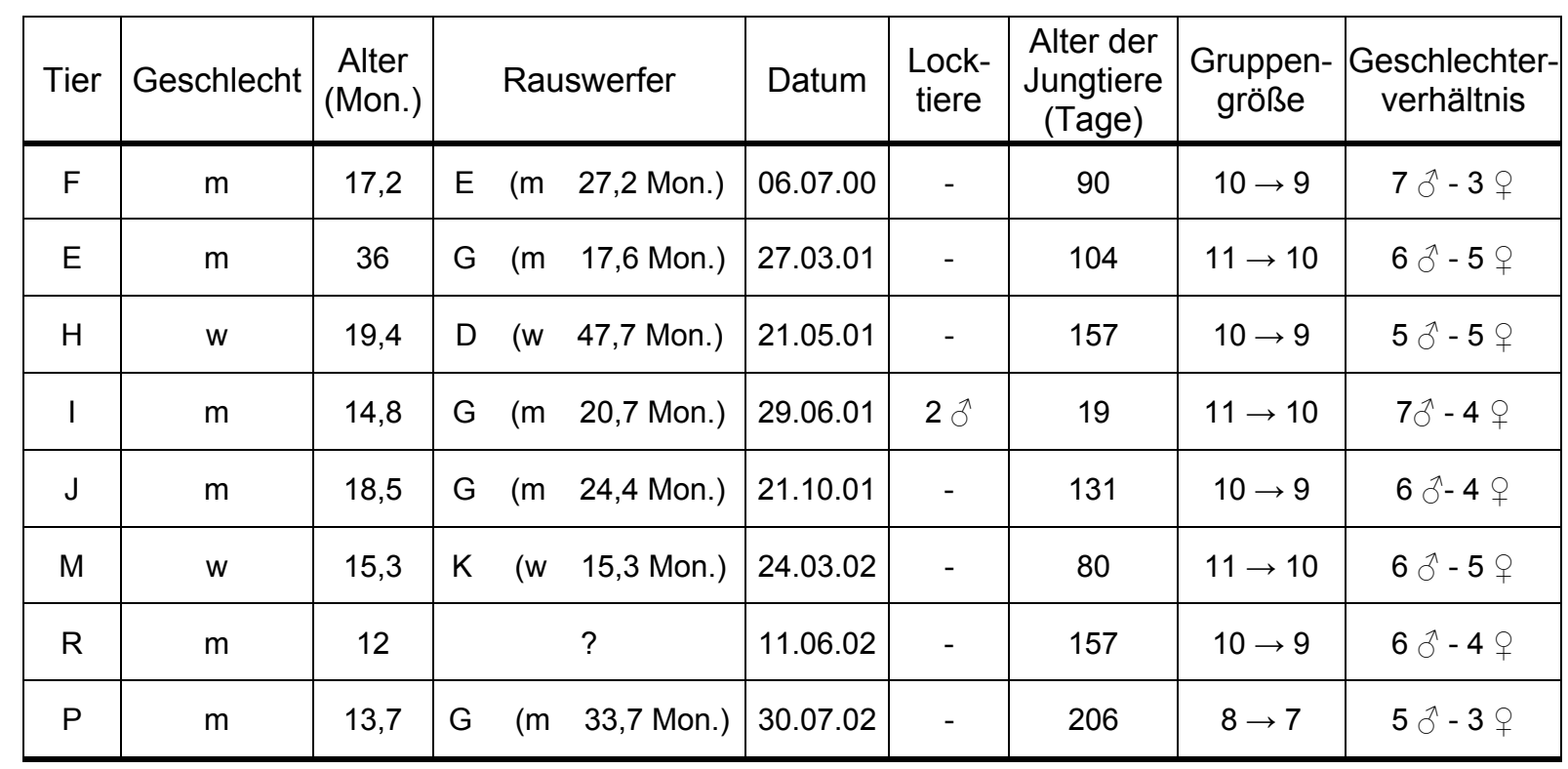

Aus den Datenerhebungen von 1972-2003 aus der Weißbüschelaffen-Kolonie des Instituts für Anthropologie der Universität Göttingen (IfA) ergab sich das folgende Bild über Rauswürfe bei Callithrix jacchus (Tabelle 3.4).

Tabelle 3.4 Anzahl der Rauswürfe von Männchen und Weibchen vollständiger Gruppen ${ }^{15}$ aus der Callithrix jacchus-Kolonie des IfA in dem Zeitraum von 1972 bis 2003. Das gemittelte Alter der rausgeworfenen Tiere in Monaten ist ebenfalls dargestellt (Rundungen: abgerundet $<0,5$; aufgerundet $\geq 0,5$ ) .

(Median) Anzahl der Rauswürfe Alter (Monate)

\begin{tabular}{lcc}
\hline Männchen & 103 & 19 \\
Weibchen & 101 & 22 \\
insgesamt & 204 & 21 \\
\hline
\end{tabular}

Im Zeitraum von 1972 bis 2003 wurden in der Callithrix jacchus-Kolonie aus vollständigen Gruppen (siehe Fußnote 13) 204 Tiere rausgeworfen, von denen 50,5\% männlich und 49,5\% weiblich waren. Drei der rausgeworfenen Männchen waren Alpha-Männchen ihrer jeweiligen Gruppe (2,9\% der rausgeworfenen Männchen). Es wurde kein Alpha-Weibchen von einer Gruppe ausgestoßen. Das durchschnittliche Alter der rausgeworfenen Tiere betrug 21 Monate, wobei die Männchen drei Monate jünger waren als die Weibchen (Männchen = 19 Monate, Weibchen $=22$ Monate). In 100 Fällen waren der bzw. die Rauswerfer bekannt; in 104 Fällen

\footnotetext{
${ }^{15}$ Es wurden nur Gruppen gewertet, in denen das reproduktive Alpha-Paar anwesend war.
} 
blieben die Rauswerfer unbekannt (Tab. 3.5). Sieben Rauswürfe wurden von der gesamten Gruppe durchgeführt. Der Anteil der Männchen an den Rauswerfern betrug 58,1\% und der Anteil der Weibchen 41,9\%. Von den männlichen Rauswerfern waren 29,6\% Alpha-Tiere und 68,5\% Subordinierte bzw. Geschwister. Der Anteil an Alpha-Tieren bei den rauswerfenden Weibchen betrug 79,5\% und der Anteil an Subordinierten bzw. Geschwistern 17,9\%. Es wurde einmal der Rauswurf eines männlichen Familienmitgliedes durch ein subordiniertes Männchen und das Alpha-Männchen (1,9\%) und ein Rauswurf eines weiblichen Familienmitgliedes durch ein subordiniertes Weibchen und das Alpha-Weibchen $(2,5 \%)$ beobachtet.

Tabelle 3.5 Anzahl der Rauswerfer (Männchen, Weibchen, Gruppe und unbekannt) aus der Callithrix jacchusKolonie des IfA in dem Zeitraum von 1972 bis 2003. Es wurde außerdem unterschieden, ob die Rauswürfe von Alpha-Tieren, von Geschwistern oder von beiden gleichzeitig durchgeführt wurden.

\begin{tabular}{lcccc} 
(Median) & $\begin{array}{c}\text { Anzahl der } \\
\text { Rauswerfer }\end{array}$ & $\begin{array}{c}\text { Anzahl Alpha- } \\
\text { Tiere }\end{array}$ & $\begin{array}{c}\text { Anzahl } \\
\text { Geschwister }\end{array}$ & $\begin{array}{c}\text { Anzahl Alpha-Tier } \\
\text { und Geschwister }\end{array}$ \\
\hline Männchen & 54 & 16 & 37 & 1 \\
Weibchen & 39 & 31 & 7 & 1 \\
Gruppe & 7 & & & \\
unbekannt & 104 & & 44 & 2 \\
insgesamt & 204 & 47 & 44 & \\
\hline
\end{tabular}

\subsubsection{Rauswürfe in Abhängigkeit von individuen-externen Faktoren}

Hypothese 1: Die Wahrscheinlichkeit, dass ein Rauswurf geschieht, ist von individuenexternen Faktoren unabhängig.

\subsubsection{Abhängigkeit der Rauswurfwahrscheinlichkeit von den Locktieren (Experimenten)}

Hypothese 1a: Die Wahrscheinlichkeit, dass ein Rauswurf geschieht, ist von den Locktieren (Experimenten) unabhängig.

Zwei von acht Rauswürfen (25\%) ereigneten sich während der Semifreilandphasen, in denen die Experimente durchgeführt wurden. Der Rauswurf von F geschah direkt nach der ersten Kontrollphase im ersten Beobachtungsjahr als noch keine Locktiere präsentiert wurden. Der Rauswurf von I erfolgte unter den Bedingungen des zweiten Experiments im zweiten Beobachtungsjahr, als der Fokusfamilie zwei Lockmännchen präsentiert wurden. Sechs von acht Rauswürfen (75\%) ereigneten sich außerhalb der Experimentalphasen. Es wird somit 
deutlich, dass keine familienexternen Auslöser, wie die Anwesenheit fremder Artgenossen für die Entstehung eines Rauswurfes notwendig sind oder die Rauswurfwahrscheinlichkeit in der Familie erhöhen. Hypothese 1a kann damit nicht zurückgewiesen werden.

\subsubsection{Abhängigkeit der Rauswurfwahrscheinlichkeit von der Jahreszeit}

Hypothese 1b: Die Wahrscheinlichkeit, dass ein Rauswurf geschieht, ist von der Jahreszeit unabhängig.

Legt man die kalendarische Einteilung der Jahreszeiten zugrunde und setzt voraus, dass die Wahrscheinlichkeit für einen Rauswurf in allen Jahreszeiten gleich groß ist, so würde die Rauswurfwahrscheinlichkeit pro Jahreszeit 0,25 betragen. In der Studie ereigneten sich drei Rauswürfe im Frühjahr (Zeitraum vom 20.03.-20.06.), vier Rauswürfe im Sommer (Zeitraum vom 21.06.-21.09.) und ein Rauswurf im Herbst (Zeitraum vom 22.09.-21.12.). Im Winter (Zeitraum vom 22.12.-19.03.) wurde kein Tier aus der Sozietät ausgeschlossen. Der beobachtete Anteil (Wahrscheinlichkeit für Rauswürfe) der Rauswürfe im Frühjahr betrug demnach 0,375, im Sommer 0,5, im Herbst 0,125 und im Winter 0. Der größte Anteil der Rauswürfe trat in den Sommermonaten auf. Da die Familie während der Sommermonate im Semifreiland lebte und während der Wintermonate im Hütten-Käfig-Komplex, erhöht die Vergrößerung des Streifgebietes möglicherweise die Rauswurfwahrscheinlichkeit. Unter der Annahme dieses Zusammenhanges weisen die mittleren Rauswurfwahrscheinlichkeiten der Frühjahrs- und Herbstmonate darauf hin, dass die Familie ca. jeweils eine Hälfte dieser Monate im Semifreiland verbrachte. Aufgrund der geringen Stichprobenzahl kann nicht ermittelt werden, ob das vorliegende Ergebnis zufällig ist, so dass die Hypothese $1 \mathrm{~b}$ nicht überprüft werden kann.

\subsubsection{Abhängigkeit der Rauswurfwahrscheinlichkeit von der Gruppengröße}

Hypothese 1c: Die Wahrscheinlichkeit, dass ein Rauswurf geschieht, ist von der Gruppengröße unabhängig.

Die Wahrscheinlichkeit für einen Rauswurf in Abhängigkeit von der Gruppengröße wird anhand empirischer Befunde aus Aufzeichnungen über die Callithrix jacchus-Kolonie des IfA aus dem Zeitraum 1988 bis 2003 mit der Formel

$\mathrm{P}_{\text {Rauswurf }}[$ Gruppengröße $]=\mathrm{n} / \mathrm{N}$

$\mathrm{n}=$ Anzahl der Rauswürfe bei einer bestimmten Gruppengröße

$\mathrm{N}=$ Anzahl der Gruppenmonate einer bestimmten Gruppengröße 
berechnet. Die Gruppengrößen variierten zwischen 3 und 18 Mitgliedern und es fanden 106 Rauswürfe statt. Zur Berechnung der Rauswurfwahrscheinlichkeit bei einer bestimmten Gruppengröße wird die Anzahl aller Monate, in denen sich Gruppen einer bestimmten Größe in der Kolonie befanden, addiert $(=\mathrm{N})$. Die Anzahl der beobachteten Rauswürfe bzw. Emigrationen bei einer gegebenen Gruppengröße wird durch die Anzahl der Gruppenmonate dividiert, woraus die Rauswurfwahrscheinlichkeit bei der entsprechenden Gruppengröße resultiert.

Es zeigt sich dabei, dass die Wahrscheinlichkeit für einen Rauswurf bei den Gruppengrößen 11, 13 und 17 höher ist als bei anderen Gruppengrößen ${ }^{16}$ (Abb. 3.30). Bei den Gruppengrößen 3, 4 und 6 hingegen ist die Rauswurfwahrscheinlichkeit geringer als erwartet.

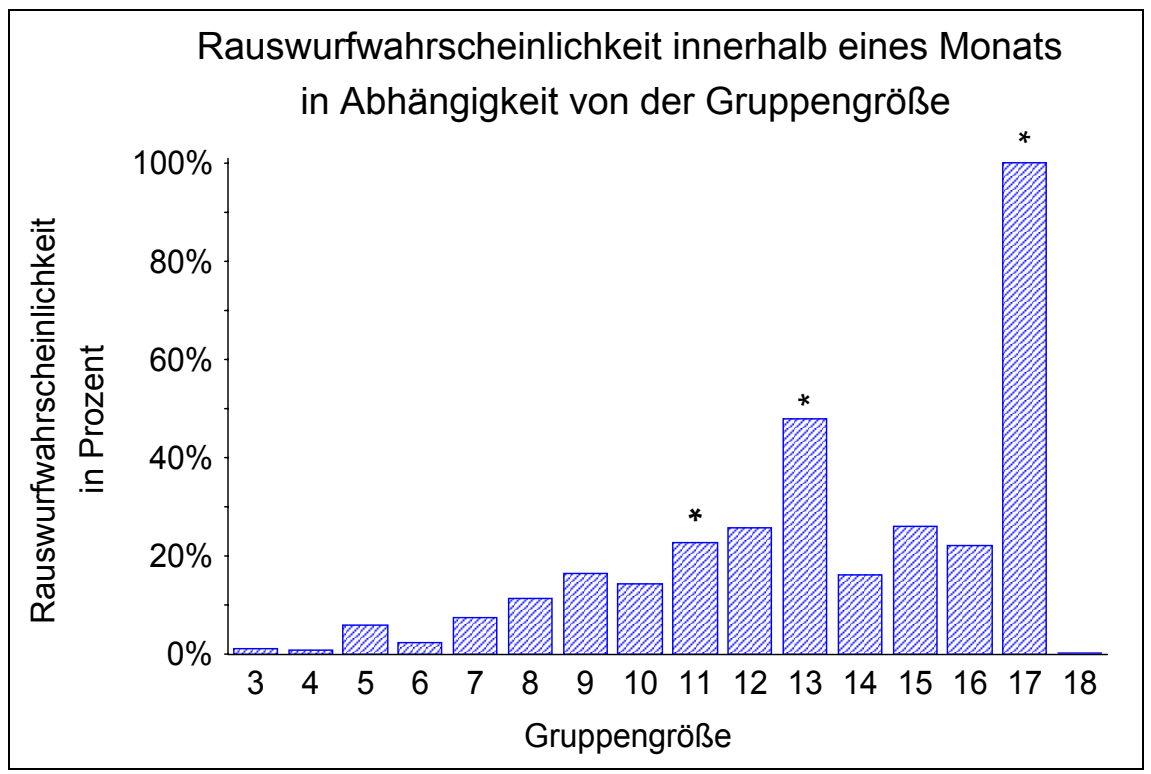

Abb. 3.30 Rauswurfwahrscheinlichkeit innerhalb eines Monats bei den Gruppengrößen 3 bis 18 in der $C$. jacchus-Kolonie des IfA im Zeitraum von 1988 bis 2003 (Anzahl der Gruppenmonate N=991,62; Anzahl der Rauswürfe $n=106)$. Die Wahrscheinlichkeit, dass innerhalb eines Monats ein Rauswurf geschieht, ist bei den Gruppegrößen 11, 13 und 17 größer als erwartet und bei den Gruppengrößen 3, 4 und 6 geringer als erwartet (KFA, EW=10,69\% n=16). Symbol: * = signifikant häufiger als erwartet.

Die Rauswürfe der vorliegenden Studie geschahen bei den Gruppengrößen 8, 10 und 11, wobei sich der einzige Rauswurf bei der Gruppengröße 8 nach dem Tod des AlphaWeibchens zutrug und er somit nicht unter den Bedingungen vollständiger Familien stattfand.

Da eine Abhängigkeit der Rauswurfwahrscheinlichkeit von der Gruppengröße ermittelt werden kann, muss die Hypothese 1c zurückgewiesen werden.

\footnotetext{
${ }^{16}$ Die hohe Rauswurfwahrscheinlichkeit bei einer Gruppengröße von 17 liegt möglicherweise an der geringen Anzahl (2) dieser Gruppengröße in der statistischen Analyse.
} 


\subsubsection{Abhängigkeit der Wahrscheinlichkeit eines Rauswurf vom Alter der Jungtiere}

Hypothese 1d: Die Wahrscheinlichkeit, dass ein Rauswurf geschieht, besteht nur während Zeiträumen, in denen Jungtiere nicht getragen werden müssen.

Während der zweieinhalb Beobachtungsjahre gab es drei Geburten, und ein Jungtierzwillingspaar war zu Beginn der ersten Beobachtungsperiode bereits 51 Tage alt. Rothe et al. (1993) fanden, dass die Entlastung der Eltern durch Jungtierträger in der Periode zwischen der vierten und siebenten Lebenswoche (28 - 49 Tage) der Jungtiere am größten ist. Es ist daher $\mathrm{zu}$ erwarten, dass Rauswürfe vorwiegend auftreten, wenn die Jungtiere die siebte Lebenswoche bereits überschritten haben, da von diesem Zeitpunkt an eher auf zusätzliche Helfer verzichtet werden kann als vorher.

Ausgehend von einem Inter-Geburten-Intervall von 150-157 Tagen (König et al. 1990) kann für die C. jacchus-Kolonie des IfA ermittelt werden, dass Rauswürfe von Gruppenmitgliedern am häufigsten innerhalb der ersten 20 Lebenstage der Jungtiere geschehen (Abb. 3.31). Die Wahrscheinlichkeit, dass sich ein Rauswurf ereignet, ist demnach während der Jungtieraltersklasse 0-20 am größten, während die Rauswurfwahrscheinlichkeit in allen anderen Jungtieraltersklassen gleich groß ist.

In der Familie J18 traten die Rauswürfe auf, als die jeweiligen Jungtiere zwischen 19 und 160 Tage alt waren. Es gab somit einen Rauswurf in der Jungtieraltersklasse 0-20, zwei Rauswürfe in der Alterklasse 60-80, einen Rauswurf in der Altersklasse 100-120 und zwei Rauswürfe in der Altersklasse 140-160. Für eine statistische Analyse ist die Datengrundlage zu gering.

Die Hypothese 1d muss abgelehnt werden, da die Rauswurfwahrscheinlichkeit am größten ist, wenn die Jungtiere noch getragen werden müssen. 


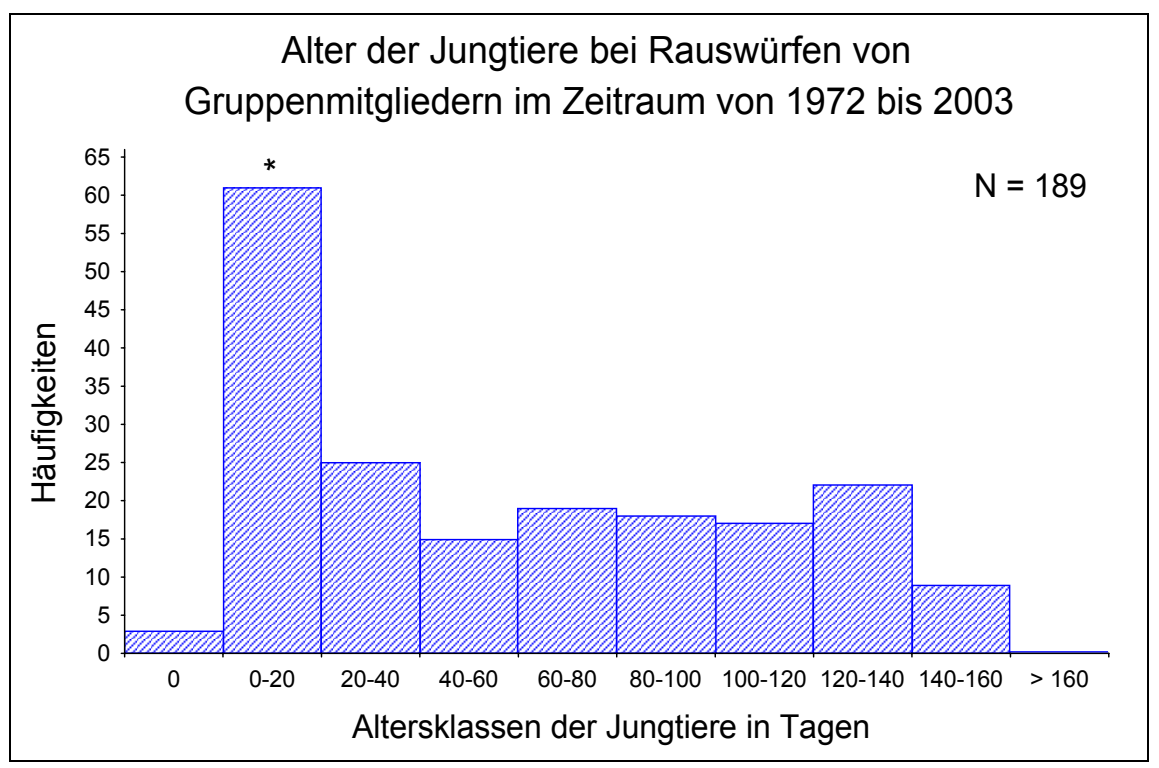

Abb. 3.31 Alter der Jungtiere bei Rauswürfen von Gruppenmitgliedern aus Gruppen der C. jacchus-Kolonie des IfA im Zeitraum von 1972-2003; N=189. (* = signifikant häufiger als erwartet nach KFA, EW=21 n=9).

\subsubsection{Abhängigkeit der Rauswurfwahrscheinlichkeit vom Östrus des Alpha- Weibchens}

Hypothese 1e: Die Wahrscheinlichkeit, dass ein Rauswurf geschieht, ist vom Östrus des Alpha-Weibchens unabhängig.

Es wurde getestet, ob ein zeitlicher Zusammenhang zwischen den Rauswürfen und dem Östrus des Alpha-Weibchens der Fokusfamilie bestand. Grooming zwischen den AlphaTieren ist eine wesentliche Komponente des Verhaltens-Östrus bei Callithrix jacchus. Eine Erhöhung der Grooming-Rate des Alpha-Männchens gegenüber dem Alpha-Weibchen kann auf einen Postpartum-Östrus des Weibchens hindeuten (Rothe 1974). Die GroomingHäufigkeit von A gegenüber B wurde daher zur Ermittlung des Östrus von B herangezogen. Zusätzlich deuten die Partnerbewachung des Alpha-Männchens (dissoziatives Verhalten gegenüber seinen Söhnen in der Nähe des Alpha-Weibchens) und Kopulationen auf einen Östrus $\operatorname{hin}^{17}$.

Mate-guarding konnte ad libitum im ersten Beobachtungsjahr an den statistischen Tagen B23, B2-4 und C1-3 beobachtet werden. Kopulationen traten in B1-5, B2-4 und C1-4 auf, d.h. einmal ohne vorheriges mate-guarding, einmal einen statistischen Tag nachdem mateguarding beobachtet wurde und einmal während des statistischen Tages, an dem auch mate-

\footnotetext{
${ }^{17}$ Auf eine hormonelle Bestimmung des Östrus des Alpha-Weibchens musste in dieser Studie verzichtet werden, da die Gewinnung von Blut- oder Urinproben regelmäßig Eingriffe in die Fokusfamilie vorausgesetzt hätte, deren Auswirkungen auf die Soziodynamik nicht kalkulierbar sind.
} 
guarding auftrat. Die durch instantaneous sampling gewonnen Daten über GroomingHäufigkeiten geben im ersten Beobachtungsjahr die höchste Grooming-Rate in B1-5, relativ hohe Raten in B2-3 bis B2-5 und in C1-4 an (Abb. 3.32). In allen drei Zeiträumen wurden auch Kopulationen beobachtet. Die höchste Grooming-Rate trat 85-88 Tage nach der letzten Geburt auf. Ein Postpartum-Östrus beginnt ca. fünf Tage nach der Geburt und hält 9 bis 11 Tage an (Rothe 1974). Da die erhöhte Grooming-Rate erst sehr viel später beobachtet wurde, handelte es sich damit nicht um einen Postpartum-Östrus. Eine Rückrechnung von der darauffolgenden Geburt mit einer Gestationsphase von 144-150 Tagen ließ darauf schließen, dass die Konzeption innerhalb des statistischen Tages B2-4 lag, in dem auch Kopulationen und mate-guarding dokumentiert wurden. Es kann damit nachgewiesen werden, dass das Alpha-Weibchen im Zeitraum der hohen Grooming-Raten östrisch war.

Der einzige Rauswurf im ersten Beobachtungsjahr ereignete sich zwischen den statistischen Tagen B1-5 und B2-1, also direkt nachdem die höchste Grooming-Rate bei den Alpha-Tieren beobachtet wurde, jedoch vor der errechneten Konzeption.

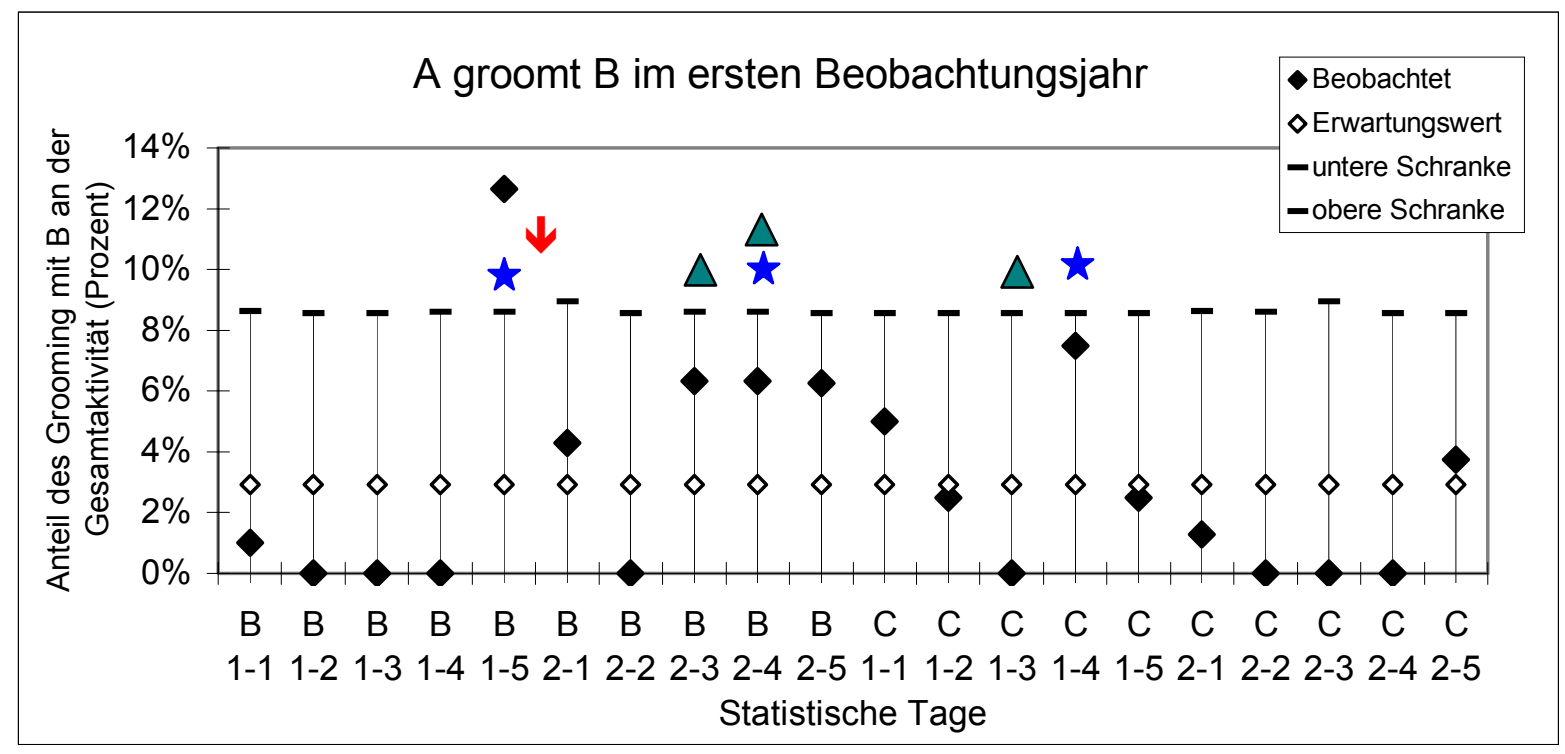

Abb. 3.32 Prozentualer Anteil des Grooming des Alpha-Weibchen durch das Alpha-Männchen an der Gesamtaktivität im ersten Beobachtungsjahr. Abkürzungen: B1-1 ist der erste statistischen Tag in der ersten Kontrollphase, B1-2 ist der zweite statistische Tag in der ersten Kontrollphase etc. Die Werte sind für jeweils fünf statistische Tage der vier Phasen B1, B2, C1 und C2 angegeben. Roter Pfeil: Zeitpunkt Rauswurf von F; blaue Sterne: Kopulationen, grüne Dreiecke: mate-guarding (KFA, EW=2,93 n=20).

Im zweiten Beobachtungsjahr war die Grooming-Rate während der statistischen Tage E1-3 und E2-2 überdurchschnittlich erhöht (Abb. 3.33). Einen statistischen Tag nach dem ersten Peak (in E1-4) wurden Jungtiere geboren. Während der Phase E2-1 und E3-4 kopulierten A und B und in E3-4 wurde mate-guarding beobachtet. In E2-2 wurde der männliche Nachkomme I aus der Familie geworfen. Die Beobachtungen deuten daher auf einen 
Zusammenhang zwischen dem Rauswurf von I und dem Postpartum-Östrus des AlphaWeibchens hin. H wurde am Ende der Hüttenphase D1 (2001) aus der Familie verstoßen. Da das Alpha-Männchen B während dieser Zeit fast gar nicht groomte und 20 Tage nach dem Rauswurf Jungtiere geboren wurde, gab es keinen Hinweis auf einen Zusammenhang zwischen einem Östrus des Alpha-Weibchens und dem Rauswurf von $\mathrm{H}$.

Der Rauswurf von I deutete auf einen Zusammenhang mit dem Postpartum-Östrus des AlphaWeibchens hin. Der Rauswurf von F stand möglicherweise auch im Zusammenhang mit einem Östrus des Alpha-Weibchens. Bevor E ausgestoßen wurde, waren keine Anzeichen eines Östrus des Alpha-Weibchens zu erkennen. Auffällig war jedoch ein erhöhter Anteil an lokomotorischen und Markier-Aktivitäten der ältesten Tochter D und zeitgleich auftretender Aggression der Familie gegen sie. Später war E auch von dissoziativen Aktionen der Familienmitglieder betroffen. Da sich die Anwendung der Temperaturbuttons für die Erkennung von Ovulationen in dieser Studie als unbrauchbar erwies, konnte bei D kein Östrus nachgewiesen werden. Es bleibt daher ungeklärt, ob die älteste Tochter ovulierte und der Rauswurf von E damit in Zusammenhang gebracht werden könnte.

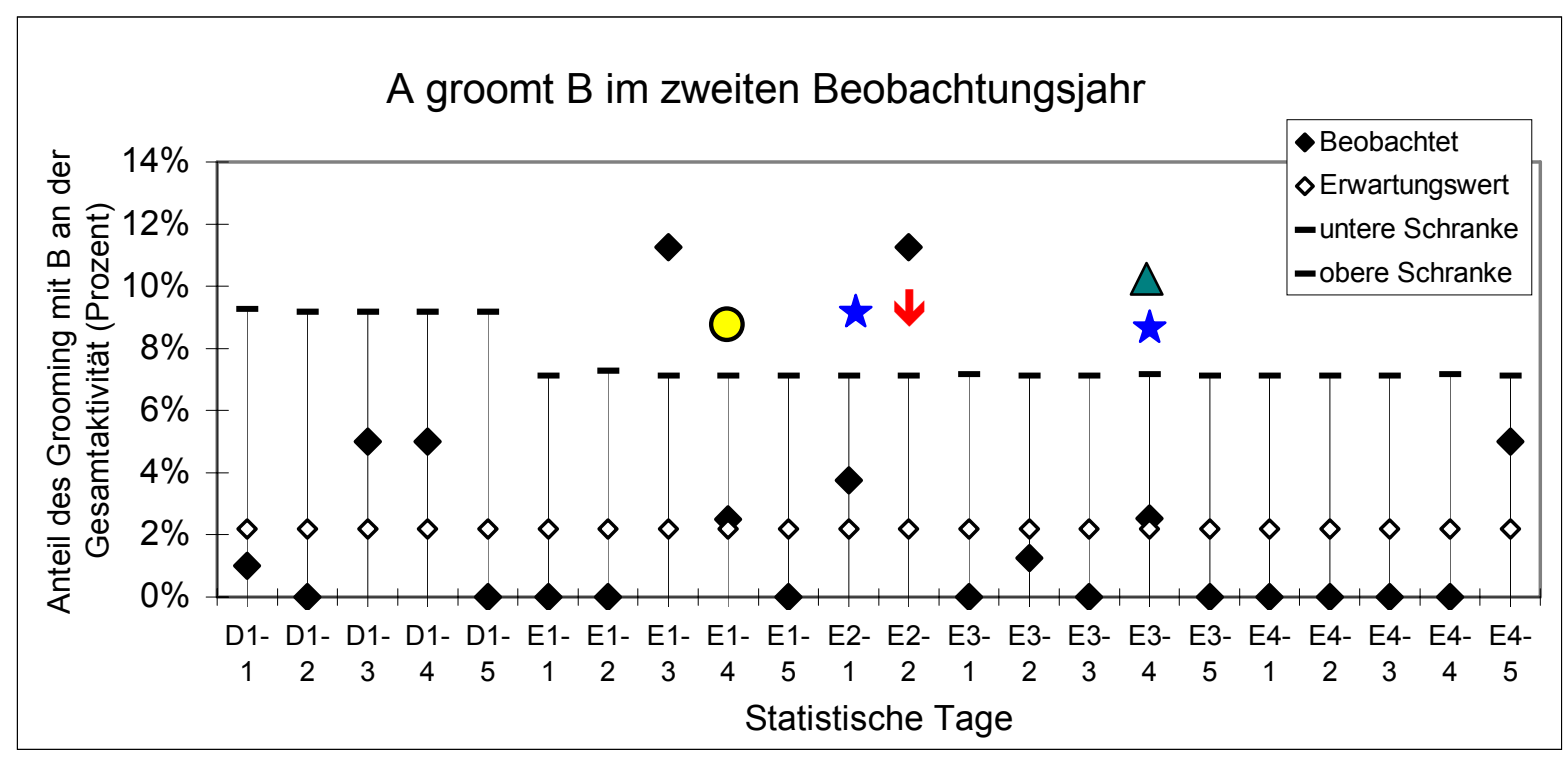

Abb. 3.33 Prozentualer Anteil des Grooming des Alpha-Weibchen durch das Alpha-Männchen an der Gesamtaktivität im zweiten Beobachtungsjahr. Abkürzungen: D1-1 ist der erste statistischen Tag in der Hüttenphase, D1-2 ist der zweite statistische Tag in der Hüttenphase etc. Die Werte sind für jeweils fünf statistische Tage der Phasen D1, E1, E2, E3 und E4 angegeben. Roter Pfeil: Zeitpunkt Rauswurf von I; blaue Sterne: Kopulationen, grüne Dreiecke: mate-guarding, gelber Kreis: Geburt (KFA, EW=2,19 n=22).

Fünf Tage vor dem Rauswurf von M machten sich Anzeichen für einen Östrus von B bemerkbar, indem A mate-guarding betrieb und häufig an der Genitalregion des AlphaWeibchens leckte. Die letzte Geburt lag vor dem Rauswurf 79 Tage zurück. Da das AlphaWeibchen nach dem Rauswurf von M keine Geburt mehr hatte und das Verhalten der Familie 
während dieser Zeit ad libitum erhoben wurde, war es nicht möglich, den rezeptiven Zeitraum des Alpha-Weibchens genauer zu ermitteln.

Zwei der beobachteten Rauswürfe männlicher Familienmitglieder (F und I) lassen einen Zusammenhang der Rauswurfwahrscheinlichkeit und dem (Postpartum)-Östrus des AlphaWeibchens vermuten. Da während des instantaneous sampling nur diese beiden Rauswürfe auftraten, ist die Wahrscheinlichkeit, dass ein Rauswurf von dem Östrus des AlphaWeibchens abhängt, sehr groß. Zur Berechnung der Wahrscheinlichkeit eines Rauswurfs muss die Annahme zugrunde gelegt werden, dass alle Östren des Alpha-Weibchens erkannt wurden. Unter Vorbehalt dieser Annahme traten in einem Zeitrahmen von N=22 Monaten zwei Rauswürfe während eines Östrus auf und drei Rauswürfe in Monaten, in denen das Alpha-Weibchen nicht östrisch war. Innerhalb drei weiterer Monate zeigte das AlphaWeibchen östrisches Verhalten, in denen aber kein Rauswurf vorkam. Während der restlichen 14 Monate konnte kein östrisches Verhalten beim Alpha-Weibchen beobachtet werden und es gab auch keinen Rauswurf.

Nach der statistischen Analyse (KFA, EW=29,41 n=2) ist die Wahrscheinlichkeit, dass ein Rauswurf während eines Östrus geschieht ebenso groß wie die Wahrscheinlichkeit, dass ein Rauswurf stattfindet, wenn das Alpha-Weibchen nicht östrisch ist. Unter der Annahme, dass alle Östren erkannt wurden, kann Hypothese 1e nicht abgelehnt werden. Werden nur Rauswürfe betrachtet, welche im Zeitraum des instantaneous sampling geschahen, kann die Hypothese zurückgewiesen werden.

\subsubsection{Rauswürfe in Abhängigkeit von individuellen Faktoren}

Bei der Suche nach Gründen und Voraussetzungen, die dazu führen, dass ein Familienmitglied ausgeschlossen wird, erhebt sich die Frage, ob bestimmte Individuen aufgrund ihres Verhaltens, Alters, Geschlechts etc. von der Familie ausgeschlossen werden. Anhand der folgenden Hypothese wurde die Analyse der Beobachtungen für dieses Kapitels durchgeführt:

Hypothese 2: Die Wahrscheinlichkeit, rausgeworfen zu werden, ist für alle Nachkommen gleich groß. 


\subsubsection{Abhängigkeit der Wahrscheinlichkeit, rausgeworfen zu werden, vom Alter der Nachkommen}

Hypothese 2a: Die Wahrscheinlichkeit, rausgeworfen $\mathrm{zu}$ werden, ist vom Alter der Nachkommen unabhängig.

Das Alter der rausgeworfenen Gruppenmitglieder der C. jacchus-Kolonie des IfA wird Alterklassen zugeordnet. Da das jüngste rausgeworfene Tier 5,5 Monate alt war, beginnt die Einteilung bei 5 Monaten und geht dann in 5-Monats-Schritten bis zum Alter von 60 Monaten. Die letzte Altersklasse fasst alle Tiere, die älter als 60 Monate alt waren, zusammen. Die Altersklassen 10-15, 15-20, 20-25 und 25-30 Monate wurden häufiger als erwartet rausgeworfen, während Tiere der Altersklassen 0-5, 45-50, 50-55, 55-60 und > 60 seltener als erwartet rausgeworfen wurden (Abb. 3.34).

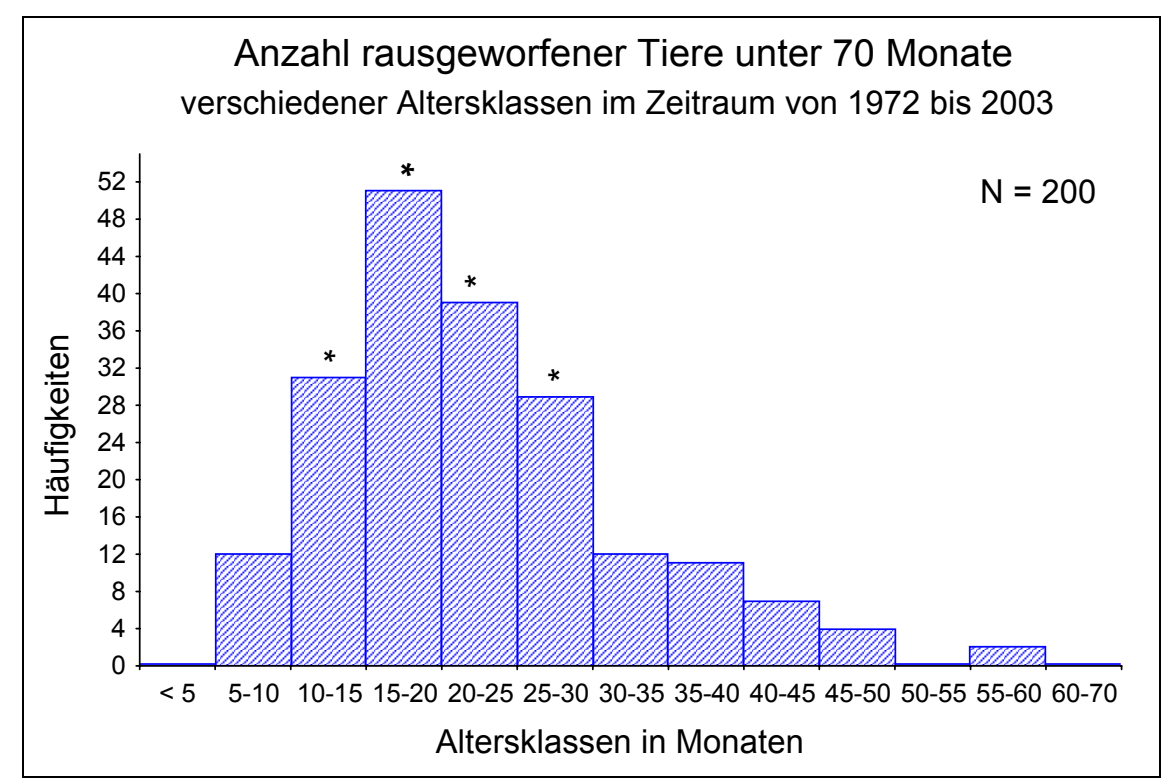

Abb. 3.34 Alter der Tiere, die im Zeitraum von 1972-2003 in der C. jacchus-Kolonie des IfA aus ihrer Gruppe herausgeworfen worden sind. Vier männliche Tiere sind nicht abgebildet, die zu den Altersklassen über 70 Monaten gehörten: 70-75; 85-90, 100-105 und 125-130; N=200. Gruppenmitglieder der Altersklassen 10-15, 1520, 20-25 und 25-30 wurden häufiger als erwartet rausgeworfen (KFA, EW=15,23 n=13). * = signifikant häufiger als erwartet.

Bei der Berechnung der Rauswurfwahrscheinlichkeit für ein bestimmtes Alter werden Männchen und Weibchen getrennt betrachtet. Dabei wird die Anzahl der männlichen rausgeworfenen Tiere durch die Anzahl der Tiere geteilt, welche innerhalb der Population die entsprechende Altersklasse erreicht hatten. Die Rauswurfwahrscheinlichkeit der Männchen ist in den Altersklassen 15-20 und 20-25 Monate höher als in den anderen Altersklassen, während sie in den Altersklassen 5-10 und >60 Monate geringer als erwartet ist (Abb. 3.35). Die Rauswurfwahrscheinlichkeit der Weibchen ist in den Altersklassen 15-20, 20-25 und 25- 
30 Monate höher als in den anderen Altersklassen und in den Altersklassen 5-10 und >60 geringer als erwartet (Abb. 3.36). Es gibt keine Unterschiede in der Rauswurfwahrscheinlichkeit zwischen Männchen und Weibchen in Abhängigkeit vom Alter (Mann-Whitney-UTest: $U=3186, Z=-1,91, p=0,055)$.

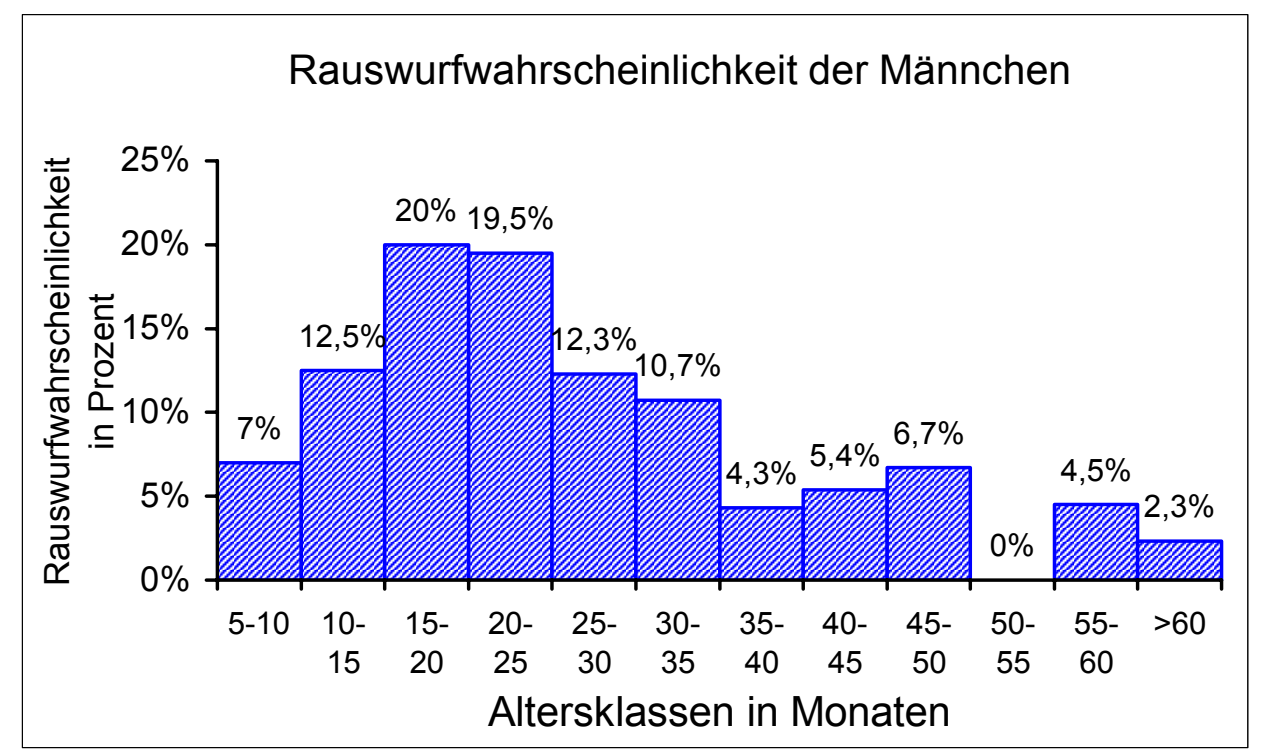

Abb. 3.35 Rauswurfwahrscheinlichkeit für Männchen, die älter als 5 Monate waren, im Zeitraum von 1972-2003 in $\operatorname{der}$ C. jacchus-Kolonie des IfA in den verschiedenen Altersklassen. Die Rauswurfwahrscheinlichkeit ist in den Altersklassen 15-20 und 20-25 Monate höher als in den anderen Altersklassen. In den Altersklassen 5-10 und $>60$ Monate ist die Rauswurfwahrscheinlichkeit für Männchen geringer als erwartet (KFA, EW=9,95 n=12).

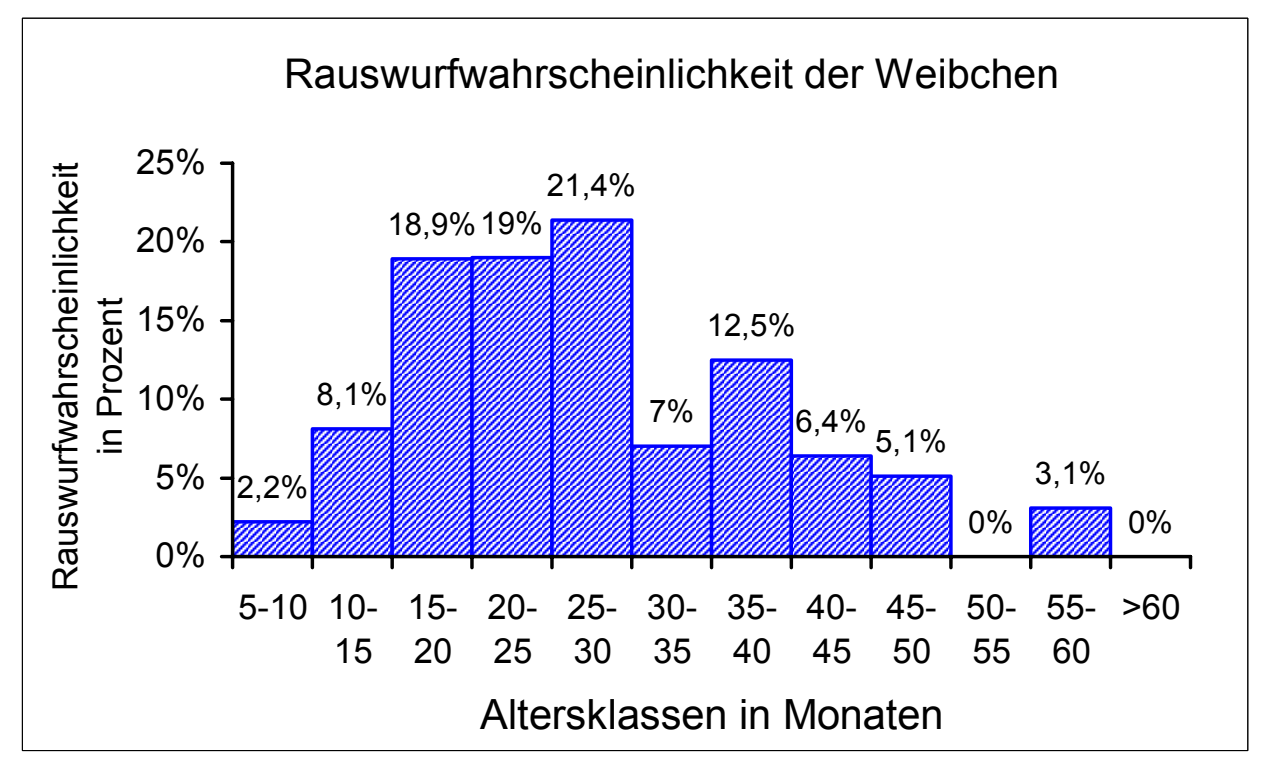

Abb. 3.36 Rauswurfwahrscheinlichkeit für Weibchen, die älter als 5 Monate waren, im Zeitraum von 1972-2003 in der C. jacchus-Kolonie des IfA in den verschiedenen Altersklassen. Die Rauswurfwahrscheinlichkeit ist in den Altersklassen 15-20, 20-25 und 25-30 Monate höher als in den anderen Altersklassen. In den Altersklassen 5-10 und >60 Monate ist die Rauswurfwahrscheinlichkeit für Weibchen geringer als erwartet (KFA, EW=9,12 $\mathrm{n}=12)$.

Drei der in dieser Studie rausgeworfenen Männchen waren der Altersklasse 10-15 Monate zuzuordnen, zwei Männchen der Altersklasse 15-20 Monate und ein Männchen der Altersklasse 35-40 Monate. Demnach wurden nur zwei Männchen in der Altersklasse rausgeworfen, 
in der die Rauswurfwahrscheinlichkeit am größten ist. Die beiden rausgeworfenen Weibchen gehörten der Altersklasse 15-20 Monate an, für die ebenfalls die Rauswurfwahrscheinlichkeit am größten ist.

Hypothese 2a kann abgelehnt werden, da sich die Wahrscheinlichkeit, rausgeworfen zu werden, zwischen den Altersklassen unterscheidet. Das Risiko für einen Rauswurf ist bei Männchen im Alter von 15-25 Monaten und bei Weibchen im Alter von 15-30 Monaten am größten.

\subsubsection{Abhängigkeit der Wahrscheinlichkeit, rausgeworfen zu werden, vom Geschlecht der Nachkommen}

Hypothese 2b: Die Wahrscheinlichkeit, rausgeworfen zu werden, ist vom Geschlecht der Nachkommen unabhängig.

Obwohl nach dem Modell bekannt ist, dass beide Geschlechter aus ihren Natalfamilien emigrieren, blieb bisher unklar, ob ein bestimmtes Geschlecht bevorzugt rausgeworfen wird. Aus Arbeiten von König (1995) und Garber et al. (1984 für S. mystax) geht hervor, dass der Aufzuchtserfolg vom Geschlechterverhältnis beeinflusst werden kann. Es wird daher getestet, ob Unterschiede in der Rauswurfwahrscheinlichkeit der beiden Geschlechter existieren. Die während der Beobachtungsperiode dieser Studie herausgeworfenen Tiere waren zu 75\% männlichen und zu 25\% weiblichen Geschlechts. Um eine Abschätzung des Rauswurfrisikos vorzunehmen, ist die Stichprobengröße zu gering. Rauswürfe, die in der WeißbüschelaffenKolonie des IfA während des Zeitraums von 1972 bis 2003 beobachtet wurden, lassen jedoch eine Abschätzung des Risikos zu.

Es gab innerhalb des Zeitraumes von 1972-2003 insgesamt 724 Tiere in der Kolonie, die mindestens 5 Monate alt waren. Die Anzahl der Männchen betrug 371 und die Anzahl der Weibchen 353. Somit wurden 103/371 = 27,76\% der Männchen und 101/353 = 28,61\% der Weibchen aus ihren Gruppen geworfen. Es gibt keinen Unterschied zwischen den Anteilen der rausgeworfenen Männchen und Weibchen an der Gesamtzahl der Geschlechtsklassen, die älter als 5 Monate waren (Abb. 3.37). Es kann damit belegt werden, dass die Rauswurfwahrscheinlichkeit für einen Weißbüschelaffen in Laborhaltung von seinem Geschlecht unabhängig ist, so dass die Hypothese $2 \mathrm{~b}$ nicht zurückgewiesen werden kann. 


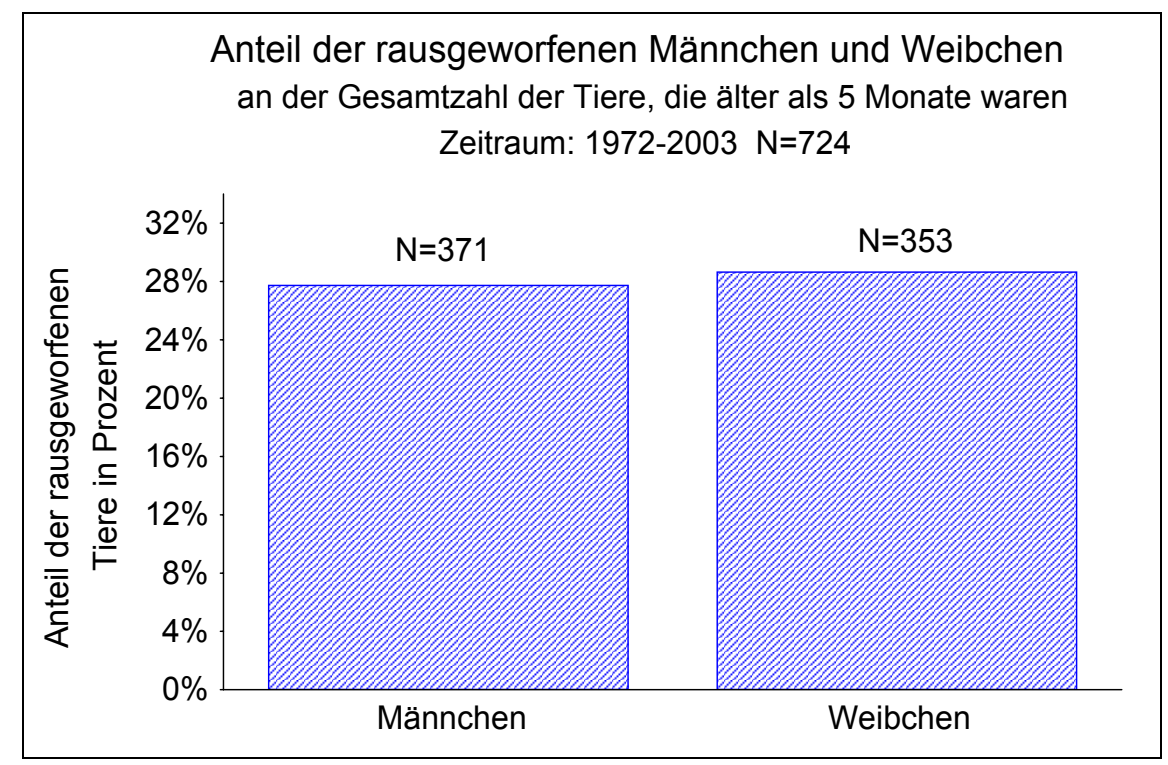

Abb. 3.37 Anteil der rausgeworfenen Männchen und Weibchen an der Gesamtzahl der Männchen und Weibchen, die älter als 5 Monate waren. Die Daten stammen aus der C. jacchus-Kolonie des IfA aus dem Zeitraum 1972-2003 (KFA, EWB=23,81-32,54\%, n=2). Es gibt keinen Unterschied zwischen den Anteilen der rausgeworfenen Männchen und Weibchen an der Gesamtzahl der Tiere, die älter als 5 Monate waren.

\subsubsection{Abhängigkeit der Wahrscheinlichkeit, rausgeworfen zu werden, von der Häufigkeit des Jungtiertragens}

Hypothese 2c: Tiere, die rausgeworfen werden, beteiligten sich ebenso häufig wie andere Nachkommen an der Jungtierfürsorge.

Im ersten Beobachtungsjahr waren die Jungtiere bereits so alt, dass sie nur noch in der ersten Kontrollphase (B1) von Familienmitgliedern getragen wurden. Da das Jungtiertragen den größten Teil der Jungtierfürsorge ausmachte, wurden die Nachkommen nur in dieser Phase miteinander verglichen. Der älteste Sohn C betrieb häufiger Jungtierfürsorge als die anderen adulten und subadulten Geschwister. Die Beobachtungswerte von D und E entsprachen dem Erwartungswert, während F, G und H gar nicht an der Jungtierfürsorge beteiligt waren (Abb. $3.38)$.

Im zweiten Beobachtungsjahr wurden von einer gesamten Trageperiode, beginnend mit der Geburt der Jungtiere (P und R), Daten erhoben. Im ersten Experiment (E1) betrieb C häufiger Jungtierfürsorge als die anderen Nachkommen (Abb. 3.39). Im zweiten Experiment (E2) übten C und M gemeinsam häufiger als erwartet Jungtierfürsorge aus (Abb. 3.40), während C im dritten Experiment (E3) sich wieder als einziger aus den Nachkommen mit häufigerer Jungtierfürsorge heraushob (Abb.3.41). 


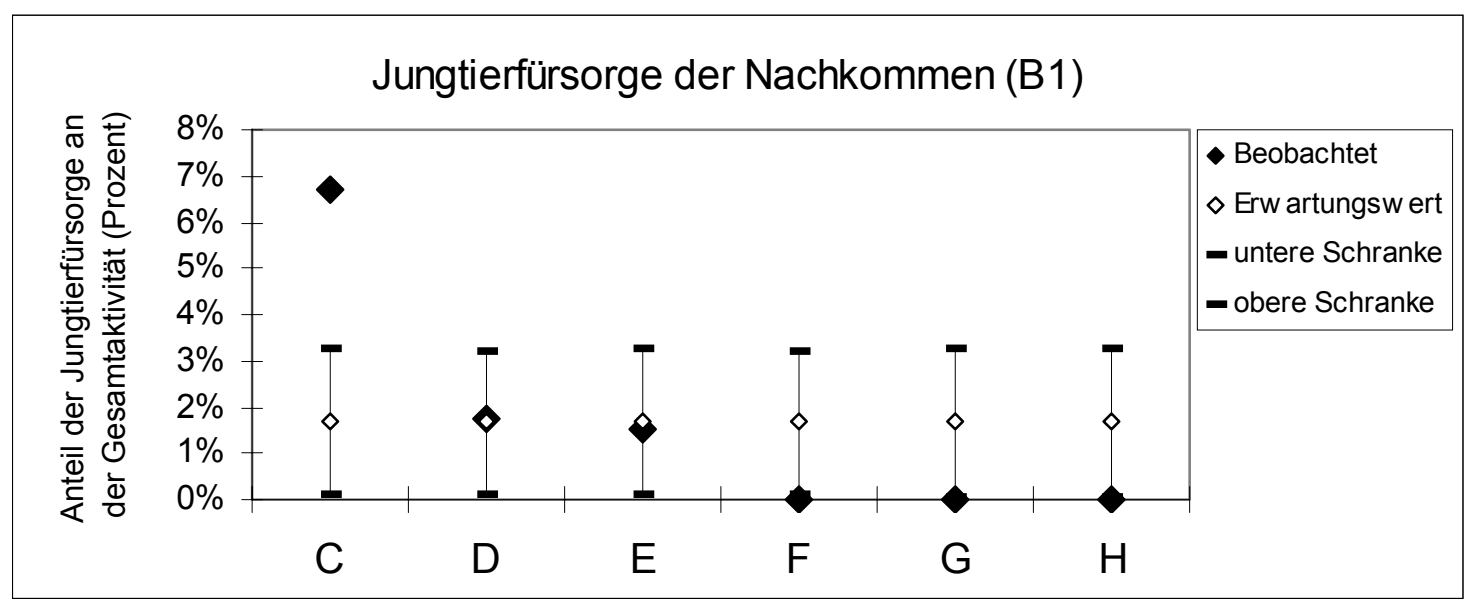

Abb. 3.38 Jungtierfürsorge der adulten und nicht-adulten Nachkommen während der ersten Kontrollphase des ersten Beobachtungsjahres (KFA, EW=1,67 n=6). Abkürzungen: C-H: Nachkommen.

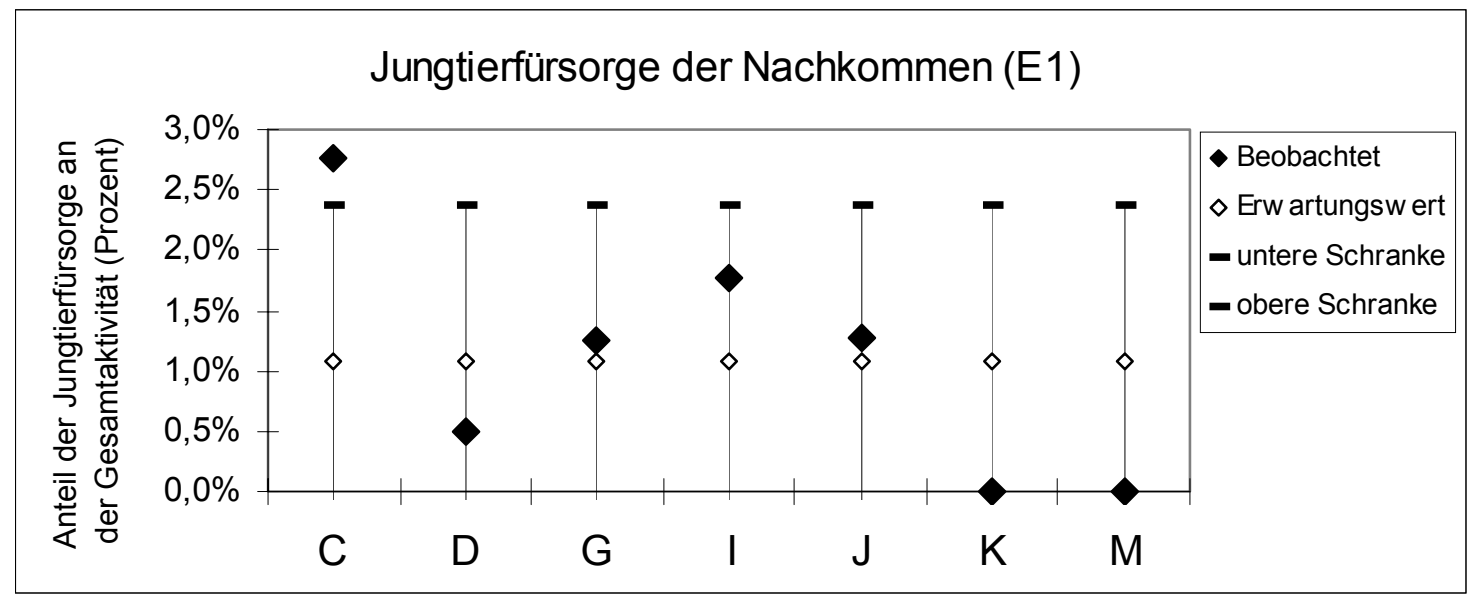

Abb. 3.39 Jungtierfürsorge der adulten und nicht-adulten Nachkommen im ersten Experiment des zweiten Beobachtungsjahres (KFA: EW=1,08 n=7). Abkürzungen: C-M: Nachkommen.

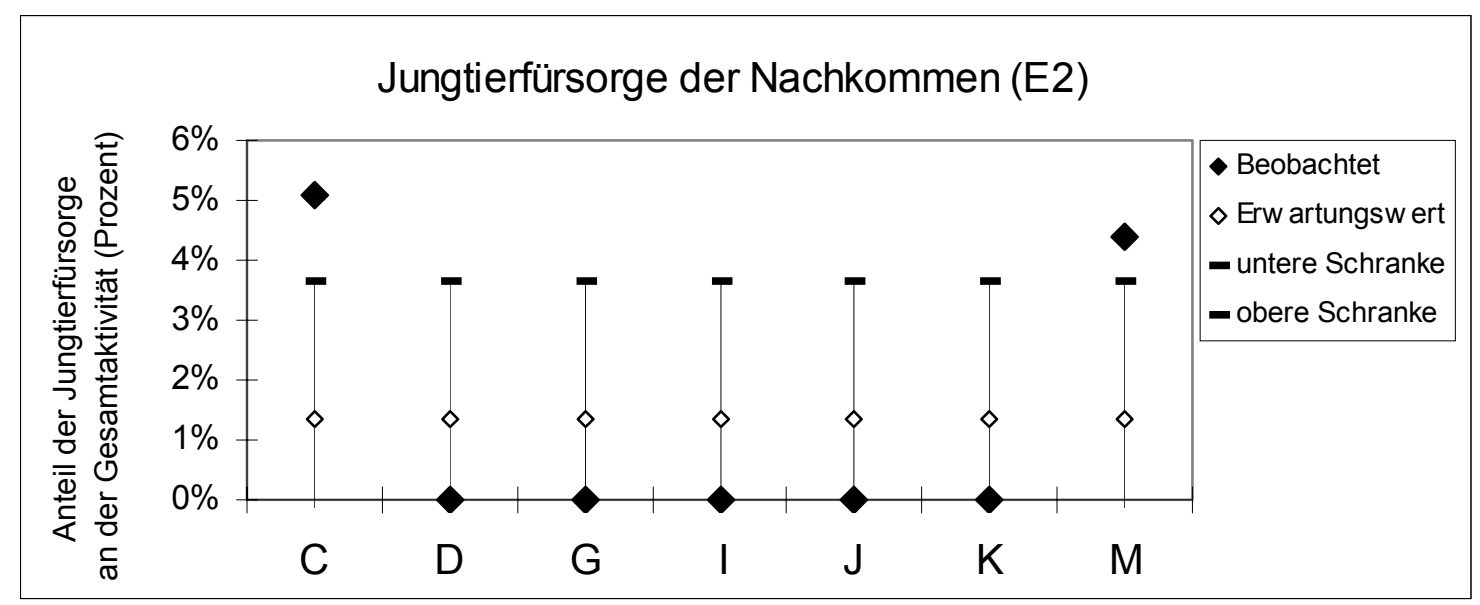

Abb. 3.40 Jungtierfürsorge der adulten und nicht-adulten Nachkommen im zweiten Experiment des zweiten Beobachtungsjahres (KFA: EW=1,35 n=7). Abkürzungen: C-M: Nachkommen. 


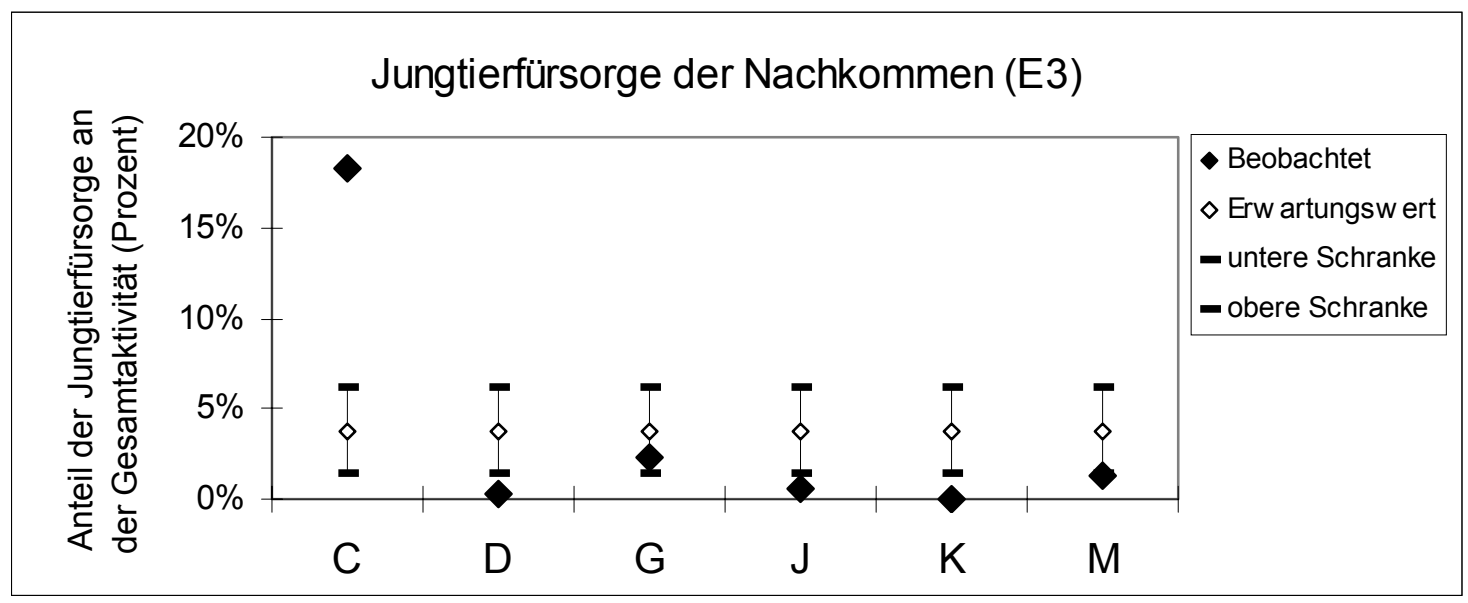

Abb. 3.41 Jungtierfürsorge der adulten und nicht-adulten Nachkommen im dritten Experiment des zweiten Beobachtungsjahres (KFA: EW=3,81 n=6). Abkürzungen: C-M: Nachkommen.

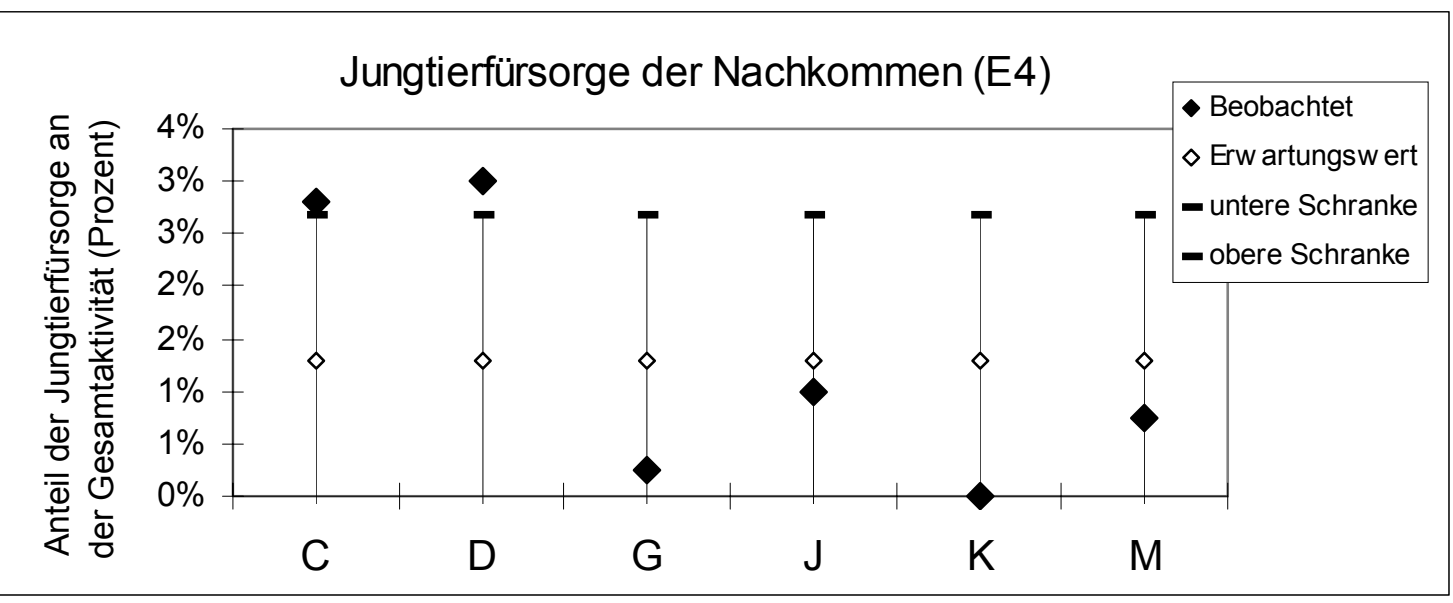

Abb. 3.42 Jungtierfürsorge der adulten und nicht-adulten Nachkommen im vierten Experiment des zweiten Beobachtungsjahres (KFA: EW=1,3 n=6). Abkürzungen: C-M: Nachkommen.

Während des vierten Experiments (E4) betrieben C und D häufiger Jungtierfürsorge, wobei der Anteil von D geringfügig höher war als der von C (Abb. 3.42). Da I am Ende des zweiten Experiments aus der Familie geworfen wurde, gilt ihm besondere Aufmerksamkeit. Seine Jungtierfürsorge lag während des ersten Experiments zwar im Erwartungsbereich, war jedoch höher als bei allen übrigen Nachkommen (außer C). Im zweiten Experiment beteiligte er sich nicht mehr an der Jungtierfürsorge.

Nachdem die zweite quantitative Beobachtungsphase im dritten Jahr beendet war, wurden noch einmal Jungtiere ( $\mathrm{S}$ und $\mathrm{T}$ ) geboren. Da für diese Periode nur ad libitum-Protokolle angefertigt wurden, kann keine statistische Analyse durchgeführt werden. Die erste und anfangs einzige Jungtierträgerin nach dem Alpha-Weibchen war die subadulte K, die auffällig häufig die Jungtiere übernahm. Später wurden die Neugeborenen zeitweise minütlich zwischen den Familienmitgliedern hin- und hergereicht. 
Die Ergebnisse verdeutlichen, dass sich überwiegend ein bis zwei Nachkommen an der Jungtierfürsorge beteiligt hatten und die Jungtierfürsorgeleistung der rausgeworfenen Familienmitglieder F, I, J und M während aller Experimentalphasen innerhalb des Erwartungsbereichs lag. Um herauszufinden, ob sich rausgeworfene und verbliebene Tiere in ihrer Jungtierfürsorgehäufigkeit unterschieden, wird ein Mann-Whitney-U-Test durchgeführt, der jedoch keine Unterschiede nachweisen kann $(U=11397 ; Z=0,68 ; p=0,494)$. Die Hypothese 2c kann nicht zurückgewiesen werden.

\subsubsection{Abhängigkeit der Wahrscheinlichkeit, rausgeworfen zu werden, von der Spielhäufigkeit mit den Jungtieren}

Hypothese 2d: Tiere, die rausgeworfen werden, haben ebenso häufig mit Jungtieren gespielt wie andere Nachkommen.

In der ersten Kontrollphase (B1) des Jahres 2000 spielte $\mathrm{F}$ häufiger als erwartet mit den Jungtieren I und $\mathrm{J}(\mathrm{KFA}, \mathrm{EW}=0,45, \mathrm{n}=8)$. Außer $\mathrm{F}$ spielten noch $\mathrm{A}$, $\mathrm{G}$ und $\mathrm{H}$ mit den Jungtieren. Ihre Werte blieben aber innerhalb des Erwartungsbereiches. Ein Vergleich über das gesamte erste Beobachtungsjahr zeigt, dass $\mathrm{G}$ und $\mathrm{H}$, also die nächst älteren Geschwister, häufiger als die übrigen Familienmitglieder mit den Jungtieren spielten. C und D spielten seltener als erwartet mit I und $\mathrm{J}(\mathrm{KFA}, \mathrm{EW}=1,08, \mathrm{n}=8)$. Hier sei allerdings angemerkt, dass I und $\mathrm{J}$ während der ersten Kontrollphase noch infantil und am Ende des Beobachtungsjahres bereits adoleszent waren.

Im zweiten Beobachtungsjahr waren während der Hüttenphase $\mathrm{K}$ und $\mathrm{M}$ die jüngsten Mitglieder der Familie. G spielte mit ihnen häufiger als alle anderen Familienmitglieder (KFA, EW=1,69, n=8). Während des ersten Experiments spielte $J$ häufiger als die übrigen mit $\mathrm{K}$ und $\mathrm{M}(\mathrm{KFA}, \mathrm{EW}=0,43, \mathrm{n}=7)$. Die neuen Jungtiere $\mathrm{P}$ und $\mathrm{R}$ wurden im Laufe des ersten Experiments geboren, waren für Spiele aber noch zu jung. Im zweiten und dritten Experiment lagen die Werte aller Familienmitglieder im Erwartungsbereich. Während des vierten Experiments begannen $\mathrm{P}$ und $\mathrm{R}$ mit den Familienmitgliedern $\mathrm{zu}$ spielen. Nur $\mathrm{M}$ konnte während des instantaneous sampling beim Spiel mit P und R beobachtet werden (KFA, $\mathrm{EW}=0,13, \mathrm{n}=8$ ).

Kein später rausgeworfenes Familienmitglied spielte seltener als erwartet mit den Jungtieren. Die rausgeworfenen Tiere F, H, J und M spielten in einigen Experimentalphasen häufiger als erwartet mit den Jungtieren, so dass dies ein Hinweis auf einen Zusammenhang zwischen der Rauswurfwahrscheinlichkeit und der Spielhäufigkeit mit den Jungtieren sein könnte. Dagegen 
spricht jedoch, dass G während der beiden Beobachtungsjahre auch häufiger als erwartet mit den Jungtieren spielte. Das fortschreitende Alter der Jungtiere im Laufe eines Beobachtungsjahres lassen eine Beschränkung der Analyse auf die infantile Phase der Jungtiere sinnvoll erscheinen. Danach hätten nur F und M häufiger als erwartet mit den Jungtieren gespielt.

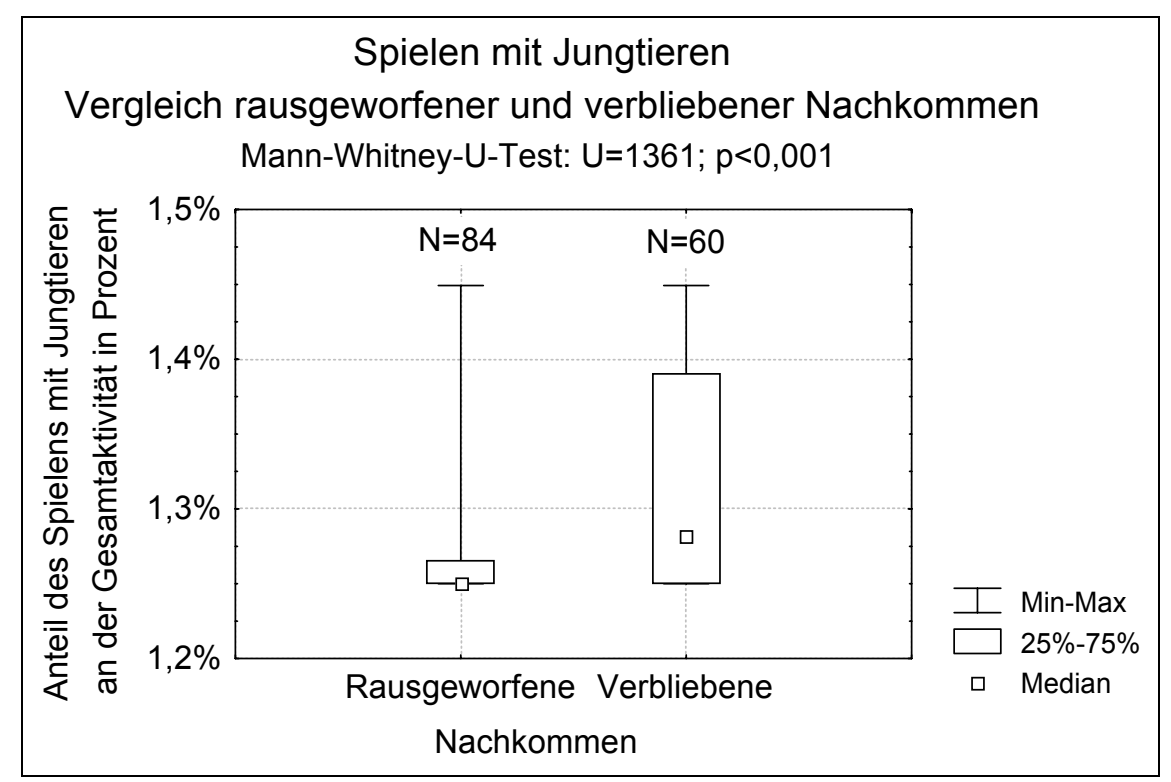

Abb. 3.43 Anteil des Spielens mit Jungtieren an der Gesamtaktivität: Vergleich rausgeworfener und verbliebender Nachkommen (Mann-Whitney-U-Test: $U=1361 ; Z=-4,69 ; p<0,001$ ).

Aus der statistischen Analyse geht hervor, dass Nachkommen, die rausgeworfen wurden seltener mit den Jungtieren spielten als Nachkommen, welche bis zum Ende der Studie in der Familie geblieben sind (Abb. 3.43). Hypothese 2d kann damit zurückgewiesen werden.

\subsubsection{Abhängigkeit der Wahrscheinlichkeit, rausgeworfen zu werden, von der Häufigkeit der Ressourcensicherung und Raubfeindvermeidung}

Hypothese 2e: Tiere, die rausgeworfen werden, verbrachten ebenso viel Zeit wie andere Familienmitglieder mit Ressourcensicherung und Raubfeindvermeidung.

Die prozentualen Anteile des Verhaltensbereichs Ressourcensicherung und Raubfeindvermeidung am Zeitbudget der Familienmitglieder werden verglichen. Das ressourcensichernde und raubfeindvermeidende Verhalten der $\mathrm{zu}$ einem späteren Zeitpunkt ausgestoßenen Tiere E und F entsprach im ersten Beobachtungsjahr dem Erwartungsbereich. H zeigte seltener als erwartet ressourcensicherndes und raubfeindvermeidendes Verhalten, was ihr Zwillings-Bruder G jedoch ebenfalls tat. Die adulten Nachkommen C, D und E investierten mehr Zeit als erwartet in den Verhaltensbereich Ressourcensicherung und 
Raubfeindvermeidung, mit Ausnahme des zweiten Experiments (KFA, B1:EW=38,66 n=6, $\mathrm{B} 2: \mathrm{EW}=44,17 \mathrm{n}=5, \mathrm{C1}: \mathrm{EW}=43,37 \mathrm{n}=5, \mathrm{C} 2: \mathrm{EW}=44,83 \mathrm{n}=5)$.

Während des zweiten Jahres lag der Anteil des Verhaltensbereichs Ressourcensicherung und Raubfeindvermeidung an der Gesamtaktivität bei $\mathrm{C}$ und $\mathrm{K}$ innerhalb des Erwartungsbereichs. Bei D und $G$ befand sich der Anteil an Ressourcensicherung und Raubfeindvermeidung in den ersten beiden Experimenten ebenfalls im Erwartungsbereich, im dritten Experiment bei beiden und im vierten Experiment bei D häufiger als erwartet. G's Anteil an Ressourcensicherung und Raubfeindvermeidung war im vierten Experiment seltener als erwartet. Der Anteil von Ressourcensicherung und Raubfeindvermeidung lag bei I während des ersten Experiments innerhalb des Erwartungsbereichs, war dann im zweiten Experiment jedoch häufiger als erwartet. Der Anteil an Ressourcensicherung und Raubfeindvermeidung war bei J dagegen im ersten Experiment häufiger als erwartet und lag in den übrigen Experimenten innerhalb des Erwartungsbereichs. M, die während des zweiten Beobachtungsjahres noch juvenil bis adoleszent war und erst im darauffolgenden Jahr rausgeworfen wurde, wich in ihrem Anteil an Ressourcensicherung und Raubfeindvermeidung in keinem Experiment vom Erwartungsbereich ab (KFA, E1: EW=31,63 n=7, E2: EW=27,07 n=7, E3: EW=34,26 n=6, $\mathrm{E} 4: \mathrm{EW}=27,88 \mathrm{n}=6$ ).

Ein Vergleich der rausgeworfenen und verbliebenen Nachkommen ergibt keinen Unterschied in der Ressourcensicherung und dem Raubfeindvermeidungsverhalten (Mann-Whitney-UTest: $U=7377 ; Z=-0,68 ; p=0,493)$. Hypothese 2e kann deshalb nicht abgelehnt werden.

\subsubsection{Abhängigkeit der Wahrscheinlichkeit, rausgeworfen zu werden, von der Häufigkeit der nicht-taktilen Interaktion mit fremden Artgenossen}

Hypothese 2f: Tiere, die rausgeworfen werden, wiesen einen ebenso hohen ZeitbudgetAnteil an nicht-taktiler Interaktion mit fremden Artgenossen wie andere Familienmitglieder auf.

Geht man davon aus, dass Interaktionen mit fremden Artgenossen dazu dienen, Kontakte mit zukünftigen Paarungspartnern zu knüpfen und die Chancen der eigenen Reproduktion abzuschätzen, kann die nicht-taktile Interaktion mit fremden Artgenossen als Vorbereitung für eine Emigration aus der Natalfamilie aufgefasst werden. Familienmitglieder, die besonders an fremden Artgenossen interessiert sind, sollten dann bevorzugt rausgeworfen werden, weil sie der Familie durch ihr Interesse an fremden Artgenossen eine Bereitschaft zur Emigration signalisieren. Sollte das Interesse an fremden Artgenossen einen Aspekt der Verteidigung des 
Streifgebietes darstellen, wäre von der Natalfamilie kein dissoziatives Verhalten gegenüber den Nachkommen zu erwarten.

Bei einem Vergleich der adulten Artgenossen hinsichtlich ihrer nicht-taktilen Interaktion mit fremden Artgenossen wurden im Experiment (C1) des ersten Beobachtungsjahres Unterschiede deutlich. E interagierte häufiger als erwartet mit fremden Artgenossen, während die anderen adulten und subadulten Nachkommen keine Abweichungen vom Erwartungsbereich zeigten (Abb.3.44). Da E später von der Familie ausgestoßen wurde, könnte ein Zusammenhang zwischen seinem Verhalten und dem Rauswurf vermutet werden.

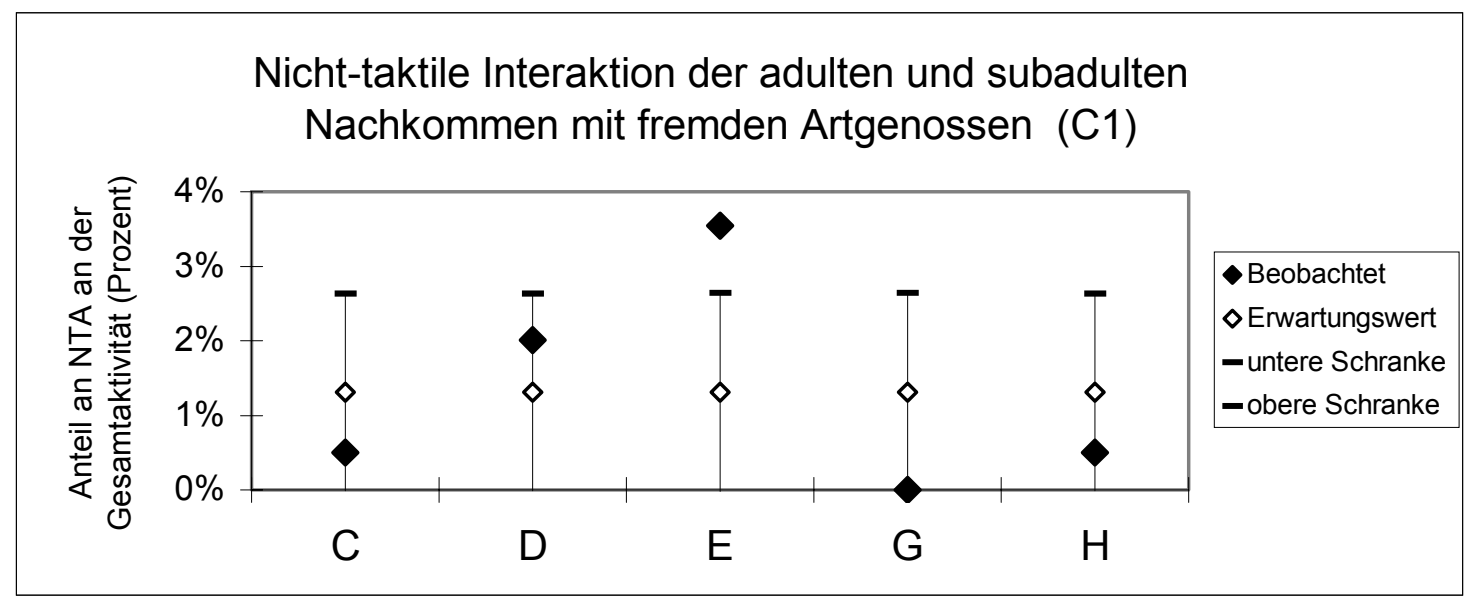

Abb. 3.44 Nicht-taktile Interaktion der adulten und subadulten Nachkommen mit fremden Artgenossen im ersten Experiment des ersten Beobachtungsjahres (KFA, EW=1,31, $\mathrm{n}=5$ ). Abkürzungen: $\mathrm{C} 1$ : erstes Experiment; $\mathrm{C}-\mathrm{E}=$ adulte Nachkommen; G-H = subadulte Nachkommen. Der Anteil an nicht-taktiler Interaktion mit fremden Artgenossen lag bei C, D, G und H innerhalb und bei E über dem Erwartungsbereich.

Im zweiten Beobachtungsjahr war $\mathrm{G}$ bereits adult und I und J subadult. G zeichnete sich während des ersten und dritten Experiments gegenüber seinen adulten und subadulten Geschwistern dadurch aus, dass er häufiger als erwartet mit fremden Artgenossen in Interaktion trat. Die Werte der Nachkommen C, D und I lagen während des ersten Experiments innerhalb des Erwartungsbereichs, ebenso im dritten Experiment die Werte von J (Abb. 3.45 und 3.46).

G zeigte das größte Interesse an den fremden Artgenossen und wurde im Gegensatz zu E nicht ausgestoßen, sondern warf seinerseits andere Geschwister aus der Familie. Obwohl I ausgestoßen wurde, blieb sein Anteil an nicht-taktiler Interaktion mit fremden Artgenossen im Familiendurchschnittsbereich. J, der auch rausgeworfen wurde, hatte geringeres Interesse als erwartet oder zeigte keinen Unterschied zu den adulten Geschwistern C und D, deren Anteil an nicht-taktiler Interaktion mit fremden Artgenossen dem Erwartungsbereich entsprach. Für das zweite Beobachtungsjahr kann daher kein Hinweis dafür gefunden werden, dass die nicht- 
taktile Interaktion mit fremden Artgenossen die Wahrscheinlichkeit, rausgeworfen zu werden, beeinflusst.

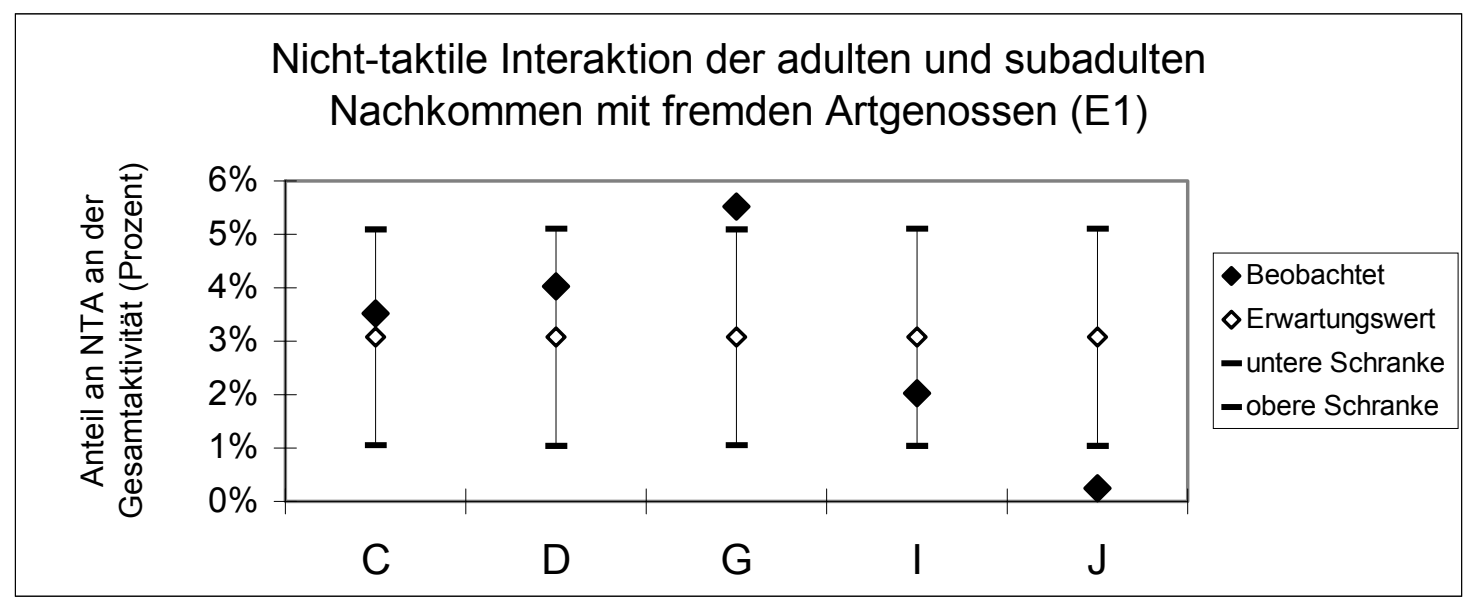

Abb. 3.45 Nicht-taktile Interaktion der adulten Nachkommen mit fremden Artgenossen im ersten Experiment des zweiten Beobachtungsjahres (KFA, EW=3,07 n=5). Abkürzungen: $\mathrm{E} 1=$ erstes Experiment; $\mathrm{C}-\mathrm{G}=$ adulte Nachkommen, I-J = subadulte Nachkommen. Der Anteil an nicht-taktiler Interaktion mit fremden Artgenossen war bei $\mathrm{G}$ häufiger und bei J seltener als erwartet. Die Werte von C, D und I lagen innerhalb des Erwartungsbereichs.

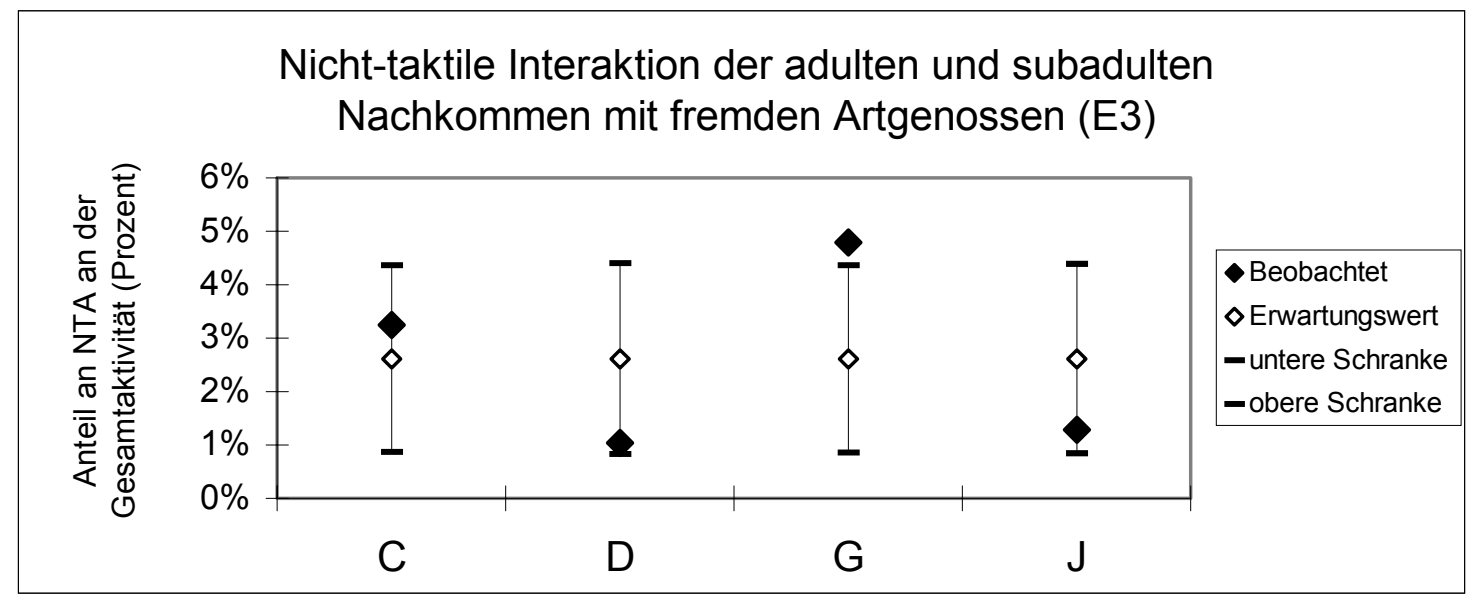

Abb. 3.46 Nicht-taktile Interaktion der adulten Nachkommen mit fremden Artgenossen im dritten Experiment des zweiten Beobachtungsjahres (KFA, EW=2,61 n=4). Abkürzungen: $\mathrm{E} 3=$ drittes Experiment; $\mathrm{C}-\mathrm{G}=$ adulte Nachkommen, $\mathrm{J}=$ subadulter Nachkomme. Der Anteil an nicht-taktiler Interaktion mit fremden Artgenossen war bei G häufiger als erwartet. Die Werte von C, D und J lagen innerhalb des Erwartungsbereichs.

Die rausgeworfenen und verbliebenen Nachkommen unterscheiden sich nicht in ihrem Anteil der nicht-taktilen Interaktion mit fremden Artgenossen an der Gesamtaktivität (MannWhitney-U-Test: $U=4787,5 ; Z=1,13 ; p=0,255)$. Hypothese $2 f$ kann daher nicht abgewiesen werden, wobei die Einzelbetrachtung von E eine Zurückweisung der Hypothese zulässt. 


\subsubsection{Abhängigkeit der Wahrscheinlichkeit, rausgeworfen zu werden, von der Häufigkeit affiliativer Interaktionen}

Hypothese 2g: Tiere, die rausgeworfen werden, hatten ebenso viele affiliative Interaktionen mit Familienmitgliedern wie die übrigen adulten Nachkommen.

In der Literatur über dispersal bei Caniden und Murmeltieren wurde unter anderem vermutet, dass Individuen, denen es nur schwer gelang mit Sozialpartnern zu interagieren, weniger starke Bindungen mit Gruppenmitgliedern eingingen und mit der größten Wahrscheinlichkeit emigrierten (Bekoff 1977). Auf das Sozialsystem der Weißbüschelaffen übertragen ist entsprechend hypothetisierbar, dass Gruppenmitglieder, die nur wenig mit Gruppenmitgliedern affiliativ interagieren, eher aus dem Gruppenverband ausgeschlossen werden als solche, die häufiger in affiliative Interaktionen eingebunden sind.

Die verbliebenen und rausgeworfenen adulten und subadulten Nachkommen unterscheiden sich jedoch nicht in dem Anteil an affiliativer Interaktion mit Familienmitgliedern an der Gesamtaktivität (Mann-Whitney-U-Test: $U=5181,5 ; Z=-1,61 ; p=0,106$ ). Hypothese $5 \mathrm{~g}$ kann daher nicht abgewiesen werden.

Das Tier I zeigte jedoch während des Experiments direkt vor seinem Rauswurf eine deutliche Verminderung der affiliativen Kontakte mit Familienmitgliedern. Sein Anteil affiliativer Kontakte war während des ersten Experiments höher und während des zweiten Experiments seltener als erwartet (Abb.3.47). Alle übrigen Familienmitglieder, die von der Familie ausgestoßen wurden, wiesen vor ihrem Rauswurf keine Veränderungen der affiliativen Kontakte zu Familienmitgliedern auf (KFA, F:EW=7,35 n=2; E:EW=5,08 n=4; H:EW=7,11 $\mathrm{n}=5 ; \mathrm{J}: \mathrm{EW}=10,40 \mathrm{n}=4 ; \mathrm{M}: \mathrm{EW}=10,69 \mathrm{n}=4$ ). Es muss allerdings angemerkt werden, dass für einige der rausgeworfenen Tiere $(\mathrm{E}, \mathrm{J}$ und $\mathrm{M})$ keine quantifizierbare Datengrundlage für den Zeitraum direkt vor dem Rauswurf vorlag, weil sich die Rauswürfe außerhalb der instantaneous sampling-Datenerhebungsphase ereigneten. Vom Zeitpunkt des Rauswurfes aus gesehen lag somit die Datenerhebung z.T. mehrere Wochen zurück. 


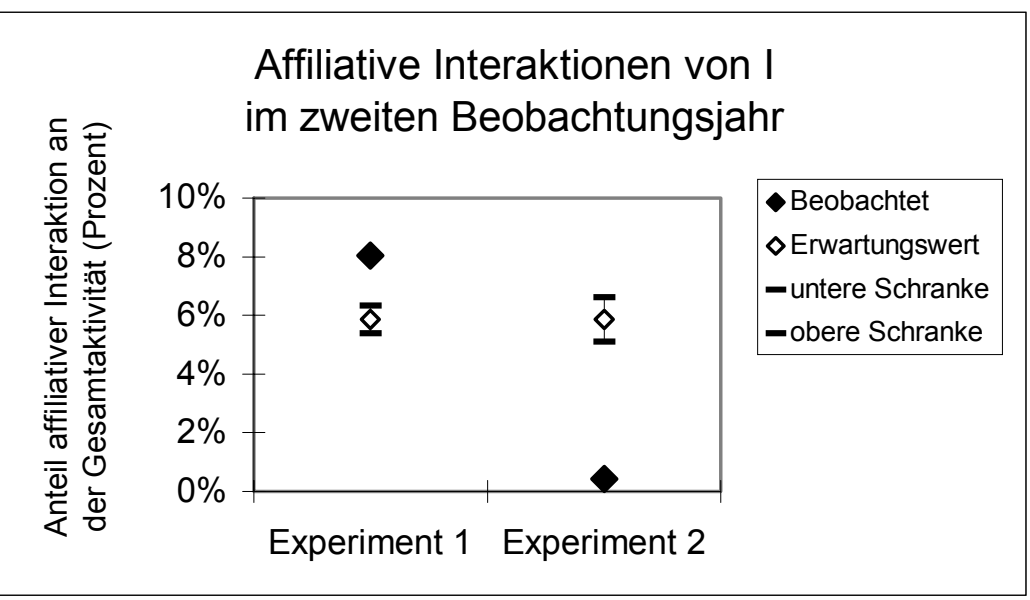

Abb. 3.47 Affiliative Kontakte zwischen I und den Familienmitgliedern vor seinem Rauswurf im zweiten Beobachtungsjahr (KFA, EW=5,86\%, Beobachtungswerte: $E 1=8,04 \%$, E2 $=0,43 \%$ als prozentuale Anteile an der Gesamtaktivität).

Die Ergebnisse aus 3.2.4 lassen eine Ablehnung der Hypothese $2 \mathrm{zu}$, da das Alter eines Nachkommen und seine Spielhäufigkeit mit den Jungtieren im Zusammenhang mit der Rauswurfwahrscheinlichkeit stehen.

\subsubsection{Spontaneität von Rauswürfen}

Hypothese 3: Rauswürfe deuten sich nicht durch einen Anstieg der Körperkerntemperatur des rausgeworfenen Tieres an.

Ein Ziel der Studie war es, herauszufinden, ob Rauswürfe spontan geschehen oder ob sie zuvor durch graduelle Verhaltensänderungen von Familienmitgliedern vorbereitet werden bzw. sich anbahnen und so für den Beobachter vorhersehbar werden.

Mit Hilfe von Temperaturbuttons, die fünf adulten Sozietätsmitgliedern kurz vor dem zweiten Beobachtungsjahr implantiert worden waren, sollten stress- oder ovulationsbedingte Temperaturveränderungen angezeigt werden. Die Änderungen der Körperkerntemperaturen sollten Aufschluss über den physiologischen Zustand eines Tieres geben. So ist z.B. bekannt, dass bei weiblichen Callithrix jacchus hormonell bedingte Temperaturschwankungen auftreten (Ruiz und Colillas 1962). Die Auswirkungen von Stress auf die Körpertemperatur von Krallenaffen konnten unter anderem von Maier (1988) bei C. jacchus und von Werthern (1990) bei S. fuscicollis bestätigt werden. Beide Autoren fanden erhöhte Temperaturen bei Tieren in Stresssituationen.

Die angewandte Methode der Temperaturmessung in dieser Studie kann keine Zusammenhänge zwischen der Körpertemperatur der Tiere mit sozialem Stress einerseits und östrischem Verhalten andererseits nachweisen (siehe Thieß und Rothe, 2002). 
Zur Ermittlung von Stresssituationen der Familienmitglieder erwiesen sich die Abstände zwischen den einzelnen Messvorgängen (alle drei Stunden eine Messung) als zu lang. Stressbedingte Temperaturerhöhungen verlaufen sehr kurzfristig, so dass möglicherweise nur bei minütlichen Abständen zwischen den Messeinheiten Zusammenhänge erhöhter Temperatur mit Stress nachgewiesen werden können (Steinlechner, pers. Mtlg). Die Hypothese 3 kann daher nicht überprüft werden.

\subsubsection{Kapiteldiskussion}

In den beiden Beobachtungsjahren konnten acht Rauswürfe, jedoch keine freiwillige Emigration aus der Natalfamilie beobachtet werden. Bei allen Nachkommen der Familie fiel die Entscheidung daher für die Philopatrie. Die Gründe für das Ausbleiben von freiwilligen Emigrationen werden im Folgenden erörtert.

\subsubsection{Philopatrie der adulten Nachkommen}

Rylands (1982) und Ferrari (1988a) stellten bei freilebenden Callithrix-Gruppen (C. flaviceps und C. humeralifer) generell eine hohe Gruppenstabilität fest, die sich durch eine geringe Rate an Emigrationen bzw. Immigrationen auszeichnete. Die Vorteile des philopatrischen Verhaltens werden vor allem darin gesehen, dass Emigrationen aus der Natalfamilie meist hohe Risiken in sich bergen, die von Prädation über Verhungern bis zu Angriffen durch fremde Artgenossen reichen (Caine 1993, Cheney und Seyfarth 1983, Gaines und McClenaghan 1980, Goldizen und Terborgh, 1984, 1989, Harcourt 1978, Waser 1988, Waser und Jones 1983). Die im Semifreiland gehaltenen Affen waren durch die Anwesenheit von Greifvögeln einem ständigen Prädationsrisiko ausgesetzt. Da die Streifgebiete von mindestens $30 \mathrm{~m}$ breiten Grasgürteln umgeben waren, in denen sich ebenso bodenlebende Beutegreifer, wie Katzen und Füchse verbergen konnten, stellte ihre Durchquerung für die Affen ein unüberschaubares Risiko dar. Habitatsättigung und ein gleichzeitig ressourcenreiches Streifgebiet der Natalfamilie sind nach Waser und Jones (1983), Pusey und Packer (1987) und Ferrari und Digby (1996) Faktoren, die philopatrisches Verhalten fördern. Die Versuchsanlage in Sennickerode war nicht lückenlos mit Gruppen besetzt, so dass genügend freie Gebiete für Emigranten zugänglich waren. Habitatsättigung kann daher keine Ursache für das Verhalten der Nachkommen sein. Carr und Macdonald (1986) favorisieren die Aufrechterhaltung von Systemen mit Philopatrie und cooperative breeding in Habitaten, in denen die Individuen ausreichende Nahrungsmengen vorfinden. Durch die Anpassung von Callithrix 
jacchus an Gummivorie (Rylands 1996) ist im Freiland auch während fruchtarmer Perioden das Überleben einer größeren Gruppe von Tieren in einem relativ kleinen Streifgebiet gesichert (Ferrari und Digby 1996). Die Fokusfamilie dieser Studie wurde während der gesamten Beobachtungszeit ausreichend mit Nahrung und Wasser versorgt, so dass extreme Bedingungen der Nahrungsknappheit durch Trockenheit etc. (Terborgh und Stern 1987) nicht eintreten konnten und die genannten Voraussetzungen für Philopatrie gewährleistet waren. Erfolgreich kann das Zusammenleben von Eltern und Nachkommen allerdings nur sein, wenn das reproduktive Paar, also die Eltern, durch philopatrisches Verhalten der Nachkommen keine negative Kostenbilanz hat (Carr und Macdonald 1986 und Waser 1988). Vermutlich minimiert die reproduktive Unterdrückung der Nachkommen die Kosten der Eltern während der Anwesenheit der Nachkommen. Die Fokusfamilie war Bedingungen ausgesetzt, von denen angenommen wird, dass sie philopatrisches Verhalten von Krallenaffen im Freiland fördern.

Obwohl die Nachkommen ihre eigene Reproduktion verzögerten, entschieden sie sich während des Beobachtungszeitraumes für Philopatrie. Einen Ausgleich für die offensichtlichen Nachteile konnten die Nachkommen durch inklusive Fitnessgewinne erzielen, indem sie bei der Jungtieraufzucht halfen und das Streifgebiet verteidigen konnten. An der Jungtierfürsorge beteiligte sich im ersten Jahr jedoch fast ausschließlich der älteste Sohn C, in geringem Maße auch E und D. Im zweiten Jahr wurden zwar mehrere Nachkommen bei der Jungtierfürsorge beobachtet, doch den höchsten Anteil investierte wieder fast ausschließlich der älteste Sohn C. M unterstützte C im zweiten Experiment und D half ihm im vierten Experiment. Im Durchschnitt waren quantitativ nachweisbar also immer nur ein bis zwei Nachkommen an der direkten Jungtierfürsorge beteiligt. Diese Ergebnisse bestätigen die Befunde von König und Rothe (1991) und Rothe et al. (2003). Rothe et al. (1993) wiesen darauf hin, dass eine zunehmende Anzahl von Helfern die Häufigkeit des Jungtiertragens je Helfer verringert. Mit steigender Anzahl von Helfern kann daher eine Konkurrenz um Helferpositionen entstehen (König und Rothe 1991). Außerdem sinkt die inklusive Fitness eines nicht-reproduktiven Helfers pro aufgezogenem Nachkommen, da die Gewinne der Aufzucht durch die Anzahl der Helfer geteilt werden muss (Lucas et al. 1997). Ab einer bestimmten Gruppengröße könnte der Vorteil des Helfens gegenüber dem Nachteil der Aufgabe eigener Reproduktion nicht mehr überwiegen, so dass möglicherweise die Emigration wieder zur fitnesssteigernden Alternative würde. Da adulte Nachkommen aber auch anders den Aufzuchtserfolg steigern können, z.B. durch Raubfeindvermeidung oder 
Streifgebietsicherung, konnten sie möglicherweise über diese Hilfeleistung Fitnessgewinne erzielen.

Die älteste Tochter D hatte im zweiten Beobachtungsjahr häufig versucht, die Jungtiere von Familienmitgliedern zu übernehmen. Das Alpha-Männchen ließ D jedoch nicht an sie heran. Nach kurzer Zeit verscheuchten sogar alle übrigen Familienmitglieder D aus der Nähe der Jungtiere. In der Studiengruppe von König und Rothe (1991) gewährten die Eltern nur dem ältesten Sohn und einer subadulten Tochter die Aufnahme der Jungtiere. Das AlphaWeibchen verhinderte hier ebenfalls die Jungtieraufnahme durch die älteste Tochter. Price (1990) beobachtete auch bei adulten S. oedipus-Töchtern eine verminderte Jungtierfürsorge im Vergleich zu adulten Söhnen und vermutete, dass andere Familienmitglieder die Annäherung der ältesten Töchter an die Jungtiere unterbanden, was sie ein Jahr später auch belegen konnte (Price 1991; siehe auch Snowdon et al. 1993). In einer späteren Entwicklungsphase der Jungtiere ließen die Familienmitglieder der vorliegenden Studie zu, dass D die Jungtiere aufnahm und häufig trug (Experiment 4/2001). Sie konnte somit auch in einer späteren Entwicklungsstufe der Jungtiere bei der Aufzucht helfen. Vergleichbare Beobachtungen wurden bereits in mehreren Untersuchungen an Marmosetten gemacht (z.B. Ferrari 1988b für C. flaviceps, Rylands 1982 für C. humeralifer intermedius).

Ferrari und Diego (1992) beobachteten bei C. flaviceps, wie eine adulte Tochter mit einem immigrierten Männchen erfolgreich Nachkommen aufzog, während sich die Mutter in der Gruppe aufhielt und sich nicht mehr reproduzierte. Die Autoren halten es daher für möglich, dass weibliche Nachkommen in der Natalfamilie verweilen, um die reproduktive Position der Mutter zu erben (Ferrari und Digby 1996, Ferrari und Lopes Ferrari 1989, Garber 1997 für Saguinus, Goldizen et al. 1996 für S. fuscicollis, Savage et al. 1996 für S. oedipus, siehe auch Clutton-Brock et al. 1999 für Suricatta). Alternative Strategien wären die Emigration zur Gründung einer neuen Gruppe oder die Immigration in eine etablierte Gruppe um die reproduktive Position zu erlangen. Ferrari und Digby (1992) weisen jedoch darauf hin, dass die genannten Alternativen in Habitaten mit hoher Populationsdichte und etablierten Gruppen für Callithrix-Weibchen eher unwahrscheinlich sind. Emigrierte Weibchen gehörten mindestens zweieinhalb Monate keiner Gruppe an und es konnten keine Immigrationen von Weibchen in Gruppen dokumentiert werden (siehe auch Savage et al. 1996). Die Möglichkeit des Erbens der reproduktiven Position durch D muss für diese Studie ebenfalls in Betracht gezogen werden, zumal das Alpha-Weibchen bereits relativ alt war (10 Jahre im Jahr 2001). Am Ende der Studie (2002) starb das Alpha-Weibchen. Während der darauffolgenden 
dreieinhalb Monate übernahm D die reproduktive Position jedoch nicht. Da die Studie nach dieser Zeit beendet war, muss diese Begründung für ihr philopatrisches Verhalten hypothetisch bleiben.

Ein weiterer Aspekt, der berücksichtigt werden muss, sind individuelle Charakteristika und $\mathrm{Zu}$ - oder Abneigungen zwischen den Nachkommen der Fokusfamilie und den Locktieren. Geyer (1993) fand einen Hinweis darauf, dass bestimmte fremde Weibchen auf die Söhne einer Sozietät eine größere Attraktivität ausübten als andere. Die Emigrationsneigung und damit die Entscheidung über Philopatrie oder Emigration könnte daher durch individuelle Charakteristika stärker oder schwächer ausfallen. In Laborhaltung zusammengesetzte Paare zeigten fast durchgehend eine gute Verträglichkeit und schon nach wenigen Stunden des Zusammenseins erste sexuelle Interaktionen (z.B. Evans und Poole 1983). Es sind daher weitere Untersuchungen zur Partnerwahl von C. jacchus in der Laborhaltung und im Freiland erforderlich.

\subsubsection{Rauswürfe}

Innerhalb der zwei Beobachtungsjahre kam es zu acht Rauswürfen aus der Natalfamilie, deren mögliche Ursachen untersucht wurden. Während der Anwesenheit von Locktieren, welche den Nachkommen der Fokusfamilie als potentielle Reproduktionspartner dienen sollten, gab es keine freiwillige Abwanderung und nur einen Rauswurf eines geschlechtsreifen Familienmitgliedes. Die Tatsache, dass Rauswürfe vorwiegend während anderer Phasen geschahen, macht deutlich, dass die Familie (als rauswerfende Einheit gegenüber dem Individuum) offenbar kein „Interesse“ daran hatte, das ausgeschlossene verwandte Individuum zu einem für ihn optimalen Zeitpunkt aus der Familie zu drängen. Als optimale Bedingung wurde die Anwesenheit des potentiellen Reproduktionspartners bewertet, mit dem das Individuum direkt nach der Emigration die eigene Reproduktion hätte aufnehmen können. Möglicherweise wirkten auch andere Faktoren der Emigrations- bzw. Rauswurfentscheidung während der Anwesenheit der Locktiere entgegen, so dass die Bedingungen eben doch nicht optimal für eine Emigration waren.

Die Ergebnisse liefern Hinweise auf einen möglichen Einfluss der Jahreszeit auf die Rauswurfwahrscheinlichkeit, da 50\% der Rauswürfe während der Sommermonate auftraten. Falls Korrelationen in weiteren Semifreilanduntersuchungen gefunden würden, bleibt der Nutzen dieser Erkenntnis jedoch fraglich, da der natürliche Lebensraum von C. jacchus nicht den extremen jahreszeitlichen Schwankungen, wie sie in Mitteleuropa zu finden sind, 
unterworfen ist. Da sich die Haltungsbedingungen und damit das Raumangebot für die Familie J18 mit den Jahreszeiten änderten, sind die Unterschiede in der Rauswurfwahrscheinlichkeit möglicherweise eher hierauf zurückzuführen.

Die Auswirkungen der Gruppengröße auf die Wahrscheinlichkeit, dass ein Ausschluss geschieht wird in Kapitel 3.5.5 diskutiert.

Price und McGrew (1991) fanden für S. oedipus Zusammenhänge zwischen Ausschlüssen und den Geburten von Jungtieren. Rauswürfe von gegengeschlechtlichen Tieren oder zwischen Männchen fanden immer dicht vor Geburten von Jungtieren und zwischen Weibchen immer nach Geburten statt. Auch Digby und Barreto (1993) beobachteten bei C. jacchus zweimal nach Geburten Emigrationen von Gruppenmitgliedern (ein Männchen und ein Weibchen). Snowdon und Pickhard (1999) hingegen fanden bei S. oedipus, dass nur 17\% der beobachteten Aggressionen um Jungtiergeburten herum stattfanden. Die Analyse der Daten aus der C. jacchus-Kolonie des IfA ergab, dass die Wahrscheinlichkeit von Rauswürfen innerhalb der ersten 20 Lebenstage der Jungtiere größer ist, als während anderer Zeiten. Von der vierten Lebenswoche an beginnen die Jungtiere selbständig von ihren Trägern abzusteigen und ihre Umwelt zu erkunden (Missler et al. 1992). Rauswürfe geschehen also häufiger, bevor die Jungtiere mobiler werden. Einer der acht beobachteten Rauswürfe (Männchen) dieser Studie ereignete sich innerhalb der ersten 20 Tage nach einer Geburt, wobei die übrigen sieben Rauswürfe während Zeiten auftraten, in denen die Jungtiere ein anderes Alter hatten. Obwohl das Rauswurfrisiko während der ersten 20 Tage nach einer Geburt am größten ist, muss davon ausgegangen werden, dass Rauswürfe mehrere Ursachen haben.

Möglicherweise war der Auslöser für die Rauswürfe nicht die Anwesenheit bzw. das Alter der Jungtiere, sondern ein Aspekt, der mit dieser Entwicklungsstufe der Jungtiere einherging, z.B. der Postpartum-Östrus des Alpha-Weibchens. Kleiman (1979) berichtete, dass sechs von sieben beobachteten Konfliktsituationen in L. rosalia-Familien während Postpartum- und Postkonzeptions-Östren geschahen. Sie zog daraus die Schlussfolgerung, dass Östren des Alpha-Weibchens proximate Auslöser für Intragruppen-Konflikte sind. Die Untersuchungen der vorliegenden Studie deuten darauf hin, dass Ovulationen des Alpha-Weibchens einen Einfluss auf die Wahrscheinlichkeit von Rauswürfen hatten, da drei Rauswürfe während oder direkt nach einem Östrus stattfanden (Rauswürfe F, I und M). Nach der Berechnung für die Wahrscheinlichkeit von Rauswürfen konnte jedoch keine Abhängigkeit von dem Östrus des Alpha-Weibchens festgestellt werden. Da die Stichprobengröße von acht Rauswürfen allerdings sehr klein war und vorausgesetzt wurde, dass alle Ovulationen erkannt wurden, 
können aus den Ergebnissen der Studie keine allgemeinen Schlussfolgerungen gezogen werden. Es bleibt zum Beispiel offen, ob die älteste Tochter ovuliert hatte oder nicht. Der Rauswurf von E folgte auf Aggressionen der Familie mit D und einer starken Unruhe der ältesten Tochter, geäußert durch gehäufte Lokomotion und Fellsträuben. Verschiedene Studien aus dem Freiland berichten von mehreren reproduktiven Weibchen in einer Gruppe (Scanlon et al. 1988; Digby und Ferrari 1994; Ferrari und Digby 1996; Smith et al. 1997 für C. kuhli; Chaoni und Gallusser 1999 für Leontopithecus), in denen meist die älteste Tochter neben dem Alpha-Weibchen reproduktiv aktiv war. Abbott (1984), Saltzman et al. (1997b) und Rothe (pers. Mtlg.) berichten aus der Laborhaltung von Ovulationen bei den ältesten Töchtern. Yamamoto et al. (2001) wurden Zeugen, wie subordinierte C. jacchus-Weibchen im Freiland mit gruppenfremden Männchen kopulierten, jedoch weiterhin in der Natalgruppe blieben und trächtig wurden (siehe auch Hubrecht 1985). Da Ovulationen bei den ältesten Töchtern auftreten können, wäre es auch in dieser Studie möglich, dass die Tochter D ovuliert hatte. Solange der Reproduktionserfolg eines Alpha-Weibchens nicht gefährdet ist, würde die Reproduktion einer Tochter die inklusive Fitness des Alpha-Weibchens erhöhen (Digby und Ferrari 1994, Schaffner und French 1997). Da nach Rylands (1996) und Ferrari und Digby (1996) die reproduktive Inhibition vor allem auf Limitation von Habitaten zurückzuführen ist, wäre vorstellbar, dass in einer seminatürlichen Umgebung mit ausreichenden Ressourcen die hormonelle Unterdrückung der ältesten Tochter aufgehoben würde (Rothe und König 1987, Rylands 1996). Setzte man den Gedankengang fort, könnte die Aufhebung der reproduktiven Suppression der Töchter allerdings zur Inzuchtdepression führen (Ralls und Ballou 1982). Zur Vermeidung von Inzucht wäre dann die Abwanderung aller männlichen Nachkommen aus dem Geburtsgebiet notwendig, was aber das gesamte Sozialsystem der Weißbüschelaffen in Frage stellen würde. Falls D während ihrer Unruhephase ovuliert hatte, könnte der Rauswurf von E damit im Zusammenhang stehen.

Inwiefern das Alter und Geschlecht eines Individuums Auswirkungen auf seine Rauswurfwahrscheinlichkeit haben, wird in Kapitel 3.5 diskutiert. Bei den weiblichen Tieren, die ausgeschlossen wurden, zeigte sich ein zeitlicher Zusammenhang mit ihrer kurzfristigen Abwesenheit von der Familie zur Implantation der Temperaturbuttons. Jeweils nachdem die Familie nach Operationen wieder zusammengebracht wurde, entstanden starke Aggressionen zwischen den Schwestern, die zum Rauswurf eines weiblichen Nachkommen führten. Es wurden vor dem Rauswurf keine Peripheralisationen oder Verhaltensänderungen der rausgeworfenen Schwestern beobachtet, so dass von einem entscheidenden Einfluss der Abwesenheit von der Familie (z.B. durch invasive Eingriffe, wie Operationen) auf die 
Dynamik von Weibchen-Dyaden ausgegangen werden kann. Der Rauswurf von J verdeutlicht aber auch Auswirkungen der Abwesenheit von der Familie auf die Dynamik der MännchenDyaden. Die Entfernung eines männlichen Nachkommen löste nach dem Zurückbringen (Entfernungszeitraum: 1 Woche) bei dem nächst älteren Bruder eine dissoziative Reaktion aus, die zu einem Ausschluss von J aus der Familie führte. Die kurzfristige Veränderung der Familienzusammensetzung wurde damit bei beiden Geschlechtern eine proximate Ursache für Rauswürfe.

Mousa (2003) fand bei S. midas eine Abhängigkeit der Häufigkeit aggressiver Verhaltensweisen vom Alter eines Tieres. Jüngere Tiere waren gegenüber älteren Gruppenmitgliedern wesentlich aggressiver als umgekehrt. Es wird hypothetisiert, dass Männchen, die in größere Gruppen hineingeboren werden, geringere Chancen haben, die reproduktive Position zu erben. Verstärktes Aggressionsverhalten gegenüber älteren Brüdern würde ihren Rang innerhalb der Gruppe erhöhen und somit das Erbe einer reproduktiven Position wahrscheinlicher machen. Diese Erklärung macht die Eroberung eines hohen sozialen Ranges von G durch die Rauswürfe mehrerer Brüder (darunter ein älterer) plausibel. Die ErbschaftsHypothese muss daher auch für Männchen in Betracht gezogen werden.

Eine genauere Betrachtung des Verhaltens der ausgestoßenen Tiere vor ihrem Rauswurf, sollte Hinweise dafür liefern, dass Nachkommen aufgrund ihres Verhaltens ihren eigenen Emigrations- bzw. Rauswurfzeitpunkt beeinflussen. Eine Hypothese lautete, dass Nachkommen nur dann rausgeworfen werden, wenn sie sich nicht an gruppendienlichem Verhalten beteiligen. Es stellte sich heraus, dass zwischen Tieren, die rausgeworfen wurden und denjenigen, die in der Familie bleiben konnten, keine Unterschiede in der Jungtierfürsorge und im ressourcensichernden und raubfeindvermeidendem Verhalten bestanden. Es wurde jedoch deutlich, dass Nachkommen, die rausgeworfen wurden, seltener mit Jungtieren spielten als Nachkommen, die in der Familie blieben. Dies deutet auf einen Hinweis für einen Verhaltensunterschied zwischen verbliebenen und rausgeworfenen Nachkommen hin. Nach Lee (1983) besteht eine Korrelation zwischen dem Alter der Gruppenmitglieder und ihrer Spielhäufigkeit, so dass möglicherweise eher das Alter einen Einfluss auf das Rauswurfrisiko hat. $\mathrm{Zu}$ diskutieren bleibt trotzdem, ob Spielen als eine Form des Helfens bei der Jungtieraufzucht verstanden werden kann. Ist Spielen vorwiegend Selbstzweck des Individuums und leistet keinen Beitrag zur Überlebenswahrscheinlichkeit der Jungtiere, dann widerlegen die Ergebnisse die Hypothese, dass Helfen eine Voraussetzung ist, um in der Gruppe zu bleiben. 
Tardif (1997) und Price und McGrew (1991) beobachteten sogar vermehrt Rauswürfe von Tieren, die mehr als andere Jungtiere getragen hatten.

Die Häufigkeit und Dauer von (affiliativen) Interaktionen mit Gruppenmitgliedern lassen bei Wölfen und Kojoten Voraussagen darüber zu, ob ein Individuum im Vergleich zu seinen Wurfgeschwistern früh oder spät das heimatliche Streifgebiet verlässt (Bekoff 1977). Tiere, die als Jungtiere am wenigsten mit anderen gespielt und sozial interagiert hatten, bauten die wenigsten Bindungen auf und wanderten damit auch als erste ab. Digby (1995b) verglich bei freilebenden $C$. jacchus die Grooming-Häufigkeiten zwischen Individuen, die in der Gruppe blieben und jenen, die emigrierten und konnte keine Unterschiede feststellen. Der Vergleich affiliativer Interaktionen für die Nachkommen der Fokusfamilie dieser Studie ergab ebenfalls keinen Unterschied zwischen verbliebenen und rausgeworfenen Nachkommen. Es konnten damit für $C$. jacchus keine Hinweise gefunden werden, die auf einen Zusammenhang zwischen der Häufigkeit affiliativer Interaktionen und der Wahrscheinlichkeit, die Familie früher als andere Geschwister zu verlassen (z.B. rausgeworfen zu werden), hindeuten. Die Verringerung affiliativer Kontakte direkt vor dem Rauswurf war bei einem von zwei männlichen Nachkommen zu beobachten. Bekoff (1977) weist jedoch darauf hin, dass bestimmte Interaktionen zum Emigrationszeitpunkt bei Caniden, Sciuriden und Microtiden wahrscheinlich keinen Emigrationsauslöser darstellen. Inwiefern bestimmte Interaktionen der Familienmitglieder eine freiwillige oder erzwungene Emigration (Rauswurf) bei Weißbüschelaffen auslösen, kann nur durch eine größere Anzahl von weiteren intensiven Verhaltensbeobachtungen frei- oder semifreilebender Gruppen herausgefunden werden.

Die Beobachtung der Rauswürfe erbrachte Hinweise für die Annahme, dass die Reaktion eines Tieres auf Mobbing und Angriffe von Familienmitgliedern einen Einfluss auf den Ausgang der Auseinandersetzung hatte. Vor dem Rauswurf von M zeigten viele Familienmitglieder dissoziatives Verhalten gegenüber $\mathrm{K}$ und $\mathrm{M}$, woraufhin die Zwillingsschwestern unterschiedlich reagierten. K reagierte auf Drohgebärden, indem sie den Familienmitgliedern, die ihr gedroht hatten, katzbuckelnd folgte. M reagierte auf Drohgebärden mit Quärren (submissives Verhalten) und Fliehen. Möglicherweise werden eher Tiere von der Familie ausgestoßen, die sich einschüchtern und verjagen lassen, als jene, die auf Drohungen von Familienmitglieder anders reagieren d.h., sich nicht verjagen lassen und sich mit dissoziativem Verhalten wehren. Die älteste Tochter D wurde häufig von ihren Brüdern oder der ganzen Familie angegriffen und gejagt. Sie wehrte die Angriffe jedoch ab und ließ sich nicht derart einschüchtern wie $\mathrm{M}$ und blieb bis an das Ende der Studie in der Familie. Gaines 
und McClenaghan (1980) vermuteten, dass bei kleinen Säugern (z.B. Microtus) Emigranten eher subordiniert sind, wobei jedoch in ihren Untersuchungen bei einer Art (M. pennsylvanicus) Emigranten sich als aggressiver (und möglicherweise dominanter) als bleibende Tiere herausstellten. Beim Vergleich der Zwillingsbrüder I und J fiel auf, dass I sich schon im juvenilen Alter den Vorrang am Futternapf erkämpfte (dies wird in 3.3.1.9 gezeigt) und Konflikten nicht aus dem Weg ging wie sein Bruder J, der häufiger submissives Verhalten gezeigt hatte. I wurde jedoch vor $\mathrm{J}$ aus der Familie geworfen. Auch wenn das Verhalten, welches anthropomorph am ehesten mit „Selbstsicherheit“ zu beschreiben ist, nicht direkt mit der Dominanz eines Tieres verglichen werden kann, geht die Annahme von Gaines und McClenaghan (1980) in eine ähnliche Richtung wie die Vermutung, dass die Reaktion eines Individuums Einfluss auf seine Rauswurfwahrscheinlichkeit nehmen kann. Kleiman (1979) fand Hinweise für die Gültigkeit der Hypothese, dass dominante und selbstsichere Nachkommen von L. rosalia bevorzugt von den Eltern ausgestoßen werden, während submissive, ängstlichere Nachkommen eher durch Geschwister-Konflikte aus der Gruppe zur Emigration getrieben werden. In dieser Studie können dazu nur Vermutungen angestellt werden. Die Eltern hatten sich an den Rauswürfen von zwei Nachkommen beteiligt, von denen eines subjektiv betrachtet, sehr selbstsicher wirkte, während das andere häufig submissives Verhalten gezeigt hatte. Nachkommen, die nur von ihren Geschwister aus der Gruppe getrieben wurden, waren ebenfalls zum Teil selbstsicher und teilweise ängstlich. Es fanden sich damit keine Anzeichen für die Bestätigung der Hypothese von Kleiman (1979). Solange die Attribute Selbstsicherheit und Ängstlichkeit bei Mitgliedern von C. jacchusGruppen jedoch nicht objektiv nachvollzogen werden können, bleiben die Diskussionen spekulativ.

Gezielte hormonelle und genetische Untersuchungen können in Zukunft möglicherweise allgemeingültige Aussagen über die Persönlichkeit eines Tieres und die Emigrations- bzw. Rauswurfwahrscheinlichkeit erbringen. Snowdon und Pickhard (1999) vermuten z.B., dass individuelle Unterschiede in der hormonellen und physischen Entwicklung eventuell Voraussagen darüber zulassen, ob ein Tier zum Aggressor oder Unterlegenen werden würde und Schmidtke et al. (2003) fanden bei Rhesusaffen einen Zusammenhang zwischen dem Abwanderungsalter und dem Serotonintransporter-Gen. Dieses Gen kann in einer kurzen Variante (ss) und in einer langen Variante (11) ausgebildet sein. Die kurze Variante bewirkt beim Menschen Neurotizismus, welche mit Angst und Depressivität einhergeht. Die reinerbigen (ss)-Tiere verließen ihre Geburtsgruppe früher als mischerbige (ls)-Tiere, und diese wiederum früher als reinerbige (11)-Tiere. Es konnte damit eine Verbindung zwischen 
einem genetischen und einem Verhaltensmerkmal nachgewiesen werden. Über die Analyse des Serotonintransporter-Gens können in zukünftigen Studien eventuell auch genetische Zusammenhänge mit dem Emigrationsverhalten der Weißbüschelaffen ermittelt werden.

\subsection{Untersuchungen zur Geschwister-Konkurrenz und zum Eltern- Nachkommen-Konflikt}

\subsubsection{Untersuchungen zur Familienstruktur}

Hypothese 4: Rauswürfe ereignen sich unabhängig von Geschwister-Konkurrenz.

Die Untersuchung von Interaktionen sind das Fundament der Analyse von sozialen Beziehungen, die innerhalb einer Sozietät vorkommen (Lehner 1996). Hinde und StevensonHinde (1976) stellen die Struktur einer Gruppe als Netzwerk sozialer Beziehungen dar, welche bestimmte Qualitäten und Häufigkeiten von Interaktionen beinhalten. Inwiefern Konkurrenz (vgl. Fußnote 6, S. 16) innerhalb der Natalfamilie zu Emigrationen oder Rauswürfen führte, wurde durch die Beobachtungen zu Rangpositionen anhand von affiliativen Interaktionen, zur Bildung von Cliquen über affiliative Interaktionen, zu Grooming-Cliquen, zu dissoziativen Interaktionen und Hierarchien an den Futterplätzen untersucht. Da für die Bewertung der Hypothese ein Gesamtbild der Ergebnisse notwendig ist, wird die Hypothese 4 erst im letzten Ergebniskapitel unter 3.5.6 überprüft.

\subsubsection{Ermittlung von Rangpositionen anhand affiliativer Interaktionen im ersten Beobachtungsjahr}

Die Analyse von Rangpositionen, die anhand der Häufigkeit von affiliativen Interaktionen ermittelt wurden, zeigt, dass in einigen Experimentalphasen alle Tiere die gleiche Rangposition hatten, z.B. in der ersten Kontrollphase (B1). In der zweiten Kontrollphase hatte nur E eine niedrigere Rangposition als die anderen Familienmitglieder, welche alle weiterhin die gleiche Rangposition belegten. Während des ersten Experiments gab es mehr Unterschiede in den Rangpositionen der Tiere. H, I und J besetzten gemeinsam die erste (höchste) Position, A, $\mathrm{B}, \mathrm{C}$ und $\mathrm{E}$ die zweite, $\mathrm{G}$ die vierte und $\mathrm{D}$ die fünfte Position. Während des zweiten Experiments hatten alle Familienmitglieder wieder eine gemeinsame Rangposition. In Tabelle 3.6 sind die Rangpositionen, die anhand der Häufigkeit von affiliativen Interaktionen erstellt wurden für die vier Experimentalphasen des ersten Beobachtungsjahres abgebildet. 
Tabelle 3.6 Rangpositionen der Familienmitglieder, die anhand der Häufigkeit von affiliativen Interaktionen erstellt wurden (erstes Beobachtungsjahr). Die Ziffern beschreiben die Rangpositionen der Tiere. Alle Tiere, denen die gleiche Ziffer zugeordnet wurde, haben somit die gleiche Rangposition. Die kleinste Ziffer (1) gibt die höchste Position an; mit aufsteigender Ziffer vermindert sich die Position. Abkürzungen: B1=erste Kontrollphase; B2=zweite Kontrollphase; C1=erstes Experiment, C2=zweites Experiment.

\begin{tabular}{ccccc} 
& \multicolumn{5}{c}{ Experimente } \\
\cline { 2 - 5 } Tiere & B1 & B2 & C1 & C2 \\
\hline A & 1 & 1 & 2 & 1 \\
B & 1 & 1 & 2 & 1 \\
C & 1 & 1 & 2 & 1 \\
D & 1 & 1 & 4 & 1 \\
E & 1 & 2 & 2 & 1 \\
F & 1 & - & - & - \\
G & 1 & 1 & 3 & 1 \\
H & 1 & 1 & 1 & 1 \\
I & 1 & 1 & 1 & 1 \\
J & 1 & 1 & 1 & 1 \\
\hline
\end{tabular}

\subsubsection{Ermittlung von Rangpositionen anhand von affiliativen Interaktionen im zweiten Beobachtungsjahr}

In der Hüttenphase D1 des zweiten Beobachtungsjahres hatte I die erste Rangposition anhand der Häufigkeiten von affiliativen Interaktionen. A, G, H, J, K und M besetzten die zweite Rangposition und die folgenden Rangpositionen wurden von C, B und D besetzt. Während des ersten Experiments belegten A, C, G, I, K und M die erste Position, gefolgt von D und J auf der zweiten und $\mathrm{B}$ auf der letzten Position. $\mathrm{K}$ hatte im zweiten Experiment die erste Rangposition, I und $\mathrm{M}$ die zweite, $\mathrm{B}$ und $\mathrm{J}$ die dritte und $\mathrm{C}$ und $\mathrm{G}$ die vierte Position. A besetzte die vierte und $\mathrm{D}$ die fünfte Rangposition. Während der Experimente 3 und 4 gab es keine Rangfolge mehr zwischen den Familienmitgliedern. Die Ergebnisse der Analyse befinden sich in Tabelle 3.7 . 
Tabelle 3.7 Rangpositionen der Familienmitglieder, die anhand der Häufigkeiten von affiliativen Interaktionen erstellt wurden (zweites Beobachtungsjahr). Die Ziffern beschreiben die Rangpositionen der Tiere. Alle Tiere, denen die gleiche Ziffer zugeordnet wurde, haben somit die gleiche Rangposition. Die kleinste Ziffer (1) gibt die höchste Position an; mit aufsteigender Ziffer vermindert sich die Position. Abkürzungen: D1=Hüttenphase; E1=erstes Experiment; E2=zweites Experiment; E3=drittes Experiment, E4=zweites Experiment.

\begin{tabular}{cccccc} 
& \multicolumn{5}{c}{ Experimente } \\
\cline { 2 - 6 } Tiere & D1 & E1 & E2 & E3 & E4 \\
\hline A & 2 & 1 & 5 & 1 & 1 \\
B & 4 & 3 & 3 & 1 & 1 \\
C & 3 & 1 & 4 & 1 & 1 \\
D & 5 & 2 & 6 & 1 & 1 \\
G & 2 & 1 & 4 & 1 & 1 \\
H & 2 & - & - & - & - \\
I & 1 & 1 & 2 & - & - \\
J & 2 & 2 & 3 & 1 & 1 \\
K & 2 & 1 & 1 & 1 & 1 \\
M & 2 & 1 & 2 & 1 & 1 \\
\hline
\end{tabular}

\subsubsection{Clique-Analyse über affiliative Interaktionen im ersten Beobachtungsjahr}

Um herauszufinden, wer mit wem affiliativ interagierte, wurden Clique-Analysen zum affiliativen Verhalten durchgeführt. In der ersten Kontrollphase waren alle Familienmitglieder an einer einzigen extremen Clique (1-Clique) beteiligt. Während der zweiten Kontrollphase traten zwei 1-Cliquen auf (Tab. 3.8). An beiden Cliquen waren die Familienmitglieder B, C, D, G, H, I und J beteiligt, während A und E mit den zuvor genannten Familienmitgliedern jeweils eine Clique bildeten, d.h. nur A und E waren nicht gemeinsam in einer Clique.

Tabelle 3.8 1-Cliquen aus affiliativem Verhalten in der zweiten Kontrollphase (B2) im ersten Beobachtungsjahr. Abkürzungen: Großbuchstaben = Familienmitglieder. Die linke Spalte der Tabelle beschreibt die Anzahl der Cliquen. Rechts davon sind alle Familienmitglieder in einer Reihe aufgelistet, die zu der Clique gehören. Diese Tabelle stellt zwei Cliquen mit jeweils 8 Familienmitgliedern dar.

\begin{tabular}{llllllllll}
\hline 1. Clique: & A & B & C & D & G & H & I & J & \\
2. Clique: & & B & C & D & G & H & I & J & E \\
\hline
\end{tabular}

Im ersten Experiment $(\mathrm{C} 1)$ bildeten $\mathrm{A}, \mathrm{B}, \mathrm{C}$, und $\mathrm{H}$ die einzige 1-Clique. Alle Familienmitglieder waren jedoch an einer 2-Clique beteiligt. Im zweiten Experiment bildeten alle Familienmitglieder wieder wie in der ersten Kontrollphase eine gemeinsame 1-Clique. 


\subsubsection{Clique-Analyse über affiliative Interaktionen im zweiten Beobachtungsjahr}

Während der Hüttenphase am Anfang des zweiten Beobachtungsjahres (D1) gab es innerhalb der Familie vier 1-Cliquen über affiliative Interaktionen (Tab. 3.9). A, B, I, K und M bildeten eine Clique, A, G, H, I, J, K und $\mathrm{M}$ die zweite, C, G, H und $\mathrm{J}$ die dritte und $\mathrm{D}, \mathrm{G}, \mathrm{J}$ und $\mathrm{K}$ die vierte Clique. Die Nachkommen G, J und K waren damit jeweils an drei Cliquen beteiligt, A, $\mathrm{H}$, I und $\mathrm{M}$ jeweils an zwei Cliquen und $\mathrm{B}, \mathrm{C}$ und $\mathrm{D}$ waren nur an jeweils einer Clique beteiligt.

Tabelle 3.9 1-Cliquen aus affiliativem Verhalten in der Hüttenphase (D1) des zweiten Beobachtungsjahres. Abkürzungen: Großbuchstaben = Familienmitglieder. Erläuterungen wie in Tab. 3.8.

\begin{tabular}{|c|c|c|c|c|c|c|c|c|c|}
\hline 1. Clique: & B & A & & & I & & $\mathrm{K}$ & $\mathrm{M}$ & \\
\hline 2. Clique: & & A & G & $\mathrm{H}$ & I & $J$ & $\mathrm{~K}$ & $M$ & \\
\hline 3. Clique: & & & G & $\mathrm{H}$ & & $\mathrm{J}$ & & & C \\
\hline 4. Clique: & & & G & & & $\mathrm{J}$ & $\mathrm{K}$ & & $\mathrm{D}$ \\
\hline
\end{tabular}

Im ersten Experiment des ersten Beobachtungsjahres traten zwei 1-Cliquen auf, an denen A, $\mathrm{G}, \mathrm{I}, \mathrm{K}$ und $\mathrm{M}$ beteiligt waren. $\mathrm{C}$ und $\mathrm{J}$ bildeten eine Clique mit ihnen und $\mathrm{D}$ die zweite Clique (Tab. 3.10).

Tabelle 3.10 1-Cliquen aus affiliativem Verhalten im ersten Experiment (E1) des zweiten Beobachtungsjahres. Abkürzungen: Großbuchstaben = Familienmitglieder. Erläuterungen wie in Tab. 3.8.

\begin{tabular}{lllllllll}
\hline 1. Clique. & C & A & G & I & J & K & M & \\
2. Clique: & & A & G & I & & K & M & D \\
\hline
\end{tabular}

Während des zweiten Experiments des zweiten Beobachtungsjahres gab es fünf 1-Cliquen innerhalb der Familie mit jeweils 4 bzw. 3 Mitgliedern (Tab. 3.11). Zur ersten Clique gehörten A, B, M und K, zur zweiten Clique B, J, M und K, zur dritten Clique M, K, I und C, zur vierten Clique K, I, C und G und zur fünften Clique I, C und D. K war demnach an vier Cliquen, $\mathrm{C}, \mathrm{I}$ und $\mathrm{M}$ an drei Cliquen, $\mathrm{B}$ an zwei Cliquen und $\mathrm{A}, \mathrm{D}, \mathrm{G}$ und $\mathrm{J}$ an jeweils einer Cliquen beteiligt.

Tabelle 3.11 1-Cliquen aus affiliativem Verhalten im zweiten Experiment (E2) des zweiten Beobachtungsjahres. Abkürzungen: Großbuchstaben = Familienmitglieder. Erläuterungen wie in Tab. 3.8.

\begin{tabular}{lllllllllll}
\hline 1. Clique: & A & B & M & K & & & & & \\
2. Clique: & & B & M & K & J & & & & \\
3. Clique: & & & $M$ & $K$ & & I & C & & \\
4. Clique: & & & $K$ & & I & C & G & \\
5. Clique: & & & & & I & C & & D \\
\hline
\end{tabular}


Während des dritten und vierten Experiments im zweiten Beobachtungsjahr waren alle Familienmitglieder an einer einzigen gemeinsamen 1-Clique beteiligt.

\subsubsection{Grooming-Cliquen im ersten Beobachtungsjahr}

In der ersten Kontrollphase kamen keine 1-Cliquen vor, jedoch drei 2-Cliquen, deren Zusammensetzung in Tab. 3.12 aufgeführt sind. An der ersten Clique waren die Tiere E, H und $\mathrm{C}$, an der zweiten Clique die Tiere D, G und $\mathrm{E}$ und an der dritten Clique waren die Tiere D, G und $\mathrm{H}$ beteiligt. Auffällig war bei der Cliquenbildung, dass die Nachkommen D, E, G und $\mathrm{H}$ jeweils von zwei Cliquen Mitglieder waren, der älteste Sohn $\mathrm{C}$ jedoch nur von einer Clique. Die Eltern und Jungtiere waren an den Cliquen nicht beteiligt. Nachdem F aus der Familie geworfen worden war, gab es in der zweiten Kontrollphase keine Grooming-Clique mehr.

Tabelle 3.12 Grooming-2-Cliquen in der ersten Kontrollphase (B1) im ersten Beobachtungsjahr. Abkürzungen: Großbuchstaben $=$ Familienmitglieder. Erläuterungen wie in Tab. 3.8.

\begin{tabular}{llllll}
\hline 1. Clique: & & & E & H & C \\
2. Clique: & D & G & E & & \\
3. Clique: & D & G & & H & \\
\hline
\end{tabular}

Im ersten Experiment fanden sich vier 1-Cliquen (Tab. 3.13), in denen der älteste Sohn C immer beteiligt war. Der Vater A gehörte dreien dieser Cliquen an und die älteste Tochter D zweien. $\mathrm{B}$ und $\mathrm{H}$ bildeten jeweils mit $\mathrm{A}$ und $\mathrm{C}$ eine Clique und $\mathrm{E}$ mit $\mathrm{C}$ und $\mathrm{D}$. Zusätzlich gab es im ersten Experiment zwei 2-Cliquen, in denen A, C, E und H beteiligt waren. Sie formten mit $\mathrm{G}$ eine und mit $\mathrm{B}$ und $\mathrm{D}$ die andere Clique (Tab. 3.14). G war im ersten Experiment das einzige Familienmitglied, welches nur an einer 2-Clique beteiligt war. Die Jungtiere kamen in keiner Clique vor.

Tabelle 3.13 Grooming-1-Cliquen im ersten Experiment (C1) im ersten Beobachtungsjahr. Abkürzungen: Großbuchstaben $=$ Familienmitglieder. Erläuterungen wie in Tab. 3.8.

\begin{tabular}{lllllll}
\hline 1. Clique: & B & A & C & & & \\
2. Clique: & & & C & D & E & \\
3. Clique: & & A & C & & & H \\
4. Clique. & A & C & D & & \\
\hline
\end{tabular}

Tabelle 3.14 Grooming-2-Cliquen im ersten Experiment (C1) im ersten Beobachtungsjahr. Abkürzungen: Großbuchstaben $=$ Familienmitglieder. Erläuterungen wie in Tab. 3.8.

\begin{tabular}{llllllll}
\hline 1. Clique: & & G & A & C & E & $H$ & \\
2. Clique: & B & & A & C & E & H & D \\
\hline
\end{tabular}


Im zweiten Experiment (C2) tauchten wieder nur vier 2-Cliquen auf. Drei Cliquen bildeten sich um die älteste Tochter D. C und G waren an zwei dieser Cliquen ebenso beteiligt und $\mathrm{E}$ und $\mathrm{H}$ an jeweils einer Clique. Die Alpha-Tiere bildeten mit den Jungtieren eine eigene Clique (Tab. 3.15).

Tabelle 3.15 Grooming-2-Cliquen im zweiten Experiment (C2) im ersten Beobachtungsjahr. Abkürzungen: Großbuchstaben $=$ Familienmitglieder.

\begin{tabular}{lllllllllll}
\hline 1. Clique. & A & B & I & J & & & & & \\
2. Clique: & & & & & E & D & C & & \\
3. Clique: & & & & & & D & & G & H \\
4. Clique: & & & & & D & C & G & \\
\hline
\end{tabular}

\subsubsection{Grooming-Cliquen im zweiten Beobachtungsjahr}

In der Hüttenphase (D1) bestanden zwei 2-Cliquen um C und I herum (Tab. 3.16). A war Mitglied der einen, G und $\mathrm{H}$ Mitglieder der anderen Clique. B, D, J, K und M waren keine Cliquenmitglieder.

Nachdem $\mathrm{H}$ im ersten Experiment nicht mehr in der Familie anwesend war, stieß J zu den beiden 2-Cliquen und $\mathrm{M}$ wurde Teil der Clique, in der zuvor $\mathrm{H}$ beteiligt war. D, M und I bildeten die dritte 2-Clique, während B und K keiner Clique angehörten (Tab. 3.17).

Tabelle 3.16 Grooming-2-Cliquen in der Hüttenphase (D1) im zweiten Beobachtungsjahr. Abkürzungen: Großbuchstaben $=$ Familienmitglieder. Erläuterungen wie in Tab. 3.8.

\begin{tabular}{llllll}
\hline 1. Clique: & A & C & I & & \\
2. Clique: & & C & I & G & $H$ \\
\hline
\end{tabular}

Tabelle 3.17 Grooming-2-Cliquen im ersten Experiment (E1) im zweiten Beobachtungsjahr. Abkürzungen: Großbuchstaben $=$ Familienmitglieder. Erläuterungen wie in Tab. 3.8.

\begin{tabular}{llllllll}
\hline 1. Clique: & $\mathrm{D}$ & $\mathrm{M}$ & $\mathrm{I}$ & & & & \\
2. Clique: & & $\mathrm{M}$ & $\mathrm{I}$ & $\mathrm{J}$ & $\mathrm{C}$ & $\mathrm{G}$ & \\
3. Clique: & & & $\mathrm{I}$ & $\mathrm{J}$ & $\mathrm{C}$ & & $\mathrm{A}$ \\
\hline
\end{tabular}

Während des zweiten Experiments entwickelten sich vier 2-Cliquen, an denen I beteiligt war. C, J und M gehörten jeweils zu zwei einander ausschließenden 2-Cliquen. G und D bildeten zusammen mit $\mathrm{C}$ und I eine Clique und $\mathrm{K}$ mit I und $\mathrm{M}$ eine andere. Die Alpha-Tiere gehörten keiner Clique an (siehe Tab. 3.18). 
Tabelle 3.18 Grooming-2-Cliquen im zweiten Experiment (E2) im zweiten Beobachtungsjahr. Abkürzungen: Großbuchstaben $=$ Familienmitglieder. Erläuterungen wie in Tab. 3.8.

\begin{tabular}{lllllllll}
\hline 1. Clique: & G & D & C & I & & & \\
2. Clique: & & & $C$ & I & J & & \\
3. Clique: & & & I & J & $M$ & \\
4. Clique: & & & I & & $\mathrm{M}$ & $\mathrm{K}$ \\
\hline
\end{tabular}

Direkt nach dem Rauswurf von I im dritten Experiment konnten weder 1-, noch 2-Cliquen nachgewiesen werden. Erst im vierten Experiment des Jahres bildeten sich drei neue 1Cliquen heraus, an denen $G$ beteiligt war (siehe Tab. 3.19). A und $K$ bildeten mit $G$ eine gemeinsame und jeweils eine eigene extreme Clique, an denen J bzw. M beteiligt waren. B, C und D gehörten zu keiner Clique.

Tabelle 3.19 Grooming-1-Cliquen im vierten Experiment (E4) im zweiten Beobachtungsjahr. Abkürzungen: Großbuchstaben $=$ Familienmitglieder. Erläuterungen wie in Tab. 3.8.

\begin{tabular}{llllll}
\hline 1. Clique: & A & G & & $\mathrm{J}$ & \\
2. Clique: & $\mathrm{A}$ & $\mathrm{G}$ & $\mathrm{K}$ & & \\
3. Clique: & & $\mathrm{G}$ & $\mathrm{K}$ & & $\mathrm{M}$ \\
\hline
\end{tabular}

\subsubsection{Dissoziatives Verhalten im ersten Beobachtungsjahr}

Dissoziatives Verhalten wurden von den Familienmitgliedern nur sehr selten geäußert, so dass die Daten dazu nicht quantifizierbar waren. Die Ergebnisse verdeutlichen damit, dass in Callithrix-Sozietäten generell wenig dissoziative Interaktionen stattfinden (Ferrari 1988a, Lazaro-Perea et al. 1997). Im ersten Beobachtungsjahr waren 0,62\% und im zweiten Beobachtungsjahr 0,58\% aller beobachteten Verhaltenseinheiten dissoziativ. Da dissoziatives Verhalten für die Analyse der Gruppendynamik aber von großer Bedeutung war, werden die vorwiegend ad libitum beobachteten dissoziativen Interaktionen in den folgenden beiden Kapiteln deskriptiv dargestellt. Protokolle der acht beobachteten Rauswürfe, die mit dissoziativem Verhalten einher gingen, befinden sich im Anhang auf den Seiten 242ff. Zu dissoziativen Verhaltenseinheiten zählen Büschelklappen, Schlitzaugen machen, Keckern (stark aggressive Vokalisation), Genitalpräsentieren, Angreifen, Beißen und Wegjagen.

In der ersten Hüttenphase vor der ersten Kontrollphase konnte dissoziatives Verhalten von A gegenüber seinem Sohn F beobachtet werden. In Kontrollphase 1 zeigte $\mathrm{E}$ dissoziative Aktivitäten gegen F. Das Alpha-Weibchen beteiligte sich zum Teil an dem dissoziativen Verhalten, indem sie gemeinsam mit E F durch das Streifgebiet verfolgte. F zeigte dabei Fluchtverhalten und quärrte viel. Die älteste Tochter D wurde von A, C und E viel gemobbt 
und jagte selbst G. Direkt nach der ersten Kontrollphase verließ F das Streifgebiet seiner Natalfamilie, nachdem er von E gejagt und attackiert worden war und lief in ein unbewohntes, benachbartes Streifgebiet. Die Abwanderung wurde infolge der Aggressionen seines älteren Bruders E als Rauswurf bewertet.

In der zweiten Kontrollphase gab es starke Aggression der Familienmitglieder gegen D, vorwiegend durch die Brüder C und E. Die dissoziativen Interaktionen eskalierten im ersten Experiment $(\mathrm{C} 1)$ zu langen Verfolgungsjagden durch das Streifgebiet mit Angriffen der gesamten Familie auf D. Das Alpha-Weibchen genitalpräsentierte während dieser Phase gegen D, beobachtete und verfolgte ihre Tochter häufig. Im zweiten Experiment ließ die Spannung geringfügig nach, und D wurde etwas seltener und weniger aggressiv von den Familienmitgliedern gejagt.

In der Herbst-Winterphase, als die Fokusfamilie keinen Zugang mehr zum Semifreigelände hatte und im Hütten-Käfig-Komplex untergebracht war, wurde E zunehmend bei dissoziativen Handlungen gegenüber $\mathrm{G}$ beobachtet, welcher quärrend versuchte, Kontakt zu E aufzunehmen. Zehn Tage vor seinem Rauswurf verhielt sich E nochmals stark dissoziativ gegenüber G, während häufig Aggressionen durch mehrere Familienmitglieder gegenüber D und E geäußert wurden. Obwohl die Schwestern D und H vorwiegend affiliativ miteinander interagierten, konnten auch schwach dissoziative Handlungen von D gegenüber ihrer Schwester in Spielphasen beobachtet werden. Nachdem E rausgeworfen worden war, verringerte sich die Anzahl der dissoziativen Handlungen in der Familie. Im Frühjahr 2001 zeigten viele Familienmitglieder spontan dissoziatives Verhalten gegenüber I, die jedoch nach wenigen Tagen wieder verschwanden. Darauf häuften sich die Katzbuckelphasen der gesamten Familie (alle Familienmitglieder liefen katzbuckelnd mit gesträubtem Fell mehrere Minuten lang hintereinander her), oft von D initiiert, die dissoziatives Verhalten von A gegenüber D zur Folge hatten.

\subsubsection{Dissoziatives Verhalten im zweiten Beobachtungsjahr}

Während der Hüttenphase (D1) konnte von allen Familienmitgliedern (ausgenommen Jungtiere) häufiges Katzbuckeln und Fellsträuben beobachtet werden. G zeigte gegenüber D häufig Schlitzaugen, Genitalpräsentieren und jagte sie durch den Hütten-Käfig-Komplex. Er kontrollierte während dieser Phase die affiliativen Interaktionen seiner Schwester D mit anderen Familienmitgliedern und versuchte vor allem, affiliative Interaktionen zwischen $\mathrm{C}$ und D zu unterbinden. Wenn C D groomte, beendete dieser abrupt die Handlung und entfernte 
sich von seiner Schwester, sobald G ihm dabei zusah. Als D I groomte, näherte sich G an, woraufhin D das Grooming beendete und I sich G zuwendete. H und I schlossen sich den Jagden von $G$ auf D immer häufiger an. Als I zum ersten Mal alleine D angriff, wurde er sofort von ihr vertrieben. Nachdem G D jagte, verhielt D sich mehrfach dissoziativ gegenüber I. Die Beteiligung von I an den dissoziativen Interaktionen zwischen G und D führten letztendlich dazu, dass G seine Aggression auf I umlenkte und ihn schlug. Direkt nach der Implantation der Temperaturbuttons startete D dissoziatives Verhalten durch Keckern, Genitalpräsentieren, Schlitzaugen machen und Angriffe gegenüber H. Nachdem das AlphaWeibchen die Angriffe mehrere Minuten beobachtet hatte, begann auch sie mit Keckern, Genitalpräsentieren und Angriffen auf ihre jüngere Tochter H. Am nächsten Morgen musste $\mathrm{H}$ verletzt aus der Familie entfernt werden.

Während des ersten Experiments im Semifreiland gab es weiterhin Spannungen, häufig geäußert durch Büschelklappen, Fellsträuben, Keckern und Wegjagen, zwischen D und ihren Brüdern (häufig mit J). Nach der Geburt der Jungtiere P und R unterbanden vor allem C, G und I die Annäherung von D zu den Neugeborenen. I ließ auch J nicht an die Jungtiere heran. Während des Postpartum-Östrus des Alpha-Weibchens zeigte A dissoziatives Verhalten gegenüber I, den er sonst beim Grooming anderen Partnern vorzog.

Im zweiten Experiment steigerten sich dissoziative Interaktionen zwischen D und I, indem vorwiegend D I angriff und ihn umherjagte. Die Familie unterstützte I in den Auseinandersetzungen mit D. Gegenseitige Jagden zwischen I und D traten vor allem auf, wenn beide mit den Locktieren kommunizierten. Die Beobachtungen erheben die Vermutung, dass beide die Anwesenheit des jeweils anderen während der nicht-taktilen Interaktion mit den fremden Artgenossen verhindern wollten. Während $G$ seine ältere Schwester häufig jagte und nur zeitweise attackierte, jagte $\mathrm{C}$ sie weniger, griff aber dafür häufiger mit kurzen Beißattacken an. Dann richtete G sein dissoziatives Verhalten spontan auf I und jagte ihn, z.T. mit Hilfe von $\mathrm{C}$ und D solange, bis I sich räumlich von der Familie entfernte, indem er sich nur noch an den äußersten Rändern des Streifgebietes aufhielt. J intervenierte bei den Angriffen auf I, indem er laut chatterte und sich neben I setzte. Am Abend desselben Tages unterstützte er dann $G$ bei den Verfolgungen auf I. D setzte ihre Angriffe auf I fort, woraufhin A ihr bei jedem Angriff auf I mit Schlitzaugen und Büschelklappen begegnete und C und G begannen, D gemeinsam wegzujagen. Als D auch $\mathrm{J}$ bedrohte, griffen die Eltern ein, indem sie sich zwischen D und J positionierten, D gegenüber Schlitzaugen machten und mit den Büscheln klappten, so dass D sich von J entfernte. G verfolgte I solange, bis I den Grasgürtel 
überquerte, der das heimatliche Streifgebiet umgab, und am Zaun des Semifreigeländes entlang zu anderen C. jacchus-Gruppen der Kolonie im Gewächshaus lief (vgl. Abb. 2.2 Übersichtsskizze, S.23). Dort nahm er akustischen und visuellen Kontakt zu den fremden Artgenossen auf und wurde in einem Käfig im Gewächshaus untergebracht.

Im dritten Experiment kontrollierte G die Sozialspiele und verjagte J, sobald dieser mitspielen wollte. D durfte mitspielen, wurde jedoch auch von $\mathrm{G}$ gejagt und bedroht, wenn sie J verfolgte.

Im letzten Experiment jagte C D zeitweise und zeigte sexuelles Interesse an ihr. D und J interagierten weiterhin häufig dissoziativ. Am Ende dieser Phase war D immer wieder in dissoziative Interaktionen mit Familienmitgliedern, inzwischen vermehrt mit den jüngeren Schwestern K und M involviert. B intervenierte, als G M mit Schlitzaugen und Büschelklappen bedrohte, indem B sich zwischen $G$ und $M$ stellte. G drohte B daraufhin ebenfalls mit Schlitzaugen und Büschelklappen, ließ M aber danach in Ruhe.

Nach Beendigung der Beobachtungsphase wurde J krank und für einige Tage aus der Familie genommen. Nachdem er zurückgebracht wurde, zeigte $G$ ihm gegenüber dissoziatives Verhalten (siehe Rauswurf $\mathrm{J}$ ) und verhinderte alle affiliativen Interaktionen, die $\mathrm{J}$ mit Familienmitgliedern einging, indem er zwischen $\mathrm{J}$ und die Familienmitglieder trat, keckerte und J wegjagte. Als G am nächsten Morgen J mehrere Stunden hart attackierte, musste J aus der Familie entfernt werden, um Verletzungen zu vermeiden.

Ende Oktober 2001 zeigten sich im Spiel zwischen K und M schwach dissoziative Handlungen, z.B. Büschelklappen, Schlitzaugen-machen und Genitalpräsentieren, so dass nicht mehr eindeutig war, ob ihre Interaktionen spielerisch oder dissoziativ waren. Ebenso wurde das Spiel zwischen D und M Ende November zunehmend aggressiver. Zur gleichen Zeit nahmen die Katzbuckelepisoden in der Familie zu. D störte mehrmals affiliative Kontaktaufnahmen zwischen K und M. M sprang häufig katzbuckelnd vor die Familienmitglieder, woraufhin diese mit Katzbuckeln antworteten. K quärrte D einige Wochen lang an. D reagierte auf das Quärren mit Keckern. A attackierte D wieder, sobald diese sich den neugeborenen Jungtieren näherte. Ende Januar 2002 hatten K und R eine Auseinandersetzung die von $\mathrm{M}$ geschlichtet wurde, indem $\mathrm{M}$ mit gehobenen Büscheln zwischen $\mathrm{K}$ und $\mathrm{R}$ sprang, woraufhin die Kontrahenten voneinander abließen. Anfang Februar begann die Familie wieder, D zu jagen (A keckerte und G griff sie an). Fünf Tage vor M's Rauswurf wurde M von $\mathrm{K}$ im Spiel angegriffen und floh vor ihr. Die Familie begann, um $\mathrm{M}$ herum zu katzbuckeln, was M verängstigte. Sie folgte von da an häufig ihrer Zwillingsschwester K, die 
ihr mit Trillern, Handauflegen und Kuscheln außergewöhnlich freundlich begegnete. D katzbuckelte dicht vor M, woraufhin K katzbuckelnd D nachfolgte. A stellte sich vor M und zeigte Schlitzaugen und Büschelklappen gegenüber D. Etwas später gab es viel Katzbuckeln in der Familie mit Verfolgung auf D und teilweise auf M. Für die Explantation der Temperaturbuttons wurde die gesamte Familie eingefangen und danach wieder zusammengebracht. Direkt danach begannen Verfolgungen von K auf M, die letztendlich zum Rauswurf von M führten. Daraufhin verringerte sich der Anteil der dissoziativen Interaktionen innerhalb der Familie wieder.

\subsubsection{Rangpositionen in der Hierarchie an den Futterplätzen im ersten Beobachtungsjahr}

An den Futterplätzen waren während der beiden Beobachtungsjahre häufig Auseinandersetzungen zwischen Familienmitgliedern um den Zugang zum Futter zu beobachten. Die ad libitum erhobenen Daten wurden für jede experimentelle Phase dokumentiert und in den Tabellen 3.20 und 3.21 dargelegt. Es wurden nur dissoziative Interaktionen bewertet, in denen die Aggressoren mit Büschelklappen, Schlitzaugen-machen, Über-die-Schulter-blicken Keckern, Genitalpräsentieren und Angriffen die Rezipienten vom Futter vertrieben oder wenn Familienmitglieder submissives Verhalten, wie Ausweichen und Quärren gegenüber anderen Familienmitgliedern zeigten, denen sie den Vorrang beim Fressen ließen. Die Aufstellung der Rangpositionen an den Futterplätzen erhebt keinen Anspruch auf Vollständigkeit, da die Reihenfolge beim Zugang zum Futter nicht regelmäßig und vollständig protokolliert wurde ${ }^{18}$. Sie soll als Ergänzung zur Analyse der Familienstruktur dienen.

Überwiegend wurden jüngere Familienmitglieder von älteren an den Futterplätzen verdrängt. Dies ähnelt der Dominanz-Hierarchie entsprechend der Seniorität. Das dissoziative Verhalten wurde unabhängig vom Geschlecht geäußert. Die einzigen Verdrängungen vom Futterplatz, die von jüngeren gegenüber älteren Familienmitgliedern geschahen, waren im ersten Experiment von D gegenüber $\mathrm{C}$ und von $\mathrm{E}$ gegenüber D und im zweiten Experiment von I gegenüber A, B, G und $\mathrm{H}$.

\footnotetext{
${ }^{18}$ Die Verteilung der Futterplätze über das gesamte Streifgebiet der Familie machte es teilweise unmöglich, regelmäßig und gleichzeitig an jedem Futterplatz die Fressreihenfolge zu erfassen. Da mit dem instantaneous sampling die gesamte Tagesaktivität abgedeckt wurde, gab es häufig Überschneidungen dieser Beobachtungen mit der Fütterung der Affen.
} 
Tabelle 3.20 Dissoziatives Verdrängen an den Futterplätzen während der Kontrollphasen und Experimente im ersten Beobachtungsjahr. Aggressor: Familienmitglied, welches ein anderes Familienmitglied, den Rezipienten, vom Futter durch dissoziatives Verhalten verdrängt. Linien: dissoziative Interaktion.

\section{Kontrollphase 1}

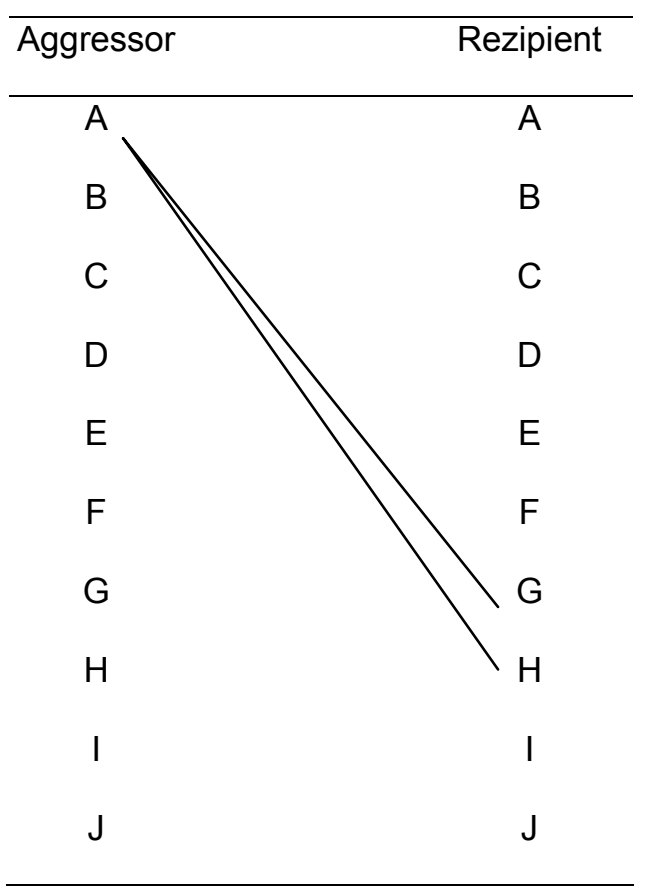

\section{Experiment 1}

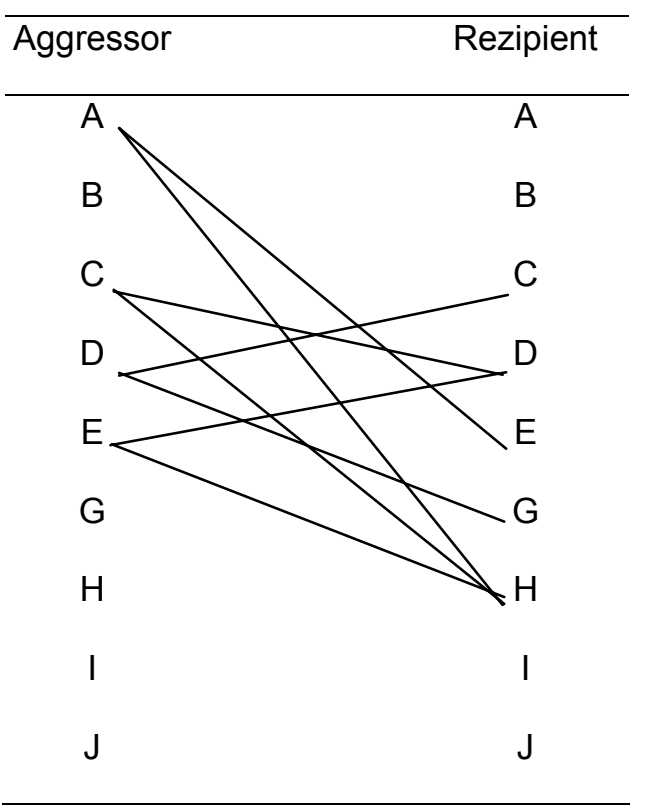

\section{Kontrollphase 2}

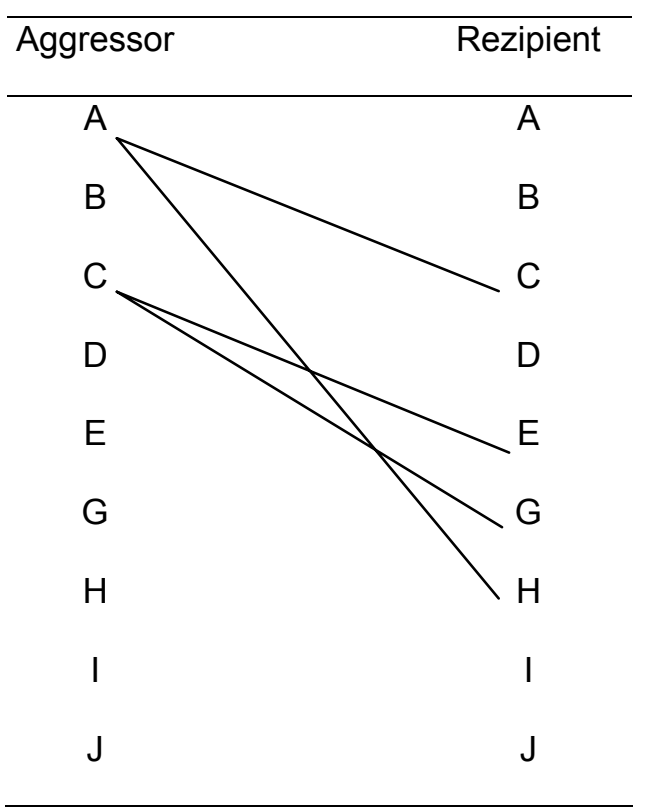

\section{Experiment 2}

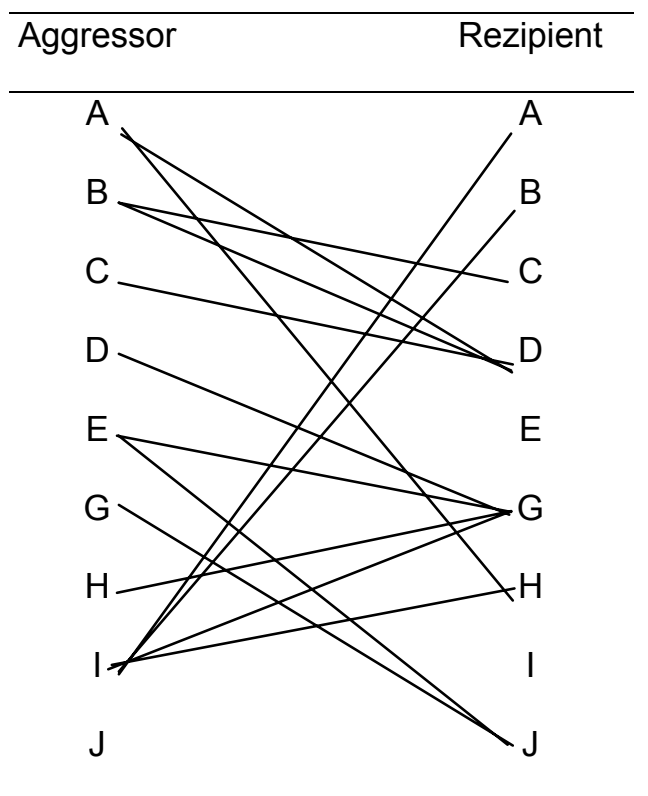




\subsubsection{Rangpositionen in der Hierarchie an den Futterplätzen im zweiten Beobachtungsjahr}

Während die älteste Tochter D im ersten Jahr am Futterplatz noch ihren jüngeren Bruder G verdrängte, hatte $\mathrm{G}$ im ersten Experiment des zweiten Jahres eine höhere Rangposition in der Hierarchie am Futterplatz, was sich im Laufe des Jahres auch nicht mehr veränderte. M verdrängte in den Experimenten E1 und E2 ihren älteren Bruder J und in E1 ihre Schwester D vom Futter. Im dritten Experiment wurde sie dann jedoch von D verdrängt. Das Verhältnis der Rangpositionen von D und J in der Futterplatz-Hierarchie unterlag im zweiten Beobachtungsjahr zwei Wechseln. Zunächst war D an den Futterplätzen dominant über J. Im zweiten Experiment verdrängte $J$ dann D vom Futter und im vierten Experiment hatte D wieder das Vorrecht in der Fresshierarchie.

Während des dritten Experiments wurde nur wenig dissoziatives Verhalten an den Futterplätzen beobachtet. Während der Experimente 1 bis 3 konnte zwischen den Brüdern $\mathrm{C}$ und $\mathrm{G}$ kein hierarchisches Verhältnis erkannt werden. Im vierten Experiment gab es Auseinandersetzungen zwischen $\mathrm{C}$ und $\mathrm{G}$, die aber keinen Rückschluss auf die Rangpositionen der beiden Brüder in der Hierarchie am Futterplatz zuließen. Bei allen übrigen Dyaden kamen Verdrängungen vom Futter nur abwärts in der Seniorität vor. 
Tabelle 3.21 Dissoziatives Verdrängen an den Futterplätzen während der vier Experimente im zweiten Beobachtungsjahr. Aggressor: Familienmitglied, welches ein anderes Familienmitglied, den Rezipienten, vom Futter durch dissoziatives Verhalten verdrängt. Linien: dissoziative Interaktion.

\section{Experiment 1}

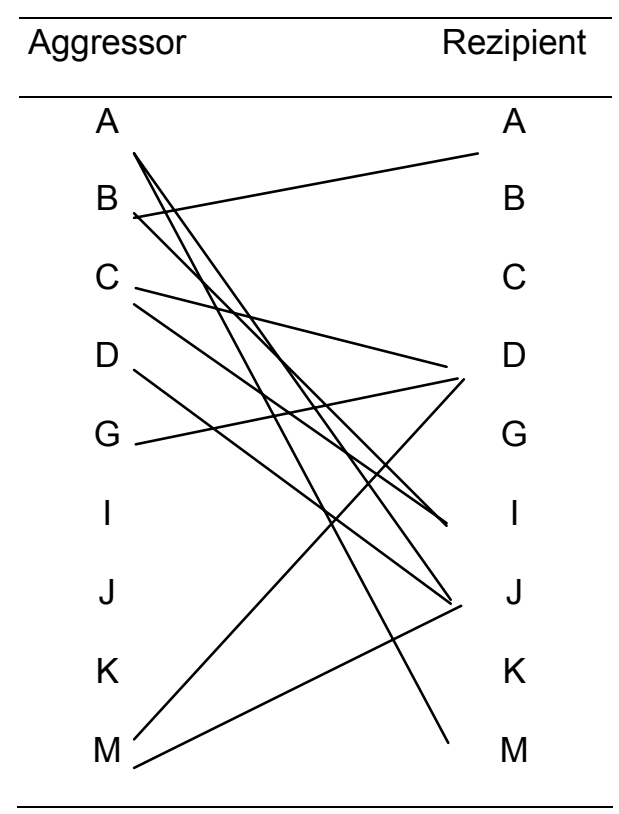

\section{Experiment 3}

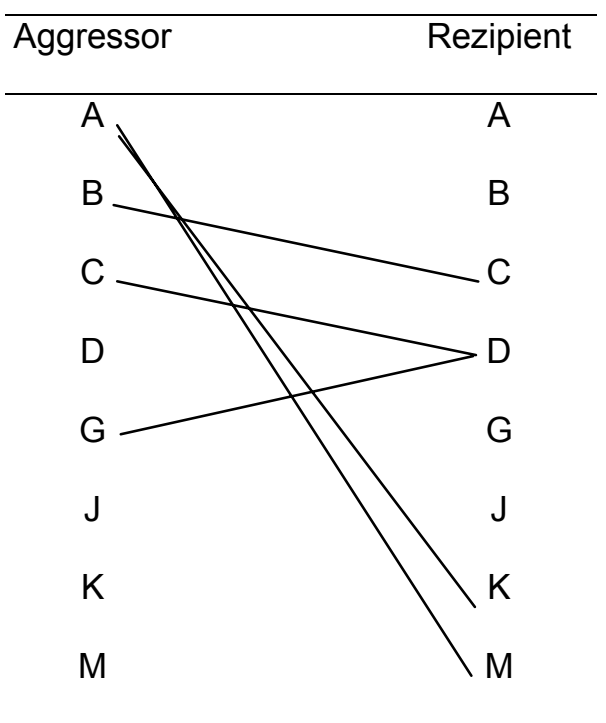

\section{Experiment 2}

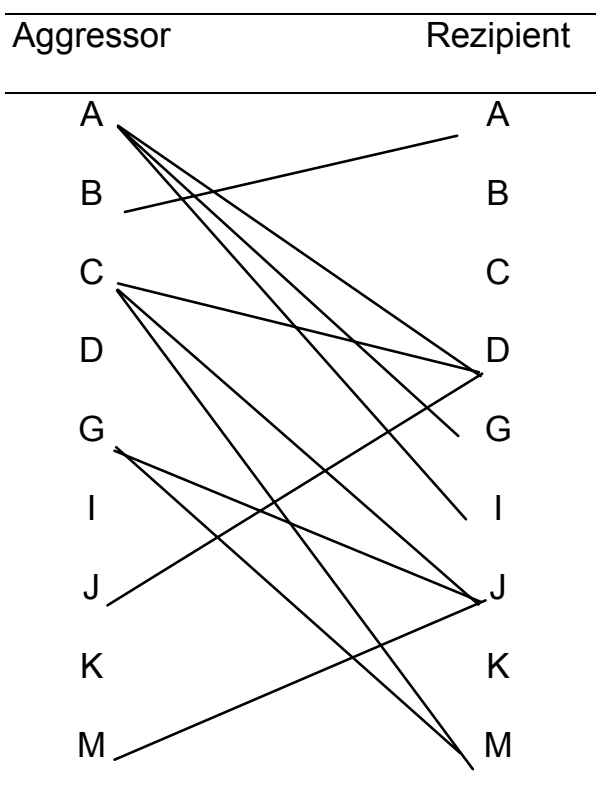

\section{Experiment 4}

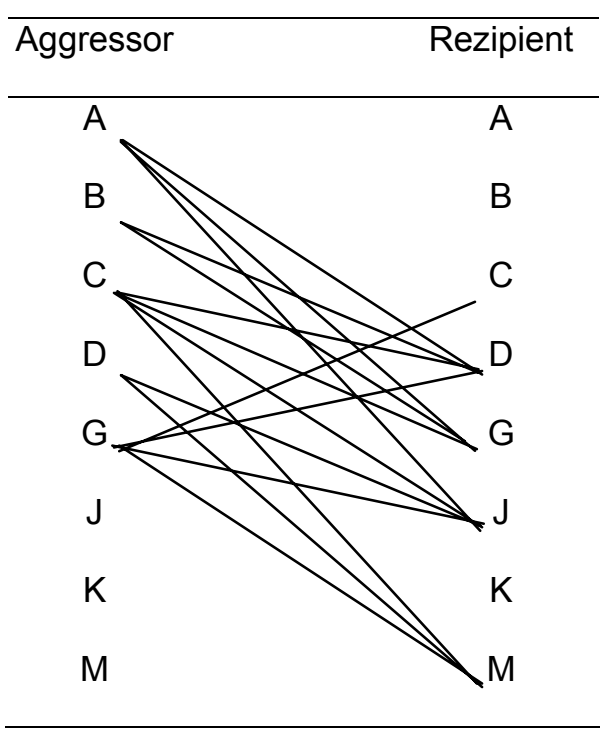




\subsubsection{Einfluss der Locktiere auf die Beziehungen der Geschwister}

Den folgenden drei Kapiteln liegt die Hypothese 5 zugrunde. Es wurden die räumliche Assoziation und affiliative Interaktionen der Bruder-Dyaden, der Schwester-Dyaden und der Bruder-Schwester-Dyaden über die unterschiedlichen Bedingungen der Kontrollen und Experimente miteinander verglichen.

Hypothese 5: Die Beziehungen der Geschwister (räumliche Assoziation und affiliative Interaktionen) bleiben von wechselnden Experimentalbedingungen unbeeinflusst.

Bei der Präsentation der Lockweibchen im ersten Experiment (C1) des Jahres 2000 wurden Veränderungen in den Interaktionen zwischen den Brüdern der Familie erwartet. Rückläufige soziopositive Kontakte und die Zunahme dissoziativer Interaktionen könnten daher als Konkurrenz interpretiert werden. Im Gegensatz dazu würde unter der Voraussetzung eines hohen Prädationsrisikos eine Zunahme der freundschaftlichen Kontakte und die Erhöhung der räumlichen Nähe dann erwartet, wenn Brüder Emigrationsgemeinschaften bilden (vgl. Garber et al. 1993 und Goldizen et al. 1996 für Saguinus). Nach welchen Kriterien ein Weißbüschelaffe seine(n) Emigrationspartner wählen würde, wurde unter 1.2 bereits dargelegt. Die Intensivierung der affiliativen Interaktionen zu einem jüngeren Geschwister erscheint dabei am ehesten adaptiv. Um während oder nach einer Emigration möglichst wenig Konflikten ausgesetzt zu sein, sollte er einen Partner suchen, der ihm vertraut ist und mit dem er schon vor der Emigration konfliktarme, positive Erfahrungen machen konnte. Deshalb wäre es denkbar, dass Geschwister, die häufig affiliative Kontakte hatten, gemeinsam emigrieren. Persönliche Vorlieben und Abneigungen könnten ebenso relevant in der Wahl eines Emigrationspartners sein. Die meisten in Labor- und Freilandstudien beobachteten Emigrationen betrafen jedoch nur Einzeltiere (Soini 1982, 1987, 1988; Rothe et al. 1986; Darms 1987, 1989; Goldizen und Terborgh 1989; Stevenson and Rylands 1988), so dass gemeinschaftliche Emigrationen (Nievergelt et al. 2000, Digby und Ferrari 1994, Ferrari und Diego 1992) möglicherweise nur unter bestimmten Voraussetzungen auftreten.

$\mathrm{Da}$ in den genannten Studien auch gemeinschaftliche Emigrationen von Schwestern beobachtet wurden, gelten diese Überlegungen auch für Schwestern.

Die Experimente des zweiten Beobachtungsjahres sollten Töchter stimulieren, ihre Natalfamilie zu verlassen. Da direkt vor Beginn der Experimente im zweiten Jahr die ältere Tochter 
D ihre jüngere Schwester H aus der Familie geworfen hatte und die Schwestern K und M noch nicht subadult waren, konnte nur noch von D eine Peripheralisation und möglicherweise eine Emigration erwartet werden.

Es wurde während der Anwesenheit von Locktieren auch erwartet, dass sich die Beziehungen zwischen Brüdern und ihren Schwestern destabilisieren, falls in den Locktieren potentielle Paarungspartner gesehen werden. Brüder sollten sich in der Anwesenheit von Lockweibchen dissoziativ und weniger affiliativ gegenüber Schwestern verhalten, damit die Nähe zur Schwester beim Lockweibchen nicht den Eindruck eines etablierten Paares hinterlässt (Darms 1987). Bei der Präsentation der Lockmännchen ist von den Schwestern das entsprechende Verhalten gegenüber den Brüdern zu erwarten. Somit entspricht eine räumliche Distanzierung zwischen Brüdern und Schwestern bei der Anwesenheit von Locktieren der Erwartung.

\subsubsection{Räumliche Assoziationen der Brüder im ersten Beobachtungsjahr}

Hypothese 5a : Die Anwesenheit von Locktieren hat keinen Einfluss auf die räumliche Assoziation der Brüder.

Von der elektronischen Aufzeichnung der Transponderdaten ließ sich die räumliche Assoziation der einzelnen Bruder-Dyaden erfassen. Gemittelt über das ganze Jahr gab es Unterschiede zwischen den Dyaden (Abb. 3.48). Die Dyaden C/F, E/F und C/E hatten dabei relativ hohe Assoziationskoeffizienten und die Dyaden F/G, C/G und E/G eher niedrige Werte. Es konnte keine Abhängigkeit der räumlichen Assoziation von den Experimenten ermittelt werden (Kruskal-Wallis-Test: $\mathrm{H}=3,95 ; \mathrm{N}=297$; $\mathrm{p}=0,267$ ). Da $\mathrm{F}$ nach der ersten Kontrollphase aus der Familie geworfen wurde, musste die detailliertere Analyse über die Abhängigkeit der räumlichen Assoziation der Dyaden von den Experimenten ohne $\mathrm{F}$ durchgeführt werden. Während der Experimente (C1 und C2) konnten im Vergleich zu den Kontrollphasen jedoch keine Veränderungen in der räumlichen Beziehung zwischen den einzelnen Bruder-Dyaden festgestellt werden (ANOVA, F=1,42, n.s.). 


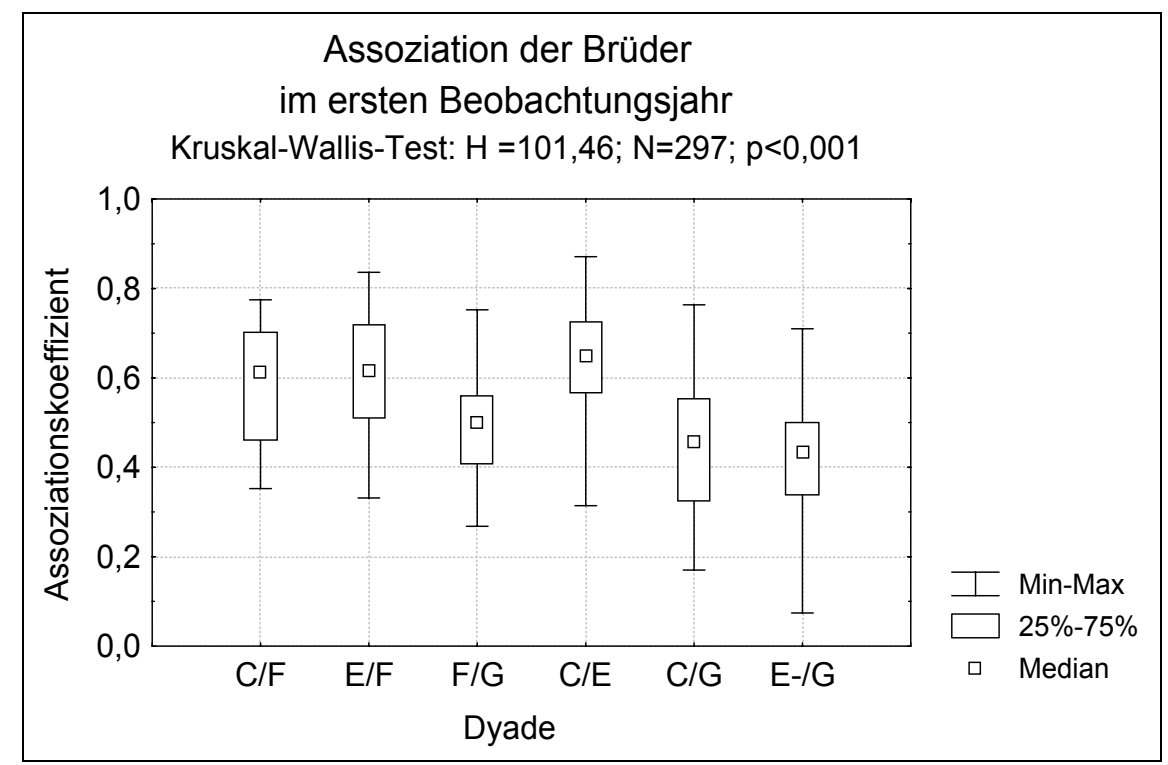

Abb. 3.48 Assoziation der Brüder im ersten Beobachtungsjahr. Der Assoziationskoeffizient ist ein Maß für die Stärke der Assoziation bzw. Kohäsion zweier Tiere (Dyade). Der Koeffizient liegt zwischen 0 und 1, wobei 1 die engste Assoziation (Tiere jederzeit gleichzeitig in denselben Arealen) und 0 die lockerste Assoziation (Tiere niemals gleichzeitig in einem Areal) beschreibt. Der Assoziationskoeffizient der Dyade C/F beschreibt die räumliche Nähe von $C$ und $F$ (das gleiche gilt für die Dyaden $E / F, F / G, C / E, C / G$ und E/G). Die Dyaden unterschieden sich in ihrer räumlichen Assoziation (Kruskal-Wallis-Test: $\mathrm{H}=101,46 ; \mathrm{N}=297 ; \mathrm{p}<0,001$ ).

Die Brüder $\mathrm{C}$ und $\mathrm{E}$ blieben während des gesamten Beobachtungsjahres räumlich enger verbunden als die Brüder $C$ und $G$ und die Brüder $E$ und $G$ (ANOVA, $F=61,94, p<0,001$ ). Abb. 3.49 zeigt die Assoziation der Brüder in den vier Experimenten des ersten Beobachtungsjahres.

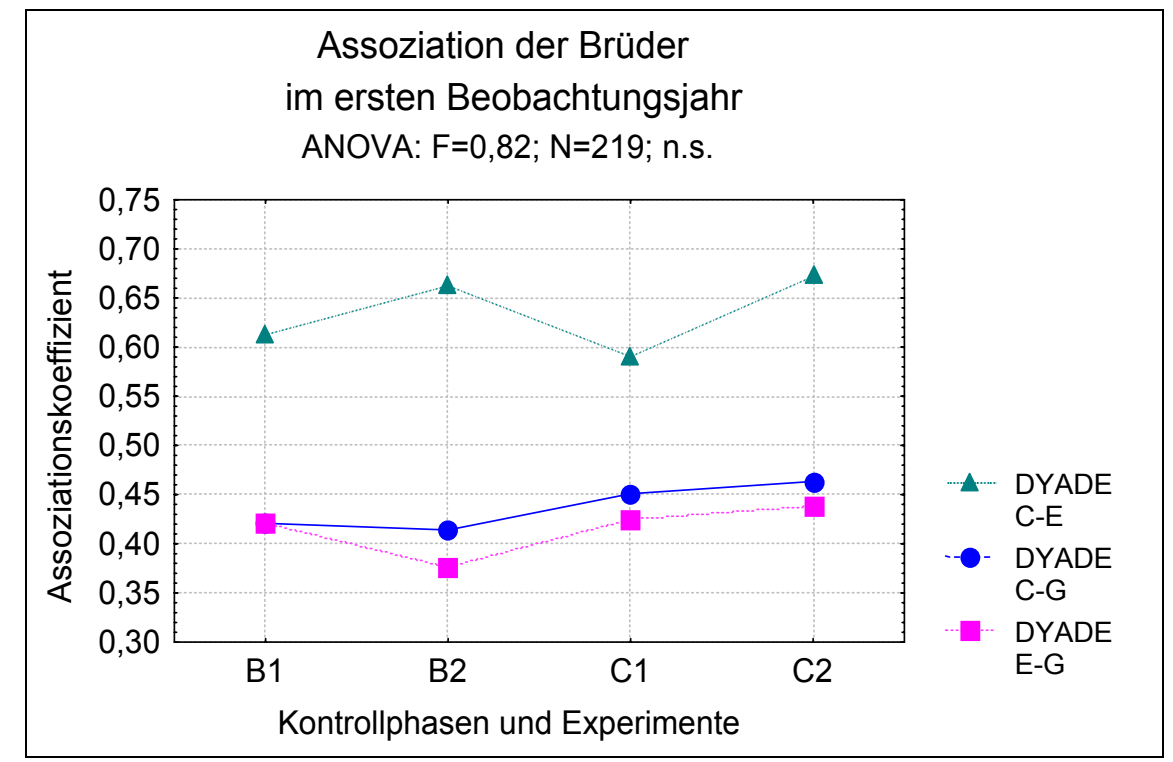

Abb. 3.49 Assoziation der Brüder im ersten Beobachtungsjahr (F ausgenommen). Der Assoziationskoeffizient der Dyade $\mathrm{C} / \mathrm{E}$ beschreibt die räumliche Assoziation der beiden Brüder $\mathrm{C}$ und $\mathrm{E}$ zueinander (das gleiche gilt für die beiden anderen Dyaden $\mathrm{C} / \mathrm{G}$ und $\mathrm{E} / \mathrm{G}$ ). Die Assoziationskoeffizienten der verschiedenen Dyaden sind mit unterschiedlichen Symbolen gekennzeichnet (siehe Legende) und für jedes Experiment (B1-C2) parallel zueinander (also übereinander) dargestellt. Es gab keine gegenseitige Beeinflussung zwischen den Faktoren Dyade und Experiment (ANOVA, $\mathrm{F}=0,82 ; \mathrm{N}=219 ;$ n.s.). 


\subsubsection{Räumliche Assoziationen der Brüder im zweiten Beobachtungsjahr}

Im zweiten Beobachtungsjahr wurden als Locktiere in E1 eine Kleinfamilie, in E2 und E3 zwei und in E4 ein Männchen verwendet. Es gab sowohl Unterschiede in der räumlichen Nähe der verschiedenen Dyaden (Abb. 3.50), als auch zwischen den vier Experimenten (Kruskal-Wallis-Test, $\mathrm{H}=16,40, \mathrm{p}<0,001$ ). Die Brüder I und $\mathrm{J}$ und die Brüder $\mathrm{C}$ und $\mathrm{I}$ hatten im Vergleich zu den restlichen Dyaden eine eher enge räumliche Assoziation.

Um die Veränderungen der Assoziation der einzelnen Dyaden eingehender analysieren zu können, wurden die Zeiträume vor und nach dem Rauswurf von I separat betrachtet. Die Assoziationen der Brüder waren im zweiten Experiment bei Anwesenheit zweier Lockmännchen lockerer als im ersten Experiment bei Präsenz der Kleinfamilie. Die Dyaden unterschieden sich nicht voneinander, und zwischen den Dyaden und den Experimenten gab es keine wechselseitige Beeinflussung (Abb. 3.51). Beim Vergleich des dritten und vierten Experiments zeigten sich weder Unterschiede zwischen den Experimenten noch zwischen den Dyaden (Abb. 3.52). Ein Vergleich der Assoziation zwischen C und G im Jahresverlauf macht deutlich, dass sich vom dritten zum vierten Experiment, also von den Bedingungen mit zwei Lockmännchen zu den Bedingungen mit einem Lockmännchen, die räumliche Assoziation der Dyade lockerte (ANOVA, Least Significant Difference-Test, F=2,74, $\mathrm{p}<0,05$ ).

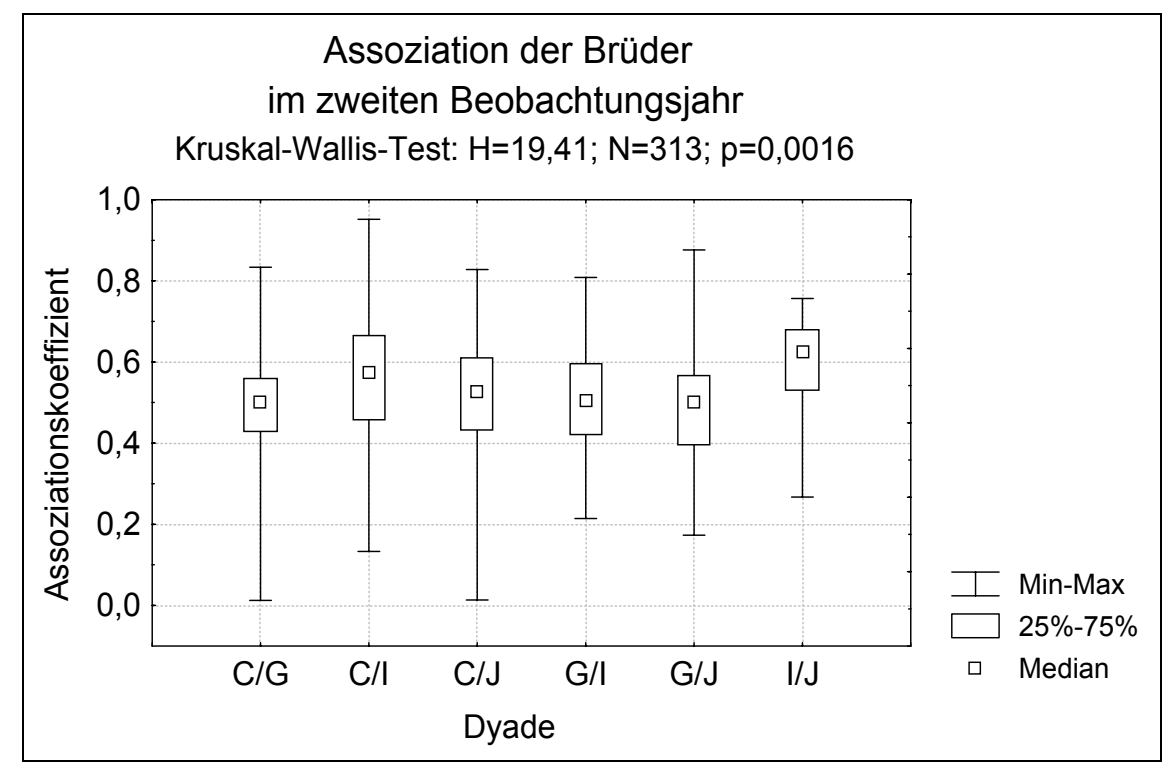

Abb. 3.50 Assoziation der Brüder im zweiten Beobachtungsjahr. Der Assoziationskoeffizient der Dyade C/G beschreibt die räumliche Assoziation von $\mathrm{C}$ und $\mathrm{G}$ (das gleiche gilt für die Dyaden $\mathrm{C} / \mathrm{I}, \mathrm{C} / \mathrm{J}, \mathrm{G} / \mathrm{I}, \mathrm{G} / \mathrm{J}$ und I/J). Die Dyaden unterschieden sich in ihrer räumlichen Assoziation (Kruskal-Wallis-Test: $H=19,41 ; N=313$; $\mathrm{p}<0,01)$. 


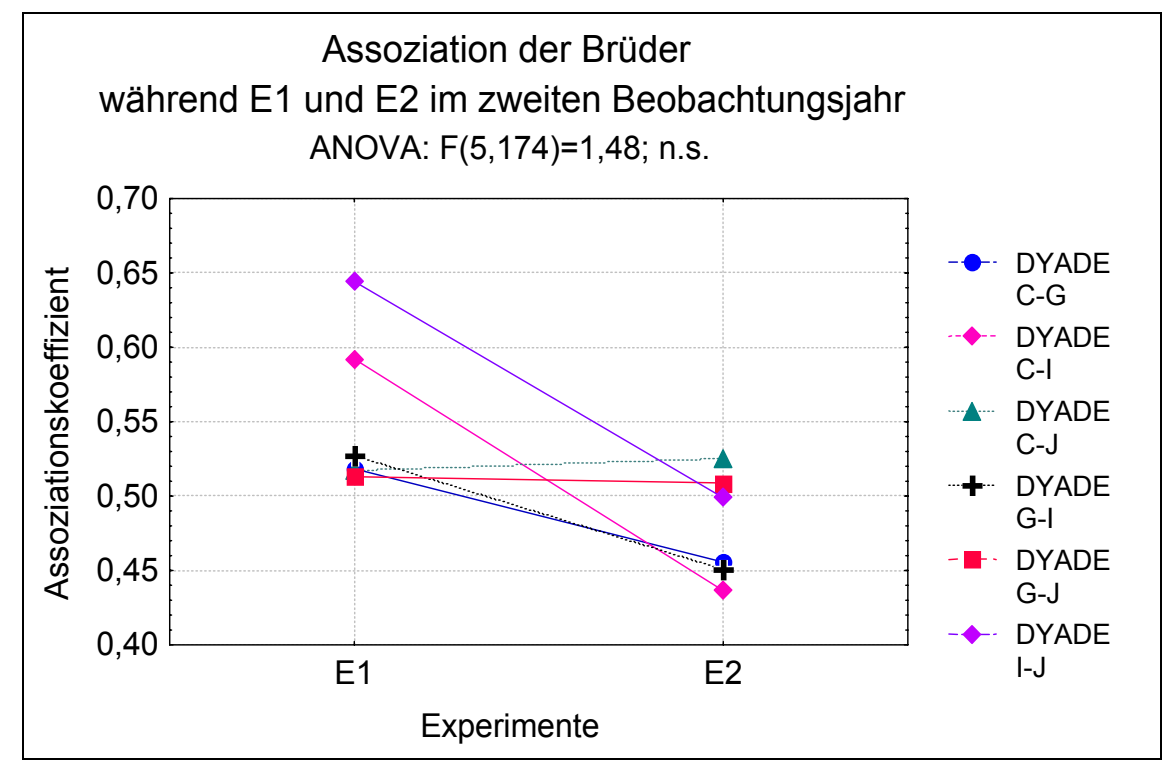

Abb. 3.51 Assoziation der Brüder in den Experimenten E1 und E2 (vor dem Rauswurf von I) im zweiten Beobachtungsjahr. Abkürzungen: Dyade C/G beschreibt z.B. den Assoziationskoeffizienten (Abzisse) zwischen den Brüdern C und G. Die unterschiedlichen Symbole stehen für die verschiedenen Bruder-Dyaden. Gestrichelte Linien stellen die Veränderungen der Assoziation einer Dyade von einem Experiment zum nächsten dar. Die Assoziationen der Bruder-Dyaden waren im zweiten Experiment geringer als im ersten Experiment (ANOVA, $F=9,52 ; p=0,002$ ). Die Dyaden unterschieden sich nicht voneinander (ANOVA, $F=2,14 ; p=0,06$ ) und zwischen den Dyaden und den Experimenten gab es keine wechselseitige Beeinflussung (ANOVA, F=1,48; n.s.).

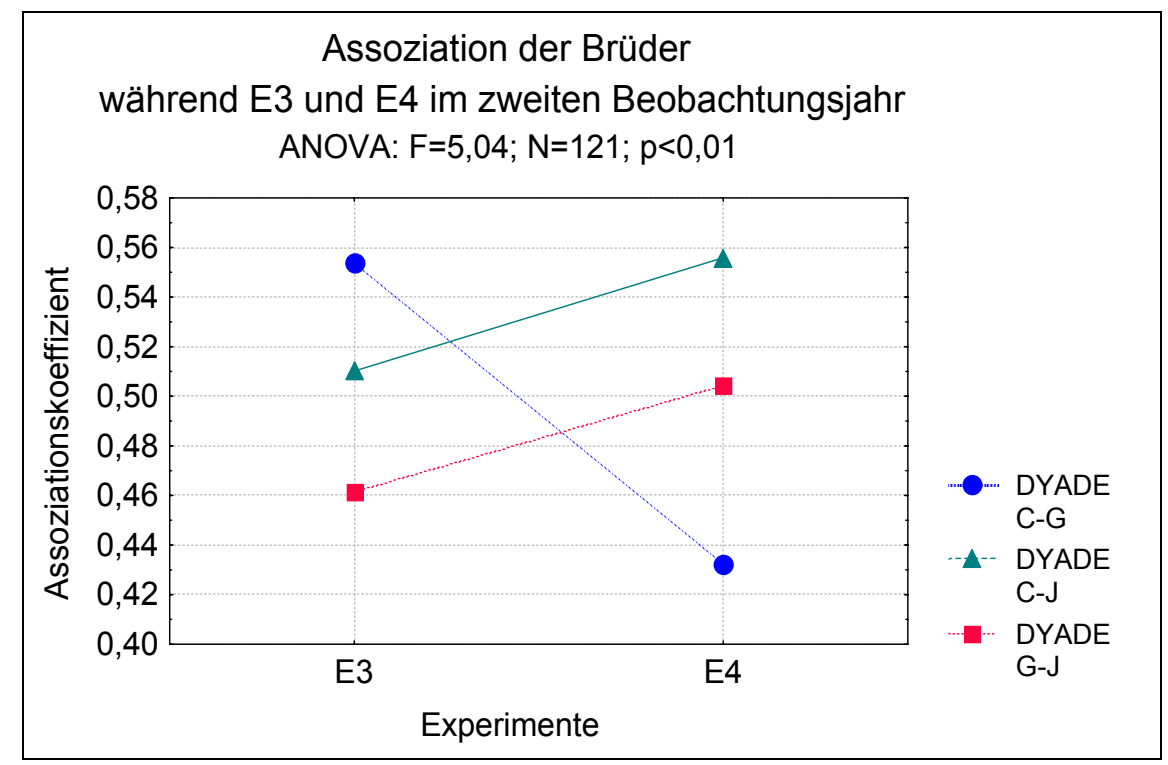

Abb. 3.52 Assoziation der Brüder in den Experimenten E3 und E4 (nach dem Rauswurf von I) im zweiten Beobachtungsjahr. Abkürzungen: Dyade C/G beschreibt z.B. den Assoziationskoeffizienten (Abzisse) zwischen den Brüdern C und G. Die unterschiedlichen Symbole stehen für die verschiedenen Bruder-Dyaden. Gestrichelte Linien stellen die Veränderungen der Assoziation einer Dyade von einem Experiment zum nächsten dar. Es gab weder Unterschiede zwischen den Experimenten (ANOVA, $\mathrm{F}=0,20 ; \mathrm{N}=121$; n.s.), noch zwischen den Dyaden (ANOVA, F1,56; $\mathrm{N}=121$; n.s.). In Abhängigkeit von den Experimenten hatten die Dyaden jedoch Auswirkungen auf die Assoziationskoeffizienten (ANOVA, $\mathrm{F}=5,04 ; \mathrm{N}=121 ; \mathrm{p}<0,01$ ).

Im ersten Beobachtungsjahr war die räumliche Assoziation der Brüder von den Experimenten unabhängig, während es im zweiten Beobachtungsjahr eine Abhängigkeit der räumlichen Assoziation der Brüder von den Experimenten gab. Unter den experimentellen Voraus- 
setzungen des ersten Beobachtungsjahres (Präsentation von Lockweibchen) kann somit die Hypothese 5a nicht abgelehnt werden. Unter den experimentellen Voraussetzungen des zweiten Beobachtungsjahres (Präsentation einer Kleinfamilie und Lockmännchen in unterschiedlicher Anzahl) ist Hypothese 5a jedoch zurückzuweisen.

\subsubsection{Affiliative Interaktionen der Brüder im ersten Beobachtungsjahr}

Hypothese 5b: Die Anwesenheit von Locktieren hat keinen Einfluss auf die Häufigkeit affiliativer Interaktionen der Brüder.

In der ersten Kontrollphase hatten $G$ und $F$ überdurchschnittlich häufig affiliative Kontakte (Abb. 3.53). Nachdem F die Familie verlassen hatte, interagierten $C$ und $G$ in der zweiten Kontrollphase häufiger als erwartet affiliativ und $E$ und $G$ seltener als erwartet (Abb. 3.54). Während der Experimente C1 und C2, also während der Präsentation der Lockweibchen, sind die affiliativen Interaktionen aller Brüder gleich häufig (Abb. 3.55 und Abb. 3.56). Die nach dem Rauswurf von F entstandenen Unterschiede in der Häufigkeit der affiliativen Interaktionen zwischen den Brüdern hoben sich während der Experimente, in denen Lockweibchen präsentiert werden, wieder auf. Wird jede Dyade einzeln für sich über das Beobachtungsjahr betrachtet, so fällt auf, dass alle Dyaden im ersten Experiment (C1) seltener affiliative Kontakte hatten, als erwartet (Abb. 3.57-59). Während der Anwesenheit des Lockweibchens im Experimentalareal war also bei allen Brüdern die Häufigkeit affiliativer Interaktionen vermindert.

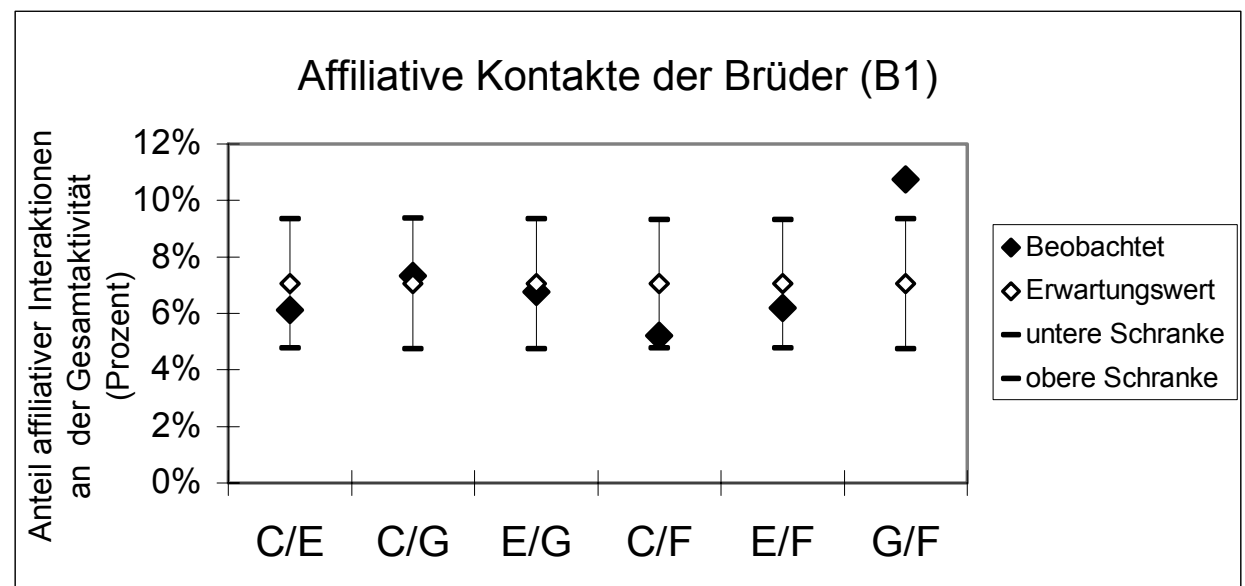

Abb. 3.53 Affiliative Interaktionen zwischen jeweils zwei Brüdern (Bruder-Dyaden) in der ersten Kontrollphase des ersten Beobachtungsjahres (KFA, $E W=7,05 n=6$ ). Abkürzungen: $C / E$ bedeutet, dass alle beobachteten affiliativen Interaktionen zwischen den Brüdern $\mathrm{C}$ und $\mathrm{E}$ gewertet wurden; etc. Die Dyade $\mathrm{G} / \mathrm{F}$ hatte als einzige der getesteten Dyaden häufiger affiliative Kontakte als erwartet. 


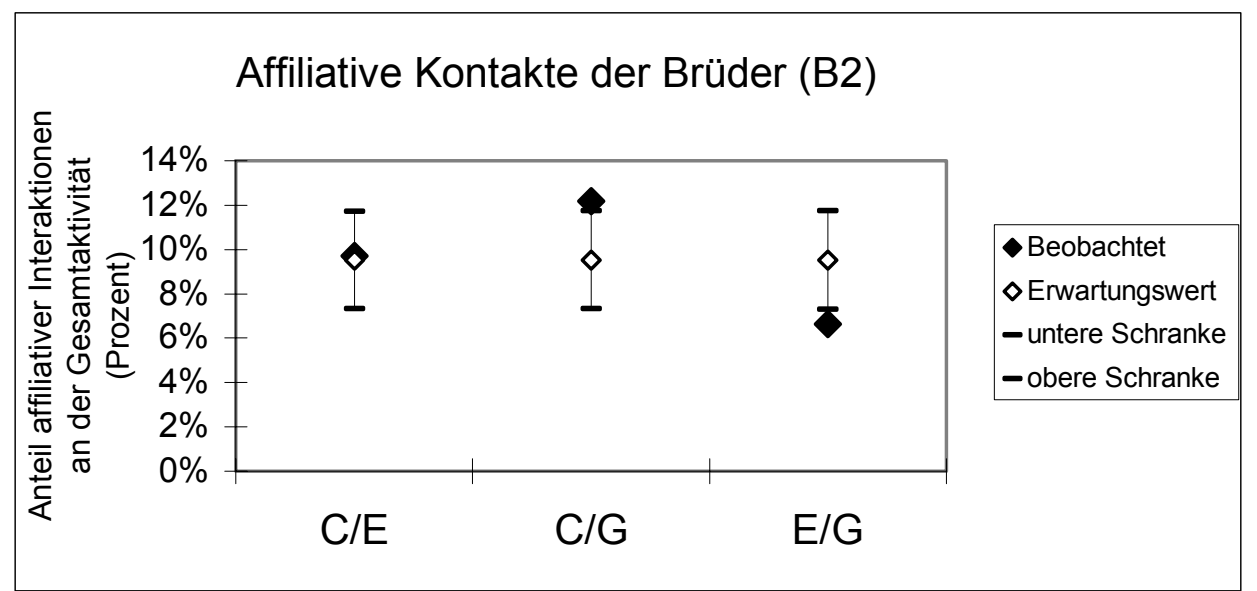

Abb. 3.54 Affiliative Interaktionen zwischen jeweils zwei Brüdern (Bruder-Dyaden) in der zweiten Kontrollphase des ersten Beobachtungsjahres (KFA, EW=9,53 $n=3$ ). Abkürzungen: $\mathrm{C} / \mathrm{E}$ bedeutet, dass alle beobachteten affiliativen Interaktionen zwischen den Brüdern $\mathrm{C}$ und $\mathrm{E}$ gewertet wurden; etc. Die Dyade $\mathrm{C} / \mathrm{G}$ hatte häufiger und die Dyade E/G seltener affiliative Kontakte als erwartet.

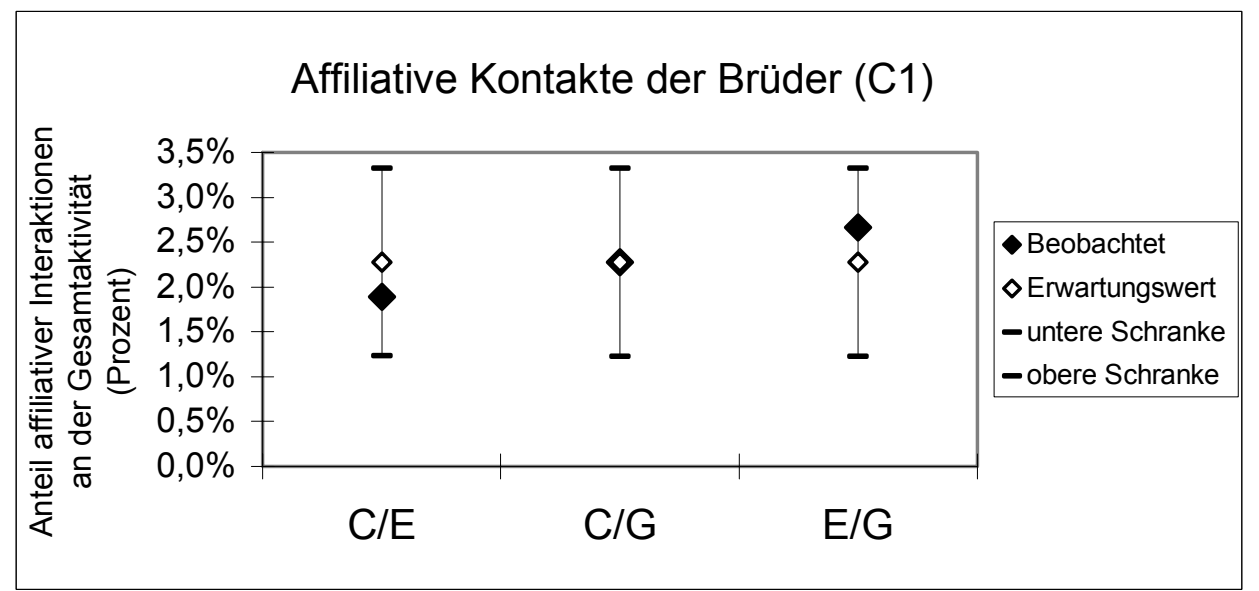

Abb. 3.55 Affiliative Interaktionen zwischen jeweils zwei Brüdern (Bruder-Dyaden) im ersten Experiment des ersten Beobachtungsjahres (KFA, $\mathrm{EW}=2,27 \mathrm{n}=3$ ). Abkürzungen: $\mathrm{C} / \mathrm{E}$ bedeutet, dass alle beobachteten affiliativen Interaktionen zwischen den Brüdern $\mathrm{C}$ und $\mathrm{E}$ gewertet wurden, etc. Die affiliativen Interaktionen aller drei Dyaden entsprachen dem Erwartungsbereich.

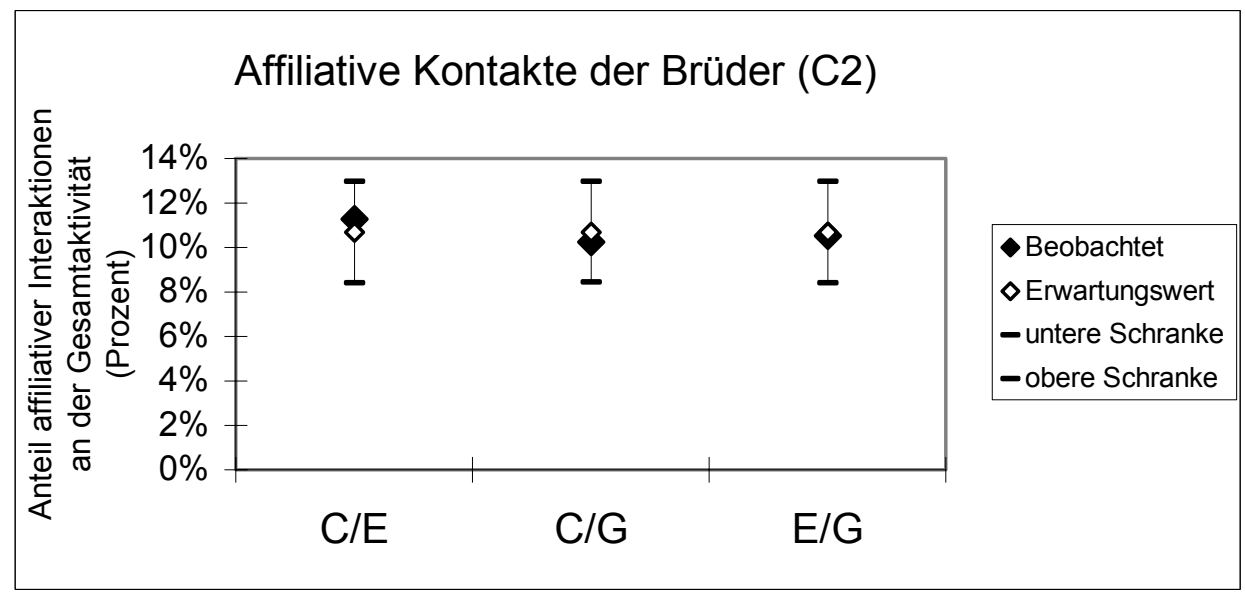

Abb. 3.56 Affiliative Interaktionen zwischen jeweils zwei Brüdern (Bruder-Dyaden) im ersten Experiment des ersten Beobachtungsjahres (KFA, $\mathrm{EW}=10,70 \mathrm{n}=3$ ). Abkürzungen: $\mathrm{C} / \mathrm{E}$ bedeutet, dass alle beobachteten affiliativen Interaktionen zwischen den Brüdern $\mathrm{C}$ und $\mathrm{E}$ gewertet wurden; etc. Die affiliativen Interaktionen aller drei Dyaden entsprachen dem Erwartungsbereich. 
Des weiteren fällt auf, dass die räumliche Nähe der Brüder zueinander nicht mit dem Anteil an affiliativen Kontakten einherging. So zeichnete sich das Verhältnis zwischen C und E vorwiegend durch räumliche Nähe, nicht aber durch überdurchschnittlich häufiges affiliatives Verhalten aus. Obwohl $\mathrm{C}$ und $\mathrm{G}$ in der zweiten Kontrollphase vermehrt affiliative Kontakte hatten, wurde ihre räumliche Nähe jedoch nicht größer. Das Verhältnis zwischen E und G war während des ersten Beobachtungsjahres mit einer Ausnahme unauffällig. In der zweiten Kontrollphase hatten E und G seltener affiliative Kontakte als die anderen Bruder-Dyaden.

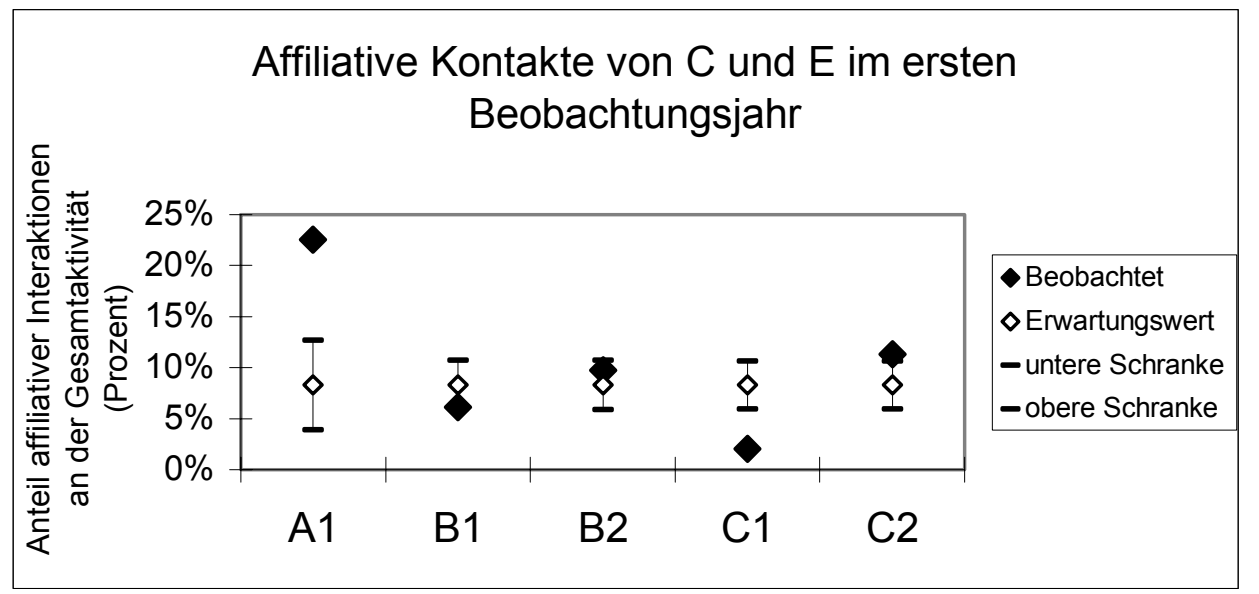

Abb. 3.57 Affiliative Kontakte von $\mathrm{C}$ und $\mathrm{E}$ im ersten Beobachtungsjahr (KFA, EW=8,29 n=5). Abkürzungen: A1: Hüttenphase 1, B1: erste Kontrollphase, B2: zweite Kontrollphase, C1: erstes Experiment, C2: zweites Experiment. Der Anteil an affiliativer Interaktion lag während der Hüttenphase weit über und im ersten Experiment unter dem Erwartungswert. Im zweiten Experiment hatten $\mathrm{C}$ und $\mathrm{E}$ wieder häufiger affiliative Kontakte als erwartet.

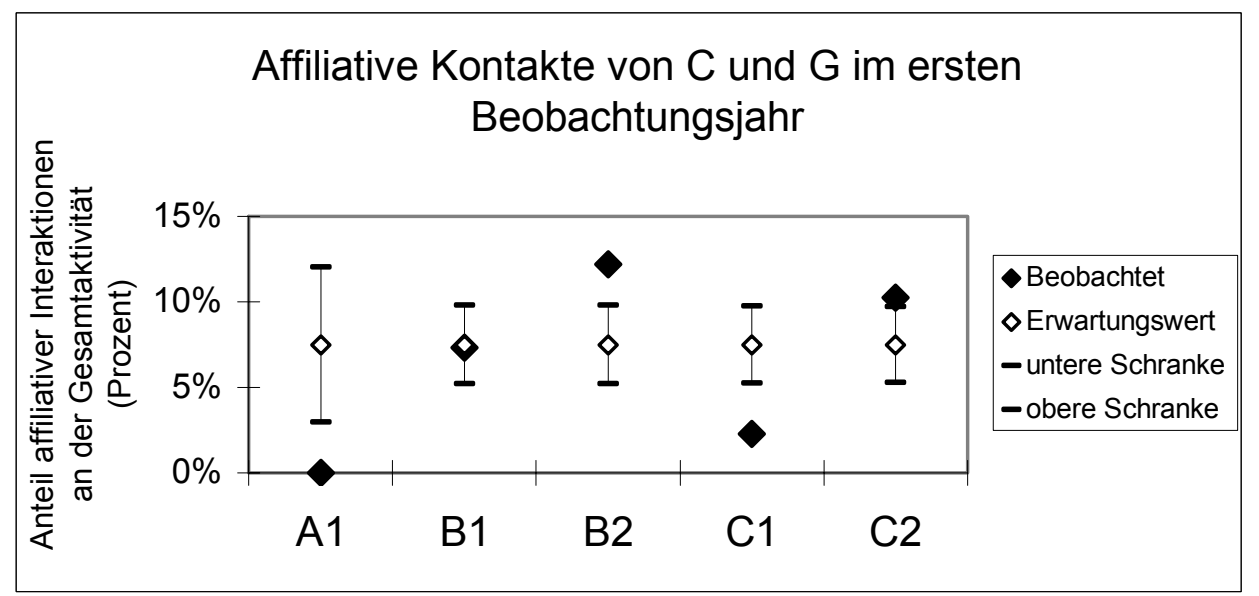

Abb. 3.58 Affiliative Kontakte von $\mathrm{C}$ und $\mathrm{G}$ im ersten Beobachtungsjahr (KFA, EW=7,5, n=5). Abkürzungen: A1: Hüttenphase 1, B1: erste Kontrollphase, B2: zweite Kontrollphase, C1: erstes Experiment, C2: zweites Experiment. In der Hüttenphase und im ersten Experiment lag der beobachtete Wert signifikant unter dem Erwartungswert, während er in der zweiten Kontrollphase und im zweiten Experiment über dem erwarteten Wert lag. 


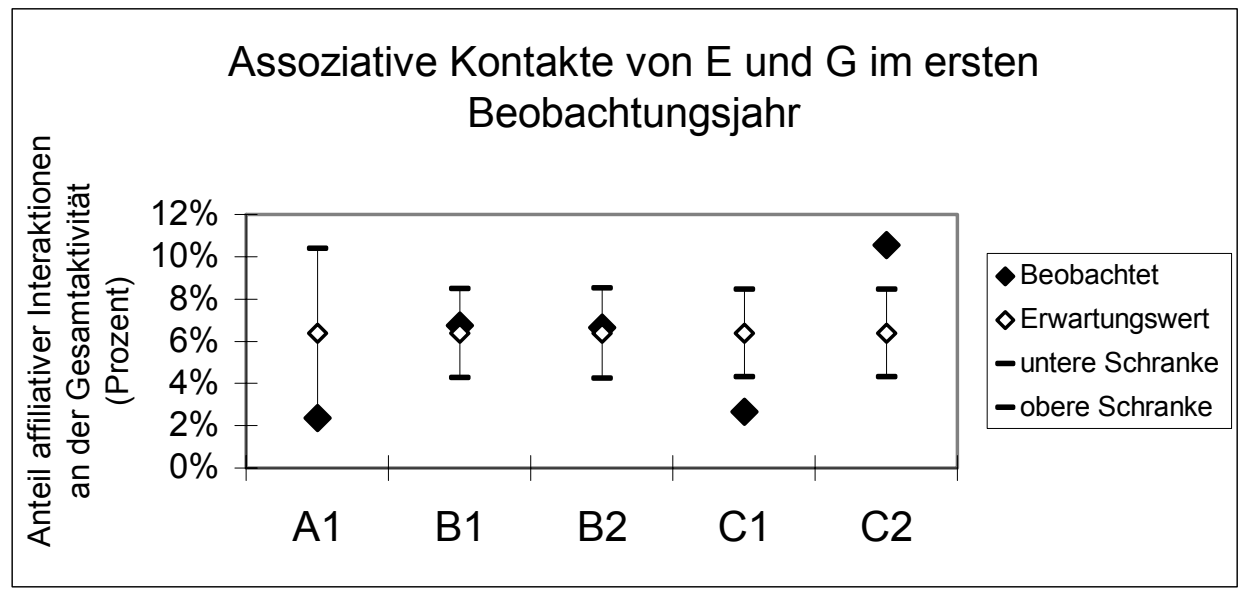

Abb. 3.59 Affiliative Kontakte von $E$ und $G$ im ersten Beobachtungsjahr (KFA, EW=6,37, n=5). Abkürzungen: A1: Hüttenphase 1, B1: erste Kontrollphase, B2: zweite Kontrollphase, C1: erstes Experiment, C2: zweites Experiment. In der Hüttenphase und im ersten Experiment hatten E und $\mathrm{G}$ seltener als erwartet affiliative Interaktionen. Im zweiten Experiment lag der beobachtete Wert über dem Erwartungswert.

\subsubsection{Affiliative Interaktionen der Brüder im zweiten Beobachtungsjahr}

Während der Hüttenphase (D1) lagen die affiliativen Interaktionen der Brüder C und I und der Brüder C und J unter dem Erwartungsbereich. I und J hatten häufiger affiliative Interaktionen als erwartet. Die affiliativen Kontakte der Dyaden C/G, G/I und G/J lagen innerhalb des Erwartungsbereichs (Abb. 3.60). Im ersten und zweiten Experiment hatten die Brüder C und I und die Brüder $\mathrm{C}$ und $\mathrm{J}$ seltener als erwartet und die Brüder $\mathrm{C}$ und $\mathrm{G}$ und die Brüder $\mathrm{I}$ und $\mathrm{J}$ häufiger als erwartet affiliative Interaktionen. Die affiliativen Interaktionen von G und I und von $\mathrm{G}$ und $\mathrm{J}$ lagen im Erwartungsbereich (Abb. 3.61 und Abb. 3.62). Während des dritten Experiments hatten die Brüder $\mathrm{C}$ und $\mathrm{J}$ seltener und die Brüder $\mathrm{G}$ und $\mathrm{J}$ häufiger als erwartet affiliative Interaktionen. Die affiliativen Kontakte von $\mathrm{C}$ und $\mathrm{G}$ lagen innerhalb des Erwartungsbereichs (Abb. 3.63). Die affiliativen Interaktionen der Brüder $\mathrm{C}$ und $\mathrm{G}$ und der Brüder $\mathrm{C}$ und $\mathrm{J}$ lagen während des vierten Experiments unter und jene von $\mathrm{G}$ und $\mathrm{J}$ über dem Erwartungsbereich (Abb. 3.64). 


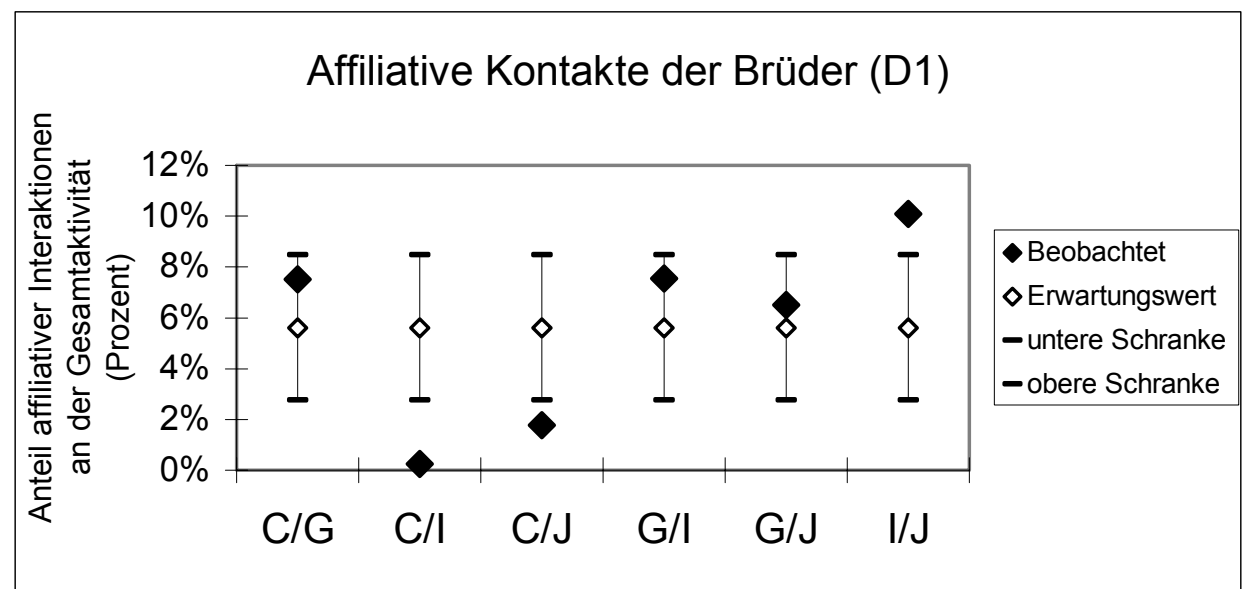

Abb. 3.60 Affiliative Interaktionen zwischen jeweils zwei Brüdern (Bruder-Dyaden) in der Hüttenphase D1 des zweiten Beobachtungsjahres (KFA, $\mathrm{EW}=5,61 \mathrm{n}=6$ ). Abkürzungen: $\mathrm{C} / \mathrm{G}$ bedeutet, dass alle beobachteten affiliativen Interaktionen zwischen den Brüdern $\mathrm{C}$ und $\mathrm{G}$ gewertet wurden. Die affiliativen Interaktionen der Dyaden $\mathrm{C} / \mathrm{I}$ und $\mathrm{C} / \mathrm{J}$ lagen unter dem Erwartungsbereich. I und $\mathrm{J}$ hatten häufiger affiliative Interaktionen als erwartet.

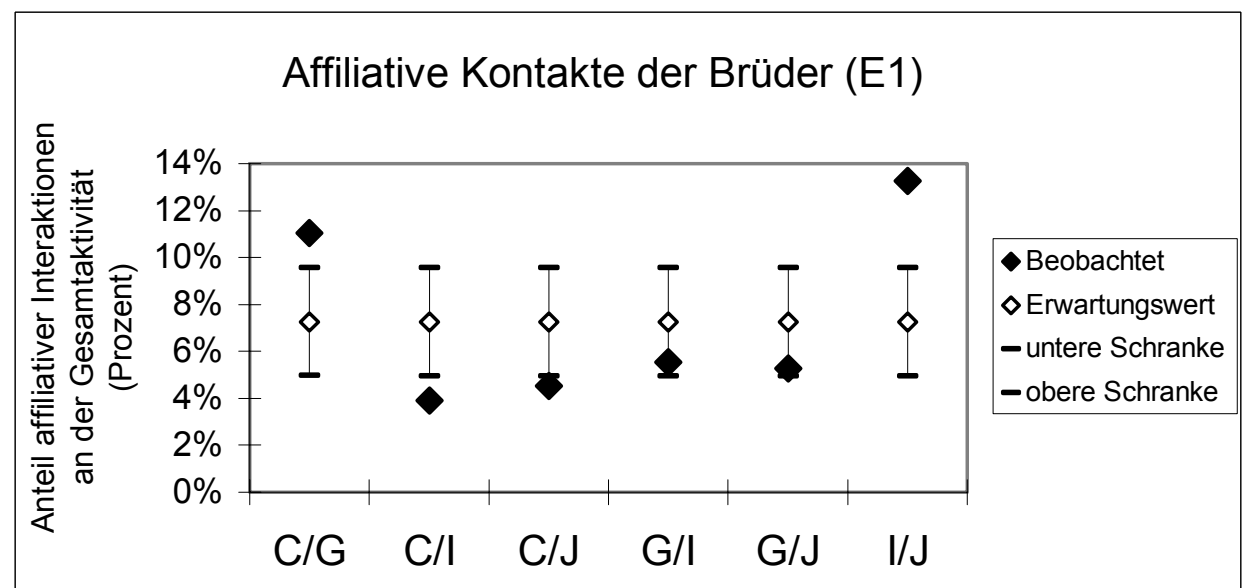

Abb. 3.61 Affiliative Interaktionen zwischen jeweils zwei Brüdern (Bruder-Dyaden) im ersten Experiment E1 des zweiten Beobachtungsjahres (KFA, $E W=7,26 \mathrm{n}=6$ ). Abkürzungen: $\mathrm{C} / \mathrm{G}$ bedeutet, dass alle beobachteten affiliativen Interaktionen zwischen den Brüdern $\mathrm{C}$ und $\mathrm{G}$ gewertet wurden. Die affiliativen Interaktionen der Dyaden C/I und C/J lagen unter dem Erwartungsbereich. Die Dyaden C/G und I/J hatten häufiger affiliative Interaktionen als erwartet. Die Beobachtungswerte der Dyaden $\mathrm{G} / \mathrm{I}$ und $\mathrm{G} / \mathrm{J}$ lagen innerhalb des Erwartungsbereichs. 


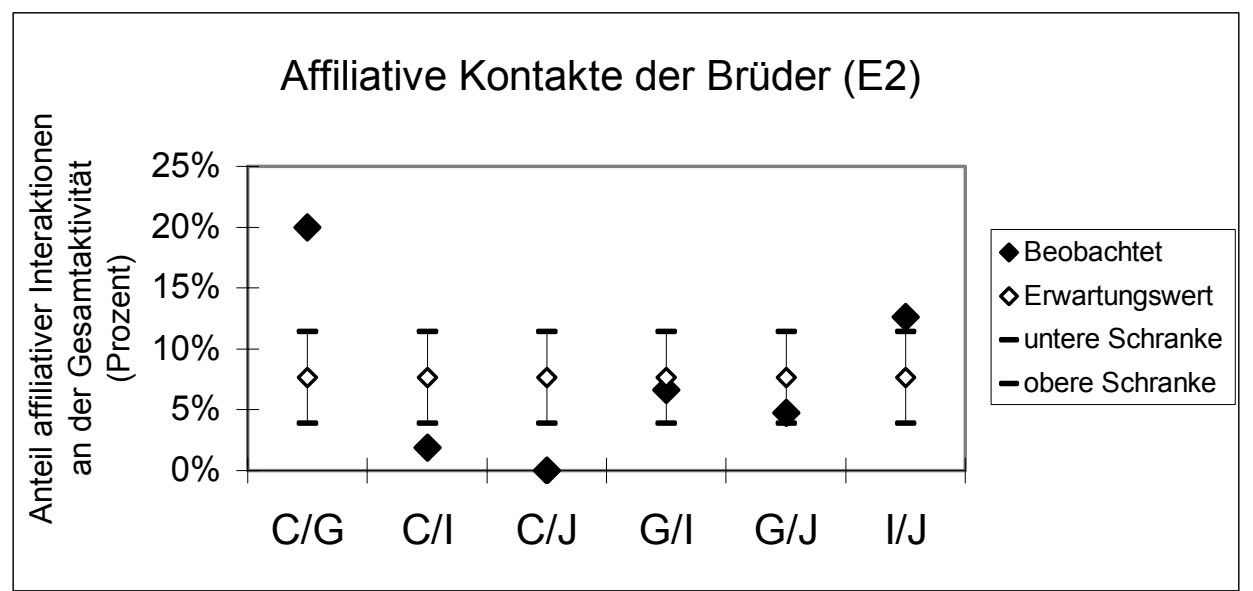

Abb. 3.62 Affiliative Interaktionen zwischen jeweils zwei Brüdern (Bruder-Dyaden) im zweiten Experiment E2 des zweiten Beobachtungsjahres (KFA, $E W=7,65 n=6$ ). Abkürzungen: $C / G$ bedeutet, dass alle beobachteten affiliativen Interaktionen zwischen den Brüdern $\mathrm{C}$ und $\mathrm{G}$ gewertet wurden. Die affiliativen Interaktionen der Dyaden $\mathrm{C} / \mathrm{G}$ und $\mathrm{I} / \mathrm{J}$ lagen über und der Dyaden $\mathrm{C} / \mathrm{I}$ und $\mathrm{C} / \mathrm{J}$ lagen unter dem Erwartungsbereich. Die Beobachtungswerte der Dyaden G/I und G/J lagen innerhalb des Erwartungsbereichs.

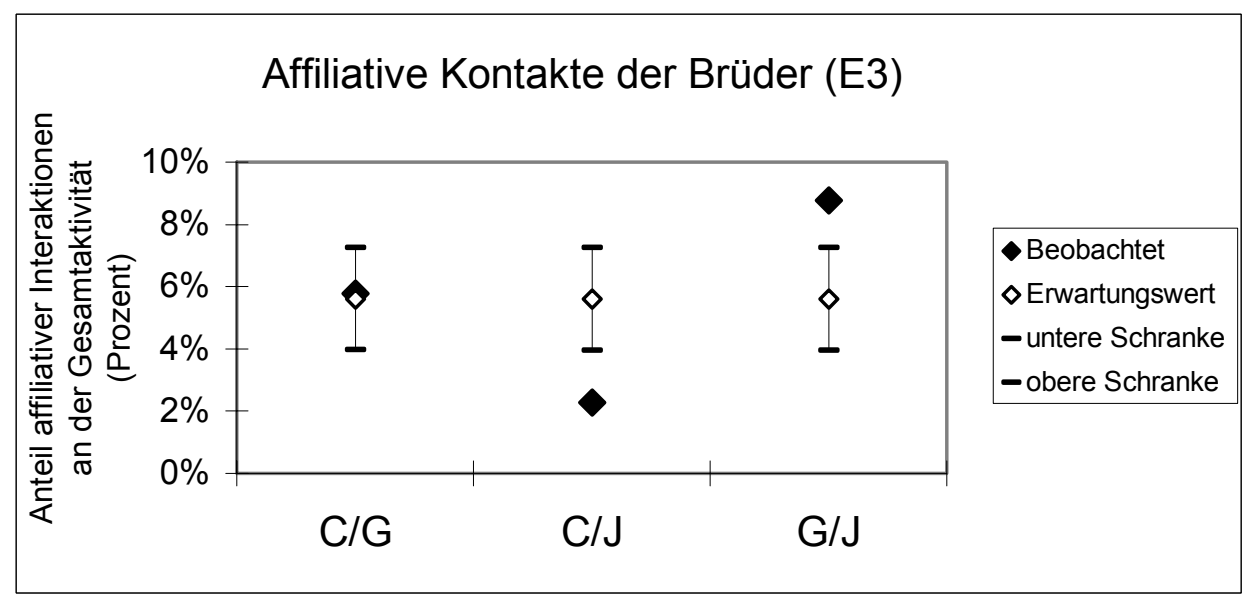

Abb. 3.63 Affiliative Interaktionen zwischen jeweils zwei Brüdern (Bruder-Dyaden) im dritten Experiment E3 des zweiten Beobachtungsjahres (KFA, $E W=5,61 \mathrm{n}=3$ ). Abkürzungen: $\mathrm{C} / \mathrm{G}$ bedeutet, dass alle beobachteten affiliativen Interaktionen zwischen den Brüdern $\mathrm{C}$ und $\mathrm{G}$ gewertet wurden. Die affiliativen Interaktionen der Dyade C/J lag unter, die der Dyade G/J über und jene der Dyade C/G innerhalb des Erwartungsbereichs.

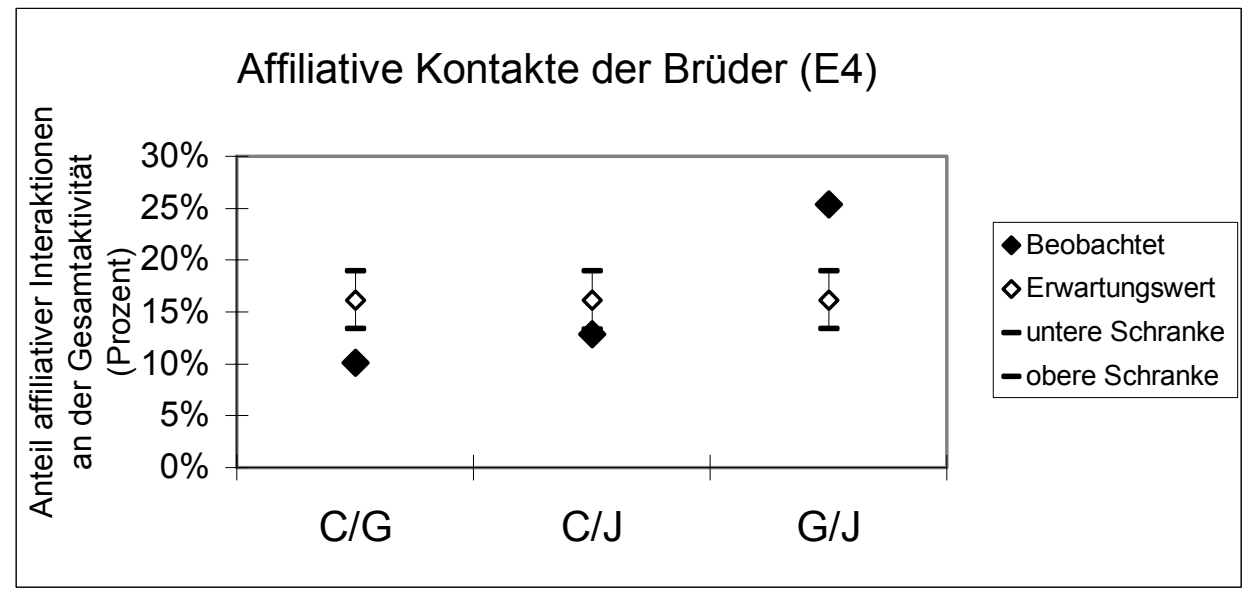

Abb. 3.64 Affiliative Interaktionen zwischen jeweils zwei Brüdern (Bruder-Dyaden) im vierten Experiment E4 des zweiten Beobachtungsjahres (KFA, $\mathrm{EW}=16,15 \mathrm{n}=3$ ). Abkürzungen: $\mathrm{C} / \mathrm{G}$ bedeutet, dass alle beobachteten affiliativen Interaktionen zwischen den Brüdern $\mathrm{C}$ und $\mathrm{G}$ gewertet wurden. Die affiliativen Interaktionen der Dyaden $\mathrm{C} / \mathrm{G}$ und $\mathrm{C} / \mathrm{J}$ lagen unter dem Erwartungsbereich und die von G/J lagen über dem Erwartungsbereich. 
Zwischen $\mathrm{C}$ und $\mathrm{G}$ nahmen die affiliativen Kontakte im zweiten Experiment zu, wurden im dritten Experiment (nach I's Rauswurf) seltener als erwartet und lagen dann im vierten Experiment wieder im Erwartungsbereich (Abb. 3.65). C und I hatten im ersten Experiment überdurchschnittlich häufig affiliative Kontakte, deren Häufigkeit sich im zweiten Experiment aber innerhalb des Erwartungsbereiches befand (Abb. 3.66). Die recht seltenen affiliativen Kontakte zwischen $\mathrm{C}$ und $\mathrm{J}$ sanken im zweiten Experiment auf Null, nahmen danach wieder zu und waren im vierten Experiment überdurchschnittlich häufig (Abb. 3.67). Die affiliativen Kontakte zwischen $\mathrm{G}$ und I zeigten zwischen den Experimenten keinen bedeutenden Unterschied (Abb. 3.68). Die Beziehung zwischen $G$ und J veränderte sich hingegen nach dem Rauswurf von I stark. Die zuvor unterdurchschnittlich häufigen Kontakte stiegen im vierten Experiment weit über den Erwartungsbereich an (Abb. 3.69). I und J hatten über die Experimente hinweg gleichbleibend häufig affiliative Kontakte (Abb. 3.70).

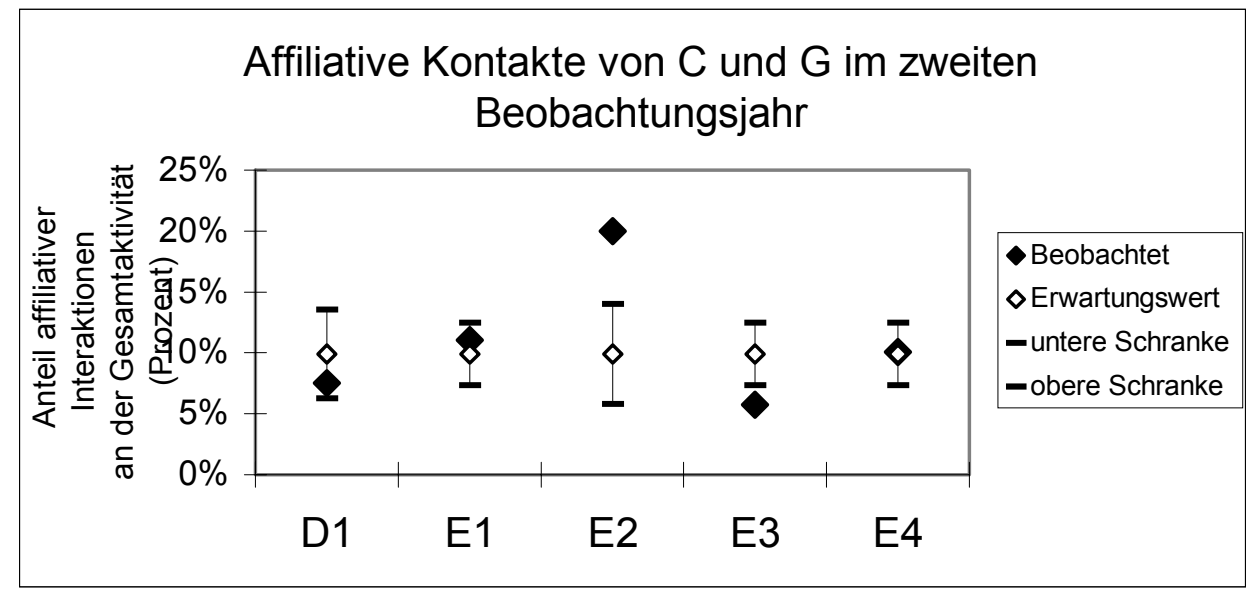

Abb. 3.65 Affiliative Kontakte von $C$ und $G$ im zweiten Beobachtungsjahr (KFA, EW=9,9, n=5). Abkürzungen: D1: Hüttenphase 1, E1: erstes Experiment, E2: zweites Experiment, E3: drittes Experiment, E4: viertes Experiment. Im zweiten Experiment lag der beobachtete Wert signifikant über dem Erwartungswert, während er im dritten Experiment unter dem erwarteten Wert lag.

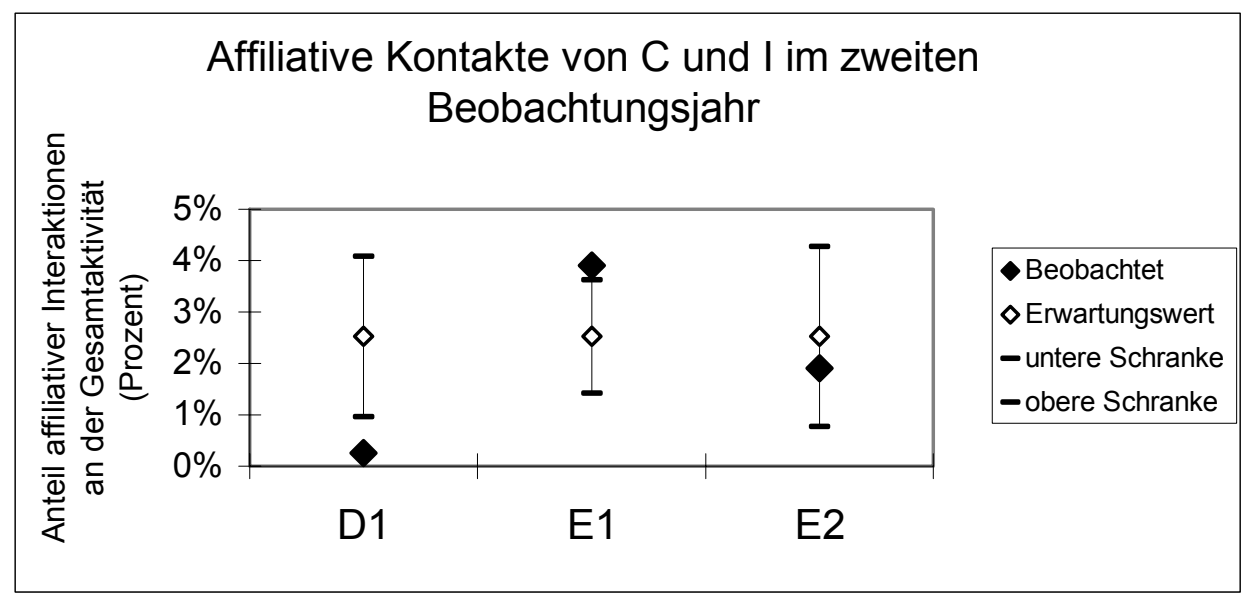

Abb. 3.66 Affiliative Kontakte von C und I im zweiten Beobachtungsjahr (KFA, EW=2,52, n=3). Abkürzungen: D1: Hüttenphase 1, E1: erstes Experiment, E2: zweites Experiment. In der Hüttenphase lag der beobachtete Wert signifikant unter dem Erwartungswert, während er im ersten Experiment über dem erwarteten Wert lag. 


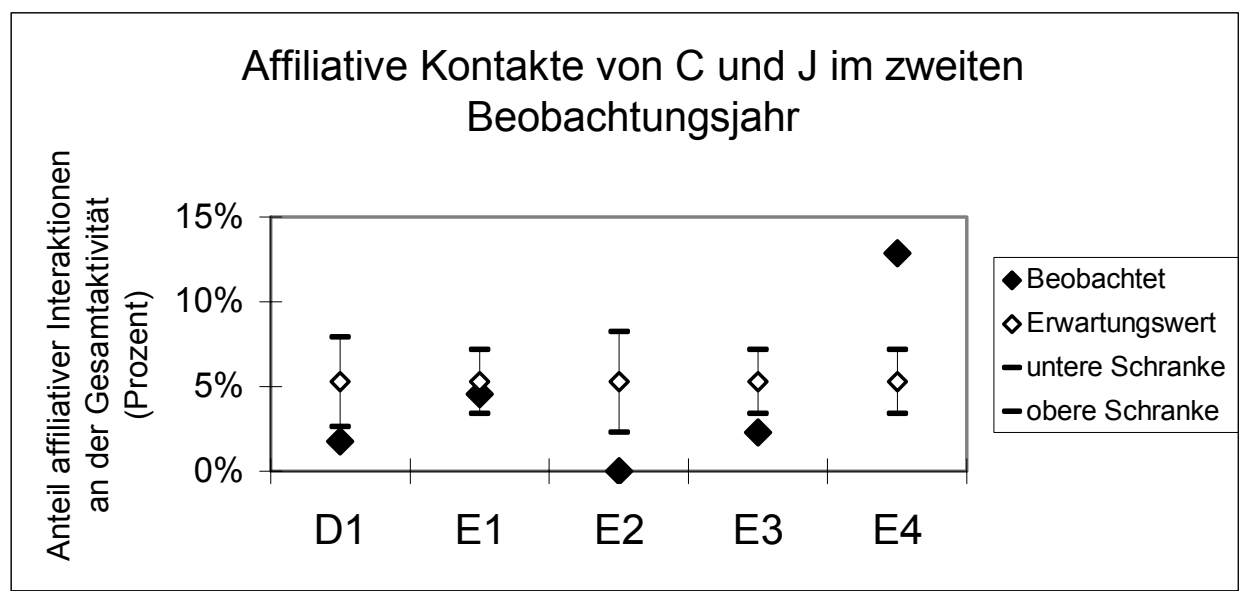

Abb. 3.67 Affiliative Kontakte von $\mathrm{C}$ und $\mathrm{J}$ im zweiten Beobachtungsjahr (KFA, EW=5,28, n=5). Abkürzungen: D1: Hüttenphase 1, E1: erstes Experiment, E2: zweites Experiment, E3: drittes Experiment, E4: viertes Experiment. In der Hüttenphase und im zweiten und dritten Experiment lag der beobachtete Wert signifikant unter dem Erwartungswert, während er im vierten Experiment über dem erwarteten Wert lag.

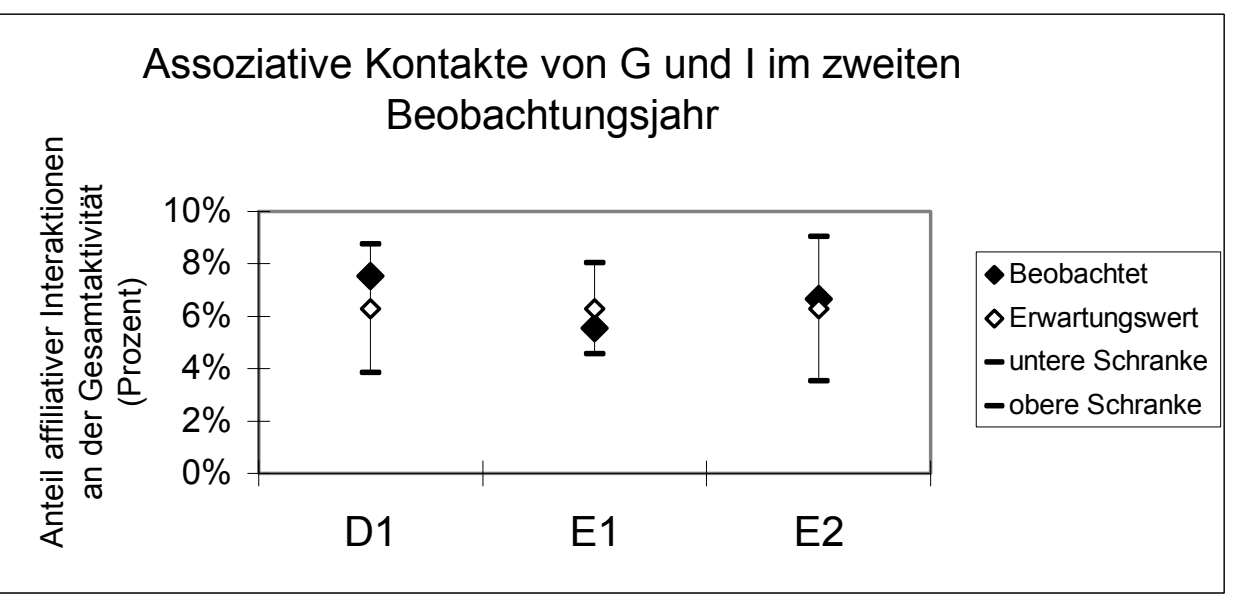

Abb. 3.68 Affiliative Kontakte von $G$ und I im zweiten Beobachtungsjahr (KFA, EW=6,3, n=3). Abkürzungen: D1: Hüttenphase 1, E1: erstes Experiment, E2: zweites Experiment. Alle beobachteten Werte lagen im Erwartungsbereich.

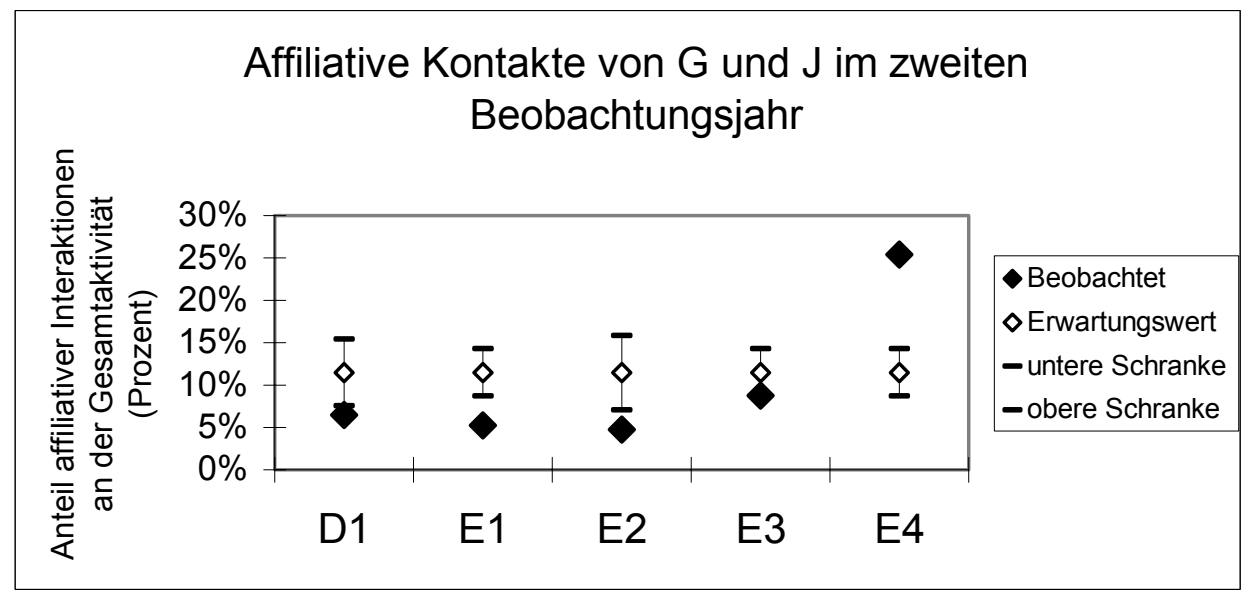

Abb. 3.69 Affiliative Kontakte von $G$ und $J$ im zweiten Beobachtungsjahr (KFA, EW=11,47, n=5). Abkürzungen: D1: Hüttenphase 1, E1: erstes Experiment, E2: zweites Experiment, E3: drittes Experiment, E4: viertes Experiment. In der Hüttenphase und den ersten beiden Experimenten lag der beobachtete Wert signifikant unter dem Erwartungswert, während er im vierten Experiment über dem erwarteten Wert lag. 


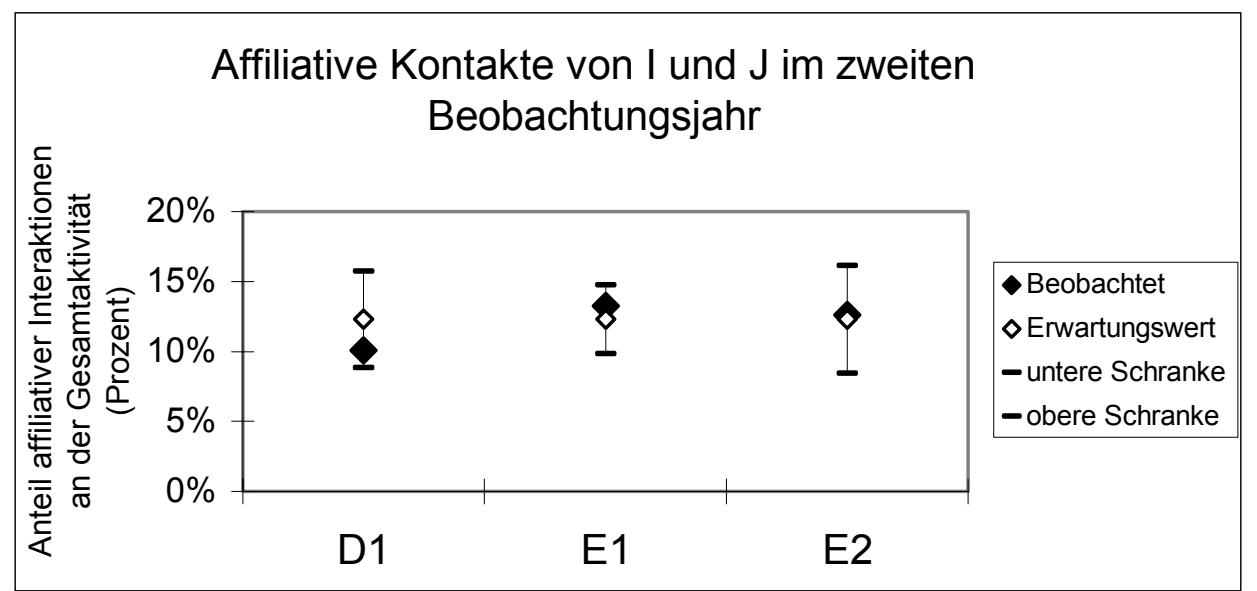

Abb. 3.70 Affiliative Kontakte von I und J im zweiten Beobachtungsjahr (KFA, EW=12,28, n=3). Abkürzungen: D1: Hüttenphase 1, E1: erstes Experiment, E2: zweites Experiment. Alle beobachteten Werte lagen im Erwartungsbereich.

Die Hypothese 5b kann für die Befunde aus dem ersten Beobachtungsjahr zurückgewiesen werden, da die Unterschiede in der Häufigkeit der affiliativen Kontakte der Brüder in den Kontrollphasen während der Experimente verschwinden und bei allen Bruder-Dyaden die Häufigkeit der affiliativen Interaktionen im ersten Experiment gegenüber den anderen Phasen vermindert war.

Im zweiten Beobachtungsjahr gab es Unterschiede zwischen den Bruder-Dyaden in der affiliativen Interaktion. Bei allen Bruder-Dyaden mit Ausnahme der Dyaden G/I und I/J zeigten sich Unterschiede in der affiliativen Interaktion in Abhängigkeit von den Experimenten. Für die Dyaden C/G, C/I, C/J und G/J kann im zweiten Beobachtungsjahr die Hypothese $5 b$ demnach abgelehnt werden. Für die Dyaden G/I und I/J ist die Hypothese allerdings nicht zurückzuweisen, da bei ihnen die Häufigkeit der affiliativen Kontakte von den Experimenten unabhängig war.

\subsubsection{Räumliche Assoziationen der Schwestern im ersten Beobachtungsjahr}

Hypothese 5c: Die Anwesenheit von Locktieren hat keinen Einfluss auf die räumliche Assoziation der Schwestern.

In der räumlichen Assoziation zwischen den Schwestern $\mathrm{D}$ und $\mathrm{H}$ war im ersten Beobachtungsjahr in Abhängigkeit von den Experimenten kein Unterschied festzustellen (ANOVA, $\mathrm{F}=0,76 ; \mathrm{N}=73 ;$ n.s.). 


\subsubsection{Räumliche Assoziationen der Schwestern im zweiten Beobachtungsjahr}

Die Assoziationen der Schwester-Dyaden unterschieden sich im zweiten Beobachtungsjahr voneinander und waren zudem von den Experimenten abhängig (Abb. 3.71). Die Schwestern $\mathrm{K}$ und $\mathrm{M}$ waren enger räumlich assoziiert als die Schwestern D und K und D und M. Während des dritten Experiments waren die Assoziationskoeffizienten insgesamt höher als im zweiten Experiment. Ebenso gab es auch in Abhängigkeit von den Experimenten einen Einfluss der Dyaden auf die Assoziationskoeffizienten.

Unter den experimentellen Voraussetzungen des ersten Beobachtungsjahres (Präsentation von Lockweibchen) kann Hypothese 5c nicht zurückgewiesen werden. Unter den experimentellen Voraussetzungen des zweiten Beobachtungsjahres (Präsentation einer Kleinfamilie und Lockmännchen in unterschiedlicher Anzahl) muss Hypothese 5c jedoch abgelehnt werden.

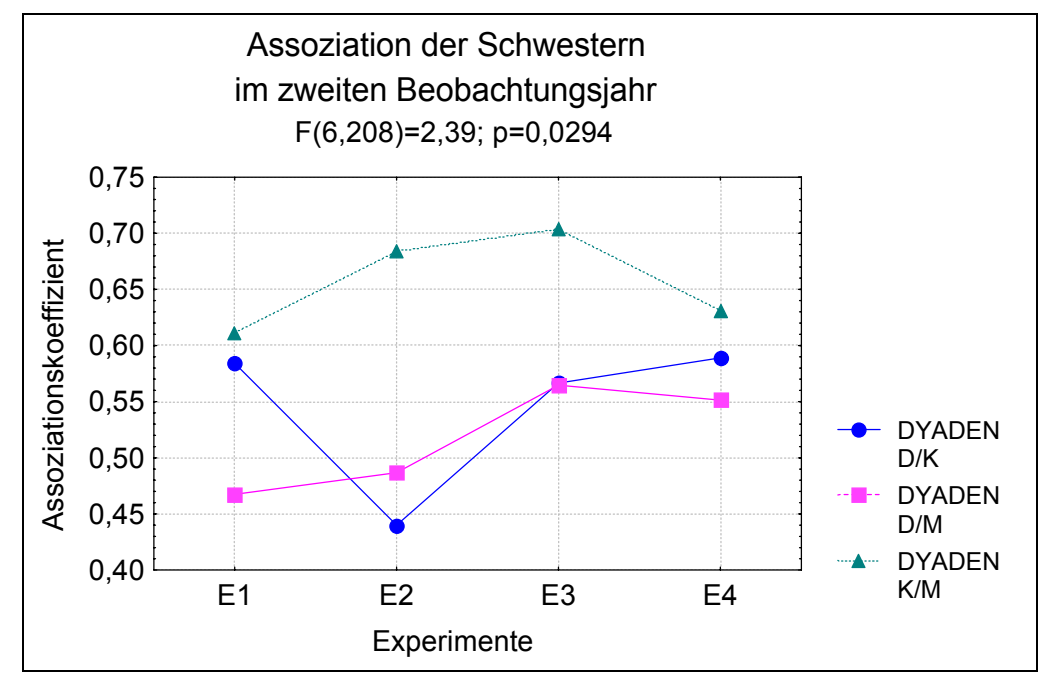

Abb. 3.71 Assoziation der Schwestern $\mathrm{D} / \mathrm{K}, \mathrm{D} / \mathrm{M}$ und $\mathrm{K} / \mathrm{M}$ im Laufe der vier Experimente im zweiten Beobachtungsjahr (ANOVA, Experimente: $\mathrm{F}=3,26 ; \mathrm{N}=208 ; \mathrm{p}=0,0224$; Dyaden: $\mathrm{F}=20,59 ; \mathrm{N}=208 ; \mathrm{p}<0,001$; Wechselseitige Beeinflussung von Experimenten und Dyaden: $F=2,39 ; \mathrm{N}=208 ; \mathrm{p}=0,0294)$. Es gab Unterschiede in der Assoziation der Schwester-Dyaden untereinander, als auch verglichen zwischen den Experimenten. Die Dyade K/M hatte höhere Assoziationskoeffizienten als die Dyaden D/K und D/M. Experiment 3 unterschied sich von Experiment 2 durch eine engere Assoziation der Dyaden. Abhängig von den Dyaden wirkten sich die Experimente unterschiedlich auf die Assoziationskoeffizienten aus.

\subsubsection{Affiliative Interaktionen der Schwestern im ersten Beobachtungsjahr}

Hypothese 5d: Die Anwesenheit von Locktieren hat keinen Einfluss auf die Häufigkeit affiliativer Interaktionen der Schwestern.

Die Schwestern D und H hatten während der ersten Kontrollphase und im ersten Experiment bei der Präsentation des ersten Lockweibchens seltener und in der zweiten Kontrollphase und 
im zweiten Experiment bei der Anwesenheit des zweiten Lockweibchens häufiger als erwartet affiliative Kontakte (Abb. 3.72).

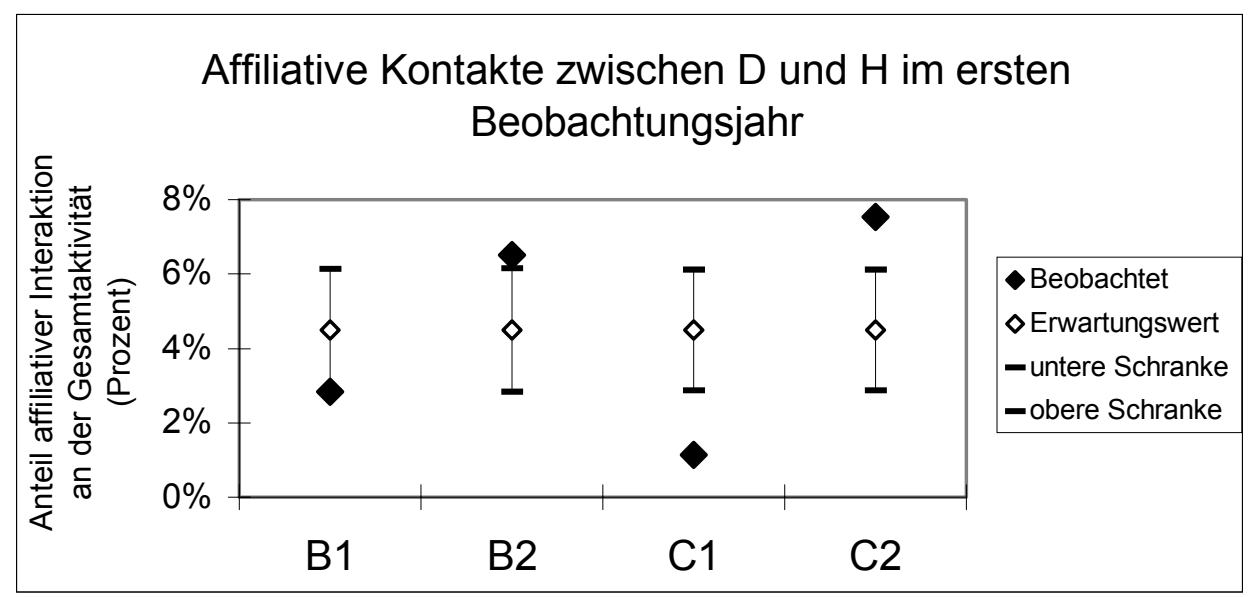

Abb. 3.72 Affiliative Kontakte zwischen D und H im ersten Beobachtungsjahr (KFA, EW=4,49 n=4). Abkürzungen: $\mathrm{B} 1=$ erste Kontrollphase, B2 $=$ zweite Kontrollphase, $\mathrm{C} 1=$ erstes Experiment, $\mathrm{C} 2=\mathrm{zweites}$ Experiment. Die affiliativen Kontakte lagen in der ersten Kontrollphase und während des ersten Experiments unter und während der zweiten Kontrollphase und des zweiten Experiments über dem Erwartungsbereich.

\subsubsection{Affiliative Interaktionen der Schwestern im zweiten Beobachtungsjahr}

Die affiliativen Kontakte zwischen D und K bzw. D und M waren während des gesamten zweiten Jahres seltener als zwischen den Zwillings-Schwestern K und M. Der Anteil der affiliativen Interaktionen lag bei den Dyaden $\mathrm{D} / \mathrm{K}$ und $\mathrm{D} / \mathrm{M}$ in allen Experimenten unter dem Erwartungswert, während der Koeffizient der Dyade $\mathrm{K} / \mathrm{M}$ immer weit über dem Erwartungswert lag (siehe Abb. 3.73 - 3.76).

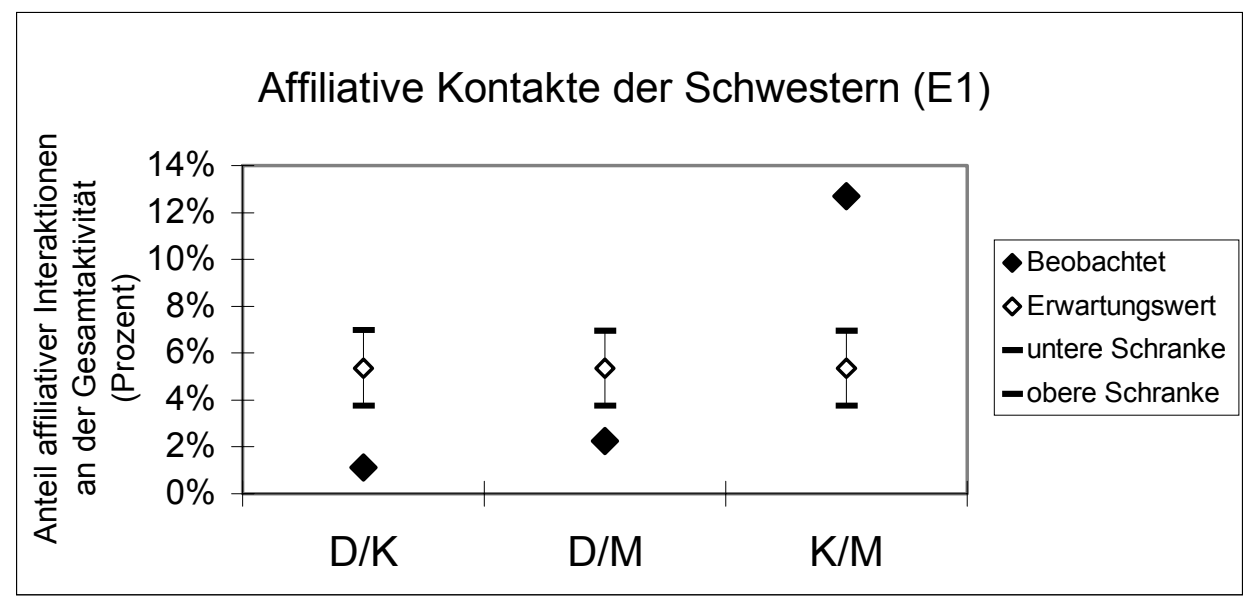

Abb. 3.73 Affiliative Kontakte zwischen den Schwestern im ersten Experiment des zweiten Beobachtungsjahres. (KFA, EW=5,36 n=3). Abkürzungen: $\mathrm{D} / \mathrm{K}$ steht für die Interaktion zwischen den Schwestern $\mathrm{D}$ und K. Die Dyade K/M interagierte häufiger affiliativ als erwartet, während die Dyaden $\mathrm{D} / \mathrm{K}$ und $\mathrm{D} / \mathrm{M}$ seltener als erwartet affiliative Kontakte hatten. 


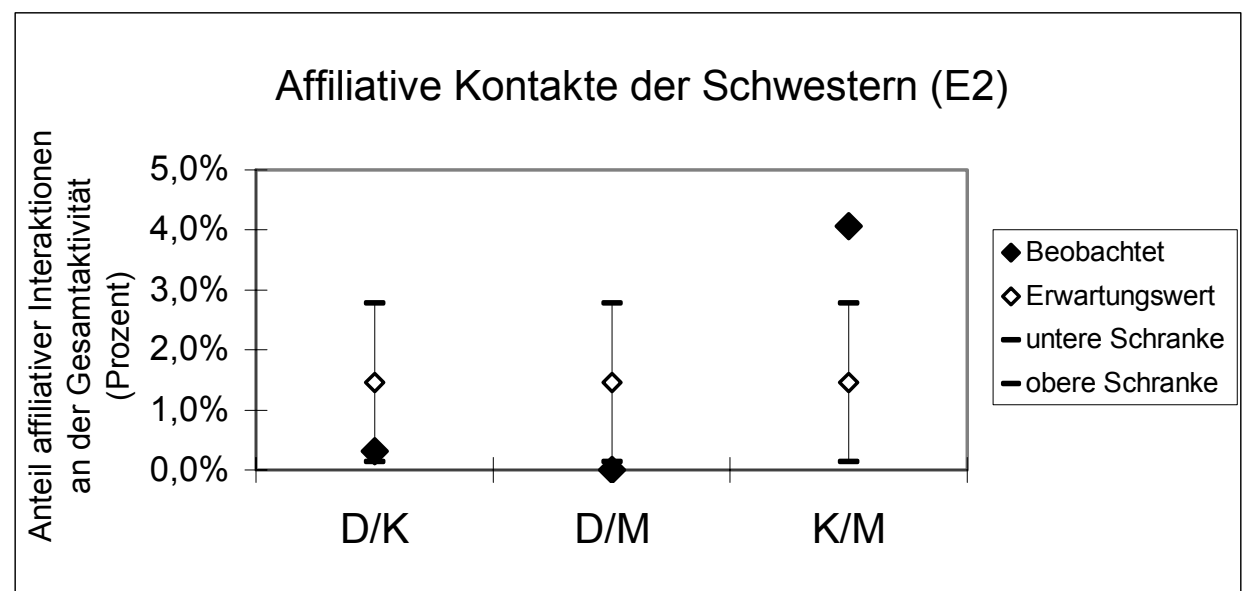

Abb. 3.74 Affiliative Kontakte zwischen den Schwestern im zweiten Experiment des zweiten Beobachtungsjahres. (KFA, $\mathrm{EW}=1,46 \mathrm{n}=3$ ). Abkürzungen: $\mathrm{D} / \mathrm{K}$ steht für die Interaktion zwischen den Schwestern D und K. Die Dyade K/M interagierte häufiger affiliativ als erwartet, während die Dyade D/M seltener als erwartet affiliative Kontakte hatte. Der Wert der Dyade D/K lag im Erwartungsbereich.

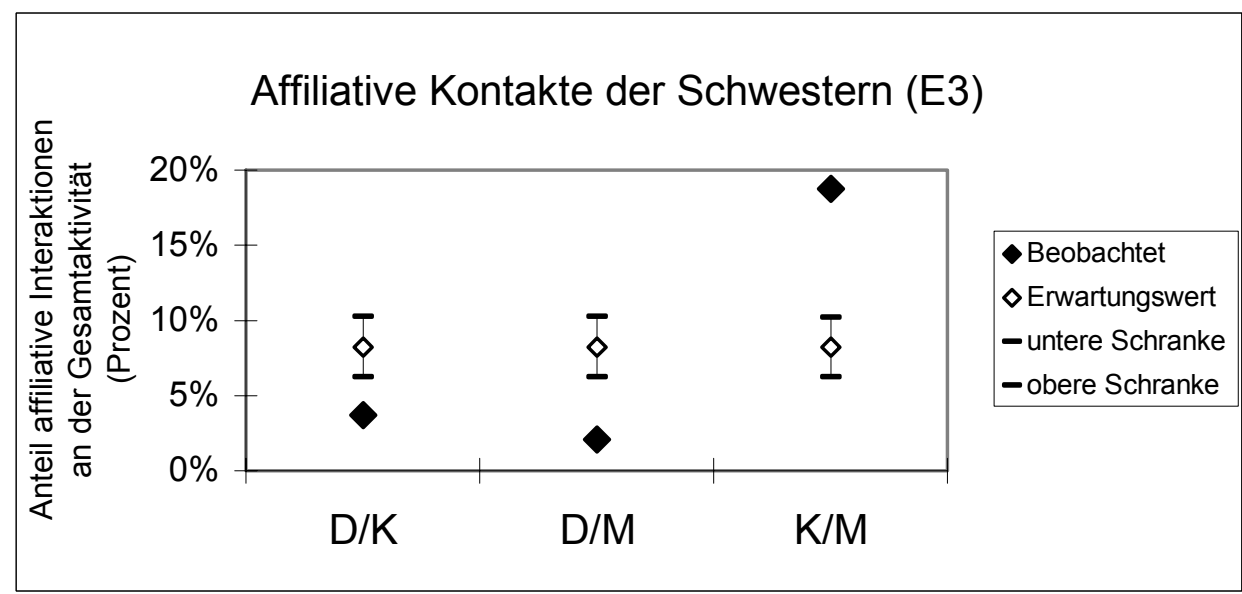

Abb. 3.75 Affiliative Kontakte zwischen den Schwestern im dritten Experiment des zweiten Beobachtungsjahres. (KFA, $E W=8,23 \mathrm{n}=3$ ). Abkürzungen: $\mathrm{D} / \mathrm{K}$ steht für die Interaktion zwischen den Schwestern D und K. Die Dyade K/M interagierte häufiger affiliativ als erwartet, während die Dyaden D/K und $\mathrm{D} / \mathrm{M}$ seltener als erwartet affiliative Kontakte hatten.

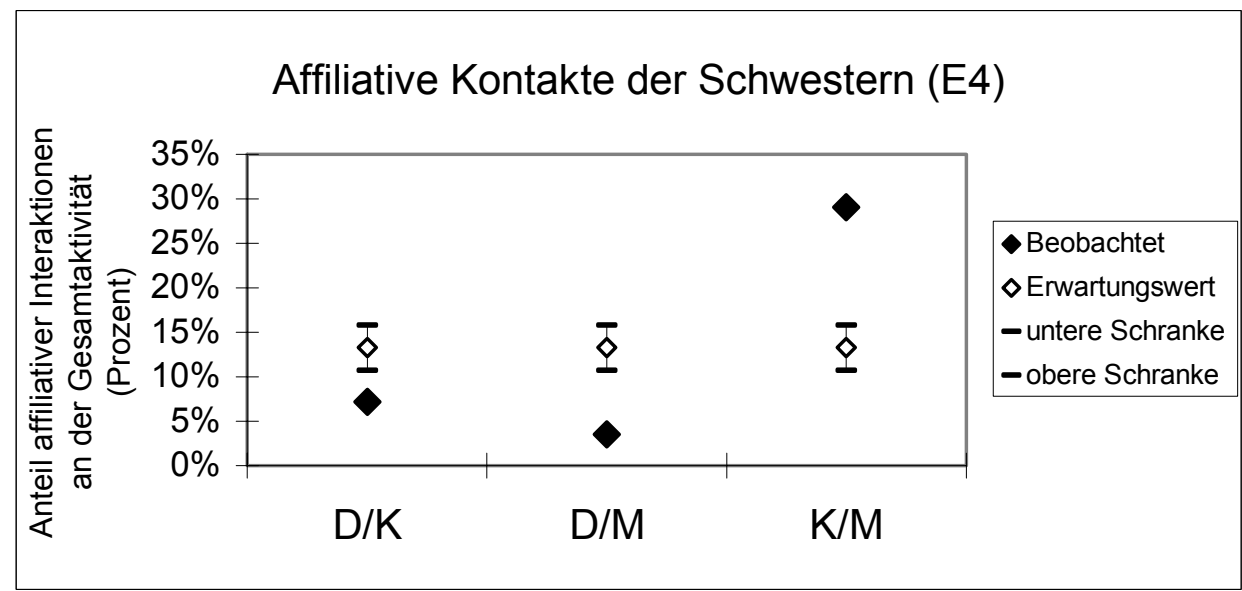

Abb. 3.76 Affiliative Kontakte zwischen den Schwestern im vierten Experiment des zweiten Beobachtungsjahres. (KFA, $\mathrm{EW}=13,24 \mathrm{n}=3$ ). Abkürzungen: $\mathrm{D} / \mathrm{K}$ steht für die Interaktion zwischen den Schwestern D und K. Die Dyade K/M interagierte häufiger affiliativ als erwartet, während die Dyaden D/K und $\mathrm{D} / \mathrm{M}$ seltener als erwartet affiliative Kontakte hatte. 
Während der Hüttenphase und den ersten beiden Experimenten waren die affiliativen Interaktionen von $\mathrm{D}$ und $\mathrm{K}$ seltener als erwartet und während des vierten Experiments häufiger als erwartet (Abb. 3.77). Die affiliativen Kontakte von D und $M$ waren während der Hüttenphase und des zweiten Experiments seltener, im ersten und dritten Experiment innerhalb des Erwartungsbereichs und während des vierten Experiments häufiger als erwartet (Abb. 3.78). Die affiliativen Kontakte von $\mathrm{K}$ und $\mathrm{M}$ waren während der Hüttenphase und der ersten beiden Experimente seltener als erwartet und während des vierten Experiments häufiger als erwartet (Abb. 3.79). Alle Dyaden hatten daher bei der Anwesenheit der zwei Lockmännchen und vor dem Rauswurf von I seltener und bei der Präsentation des einzelnen Lockmännchens häufiger als erwartet affiliative Kontakte.

Da die affiliativen Interaktionen aller Schwester-Dyaden während der verschiedenen Experimente unterschiedlich häufig waren, kann Hypothese 5d für beide Beobachtungsjahre abgewiesen werden.

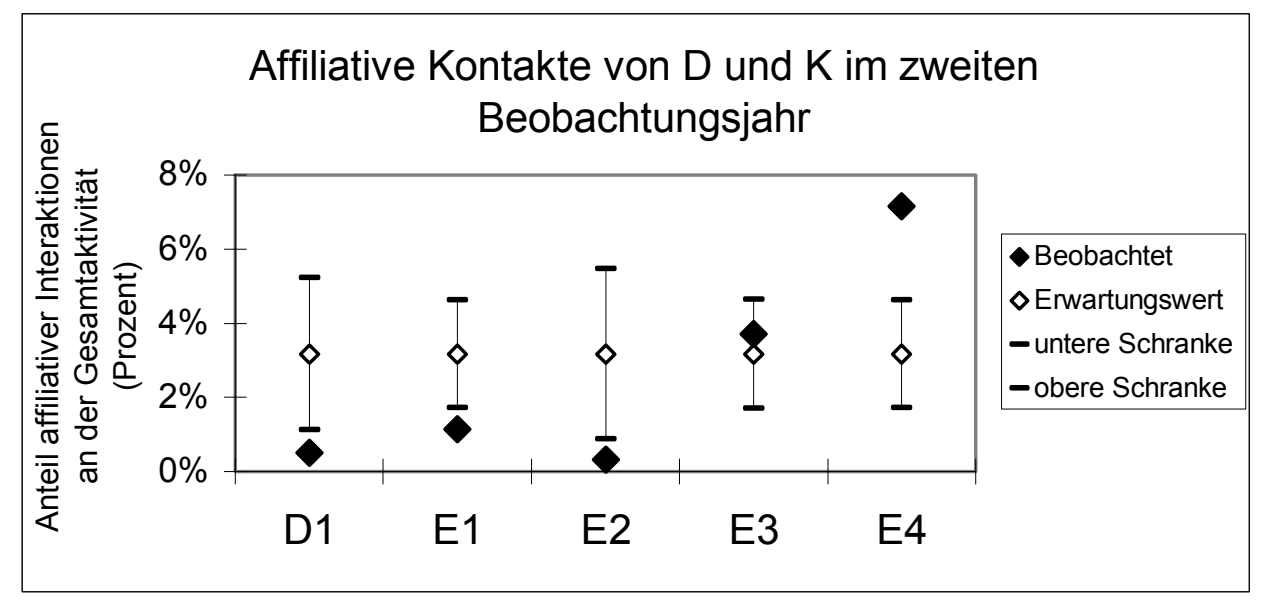

Abb. 3.77 Affiliative Kontakte von D und K im zweiten Beobachtungsjahr (KFA, EW=3,17 n=5). Abkürzungen: D1: Hüttenphase 1, E1: erstes Experiment, E2: zweites Experiment, E3: drittes Experiment, E4: viertes Experiment. Während der Hüttenphase und den ersten beiden Experimenten lag der beobachtete Wert signifikant unter dem Erwartungswert, während er im dritten Experiment innerhalb des Erwartungsbereichs und während des vierten Experiments über dem Erwartungsbereich lag. 


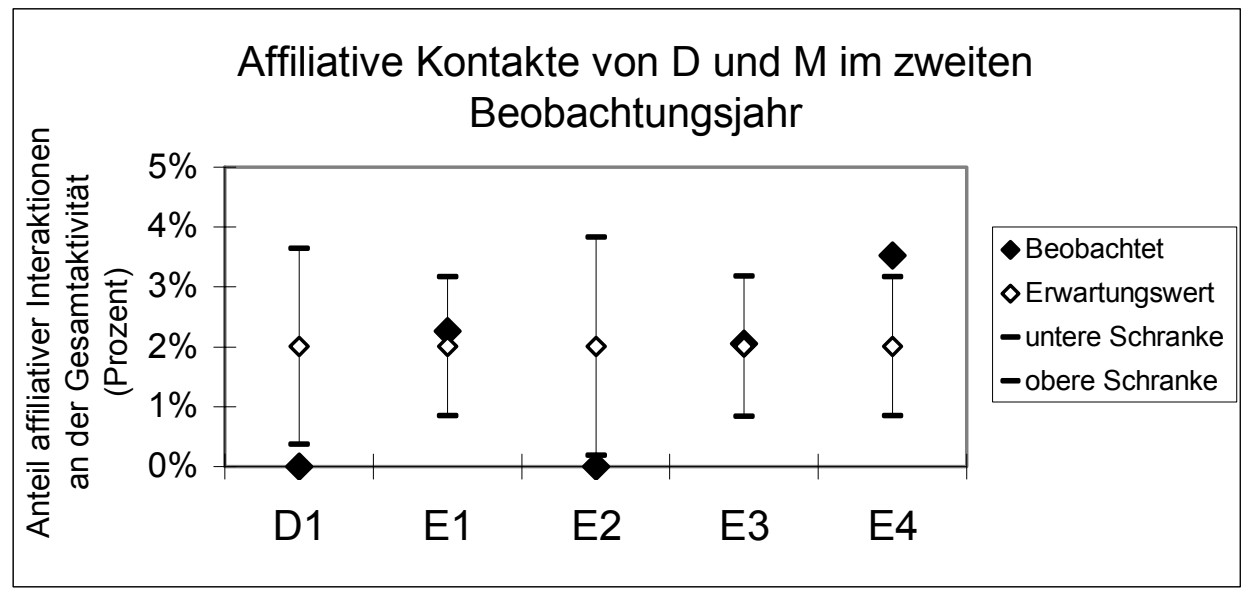

Abb. 3.78 Affiliative Kontakte von $D$ und $M$ im zweiten Beobachtungsjahr (KFA, EW=2,01 $n=5$ ). Abkürzungen: D1: Hüttenphase 1, E1: erstes Experiment, E2: zweites Experiment, E3: drittes Experiment, E4: viertes Experiment. Während der Hüttenphase und des zweiten Experiments lag der beobachtete Wert signifikant unter dem Erwartungswert, während er im ersten und dritten Experiment innerhalb des Erwartungsbereichs und während des vierten Experiments über dem Erwartungsbereich lag.

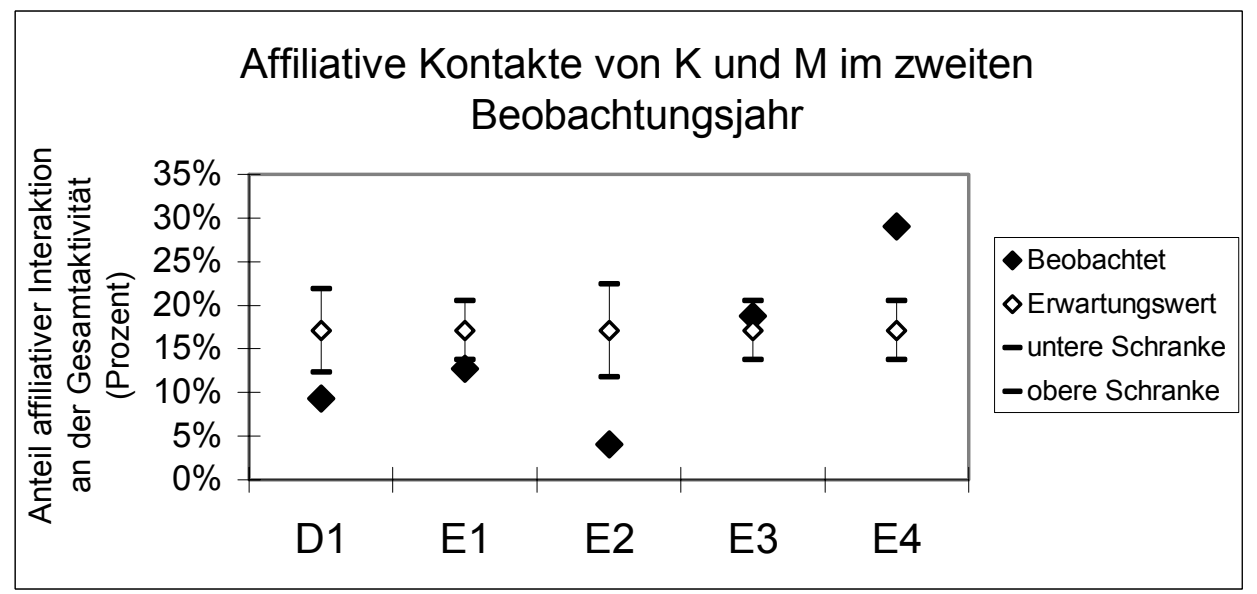

Abb. 3.79 Affiliative Kontakte von $K$ und $M$ im zweiten Beobachtungsjahr (KFA, EW=17,11 n=5). Abkürzungen: D1: Hüttenphase 1, E1: erstes Experiment, E2: zweites Experiment, E3: drittes Experiment, E4: viertes Experiment. Während der Hüttenphase und den ersten beiden Experimenten lag der beobachtete Wert signifikant unter dem Erwartungswert, während er im dritten Experiment innerhalb des Erwartungsbereichs und während des vierten Experiments über dem Erwartungsbereich lag.

\subsubsection{Räumliche Assoziationen von Brüdern und Schwestern im ersten Beobachtungsjahr}

Hypothese 5e: Die Anwesenheit von Locktieren hat keinen Einfluss auf die räumliche Assoziation von Brüdern und Schwestern.

Ein Vergleich der räumlichen Assoziation von D mit ihren Brüdern im ersten Beobachtungsjahr machte Unterschiede zwischen den Dyaden deutlich (Abb. 3.80). Es gab jedoch keine Abhängigkeit der räumlichen Assoziation von den Experimenten (Kruskal-Wallis-Test: $\mathrm{H}=$ $7,45 ; \mathrm{N}=253 ; \mathrm{p}=0,058)$. 


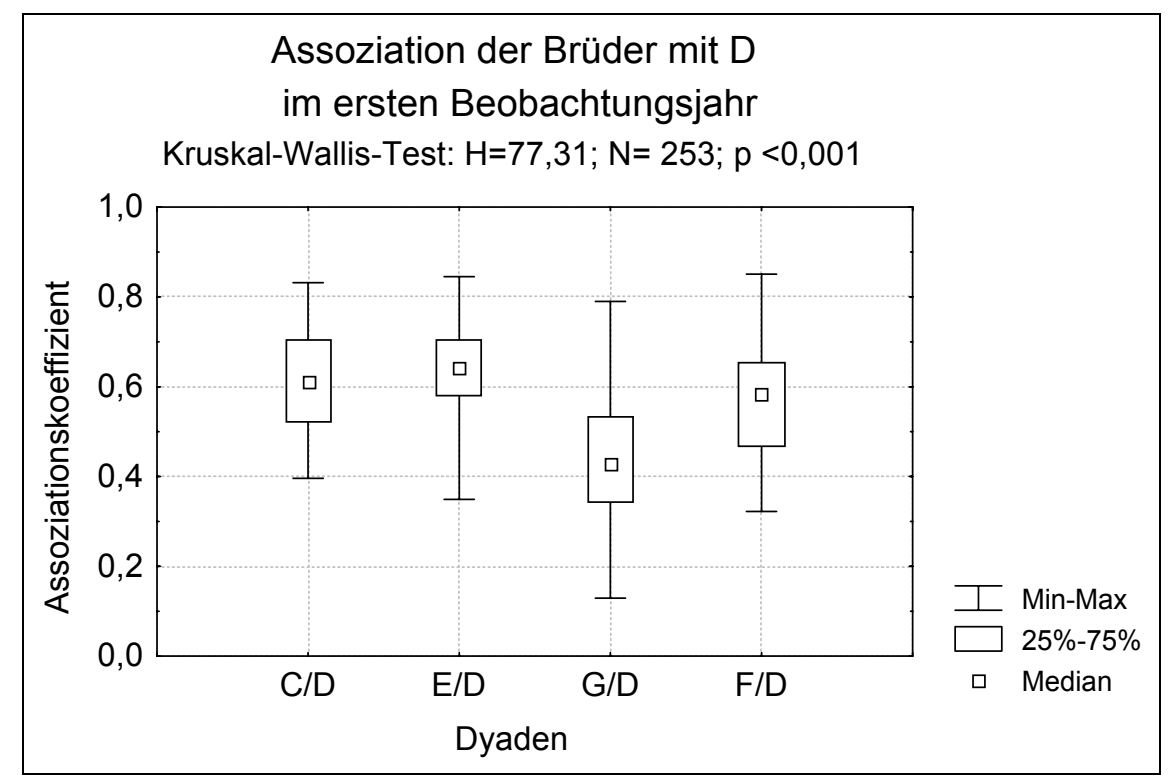

Abb. 3.80 Assoziation der Brüder mit D im ersten Beobachtungsjahr. Der Assoziationskoeffizient der Dyade $\mathrm{C} / \mathrm{D}$ beschreibt die räumliche Assoziation von $\mathrm{C}$ und $\mathrm{D}$ (das gleiche gilt für die Dyaden $\mathrm{E} / \mathrm{D}, \mathrm{G} / \mathrm{D}$ und F/D). Die Dyaden unterschieden sich in ihrer räumlichen Assoziation (Kruskal-Wallis-Test: $\mathrm{H}=77,31 ; \mathrm{N}=253 ; \mathrm{p}<0,001$ ).

Um die Assoziationskoeffizienten auf eine wechselseitige Beeinflussung der Dyaden und Experimente zu testen, wurde mit den Bruder-D-Dyaden eine ANOVA durchgeführt. Die Dyade D/F musste dafür jedoch weggelassen werden, weil F nach der ersten Kontrollphase rausgeworfen worden war. Die Analyse ergab Unterschiede sowohl im Vergleich der Experimente, als auch zwischen den Dyaden (Abb. 3.81). Die räumliche Assoziation war gemittelt über alle Dyaden im zweiten Experiment enger als während der anderen Phasen (ANOVA, $\mathrm{F}=3,03 ; \mathrm{p}<0,05$ ). D war mit $\mathrm{C}$ und mit $\mathrm{E}$ enger assoziiert als mit $\mathrm{G}$ (ANOVA, $\mathrm{F}=71,32 ; \mathrm{p}<0,01)$. In Abhängigkeit von den Experimenten beeinflussten die Dyaden die Assoziationskoeffizienten. 


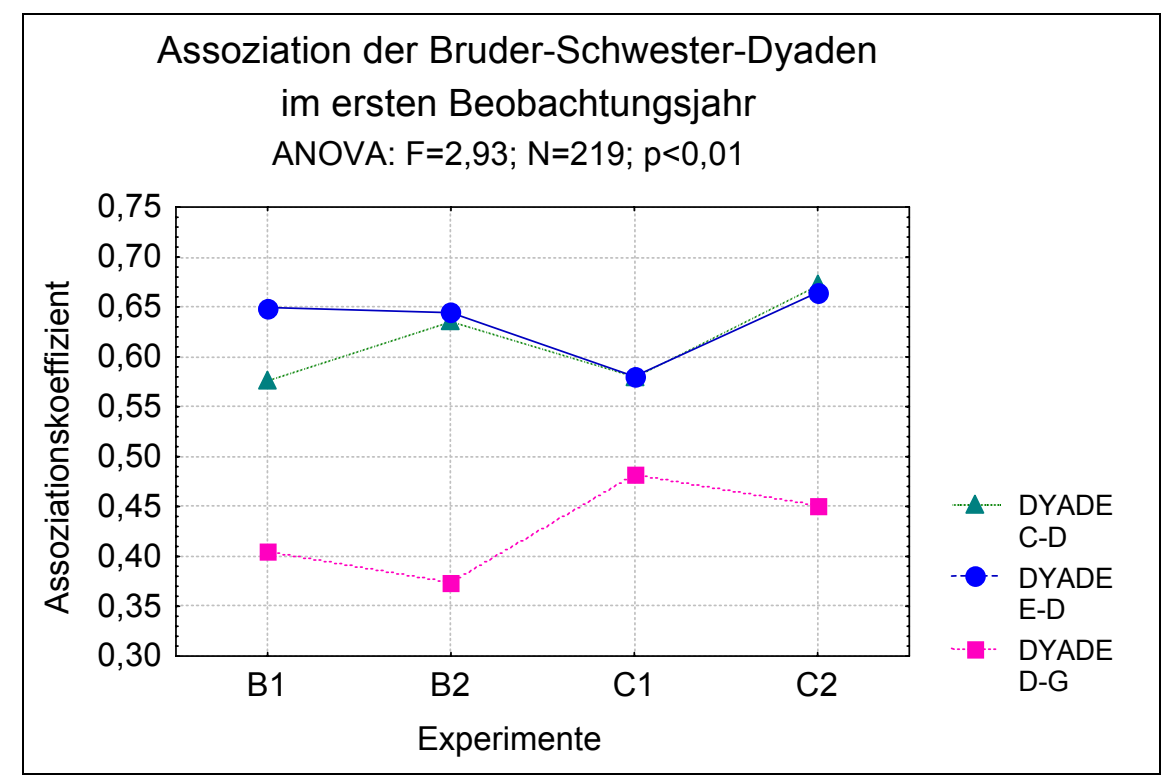

Abb. 3.81 Assoziation der Brüder mit D im ersten Beobachtungsjahr (ohne F). Der Assoziationskoeffizient der Dyade C/D beschreibt die räumliche Assoziation von C und D etc. Die Assoziationskoeffizienten der verschiedenen Dyaden sind mit unterschiedlichen Symbolen gekennzeichnet (siehe Legende) und für jedes Experiment (B1-C2) parallel zueinander (also übereinander) dargestellt. Es bestand eine wechselseitige Abhängigkeit zwischen den Dyaden und Experimenten (ANOVA, F=2,93; N=219; $<<0,01$ ).

Ein Vergleich der räumlichen Assoziation von H mit ihren Brüdern im ersten Beobachtungsjahr ergab Unterschiede zwischen den Dyaden (Abb. 3.82), jedoch keine Abhängigkeit der räumlichen Assoziation der Tiere von den Experimenten (Kruskal-Wallis-Test: $\mathrm{H}=2,85$; $\mathrm{N}=253$; n.s.).

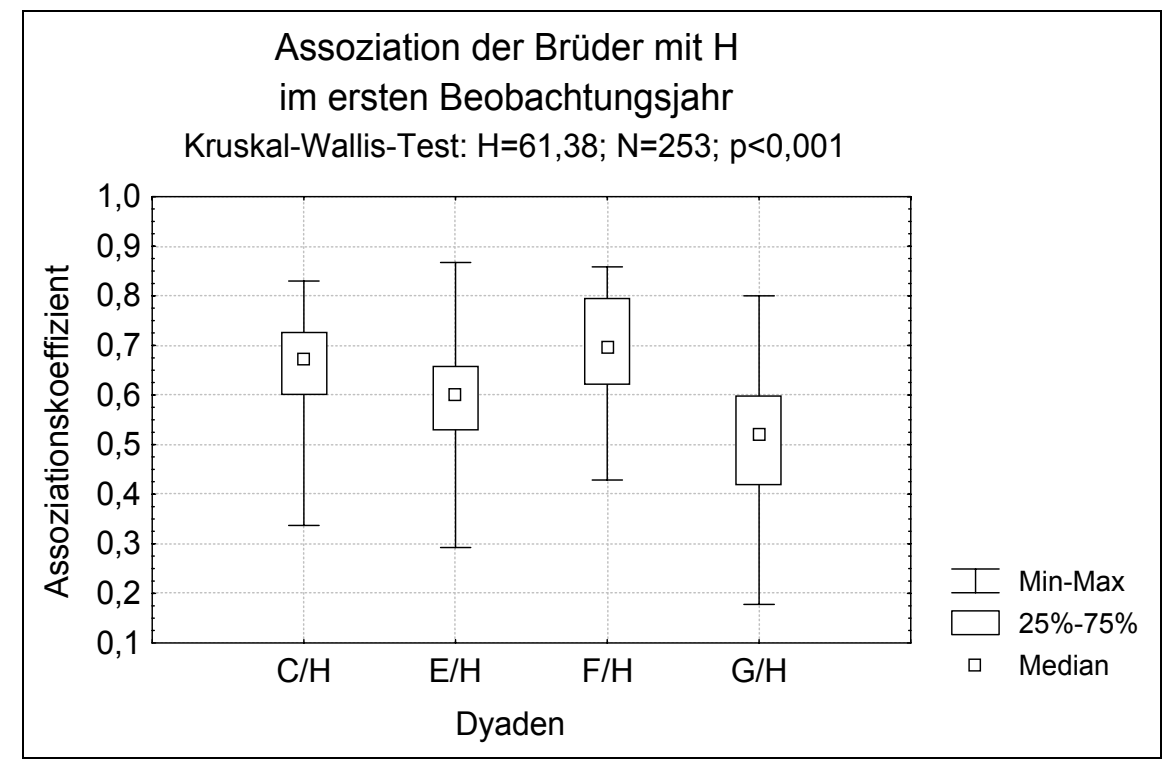

Abb. 3.82 Assoziation der Brüder mit H im ersten Beobachtungsjahr. Der Assoziationskoeffizient der Dyade $\mathrm{C} / \mathrm{H}$ beschreibt die räumliche Assoziation von $\mathrm{C}$ und $\mathrm{H}$ etc. Die Dyaden unterschieden sich in ihrer räumlichen Assoziation (Kruskal-Wallis-Test: $\mathrm{H}=61,38 ; \mathrm{N}=253$; $\mathrm{p}<0,001$ ).

Um die Assoziationskoeffizienten auf eine wechselseitige Beeinflussung der Dyaden und Experimente zu testen, wurde mit den Bruder-H-Dyaden eine ANOVA durchgeführt. Die 
Dyade $\mathrm{F} / \mathrm{H}$ musste dafür jedoch weggelassen werden. Bei den Bruder-H-Dyaden hatten die Experimente keinen Einfluss auf die räumliche Assoziation der Tiere zueinander (ANOVA, $\mathrm{F}=0,22$, n.s.). Ein Unterschied in der Assoziation bestand jedoch zwischen allen beteiligten Dyaden $\mathrm{C} / \mathrm{H}, \mathrm{E} / \mathrm{H}$ und $\mathrm{G} / \mathrm{H}$ (Abb. 3.83). Die Dyade $\mathrm{C} / \mathrm{H}$ hat eine engere räumliche Assoziation als die Dyade E/H und letztere wiederum eine engere Assoziation als die Dyade $\mathrm{G} / \mathrm{H}$.

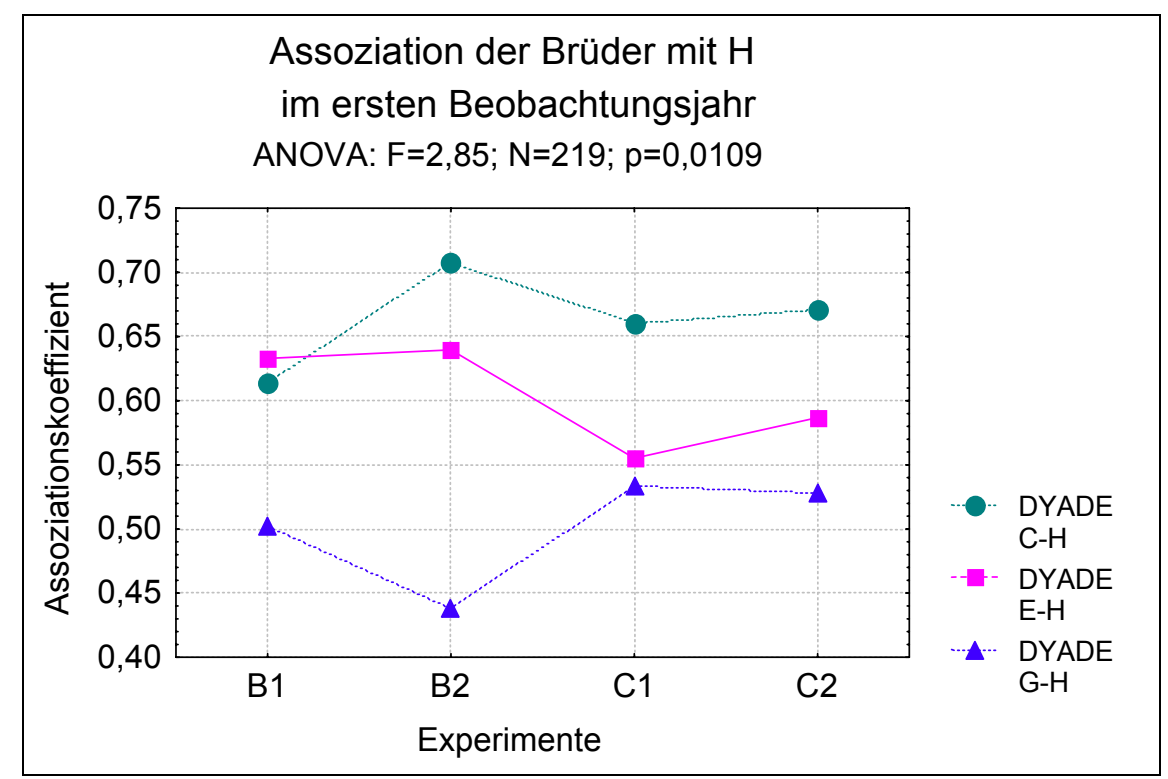

Abb. 3.83 Assoziation der Brüder mit H im ersten Beobachtungsjahr (ohne F). Der Assoziationskoeffizient der Dyade $\mathrm{C} / \mathrm{H}$ beschreibt die räumliche Assoziation von $\mathrm{C}$ und $\mathrm{H}$ etc. Die Assoziationskoeffizienten der verschiedenen Dyaden sind mit unterschiedlichen Symbolen gekennzeichnet (siehe Legende) und für jedes Experiment (B1-C2) parallel zueinander übereinander dargestellt. Es bestand ein wechselseitige Abhängigkeit zwischen den Dyaden und Experimenten (ANOVA, $F=2,85 ; \mathrm{N}=219 ; \mathrm{p}=0,0109$ ).

\subsubsection{Räumliche Assoziationen von Brüdern und Schwestern im zweiten Beobachtungsjahr}

Die räumliche Nähe von D zu ihren Brüdern unterschied sich im zweiten Beobachtungsjahr beim Vergleich der Experimente (Abb. 3.84), wobei das zweite Experiment die lockerste und das erste und vierte die engste räumliche Kohäsion zwischen D und ihren Brüdern aufwies. Ein Unterschied bestand auch zwischen den einzelnen Dyaden. G und D wiesen die lockerste und I und D die engste Assoziation zueinander auf (Abb. 3.85). Dieses Ergebnis kann auch durch ad libitum Beobachtungen bestätigt werden, da I und D auffällig häufig in Areal 6 ihres Streifgebietes gemeinsam gesehen wurden. 


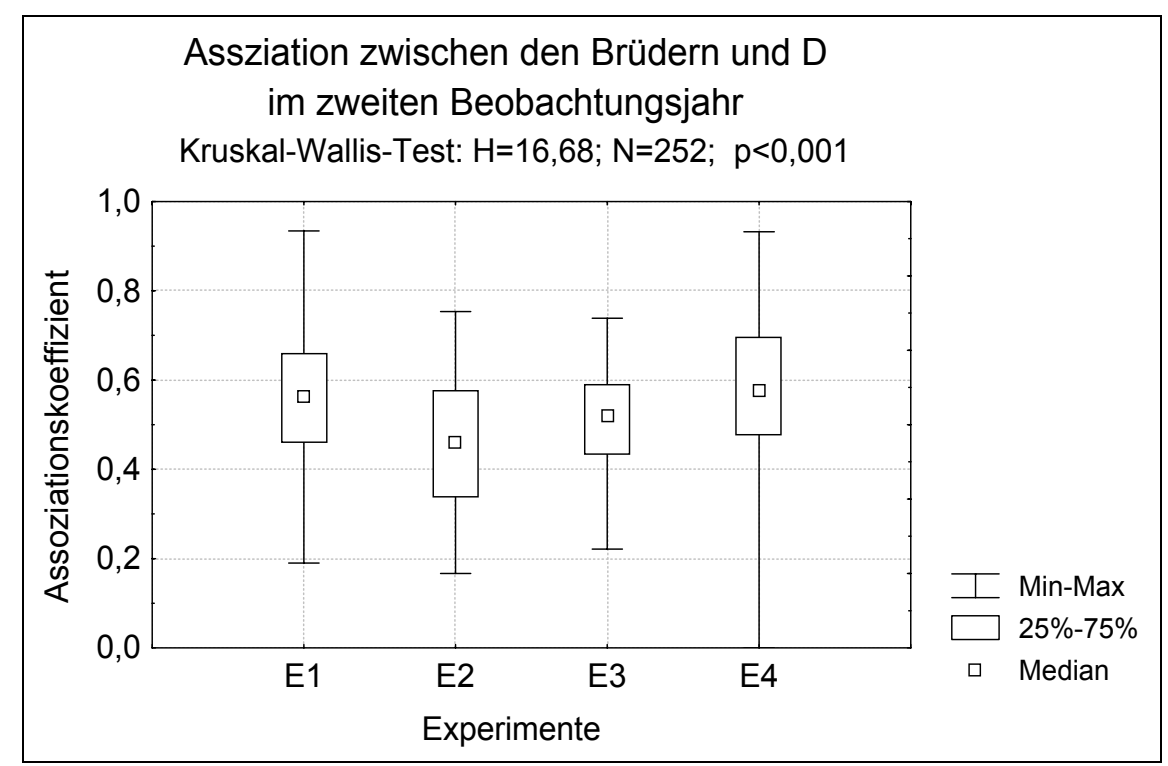

Abb. 3.84 Assoziation der Brüder mit D in den vier Experimenten des zweiten Beobachtungsjahres. Die Assoziationskoeffizienten zeigten eine Abhängigkeit von den Experimenten (Kruskal-Wallis-Test, H=16,68; $\mathrm{N}=252 ; \mathrm{p}<0,001)$.

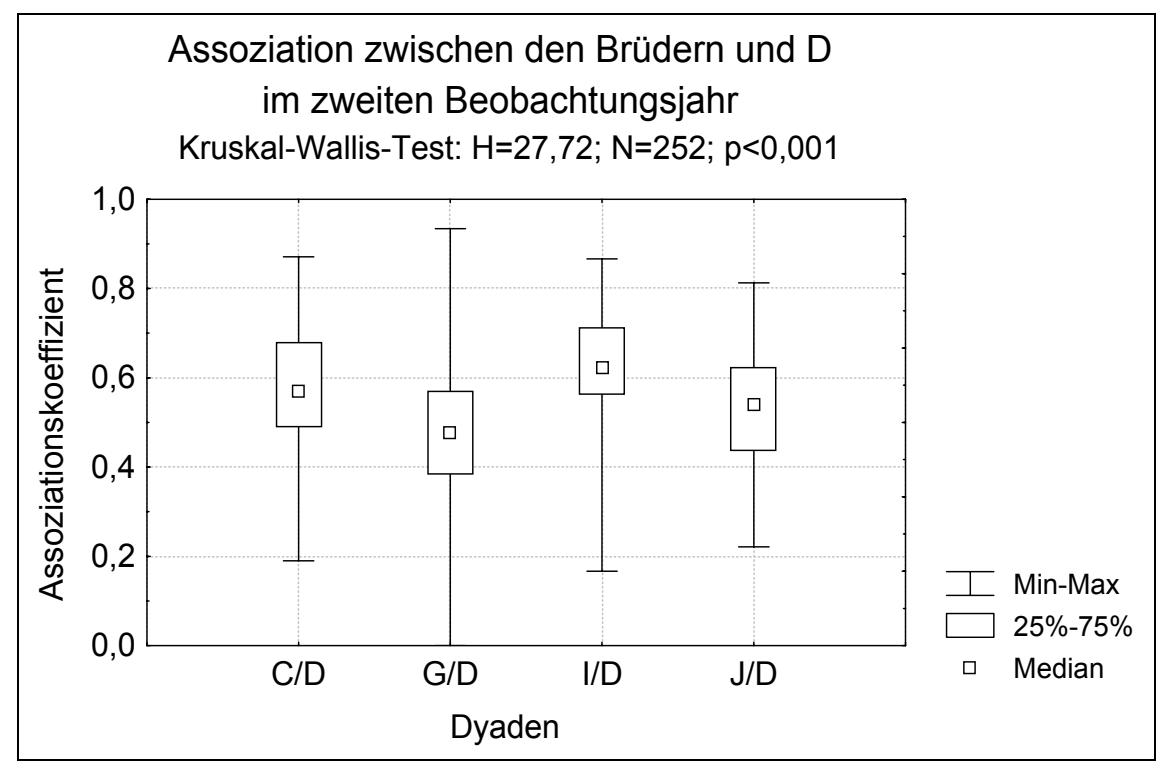

Abb. 3.85 Assoziation der Brüder mit D im zweiten Beobachtungsjahr. Der Assoziationskoeffizient der Dyade $\mathrm{C} / \mathrm{D}$ beschreibt die räumliche Assoziation von $\mathrm{C}$ und $\mathrm{D}$ etc. Die Assoziationskoeffizienten der Bruder-D-Dyaden unterscheiden sich voneinander (Kruskal-Wallis-Test, $\mathrm{H}=27,72 ; \mathrm{N}=252 ; \mathrm{p}<0,001$ ).

Im ersten Beobachtungsjahr gab keine Abhängigkeit der räumlichen Assoziation der BruderSchwester-Dyaden von den Experimenten. Hypothese 5e kann daher nicht abgelehnt werden. Im zweiten Beobachtungsjahr gab es jedoch Abhängigkeiten der Assoziationskoeffizienten der Bruder-D-Dyaden von den Experimenten, so dass für das zweite Beobachtungsjahr Hypothese 5e zurückgewiesen werden kann. 


\subsubsection{Affiliative Interaktionen von Brüdern und Schwestern im ersten}

\section{Beobachtungsjahr}

Hypothese 5f: Die Anwesenheit von Locktieren hat keinen Einfluss auf die Häufigkeit affiliativer Interaktionen von Brüdern und Schwestern.

Die Brüder C, F und G hatten mit D in der ersten Kontrollphase seltener affiliative Kontakte als erwartet, mit $\mathrm{H}$ hingegen interagierten $\mathrm{E}, \mathrm{F}$ und $\mathrm{G}$ häufiger als erwartet affiliativ (Abb. 3.86). Nach dem Rauswurf von $F$ änderte sich an den affiliativen Interaktionen nur wenig. D interagierte mit ihren Brüdern C, E und G seltener als erwartet, ebenso wie H und E. Der Wert der Dyade H/C lag im Erwartungsbereich und $\mathrm{H}$ und $\mathrm{G}$ interagierten häufiger affiliativ als erwartet (Abb. 3.87). Bei der Präsentation des Lockweibchens im ersten Experiment (C1) lag der Anteil an affiliativen Interaktionen der Dyaden C/D und H/E wieder im Erwartungsbereich (Abb. 3.88). Im zweiten Experiment traten keine Unterschiede mehr zwischen den Bruder-Schwester-Dyaden auf (Abb. 3.89).

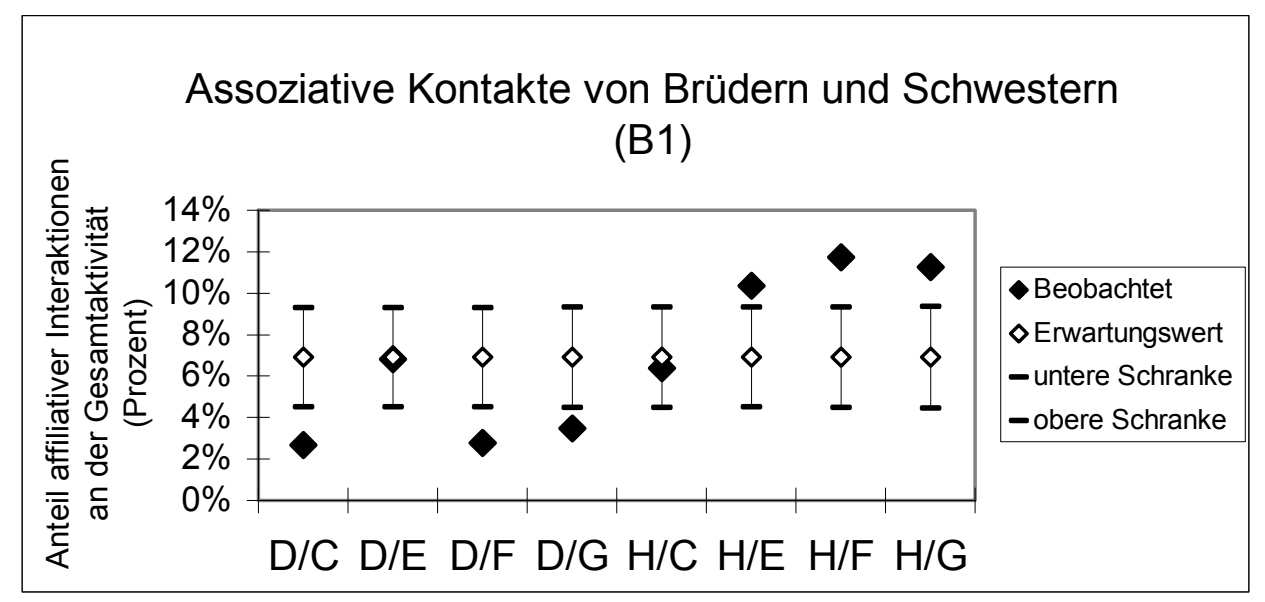

Abb. 3.86 Affiliative Kontakte von Brüdern und Schwestern in der ersten Kontrollphase des ersten Beobachtungsjahres (KFA, EW=6,9 $\mathrm{n}=6$ ). Abkürzungen: $\mathrm{D} / \mathrm{C}=$ Interaktion zwischen den Tieren $\mathrm{D}$ und $\mathrm{C}$ etc. . Die Dyaden D/C, D/F und D/G hatten seltener als erwartet affiliative Kontakte, während die Dyaden H/E, H/F und $\mathrm{H} / \mathrm{G}$ häufiger als erwartet affiliativ interagierten. Die Beobachtungswerte der Dyaden D/E und H/C lagen im Erwartungsbereich. 


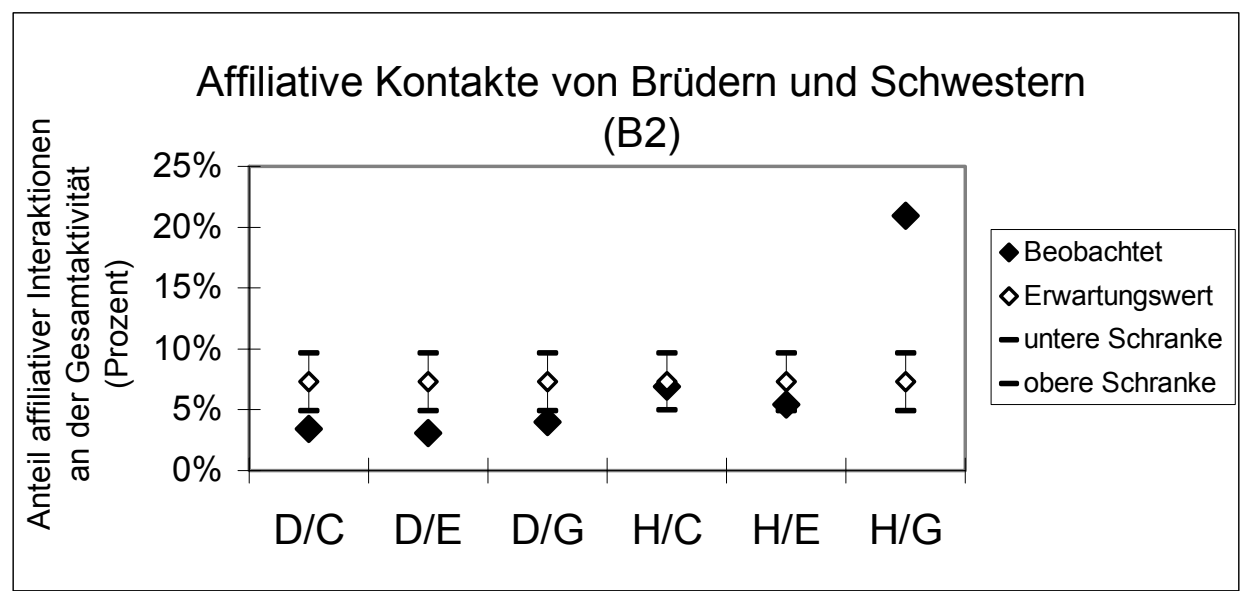

Abb. 3.87 Affiliative Kontakte von Brüdern und Schwestern in der zweiten Kontrollphase des ersten Beobachtungsjahres (KFA, EW=7,29 n=6.) Abkürzungen: $\mathrm{D} / \mathrm{C}=$ Interaktion zwischen den Tieren $\mathrm{D}$ und $\mathrm{C}$ etc. Die Dyaden D/C, D/E und D/G hatten seltener als erwartet affiliative Kontakte, während die Dyade H/G häufiger als erwartet affiliativ interagierte. Die Beobachtungswerte der Dyaden H/C und H/E lagen im Erwartungsbereich.

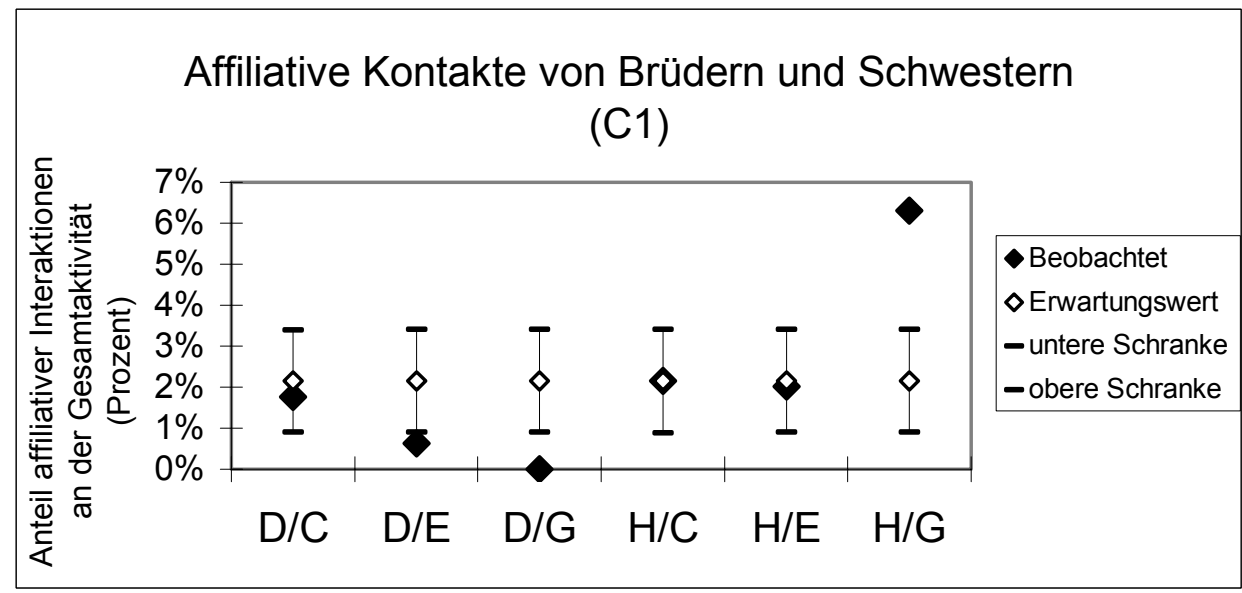

Abb. 3.88 Affiliative Kontakte von Brüdern und Schwestern im ersten Experiment des ersten Beobachtungsjahres (KFA, $E W=2,15 n=6)$. Abkürzungen: $D / C=$ Interaktion zwischen den Tieren $D$ und $C$ etc.. Die Dyaden $\mathrm{D} / \mathrm{E}, \mathrm{D} / \mathrm{G}$ und hatten seltener als erwartet affiliative Kontakte, während die Dyade H/G häufiger als erwartet affiliativ interagierte. Die Beobachtungswerte der Dyaden D/C, H/C und H/E lagen im Erwartungsbereich.

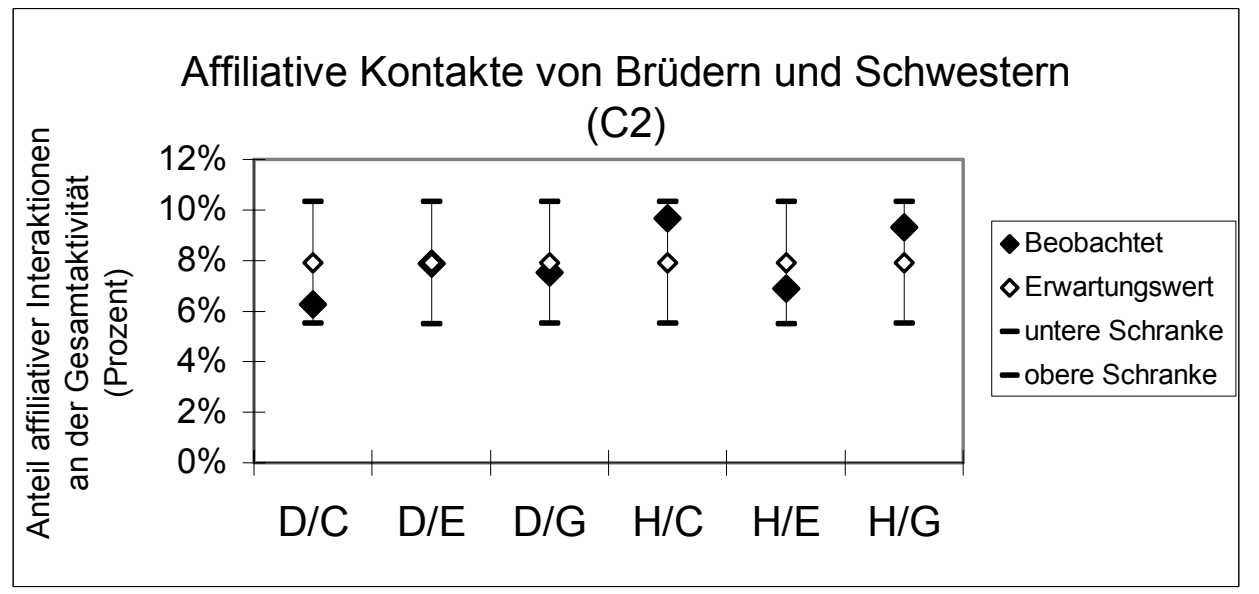

Abb. 3.89 Affiliative Kontakte von Brüdern und Schwestern im zweiten Experiment des ersten Beobachtungsjahres (KFA, EW=7,92 $\mathrm{n}=6$ ). Abkürzungen: $\mathrm{D} / \mathrm{C}=$ Interaktion zwischen den Tieren $\mathrm{D}$ und $\mathrm{C}$ etc.. Die Beobachtungswerte aller Dyaden lagen im Erwartungsbereich. 
Beim Vergleich der affiliativen Kontakte der einzelnen Dyaden im Jahresverlauf fiel auf, dass bei allen Dyaden im ersten Experiment die affiliativen Kontakte seltener als erwartet und im zweiten Experiment dann häufiger als erwartet waren mit Ausnahme der Dyaden E/H und G/H, deren Werte während des zweiten Experiments im Erwartungsbereich angesiedelt waren (Abb.3.90-95). Die affiliativen Interaktionen zwischen Brüdern und Schwestern waren also während des ersten Experiments in der Anwesenheit des Lockweibchens vermindert.

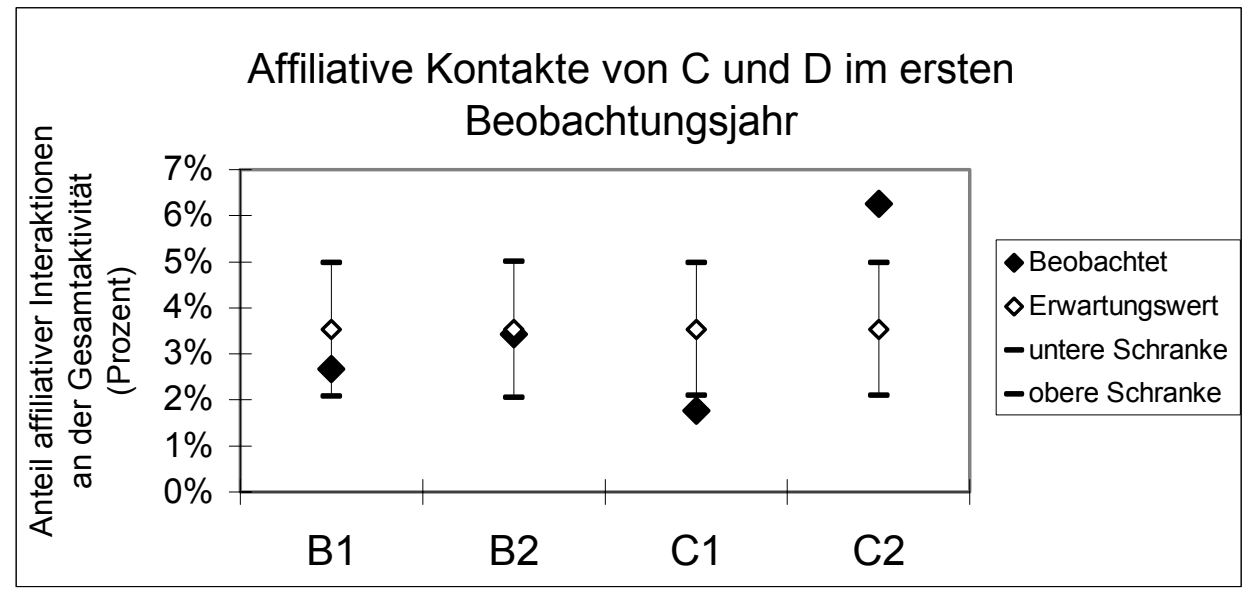

Abb. 3.90 Affiliative Kontakte zwischen C und D im ersten Beobachtungsjahr (KFA, EW=3,53 n=4). Abkürzungen: B1: erste Kontrollphase, B2: zweite Kontrollphase, C1: erstes Experiment, C2: zweites Experiment. In den Kontrollphasen befanden sich die Beobachtungswerte im Erwartungsbereich, lagen dann im ersten Experiment unter und im zweiten Experiment über dem Erwartungsbereich.

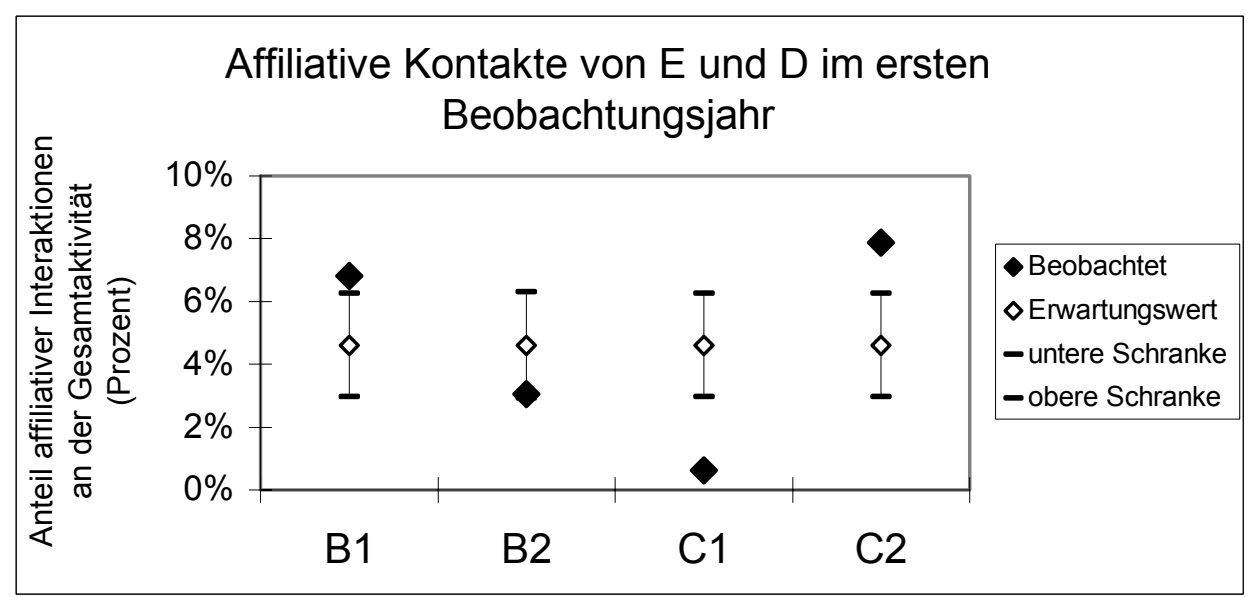

Abb. 3.91 Affiliative Kontakte zwischen E und D im ersten Beobachtungsjahr (KFA, EW=4,61 n=4). Abkürzungen: B1: erste Kontrollphase, B2: zweite Kontrollphase, C1: erstes Experiment, C2: zweites Experiment. In der ersten Kontrollphase und im zweiten Experiment lagen die Beobachtungswerte über dem Erwartungsbereich und waren in der zweiten Kontrollphase innerhalb des Erwartungsbereichs. Während des ersten Experiments hatten E und D seltener als erwartet affiliative Kontakte. 


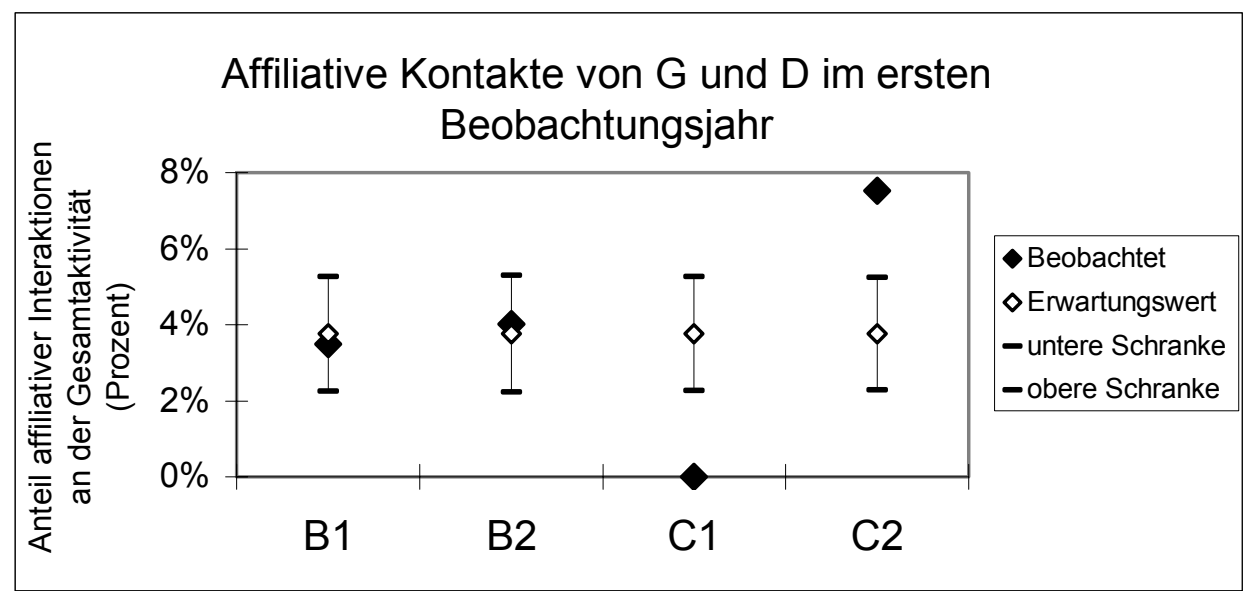

Abb. 3.92 Affiliative Kontakte zwischen G und D im ersten Beobachtungsjahr (KFA, EW=3,76 n=4). Abkürzungen: B1: erste Kontrollphase, B2: zweite Kontrollphase, C1: erstes Experiment, C2: zweites Experiment. In den Kontrollphasen befanden sich die Beobachtungswerte im Erwartungsbereich, lagen dann im ersten Experiment unter und im zweiten Experiment über dem Erwartungsbereich.

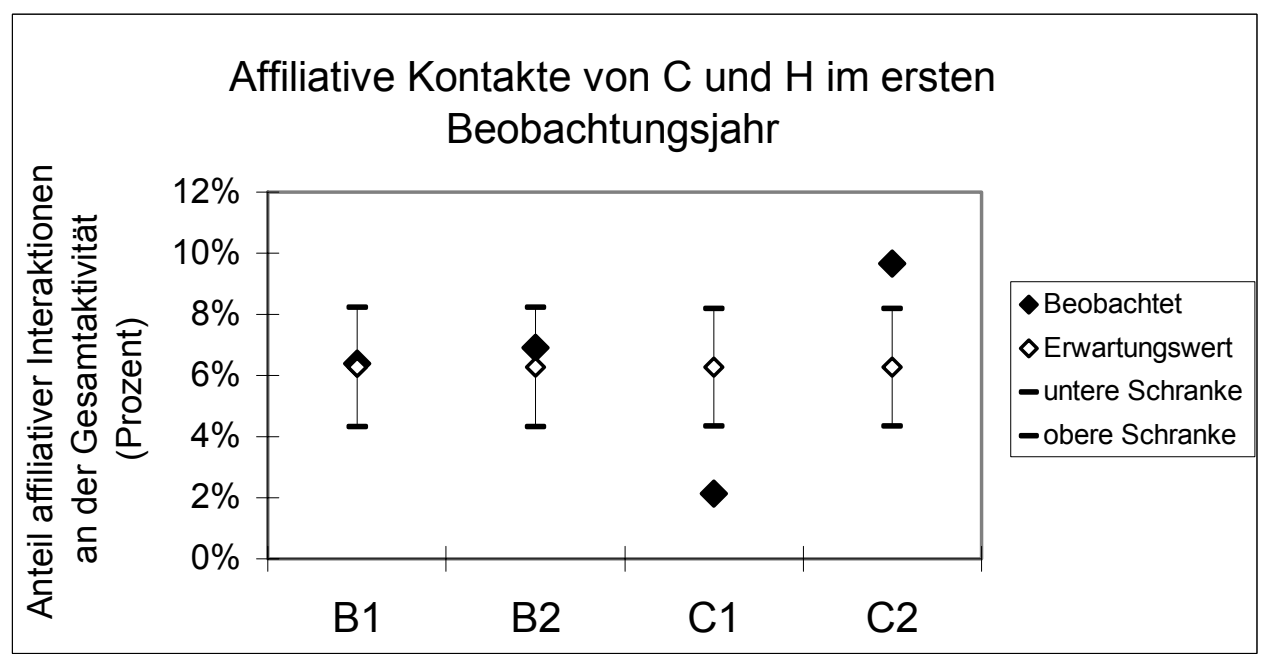

Abb. 3.93 Affiliative Kontakte zwischen $\mathrm{C}$ und $\mathrm{H}$ im ersten Beobachtungsjahr (KFA, EW=6,27 n=4). Abkürzungen: B1: erste Kontrollphase, B2: zweite Kontrollphase, C1: erstes Experiment, C2: zweites Experiment. In den Kontrollphasen befanden sich die Beobachtungswerte im Erwartungsbereich, lagen dann im ersten Experiment unter und im zweiten Experiment über dem Erwartungsbereich. 


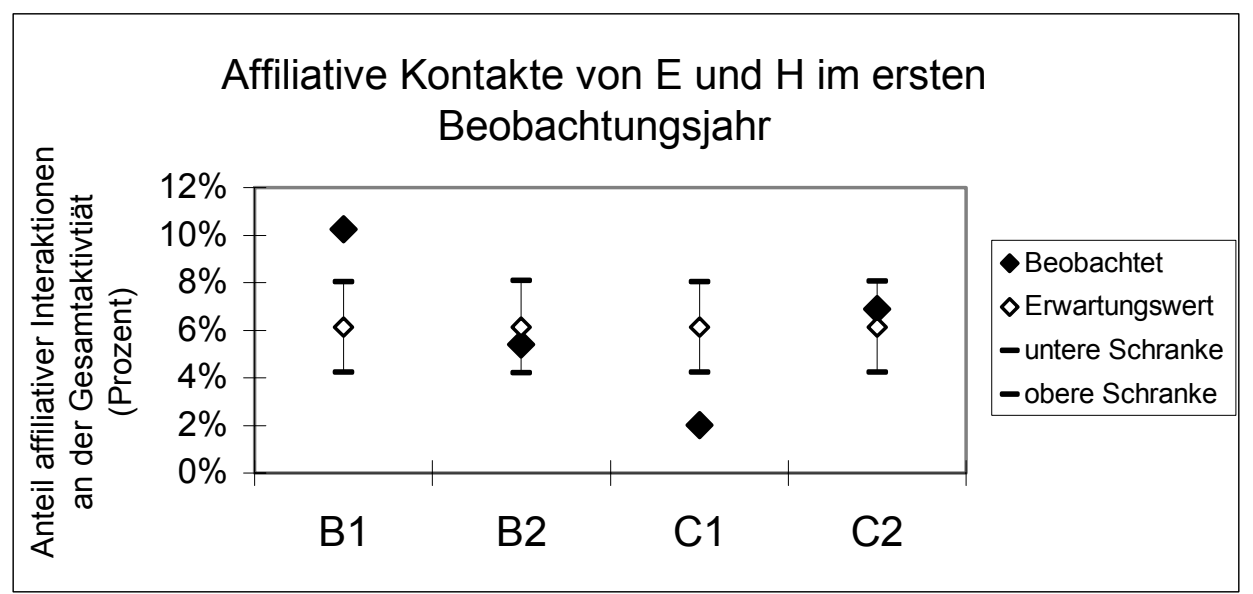

Abb. 3.94 Affiliative Kontakte zwischen $\mathrm{E}$ und $\mathrm{H}$ im ersten Beobachtungsjahr (KFA, EW=6,14 n=4). Abkürzungen: B1: erste Kontrollphase, B2: zweite Kontrollphase, C1: erstes Experiment, C2: zweites Experiment. In der ersten Kontrollphase lag der Beobachtungswert über dem Erwartungsbereich und war in der zweiten Kontrollphase und im zweiten Experiment im Erwartungsbereich. Während des ersten Experiments hatten $\mathrm{E}$ und $\mathrm{H}$ seltener als erwartet affiliative Kontakte.

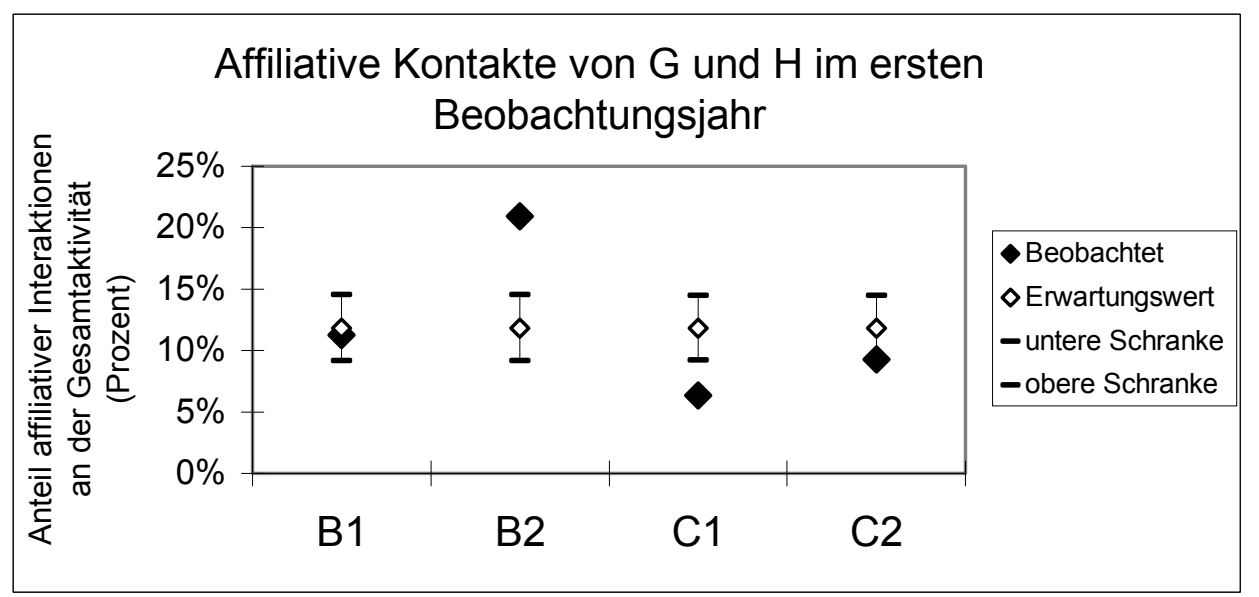

Abb. 3.95 Affiliative Kontakte zwischen $\mathrm{G}$ und $\mathrm{H}$ im ersten Beobachtungsjahr (KFA, EW=11,85 n=4). Abkürzungen: B1: erste Kontrollphase, B2: zweite Kontrollphase, C1: erstes Experiment, C2: zweites Experiment. Der beobachtete Wert befand sich in der ersten Kontrollphase im Erwartungsbereich, lag in der zweiten Kontrollphase über und während der beiden Experimente unter dem Erwartungsbereich.

\subsubsection{Affiliative Interaktionen von Brüdern und Schwestern im zweiten}

\section{Beobachtungsjahr}

Bei einem Vergleich der affiliativen Interaktionen zwischen Brüdern und Schwestern zeigte sich in der Hüttenphase des zweiten Beobachtungsjahres (D1), dass nur G mit K und I mit M häufiger als erwartet affiliativ interagierten. Der Anteil affiliativer Interaktionen an der Gesamtaktivität blieb bei allen anderen Bruder-Schwester-Dyaden im Erwartungsbereich (Abb. 3.96).

Während des ersten Experiments hatten die Dyaden $\mathrm{J} / \mathrm{K}$ und $\mathrm{J} / \mathrm{M}$ häufiger und die Dyaden C/D und J/D seltener als die anderen Dyaden affiliative Kontakte. Die Werte der übrigen Dyaden befinden sich im Erwartungsbereich (Abb. 3.97). 
Während des zweiten und dritten Experiments hatten die Dyaden C/D, G/D und J/D seltener als erwartet (G/D und J/D hatten überhaupt keine affiliativen Kontakte) und die Dyaden $\mathrm{J} / \mathrm{K}$ und J/M hatten wieder häufiger als erwartet affiliative Interaktionen (Abb. 3.98 und 3.99).

Im vierten Experiment interagierten $\mathrm{G} / \mathrm{D}, \mathrm{J} / \mathrm{D}$ und $\mathrm{C} / \mathrm{K}$ seltener affiliativ, während $\mathrm{G}$ und $\mathrm{M}$ häufiger als erwartet affiliativ interagierten. Die Werte aller anderen Dyaden liegen im Erwartungsbereich (Abb. 3.100).

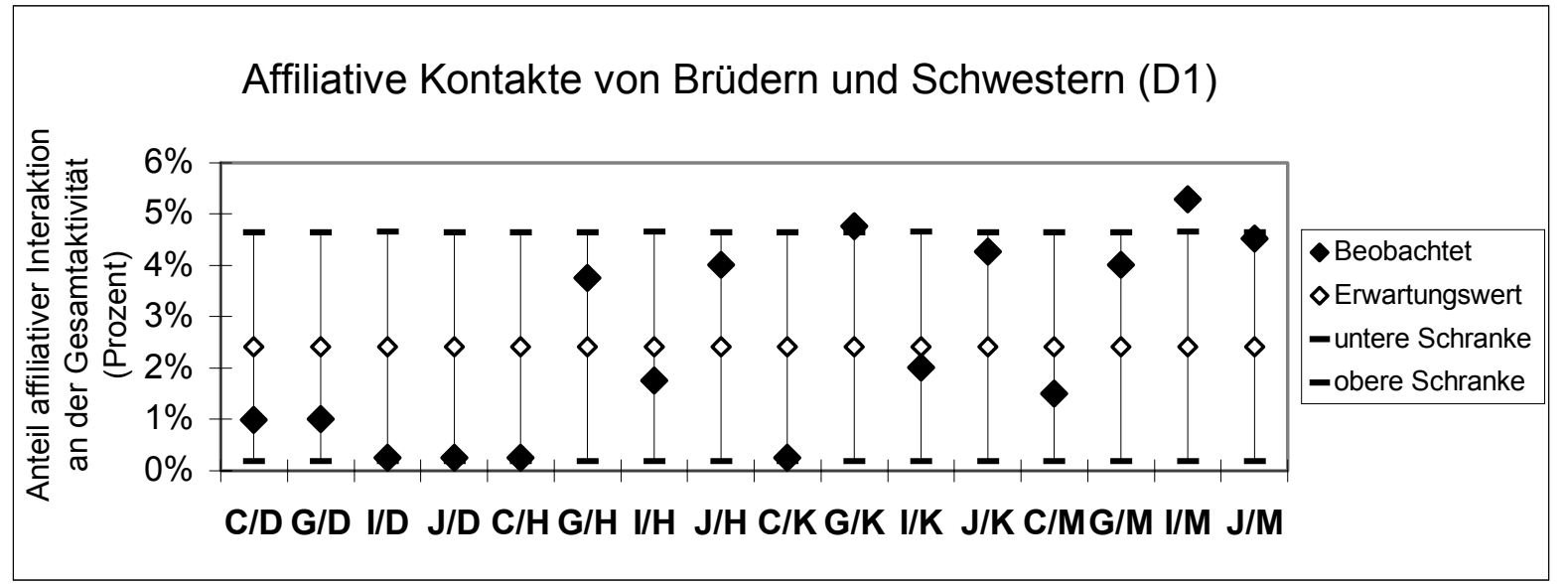

Abb. 3.96 Affiliative Kontakte von Brüdern und Schwestern in der Hüttenphase des zweiten Beobachtungsjahres (KFA, EW=2,42 $\mathrm{n}=16$ ). Abkürzungen: $\mathrm{C} / \mathrm{D}=$ Interaktion zwischen den Tieren $\mathrm{C}$ und $\mathrm{D}$ etc. Die Dyaden $\mathrm{G} / \mathrm{K}$ und I/M hatten häufiger als erwartet affiliative Kontakte. Die Beobachtungswerte der übrigen Dyaden befanden sich im Erwartungsbereich.

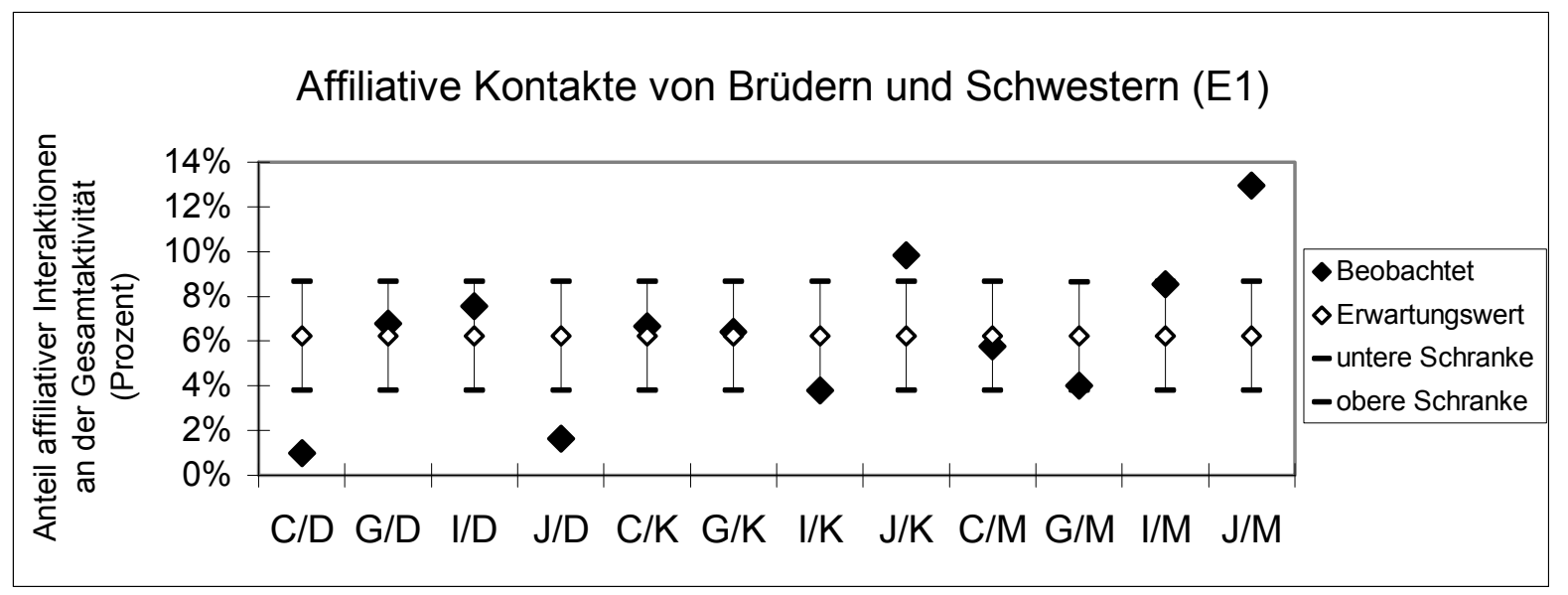

Abb. 3.97 Affiliative Kontakte von Brüdern und Schwestern im ersten Experiment des zweiten Beobachtungsjahres (KFA, EW=6,23 n=12). Abkürzungen: $C / D=$ Interaktion zwischen den Tieren $C$ und $D$ etc. Die Dyaden $\mathrm{C} / \mathrm{D}$ und $\mathrm{J} / \mathrm{D}$ hatten seltener als erwartet affiliative Kontakte, während die Dyaden $\mathrm{J} / \mathrm{K}$ und $\mathrm{J} / \mathrm{M}$ häufiger als erwartet affiliativ interagierten. Die Beobachtungswerte der übrigen Dyaden befanden sich im Erwartungsbereich. 


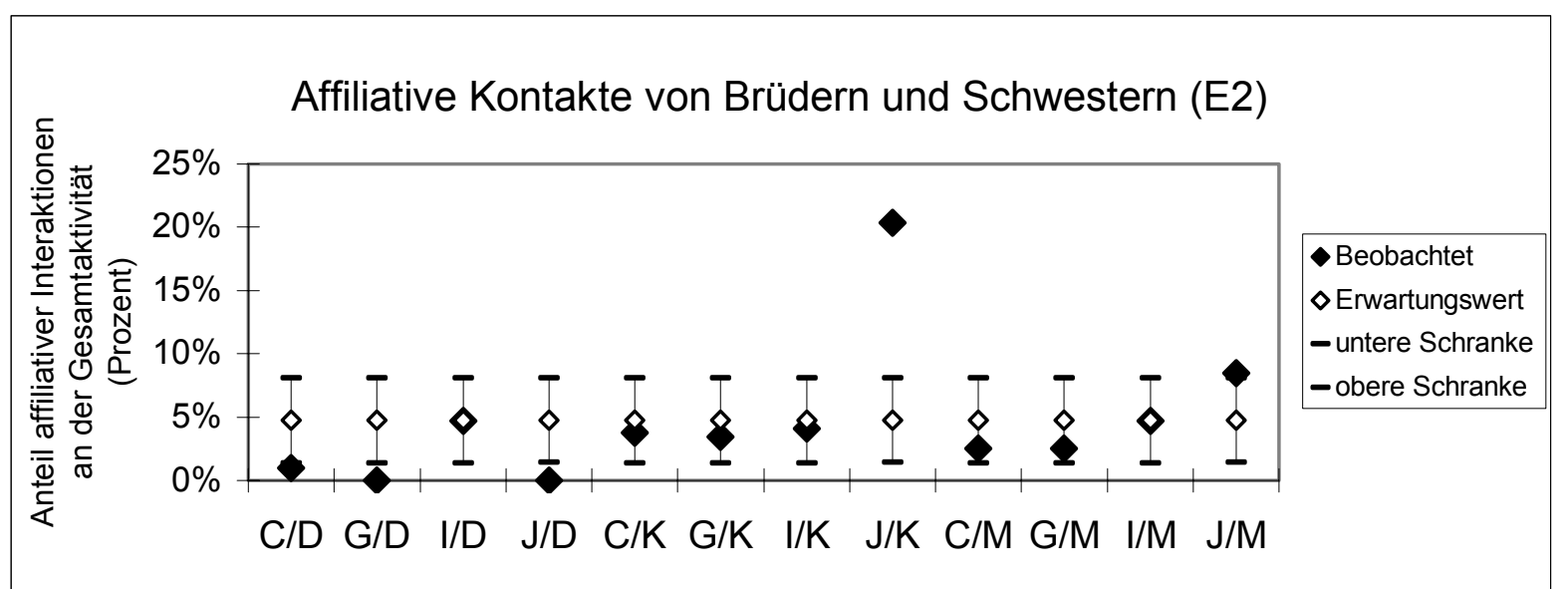

Abb. 3.98 Affiliative Kontakte von Brüdern und Schwestern im zweiten Experiment des zweiten Beobachtungsjahres (KFA, EW=4,74 n=12). Abkürzungen: $\mathrm{C} / \mathrm{D}=$ Interaktion zwischen den Tieren $\mathrm{C}$ und $\mathrm{D}$ etc. Die Dyaden C/D, G/D und J/D hatten seltener als erwartet affiliative Kontakte, während die Dyaden J/K und J/M häufiger als erwartet affiliativ interagierten. Die Beobachtungswerte der übrigen Dyaden befanden sich im Erwartungsbereich.

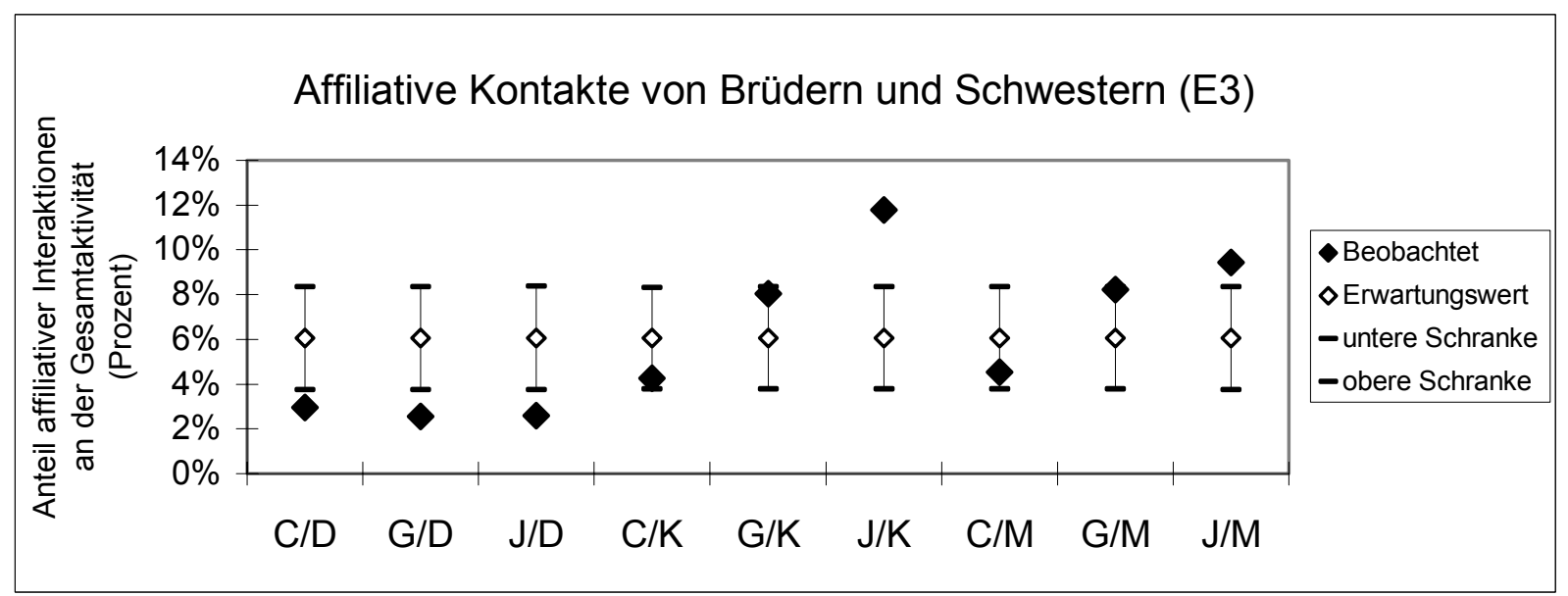

Abb. 3.99 Affiliative Kontakte von Brüdern und Schwestern im dritten Experiment des zweiten Beobachtungsjahres (KFA, EW=6,05 n=9). Abkürzungen: $C / D=$ Interaktion zwischen den Tieren $C$ und $D$ etc. Die Dyaden $\mathrm{C} / \mathrm{D}, \mathrm{G} / \mathrm{D}$ und $\mathrm{J} / \mathrm{D}$ hatten seltener als erwartet affiliative Kontakte, während die Dyaden $\mathrm{J} / \mathrm{K}$ und $\mathrm{J} / \mathrm{M}$ häufiger als erwartet affiliativ interagierten. Die Beobachtungswerte der übrigen Dyaden befanden sich im Erwartungsbereich.

Die Dyaden $\mathrm{C} / \mathrm{D}$ und $\mathrm{J} / \mathrm{D}$ hatten vom ersten bis zum dritten Experiment seltener affiliative Kontakte, die sich in E4 stark häuften und dann über dem Erwartungsbereich lagen (Abb. 3.101 und Abb. 3.104). Die affiliativen Interaktionen zwischen G und D entsprachen im ersten Experiment der Erwartung, sanken im zweiten Experiment auf Null, lagen im dritten Experiment noch unter dem Erwartungsbereich und waren im vierten Experiment häufiger als erwartet (Abb. 3.102). D interagierte während des ersten Experiments mit I häufiger affiliativ und im zweiten Experiment (E2) seltener als erwartet. Danach lag der Wert innerhalb des Erwartungsbereichs (Abb. 3.103).

Insgesamt war der Anteil an affiliativen Interaktionen zwischen den Brüdern und D im zweiten und dritten Experiment recht gering. Da $\mathrm{K}$ und $\mathrm{M}$ während der zweiten Beob- 
achtungsperiode noch nicht adult bzw. subadult waren und bei ihnen kein Interesse an den Locktieren festgestellt werden konnte (siehe 3.1.6), wurden keine Vergleiche ihrer affiliativen Interaktionen zu den Brüdern vorgenommen.

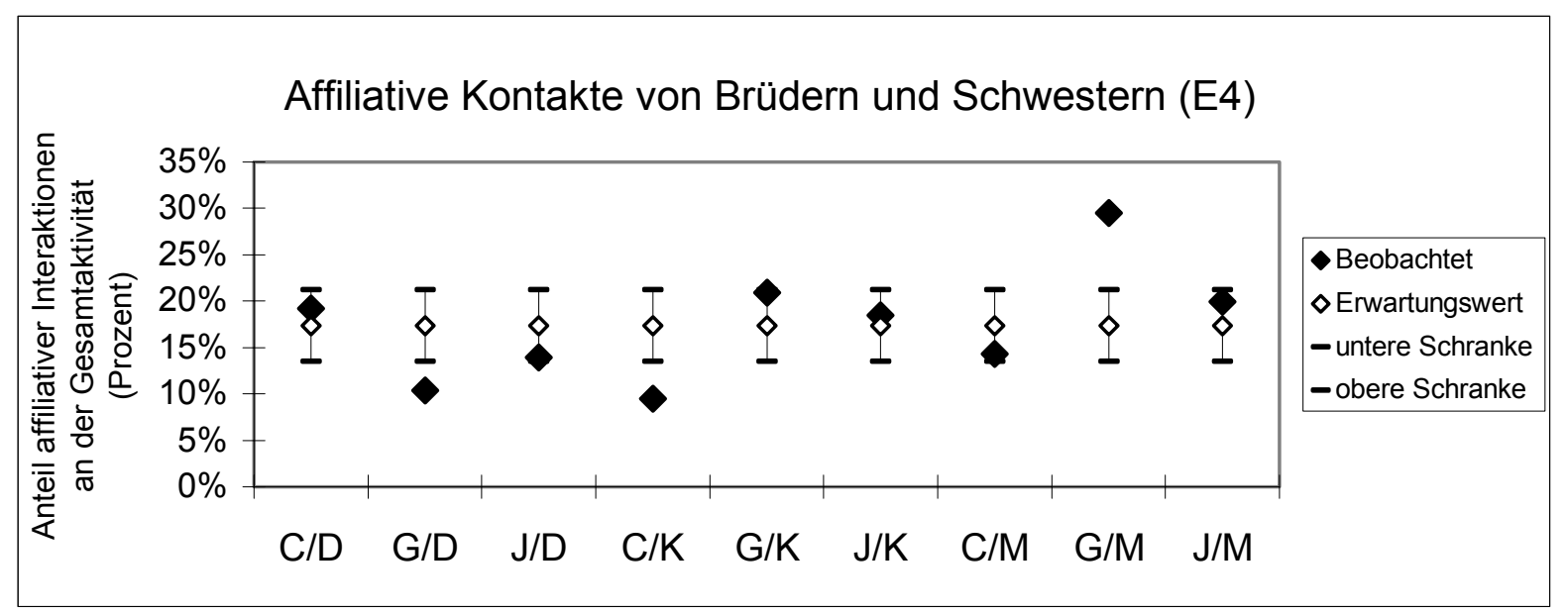

Abb. 3.100 Affiliative Kontakte von Brüdern und Schwestern im vierten Experiment des zweiten Beobachtungsjahres (KFA, EW=17,36 n=9). Abkürzungen: $C / D=$ Interaktion zwischen den Tieren $C$ und $D$ etc. Die Dyaden $\mathrm{G} / \mathrm{D}$ und $\mathrm{C} / \mathrm{K}$ hatten seltener als erwartet affiliative Kontakte, während die Dyade $\mathrm{G} / \mathrm{M}$ häufiger als erwartet affiliativ interagierte. Die Beobachtungswerte der übrigen Dyaden befanden sich im Erwartungsbereich.

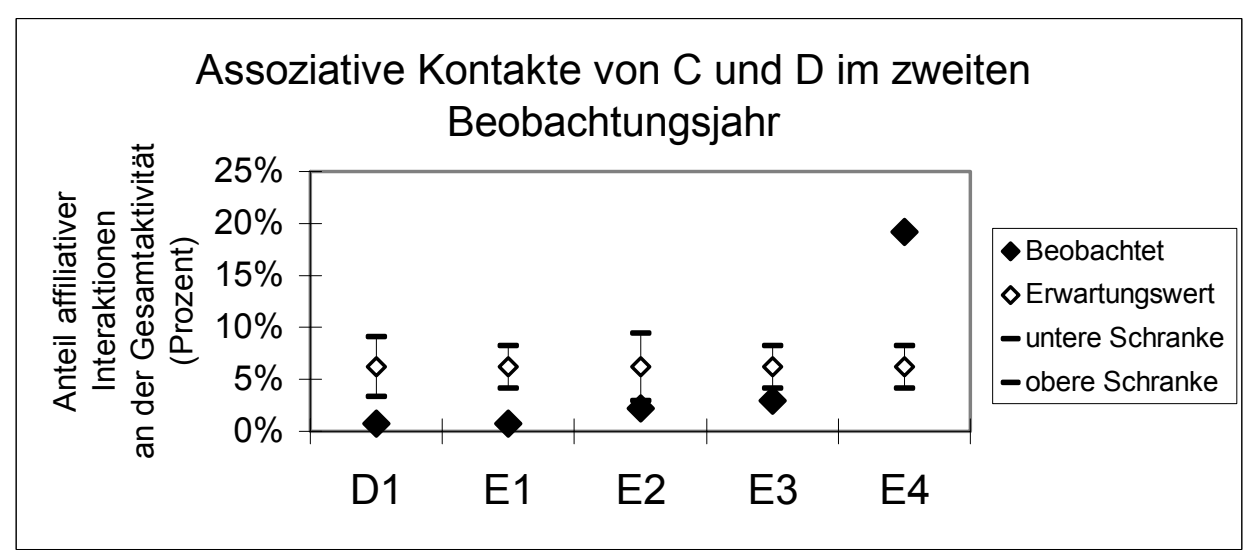

Abb. 3.101 Affiliative Kontakte zwischen C und D im zweiten Beobachtungsjahr (KFA, EW=6,19 n=5). Abkürzungen: D1=Hüttenphase, E1=erstes Experiment, E2=zweites Experiment, E3= drittes Experiment, E4=viertes Experiment. Von der Hüttenphase bis zum dritten Experiment hatten $C$ und D seltener affiliative Interaktionen als erwartet. Im vierten Experiment lag der beobachtete Wert signifikant über dem Erwartungswert. 


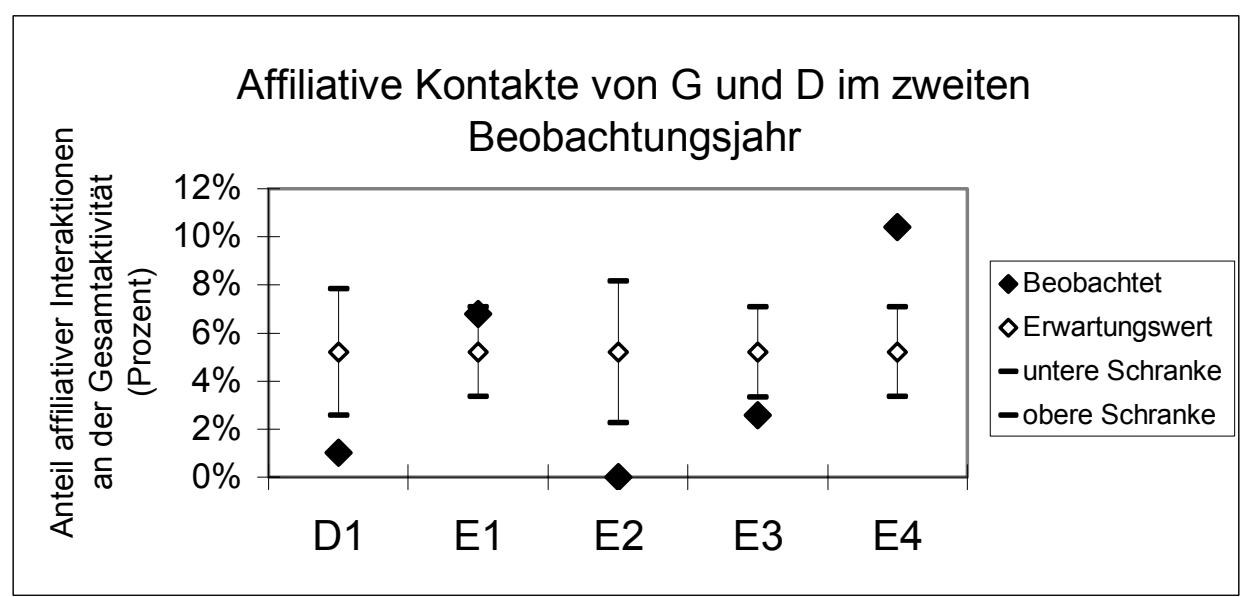

Abb. 3.102 Affiliative Kontakte zwischen G und D im zweiten Beobachtungsjahr (KFA, EW=5,21 n=5). Abkürzungen: D1=Hüttenphase, E1=erstes Experiment, E2=zweites Experiment, E3= drittes Experiment, E4=viertes Experiment. Die affiliativen Kontakte waren zwischen G und D in der Hüttenphase und in den Experimenten 2 und 3 seltener, in Experiment 4 dann häufiger als erwartet. Der beobachtete Wert lag im ersten Experiment innerhalb des Erwartungsbereichs.

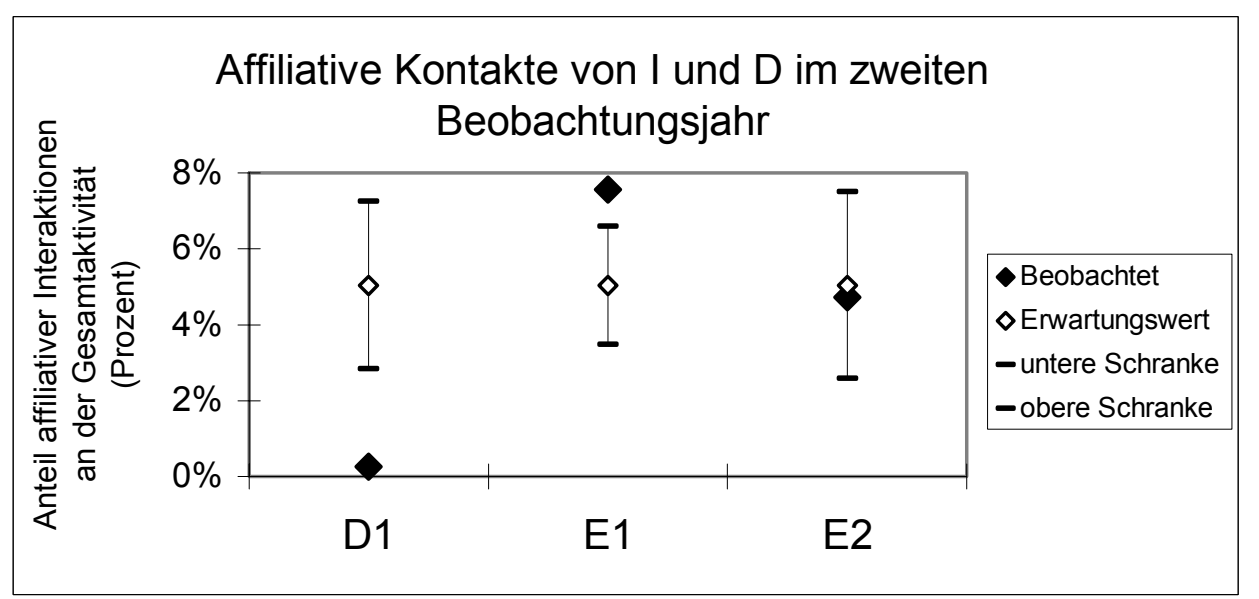

Abb. 3.103 Affiliative Kontakte zwischen I und D im zweiten Beobachtungsjahr (KFA, EW=5,04 n=3). Abkürzungen: D1=Hüttenphase, E1=erstes Experiment, E2=zweites Experiment. Die affiliativen Interaktionen von I und D lagen in der Hüttenphase weit unter dem Erwartungsbereich. Im ersten Experiment interagierte die Dyade häufiger als erwartet und im zweiten Experiment lag der beobachtete Wert innerhalb des Erwartungsbereichs.

Unter den experimentellen Bedingungen des ersten und zweiten Beobachtungsjahres traten Abhängigkeiten der affiliativen Interaktionen zwischen Brüdern und Schwestern von den Experimenten auf, so dass die Hypothese $5 \mathrm{f}$ zurückgewiesen werden muss.

Da die räumliche Assoziation und der Anteil affiliativer Interaktionen zwischen einigen Geschwistern in Abhängigkeit von den Experimenten Veränderungen unterlagen, kann Hypothese 5 zurückgewiesen werden. 


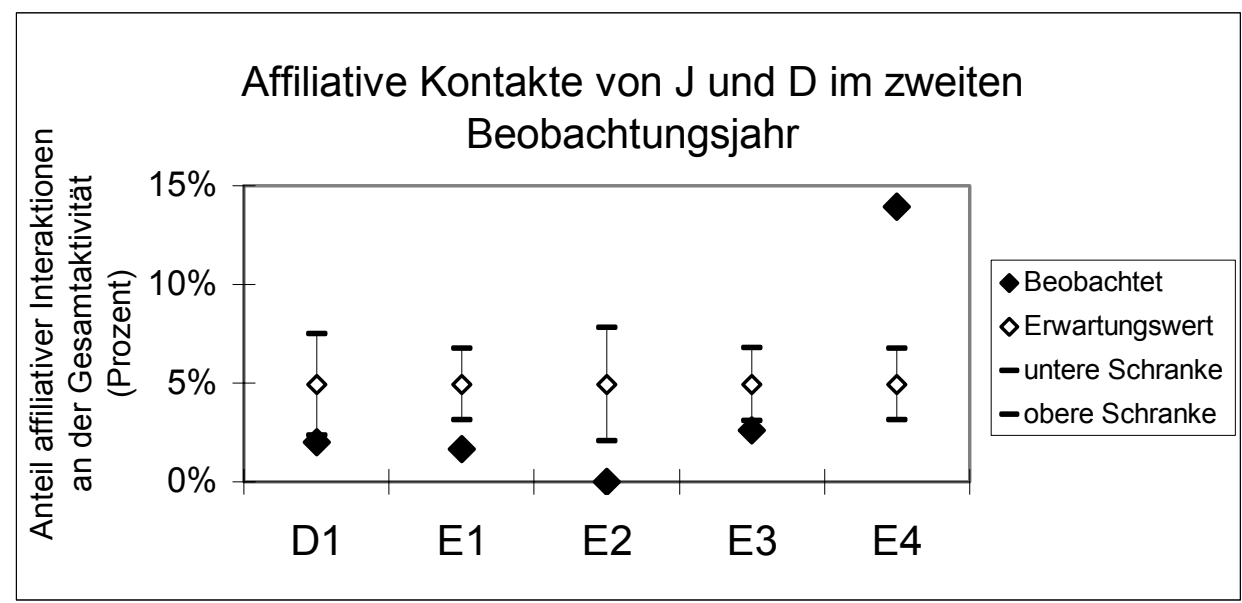

Abb. 3.104 Affiliative Kontakte zwischen J und D im zweiten Beobachtungsjahr (KFA, EW=4,94 n=5). Abkürzungen: D1=Hüttenphase, E1=erstes Experiment, E2=zweites Experiment, E3= drittes Experiment, E4=viertes Experiment. Von der Hüttenphase bis zum dritten Experiment hatten $\mathrm{J}$ und D seltener affiliative Interaktionen als erwartet. Im vierten Experiment lag der beobachtete Wert signifikant über dem Erwartungswert.

\subsubsection{Einfluss der Eltern auf die gruppendynamischen Prozesse}

Hypothese 6: Rauswürfe ereignen sich unabhängig von Eltern-Nachkommen-Konflikten.

Obwohl adulte Nachkommen in Sozietäten mit Helfersystem, wie denen der Marmosetten, normalerweise nicht reproduktiv sind, wurde in einzelnen Fällen beobachtet, wie die Eltern durch adulte Nachkommen von ihrer reproduktiven Position verdrängt wurden (Box 1977, Ferrari und Lopes Ferrari 1989, Ferrari und Diego 1992 für C. flaviceps, Rothe und König 1987, 1991, Rothe und Radespiel 1988, Rothe et al. 1986, Rylands 1982 für C. humeralifer, Scanlon et al. 1988, Spichiger-Carlsson 1982). So scheinen die adulten Nachkommen mit den Eltern in Konkurrenz um das Reproduktionsprivileg treten zu können (Abbott 1984, Emlen und Vehrenkamp 1985, Kleiman 1979 für L. rosalia). Angriffe und Rauswürfe durch die Eltern auf Nachkommen ihres eigenen Geschlechts sprechen ebenso für diese Annahme (König et al. 1988, König und Rothe 1991, eigene Beobachtungen für C. jacchus, McGrew 1997 nur für weibliche S. oedipus, Kleiman (1979) für L. rosalia).

Es ist deshalb zu erwarten, dass sich Alpha-Männchen an Rauswürfen männlicher Nachkommen und Alpha-Weibchen an Rauswürfen von Töchtern beteiligen, falls diese ihr Reproduktionsprivileg herausfordern. Da jedoch adulte Helfer eine wichtige Voraussetzung für das Funktionieren des Helfersystems sind, ist ebenso zu erwarten, dass Alpha-Tiere etwa über affiliative Interaktionen versuchen, Helfer an die Familie zu binden (vgl. Digby 1995b, Lazaro-Perea et al. 2004, Zahavi 1990), z.B. bei Emigrationsneigungen der Nachkommen, die für den Beobachter möglicherweise als Peripheralisierungen sichtbar werden (Darms 1987, 
Geyer 1993, Radespiel 1990). Eine Konsequenz aus den beiden Annahmen könnte sein, dass Alpha-Männchen versuchen, Rauswürfe und Emigrationen von Töchtern und AlphaWeibchen Rauswürfe und Emigrationen von Söhnen zu verhindern.

\subsubsection{Rauswürfe durch die Alpha-Tiere (Eltern)}

Hypothese 6a: Die Wahrscheinlichkeit, dass Alpha-Tiere Nachkommen rauswerfen ist genauso groß wie die Wahrscheinlichkeit, dass Geschwister Rauswürfe durchführen.

Die Daten über Rauswürfe in der C. jacchus-Kolonie des IfA liefern keine Unterschiede in der Rauswurfhäufigkeit durch Alpha-Tiere und durch Geschwister der rausgeworfenen Tiere. $51,65 \%$ der Rauswürfe wurden von Alpha-Tieren und 48,35\% der Rauswürfe wurden von Geschwistern durchgeführt. Innerhalb der Geschlechter traten allerdings Unterschiede auf. Alpha-Männchen warfen seltener Gruppenmitglieder aus der Gruppe als Brüder dies tun (Abb.3.105), während Alpha-Weibchen häufiger als Schwestern Gruppenmitglieder aus der Gruppe warfen (Abb. 3.106).

Hypothese 6a muss zurückgewiesen werden, da es bei einer getrennten Betrachtung der Geschlechter Unterschiede zwischen der Rauswurfhäufigkeit durch Alpha-Tiere und der durch Geschwister gibt.

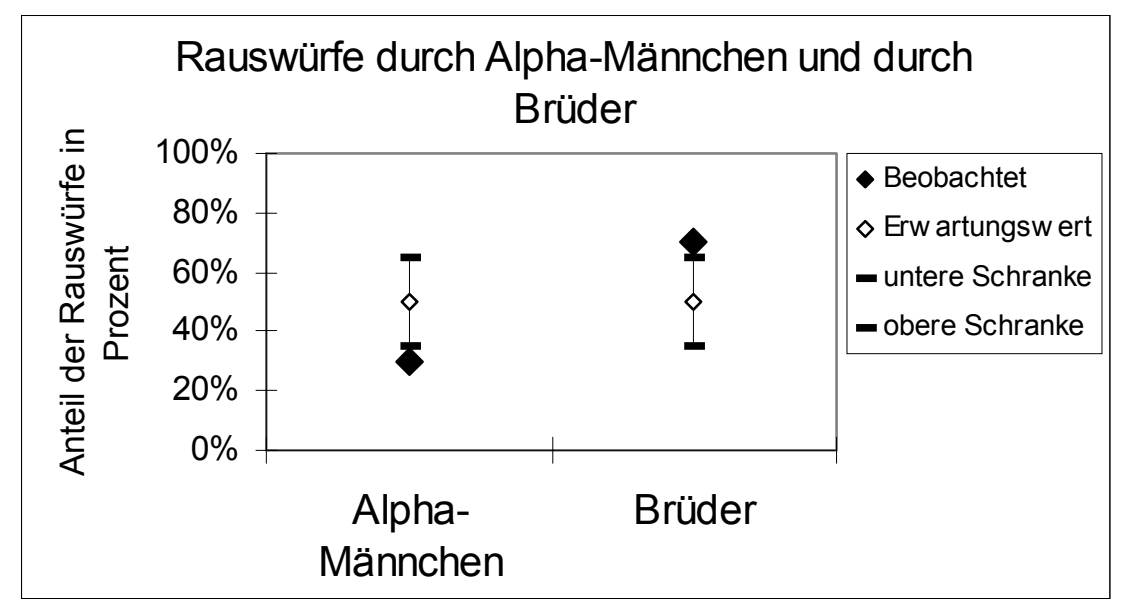

Abb. 3.105 Rauswürfe in der C. jacchus-Kolonie des IfA im Zeitraum 1972-2003 durch männliche Alpha-Tiere und männliche Geschwister. Alpha-Männchen warfen zu 26,63\% und Brüder zu 70,37\% Mitglieder aus der Gruppe (KFA, EW=50, $\mathrm{n}=2)$. 


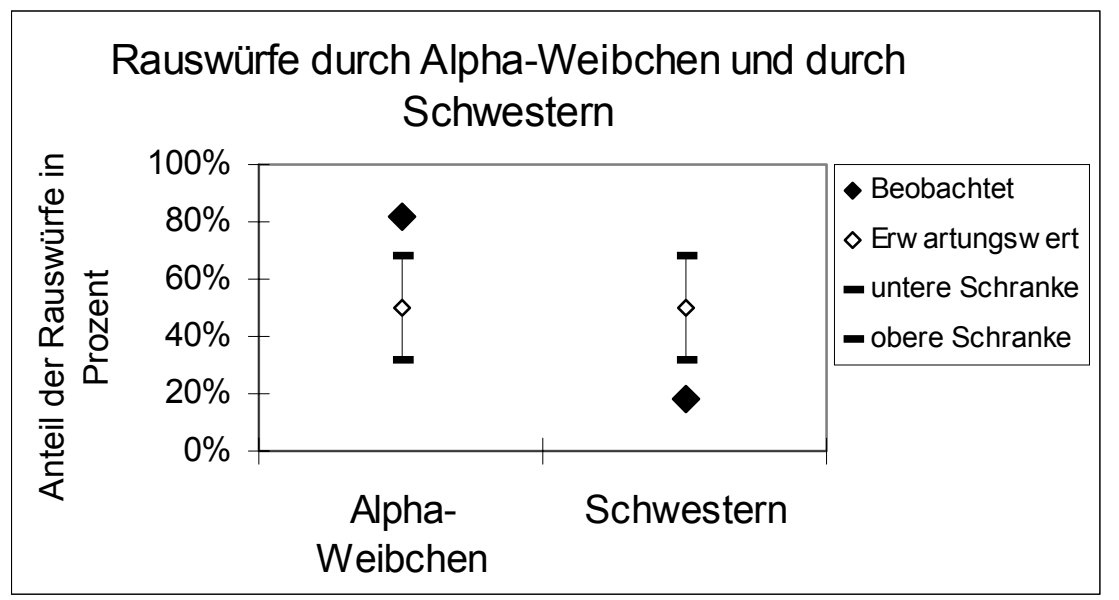

Abb. 3.106 Rauswürfe in der C. jacchus-Kolonie des IfA im Zeitraum 1972-2003 durch weibliche Alpha-Tiere und weibliche Geschwister. Alpha-Weibchen warfen zu 81,58\% und Schwestern zu 18,42\% Mitglieder aus der Gruppe (KFA, EW=50, n=2).

Hypothese 6b: Es gibt keine Unterschiede zwischen Alpha-Männchen und AlphaWeibchen, sich an Rauswürfen von Nachkommen zu beteiligen.

Alpha-Weibchen warfen häufiger als Alpha-Männchen Nachkommen aus der Gruppe (KFA, $\mathrm{EW}=50,54, \mathrm{n}=2$ ). Somit kann Hypothese $6 \mathrm{~b}$ zurückgewiesen werden.

Hypothese 6c: Die Wahrscheinlichkeit, dass die Eltern Rauswürfe durchführen bzw. sich daran beteiligen ist vom Geschlecht des rausgeworfenen Tieres unabhängig.

Während Weibchen nur Töchter bzw. Schwestern aus der Gruppe warfen, wurden von Männchen in 5 von 43 Fällen (11,63\%) auch Töchter bzw. Schwestern rausgeworfen (zwei Rauswürfe durch Alpha-Männchen und drei Rauswürfe durch Brüder). Alpha-Männchen warfen somit Nachkommen beider Geschlechter aus der Gruppe, wobei sie bevorzugt Söhne rauswarfen (Söhne: 87,5\%, Töchter: 12,5\%; KFA, EW=50\%, n=2).

Während der zweieinhalb Beobachtungsjahre kam es in der Fokusfamilie zu zwei Ausschlüssen, die dadurch gekennzeichnet waren, dass der Rauswerfer von einem Elternteil „unterstützt“ wurde. In einem Fall schloss der jung-adulte $G$ mit Hilfe des Vaters seinen älteren Bruder E aus dem Familienverband aus. In einer anderen Situation beteiligte sich das Alpha-Weibchen B solange an stark dissoziativem Verhalten (Wegjagen, Keckern, Angreifen und Beißen) der ältesten Tochter D gegenüber der jüngsten Tochter $\mathrm{H}$, bis $\mathrm{H}$ aus der Familie entfernt werden musste. Der Rauswurf der Tochter $\mathrm{H}$ zeichnete sich durch ein weiteres besonderes Ereignis aus. Das Alpha-Männchen A stellte sich während der Mobbing- und Beißattacken von $\mathrm{D}$ und $\mathrm{B}$ auf $\mathrm{H}$ den Angreiferrinnen in den Weg und drohte $\mathrm{D}$ mit Schlitzaugen-machen, Keckern und kurzen Scheinangriffen. Das Verhalten des Alpha- 
Männchens wird derart interpretiert, dass er den Rauswurf von $\mathrm{H}$ zu verhindern suchte. Als es zu einem Konflikt zwischen ihm und dem Alpha-Weibchen kam, entfernte er sich von den Kontrahenten.

Nachdem I aus der Familie verjagt worden war, griffen die Alpha-Tiere ein, als D J, den Zwillings-Bruder von I, mobbte. Obwohl D auch schon vor dem Rauswurf von I mit J dissoziativ interagierte, griffen die Eltern erst in den Konflikt ein, nachdem I die Familie verlassen hatte, indem sie zwischen die Kontrahenten katzbuckelten und D gegenüber genitalpräsentierten. In einem anderen Fall bedrohte das Alpha-Weibchen G mit Büschelklappen und Schlitzaugen-machen, als dieser seine jüngere Schwester M mobbte. G reagierte darauf, indem er das Alpha-Weibchen schlug, nachfolgend aber M in Ruhe ließ.

Die Alpha-Tiere konnten also nur bei Rauswürfen von gleichgeschlechtlichen Nachkommen beobachtet werden, während sie schwach dissoziatives Verhalten auch in Konfliktsituationen zwischen gegengeschlechtlichen Nachkommen zeigten. Das Alpha-Weibchen beteiligte sich in einem von zwei Fällen an den Rauswürfen von Töchtern, während sich das AlphaMännchen in einem von sechs Fällen (ca. 17\%) an den Rauswürfen von Söhnen teilnahm. Das Alpha-Weibchen der Fokusfamilie unterschied sich nicht von dem Alpha-Männchen in der Beteiligung an Rauswürfen (KFA, EW=25, n=2), was jedoch in der geringen Stichprobengröße begründet liegen mag und somit wahrscheinlich nicht allgemein für $C$. jacchus gültig ist.

Da sich Alpha-Weibchen nur an Rauswürfen von Töchtern beteiligen und Alpha-Männchen bevorzugt Söhne rauswerfen kann Hypothese 6c zurückgewiesen werden.

\subsubsection{Abhängigkeit elterlicher Eingriffe von verschiedenen Faktoren}

Mit Hilfe der Transponderdaten wurde ermittelt, wie häufig die Eltern jene Areale aufsuchten, in denen sich gerade ihre Nachkommen befanden. Das erwartete Interesse der Eltern an bestimmten Nachkommen könnte sowohl einer Kontroll-, als auch einer Rückholtaktik entsprechen. 


\subsubsection{Abhängigkeit elterlichen Aufsuchens ihrer Nachkommen vom Geschlecht dieser Nachkommen}

Hypothese 6d: Die Alpha-Tiere suchen männliche und weibliche Nachkommen gleich häufig auf.

Hypothese 6e: Die Alpha-Tiere unterscheiden sich nicht in der Häufigkeit, männliche und weibliche Nachkommen aufzusuchen.

In den Experimentalphasen des ersten und zweiten Beobachtungsjahres suchten das AlphaMännchen und das Alpha-Weibchen Areale, in denen sich männliche und Areale, in denen sich weibliche Nachkommen gerade befanden, jeweils gleich häufig auf. Damit bevorzugte weder das Alpha-Weibchen, noch das Alpha-Männchen männliche oder weibliche Nachkommen (siehe Tab. 3.22 und 3.23).

Tabelle 3.22 $\mathrm{Chi}^{2}$-Test für den Vergleich von Häufigkeiten, mit denen die Alpha-Tiere Areale aufsuchten, in denen sich männliche und weibliche Nachkommen aufhielten. Das Alpha-Männchen und das Alpha-Weibchen werden getrennt für jede Kontrollphase und jedes Experiment des ersten Beobachtungsjahres getestet.

\begin{tabular}{lllll}
$\begin{array}{l}\text { Kontrollphasen und } \\
\text { Experimente }\end{array}$ & \multicolumn{2}{l}{ Alpha-Männchen } & Alpha-Weibchen \\
\hline B1 & $\mathrm{Chi}^{2}=0,01$ & $\mathrm{p}=0,9228$ & $\mathrm{Chi}^{2}=0,03$ & $\mathrm{p}=0,8579$ \\
B2 & $\mathrm{Chi}^{2}=0,43$ & $\mathrm{p}=0,5109$ & $\mathrm{Chi}^{2}=0,31$ & $\mathrm{p}=0,5787$ \\
C1 & $\mathrm{Chi}^{2}=0,21$ & $\mathrm{p}=0,6469$ & $\mathrm{Chi}^{2}=0,29$ & $\mathrm{p}=0,5876$ \\
C2 & $\mathrm{Chi}^{2}=0,16$ & $\mathrm{p}=0,6872$ & $\mathrm{Chi}^{2}=0,15$ & $\mathrm{p}=0,6968$ \\
\hline
\end{tabular}

Tabelle 3.23 $\mathrm{Chi}^{2}$-Test für den Vergleich von Häufigkeiten, mit denen die Alpha-Tiere Areale aufsuchten, in denen sich männliche und weibliche Nachkommen aufhielten. Das Alpha-Männchen und das Alpha-Weibchen werden getrennt für jedes Experiment des zweiten Beobachtungsjahres getestet.

\begin{tabular}{lllll} 
Experimente & \multicolumn{2}{l}{ Alpha-Männchen } & \multicolumn{2}{l}{ Alpha-Weibchen } \\
\hline E1 & $\mathrm{Chi}^{2}=0$ & $\mathrm{p}=0,9983$ & $\mathrm{Chi}^{2}=0$ & $\mathrm{p}=0,9492$ \\
E2 & $\mathrm{Chi}^{2}=0,05$ & $\mathrm{p}=0,8185$ & $\mathrm{Chi}^{2}=0,06$ & $\mathrm{p}=0,8017$ \\
E3 & $\mathrm{Chi}^{2}=0,21$ & $\mathrm{p}=0,6455$ & $\mathrm{Chi}^{2}=0,24$ & $\mathrm{p}=0,6214$ \\
E4 & $\mathrm{Chi}^{2}=0,07$ & $\mathrm{p}=0,7954$ & $\mathrm{Chi}^{2}=0,09$ & $\mathrm{p}=0,7703$ \\
\hline
\end{tabular}

Das Verhältnis der Häufigkeiten, mit denen männliche und weibliche Nachkommen aufgesucht wurden, war beim Alpha-Männchen genauso groß wie beim Alpha-Weibchen (Tab. 3.24 und 3.25). 
Tabelle 3.24 Chi²-Test für den Vergleich des Alpha-Weibchens mit dem Alpha-Männchen in den Häufigkeiten, mit denen männliche und weibliche Nachkommen im ersten Beobachtungsjahr aufgesucht wurden. Es gibt keine Unterschiede zwischen den Alpha-Tieren.

\begin{tabular}{lll}
$\begin{array}{l}\text { Kontrollphasen und } \\
\text { Experimente }\end{array}$ & & Signifikanzniveau \\
\hline B1 & $\mathrm{Chi}^{2}=0,42$ & $\mathrm{P}=0,5151$ \\
B2 & $\mathrm{Chi}^{2}=0,55$ & $\mathrm{P}=0,4600$ \\
C1 & $\mathrm{Chi}^{2}=0,49$ & $\mathrm{P}=0,4847$ \\
C2 & $\mathrm{Chi}^{2}=0,01$ & $\mathrm{P}=0,9036$ \\
\hline
\end{tabular}

Tabelle 3.25 Chi $^{2}$-Test für den Vergleich des Alpha-Weibchens mit dem Alpha-Männchen in den Häufigkeiten, mit denen männliche und weibliche Nachkommen im zweiten Beobachtungsjahr aufgesucht wurden. Es gibt keine Unterschiede zwischen den Alpha-Tieren.

\begin{tabular}{lll} 
Experimente & & Signifikanzniveau \\
\hline E1 & $\mathrm{Chi}^{2}=0,18$ & $\mathrm{P}=0,6707$ \\
E2 & $\mathrm{Chi}^{2}=0,01$ & $\mathrm{P}=0,9132$ \\
E3 & $\mathrm{Chi}^{2}=0,05$ & $\mathrm{P}=0,8192$ \\
E4 & $\mathrm{Chi}^{2}=0,04$ & $\mathrm{P}=0,8508$ \\
\hline
\end{tabular}

Da die Alpha-Tiere männliche und weibliche Nachkommen nicht unterschiedlich häufig aufsuchten und sich darin nicht voneinander unterschieden, können die Hypothesen $6 \mathrm{~d}$ und 6e nicht zurückgewiesen werden.

\subsubsection{Abhängigkeit der räumlichen Assoziation bestimmter Nachkommen zu den Familienmitgliedern und der Häufigkeit, mit der die Alpha-Tiere Areale aufsuchen, in denen sich diese Nachkommen aufhalten}

Hypothese 6f: Es gibt keinen Zusammenhang zwischen der räumlichen Assoziation bestimmter Nachkommen zu den Familienmitgliedern und der Häufigkeit, mit der die Alpha-Tiere Areale aufsuchen, in denen sich diese Nachkommen aufhalten.

Sind die Eltern an dem Verbleib von bestimmten Nachkommen in der Familie interessiert, so sollten sie versuchen, Nachkommen zurückzuhalten, wenn sich diese von der Familie peripheralisieren (vgl. Emlen und Wrege 1992, Keller und Reeve 1994, Sherman et al. 1995). Um diese Hypothese zu testen wurde eine Korrelationsanalyse zwischen der Häufigkeit, mit der die Alpha-Tiere diejenigen Areale aufsuchten, in denen sich gerade bestimmte Nachkommen aufhielten und der räumlichen Assoziation derselben Nachkommen zur Familie durchgeführt. 
Im ersten Beobachtungsjahr gab es eine Korrelation zwischen der räumlichen Assoziation des männlichen Nachkommen E zu den übrigen Familienmitgliedern und der Häufigkeit, mit der das Alpha-Männchen Areale aufsuchte, in denen sich E aufhielt. Es besteht außerdem eine Korrelation zwischen der räumlichen Assoziation der Tochter D zur Familie und der Häufigkeit, mit der das Alpha-Weibchen Areale aufsuchte, in denen sich D gerade befand (Tab.3.26).

Tabelle 3.26 Korrelation zwischen der räumlichen Assoziation von Nachkommen zur Familie und der Häufigkeit, mit der die Eltern Areale aufsuchten, in denen sich gerade bestimmte Nachkommen aufhielten (erstes Beobachtungsjahr). Abkürzungen: $\mathrm{A}->\mathrm{C}=\mathrm{A}$ (das Alpha-Männchen) sucht Areale auf, in denen sich $\mathrm{C}$ aufhielt: $\mathrm{B}->\mathrm{C}=$ (das Alpha-Weibchen) sucht Areale auf, in denen sich $\mathrm{C}$ aufhielt, etc.; $\mathrm{n}=$ Anzahl der statistischen Tage, welche der Korrelationsanalyse zugrunde gelegt wurden.

\begin{tabular}{ccccc}
\hline Dyade & $\mathrm{n}$ & Spearman's $\mathrm{t}(\mathrm{n}-2)$ & Signifikanzniveau \\
\hline A -> C & 18 & 0,213026 & 0,872124 & $\mathrm{n} . \mathrm{s}$. \\
A -> D & 18 & 0,394790 & 1,718774 & $\mathrm{n} . \mathrm{s}$. \\
A -> E & 18 & 0,519882 & 2,434366 & $\mathrm{p}=0,027$ \\
A -> G & 18 & 0,130529 & 0,526621 & n.s. \\
A -> H & 18 & 0,012540 & 0,050163 & n.s. \\
B -> C & 18 & 0,309904 & 1,303805 & n.s. \\
B -> D & 18 & 0,478292 & 2,178506 & $\mathrm{p}=0,044$ \\
B -> E & 18 & 0,342411 & 1,457766 & n.s. \\
B -> G & 18 & 0,007874 & 0,031497 & n.s. \\
B -> H & 18 & 0,270407 & 1,123482 & n.s. \\
\hline
\end{tabular}

Für das zweite Beobachtungsjahr wurden Korrelationen zwischen der räumlichen Assoziation von $\mathrm{J}$ und von $\mathrm{M}$ zu den Familienmitgliedern und der Häufigkeit, mit der das AlphaMännchen Areale aufsuchte, in denen sich J oder M aufhielten, nachgewiesen. Es besteht ebenso eine Korrelation zwischen der Häufigkeit, mit der das Alpha-Weibchen Areale aufsuchte, in denen sich $\mathrm{M}$ befand und der räumlichen Assoziation von $\mathrm{M}$ zu den Familienmitgliedern (Tab.3.27).

In beiden Beobachtungsjahren bestehen Zusammenhänge zwischen der Aufsuchhäufigkeit bestimmter Nachkommen und deren Assoziation zur Familie (für das Alpha-Männchen in 3 von 12 Fällen und für das Alpha-Weibchen in 2 von 12 Fällen). Alle Korrelationen sind positiv, d.h., dass die Alpha-Tiere umso häufiger Areale aufgesucht haben, in denen sich bestimmte Nachkommen aufhielten, je enger die räumliche Assoziation dieser Nachkommen zur Familie war. Ob die Alpha-Tiere ihre Nachkommen häufiger aufsuchten, weil diese weniger peripher waren, oder ob die Nachkommen weniger peripher waren, weil die AlphaTiere sie aufsuchten, muss ungeklärt bleiben. Die Ergebnisse zeigen jedoch, dass das Alpha- 
Männchen bevorzugt männliche Nachkommen aufsuchte und das Alpha-Weibchen bevorzugt weibliche Nachkommen aufsuchte, wenn die jeweiligen Nachkommen mit der Familie räumlich enger assoziiert waren. Hypothese $6 f$ kann damit abgelehnt werden.

Tabelle 3.27 Korrelation zwischen der räumlichen Assoziation von Nachkommen zur Familie und der Häufigkeit, mit der die Eltern Areale aufsuchten, in denen sich gerade bestimmte Nachkommen aufhielten (zweites Beobachtungsjahr). Abkürzungen: A $->\mathrm{C}=\mathrm{A}$ (das Alpha-Männchen) sucht Areale auf, in denen sich $\mathrm{C}$ aufhielt: $\mathrm{B} \rightarrow \mathrm{C}=$ (das Alpha-Weibchen) sucht Areale auf, in denen sich $\mathrm{C}$ aufhielt, etc.; $\mathrm{n}=$ Anzahl der statistischen Tage, welche der Korrelationsanalyse zugrunde gelegt wurden.

\begin{tabular}{|c|c|c|c|c|}
\hline Dyade & $\mathrm{n}$ & Spearman's $r$ & $t(n-2)$ & Signifikanzniveau \\
\hline$A \rightarrow C$ & 17 & 0,211337 & 0,837420 & n.s. \\
\hline$A \rightarrow D$ & 17 & $-0,191904$ & $-0,757318$ & n.s. \\
\hline$A \rightarrow G$ & 17 & $-0,138941$ & $-0,543387$ & n.s. \\
\hline$A \rightarrow I$ & 7 & 0,396412 & 0,965507 & n.s. \\
\hline$A \rightarrow J$ & 17 & 0,514500 & 2,323814 & $p=0,034$ \\
\hline$A \rightarrow K$ & 17 & 0,348738 & 1,441128 & n.s. \\
\hline$A \rightarrow M$ & 17 & 0,587656 & 2,812943 & $p=0,013$ \\
\hline$B \rightarrow C$ & 17 & 0,180308 & 0,709966 & n.s. \\
\hline$B->D$ & 17 & $-0,102205$ & $-0,397922$ & n.s. \\
\hline$B->G$ & 17 & $-0,059480$ & $-0,230774$ & n.s. \\
\hline B $->$ I & 7 & 0,180187 & 0,409616 & n.s. \\
\hline$B->J$ & 17 & 0,300869 & 1,221877 & n.s. \\
\hline$B->K$ & 17 & 0,278567 & 1,123352 & n.s. \\
\hline$B \rightarrow M$ & 17 & 0,592594 & 2,849293 & $p=0,012$ \\
\hline
\end{tabular}

\subsubsection{Zusammenhänge zwischen der Häufigkeit, mit der Alpha-Tiere bestimmte} Nachkommen beobachteten und der Häufigkeit, mit der die Alpha-Tiere Areale aufsuchten, in denen sich diese Nachkommen aufhielten

Hypothese 6g: Es gibt keinen Zusammenhang zwischen der Häufigkeit, mit der die AlphaTiere bestimmte Nachkommen beobachteten und der Häufigkeit, mit der die Alpha-Tiere Areale aufsuchten, in denen sich diese Nachkommen aufhielten.

Unter der Annahme, dass die Alpha-Tiere gleichgeschlechtliche Nachkommen kontrollieren, die ihr Reproduktionsprivileg herausfordern, ist zu erwarten, dass sie diese Nachkommen auch häufiger beobachten, wenn sie diese aufsuchen. Es wurde daher untersucht, ob es Zusammenhänge zwischen der Häufigkeit gibt, mit der die Alpha-Tiere bestimmte Nachkommen aufsuchten und der Häufigkeit, mit der sie diese Nachkommen beobachteten.

Die Eltern unterschieden sich in den Experimenten des ersten Beobachtungsjahres nicht in der 
Häufigkeit, mit der sie ihre Nachkommen beobachteten. Da sich nach der ersten Kontrollphase nur noch fünf Nachkommen in der Familie befanden, deren Aufenthaltswahrscheinlichkeiten mit der Transponderanlage gelesen werden konnten (I und J hatten im ersten Beobachtungsjahr noch keinen Transponder-Chip), ist aufgrund der geringen Stichprobengröße keine Korrelationsanalyse zulässig.

Im zweiten Beobachtungsjahr beobachtete das Alpha-Männchen während des ersten Experiments den Sohn G und während des dritten Experiments den Sohn C häufiger als erwartet. Es kann jedoch weder für das Alpha-Männchen, noch für das Alpha-Weibchen eine Korrelation zwischen der Häufigkeit, mit der sie Areale aufsuchten, in denen sich bestimmte Nachkommen aufhielten und der Häufigkeit, mit der sie diese Nachkommen beobachteten gefunden werden (Tab.3.28 und 3.29). Hypothese $6 \mathrm{~g}$ kann daher nicht abgewiesen werden.

Tabelle 3.28 Korrelation zwischen der Häufigkeit, mit der das Alpha-Männchen Areale aufsuchte, in denen sich bestimmte Nachkommen aufhielten und der Häufigkeit, mit der er diese Nachkommen beobachtete (zweites Beobachtungsjahr). Abkürzungen: $\mathrm{n}=$ Anzahl der Nachkommen, die in die Analyse einbezogen wurden.

\begin{tabular}{cccc}
\hline Experiment & $\mathrm{n}$ & $\mathrm{R}$ & Signifikanzniveau \\
\hline E1 & 7 & 0,094 & $\mathrm{p}=0,841$ \\
E2 & 7 & 0,120 & $\mathrm{p}=0,797$ \\
E3 & 6 & 0,759 & $\mathrm{p}=0,080$ \\
E4 & 6 & $-0,132$ & $\mathrm{p}=0,803$ \\
\hline
\end{tabular}

Tabelle 3.29 Korrelation zwischen der Häufigkeit, mit der das Alpha-Weibchen Areale aufsuchte, in denen sich bestimmte Nachkommen aufhielten und der Häufigkeit, mit der sie diese Nachkommen beobachtete (zweites Beobachtungsjahr). Abkürzungen: $\mathrm{n}=$ Anzahl der Nachkommen, die in die Analyse einbezogen wurden.

\begin{tabular}{cccc}
\hline Experiment & $\mathrm{n}$ & $\mathrm{R}$ & Signifikanzniveau \\
\hline E1 & 7 & 0,487 & $\mathrm{p}=0,268$ \\
E2 & 7 & $-0,399$ & $\mathrm{p}=0,375$ \\
E3 & 6 & 0,412 & $\mathrm{p}=0,417$ \\
E4 & 6 & $-0,093$ & $\mathrm{p}=0,862$ \\
\hline
\end{tabular}




\subsubsection{Zusammenhang zwischen affiliativen Kontakten zwischen EItern und}

\section{Nachkommen und der Häufigkeit mit der die Nachkommen Hilfe bei der Jungtieraufzucht geleistet haben}

Hypothese 6h: Die Wahrscheinlichkeit, dass die Eltern versuchen, Rauswürfe von Nachkommen zu verhindern, ist unabhängig davon, ob sich die Nachkommen an der Jungtierfürsorge und am Scannen beteiligt haben.

Resultierend aus der Annahme (nach Gaston 1978), dass der Verbleib der Nachkommen in der Natalfamilie von den Nachkommen durch gruppendienliches Verhalten (z.B. Jungtierfürsorge und Scannen) erkauft werden muss, sind von den Eltern vermehrt affiliative Kontakte mit Nachkommen zu erwarten, die helfen (Lazaro-Perea et al. 2004). Es gibt für beide Eltern jedoch weder eine Korrelation zwischen der Wahrscheinlichkeit affiliativer Kontakte und der Jungtierfürsorge eines Nachkommen (Tab.3.30), noch eine Korrelation zwischen ihren affiliativen Kontakten und der Scanning-Häufigkeit der Nachkommen (s. Tab. 3.31). Hypothese 6h kann nicht abgewiesen werden.

Tabelle 3.30 Korrelation zwischen dem prozentualen Anteil der Jungtierfürsorge der Nachkommen und dem Anteil der affiliativen Kontakte der Eltern zu den Nachkommen an der Gesamtaktivität. Abkürzungen: A = Alpha-Männchen, B = Alpha-Weibchen; $\mathrm{n}=$ Anzahl der Nachkommen, die in die Analyse einbezogen wurden.

\begin{tabular}{ccccc}
\hline Eltern & $\mathrm{n}$ & Spearman's $\mathrm{t}$ & $\mathrm{t}(\mathrm{n}-2)$ & Signifikanzniveau \\
\hline $\mathrm{A}(2000)$ & 6 & $-0,273229$ & $-0,568075$ & n.s. \\
B (2000) & 6 & 0,576817 & 1,412258 & n.s. \\
A (2001) & 7 & $-0,684712$ & $-2,100762$ & n.s. \\
B (2001) & 7 & $-0,468487$ & $-1,185744$ & n.s. \\
\hline
\end{tabular}

Tabelle 3.31 Korrelation zwischen dem prozentualen Anteil der Scanning-Häufigkeit der Nachkommen und dem Anteil der affiliativen Kontakte der Eltern zu den Nachkommen an der Gesamtaktivität. Abkürzungen: A = Alpha-Männchen, B = Alpha-Weibchen; $\mathrm{n}$ = Anzahl der Nachkommen, die in die Analyse einbezogen wurden.

\begin{tabular}{ccccc}
\hline Eltern & $\mathrm{n}$ & Spearman's $\mathrm{t}(\mathrm{n}-2)$ & Signifikanzniveau \\
\hline A (2000) & 6 & $-0,600000$ & $-1,500000$ & n.s. \\
B (2000) & 6 & 0,257143 & 0,532181 & n.s. \\
A (2001) & 7 & $-0,392857$ & $-0,955258$ & n.s. \\
B (2001) & 7 & $-0,107143$ & $-0,240966$ & n.s. \\
\hline
\end{tabular}




\subsubsection{Kapiteldiskussion}

\subsubsection{Untersuchungen zur Familienstruktur}

Beim Vergleich der Experimentalphasen wurde deutlich, wie schwierig bei Krallenaffen die eindeutige Zuordnung der Familienmitglieder in Rangpositionen ist. Sade (1972) wendete seine Methode bei Macaca mulatta an und konnte über Grooming-Interaktionen eindeutige Dominanzhierarchien aufstellen. In Weißbüschelaffen-Gruppen sind die Alpha-Tiere über die Nachkommen und ältere in der Regel über jüngere Nachkommen dominant (Abbott 1984, Anzenberger 1983, Evans und Poole 1984, Sutcliffe und Poole 1984, Knox und Sade 1991 für S. imperator subgrisescens). Männchen und Weibchen sind nach Epple (1975) und Rothe $(1975,1978)$ in einer jeweils eigenen Hierarchie organisiert, so dass demnach die Darstellung von Rangpositionen in einer einzigen linearen Hierarchie nicht möglich ist.

In der vorliegenden Studie konnten aggressive Auseinandersetzungen zwischen männlichen und weiblichen Familienmitgliedern an den Futterplätzen, als auch in anderen Kontexten beobachtet werden. Anzenberger (1983) berichtet ebenfalls von seinen Gruppen in der Laborhaltung, dass keine nach Geschlechtern getrennten Rangordnungen vorlagen und fand in Futterrangtests gemischte Rangordnungen. Die Rangordnung an Futterplätzen stellt allerdings die Hierarchie der Familie nur in einem Kontext dar. In den Untersuchungen über das Dominanzverhalten von freilebenden Weißbüschelaffen beobachtete Digby (1995b) intra- und intergeschlechtliche Dominanz-Subordinations-Interaktionen. Sie fand, entgegen den Beschreibungen von Rothe (1975) und Epple (1975a) für C. jacchus in der Laborhaltung, keine getrennten Hierarchien für die einzelnen Geschlechter. In der vorliegenden Studie spiegelt die Hierarchie an den Futterplätzen mit wenigen Ausnahmen, in denen jüngere Familienmitglieder ältere dominieren, die Seniorität wieder.

Freilebende $C$. flaviceps-Alpha-Weibchen und Jungtiere (infantil) nehmen anderen Gruppenmitgliedern häufig das Futter weg (Ferrari 1987), was darauf hinweist, dass Jungtiere noch gar nicht in die Hierarchie am Futterplatz eingebunden sind. In beiden Beobachtungsjahren konnte jeweils eines von zwei Jungtieren dabei beobachtet werden, Familienmitglieder von Futterstellen zu vertreiben bzw. sich den Vorrang beim Fressen zu sichern. Es waren die Nachkommen I und M, die später aus der Familie ausgestoßen wurden. J wurde nach einer krankheitsbedingten einwöchigen Abwesenheit von der Familie ebenfalls rausgeworfen. Allerdings konnte dieser Rauswurf wegen des anthropogenen Einflusses nicht weiter bewertet werden. 
Sutcliffe und Poole (1984) stellten anhand dissoziativer Verhaltensweisen die Rangpositionen (status) der Familienmitglieder dar und fanden, dass sie von Grooming-Präferenzen unabhängig waren. Sie nehmen an, dass die Rangpositionen über dissoziatives Verhalten mit der Seniorität gleichgesetzt werden können (Ausnahme bei Zwillingen). Trotz höherer Seniorität hatte D z.B. eine niedrigere Grooming-Rangposition als ihr Bruder E. Die AlphaTiere, deren höchste Ränge in den Hierarchien ohne Zweifel mit der höchste Seniorität einhergingen (siehe Abbott 1984, Evans und Poole 1984, Sutcliffe und Poole 1984), haben in der ersten Kontrollphase niedrige Grooming-Rangpositionen. Die Ergebnisse sind demnach mit den Beobachtungen von Sutcliffe und Poole (1984) vereinbar.

Bei freilebenden Marmosettengruppen wurden aggressive Interaktionen nur selten beobachtet (Ferrari 1988a, Lazaro-Perea et al. 1997). Auch bei der Fokusfamilie wurde nur wenig dissoziatives Verhalten beobachtet. Garber (1997) vermutet, dass sich Konkurrenz bei Callitrichiden nicht durch aggressives Verhalten äußert, sondern über sozioendokrinologische Mechanismen und einer Reihe von affiliativen Verhaltensweisen, welche dem Individuum selbst und seiner Verwandtschaft Vorteile bringen. Die dissoziativen Handlungen männlicher Nachkommen gegenüber ihren Schwestern, wie sie in dieser Studie von den Brüdern C, E und G gegenüber D beschrieben wurden, konnte auch Heymann (1990b) bei S. mystax nachweisen. Intergeschlechtliche Aggression wurde derart gedeutet, dass Rauswürfe von Schwestern die Einwanderung nicht-verwandter Weibchen wahrscheinlicher machen, welche dann potentielle Reproduktionspartnerinnen für die männlichen Nachkommen darstellen. Dagegen ist jedoch für C. jacchus einzuwenden, dass die Familien gegenüber fremden Artgenossen geschlossen sind, d.h. nicht-verwandte Eindringlinge abgewehrt werden.

Die Untersuchungen zu Rangpositionen anhand der Häufigkeiten affiliativer Interaktionen zeigen den Einfluss dynamischer Prozesse im zeitlichen Zusammenhang mit Rauswürfen und der Anwesenheit von Locktieren auf die affiliativen Interaktionen zwischen den Familienmitgliedern. Im ersten Beobachtungsjahr traten in der zweiten Kontrollphase (B2) - also nach dem Rauswurf von F - als auch während der Präsentation des ersten Lockweibchens (C1) Unterschiede zwischen den Familienmitgliedern in den Rangpositionen (ermittelt anhand der Häufigkeiten affiliativer Interaktionen) auf. E (Rauswerfer von F) hatte in B2 eine niedrigere Rangposition als die anderen Familienmitglieder. Im zweiten Jahr unterschieden sich die Familienmitglieder in ihren Rangpositionen während der Hüttenphase und der ersten zwei Experimente. Während dieser Phasen gab es eine Häufung der dissoziativen Interaktionen in der Familie. Nach dem Rauswurf von I im zweiten Experiment wiesen alle Familien- 
mitglieder wieder die gleichen Rangpositionen auf. Es gab auch unter veränderten Experimentalbedingungen keine Änderungen mehr. Die Vermutung liegt daher nahe, dass normalerweise alle Familienmitglieder affiliativ miteinander interagieren und Unterschiede in den Rangpositionen, die anhand der Häufigkeiten von affiliativen Interaktionen ermittelt werden, auf Spannungen (dissoziativen Interaktionen) der Mitglieder hinweisen, die möglicherweise zu Rauswürfen einzelner Tiere aus der Familie führen. Die Unterschiede in den Rangpositionen im ersten Beobachtungsjahr weisen auf einen Einfluss des Lockweibchens auf die Gruppendynamik hin, auch wenn dies nicht zu Veränderungen der Gruppengröße führte.

Nachdem ein Tier aus der Familie geworfen worden war, konnten mit der Clique-Analyse keine Grooming-Cliquen ausgemacht werden. Das Ausbleiben von Cliquen ist damit zu erklären, dass sich die Stabilität der sozialen Beziehungen bei Veränderungen der Familienzusammensetzung auflöst und neu geordnet oder etabliert werden muss. Eine Weißbüschelaffenfamilie reagiert daher besonders sensibel auf Rauswürfe. Nach einer Geburt zeigten sich in den Grooming-Cliquen der Fokusfamilie keine deutlichen Reaktionen. Es ließen sich aber Veränderungen der Rangpositionen, die anhand der Häufigkeiten affiliativer Interaktionen ermittelt wurden, nachweisen (Experimente 1 und 2 im zweiten Jahr). Direkt vor Rauswürfen waren häufig dissoziative Interaktionen zu beobachten, die nach den Rauswürfen an Schärfe verloren, d.h. die Handlungen waren weniger aggressiv und seltener, wurden jedoch nicht vollkommen eingestellt. Bei der Präsentation des Lockweibchens im ersten Experiment des ersten Jahres war A auffällig häufig an Grooming-Cliquen, vor allem mit C, beteiligt, was in den anderen Phasen nicht beobachtet wurde. Die Intensivierung der Grooming-Interaktionen von A zu seinen ältesten Nachkommen könnte demnach durch die Anwesenheit des Lockweibchens ausgelöst worden sein und dazu dienen, die wichtigsten Helfer an die Familie zu binden. Während der Anwesenheit des einzelnen Lockmännchens in E4 war A in zwei Grooming-Cliquen mit seinem nächst jüngeren Sohn G, was zuvor in den Experimenten 2 und 3 bei der Präsentation der zwei Lockmännchen nicht der Fall war. Da das Lockmännchen keinen Emigrationsanreiz für die Söhne darstellte, kann die Bindung des adulten Sohnes G an die Familie keine Begründung für die häufigen Grooming-Interaktionen zwischen A und $\mathrm{G}$ sein. Möglicherweise ist das Verhalten jedoch auf G's Krankheit im vierten Experiment zurückzuführen, da sich einige Familienmitglieder (vor allem A und K) dem Kranken besonders häufig zuwandten, mit ihm kuschelten und ihn groomten. Die besondere Zuwendung zu kranken Gruppenmitgliedern wurden auch von Macdonald und Moehlmann 
(1983) bei kooperativ lebenden Caniden berichtet.

\subsubsection{Einfluss der Locktiere auf die Beziehungen der Geschwister}

Harcourt (1978) und Pusey und Packer (1987) nennen die sexuelle Attraktion von Artgenossen als einen der Gründe für Emigrationen bei Primaten. Der Zweck der Präsentation von Locktieren in dieser Studie war, den adulten (und subadulten) Nachkommen der Fokusfamilie eine solche sexuelle Attraktion zu bieten, um die Auswirkungen potentieller Paarungspartner auf die räumliche Assoziation und die affiliativen und dissoziativen Interaktionen von Brüdern, Schwestern und Brüdern und Schwestern zu testen und gegebenenfalls Emigrationen aus der Natalfamilie zu provozieren. Es wurde hypothetisiert, dass die Brüder bei der Anwesenheit von Lockweibchen die Anzahl ihrer affiliativen Interaktionen steigern würden, um Emigrationsgemeinschaften $\mathrm{zu}$ bilden. Keines der Familienmitglieder verließ jedoch das heimatliche Streifgebiet, obwohl insbesondere E vergleichsweise großes Interesse am Lockweibchen gezeigt hatte (siehe 3.1.5). Falls E aufgrund eines hohen Prädationsrisikos nicht alleine emigriert war, so hätte die gemeinsame Emigration mit einem oder mehreren Geschwistern das Risiko minimiert (Baker 1991, Garber 1994, Kretzer 1996, Pusey und Packer 1987, Sugiyama 1976). Die Vorteile der CoEmigration von Brüdern (Garber 1994) bestehen neben der Minderung des Prädationsrisikos zusätzlich in der Möglichkeit, eine fraternale polyandrische Sozietät zu etablieren (Kretzer 1996; Rothe et al. 2003). Die bei Brüdern beobachteten Co-Emigrationen (Baker 1991 für Leontopithecus, Garber 1994 und Goldizen und Terborgh 1996 für Saguinus, Scanlon et al. 1988 für C. jacchus) wurden jedoch auch bei Schwestern dokumentiert (Ferrari 1988a, Ferrari und Lopes Ferrari 1989, Nievergelt et al. 2000, Digby und Ferrari 1994 für C. jacchus). Es bleibt die Frage, warum keine Co-Emigration der Brüder stattfand, als im ersten Jahr Lockweibchen präsentiert wurden. Die von Baker (1991) beschriebenen Co-Emigrationen geschahen von Brüdern, deren Altersunterschied nicht mehr als ein Jahr betrug. Daraus resultierten vor allem Reproduktionsvorteile für das dominante, also im Regelfall für das ältere der Geschwister.

Als das Lockweibchen im ersten Experiment der Familie J18 präsentiert wurde, führte dies nicht zu einer Steigerung der affiliativen Interaktionen zwischen den Brüdern, sondern zur Verringerung der affiliativen Kontakte. Die räumliche Assoziation der Brüder zueinander veränderte sich nicht. Es gab somit keinen Hinweis darauf, dass Brüder infolge eines Emigrationsanreizes Migrationsgemeinschaften bildeten. Allenfalls weisen die Beob- 
achtungen auf eine Destabilisierung der Bruder-Beziehungen.

Für E wäre sein Bruder C nach dem Modell 1.2 kein optimaler Emigrationspartner gewesen, da dieser drei Jahre älter als er war, und in der Hierarchie an den Futterplätzen war C über E dominant. Der jüngere Bruder $G$ war noch adoleszent und für eine Abwanderung aus der Familie zu jung. Der ideale Emigrationspartner hätte für E sein subadulter Bruder F sein können, den er jedoch vor Beginn des Experiments aus der Familie verjagt hatte. Möglicherweise gab es für E keinen geeigneten Emigrationspartner. C hätte aufgrund seines höheren Alters von einer Emigrationsgemeinschaft mit E mehr profitieren können. Er war nach dem Alpha-Männchen das älteste Männchen in der Familie ( $\beta$-Position) und zeichnete sich neben den Eltern als Hauptjungtierträger aus (vgl. 3.2.4.3), so dass sich seine Investitionen in die Aufzucht der jüngeren Geschwister durch deren Überleben auszahlte. $\beta$ Tiere haben eine stabile soziale Position innerhalb einer Familie und fungieren häufig als Bindeglieder zwischen den Subgruppen. Rauswürfe oder Entfernungen von $\beta$-Tieren aus ihren Natalfamilien führen häufig zu Kreislaufschocks der $\beta$-Tiere (Rothe 1978). Aus diesen Gründen könnte man vermuten, dass $C$ aufgrund seiner $\beta$-Position nicht freiwillig emigrierte. Das von Radespiel (1990) gezeigte häufige Orientierungsverhalten des ältesten Sohnes gegenüber gruppenfremden Artgenossen konnte bei $\mathrm{C}$ ebenfalls nicht beobachtet werden, so dass das Verhalten von C eher mit den Beobachtungen von Geyer (1993) übereinstimmt. Von dem neun Monate alten G wurde erwartet, sich in seiner Alterklasse soziale Kompetenz anzueignen und noch nicht aus der Familie zu emigrieren, da der eigene Aufzuchtserfolg durch Erlernen sozialer Kompetenz in kompletten Familien entscheidend positiv beeinflusst wird (Tardif et al. 1984; Rothe und Darms 1993). Der Verbleib von G in der Familie entsprach damit der Erwartung.

Während der Präsentation der beiden Lockmännchen kam es zum Rauswurf von I. Die Veränderungen der räumlichen Assoziation zwischen den Brüdern vor dem Rauswurf von I lassen sich nicht eindeutig auf die Anwesenheit der Locktiere zurückzuführen, da zur gleichen Zeit Jungtiere geboren worden waren und das Alpha-Weibchen in den Postpartum-Östrus kam.

Die affiliativen Kontakte und räumliche Assoziation zwischen den Schwestern veränderten sich bei der Präsentation der Lockweibchen nicht, obwohl D während des ersten Experiments ein größeres Interesse an dem Lockweibchen gezeigt hatte als ihre adoleszente Schwester H. Da das Lockweibchen für die Schwestern keine sexuelle Attraktion darstellte, war weder eine Steigerung der affiliativen Interaktionen und räumlichen Assoziation zwischen den 
Schwestern (als Hinweis auf die Rekrutierung von Emigrationspartnern), noch vermehrt dissoziatives Verhalten (als Hinweis auf Konkurrenz) zu erwarten gewesen.

Im zweiten Jahr wurden während der Experimente E2 und E3 zwei Lockmännchen und in E4 ein einzelnes Lockmännchen präsentiert. Es wurde erwartet, dass bei der ältesten Tochter Abwanderungstendenzen bei der Präsenz der Männchen deutlich würden, was jedoch nicht geschah. Warum D nicht aus der Familie emigrierte, wurde in 3.2.6.1 diskutiert. Die affiliativen Interaktionen und räumliche Assoziation zwischen D und ihren adoleszenten Schwestern K und M zeigten keine Veränderung durch die Anwesenheit der Lockmännchen. Nach Garber et al. (1993) und Goldizen et al. (1996) tendieren weibliche Tamarine dazu, allein zu emigrieren, während Co-Emigrationen von Schwestern nur bei Callithrix jacchus beobachtet wurden (z.B. Nievergelt et al. 2000, Digby und Ferrari 1994). Vorausgesetzt, dass D Emigrationsabsichten hatte, wären $\mathrm{K}$ und $\mathrm{M}$ als Emigrationspartnerinnen zu jung gewesen, als dass ein Rekrutierungsversuch durch gesteigerte affiliative Interaktionen und engere räumliche Assoziation zu erwarten gewesen wäre.

Die räumliche Assoziation zwischen Brüdern und Schwestern nahm in Anwesenheit der Lockweibchen nicht ab. Im zweiten Experiment C2 war die Assoziation der Brüder zu D sogar höher als in den vorhergehenden Phasen. Demnach liegt die Vermutung nahe, dass die Präsenz potentieller Paarungspartnerinnen Männchen nicht dazu veranlasst, sich räumlich von verwandten Weibchen zu entfernen. Das aktive Lockweibchen war in C1 präsentiert worden, während sich das Lockweibchen in C2 so gut wie gar nicht zeigte. Die engere räumliche Assoziation von Brüdern und Schwestern im zweiten Experiment konnte daher nicht auf die Anwesenheit des Lockweibchens zurückgeführt werden. Eine mögliche Erklärung liegt in der Jahreszeit, da das letzte Experiment im späten September bzw. Anfang Oktober lag. Durch die kühleren Temperaturen und Regenschauer hielten sich die Affen wieder vermehrt nahe beieinander auf (in Kuschelgruppen), so dass sowohl die räumliche Assoziation, als auch die Häufigkeit der affiliativen Kontakte in dieser Phase anstieg. G war mit D weniger eng räumlich assoziiert als seine Brüder. Ebenso war die Assoziation zwischen $\mathrm{G}$ und $\mathrm{H}$ geringer als zwischen H und den anderen Brüdern. Da G im ersten Beobachtungsjahr besonders häufig peripher von der Familie war, lässt sich damit auch seine geringere Assoziation mit den Schwestern erklären. Die durchweg selteneren affiliativen Kontakte zwischen Brüdern und Schwestern in Experiment C1 wurden auch bei den Bruder-Dyaden und Schwester-Dyaden beobachtet, so dass alle dyadischen affiliativen Kontakte verringert waren, als die Lockweibchen präsentiert wurden. 
Eine verringerte Assoziation zwischen Brüdern und Schwestern im zweiten Beobachtungsjahr trat zeitgleich einerseits mit der Präsenz zweier Lockmännchen (Experiment 2) und andererseits mit Spannungen zwischen D, I und G auf. Da die Lockmännchen im dritten und vierten Experiment ebenfalls anwesend waren und hier die Assoziation zwischen Brüdern und Schwestern enger war als im zweiten Experiment, bleibt unklar, ob die Anwesenheit der Lockmännchen für die verringerte Assoziation im zweiten Experiment verantwortlich war. Dennoch schienen die Bruder-Schwester-Beziehungen von den Lockmännchen nicht unbeeinflusst zu bleiben, da zwischen D und I im zweiten Experiment dissoziatives Verhalten, insbesondere bei der Kommunikation mit den Lockmännchen, beobachtet wurde. Indem I seine ältere Schwester regelmäßig verfolgt und beobachtet hatte, wenn diese Kontakte zu den Locktieren suchte, verhinderte er eine ungestörte Begegnung zwischen D und dem fremden Männchen. Da D offensichtlich versucht hatte, I mit Keckern, kurzen Angriffen und Jagden aus dem für die Kommunikation mit Locktieren bevorzugten Areal 6 zu vertreiben, war ihr seine Anwesenheit vermutlich hinderlich. Extra-Paar-Kopulationen (EPC) oder ExtraGruppen-Kopulationen (EGC) wurden von Yamamoto et al. (2001) und Digby (1999) im Freiland bei C. jacchus bei beiden Geschlechtern beobachtet. In Digby's Beschreibungen geschahen die Kopulationen häufig versteckt bei Begegnungen zweier Gruppen an den Streifgebiet-Grenzen und wurden sofort abgebrochen, wenn ein Mitglied einer der Gruppen dazu kam. Digby sah die Vorteile der EGC's für Weibchen in einer vergrößerten Auswahl an Paarungspartnern und damit einhergehend eine Qualitätsverbesserung der väterlichen Gene, ohne die Investitionen in den derzeitigen Partner zu vernachlässigen. Da D in ihrer Familie nicht reproduktiv war, kann diese Erklärung nicht direkt für sie gelten. Wahrscheinlicher ist jedoch, dass sie ihren Bruder I von der Streifgebietsgrenze vertreiben wollte, um Kontakte mit fremden Männchen herzustellen, möglicherweise auch zu kopulieren und selber reproduktiv zu werden.

Am Ende des ersten Experiments, kurz vor dem Rauswurf von I, wurden Jungtiere geboren, und das Alpha-Weibchen kam während des zweiten Experiments in den Postpartum-Östrus. Gemeinsam mit dem Alpha-Männchen verhinderten vor allem die männlichen Nachkommen (durch Büschelklappen und Schlitzaugen-machen gegenüber D und indem sie sich D in den Weg stellten) die Annäherung von $\mathrm{D}$ zu den Jungtieren. Da die Verminderungen der affiliativen Interaktionen und räumlichen Assoziation zwischen den Brüdern und D im zweiten Experiment entstanden, konnte dieser Aspekt Auswirkungen auf das Verhältnis der Brüder mit D haben. 


\subsubsection{Einfluss der Eltern auf die gruppendynamischen Prozesse}

Die insgesamt gleich große Beteiligung von Eltern und Geschwistern an Rauswürfen von Gruppenmitgliedern verdeutlicht, dass sowohl die reproduktiven, als auch die nichtreproduktiven Gruppenmitglieder an den dynamischen Prozessen beteiligt sind. Dabei agieren bei den Weibchen vorwiegend die Alpha-Tiere und bei den Männchen überwiegend die männlichen Geschwister. Ähnliche Beobachtungen machten auch Mc Grew (1997) und Tardif und Carlson (1987). Da die Alpha-Tiere vorwiegend Nachkommen des eigenen Geschlechts rauswerfen, ergibt sich der Hinweis, dass die Rauswürfe aufgrund von sexueller Konkurrenz motiviert sind. Die Beteiligung des Alpha-Männchens am Rauswurf von E ist unter dieser Betrachtung nachvollziehbar, da E häufiges sexuelles Interesse (Genitalkontrollen und Urinlecken) an dem Alpha-Weibchen gezeigt hatte. Da das Alpha-Männchen häufiger Areale aufsuchte, in denen sich E gerade befand, liegt die Vermutung nahe, dass das AlphaMännchen seinen Sohn E kontrollierte, wobei er E jedoch nicht häufiger als erwartet beobachtete. Da die Korrelation von Aufsuchhäufigkeit und räumlicher Assoziation von E zur Familie positiv war, gibt es keinen Hinweis auf eine Rückholtaktik von A gegenüber E. A vertrieb seinen Sohn letzten Endes sogar aus der Familie, womit die Vermutung unterstützt wird, dass das häufige Aufsuchen von E nicht als Rückholtaktik interpretiert werden kann.

Es wurde beobachtet, dass Alpha-Weibchen ihre Töchter aus der Gruppe werfen, wenn diese ovulieren und damit zu Konkurrentinnen ihrer Mütter werden (z.B. Snowdon und Pickhard 1999). Eine Erklärung für die Beteiligung des Alpha-Weibchens B am Rauswurf ihrer jüngsten Tochter $\mathrm{H}$ ist damit jedoch nur ungenügend beantwortet, da es bisher keine Hinweise darauf gibt, dass auch jüngere Töchter, deren ältere Schwestern in der Gruppe anwesend sind, ovulieren (Abbott 1984, Saltzman et al. 1997a). Die nächste Konkurrentin der Mutter wäre die älteste Tochter D gewesen, mit welcher sie jedoch gemeinsam $\mathrm{H}$ aus der Familie warf. Infolgedessen muss in Betracht gezogen werden, dass die Anwesenheit von D der Mutter Vorteile bot. Da reproduktive Krallenaffen von der Hilfeleistung älterer Nachkommen mehr profitieren als von jüngeren Nachkommen besteht die Strategie eventuell darin, dass im Falle von dynamischen Prozessen die jüngsten geschlechtsreifen Nachkommen vertrieben werden, um die erfahreneren Nachkommen als nicht-reproduktive Helfer in der Gruppe zu halten (siehe Price 1991). B suchte D in beiden Jahren auch auffällig häufig auf, woraus ein besonderes Interesse der Mutter an D geschlossen werden kann. Eine mögliche Erklärung wäre die Kontrolle der Tochter, um eingreifen zu können, falls deren Verhalten sich für die Mutter nachteilig ausgewirkt hätte. Eine andere Erklärung könnte auch darin liegen, dass B in 
der Gegenwart ihrer Tochter von deren Fähigkeiten (Kenntnis über gute Nahrungsplätze, Schlafplätze, Raubfeindvermeidung etc.) profitierte und ihr deshalb häufig folgte. Gerade während der Schlafplatzsuche konnte häufig beobachtet werden, dass die Familie D nachlief, wenn diese einen aufgesuchten Schlafkasten wieder verließ (ad libitum-Beobachtungen). Geht man davon aus, dass Fitness-Optimierungen der Töchter auch der Mutter Fitness-Vorteile bringen, „kooperierte“ B möglicherweise deshalb mit D, um ihre Chancen als Erbin der breeding-position zu verbessern. So stand die Beteiligung am Rauswurf von $\mathrm{H}$ eventuell nicht mit direkter Konkurrenz von H zur Mutter in Verbindung. Eine dritte Erklärung ergibt sich aus Beobachtungen, in denen Alpha-Weibchen den Kämpfen mit ihren Töchtern unterlagen (Rothe pers. Mtlg.). Möglicherweise wagte das Alpha-Weibchen keine Auseinandersetzung mit der ältesten Tochter, um eine Niederlage zu vermeiden.

Schwerer zu interpretieren war die Intervention des Vaters während des Rauswurfes von H. Da H keine sexuelle Konkurrenz für den Vater darstellte, hätte dieser von ihrem Ausscheiden aus der Familie keine reproduktiven Vorteile erlangt. Dass er jedoch den Angreiferrinnen drohte lässt die Schlussfolgerung zu, dass er aus der Anwesenheit von H in der Familie Vorteile erzielte. Die Vorteile lagen möglicherweise im Erhalt der Gruppengröße.

\subsection{Untersuchungen zur Emigrationsneigung anhand der Aufenthaltsorte und räumlichen Assoziation der Familienmitglieder}

Hypothese 7: Die Arealnutzung ist gleichmäßig und weder von den Experimenten noch von den Familienmitgliedern abhängig.

\subsubsection{Arealnutzung der Familie J18}

Zur Feststellung von Peripheralisierungen einzelner Individuen ist es notwendig, die Aufenthaltspräferenzen der Familie und der Familienmitglieder zu untersuchen. Nutzten Nachkommen häufig bestimmte Areale, die von der Familie nie oder nur sehr selten aufgesucht wurden, waren sie mit der Familie locker assoziiert und gelten als peripher (Geyer 1993). Je häufiger sich die Nachkommen mit anderen Familienmitgliedern gleichzeitig in denselben Arealen aufhielten, desto enger war ihre räumliche Assoziation. Eine enge Assoziation zwischen den Fokustieren beruht nicht zwangsläufig auf einer persönlichen Anziehung, sondern kann auch darin begründet liegen, dass zwei Tiere denselben Ort bevorzugen (Radespiel 1990). Die Analyse der Aufenthaltswahrscheinlichkeiten wird für die 
Kontrollphasen und alle Experimente durchgeführt, so dass von den Experimenten unabhängige Präferenzen für bestimmte Areale ermittelt werden können.

Hypothese 7a: Alle sieben Areale werden von der Familie J18 gleich häufig genutzt.

Hypothese 7b: Die Arealnutzung der Familie J18 ist von den verschiedenen Experimenten unabhängig.

\subsubsection{Arealnutzung der Familie J18 im ersten Beobachtungsjahr}

Während der ersten Kontrollphase des ersten Beobachtungsjahres nutzte die Fokusfamilie J18 in ihrem Streifgebiet am häufigsten Areal 4, in dem sich auch die Schutzhütte befindet, in der die Familie im Winter untergebracht war. Am zweithäufigsten wurde Areal 3 genutzt, gefolgt von den Arealen 2 und 5. Die Areale 1 und 6 nutzte die Familie am wenigsten (Abb. 3.107). Als die Nachbarfamilie in ihr Streifgebiet gebracht wurde (Kontrollphase 2), nutzte die Fokusfamilie vermehrt Areal 5 (und Areal 6), welche der Nachbarfamilie zugewandt liegen. Insgesamt wurden die Areale 3, 4 und 5 mit einer größeren Wahrscheinlichkeit genutzt als die Areale 1, 2 und 6 (Abb. 3.108). Während des ersten Experiments nutzte die Familie die Areale 2 bis 5 mit einer gleich großen Wahrscheinlichkeit, während Areal 1 und 6 wieder seltener betreten wurden. Während des ersten Experiments hielt sich die Familie auch in Areal 7 auf (in allen anderen Phasen wurde Areal 7 von der Gruppe nicht genutzt). Die Aufenthaltswahrscheinlichkeit war jedoch geringer als in den Arealen 1 bis 6 (Abb. 3.109). Im zweiten Experiment gab es Unterschiede in der Arealnutzung. Da für die statistische Bearbeitung keine Varianzanalyse zulässig ist, kann nicht ermittelt werden, welche Areal häufiger genutzt wurden als andere. Der Aufenthalt konzentrierte sich auf die Areale 3 und 4, während die Areale 1, 5 und 6 eher gemieden wurden. Areal 7 wurde gar nicht genutzt (Abb. $3.110)$. 


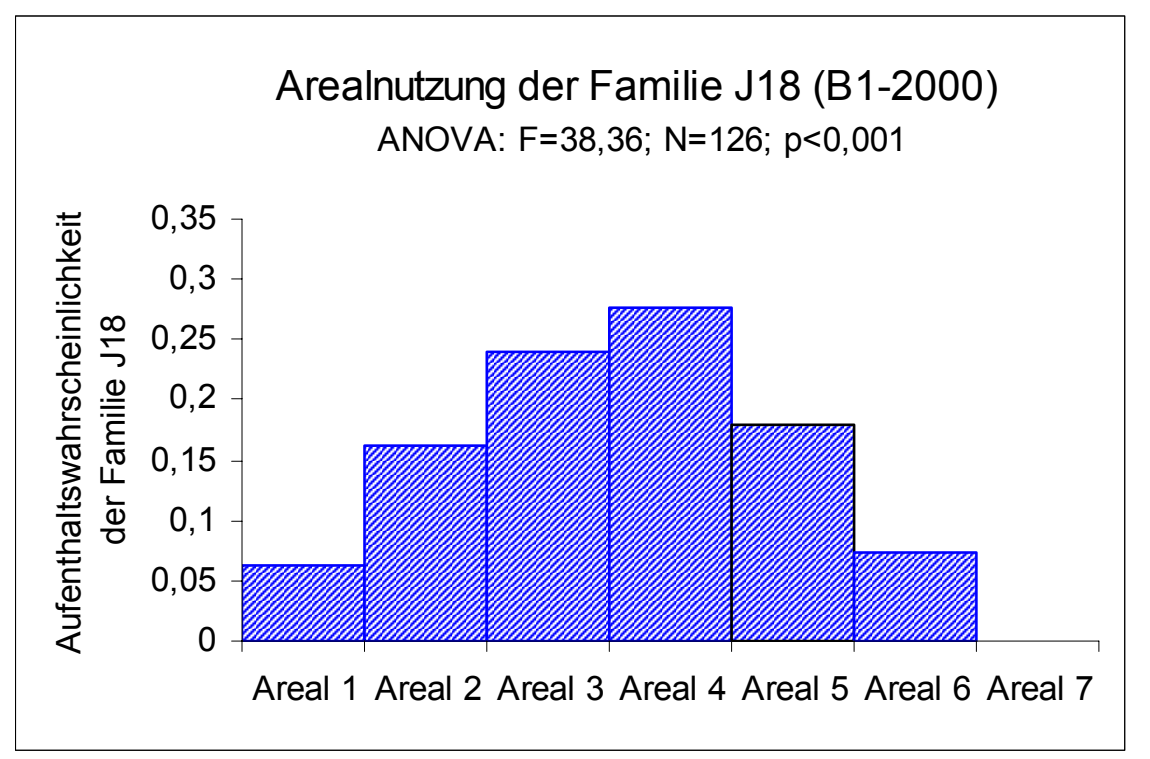

Abb. 3.107 Arealnutzung der Familie J18 in der ersten Kontrollphase des ersten Beobachtungsjahres (ANOVA, $\mathrm{F}=38,36 ; \mathrm{N}=126 ; \mathrm{p}<0,001)$. Die Familie J18 nutzte die Areale 2 und 5 mit einer größeren Wahrscheinlichkeit als die Areale 1 und 6, Areal 3 mit einer größeren Wahrscheinlichkeit als die Areale 2 und 5 und das Areal 4 mit einer größeren Wahrscheinlichkeit als Areal 3. Areal 7 wurde von der Familie nicht aufgesucht.

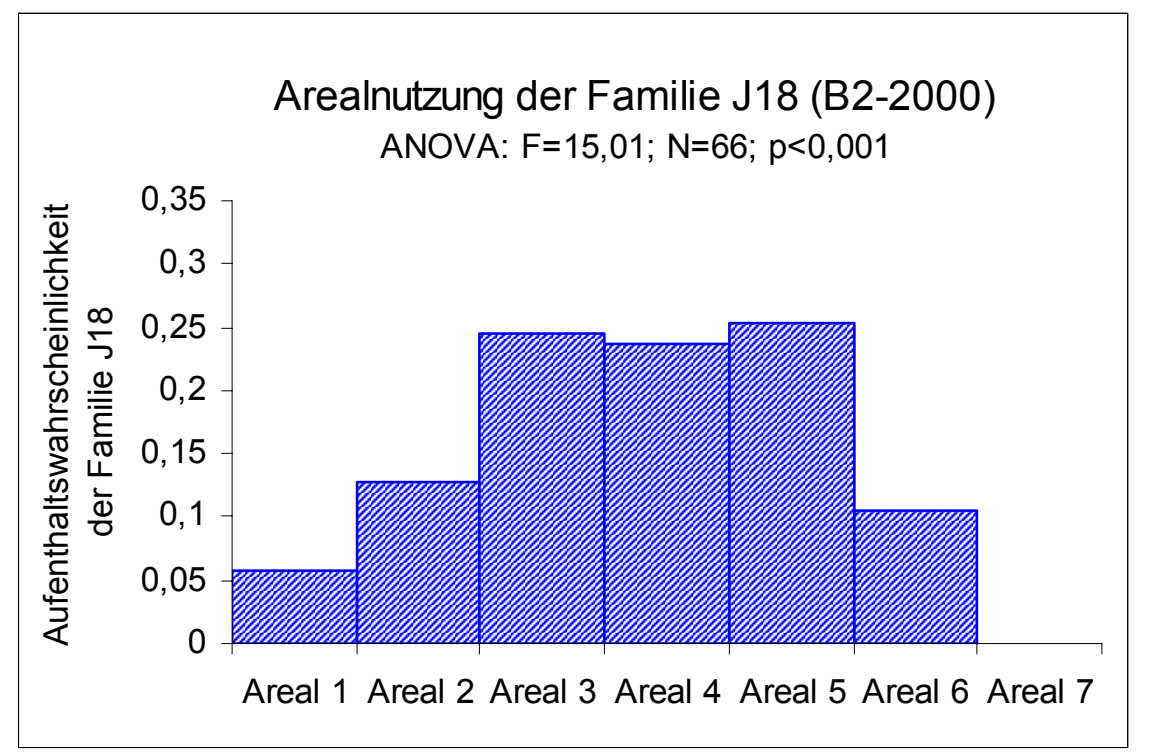

Abb. 3.108 Arealnutzung der Familie J18 in der zweiten Kontrollphase des ersten Beobachtungsjahres (ANOVA, $\mathrm{F}=15,01 ; \mathrm{N}=66 ; \mathrm{p}<0,001$ ). Die Familie J18 nutzte die Areale 3, 4 und 5 mit einer größeren Wahrscheinlichkeit als die Areale 1, 2 und 6. Areal 7 wurde von der Familie nicht aufgesucht. 


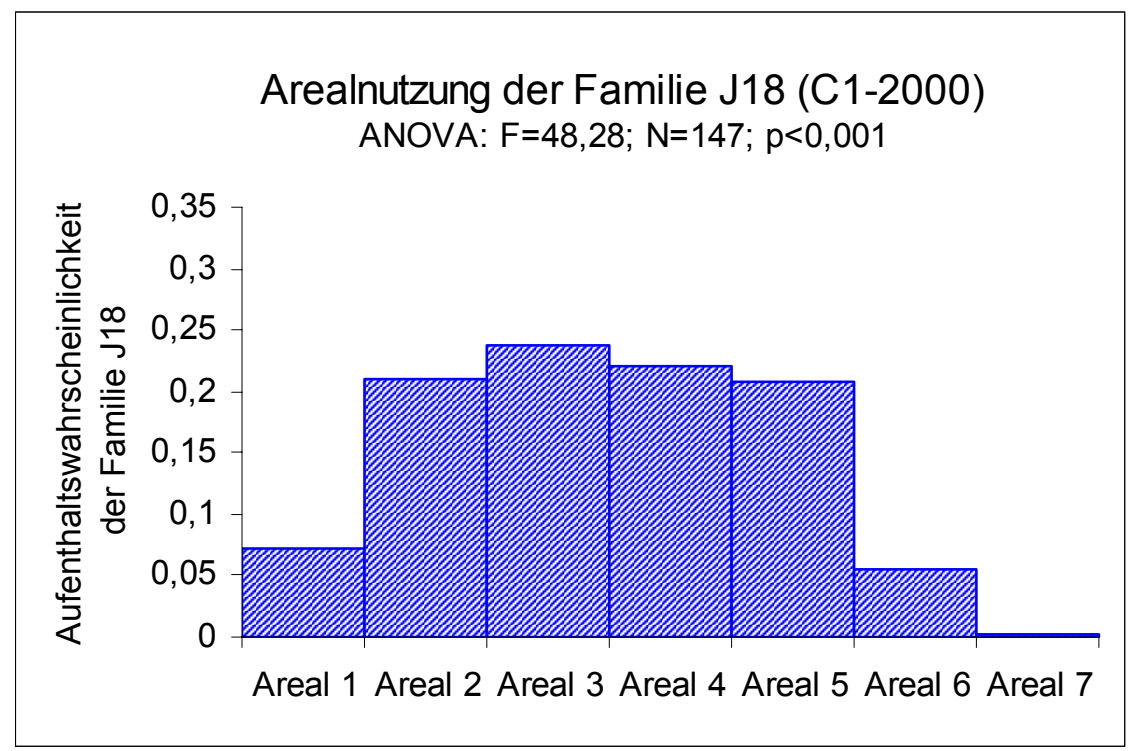

Abb. 3.109 Arealnutzung der Familie J18 im ersten Experiment des ersten Beobachtungsjahres (ANOVA, $\mathrm{F}=48,28 ; \mathrm{N}=147 ; \mathrm{p}<0,001)$. Während des ersten Experiments nutzte die Familie die Areale 2, 3, 4 und 5 mit einer gleich großen Wahrscheinlichkeit, während Areal 1 und 6 wieder seltener betreten wurden. Areal 7 wurde mit einer geringeren Wahrscheinlichkeit aufgesucht als die anderen Areale.

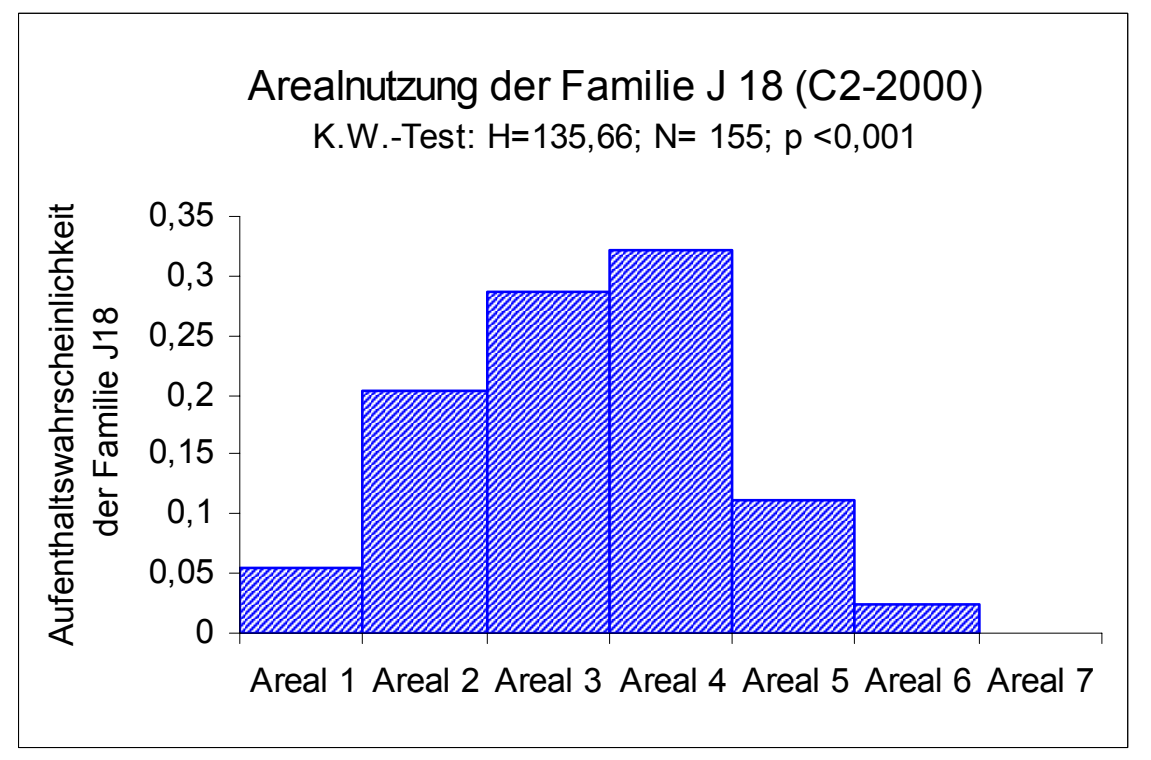

Abb. 3.110 Arealnutzung der Familie J18 im zweiten Experiment des ersten Beobachtungsjahres (KruskalWallis-Test, $\mathrm{H}=135,67 ; \mathrm{N}=155 ; \mathrm{p}<0,001)$. Es gab Unterschiede in der Arealnutzung der Familie J18. Areal 7 wurde von der Familie nicht aufgesucht.

Während des ersten Beobachtungsjahres hielt sich die Familie unterschiedlich häufig in einigen Arealen auf. Areal 1 wurde während aller Phasen gleich häufig genutzt (ANOVA, $\mathrm{F}=1,31 ; \mathrm{N}=481 ;$ n.s.). Die Aufenthaltswahrscheinlichkeit in Areal 2 war in der zweiten gegenüber der ersten Kontrollphase und der Experimente vermindert (Abb. 3.111). In Areal 3 war die Aufenthaltswahrscheinlichkeit während des zweiten Experiments im Verhältnis zu den vorhergehenden Phasen erhöht (Abb. 3.112). Areal 4 wurde von der Familie während der ersten Kontrollphase mit einer größeren Wahrscheinlichkeit als während des ersten 
Experiments genutzt. Im zweiten Experiment hielt sich die Familie in Areal 4 häufiger auf als während der drei Phasen vorher (Abb. 3.113). In Areal 5 hielt sich die Familie während der zweiten Kontrollphase (B2) mit einer höheren Wahrscheinlichkeit auf als während der anderen Phasen. Während des zweiten Experiments war die Aufenthaltswahrscheinlichkeit der Familie in Areal 5 geringer als in den übrigen Phasen (Abb. 3.114).

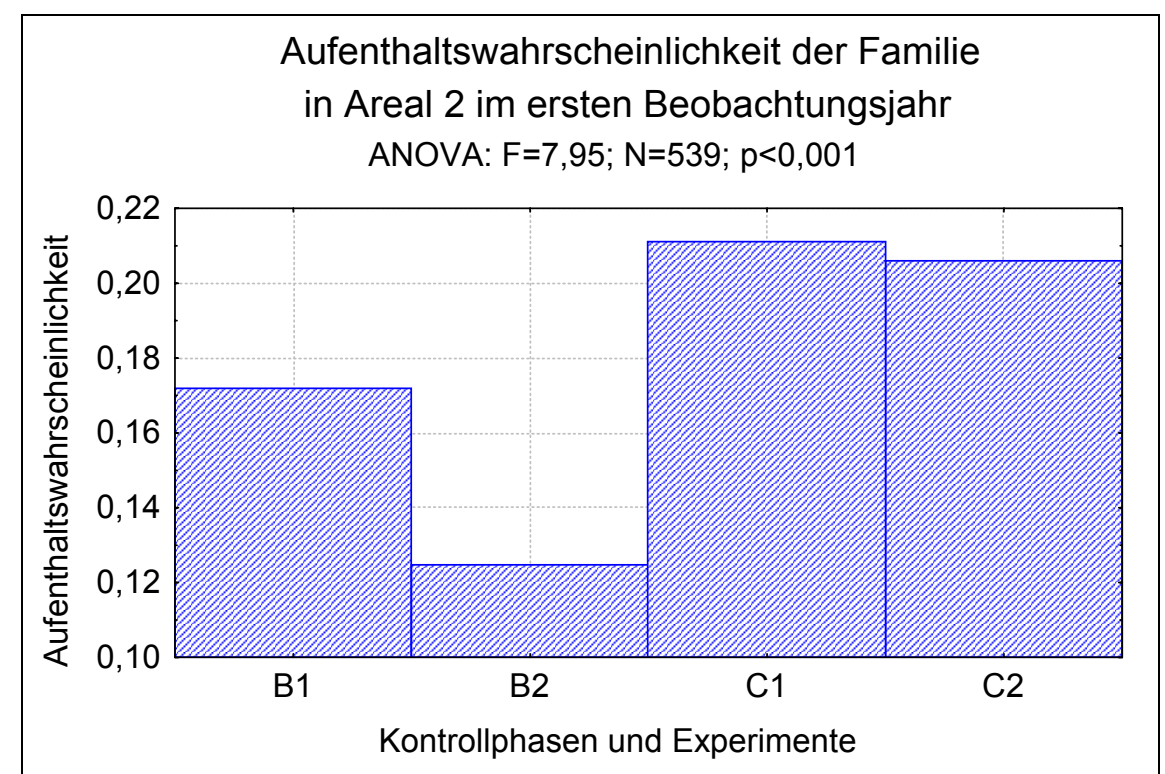

Abb. 3.111 Aufenthaltswahrscheinlichkeit der Familie in Areal 2 während der vier Phasen im ersten Beobachtungsjahr (ANOVA, F=7,95; N=539; $<<0,001$ ). Während der zweiten Kontrollphase (B2) wurde Areal 2 seltener genutzt als während der anderen Phasen. 


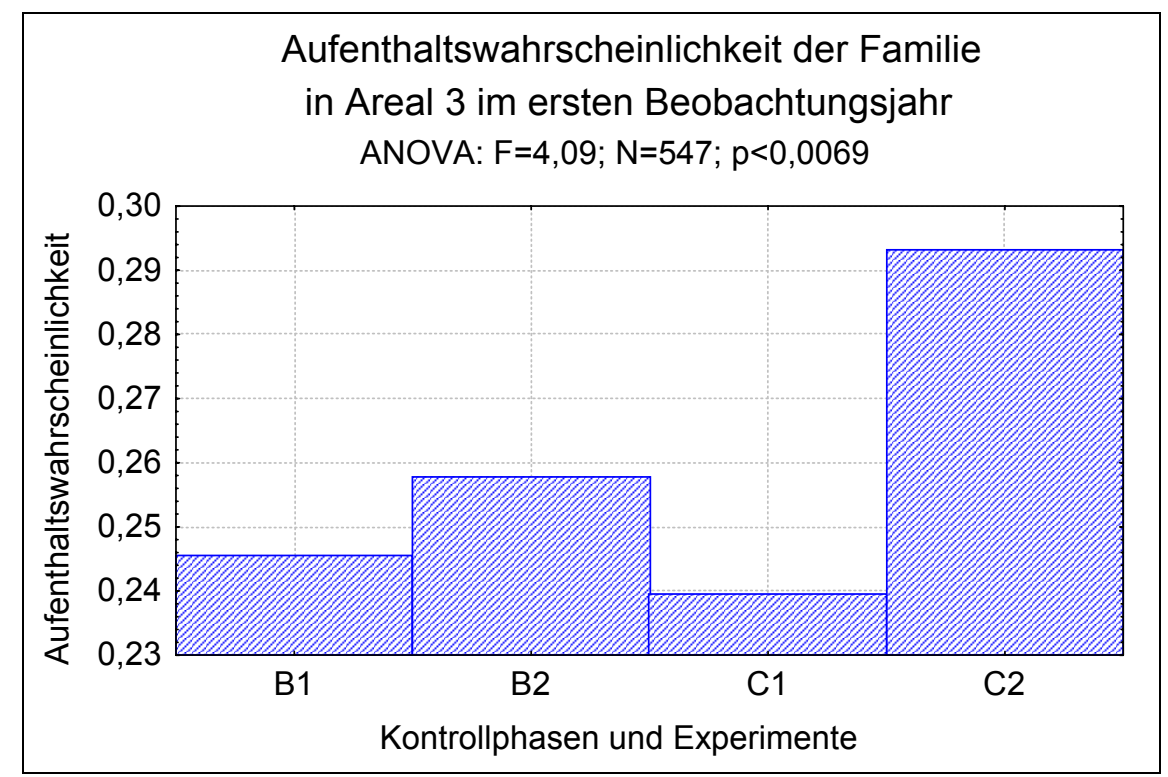

Abb. 3.112 Aufenthaltswahrscheinlichkeit der Familie in Areal 3 während der vier Phasen im ersten Beobachtungsjahr (ANOVA, $\mathrm{F}=4,09 ; \mathrm{N}=547 ; \mathrm{p}<0,01$ ). Die Aufenthaltswahrscheinlichkeit in Areal 3 war während des zweiten Experiments höher als während der vorhergehenden Phasen.

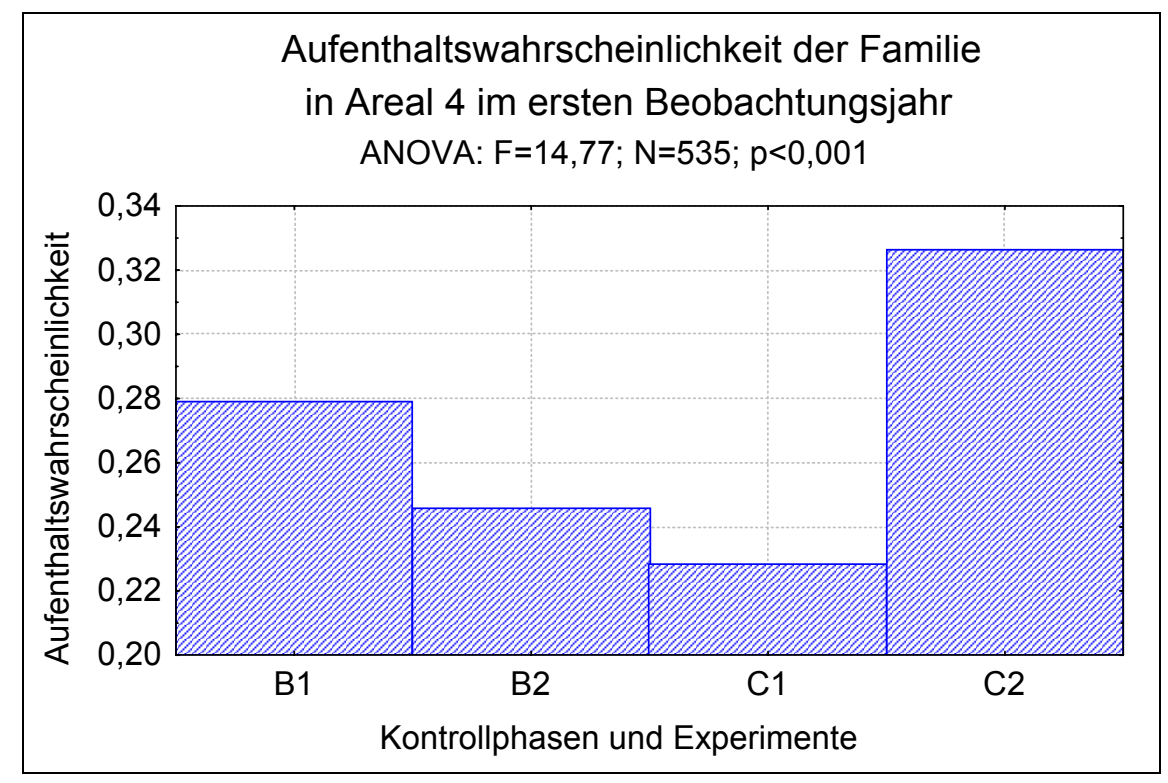

Abb. 3.113 Aufenthaltswahrscheinlichkeit der Familie in Areal 4 während der vier Phasen im ersten Beobachtungsjahr (ANOVA, $\mathrm{F}=14,77 ; \mathrm{N}=535 ; \mathrm{p}<0,001$ ). Die Aufenthaltswahrscheinlichkeit war in Areal 4 während der ersten Kontrollphase größer als während des ersten Experiments. Im zweiten Experiment hielt sich die Familie in Areal 4 häufiger auf als während der drei Phasen vorher. 


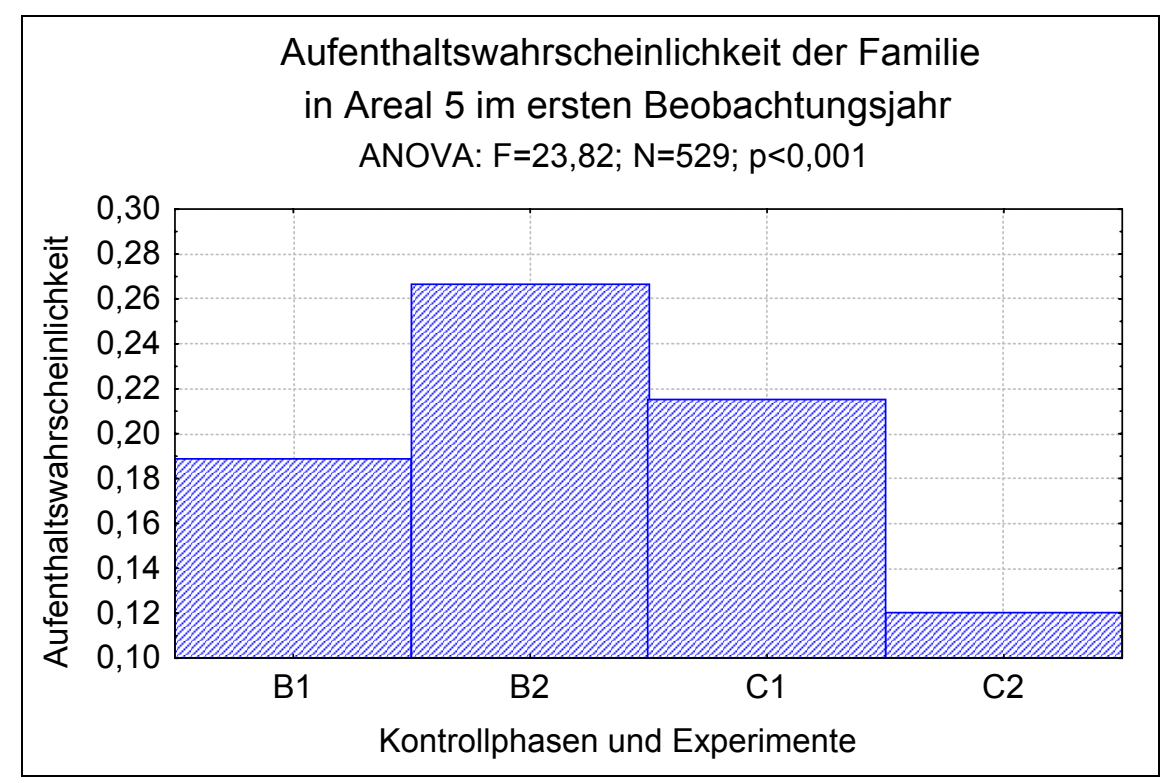

Abb. 3.114 Aufenthaltswahrscheinlichkeit der Familie in Areal 5 während der vier Phasen im ersten Beobachtungsjahr (ANOVA, $\mathrm{F}=23,82 ; \mathrm{N}=529 ; \mathrm{p}<0,001$ ). Die Familie hielt sich in der zweiten Kontrollphase (B2) mit einer höheren Wahrscheinlichkeit in Areal 5 auf als während der anderen Phasen. Während des zweiten Experiments war die Aufenthaltswahrscheinlichkeit der Familie in Areal 5 geringer als in den übrigen Phasen.

Die Aufenthaltswahrscheinlichkeit in Areal 6 war ebenso wie in Areal 5 während der zweiten Kontrollphase größer und im zweiten Experiment geringer als während der anderen Phasen (Abb. 3.115). Da Areal 7 nur in Experiment 1 genutzt wurde, kann hierzu keine statistische Analyse durchgeführt werden.

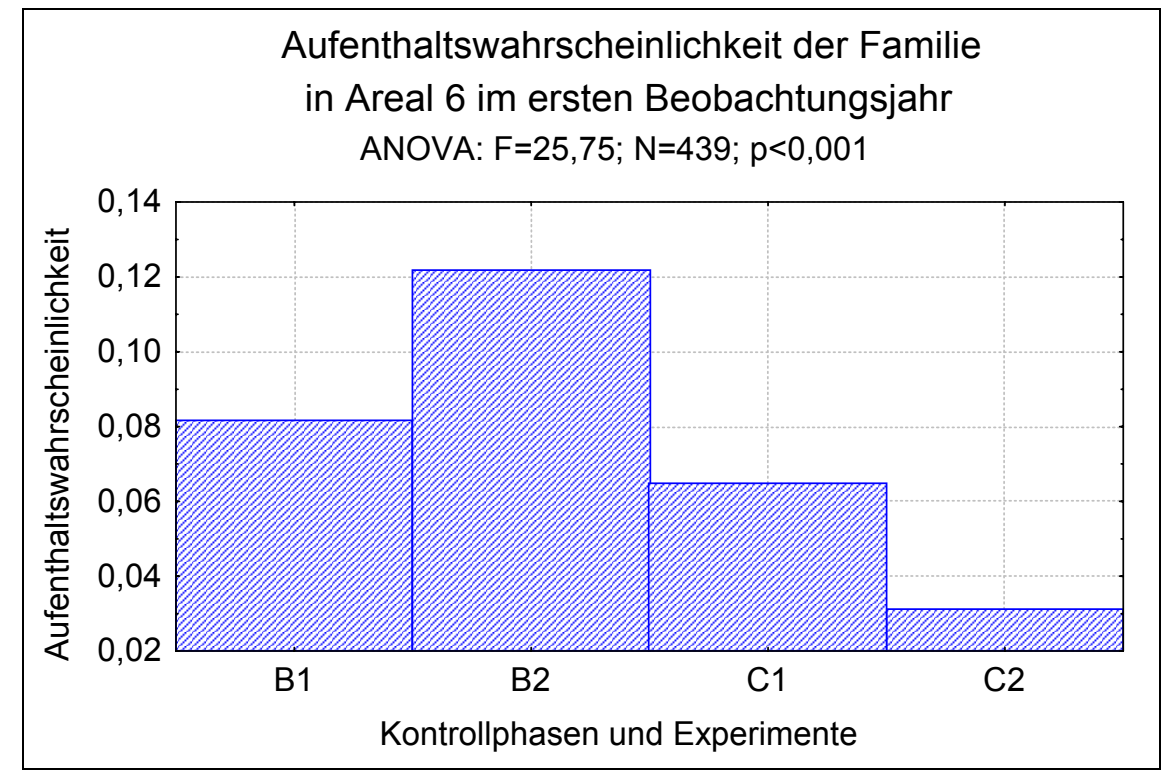

Abb. 3.115 Aufenthaltswahrscheinlichkeit der Familie in Areal 6 während der vier Phasen im ersten Beobachtungsjahr (ANOVA, F=25,75; N=439; $<<0,001$ ). Die Familie hielt sich in der zweiten Kontrollphase (B2) mit einer höheren Wahrscheinlichkeit in Areal 6 auf als während der anderen Phasen. Während des zweiten Experiments war die Aufenthaltswahrscheinlichkeit der Familie in Areal 6 geringer als in den übrigen Phasen. 


\subsubsection{Arealnutzung der Familie J18 im zweiten Beobachtungsjahr}

Während des ersten Experiments im zweiten Beobachtungsjahr war die Aufenthaltswahrscheinlichkeit in den verschiedenen Arealen unterschiedlich hoch (Abb. 3.116). Häufig wurden die Areale 3 und 4 genutzt, während Areal 7 überhaupt nicht betreten wurde. Während des zweiten Experiments war die Aufenthaltswahrscheinlichkeit der Familie in den Arealen 3 und 4 höher als in allen übrigen Arealen, während die Areale 6 und 7 seltener genutzt wurden, als die anderen Areale (Abb. 3.117). Die Aufenthaltswahrscheinlichkeit der Familie war während des dritten und vierten Experiments in den Arealen unterschiedlich groß und glich der Nutzung aus Experiment 2. Im vierten Experiment fiel allerdings auf, dass die Areale 1 und 2 relativ häufig aufgesucht wurden (Abb. 3.118 und 3.119). Areal 7 wurde nur während des vierten Experiments von der Familie aufgesucht.

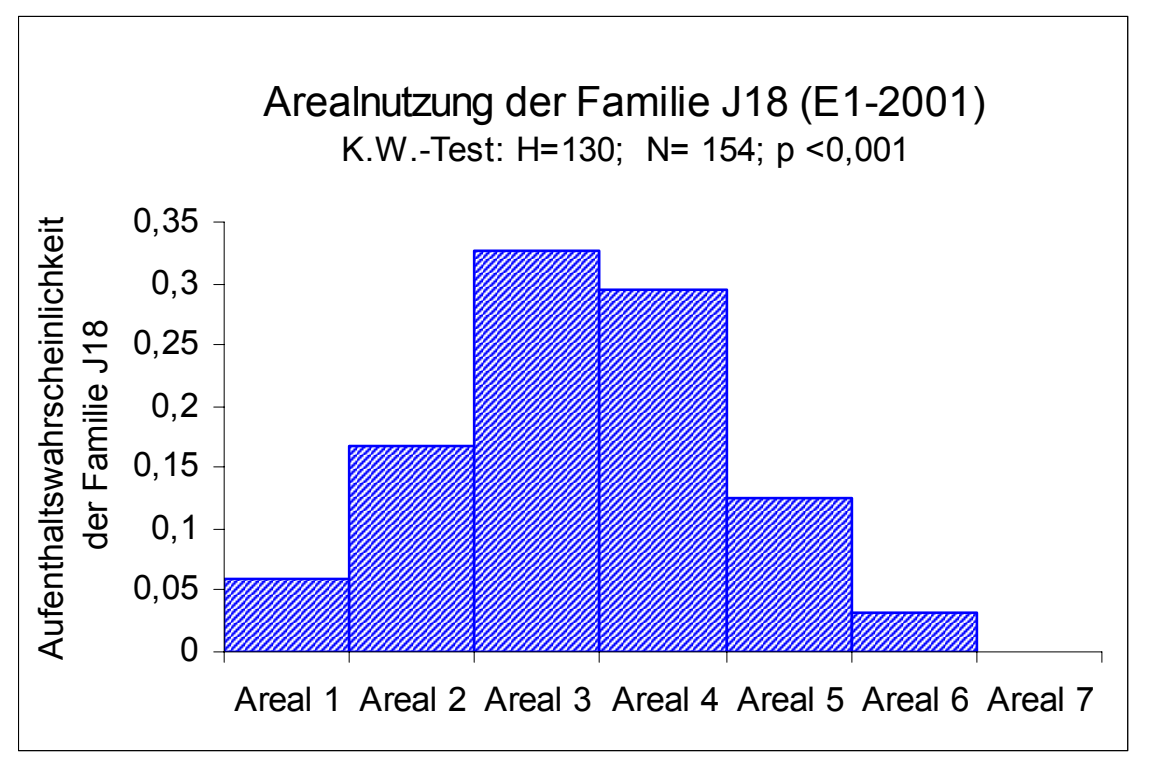

Abb. 3.116 Arealnutzung der Familie J18 im ersten Experiment des zweiten Beobachtungsjahres (KruskalWallis-Test, $\mathrm{H}=130 ; \mathrm{N}=154 ; \mathrm{p}<0,001)$. Die Aufenthaltswahrscheinlichkeit der Familie war in den einzelnen Arealen unterschiedlich groß. Da keine Varianzanalyse zulässig war, konnte nicht ermittelt werden, welche Areale sich voneinander unterschieden. Die größte Aufenthaltswahrscheinlichkeit war in den Arealen 3 und 4. Areal 7 wurde gar nicht genutzt. 


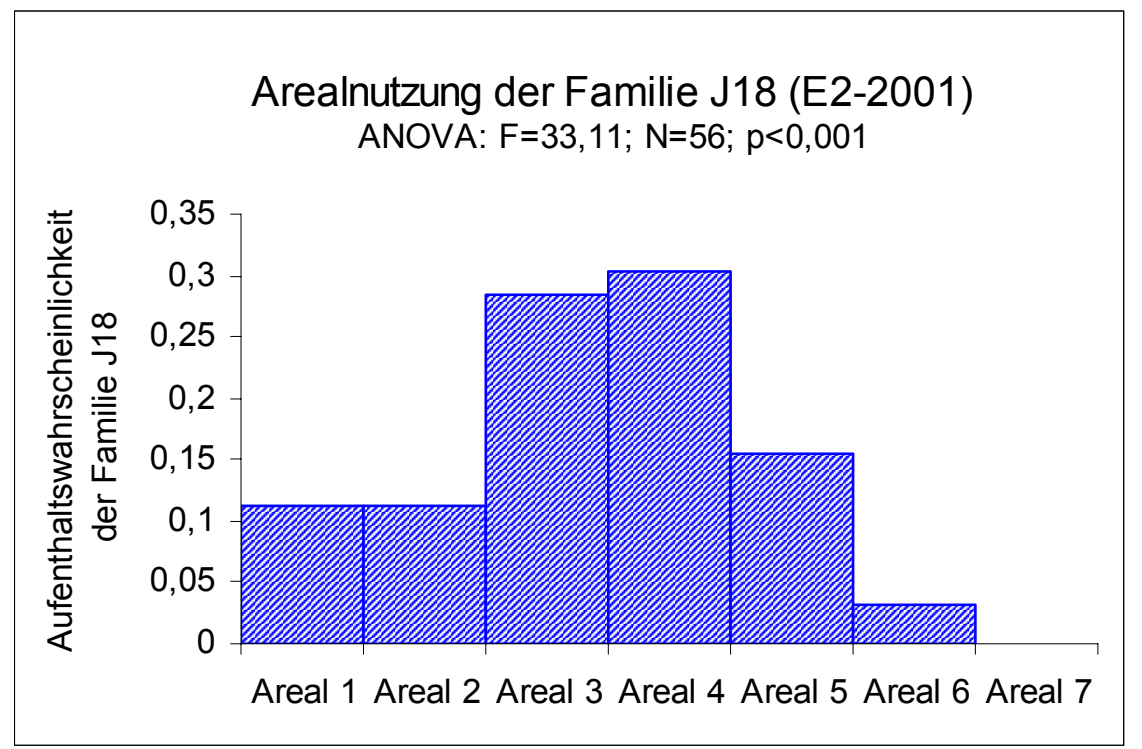

Abb. 3.117 Arealnutzung der Familie J18 im zweiten Experiment des zweiten Beobachtungsjahres (ANOVA, $\mathrm{F}=33,11 ; \mathrm{N}=56 ; \mathrm{p}<0,001)$. Die Aufenthaltswahrscheinlichkeit der Familie war in den Arealen 3 und 4 höher als in allen übrigen Arealen, während die Areale 6 und 7 seltener genutzt wurden, als die anderen Areale.

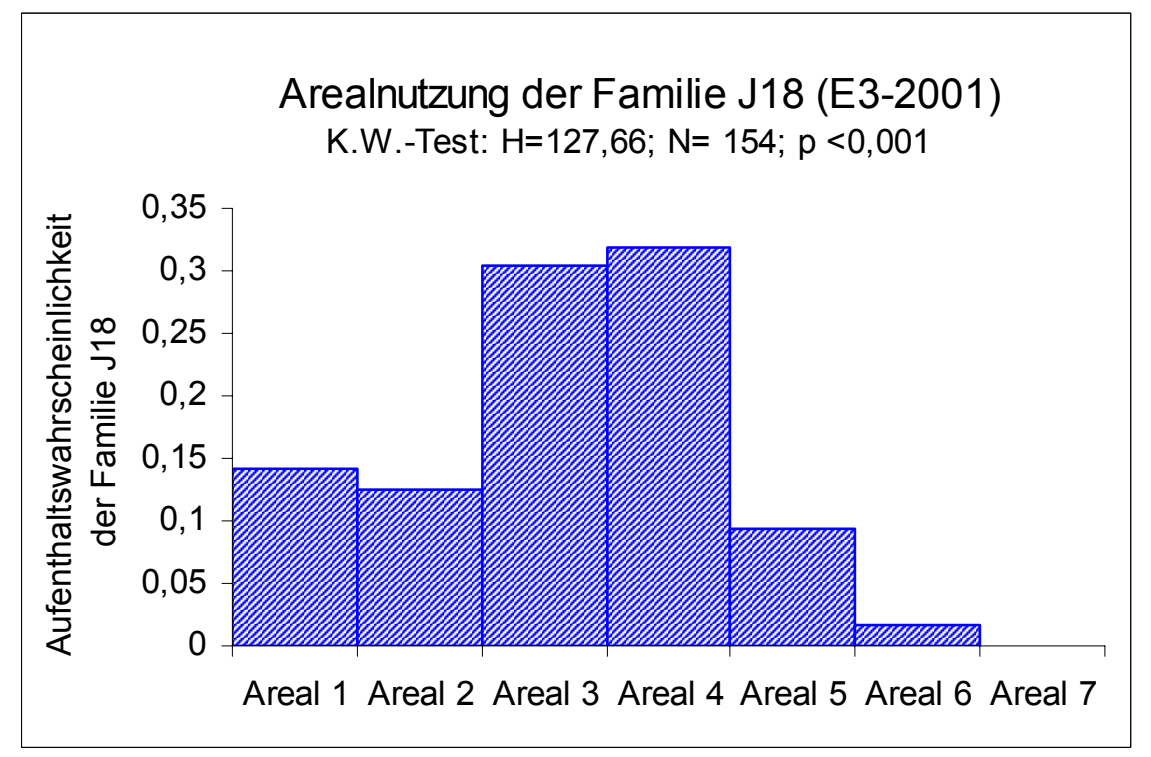

Abb. 3.118 Arealnutzung der Familie J18 im dritten Experiment des zweiten Beobachtungsjahres (KruskalWallis-Test, $\mathrm{H}=127,66 ; \mathrm{N}=154 ; \mathrm{p}<0,001)$. Die Aufenthaltswahrscheinlichkeit der Familie war in den einzelnen Arealen unterschiedlich groß. Da keine Varianzanalyse zulässig war, konnte nicht ermittelt werden, welche Areale sich voneinander unterschieden. Die größte Aufenthaltswahrscheinlichkeit war in den Arealen 3 und 4. Areal 7 wurde gar nicht genutzt. 


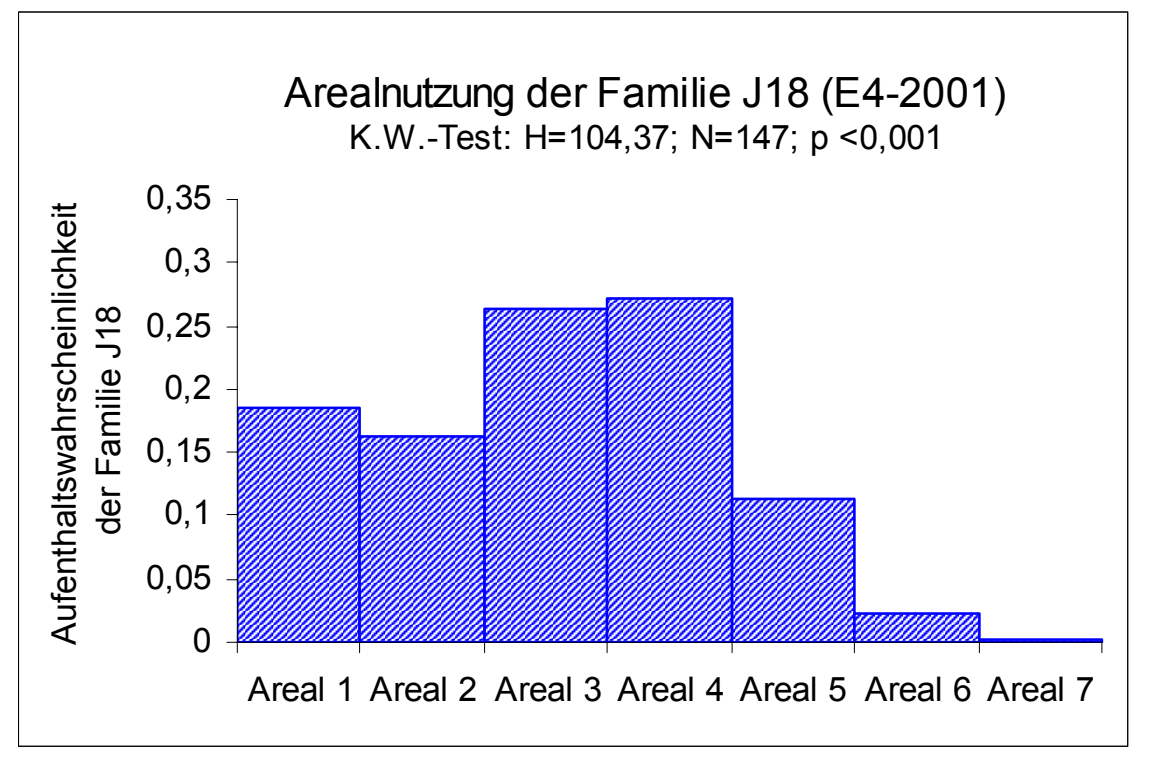

Abb. 3.119 Arealnutzung der Familie J18 im dritten Experiment des zweiten Beobachtungsjahres (KruskalWallis-Test, $\mathrm{H}=104,37 ; \mathrm{N}=147 ; \mathrm{p}<0,001)$. Die Aufenthaltswahrscheinlichkeit der Familie war in den einzelnen Arealen unterschiedlich groß. Da keine Varianzanalyse zulässig war, konnte nicht ermittelt werden, welche Areale sich voneinander unterschieden. Areal 7 wurde nur in Experiment 4 genutzt.

Auch während des zweiten Beobachtungsjahres hielt sich die Familie unterschiedlich häufig in einigen Arealen auf. In Areal 1 nahm die Aufenthaltswahrscheinlichkeit von einem Experiment zum darauffolgenden zu, d.h. während des zweiten Experiments war die Familie häufiger als während des ersten Experiments, während des dritten häufiger als während des zweiten und während des vierten häufiger als während des dritten Experiments in Areal 1 (Abb. 3.120). Die Aufenthaltswahrscheinlichkeit in Areal 2 war im ersten Experiment höher als im zweiten und dritten Experiment. Während des vierten Experiments lag die Aufenthaltswahrscheinlichkeit zwischen den Extremen und unterschied sich nicht von allen anderen Experimenten (Abb. 3.121). 


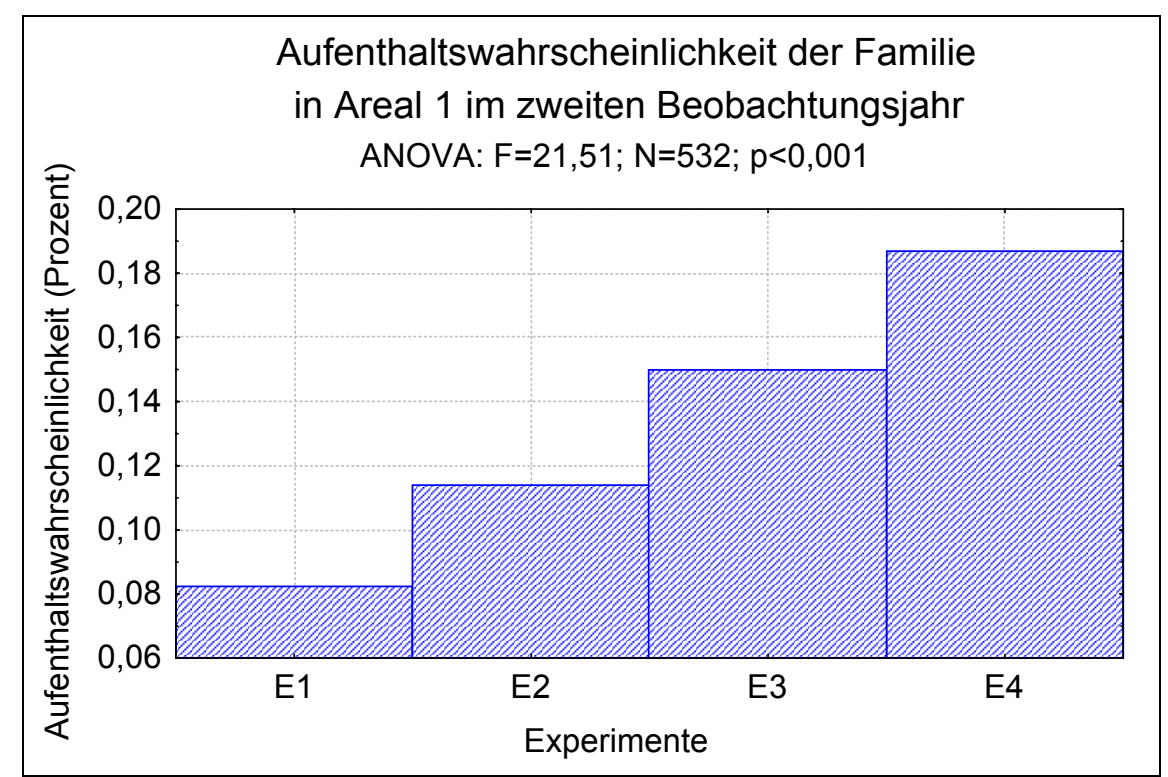

Abb. 3.120 Aufenthaltswahrscheinlichkeit der Familie in Areal 1 während der vier Phasen im zweiten Beobachtungsjahr (ANOVA, F=21,51; N=532; $<<0,001$ ). Die Aufenthaltswahrscheinlichkeit der Familie in Areal 1 unterschied sich zwischen allen Experimenten. Mit jeder Experimentalphase stieg die Aufenthaltswahrscheinlichkeit in Areal 1 immer mehr an.

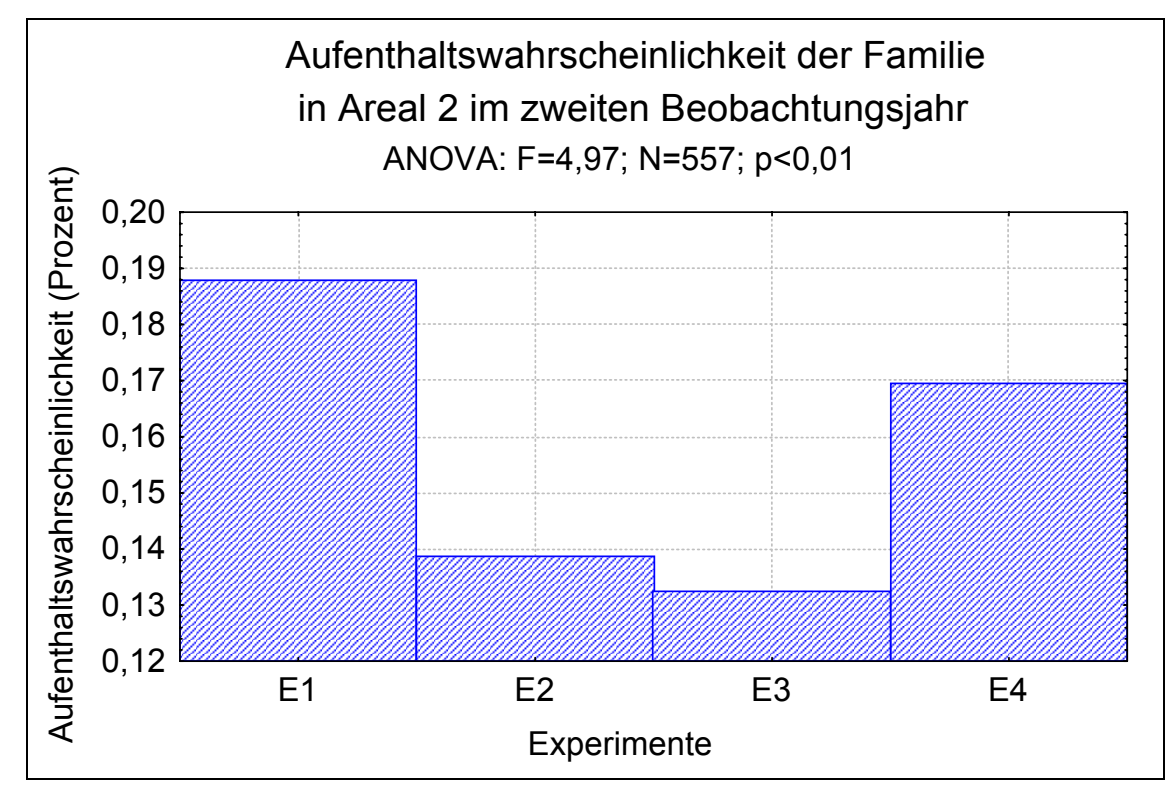

Abb. 3.121 Aufenthaltswahrscheinlichkeit der Familie in Areal 2 während der vier Phasen im zweiten Beobachtungsjahr (ANOVA, $\mathrm{F}=4,97 ; \mathrm{N}=557 ; \mathrm{p}<0,01$ ). Die Aufenthaltswahrscheinlichkeit in Areal 2 war während des ersten Experiments höher als während des zweiten und dritten Experiments.

Die Aufenthaltswahrscheinlichkeit der Familie in Areal 3 war während des ersten Experiments höher als während des vierten Experiments (Abb. 3.122). In Areal 4 hielt sich die Familie während aller Experimentalphasen gleich häufig auf (Abb. 3.123). In den Arealen 5 und 6 war die Aufenthaltswahrscheinlichkeit während des dritten Experiments gegenüber den Experimenten 1, 2 und 4 vermindert (Abb.3.124 und Abb. 3.125). 


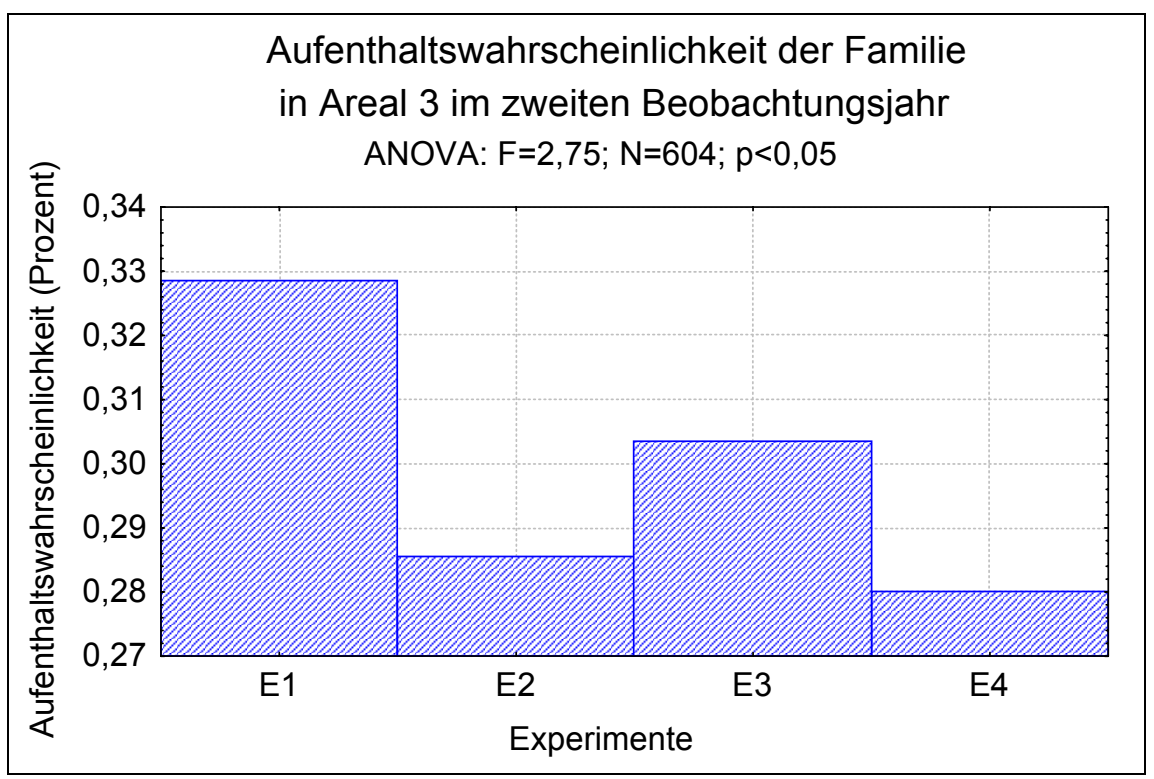

Abb. 3.122 Aufenthaltswahrscheinlichkeit der Familie in Areal 3 während der vier Phasen im zweiten Beobachtungsjahr (ANOVA, $F=2,75 ; \mathrm{N}=604 ; \mathrm{p}<0,05$ ). Die Aufenthaltswahrscheinlichkeit der Familie in Areal 3 war während des ersten Experiments höher als während des vierten Experiments.

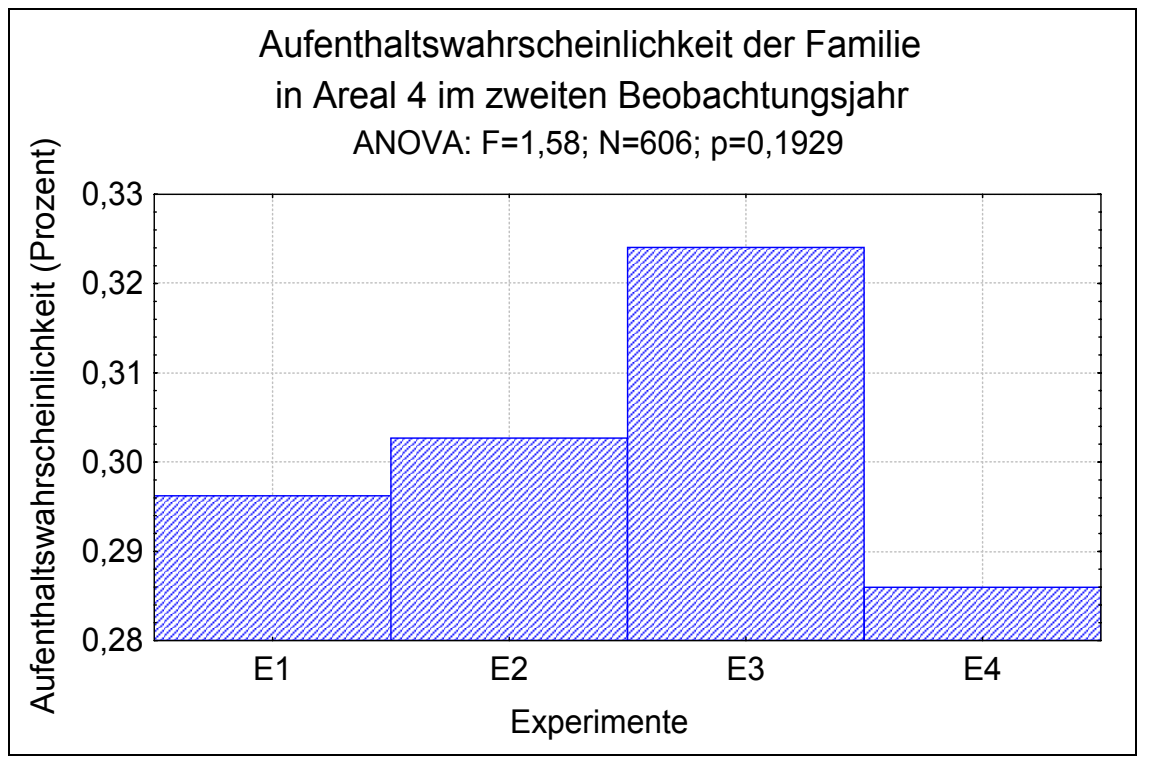

Abb. 3.123 Aufenthaltswahrscheinlichkeit der Familie in Areal 4 während der vier Phasen im zweiten Beobachtungsjahr (ANOVA, F=1,58; $\mathrm{N}=606 ; \mathrm{p}=0,1929$ ). Die Aufenthaltswahrscheinlichkeit der Familie in Areal 4 unterschied sich nicht zwischen den vier Experimentalphasen. 


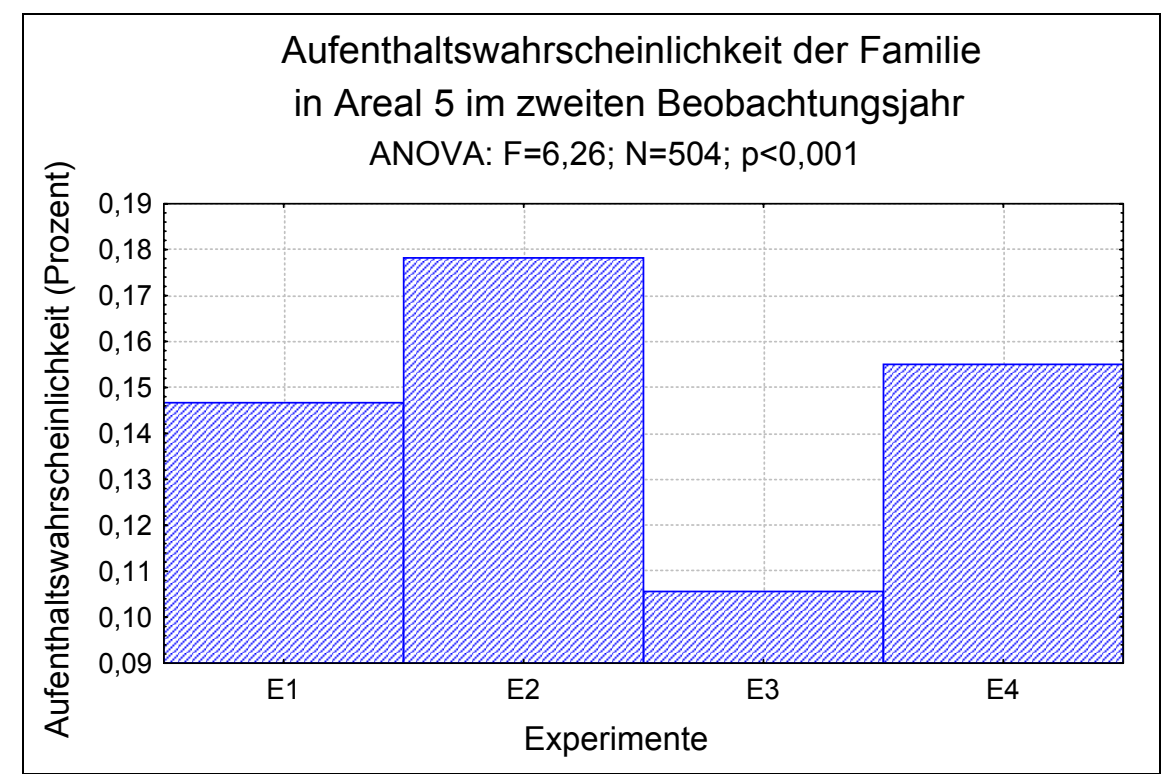

Abb. 3.124 Aufenthaltswahrscheinlichkeit der Familie in Areal 5 während der vier Phasen im ersten Beobachtungsjahr (ANOVA, $\mathrm{F}=6,26 ; \mathrm{N}=504 ; \mathrm{p}<0,001$ ). Die Aufenthaltswahrscheinlichkeit in Areal 5 war während des dritten Experiments gegenüber den anderen Phasen vermindert.

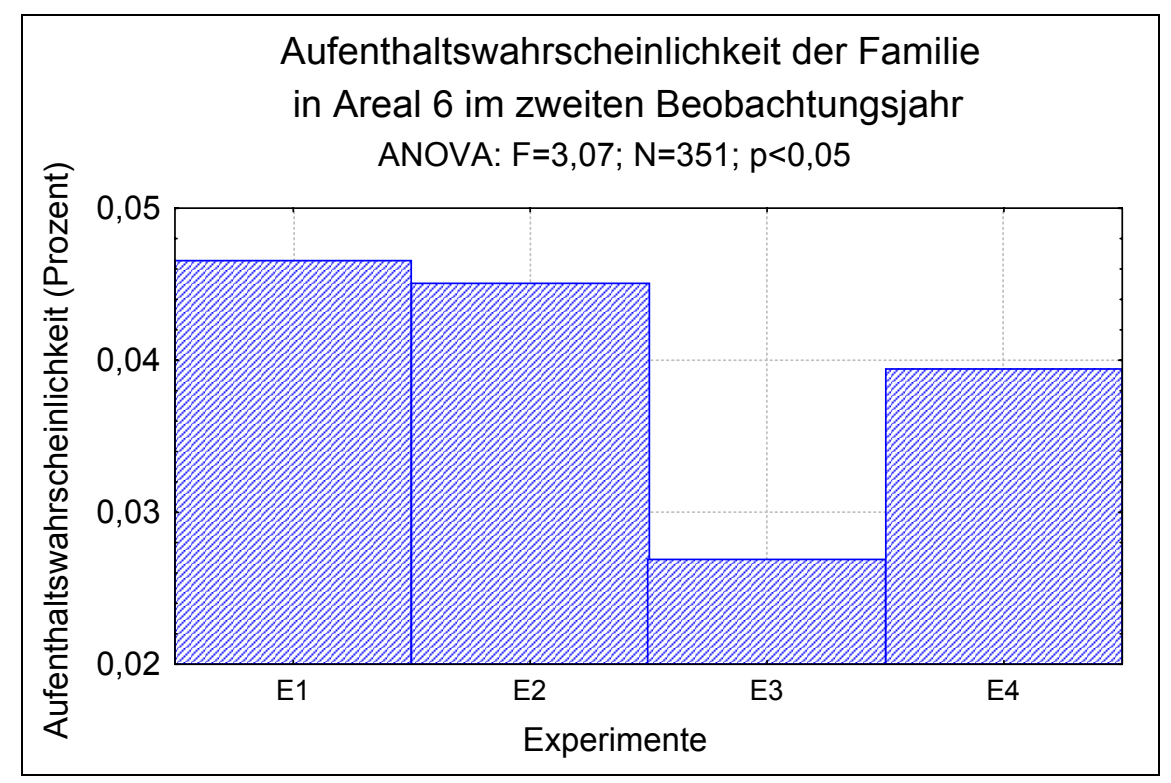

Abb. 3.125 Aufenthaltswahrscheinlichkeit der Familie in Areal 6 während der vier Phasen im ersten Beobachtungsjahr (ANOVA, $\mathrm{F}=3,07 ; \mathrm{N}=351 ; \mathrm{p}<0,05$ ). Die Aufenthaltswahrscheinlichkeit in Areal 6 war während des dritten Experiments gegenüber den anderen Phasen vermindert.

Es wird deutlich, dass die sieben Areale des Streifgebietes von der Familie während aller Experimente in beiden Beobachtungsjahren unterschiedlich häufig genutzt wurden. Hypothese $7 \mathrm{a}$ wird damit abgelehnt. Außerdem gibt es Abhängigkeiten der Nutzung einzelner Areale von den Experimenten. Bei Areal 1 besteht allerdings im ersten Beobachtungsjahr und bei Areal 4 im zweiten Beobachtungsjahr keine Abhängigkeit der Aufenthaltswahrscheinlichkeit von den Experimenten. Hypothese $7 \mathrm{~b}$ kann daher im ersten Beobachtungsjahr für die Areale 2, 3, 4, 5, 6 und 7 und im zweiten Beobachtungsjahr für die Areale 1, 2, 3, 5, 6 und 7 
zurückgewiesen werden.

\subsubsection{Arealnutzung der Familienmitglieder}

Hypothese 2c: Alle Familienmitglieder nutzen die jeweiligen Areale gleich häufig.

\subsubsection{Arealnutzung einzelner Familienmitglieder im ersten Beobachtungsjahr}

Die Familienmitglieder nutzten die Areale ihres gemeinsamen Streifgebietes im ersten Beobachtungsjahr mit einer unterschiedlich großen Wahrscheinlichkeit. G hielt sich mit einer größeren Wahrscheinlichkeit in Areal 1 auf als die anderen Familienmitglieder (Abb. 3.126). Die Aufenthaltswahrscheinlichkeit von C, D und E in Areal 2 war geringer als bei den anderen Familienmitgliedern (Abb. 3.127). In Areal 3 hielten sich D und E seltener auf als A, B, C, G und H (Abb. 3.128). Die Aufenthaltswahrscheinlichkeit von D in Areal 4 lag über der von A, B und G. E hielt sich häufiger als $G$ in Areal 4 auf (Abb. 3.129). Die Aufenthaltswahrscheinlichkeit von $\mathrm{C}$ in Areal 5 war größer als jene von A, B, F, G und H. E war häufiger, mit Ausnahme von C, als alle anderen Familienmitglieder in Areal 5 (Abb. 3.130). Es gab keinen Unterschied in der Aufenthaltswahrscheinlichkeit der Familienmitglieder in Areal 6 (Abb. 3.131).

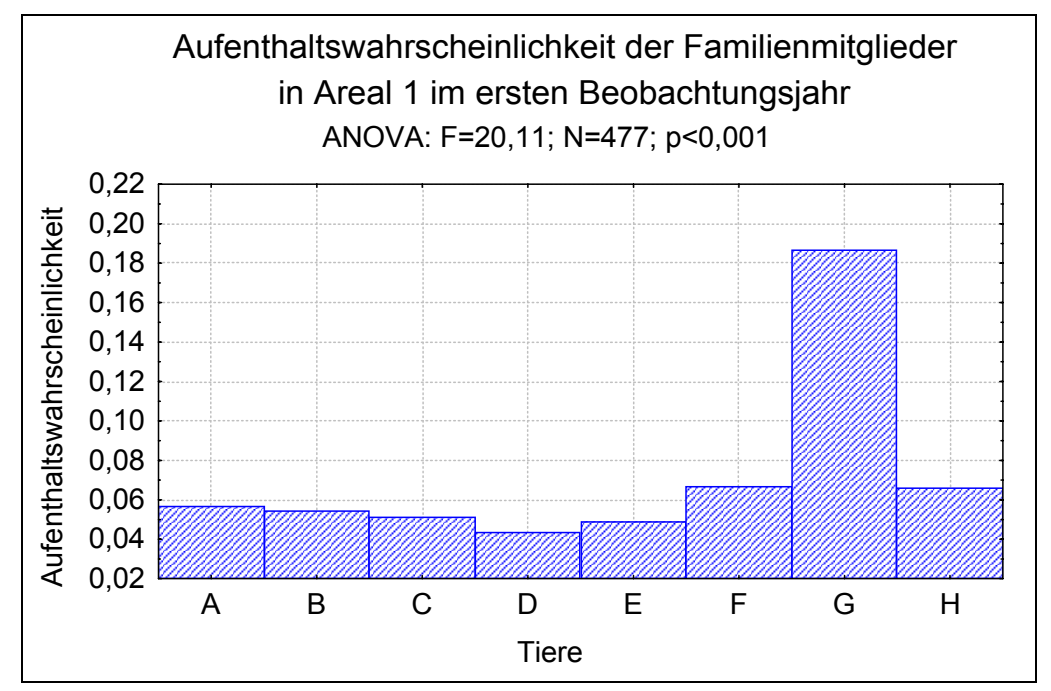

Abb. 3.126 Aufenthaltswahrscheinlichkeit der Familienmitglieder in Areal 1 ihres Streifgebietes im ersten Beobachtungsjahr (ANOVA, $\mathrm{F}=20,11, \mathrm{~N}=477$; $\mathrm{p}<0,001$ ). $\mathrm{G}$ hielt sich häufiger in Areal 1 auf als die anderen Familienmitglieder. 


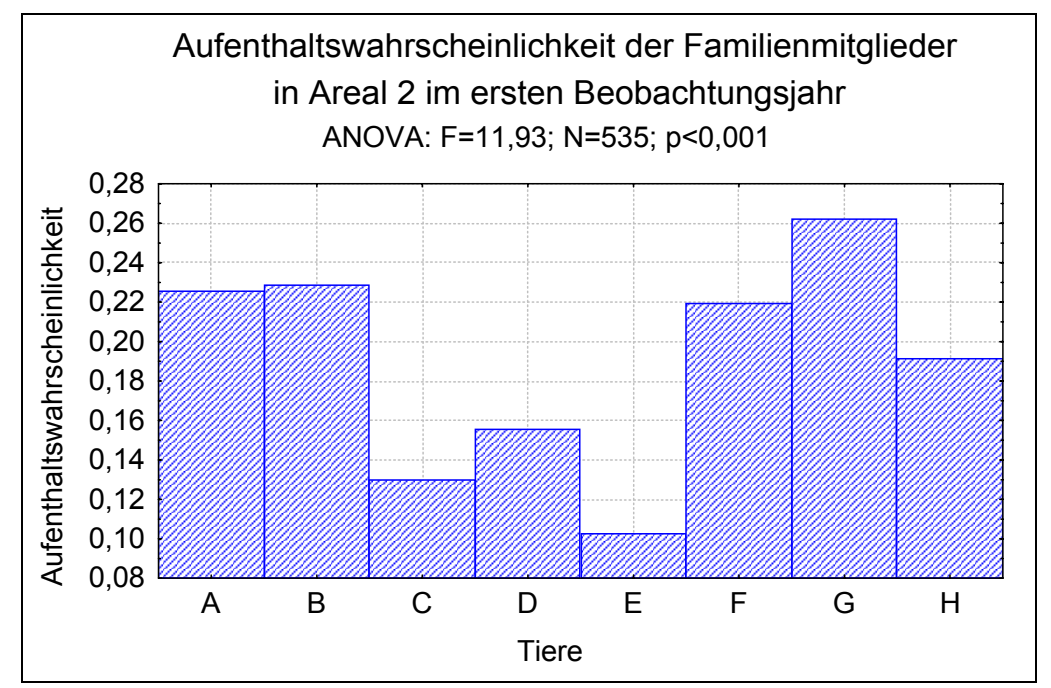

Abb. 3.127 Aufenthaltswahrscheinlichkeit der Familienmitglieder in Areal 2 ihres Streifgebietes im ersten Beobachtungsjahr (ANOVA, $\mathrm{F}=11,93, \mathrm{~N}=535 ; \mathrm{p}<0,001$ ). $\mathrm{C}, \mathrm{D}$ und $\mathrm{E}$ hielten sich seltener in Areal 2 auf als die anderen Familienmitglieder.

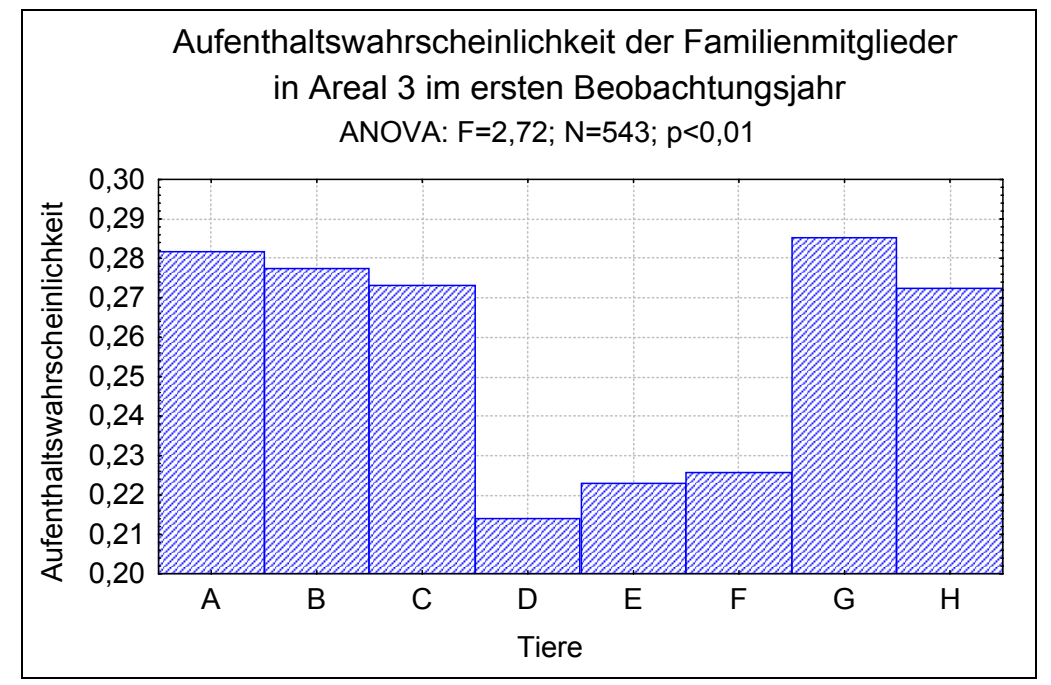

Abb. 3.128 Aufenthaltswahrscheinlichkeit der Familienmitglieder in Areal 3 ihres Streifgebietes im ersten Beobachtungsjahr (ANOVA, $\mathrm{F}=2,72, \mathrm{~N}=543 ; \mathrm{p}<0,01$ ). D und $\mathrm{E}$ hielten sich seltener als $\mathrm{A}, \mathrm{B}, \mathrm{C}, \mathrm{G}$ und $\mathrm{H}$ in Areal 3 auf. F unterschied sich von keinem Familienmitglied. 


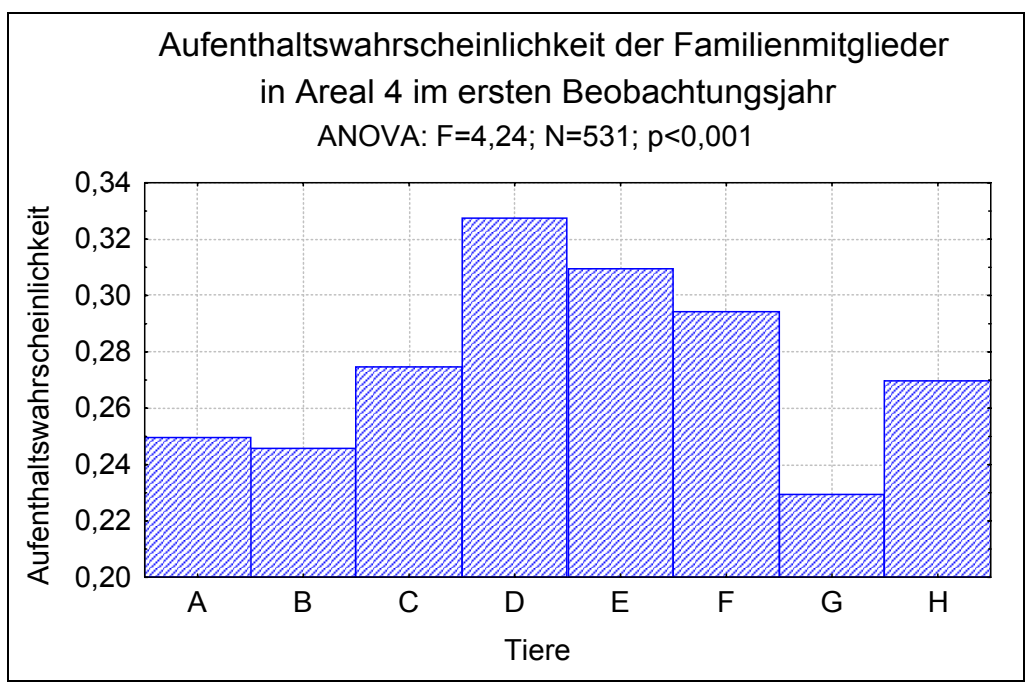

Abb. 3.129 Aufenthaltswahrscheinlichkeit der Familienmitglieder in Areal 4 ihres Streifgebietes im ersten Beobachtungsjahr (ANOVA, $\mathrm{F}=4,24, \mathrm{~N}=531$; $\mathrm{p}<0,001$ ). $\mathrm{D}$ hielt sich in Areal 4 mit einer größeren Wahrscheinlichkeit auf als A, B und G. E hielt sich häufiger als G in Areal 4 auf. F unterschied sich von keinem Familienmitglied.

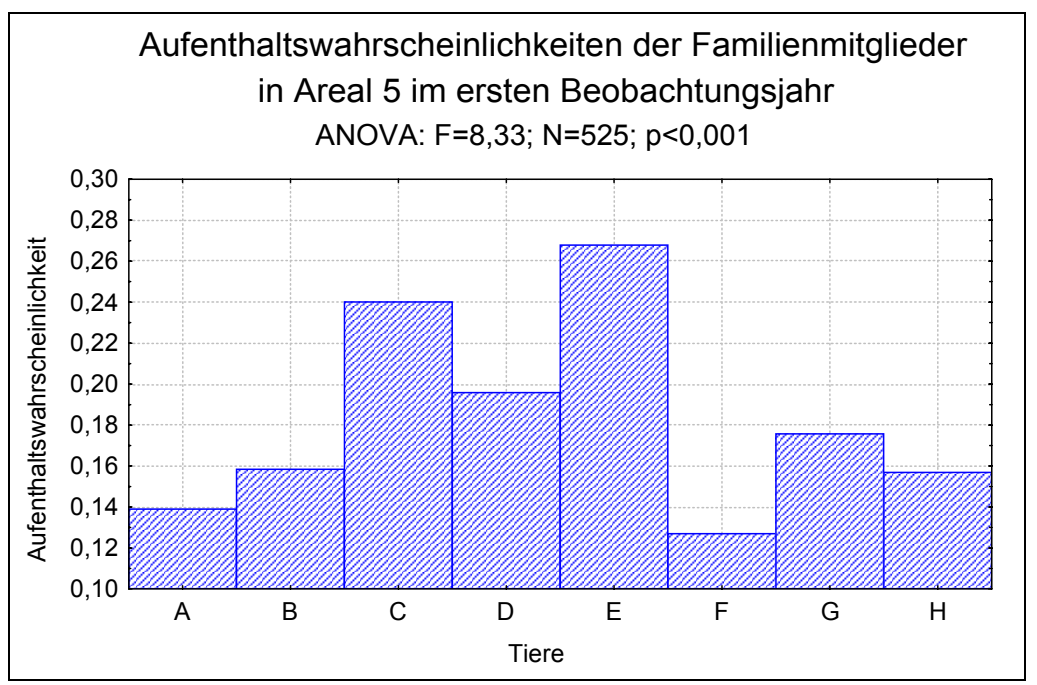

Abb. 3.130 Aufenthaltswahrscheinlichkeit der Familienmitglieder in Areal 5 ihres Streifgebietes im ersten Beobachtungsjahr (ANOVA, $F=8,33, \mathrm{~N}=525 ; \mathrm{p}<0,001$ ). Die Aufenthaltswahrscheinlichkeit von $\mathrm{C}$ in Areal 5 war höher als die von A, B, F, G und H. E war häufiger als alle anderen Familienmitglieder in Areal 5 mit Ausnahme von C. D unterschied sich von keinem Familienmitglied. 


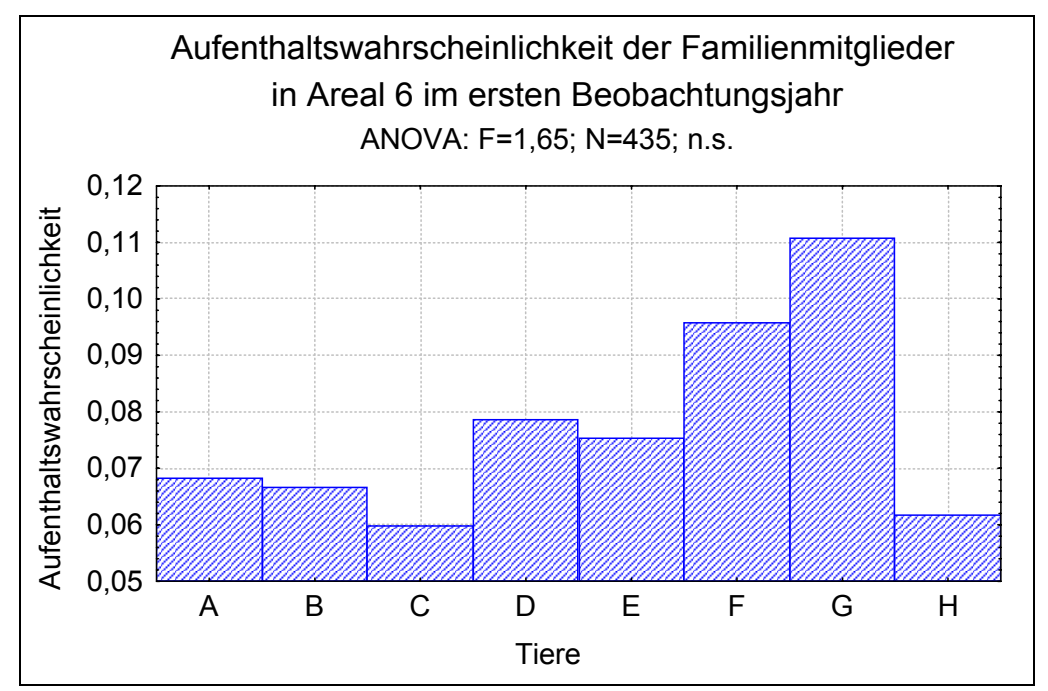

Abb. 3.131 Aufenthaltswahrscheinlichkeit der Familienmitglieder in Areal 6 ihres Streifgebietes im ersten Beobachtungsjahr (ANOVA, $\mathrm{F}=1,65, \mathrm{~N}=435$; n.s.). Es gab zwischen den Familienmitgliedern keinen Unterschied in ihrer Aufenthaltswahrscheinlichkeit in Areal 6.

\subsubsection{Arealnutzung einzelner Familienmitglieder im zweiten Beobachtungsjahr}

Die Familienmitglieder hielten sich auch im zweiten Beobachtungsjahr unterschiedlich häufig in den einzelnen Arealen ihres Streifgebietes auf. C und I hielten sich seltener als A, B, G, J, $\mathrm{K}$ und $\mathrm{M}$ in Areal 1 auf. Die Aufenthaltswahrscheinlichkeit von D in Areal 1 war geringer als von B, G, J, K und M (Abb. 3.132). In Areal 2 war die Aufenthaltswahrscheinlichkeit von C geringer als von allen anderen Familienmitgliedern. D hielt sich seltener als A in Areal 2 auf (Abb. 3.133).

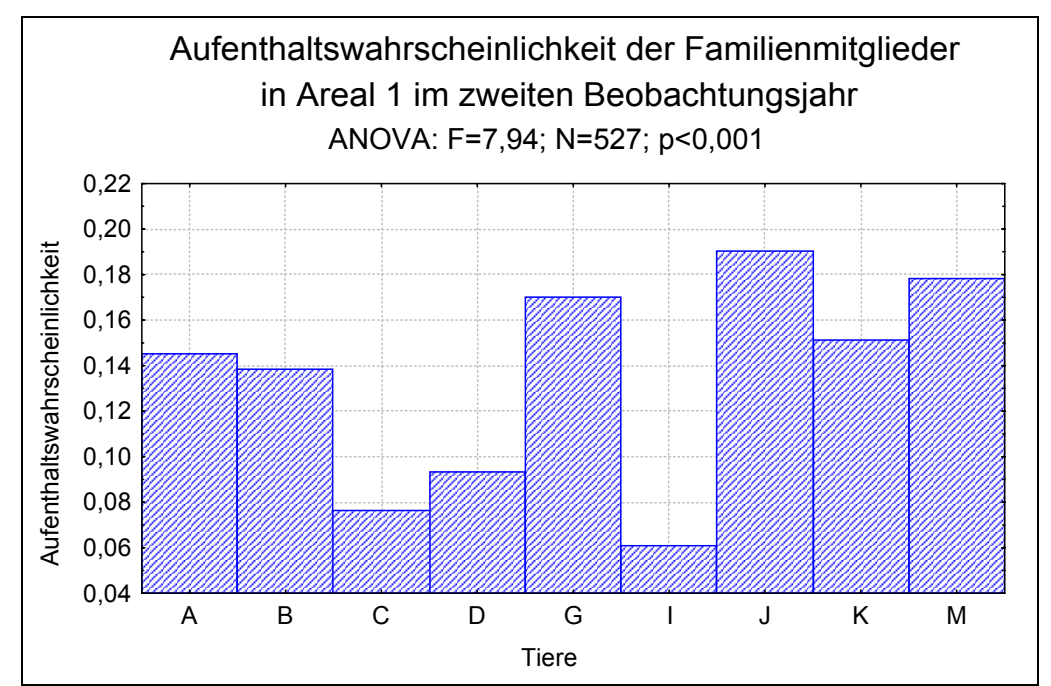

Abb. 3.132 Aufenthaltswahrscheinlichkeit der Familienmitglieder in Areal 1 ihres Streifgebietes im zweiten Beobachtungsjahr (ANOVA, F=7,94; $\mathrm{N}=527 ; \mathrm{p}<0,001$ ). $\mathrm{C}$ und I hielten sich seltener als $\mathrm{A}, \mathrm{B}, \mathrm{G}, \mathrm{J}, \mathrm{K}$ und $\mathrm{M}$ in Areal 1 auf. Die Aufenthaltswahrscheinlichkeit von D in Areal 1 war geringer als von A, B, G, J, K und M. 


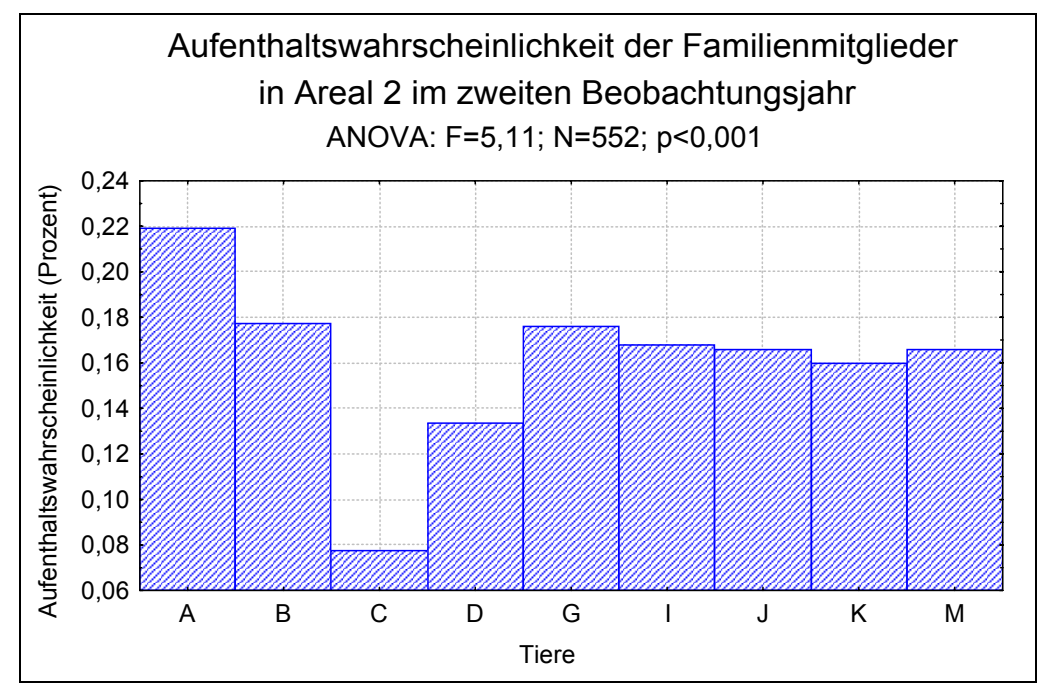

Abb. 3.133 Aufenthaltswahrscheinlichkeit der Familienmitglieder in Areal 2 ihres Streifgebietes im zweiten Beobachtungsjahr (ANOVA, F=5,11; $\mathrm{N}=552 ; \mathrm{p}<0,001$ ). Die Aufenthaltswahrscheinlichkeit von $\mathrm{C}$ in Areal 2 war geringer als von allen anderen Familienmitgliedern. D hielt sich seltener als A in Areal 2 auf.

D hielt sich mit einer geringeren Wahrscheinlichkeit in Areal 3 auf als B und K. Zwischen den anderen Familienmitgliedern gab es keine Unterschiede der Aufenthaltswahrscheinlichkeit in Areal 3 (Abb. 3.134). In Areal 4 war die Aufenthaltswahrscheinlichkeit von I höher als die von A, B, J, K und M. Die anderen Familienmitglieder unterschieden sich nicht in ihrer Aufenthaltswahrscheinlichkeit in Areal 4 (Abb. 3.135). Die adulten Nachkommen C, D und G hielten sich mit höherer Wahrscheinlichkeit in Areal 5 auf. C war außerdem häufiger in Areal 5 als I, J, K und M. D und G hielten sich häufiger als J in Areal 5 auf (Abb. 3.136). G hielt sich mit höherer Wahrscheinlichkeit in Areal 6 auf als die anderen Familienmitglieder. Die übrigen Familienmitglieder unterschieden sich nicht in ihrer Aufenthaltswahrscheinlichkeit in Areal 6 (Abb. 3.137).

Die Aufenthaltswahrscheinlichkeit der Familienmitglieder war im ersten Beobachtungsjahr für die Areale 1-5 unterschiedlich groß. Für die Areale 6 und 7 können in dem Jahr keine Unterschiede gefunden werden. Im zweiten Beobachtungsjahr hielten sich die Familienmitglieder in jedem Areal unterschiedlich häufig auf. Hypothese 7c kann damit abgelehnt werden. 


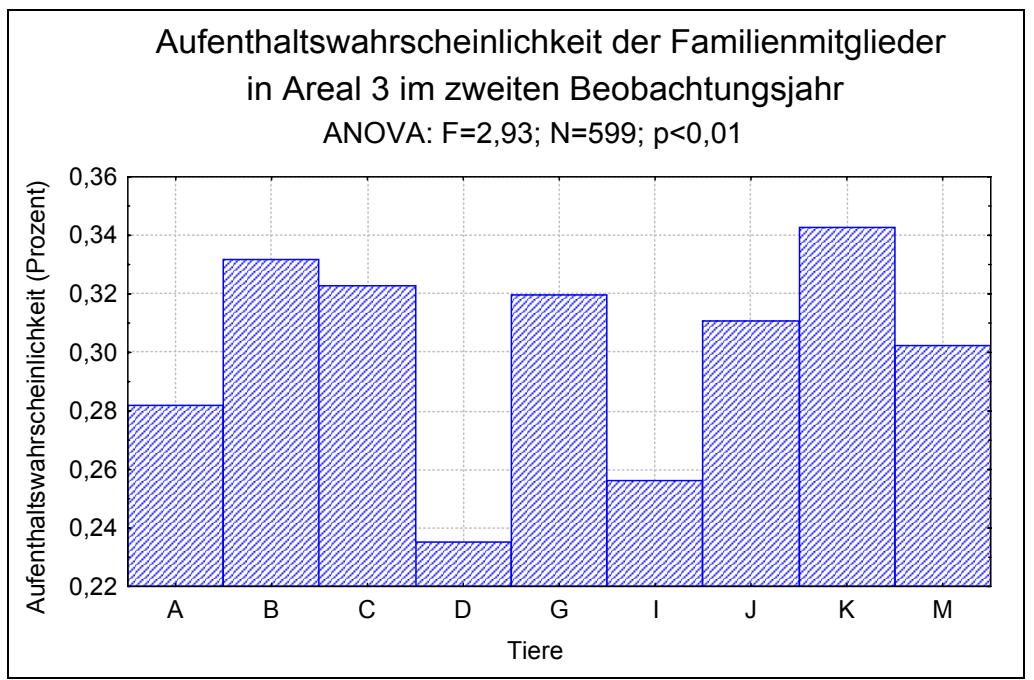

Abb. 3.134 Aufenthaltswahrscheinlichkeit der Familienmitglieder in Areal 3 ihres Streifgebietes im zweiten Beobachtungsjahr (ANOVA, $\mathrm{F}=2,93 ; \mathrm{N}=599 ; \mathrm{p}<0,01$ ). D hielt sich mit einer geringeren Wahrscheinlichkeit in Areal 3 auf als B und K. Zwischen den anderen Familienmitgliedern gab es keine Unterschiede.

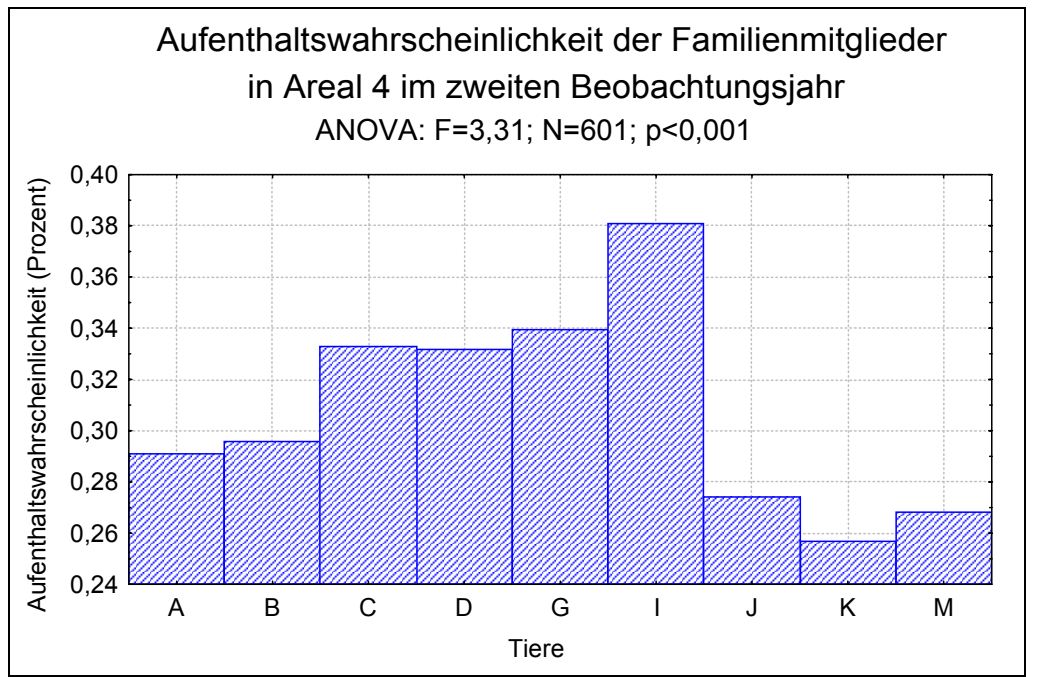

Abb. 3.135 Aufenthaltswahrscheinlichkeit der Familienmitglieder in Areal 4 ihres Streifgebietes im zweiten Beobachtungsjahr (ANOVA, F=3,31; N=601; $<<0,001$ ). Die Aufenthaltswahrscheinlichkeit von I in Areal 4 war höher als von A, B, J, K und $\mathrm{M}$. 


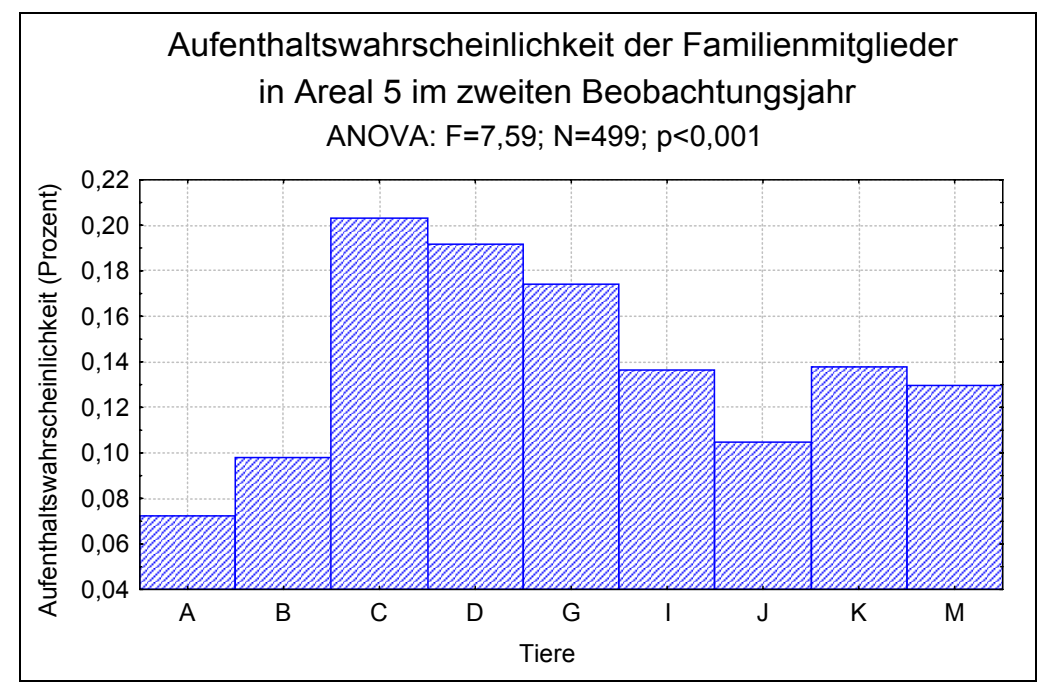

Abb. 3.136 Aufenthaltswahrscheinlichkeit der Familienmitglieder in Areal 5 ihres Streifgebietes im zweiten Beobachtungsjahr (ANOVA, F=7,59; $\mathrm{N}=499 ; \mathrm{p}<0,001$ ). Die adulten Nachkommen $\mathrm{C}$, D und $\mathrm{G}$ hielten sich mit höherer Wahrscheinlichkeit als die andern Familienmitglieder in Areal 5 auf. $\mathrm{C}$ war außerdem häufiger in Areal 5 als I, J, K und M. D und G waren mit höherer Wahrscheinlichkeit in Areal 5 als J.

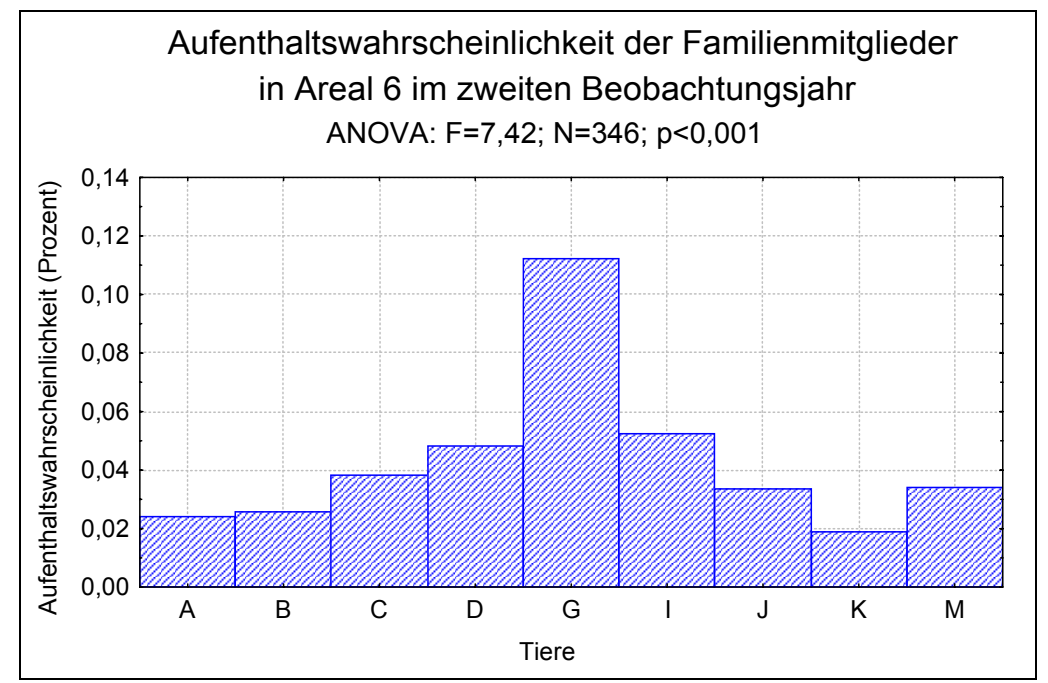

Abb. 3.137 Aufenthaltswahrscheinlichkeit der Familienmitglieder in Areal 6 ihres Streifgebietes im zweiten Beobachtungsjahr (ANOVA, F=7,42; N=346; $<<0,001$ ). G hielt sich mit höherer Wahrscheinlichkeit in Areal 6 auf als alle anderen Familienmitglieder.

\subsubsection{Peripheralisationen}

Im Freiland konnten bisher die Mechanismen, die zur Emigration einzelner Tiere aus der Gruppe führen, nicht geklärt werden (Digby 1995b, Ferrari 1988a,b, Stevenson und Rylands 1988). Rothe und Darms (1993) vermuten, dass graduelle Peripheralisationen einzelner Tiere von der Gruppe bei freilebenden Krallenaffen in Emigrationen resultieren, anstelle von Konflikten mit Beißkämpfen, die in Laborhaltung zu Ausschlüssen einzelner Familienmitglieder führen (siehe auch McGrew und McLuckie 1986, Sutcliffe und Poole 1984). McGrew (1997) betrachtet Peripheralisationen bei Saguinus als Folge von Angriffen durch 
Gruppenmitglieder. Digby (1995b) konnte nachweisen, dass sich Tiere, die aus Gruppen emigrierten, signifikant seltener im Nahbereich von Gruppenmitgliedern befanden, als solche, die in der Gruppe blieben, so dass auch hier ein Zusammenhang zwischen Peripheralisation und Emigration zu vermuten ist.

Hypothese 8: Peripheralisationen sind von individuenexternen Faktoren unabhängig.

Peripheralisationen könnten damit möglicherweise als eine Art Übergangsstadium zwischen Philopatrie und Emigration bzw. als Emigrationsbereitschaft betrachtet werden. Daraus ergaben sich folgende Erwartungen:

- Das Tier verringert die räumliche Assoziation zu seiner Natalfamilie, bleibt jedoch meist in Randbereichen des heimatlichen Streifgebietes. Aufgrund der räumlichen Trennung wird erwartet, dass sich auch die Nahbereichshäufigkeiten zu Familienmitgliedern reduzieren.

- Es wurde erwartet, dass sich adulte Nachkommen bei der Präsentation von gegengeschlechtlichen Locktieren sowie kurz vor einer freiwilligen Emigration oder einem Rauswurf räumlich von den übrigen Familienmitgliedern distanzieren.

- Es wäre auch denkbar, dass sich ein Tier nur morgens oder abends von der Familie entfernt, z.B. um von Familienmitgliedern ungesehen Kontakte zu potentiellen Reproduktionspartnern zu knüpfen. Während der übrigen Tageszeiten kann es weiterhin mit Familienmitgliedern interagieren, um soziale Integration in der Familie zu erhalten.

- Eine ausschließlich soziale Isolation könnte sich darin äußern, dass die taktilen Interaktionen zwischen einem Individuum und den anderen Familienmitgliedern verringert oder ganz einstellt werden, sich das Individuum aber trotzdem im Nahbereich anderer Familienmitglieder bewegt (siehe Hubrecht 1984, Soini 1982 für Cebuella).

Da die räumliche Assoziation zur Familie über die Messungen der Transponderanlage ermittelt wurde, ist sie eine Angabe für die großräumliche Distanz zwischen dem Familienmitglied und der Familie, d.h. ob sich Familienmitglieder im selben Areal oder in anderen Arealen aufhielten. Der Nahbereich eines Tieres ist als doppelte Armlänge definiert und umfasst einen Umkreis von ca. $50 \mathrm{~cm}$ des Tieres, so dass der Nahbereich eine wesentlich kleinere Dimension der räumlichen Assoziation beschreibt. Wenn ein Tier signifikant lockerer mit der Familie assoziiert ist als die anderen Familienmitglieder, wird es als peripher betrachtet. 


\subsubsection{Räumliche Assoziation der Familienmitglieder zur Familie}

Hypothese 8a: Die räumliche Assoziation zur Familie ist bei allen Familienmitgliedern gleich groß.

Hypothese 8b: Die räumliche Assoziation zur Familie ist von den Experimenten unabhängig.

Hypothese 8c: Die räumliche Assoziation zur Familie in den Morgen- und Abendstunden weicht bei den Familienmitgliedern nicht vom Ganztagesmittel ab.

\subsection{Räumliche Assoziation der Familienmitglieder gemittelt über den ganzen Tag im ersten Beobachtungsjahr}

Ein Vergleich der Familienmitglieder in ihrer räumlichen Assoziation zu der Gesamtheit der anderen Familienmitglieder (Restfamilie/Familie) gemittelt über den gesamten Tag (04:3022:00Uhr) ergab im ersten Beobachtungsjahr Unterschiede zwischen den Familienmitgliedern (Kruskal-Wallis-Test: $\mathrm{H}=172,80 ; \mathrm{N}=52 ; \mathrm{p}<0,001$ ). Eine Abhängigkeit der räumlichen Assoziation von den Experimenten war nicht zu verzeichnen (Kruskal-Wallis-Test: $\mathrm{H}=2,32$; $\mathrm{N}=552$; n.s.).

In der ersten Kontrollphase (Abb. 3.138a) wiesen die Eltern A und B und die Tochter H die engste Assoziation zur Familie auf, während $G$ am lockersten räumlich mit der Familie assoziiert war. Sein Wert unterschied sich von allen anderen Familienmitgliedern. Die Assoziationskoeffizienten von $\mathrm{C}$ und $\mathrm{D}$ lagen genau dazwischen und die Koeffizienten von $\mathrm{E}$ und $\mathrm{F}$ waren wiederum zwischen denen der Eltern und der adulten Geschwister angesiedelt. Mit der Ausnahme der Eltern und von G nahm der Assoziationskoeffizient mit zunehmendem Alter der Individuen ab, wobei sich der Wert von C zu D nicht mehr verringerte. Nachdem in der zweiten Kontrollphase $F$ aus der Familie geworfen worden war, zeigte nur $G$ eine geringere Assoziation zur Familie. Zwischen den anderen Familienmitgliedern war kein Unterschied festzustellen (Abb. 3.138b). 

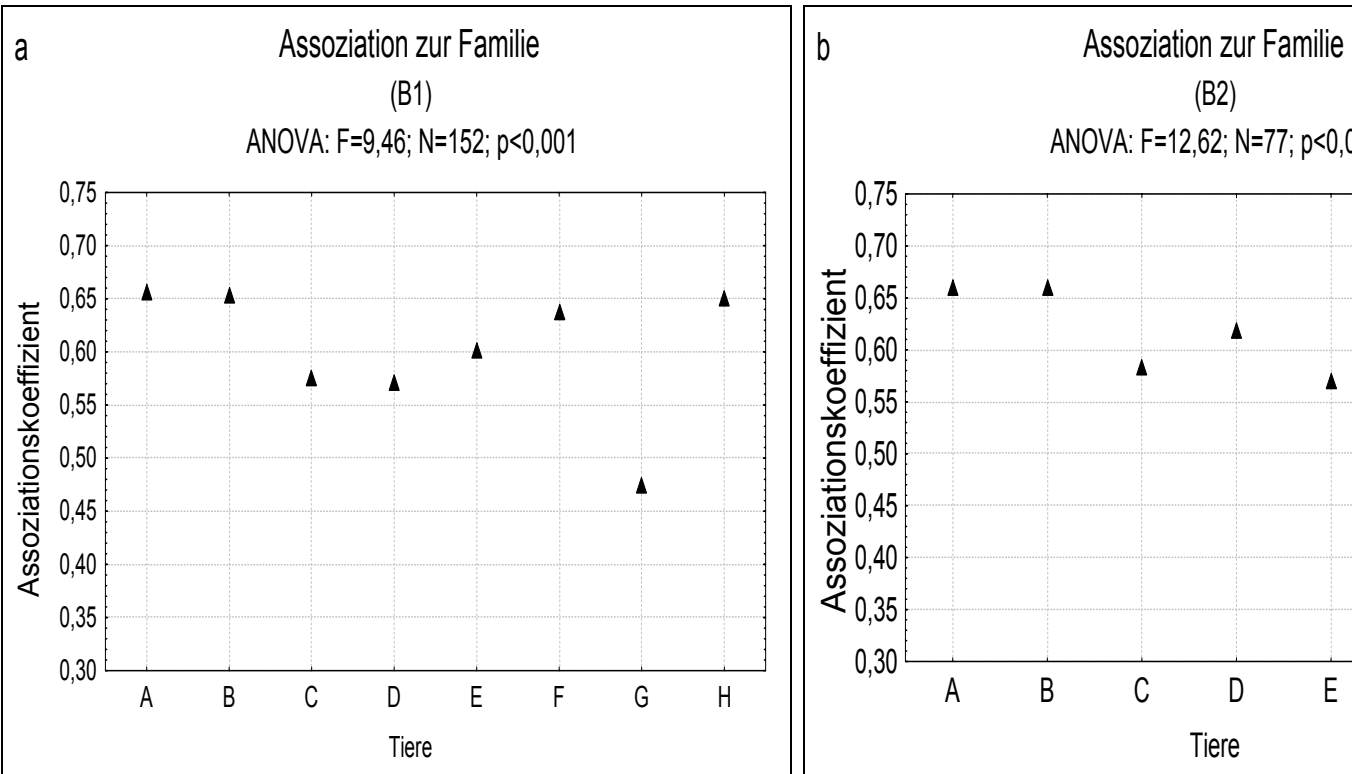

(B2)
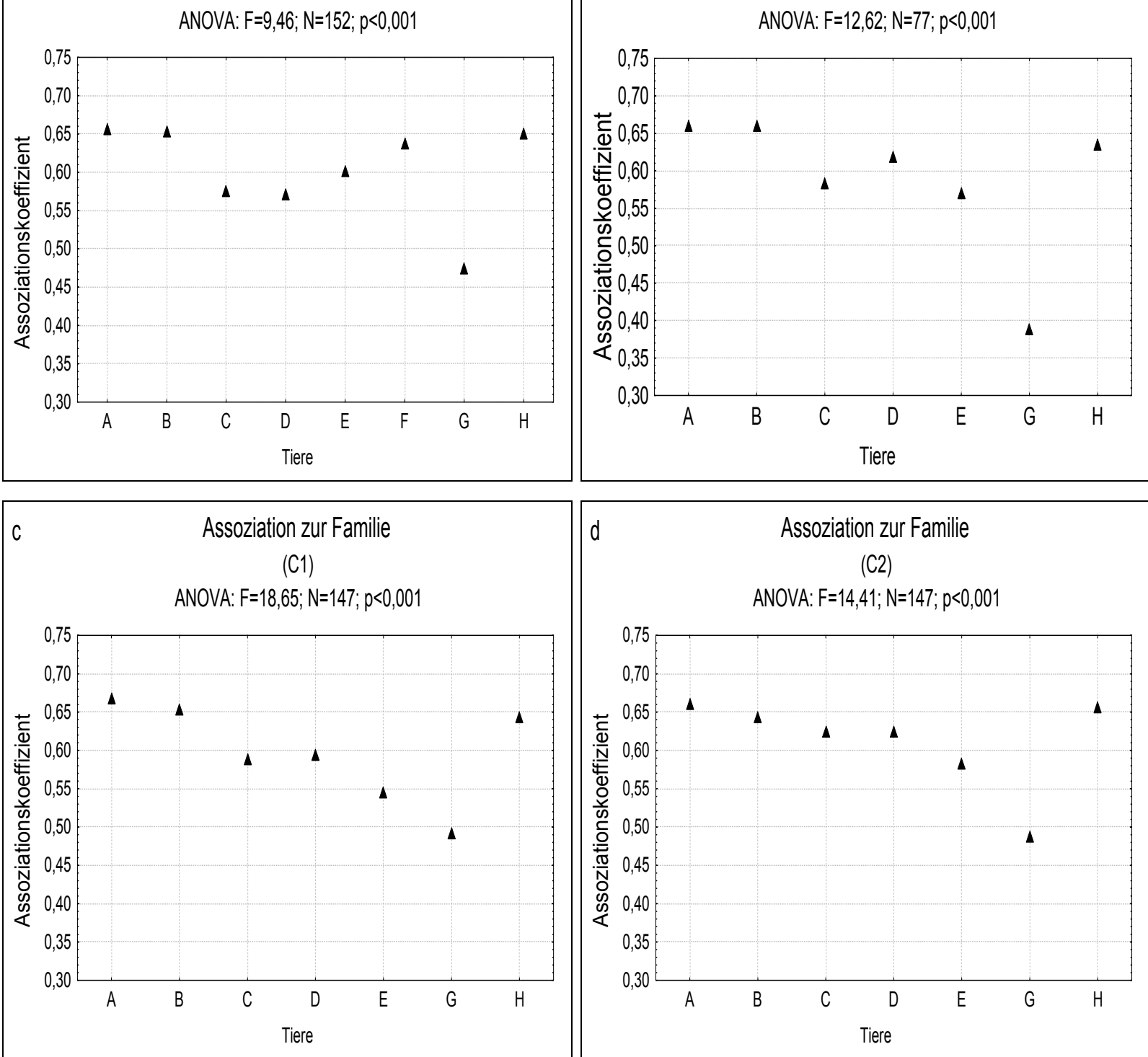

d $\quad$ Assoziation zur Familie

(C2)

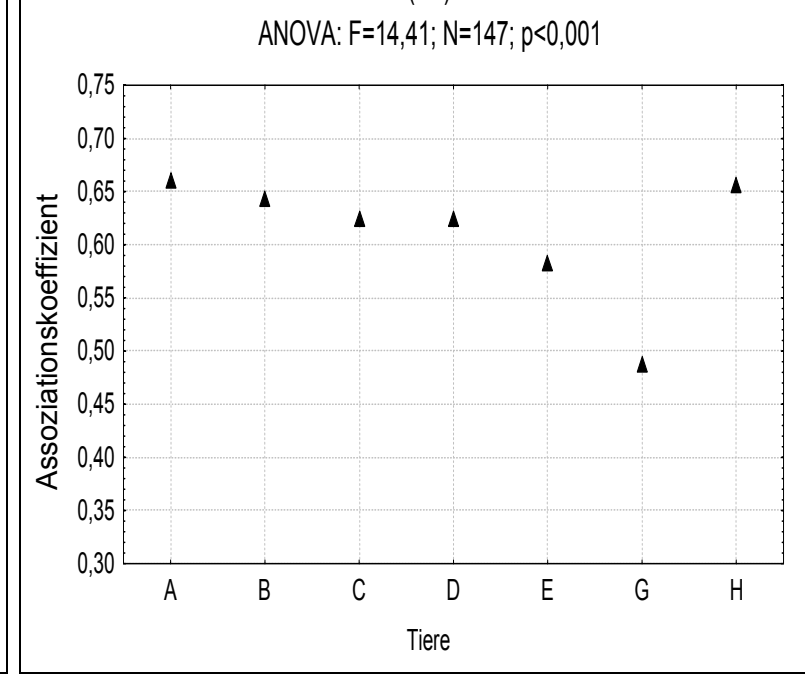

Abb. 3.138 Räumliche Assoziation der Familienmitglieder zu den übrigen Familienmitgliedern in den vier Experimentalphasen des ersten Beobachtungsjahres gemittelt über den ganzen Tag (04:30-22:00 Uhr). Der Assoziationskoeffizient ist ein $\mathrm{Ma} ß$ für die Stärke der Assoziation bzw. Kohäsion eines einzelnen Familienmitgliedes zur Gesamtheit der anderen Familienmitglieder (Restfamilie/ Familie). Der Koeffizient liegt zwischen 0 und 1, wobei 1 die engste Assoziation und 0 die lockerste Assoziation zwischen Tier und Familie beschreibt. Den Tieren A-H in der Ordinate wird jeweils ein eigener Assoziationsindex zugeordnet (Abszisse). Durch die Varianzanalyse (hier einfaktorielle ANOVA) wird deutlich, inwieweit die Unterschiede zwischen den Assoziationskoeffizienten zufällig sind.

Während des ersten Experiments waren die Nachkommen C, D, E und G lockerer mit anderen Familienmitgliedern assoziiert als die Eltern und H. Die Brüder E und G zeigten eine lockerere Assoziation zur Familie als $\mathrm{C}$ und D, wobei G noch weniger eng mit der Familie assoziiert war als E (Abb. 3.138c). Mit abnehmendem Alter der Nachkommen verringerten sich deren Assoziationskoeffizienten. $\mathrm{C}$ und $\mathrm{D}$ unterschieden sich jedoch nicht und die 
jüngste Tochter $\mathrm{H}$ hatte einen mit den Eltern vergleichbaren Assoziationskoeffizienten. Im zweiten Experiment hatte G eine lockerere Assoziation zur Familie als alle anderen Familienmitglieder. E unterschied sich in seiner räumlichen Assoziation auch von den Eltern und $\mathrm{H}$ (Abb. 3.138d).

Die räumliche Assoziation der häufig peripheren Nachkommen C, D, E und G zur Familie war von den Experimenten unabhängig (ANOVA, $C$ : $F=1,25 ; N=72 ; p<0,29 ; D: F=1,77$; $N=71 ; p<0,16 ; E: F=1,40 ; N=72 ; p<0,24$ und $G: F=2,58 ; N=72 ; p<0,06)$.

\subsection{Räumliche Assoziation der Familienmitglieder in den Morgenstunden im ersten Beobachtungsjahr}

Die räumliche Assoziation der Familienmitglieder wurde gesondert für die Morgen- und Abendstunden analysiert, da eine Peripheralisation während dieser Tageszeiten darauf hindeuten kann, dass ein Tier von der Familie, die noch (morgens) oder schon (abends) im Schlafkasten versammelt ist, ungesehen mit potentiellen Reproduktionspartnern kommunizieren kann. Während der Morgenstunden (04:30-07:30 Uhr) gab es sowohl Unterschiede zwischen den Familienmitgliedern in ihrer Assoziation zur Familie (Kruskal-Wallis-Test: $\mathrm{H}=58,44 ; \mathrm{N}=439 ; \mathrm{p}<0,001)$, als auch Abhängigkeiten der Assoziation von den Experimenten (Abb. 3.139).

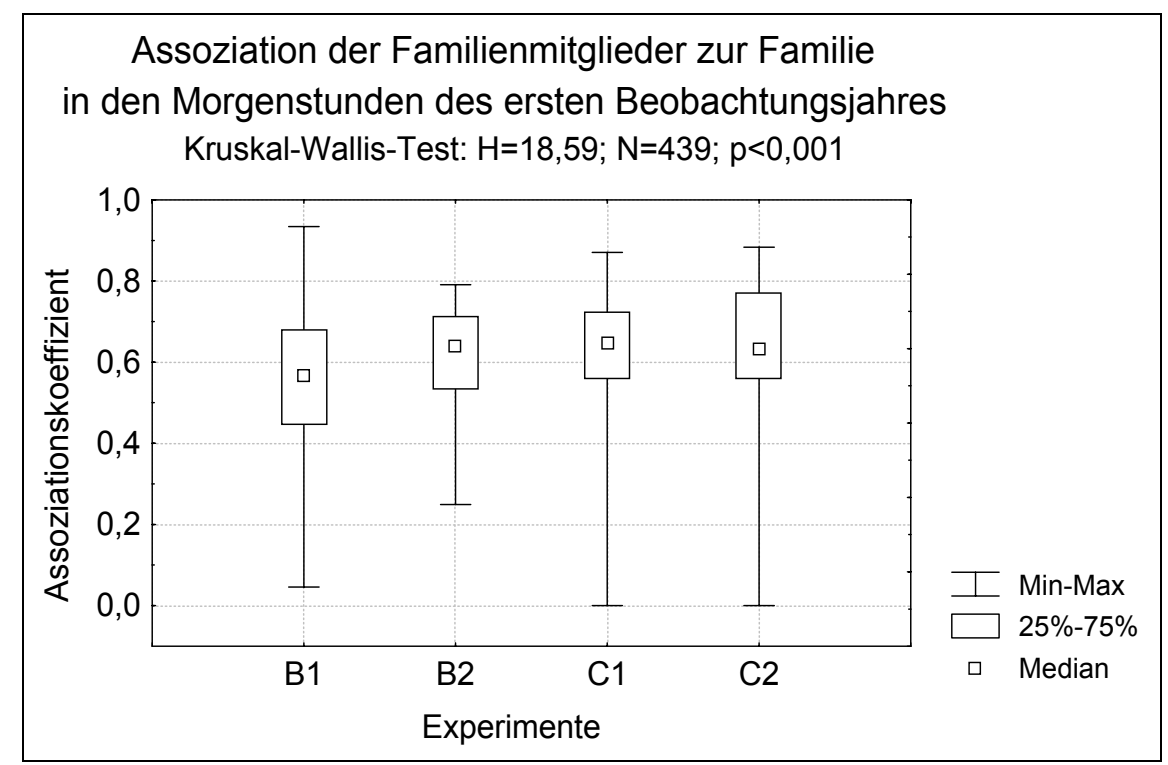

Abb. 3.139 Räumliche Assoziation der Familienmitglieder zur Familie in Abhängigkeit von den Experimenten im ersten Beobachtungsjahr (Kruskal-Wallis-Test: $\mathrm{H}=18,59$; $\mathrm{N}=439$; $\mathrm{p}=0,0003$ ). Die Assoziation der Familienmitglieder zeigt eine Abhängigkeit von den Experimenten. Die lockerste Assoziation zur Familie zeigt sich im ersten Experiment. 
Während aller Experimentalphasen unterschieden sich die Familienmitglieder in ihrer Assoziation zur Familie. In der ersten Kontrollphase (Abb. 3.140a) waren die Familienmitglieder morgens ähnlich mit der Familie assoziiert wie ganztags. C, D und G unterschieden sich durch eine lockerere räumliche Assoziation zur Familie von den Eltern und $\mathrm{C}, \mathrm{D}, \mathrm{E}$ und $\mathrm{G}$ von $\mathrm{F}$ und $\mathrm{H}$.
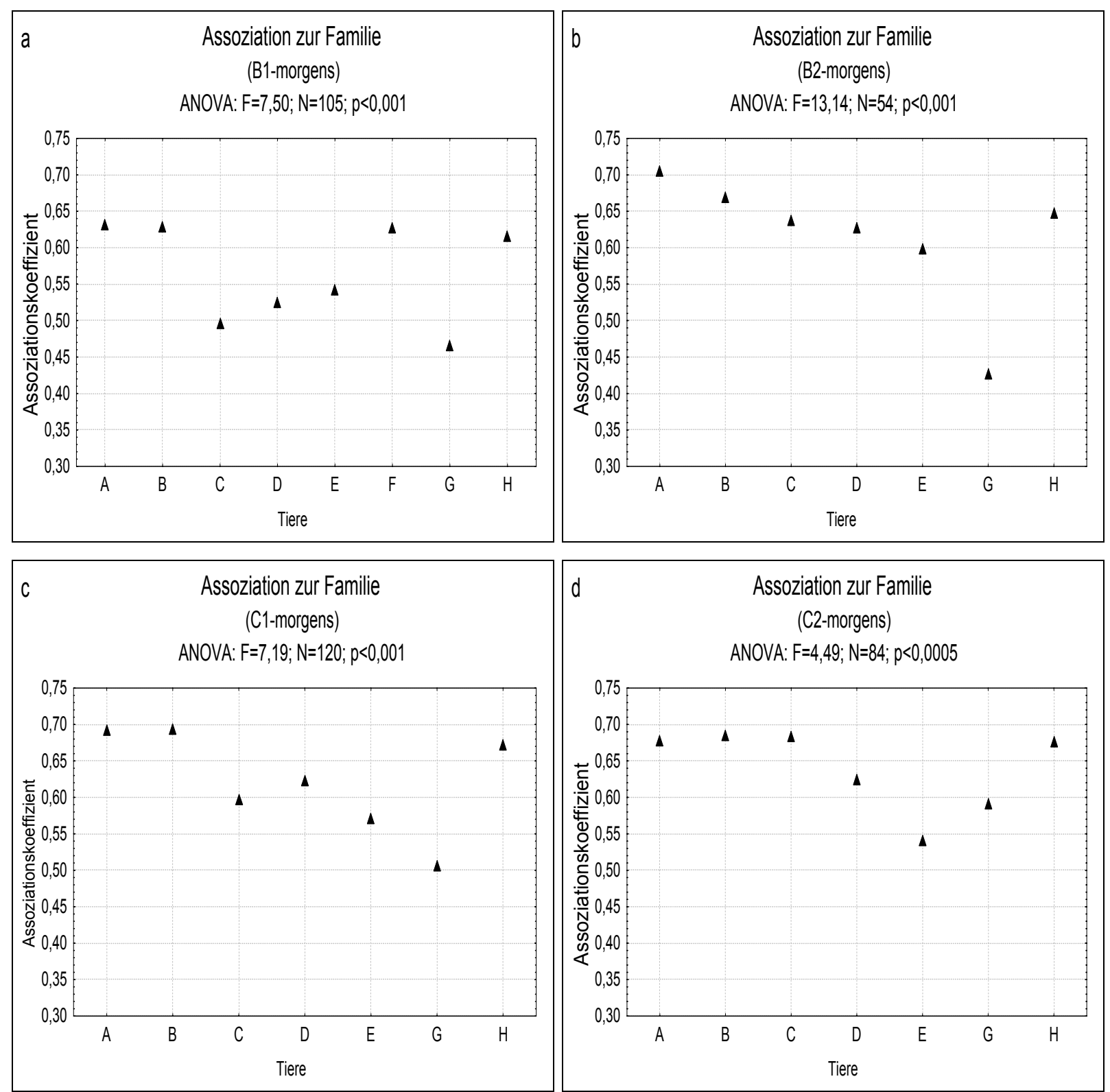

Abb. 3.140 Räumliche Assoziation der Familienmitglieder zu den übrigen Familienmitgliedern in den vier Experimentalphasen (a-d) des ersten Beobachtungsjahres in den Morgenstunden (04:30 bis 07:30 Uhr). Der Assoziationskoeffizient ist ein Maß für die Stärke der Assoziation bzw. Kohäsion eines einzelnen Familienmitgliedes zur Gesamtheit der anderen Familienmitglieder (Restfamilie/Familie). Der Koeffizient liegt zwischen 0 und 1, wobei 1 die engste Assoziation und 0 die lockerste Assoziation zwischen Tier und Familie beschreibt. Den Tieren A-H in der Ordinate wird jeweils ein eigener Assoziationsindex zugeordnet (Abszisse). Durch die Varianzanalyse (hier einfaktorielle ANOVA) wird deutlich, inwieweit die Unterschiede zwischen den Assoziationskoeffizienten zufällig sind. 
In der zweiten Kontrollphase (Abb. 3.140b) hatte $G$ einen geringeren Assoziationskoeffizienten als die anderen Familienmitglieder und E war lockerer mit der Familie assoziiert als A. Damit assoziierten sich die Männchen mit zunehmendem Alter räumlich enger zur Familie. Im ersten Experiment (Abb. 3.140c) gab es morgens keine Veränderungen der Assoziationskoeffizienten zwischen den Familienmitgliedern gegenüber der zweiten Kontrollphase, lediglich wies E einen geringeren Assoziationskoeffizienten auf als B. Im zweiten Experiment (Abb. 3.140d) war E lockerer räumlich mit der Familie assoziiert als A, B, C und H. G unterschied sich nicht mehr von den übrigen Familienmitgliedern. Die Assoziationskoeffizienten in den Morgenstunden unterschieden sich bei keinem Familienmitglied von den Assoziationskoeffizienten, die über den ganzen Tag gemittelt waren (Tab. 3.32).

Tabelle 3.32 Vergleich der über den ganzen Tag gemittelten Assoziationskoeffizienten eines Tieres mit den Assoziationskoeffizienten desselben Tieres in den Morgenstunden im ersten Beobachtungsjahr (einfaktorielle ANOVA). Abkürzungen: $\mathrm{N}$ = Anzahl der gewerteten Tage; * bei $\mathrm{C}$ war keine ANOVA zulässig, so dass bei $\mathrm{C}$ auf den Kruskal-Wallis-Test ausgewichen wurde. Daher handelt es sich bei $\mathrm{C}$ um den H-Wert.

\begin{tabular}{llll} 
Tier & $\mathrm{N}$ & $\mathrm{F}-$ Wert & $\mathrm{p}$-Wert \\
\hline A & 137 & 0,721 & 0,397 \\
$\mathrm{~B}$ & 137 & 1,247 & 0,266 \\
$\mathrm{C}$ & 139 & $1,45^{*}$ & 0,228 \\
$\mathrm{D}$ & 136 & 0,009 & 0,924 \\
$\mathrm{E}$ & 137 & 0,270 & 0,604 \\
$\mathrm{~F}$ & 35 & 0,060 & 0,807 \\
$\mathrm{G}$ & 136 & 1,808 & 0,181 \\
$\mathrm{H}$ & 136 & 0,218 & 0,641 \\
\hline
\end{tabular}

\subsection{Räumliche Assoziation der Familienmitglieder in den Abendstunden im ersten Beobachtungsjahr}

In den Abendstunden unterschieden sich die Familienmitglieder in der räumlichen Assoziation zur Familie (Kruskal-Wallis-Test: $\mathrm{H}=118,62$; $\mathrm{N}=568 ; \mathrm{p}<0,001$ ). Es gab jedoch keine Abhängigkeiten der räumlichen Assoziation von den Experimenten (Kruskal-WallisTest: $\mathrm{H}=2,09 ; \mathrm{N}=568 ;$ n.s.).

Während der ersten Kontrollphase und im ersten Experiment unterschieden sich die Familienmitglieder in ihrer Assoziation zur Familie. In beiden Fällen war G lockerer mit der Familie assoziiert als die übrigen Familienmitglieder (Abb. 3.141a und c). Während der zweiten Kontrollphase traten keine Unterschiede in der räumlichen Assoziation zur Familie zwischen 
den Familienmitgliedern auf (Abb. 3.141b). Für das zweite Experiment lagen für die Abendstunden nicht genug Daten vor, um eine statistische Analyse durchzuführen, da das zweite Experiment im Herbst stattfand und sich die Fokustiere zwischen 17:30 und 22:00 Uhr nur noch selten außerhalb ihres Schlafkastens bzw. der Schutzhütte aufhielten.

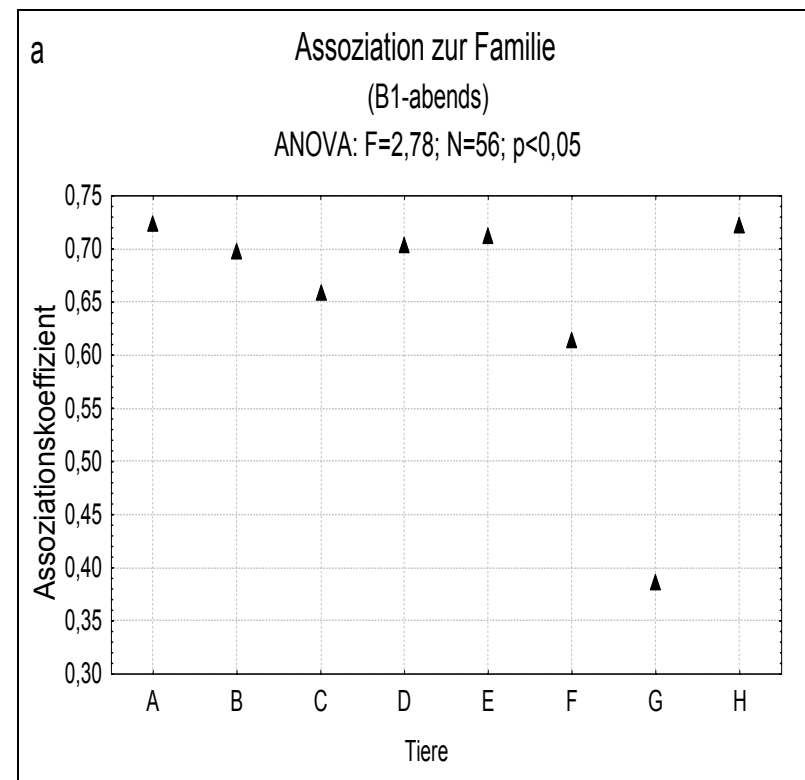

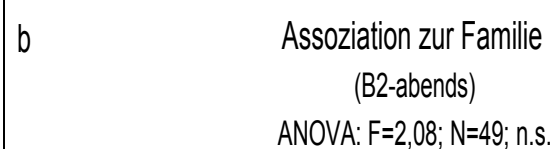

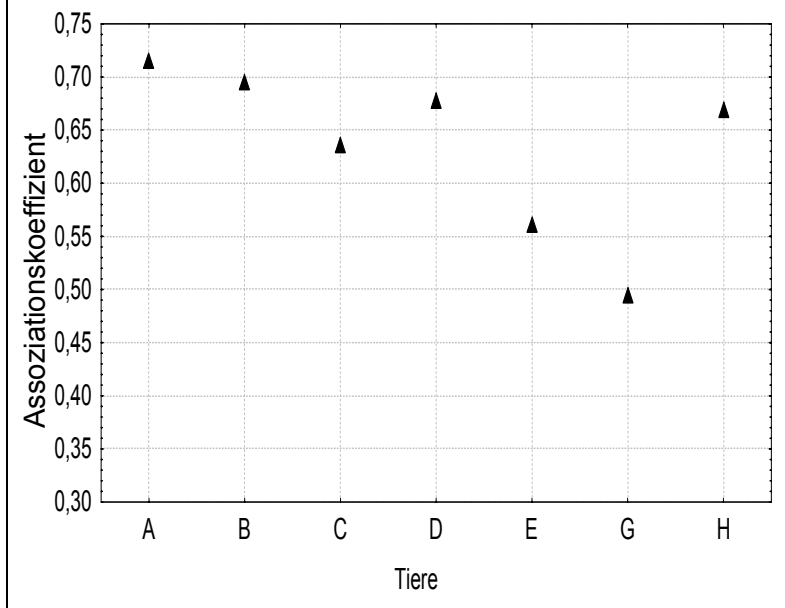

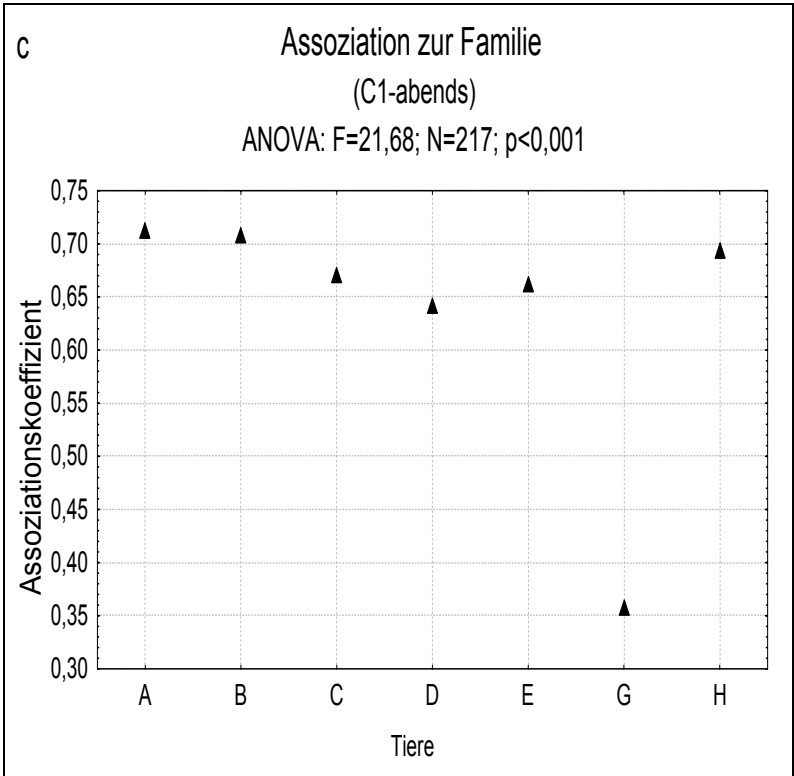

Abb. 3.141 Räumliche Assoziation der Familienmitglieder zu den übrigen Familienmitgliedern in den drei Experimentalphasen (a-c) des ersten Beobachtungsjahres in den Abendstunden (17:30 bis 22:00 Uhr). Für das Experiment C2 lagen nicht genügend Daten vor. Der Assoziationskoeffizient ist ein Maß für die Stärke der Assoziation bzw. Kohäsion eines einzelnen Familienmitgliedes zur Gesamtheit der anderen Familienmitglieder. Der Koeffizient liegt zwischen 0 und 1, wobei 1 die engste Assoziation und 0 die lockerste Assoziation zwischen Tier und Familie beschreibt. Den Tieren A-H in der Ordinate wird jeweils ein eigener Assoziationsindex zugeordnet (Abszisse). Durch die Varianzanalyse (hier einfaktorielle ANOVA) wird deutlich, inwieweit die Unterschiede zwischen den Assoziationskoeffizienten zufällig sind. 
Da G sehr häufig eine lockere Assoziation zur Familie hatte und sich in den meisten Experimenten dadurch von den anderen Familienmitgliedern unterschied, wurde getestet, in welchen Arealen er sich bevorzugt aufhielt. In der ersten Kontrollphase bevorzugte er Areal 2 und in der zweiten Kontrollphase Areale 1 und 2. Während der Experimente C1 und C2 hielt G sich dann häufiger in Areal 3 auf (Kruskal-Wallis-Test: $\mathrm{C} 1: \mathrm{H}=29,90 ; \mathrm{N}=131 ; \mathrm{p}=0,001 ; \mathrm{C} 2$ : $\mathrm{H}=58,05 ; \mathrm{N}=132 ; \mathrm{p}=0,001)$. Die Ergebnisse lassen vermuten, dass $\mathrm{G}$ trotz seiner Peripheralisation nicht an fremden Artgenossen interessiert war, da seine bevorzugten Aufenthaltsorte von der Nachbarfamilie, als auch vom Experimentalareal abgewandt lagen (Areale 1-3, vgl. Abb. 2.2, Übersichtsskizze S.23). E hingegen nutzte bevorzugt Areal 5, vor allem während des ersten Experiments (C1), was seine Orientierung $\mathrm{zu}$ den Locktieren (und Nachbarfamilien) widerspiegelt (K.-W.-Test: $\mathrm{C} 1: \mathrm{H}=108,06 ; \mathrm{N}=153 ; \mathrm{p}=0,001$ ).

Beim Vergleich der Assoziationskoeffizienten, die für den ganzen Tag gemittelt wurden und den Assoziationskoeffizienten in den Abendstunden gab es nur bei F keine Abweichung. Bei A, B, C, D, E und H war die Assoziation zur Familie in den Abendstunden höher als über das Tagesmittel. Die Assoziationskoeffizienten von $\mathrm{G}$ waren dafür abends niedriger als über den ganzen Tag gemittelt (Tab. 3.33).

Tabelle 3.33 Vergleich der Assoziationskoeffizienten eines Tieres gemittelt über den ganzen Tag mit den Assoziationskoeffizienten desselben Tieres in den Abendstunden im ersten Beobachtungsjahr (einaktorielle ANOVA). Abkürzungen: $\mathrm{N}=$ Anzahl der gewerteten Tage.

\begin{tabular}{llll} 
Tier & N & F-Wert & p-Wert \\
\hline A & 154 & 10,351 & 0,002 \\
B & 154 & 13,635 & 0,001 \\
C & 154 & 12,459 & 0,001 \\
D & 153 & 6,838 & 0,010 \\
E & 154 & 17,524 & 0,001 \\
F & 26 & 0,319 & 0,577 \\
G & 154 & 10,017 & 0,002 \\
H & 154 & 8,215 & 0,005 \\
\hline
\end{tabular}

\subsection{Räumliche Assoziation der Familienmitglieder gemittelt über den ganzen Tag im zweiten Beobachtungsjahr}

Im zweiten Beobachtungsjahr waren die Brüder $\mathrm{E}$ und $\mathrm{F}$ bereits aus der Fokusfamilie herausgeworfen worden. Kurz bevor die Familie im Juni 2001 in das Semifreigelände gelassen wurde, warf D ihre jüngere Schwester aus der Sozietät, so dass sich am Anfang der 
Beobachtungssaison 2001 die Eltern, fünf adulte (C, D, G, I und J) und zwei juvenile Nachkommen ( $\mathrm{K}$ und $\mathrm{M}$ ) in der Familie aufhielten.

Im zweiten Beobachtungsjahr unterschieden sich die Familienmitglieder in der räumlichen Assoziation der zur Familie (Kruskal-Wallis-Test; $\mathrm{H}=78,99, \mathrm{p}<0,001$ ). Es war außerdem eine Abhängigkeit der räumlichen Assoziation der Familienmitglieder zur Familie von den Experimenten festzustellen (Abb. 3.142). Während des zweiten Experiments war die räumliche Assoziation der Familienmitglieder zu den jeweils anderen Familienmitgliedern am lockersten.

Innerhalb der einzelnen Experimente unterschieden sich die Familienmitglieder in der Assoziation zur Familie. Im ersten Experiment hatten C und G eine lockerere Assoziation zur Familie als die anderen Tiere (siehe Abb. 3.143a). A, B, I, J und K waren dabei am engsten mit der Familie assoziiert. Die Assoziationskoeffizienten von D und M lagen im mittleren Bereich.

D, G und I waren im zweiten Experiment weniger eng mit der Familie assoziiert und unterschieden sich von A, B und M. G unterschied sich in der räumlichen Assoziation zur Familie außerdem von K (Abb. 3.143b).

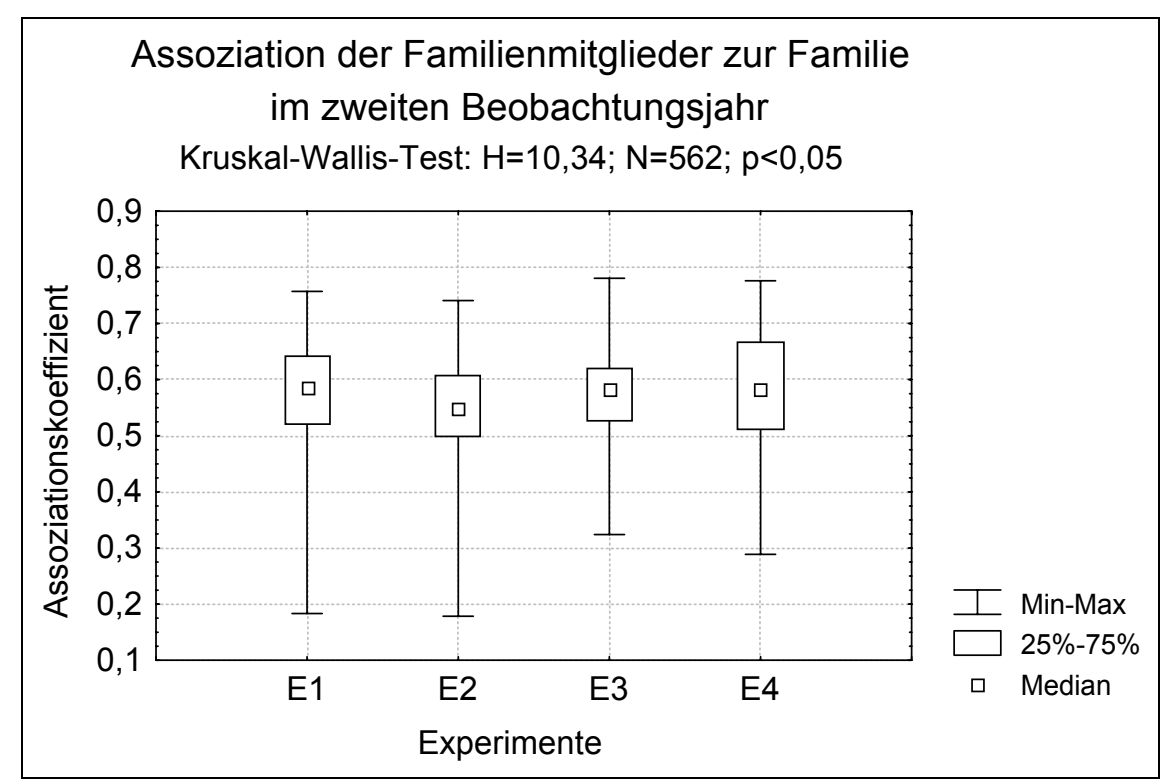

Abb. 3.142 Räumliche Assoziation der Familienmitglieder zur Familie in Abhängigkeit von den Experimenten gemittelt über den ganzen Tag im zweiten Beobachtungsjahr (Kruskal-Wallis-Test: $\mathrm{H}=10,34 ; \mathrm{N}=562 ; \mathrm{p}=0,015$ ). Die Assoziation der Familienmitglieder zeigte eine Abhängigkeit von den Experimenten. 

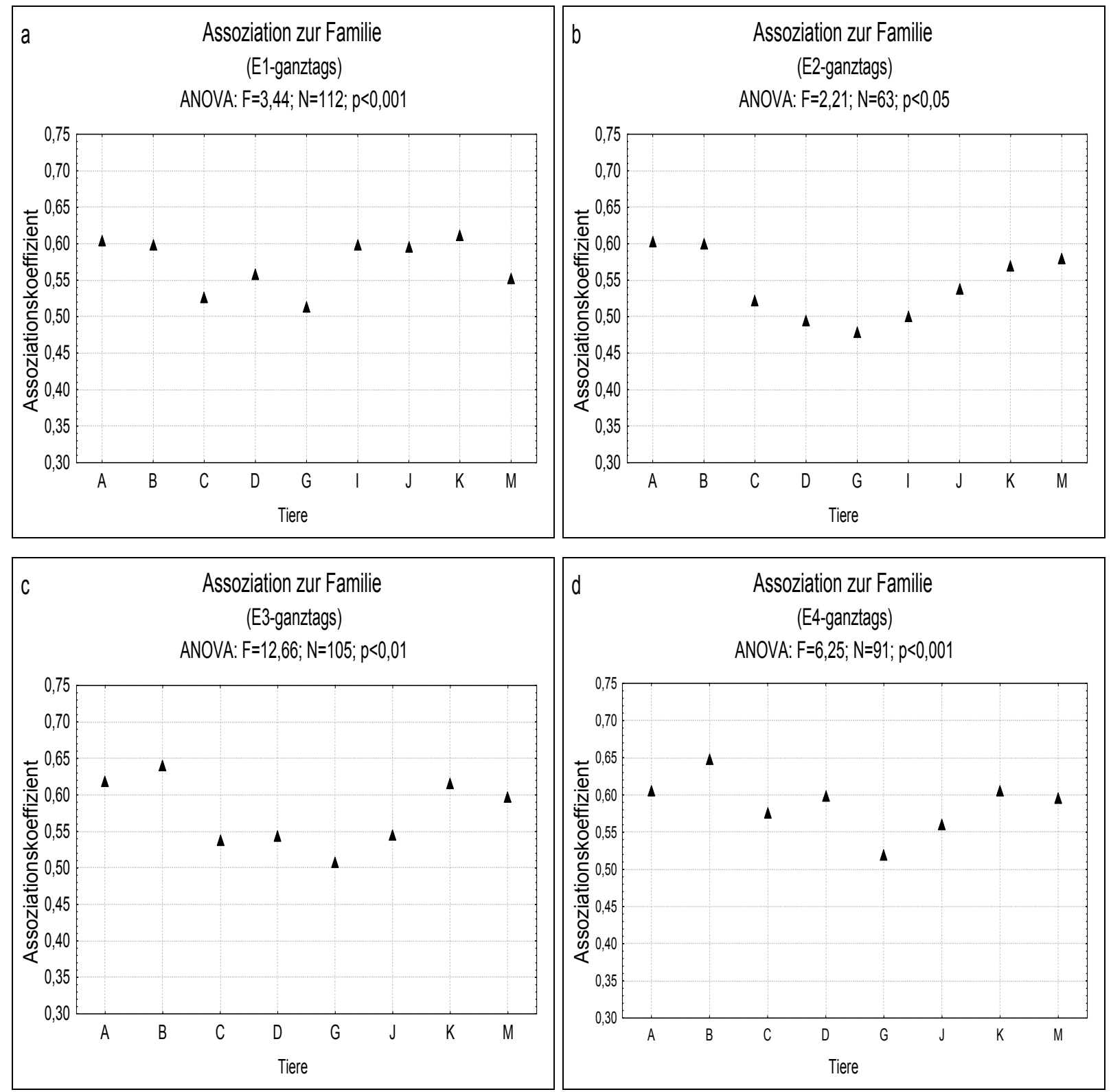

Abb. 3.143 Räumliche Assoziation der Familienmitglieder zu den übrigen Familienmitgliedern in den vier Experimentalphasen (a-d) des zweiten Beobachtungsjahres gemittelt über den ganzen Tag (04:30-22:00 Uhr). Der Assoziationskoeffizient ist ein $\mathrm{Ma} ß$ für die Stärke der Assoziation bzw. Kohäsion eines einzelnen Familienmitgliedes zur Gesamtheit der anderen Familienmitglieder. Der Koeffizient liegt zwischen 0 und 1, wobei 1 die engste Assoziation und 0 die lockerste Assoziation zwischen Tier und Familie beschreiben würde. Den Tieren A-H in der Ordinate wird jeweils ein eigener Assoziationsindex zugeordnet (Abszisse). Durch die Varianzanalyse (hier einfaktorielle ANOVA) wird deutlich, inwieweit die Unterschiede zwischen den Assoziationskoeffizienten zufällig sind.

Es fiel auf, dass die Assoziation zu den Familienmitgliedern bei den älteren adulten Nachkommen enger war als bei jüngeren adulten Nachkommen und bei den subadulten lockerer war als bei den juvenilen Nachkommen. Nach dem Rauswurf von I war die Assoziation aller adulten Nachkommen (C, D, G und J) zu den übrigen Familienmitgliedern relativ locker (Abb. 3.143c: ANOVA, F=12,66, $\mathrm{p}<0,001$ ), während $\mathrm{A}, \mathrm{B}, \mathrm{K}$ und $\mathrm{M}$ eine 
engere Assoziation zu den Familienmitgliedern aufwiesen. Im vierten Experiment wurde die Assoziation zur Familie bei C und D wieder enger. Bei G und J blieb sie weiterhin locker; ebenso zeigte B eine engere Assoziation zur Familie als A (Abb. 3.143d: ANOVA, F=6,25, $\mathrm{p}<0,001)$.

Die räumliche Assoziation zur Familie wurde für die adulten und subadulten Nachkommen auf Abhängigkeit von den Experimenten geprüft. Bei dem subadulten I lockerte sich seine räumliche Assoziation zur Familie im zweiten Experiment gegenüber dem ersten Experiment (Abb. 3.144). Bei allen anderen adulten und subadulten Nachkommen gab es keine Abhängigkeiten der räumlichen Assoziation zur Familie von den Experimenten (ANOVA, C: $F=0,50 p<0,68 ; D: F=2,44 p<0,07 ; G: F=0,33 p<0,81 ; J: F=1,52 p<0,22)$.

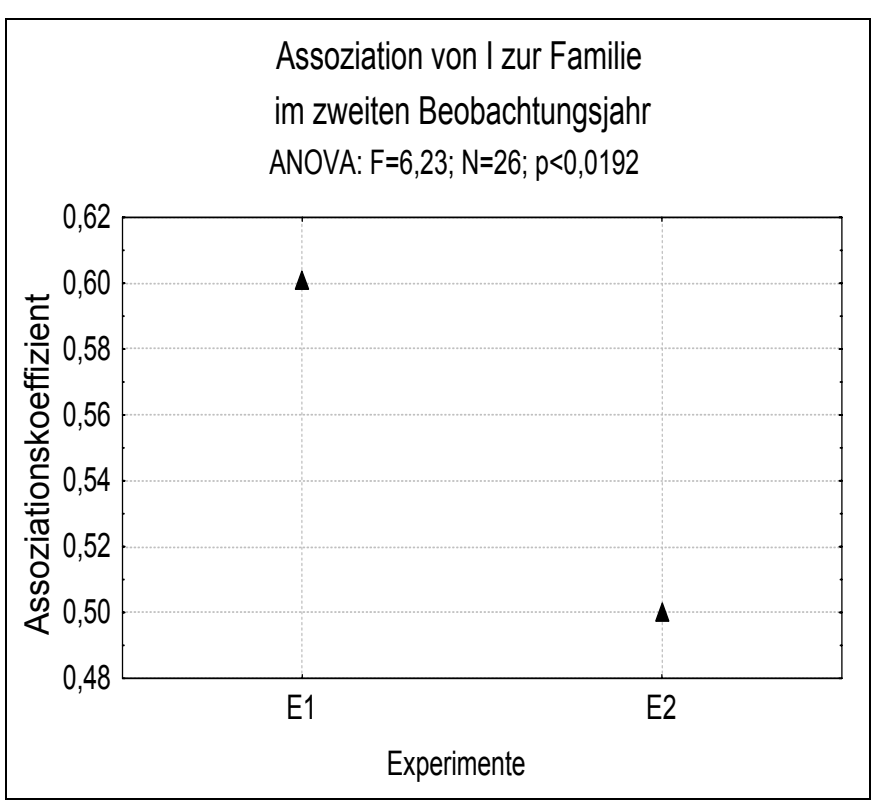

Abb. 3.144 Räumliche Assoziation von I zu den Familienmitgliedern während des ersten und zweiten Experiments im zweiten Beobachtungsjahr. Die Assoziation zur Familie war während des zweiten Experiments lockerer als während des ersten Experiments (ANOVA: $F=6,23 ; \mathrm{N}=26 ; \mathrm{p}<0,0192$ ).

\subsection{Räumliche Assoziation der Familienmitglieder in den Morgenstunden im zweiten Beobachtungsjahr}

In den Morgenstunden des zweiten Beobachtungsjahres unterschieden sich die Familienmitglieder in ihrer räumlichen Assoziation zur Familie (Kruskal-Wallis-Test: $\mathrm{H}=38,02$; $\mathrm{N}=504 ; \mathrm{p}<0,001)$ und es gab Abhängigkeiten der räumlichen Assoziation zur Familie von den Experimenten (Abb. 3.145). 


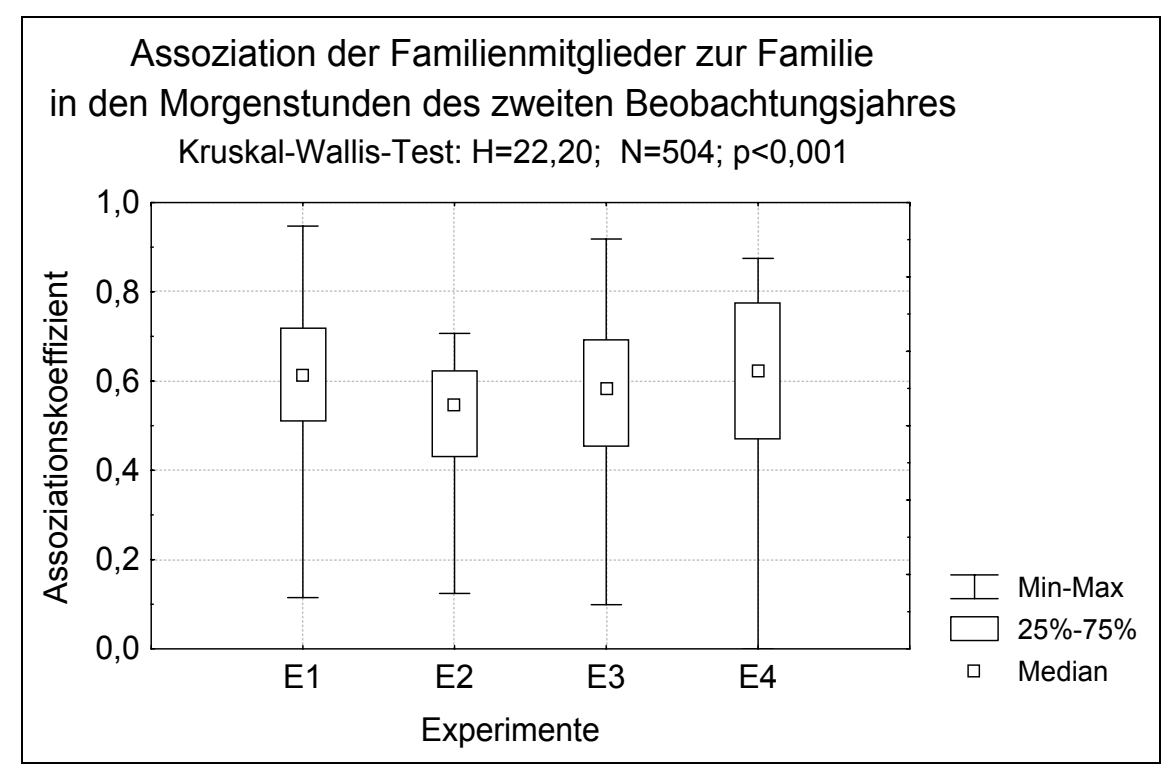

Abb. 3.145 Räumliche Assoziation der Familienmitglieder zur Familie in Abhängigkeit von den Experimenten während der Morgenstunden im zweiten Beobachtungsjahr (Kruskal-Wallis-Test: H=22,20; N=504; $<<0,001$ ). Die Assoziation der Familienmitglieder war von den Experimenten abhängig. Die lockerste Assoziation zur Familie bestand im zweiten Experiment.

In den Morgenstunden der ersten beiden Experimente unterschied sich nur D von den Eltern (Abb. 3.146a). Zwischen den anderen Familienmitgliedern gab es keine Unterschiede in ihrer Assoziation zur Familie. Die Verteilung der Assoziationskoeffizienten entsprach morgens im zweiten Experiment (Abb. 3.146b) weitestgehend der Verteilung des Tagesmittelwertes; nur D war am meisten peripher und $G$ enger mit den Familienmitgliedern assoziiert. Während des dritten Experiments (Abb. 3.146c) waren die Tiere ähnlich mit der Familie assoziiert wie im ersten Experiment, wobei J lockerer mit den Familienmitgliedern assoziiert war. Im vierten Experiment (Abb. 3.146d) gab es keine Unterschiede mehr zwischen den Familienmitgliedern in ihrer räumlichen Assoziation zur Familie. 

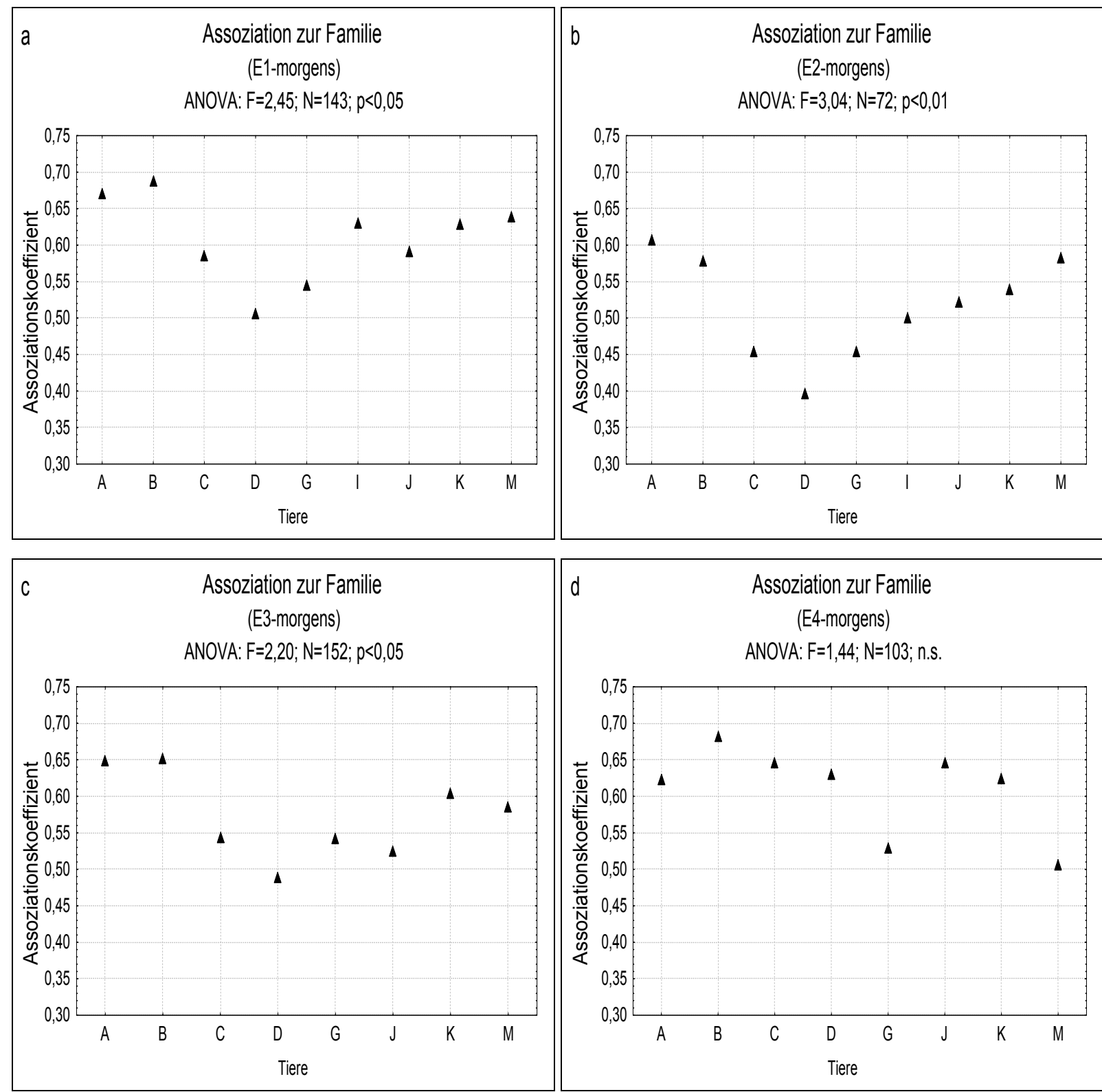

Abb. 3.146 Räumliche Assoziation der Familienmitglieder zu den übrigen Familienmitgliedern in den vier Experimentalphasen (a- d) des zweiten Beobachtungsjahres in den Morgenstunden (04:30 bis 07:30 Uhr) Der Assoziationskoeffizient ist ein $\mathrm{Ma} \beta$ für die Stärke der Assoziation bzw. Kohäsion eines einzelnen Familienmitgliedes zur Gesamtheit der anderen Familienmitglieder. Der Koeffizient liegt zwischen 0 und 1, wobei 1 die engste Assoziation und 0 die lockerste Assoziation zwischen Tier und Familie beschreiben würde. Den Tieren A-M in der Ordinate wird jeweils ein eigener Assoziationsindex zugeordnet (Abszisse). Durch die Varianzanalyse (hier einfaktorielle ANOVA) wird deutlich, inwieweit die Unterschiede zwischen den Assoziationskoeffizienten zufällig sind.

Bei keinem Familienmitglied unterschieden sich die Assoziationskoeffizienten, die über den ganzen Tag gemittelt wurden von den Assoziationskoeffizienten in den Morgenstunden (Tab. 3.34). 
Tabelle 3.34 Vergleich der Assoziationskoeffizienten eines Tieres gemittelt über den ganzen Tag mit den Assoziationskoeffizienten desselben Tieres in den Morgenstunden im zweiten Beobachtungsjahr (einfaktorielle ANOVA). Abkürzungen: $\mathrm{N}=$ Anzahl der gewerteten Tage.

\begin{tabular}{llll} 
Tier & $\mathrm{N}$ & $\mathrm{F}$-Wert & $\mathrm{p}$-Wert \\
\hline $\mathrm{A}$ & 125 & 2,769 & 0,099 \\
$\mathrm{~B}$ & 125 & 3,141 & 0,079 \\
$\mathrm{C}$ & 125 & 1,070 & 0,303 \\
$\mathrm{D}$ & 125 & 2,896 & 0,091 \\
$\mathrm{G}$ & 123 & 0,375 & 0,541 \\
$\mathrm{I}$ & 52 & 0,138 & 0,712 \\
$\mathrm{~J}$ & 125 & 0,114 & 0,736 \\
$\mathrm{~K}$ & 124 & 0,012 & 0,915 \\
$\mathrm{M}$ & 123 & 0,002 & 0,962 \\
\hline
\end{tabular}

\subsection{Räumliche Assoziation der Familienmitglieder in den Abendstunden im zweiten Beobachtungsjahr}

Während der Abendstunden des zweiten Beobachtungsjahres unterschieden sich die Familienmitglieder in ihrer räumlichen Assoziation zur Familie (Kruskal-Wallis-Test: $\mathrm{H}=24,89 ; \mathrm{N}=404 ; \mathrm{p}=0,0016$ ), und es gab auch Abhängigkeiten der räumlichen Assoziation von den Experimenten (Abb. 3.147). Dabei war die Assoziation während des zweiten Experiments am lockersten.

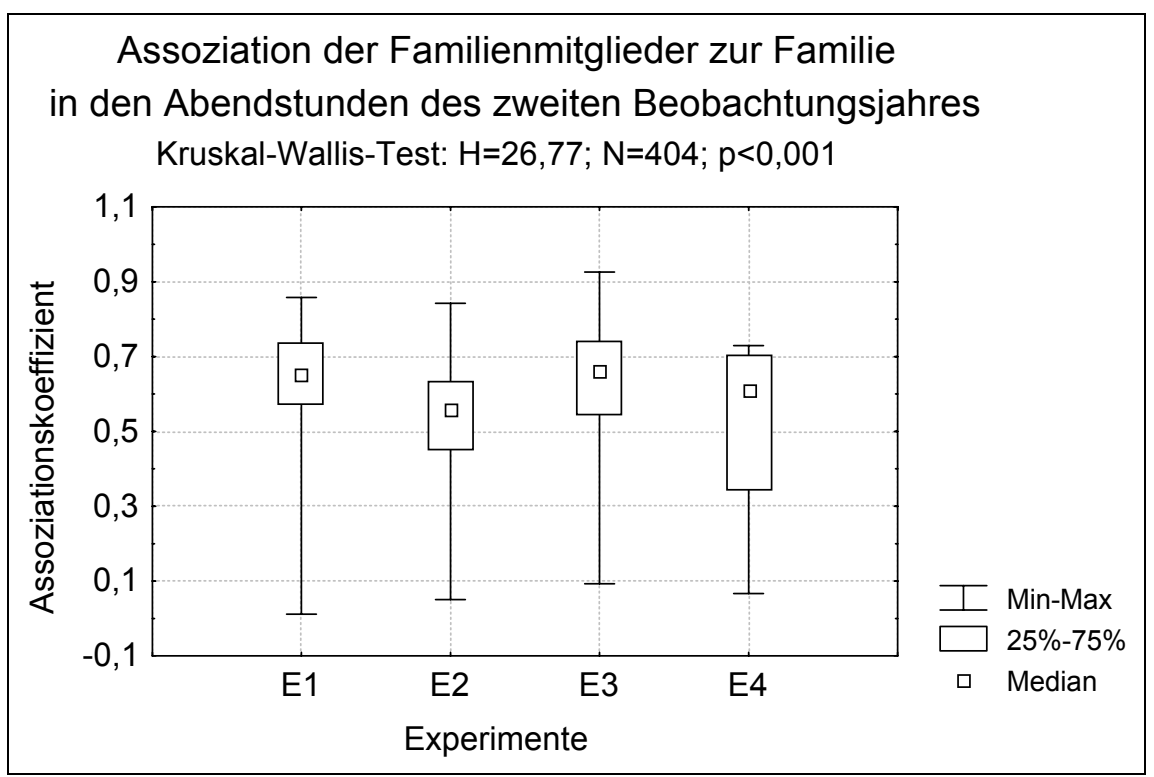

Abb. 3.147 Räumliche Assoziation der Familienmitglieder zur Familie in Abhängigkeit von den Experimenten gemittelt während der Abendstunden im zweiten Beobachtungsjahr (Kruskal-Wallis-Test: H=26,77; N=404; $\mathrm{p}<0,001)$. Die Assoziation der Familienmitglieder zeigte eine Abhängigkeit von den Experimenten. 
In den Abendstunden war $\mathrm{G}$ im ersten Experiment weniger eng mit Familienmitgliedern assoziiert als die anderen Familienmitglieder (Abb. 3.148a). Während aller anderen Experimente gab es abends zwischen den Familienmitgliedern keine Unterschiede in ihrer Assoziation zur Familie (Abb. 3.148b-d).
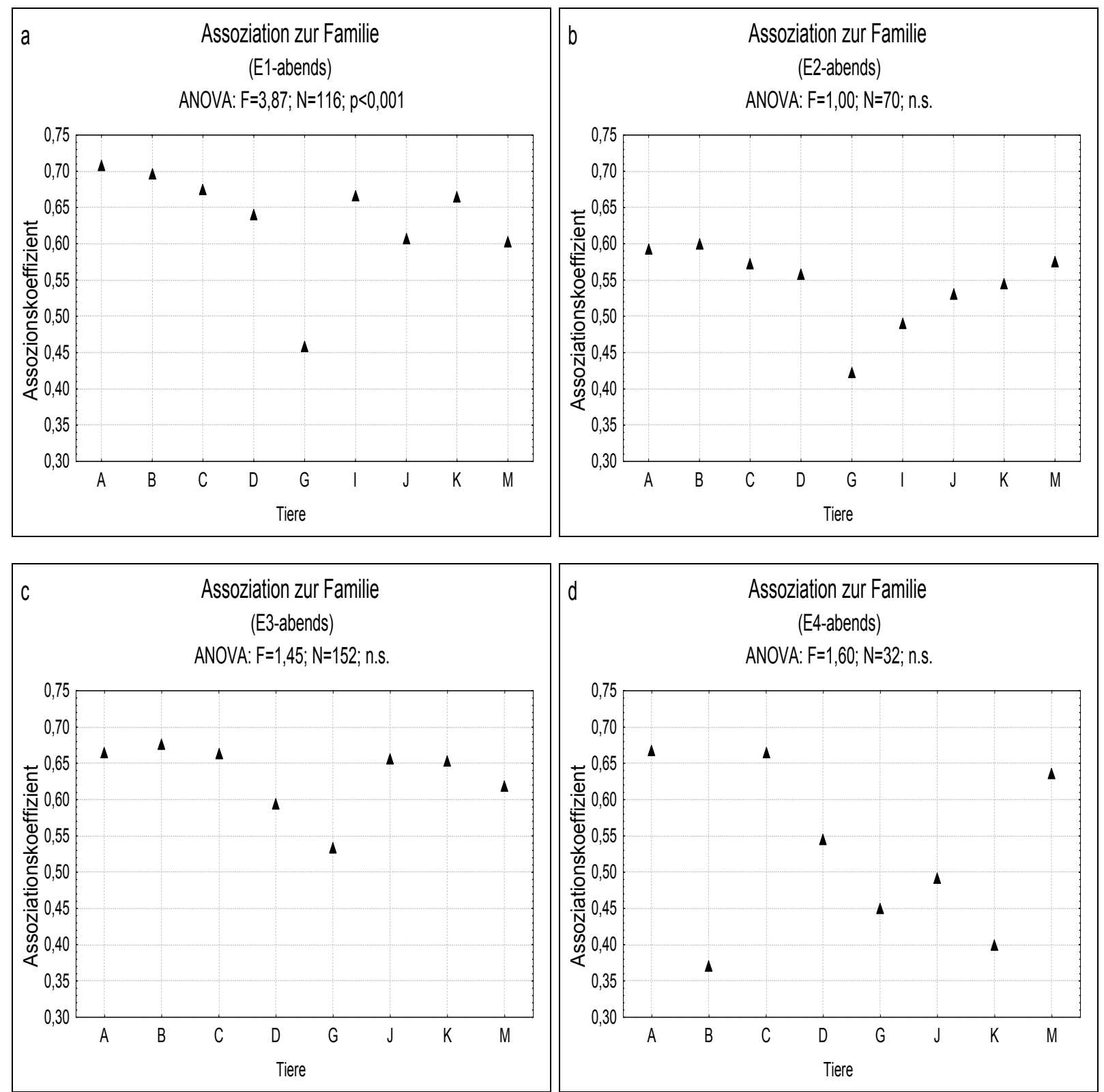

Abb. 3.148 Räumliche Assoziation der Familienmitglieder zu den übrigen Familienmitgliedern in den vier Experimentalphasen (a-d) des zweiten Beobachtungsjahres in den Abendstunden (17:30 bis 22:00 Uhr). Der Assoziationskoeffizient ist ein Maß für die Stärke der Assoziation bzw. Kohäsion eines einzelnen Familienmitgliedes zur Gesamtheit der anderen Familienmitglieder. Der Koeffizient liegt zwischen 0 und 1, wobei 1 die engste Assoziation und 0 die lockerste Assoziation zwischen Tier und Familie beschreiben würde. Den Tieren A-M in der Ordinate wird jeweils ein eigener Assoziationsindex zugeordnet (Abszisse). Durch die Varianzanalyse (hier einfaktorielle ANOVA) wird deutlich, inwieweit die Unterschiede zwischen den Assoziationskoeffizienten zufällig sind. 
Dennoch sei vermerkt, dass die jungen Männchen im zweiten Experiment geringe Assoziationskoeffizienten aufwiesen, während im vierten Experiment das Alpha-Weibchen und die adoleszente Tochter $\mathrm{K}$, als auch die Söhne $\mathrm{G}$ und $\mathrm{J}$ nur locker mit den anderen Familienmitgliedern assoziiert waren.

Bei A und C war die Assoziation zur Familie in den Abendstunden höher als im Tagesmittel, während bei den anderen Familienmitgliedern zwischen den Assoziationskoeffizienten gemittelt über den ganzen Tag und abends keine Unterschiede auftraten (Tab. 3.35).

Tabelle 3.35 Vergleich der Assoziationskoeffizienten eines Tieres gemittelt über den ganzen Tag mit den Assoziationskoeffizienten desselben Tieres in den Abendstunden im zweiten Beobachtungsjahr (einfaktorielle ANOVA). Abkürzungen: $\mathrm{N}=$ Anzahl der gewerteten Tage; * = signifikant.

\begin{tabular}{llll} 
Tier & $\mathrm{N}$ & F-Wert & p-Wert \\
\hline A & 113 & 5,963 & $0,016^{*}$ \\
B & 113 & 0,003 & 0,955 \\
C & 113 & 28,639 & $0,000^{*}$ \\
D & 113 & 2,511 & 0,116 \\
G & 111 & 0,678 & 0,412 \\
I & 52 & 0,138 & 0,712 \\
J & 113 & 2,440 & 0,121 \\
K & 112 & 0,003 & 0,955 \\
M & 111 & 1,308 & 0,255 \\
\hline
\end{tabular}

In beiden Beobachtungsjahren gab es Unterschiede zwischen den Familienmitgliedern in ihrer räumlichen Assoziation zur Familie, so dass Hypothese 8a zurückgewiesen werden kann.

Gemittelt über den ganzen Tag war die räumliche Assoziation der Familienmitglieder zur Familie im ersten Beobachtungsjahr von den Experimenten unabhängig. Während der Morgenstunden gab es jedoch Unterschiede zwischen den Experimenten. Hypothese 8b kann deshalb für die Assoziationskoeffizienten in den Morgenstunden des ersten Beobachtungsjahres abgelehnt, für das Ganztagesmittel aber nicht zurückgewiesen werden. Im zweiten Beobachtungsjahr hingegen war ganztags und in den Morgen- und Abendstunden eine Abhängigkeit der räumlichen Assoziation der Tiere zur Familie von den Experimenten festzustellen, so dass unter den Bedingungen des zweiten Jahres die Hypothese 8b abgelehnt werden kann.

In beiden Beobachtungsjahren wich bei keinem Familienmitglied die räumliche Assoziation zur Familie während der Morgenstunden von der Assoziation zur Familie im Tagesmittel ab. 
Während der Abendstunden waren im ersten Beobachtungsjahr bei allen Familienmitgliedern außer F und im zweiten Beobachtungsjahr bei A und C Veränderungen in der Assoziation zur Familie gegenüber dem Ganztagswert zu finden. Hypothese 8c kann daher auch abgewiesen werden.

\subsubsection{Nahbereichshäufigkeiten der Familienmitglieder}

Hypothese 8d: Alle Familienmitglieder befinden sich gleich häufig im Nahbereich von Familienmitgliedern.

Hypothese 8e: Es gibt keine Abhängigkeiten der Nahbereichshäufigkeiten von den Experimenten

\subsection{Nahbereichshäufigkeiten im ersten Beobachtungsjahr}

Die Nahbereichshäufigkeiten wurden mit instantaneous sampling aufgenommen, so dass es sich um keine realen Häufigkeiten, sondern um Intervallhäufigkeiten (siehe S.26) handelt. Die Alpha-Tiere befanden sich im ersten Beobachtungsjahr (Abb. 3.149-152) häufiger als erwartet im Nahbereich von Familienmitgliedern (Ausnahme A in der ersten Kontrollphase), während die adulten Nachkommen D und E sich seltener als erwartet im Nahbereich von Familienmitgliedern aufhielten. Die Nahbereichshäufigkeit von C lag während der ersten Kontrollphase und der beiden Experimente ebenso unter dem Familiendurchschnitt. Die Nahbereichshäufigkeiten der nicht-adulten Nachkommen F, G und H lagen während aller Phasen innerhalb des Erwartungsbereichs. Die juvenilen Nachkommen I und J befanden sich in der ersten Kontrollphase häufiger als erwartet im Nahbereich (Abb. 3.149). Während der zweiten Kontrollphase und dem zweiten Experiment war I häufiger als erwartet im Nahbereich, während die Werte von J innerhalb des Erwartungsbereichs lagen (Abb. 3.150 und 3.152). Im ersten Experiment lag der Wert von I im Erwartungsbereich, während sich J häufiger als erwartet im Nahbereich aufhielt (Abb. 3.151).

Verglichen mit den beiden Kontrollphasen befanden sich C und D im ersten Experiment seltener im Nahbereich zu Familienmitgliedern (KFA, C: EW=17,05; D: EW=10,73 n=4), was bei $\mathrm{C}$ im zweiten Experiment anhielt. E war schon in der zweiten Kontrollphase und auch im ersten Experiment seltener im Nahbereich von Familienmitgliedern als zuvor (KFA, E: $\mathrm{EW}=12,85 \mathrm{n}=4)$. Im zweiten Experiment lag seine Nahbereichshäufigkeit innerhalb des Erwartungsbereichs. $\mathrm{G}$ und $\mathrm{H}$ befanden sich, wie ihre Geschwister in der ersten Kontrollphase, überdurchschnittlich häufig im Nahbereich von Familienmitgliedern. 
$\mathrm{H}$ war jedoch im zweiten Experiment, wie die Alpha-Tiere, deren Werte in allen übrigen Phasen innerhalb des Erwartungsbereich lagen, seltener im Nahbereich von Familienmitgliedern als vorher (KFA, G: EW=25; H: EW=28,49; A: $E W=36,92 ; \mathrm{B}$ : $\mathrm{EW}=41,38 \mathrm{n}=4)$

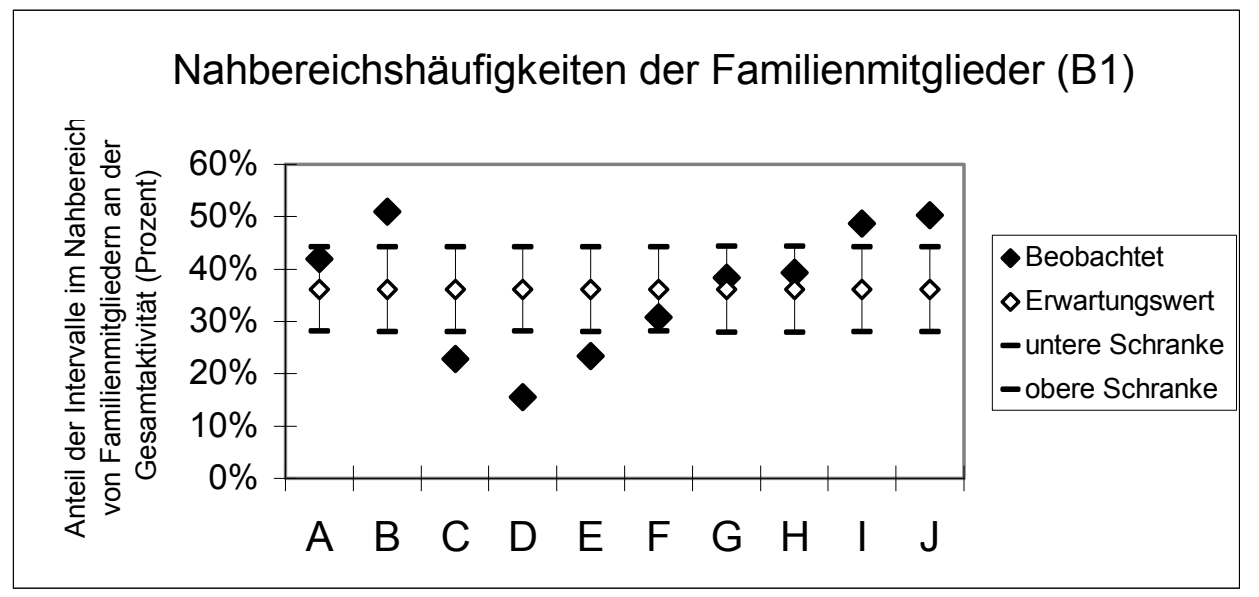

Abb. 3.149 Nahbereichshäufigkeiten der Familienmitglieder während der ersten Kontrollphase des ersten Beobachtungsjahres. Abkürzungen: A-J = Familienmitglieder; B1=erste Kontrollphase (KFA, EW=36,12 n=10).

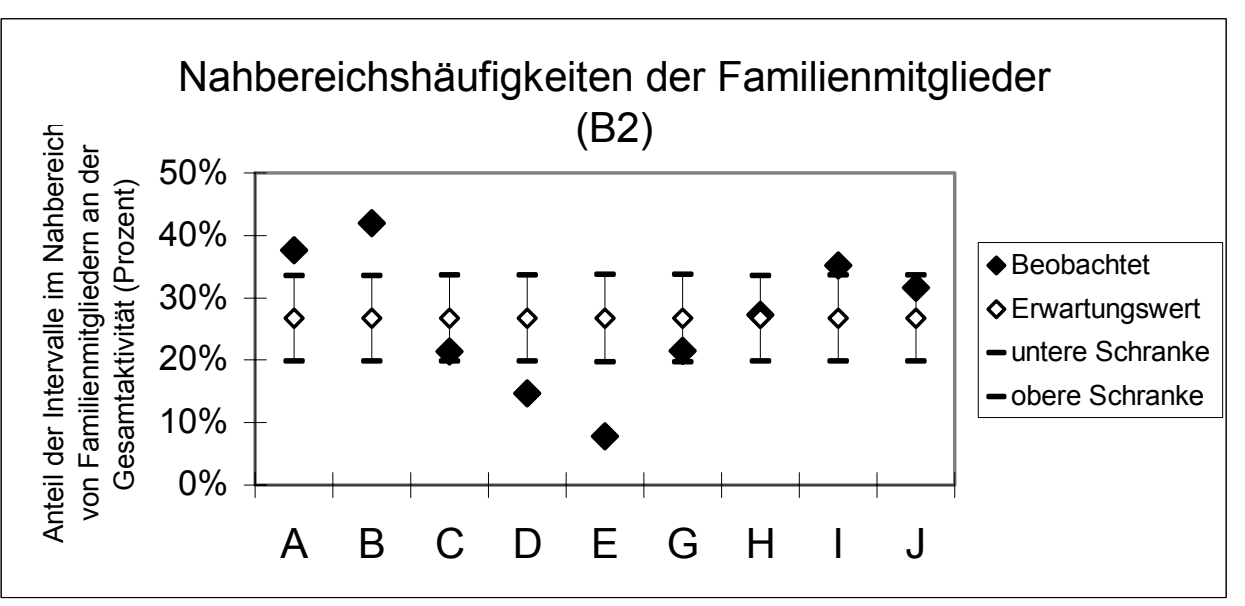

Abb. 3.150 Nahbereichshäufigkeiten der Familienmitglieder während der zweiten Kontrollphase des ersten Beobachtungsjahres. Abkürzungen: A-J = Familienmitglieder; B2=zweite Kontrollphase (KFA, EW=26,71 n=9).

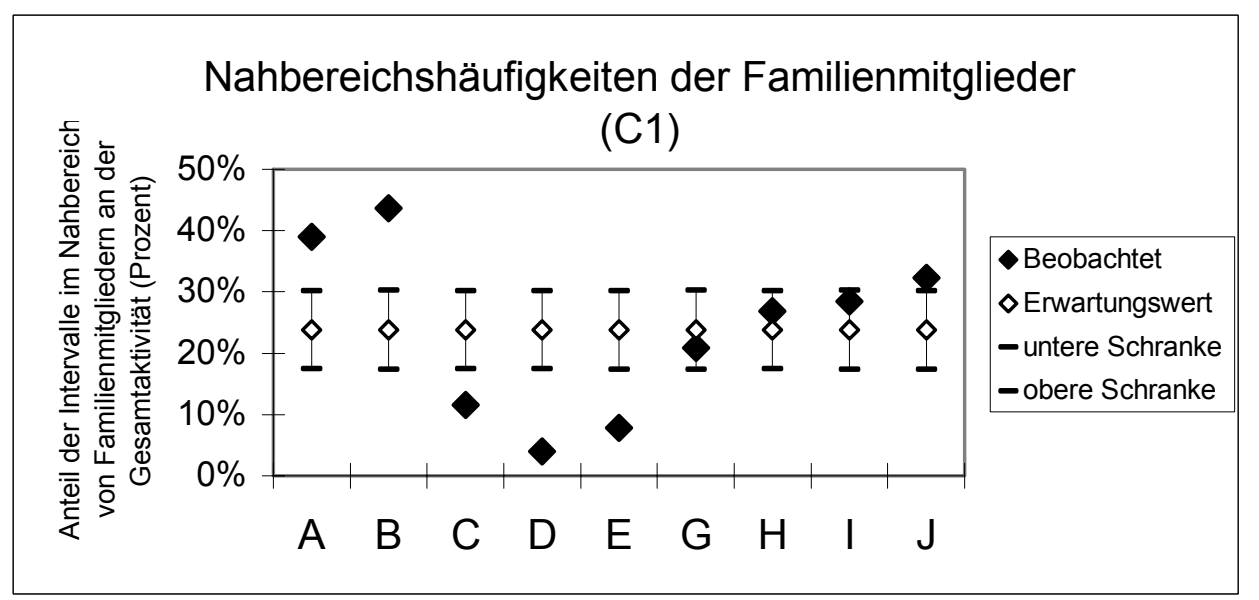

Abb. 3.151 Nahbereichshäufigkeiten der Familienmitglieder während des ersten Experiments des ersten Beobachtungsjahres. Abkürzungen: A-J = Familienmitglieder; C1=erstes Experiment (KFA, EW=23,80 n=9). 


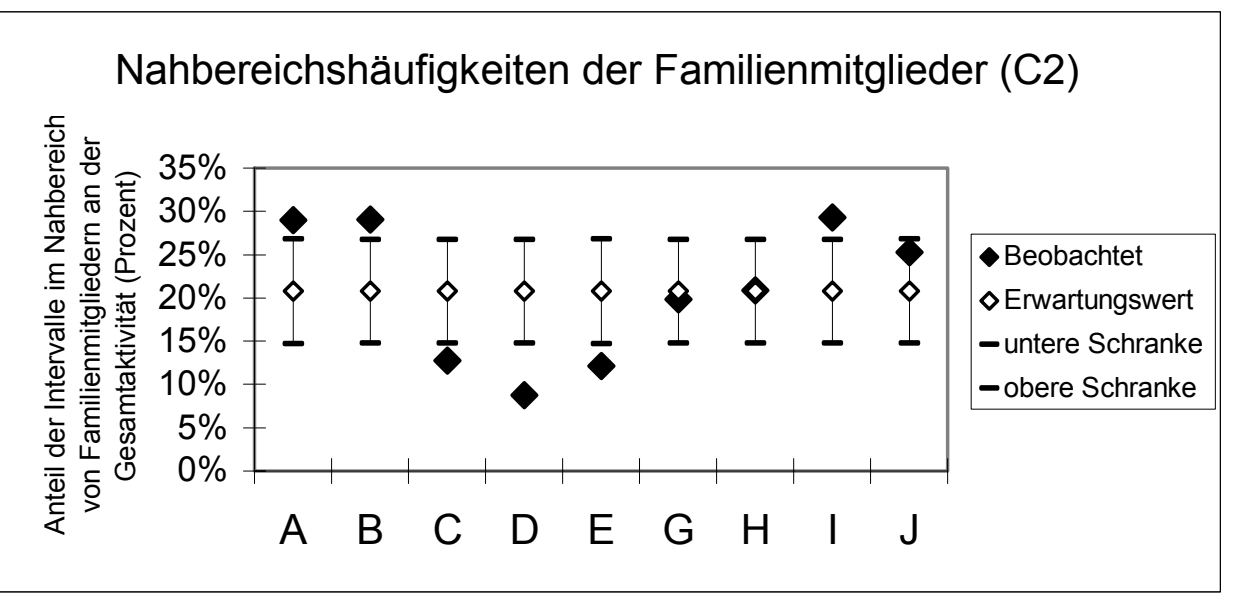

Abb. 3.152 Nahbereichshäufigkeiten der Familienmitglieder während des zweiten Experiments des ersten Beobachtungsjahres. Abkürzungen: A-J = Familienmitglieder; C2=zweites Experiment (KFA, EW=20,75 n=9).

\subsection{Nahbereichshäufigkeiten im zweiten Beobachtungsjahr}

Während der Hüttenphase (D1) des zweiten Beobachtungsjahres entsprachen die Beobachtungswerte zur Nahbereichshäufigkeit bei allen Nachkommen dem Erwartungsbereich. Die Alpha-Tiere hielten sich häufiger als erwartet im Nahbereich von Familienmitgliedern auf (Abb. 3.153). Während des ersten Experiments waren $\mathrm{K}$ häufiger und $\mathrm{D}$ seltener als erwartet im Nahbereich. Die Werte aller übrigen Familienmitglieder lagen im Erwartungsbereich (Abb. 3.154) Beim zweiten Experiment hielten sich A und $\mathrm{M}$ häufiger und D und I seltener im Nahbereich zu Familienmitgliedern auf (Abb. 3.155). Während des dritten Experiments (Abb. 3.156) waren die drei ältesten Nachkommen C, D und G und während des vierten Experiments (Abb. 3.157) nur noch C und D seltener als erwartet im Nahbereich anderer Familienmitglieder, während die Alpha-Tiere sich in beiden Experimenten häufiger als erwartet im Nahbereich aufhielten.

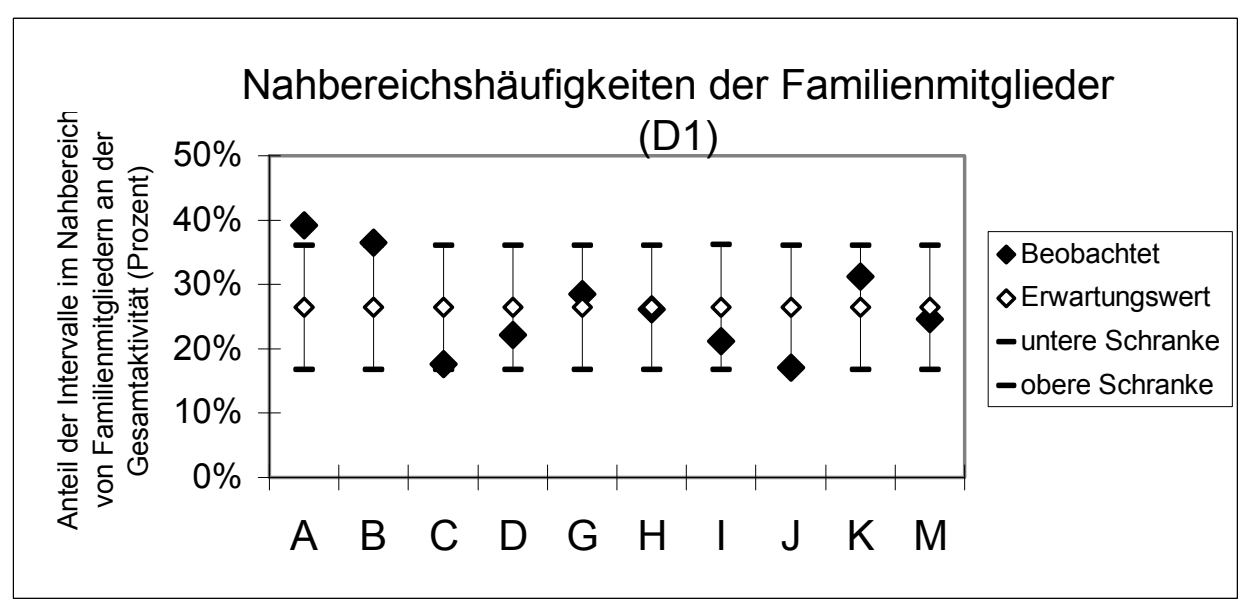

Abb. 3.153 Nahbereichshäufigkeiten der Familienmitglieder während der Hüttenphase des zweiten Beobachtungsjahres. Abkürzungen: A-M = Familienmitglieder; D1=Hüttenphase (KFA, EW=26,42 n=10). 


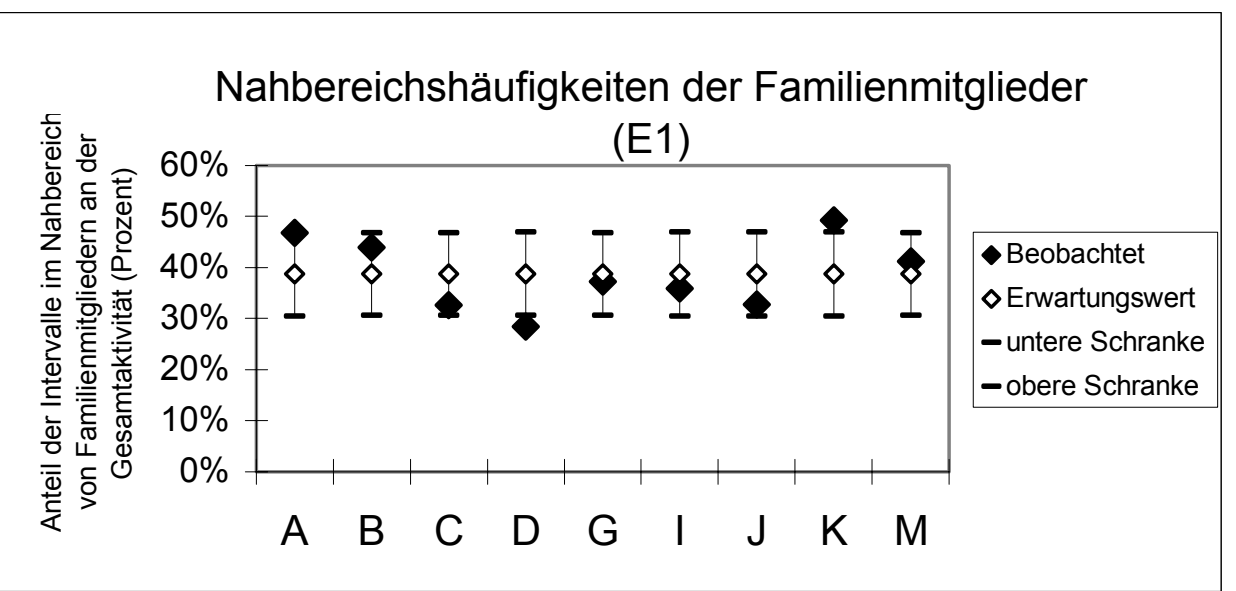

Abb. 3.154 Nahbereichshäufigkeiten der Familienmitglieder während des ersten Experiments des zweiten Beobachtungsjahres. Abkürzungen: A-M = Familienmitglieder; E1=erstes Experiment (KFA, EW=38,71 n=9).

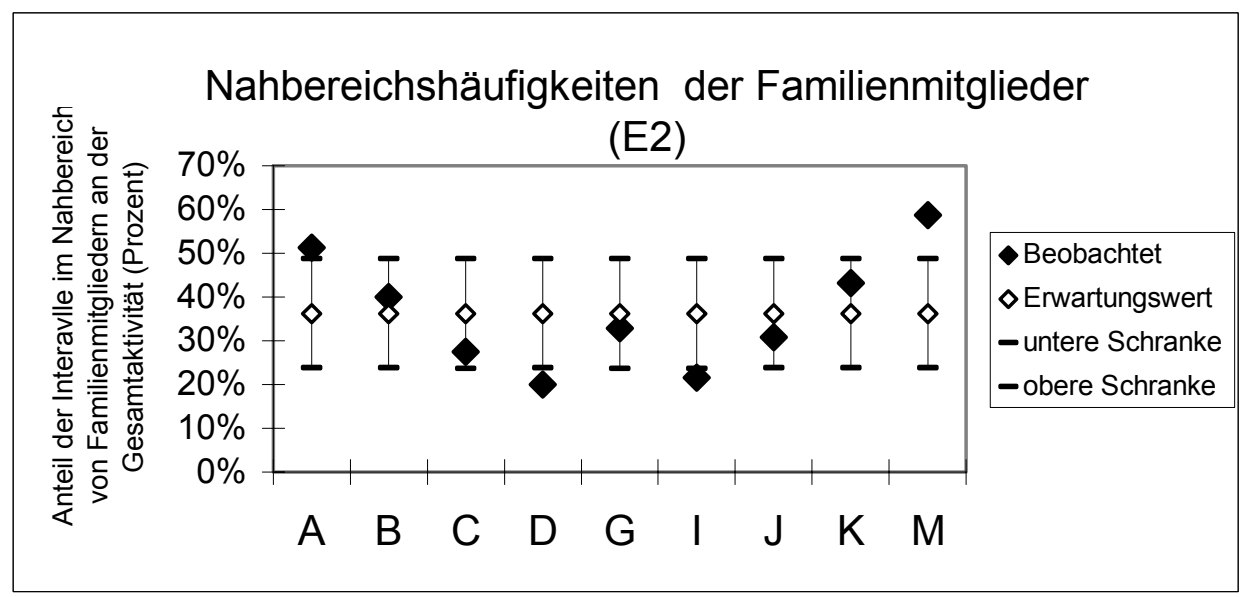

Abb. 3.155 Nahbereichshäufigkeiten der Familienmitglieder während des zweiten Experiments des zweiten Beobachtungsjahres. Abkürzungen: A-M = Familienmitglieder; E2=zweites Experiment (KFA, EW=36,24 n=9).

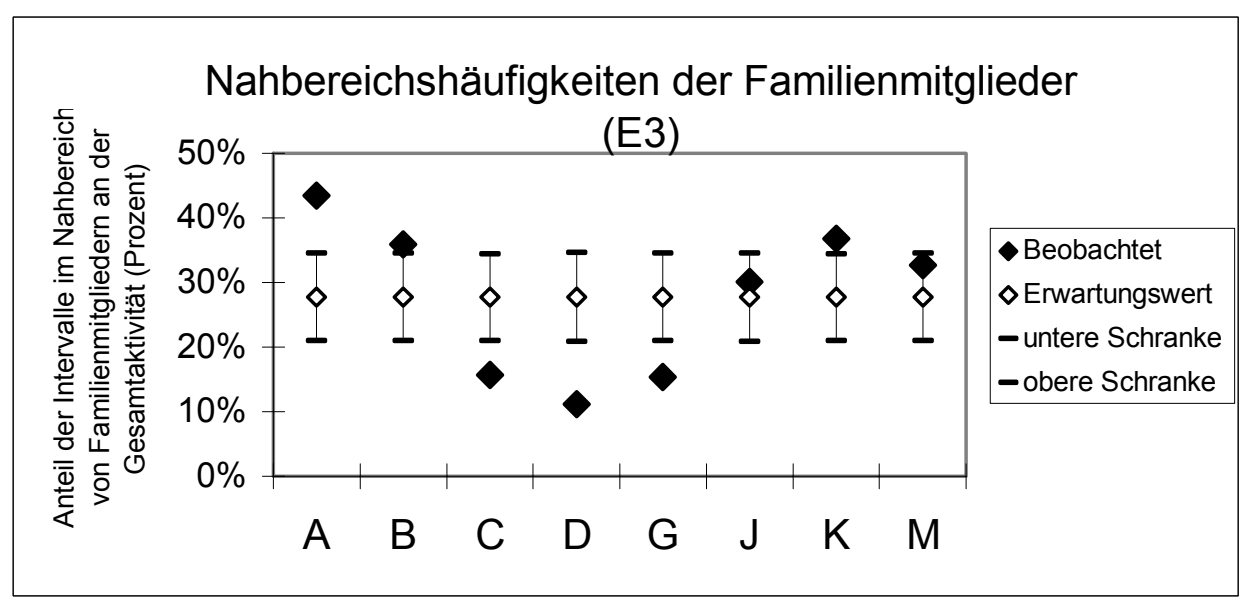

Abb. 3.156 Nahbereichshäufigkeiten der Familienmitglieder während des dritten Experiments des zweiten Beobachtungsjahres. Abkürzungen: A-M = Familienmitglieder; E3=drittes Experiment (KFA, EW=27,72 n=8). 


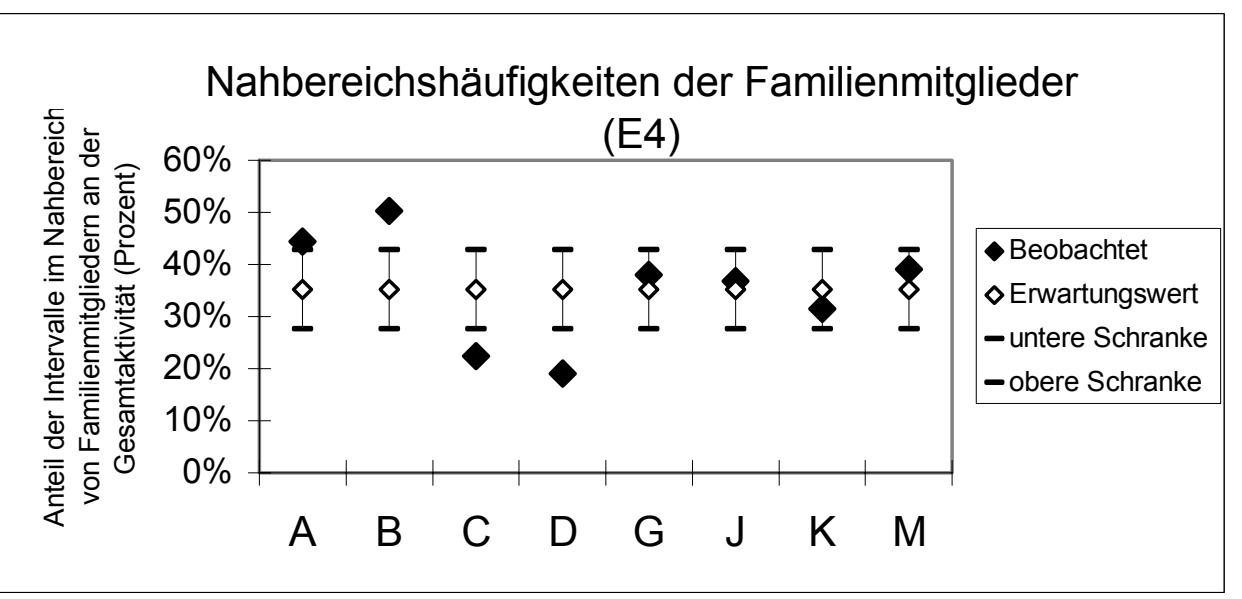

Abb. 3.157 Nahbereichshäufigkeiten der Familienmitglieder während des vierten Experiments des zweiten Beobachtungsjahres. Abkürzungen: A-M = Familienmitglieder; E4=viertes Experiment (KFA, EW=35,21 n=8).

Die adulten Nachkommen C, D und G hielten sich im ersten Experiment häufiger und im dritten Experiment, also nach dem Rauswurf von I, seltener im Nahbereich von Familienmitgliedern auf. $\mathrm{G}$ befand sich im vierten Experiment überdurchschnittlich häufig im Nahbereich von Familienmitgliedern, wie auch J, dessen Werte während der Experimente 1 bis 3 keine Abweichung vom Erwartungsbereich gezeigt hatten. Die Nahbereichshäufigkeiten von I unterschieden sich in den ersten beiden Experimenten nicht von denen der älteren Geschwister. Die Alpha-Tiere zeigten im Laufe des zweiten Beobachtungsjahres keine Veränderungen in ihren Nahbereichshäufigkeiten (KFA, A: $E W=44,81 \mathrm{n}=5, \mathrm{~B}$ : $\mathrm{EW}=42,16$ n=5, C: $E W=23,21 n=5, D: E W=20,04 n=5, G: E W=30,31 n=5$, I: $E W=28,99 n=3, J:$ $\mathrm{EW}=30,93 \mathrm{n}=5, \mathrm{~K}: \mathrm{EW}=38,51 \mathrm{n}=5, \mathrm{M}: \mathrm{EW}=38,17 \mathrm{n}=5)$.

Hypothese 8d kann zurückgewiesen werden, da während aller Phasen Unterschiede zwischen den Familienmitgliedern in ihren Nahbereichshäufigkeiten auftraten. In beiden Beobachtungsjahren gab es bei allen adulten und subadulten Nachkommen in Abhängigkeit von den Experimenten Unterschiede in den Nahbereichshäufigkeiten, so dass auch Hypothese 8e zurückgewiesen werden kann.

\subsubsection{Rückgang der taktilen Interaktion mit Familienmitgliedern}

Hypothese 8f: Der Anteil taktiler Interaktionen mit Familienmitgliedern bleibt von den Experimenten unbeeinflusst.

Zur Vermeidung von Redundanzen wird an dieser Stelle auf die Ergebnisse von Kapitel 3.1 Zeitbudgets verwiesen. 


\subsection{Rückgang der taktilen Interaktion mit Familienmitgliedern im ersten Beobachtungsjahr}

Die adulten Nachkommen C, D und E wiesen im ersten Experiment (C1) einen Rückgang der taktilen Interaktionen gegenüber den vorhergehenden, als auch nachfolgenden Phasen auf (siehe Abb. 3.14-16, S. 53f.). Unter dem Erwartungsbereich lag der Anteil an taktiler Interaktion mit Familienmitgliedern im zweiten Experiment auch bei H. Ebenso verringerte sich im ersten Experiment der Anteil an taktiler Interaktion mit Familienmitgliedern bei den Juvenilen I und J unter den Erwartungsbereich und fiel im zweiten Experiment nochmals ab.

Während des gesamten Beobachtungsjahres lag bei D der Anteil an taktiler Interaktion mit Familienmitgliedern unter dem Erwartungsbereich. Ebenso verhielt es sich mit E während der ersten drei Phasen (B1, B2 und C1) und mit C im ersten Experiment (C1). F wies ebenso wie $\mathrm{D}$ und $\mathrm{E}$ in der ersten Kontrollphase einen geringeren Anteil an taktiler Interaktion mit Familienmitgliedern auf als erwartet. Trotz der Verringerung taktiler Interaktionen der Juvenilen I und $\mathrm{J}$ im zweiten Experiment entsprachen ihre Anteile immer noch dem Erwartungsbereich. (Analysen der Zeitbudgets nach KFA, B1:EW=31,63 n=10; $\mathrm{B} 2: \mathrm{EW}=28,52 \mathrm{n}=9 ; \mathrm{C} 1: \mathrm{EW}=20,03 \mathrm{n}=9$ und $\mathrm{C} 2: \mathrm{EW}=20,64 \mathrm{n}=9$ ).

\subsection{Rückgang der taktilen Interaktion mit Familienmitgliedern im zweiten Beobachtungsjahr}

Aus einem Vergleich zwischen den Experimenten im zweiten Beobachtungsjahr für den Verhaltensbereich taktile Interaktion mit Familienmitgliedern ging hervor, dass nur bei $\mathrm{G}$ Verminderungen im dritten Experiment auftraten (siehe Abb. 3.25, S. 60). Die Anteile der taktilen Interaktion mit Familienmitgliedern von D, G und I lagen im ersten Experiment unter dem Erwartungsbereich (KFA, EW=34,46 n=9). Während des zweiten Experiments interagierten D und I, während des dritten Experiments D und G und im vierten Experiment D und $C$ seltener als erwartet taktil mit Familienmitgliedern (KFA, E2:EW=36,59 $n=9$; $\mathrm{E} 3: \mathrm{EW}=33,01 \mathrm{n}=8 ; \mathrm{E} 4: \mathrm{EW}=43,47 \mathrm{n}=8)$.

Da der Anteil an taktiler Interaktion mit Familienmitgliedern in beiden Beobachtungsjahren bei einigen Nachkommen von den Experimenten abhängig war, muss Hypothese $8 \mathrm{f}$ abgelehnt werden. 


\subsubsection{Zusammenhänge zwischen sozialen Faktoren und Peripheralisationen}

\subsubsection{Dissoziatives Verhalten und räumliche Assoziation}

Hypothese 3g: Peripheralisationen treten unabhängig von dissoziativen Interaktionen innerhalb der Familie auf.

Da familiäre Aggressionen im Zusammenhang mit Peripheralisationen beobachtet werden konnten (Sutcliffe und Poole 1984, McGrew 1997), wurden Korrelationen zwischen dissoziativem Verhalten von Familienmitgliedern und der räumlichen Assoziation der adulten Nachkommen vermutet. Es konnte jedoch in beiden Beobachtungsjahren für keines der Familienmitglieder ein Zusammenhang zwischen dissoziativem Verhalten in der Familie und dem Assoziationskoeffizienten des Tieres gefunden werden (s. Tab. 3.36 und 3.37). Aus ad libitum Beobachtungen wurde ersichtlich, dass während des ersten Experiments (C1) das Alpha-Männchen dissoziatives Verhalten gegen E und D, z.T. auch gegen C zeigte, die in dieser Phase peripher waren. Die Familie, insbesondere E und C, verhielt sich außerdem äußerst dissoziativ gegenüber D. Die dissoziativen Aktivitäten hielten im zweiten Experiment an, aber $\mathrm{C}$ war nicht mehr peripher. $\mathrm{G}$ hatte zwar durchgehend den niedrigsten Assoziationskoeffizienten, war jedoch nicht auffällig an dissoziativen Interaktionen beteiligt.

Im ersten Experiment des zweiten Jahres war D in den Morgenstunden häufiger von der Familie peripher. Während dieser Phase verhielten sich viele Familienmitglieder gegenüber D dissoziativ (Büschelklappen, Schlitzaugen-machen, Keckern, Angreifen) und hinderten D an Interaktionen mit den neugeborenen Jungtieren. Während des zweiten Experiments gab es stark dissoziative Interaktionen zwischen D und I, die beide ebenso wie G in dieser Phase nur eine lockere Assoziation zur Familie aufwiesen. Am Ende der Phase jagte G I durch das Streifgebiet. Im dritten Experiment war J zunehmend peripher von der Familie und konnte bei dissoziativen Interaktionen mit G und zeitweise mit der gesamten Familie beobachtet werden. D und $\mathrm{G}$, die ebenfalls nur locker mit der Familie assoziiert waren, beteiligten sich häufig an dissoziativen Interaktionen. 
Tabelle 3.36 Korrelation zwischen dissoziativem Verhalten der Familie und dem Assoziationskoeffizienten eines Tieres zur Familie im Jahr 2000. Da die Anzahl der quantifizierbaren dissoziativen VE pro Tier sehr gering war, wurden alle dissoziativen Verhaltenseinheiten, die in der Familie auftraten, addiert und mit den Assoziationskoeffizienten der adulten Nachkommen korreliert ( $\mathrm{n}=$ Anzahl der Korrelation zugrunde gelegten statistischen Tage).

\begin{tabular}{ccccc}
\hline Tier & $\mathrm{n}$ & Spearmans $\mathrm{r}$ & $\mathrm{t}(\mathrm{n}-2)$ & Signifikanzniveau \\
\hline C & 18 & $-0,143307$ & $-0,579206$ & n.s. \\
D & 18 & $-0,054281$ & $-0,217447$ & n.s. \\
E & 18 & $-0,048094$ & $-0,192600$ & n.s. \\
G & 18 & $-0,186266$ & $-0,758338$ & n.s. \\
H & 18 & $-0,285279$ & $-1,190592$ & n.s. \\
\hline
\end{tabular}

Tabelle 3.37 Korrelation zwischen dissoziativem Verhalten der Familie und dem Assoziationskoeffizienten eines Tieres zur Familie im Jahr 2001. Da die Anzahl der quantifizierbaren dissoziativen VE pro Tier sehr gering war, wurden alle dissoziativen Verhaltenseinheiten, die in der Familie auftraten, addiert und mit den Assoziationskoeffizienten der adulten Nachkommen korreliert $(\mathrm{n}=$ Anzahl der Korrelation zugrunde gelegten statistischen Tage).

\begin{tabular}{ccccc}
\hline Tier & $\mathrm{n}$ & Spearmans $\mathrm{r}$ & $\mathrm{t}(\mathrm{n}-2)$ & Signifikanzniveau \\
\hline C & 17 & 0,072044 & 0,279753 & n.s. \\
D & 17 & $-0,016262$ & $-0,062993$ & n.s. \\
G & 17 & $-0,048856$ & $-0,189447$ & n.s. \\
I & 7 & $-0,036363$ & $-0,081365$ & n.s. \\
J & 17 & $-0,014805$ & $-0,057349$ & n.s. \\
K & 17 & 0,154036 & 0,603785 & n.s. \\
M & 17 & $-0,016656$ & $-0,064519$ & n.s. \\
\hline
\end{tabular}

In beiden Jahren wurde beobachtet, dass Familienmitglieder, die häufig nur locker mit den übrigen Familienmitgliedern räumlich assoziiert waren, sich an dissoziativen Interaktionen beteiligt haben. Doch gab es auch periphere Tiere, die nicht in dissoziative Interaktionen eingebunden waren und an dissoziativen Interaktionen beteiligte Tiere, die nicht peripher waren. Dissoziatives Verhalten innerhalb der Familie und Peripheralisationen einzelner Familienmitglieder korrelierten nicht, so daß Hypothese 8g nicht abgewiesen werden kann. 


\subsubsection{Einfluss der Locktiere und Nachbarfamilien auf Peripheralisationen}

Hypothese 8h: Peripheralisationen treten unabhängig von nicht-taktiler Interaktion mit fremden Artgenossen auf.

Für das erste Beobachtungsjahr konnten für die Morgenstunden Abhängigkeiten der räumlichen Assoziation der Familienmitglieder zur Familie von den Experimenten festgestellt werden. Im zweiten Beobachtungsjahr gab es während aller getesteten Tageszeiten (ganztags, morgens und abends) Abhängigkeiten der räumlichen Assoziation von den Experimenten. Es wurde hypothetisiert, dass es Zusammenhänge zwischen der Änderung der räumlichen Assoziation der Familienmitglieder zur Familie und der Präsenz fremder Artgenossen gibt. Der Verhaltensbereich nicht-taktile Interaktion mit fremden Artgenossen wird daher auf Zusammenhänge mit der räumlichen Assoziation überprüft. Für das erste Beobachtungsjahr konnte kein Zusammenhang zwischen den genannten Faktoren bestätigt werden (siehe Tab. $3.38)$.

Tabelle 3.38 Korrelation zwischen nicht-taktiler Interaktion mit fremden Artgenossen und den Assoziationskoeffizienten der Nachkommen im ersten Beobachtungsjahr $(\mathrm{n}=$ Anzahl der statistischen Tage, welche der Korrelationsanalyse zugrunde gelegt wurden).

\begin{tabular}{ccccc}
\hline Tier & $\mathrm{n}$ & Spearmans $\mathrm{r}$ & $\mathrm{t}(\mathrm{n}-2)$ & Signifikanzniveau \\
\hline C & 18 & $-0,114329$ & $-0,460334$ & n.s. \\
D & 18 & 0,162801 & 0,660008 & n.s. \\
E & 18 & $-0,390710$ & $-1,697793$ & n.s. \\
G & 18 & $-0,305922$ & $-1,285312$ & n.s. \\
H & 18 & $-0,263649$ & $-1,093277$ & n.s. \\
\hline
\end{tabular}

Im zweiten Beobachtungsjahr gab es ebenfalls keine Korrelationen zwischen dem Verhaltensbereich NTA und der räumlichen Assoziation von Familienmitgliedern zur Familie (Tab.3.39).

Da für beide Beobachtungsjahre keine Korrelation zwischen nicht-taktiler Interaktion mit fremden Artgenossen und der räumlichen Assoziation von Familienmitgliedern zur Familie nachgewiesen wurde, kann die Hypothese 8h nicht zurückgewiesen werden. 
Tabelle 3.39 Korrelation zwischen nicht-taktiler Interaktion mit fremden Artgenossen und den Assoziationskoeffizienten der Nachkommen im zweiten Beobachtungsjahr $(\mathrm{n}=$ Anzahl der statistischen Tage, welche der Korrelationsanalyse zugrunde gelegt wurden).

\begin{tabular}{ccccc}
\hline Tier & $\mathrm{n}$ & Spearmans $\mathrm{r}$ & $\mathrm{t}(\mathrm{n}-2)$ & Signifikanzniveau \\
\hline C & 17 & $-0,449246$ & $-1,947513$ & n.s. \\
D & 17 & $-0,345941$ & $-1,427992$ & n.s. \\
G & 17 & $-0,465591$ & $-2,037544$ & n.s. \\
I & 7 & $-0,477084$ & $-1,213841$ & n.s. \\
J & 17 & 0,056594 & 0,219538 & n.s. \\
K & 17 & 0,281708 & 1,137104 & n.s. \\
M & 17 & $-0,128208$ & $-0,500678$ & n.s. \\
\hline
\end{tabular}

\subsubsection{Peripheralisationen und Rauswürfe}

Hypothese 8i: Tiere, die rausgeworfen werden, waren vor dem Rauswurf nicht peripher, d.h. sie waren räumlich ebenso wie die übrigen Familienmitglieder mit der Familie assoziiert, wiesen einen ebenso hohen Zeitbudget-Anteil an taktiler Interaktion und ihrer Nahbereichshäufigkeiten zu Familienmitgliedern wie andere Familienmitglieder auf.

An freilebenden $C$. jacchus-Populationen wurde beobachtet, dass Emigrationen meist Geburten vorausgingen oder Peripheralisationen der entsprechenden Tiere (Digby und Barreto 1993, Digby 1995b). Es soll getestet werden, ob Peripheralisationen einzelner Familienmitglieder im Vorwege späterer Rauswürfe geschahen. Da keine freiwilligen Emigrationen beobachtet wurden, können diese nicht auf Zusammenhänge mit Peripheralisationen untersucht werden.

Die Schwierigkeit bei der Auswahl des getesteten Zeitraums ergab sich dadurch, dass nur die männlichen Tiere F und I (in Tab.3.40 grau unterlegt) innerhalb der instantaneous samplingDatenerhebungsperiode rausgeworfen wurden. Die zugrunde liegenden Daten der anderen rausgeworfenen Tiere stammen demnach aus Zeiträumen, die nicht direkt an den Zeitpunkt des Rauswurfes angrenzten.

Anhand der Ergebnisse aus 3.4.3 wird ersichtlich, dass sich bei den männlichen Nachkommen, die von der Familie ausgestoßen wurden, entweder (1) in der räumlichen Assoziation zur Familie, (2) in der Nahbereichshäufigkeit zu Familienmitgliedern oder (3) im Anteil der taktilen Interaktion mit Familienmitgliedern an der Gesamtaktivität Hinweise auf Peripheralisation vor dem Ausschluss aus der Familie ergaben. Bei drei von vier Männchen 
konnte eine verringerte räumliche Assoziation zur Familie festgestellt werden, bei zwei Männchen eine Verringerung der Nahbereichshäufigkeiten und bei drei Männchen ein Rückgang der taktilen Interaktion mit Familienmitgliedern (siehe Tab. 3.40). Bei den weiblichen Nachkommen, die ausgeschlossen wurden, konnten keine Hinweise auf Peripheralisation festgestellt werden. Eine statistische Analyse möglicher Zusammenhänge zwischen Peripheralisationen und Rauswürfen ist wegen der geringen Anzahl an Rauswürfen nicht möglich.

Tabelle 3.40 Hinweise auf Peripheralisationen durch einen verringerten Assoziationskoeffizienten, seltenerem Aufenthalt im Nahbereich von Familienmitgliedern und den Rückgang taktiler Interaktionen der rausgeworfenen Tiere (grau unterlegt $=$ Tiere, die während der instantaneous sampling-Datenaufnahmeperiode rausgeworfen wurden).

\begin{tabular}{|c|c|c|c|}
\hline \multirow{3}{*}{$\begin{array}{c}\text { Rausgeworfenes } \\
\text { Tier }\end{array}$} & \multicolumn{3}{|c|}{ Peripheralisationen } \\
\hline & geringer & seltener im & Rückgang taktiler \\
\hline & Assoziationskoeffizient & Nahbereich & Interaktion \\
\hline$F(\lesssim)$ & nein & nein & ja \\
\hline$E(ふ)$ & ja & ja & ja \\
\hline$I\left(ठ^{\lambda}\right)$ & ja & ja & ja \\
\hline $\mathrm{J}(\overbrace{}^{\lambda})$ & ja & nein & nein \\
\hline $\mathrm{H}($ ( $)$ & nein & nein & nein \\
\hline $\mathrm{M}(q)$ & nein & nein & nein \\
\hline
\end{tabular}

Wurden die statistischen Tage des zweiten Experiments (E2) im zweiten Beobachtungsjahr getrennt betrachtet, war eine deutliche Lockerung der Assoziation von I zur Familie zu erkennen. I wurde am Ende des zweiten statistischen Tages aus der Familie geworfen. D war gleichzeitig ebenso peripher und wurde nicht ausgestoßen (Abb. 3.158). In Kapitel 3.4.3 wurde jedoch deutlich, dass nur die räumliche Assoziation von I zur Familie von den Experimenten abhängig war. Direkt vor dem Rauswurf von I lockerte sich seine räumliche Assoziation zur Familie, weshalb gefolgert werden kann, dass seine Peripheralisation im Zusammenhang mit seinem Rauswurf aus der Familie stand.

Die Hypothese 8i kann zurückgewiesen werden, da es vor mehreren Rauswürfen Hinweise auf Peripheralisationen gab. Allerdings kam es nicht vor jedem Rauswurf zu Peripheralisationen. Somit kann davon ausgegangen werden, dass Rauswürfe nicht zwingend durch Peripheralisationen vorbereitet werden. 


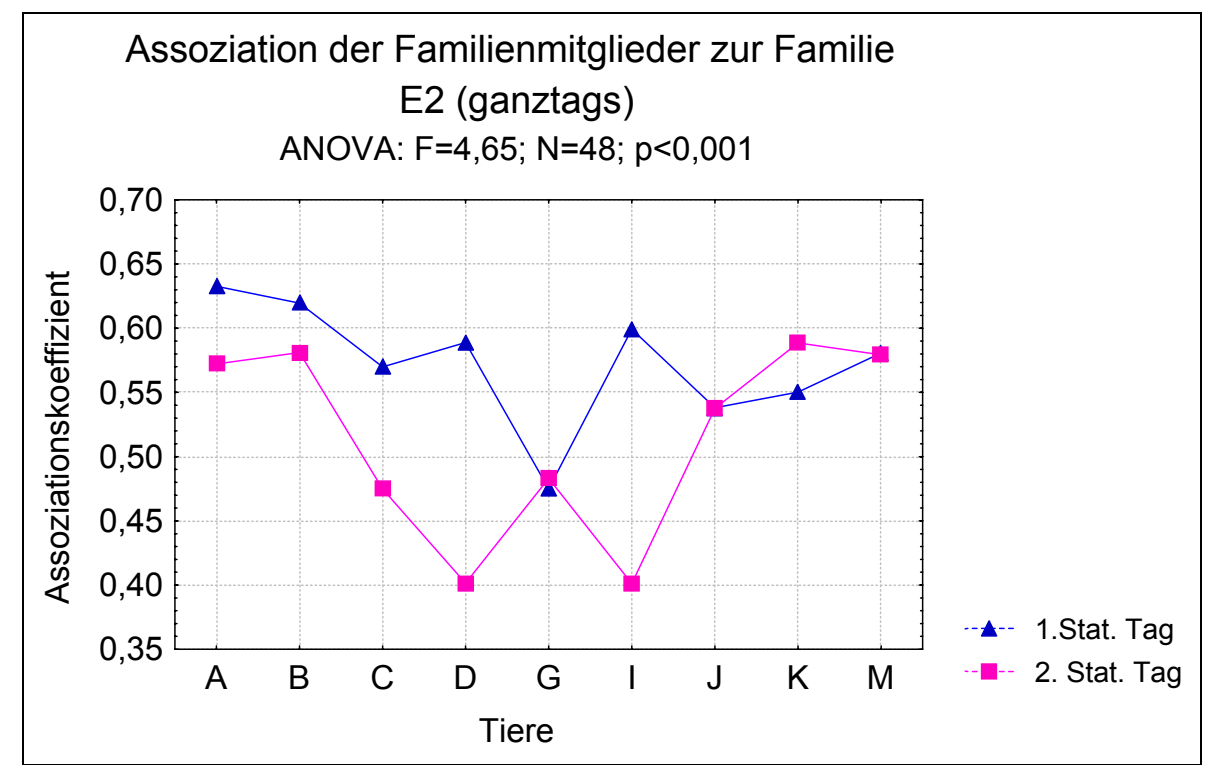

Abb. 3.158 Räumliche Assoziation der Familienmitglieder zur Familie im zweiten Experiment des zweiten Beobachtungsjahres (ANOVA, F=4,65; N=48; $<<0,001$ ). Abkürzungen: 1.Stat. Tag= erster Statistischer Tag; 2. Stat. Tag $=$ zweiter Statistischer Tag.

\subsubsection{Kapiteldiskussion}

\subsubsection{Arealnutzung der Familie}

Während aller Experimentalphasen gab es Unterschiede in der Nutzung der sieben Areale des Streifgebietes durch die Fokusfamilie. Areal 4, welches in der Mitte des Streifgebietes liegt, stellte sich als Nutzungszentrum (core-area) der Fokusfamilie heraus. Die angrenzenden Areale wurden mit zunehmender Entfernung vom Zentrum auch mit geringerer Wahrscheinlichkeit von der Familie betreten. Die größere Aufenthaltswahrscheinlichkeit der Familie in der zweiten Kontrollphase des ersten Beobachtungsjahres in Areal 5, welches dem Nachbarfamilienstreifgebiet zugewandt lag, lässt sich damit erklären, dass die Nachbarfamilie während dieser Phase in ihr Streifgebiet gebracht worden war und die Fokusfamilie ihr Streifgebiet durch ihre vermehrte Präsenz in diesem Areal verteidigte bzw. sicherstellte. Die Anwesenheit der Nachbarfamilie bewirkte in der Familie also eine kurzfristige Verschiebung der Aufenthaltswahrscheinlichkeiten. Während des ersten Experiments war die Aufenthaltswahrscheinlichkeit in den Arealen 2 bis 5 gleich groß. In dieser Phase war das Lockweibchen im Experimentalareal anwesend. Dies war die einzige Phase, in der sich die Familie auch in Areal 7 aufhielt, welches dem Streifgebiet der Nachbarfamilie zugewandt lag. Da der Aufenthaltsschwerpunkt der Familie sich über die mittleren Areale verteilte, war keine besondere Anziehung des Lockweibchens auf die Familie zu verzeichnen. Da innerhalb des zweiten Experiments wie in der ersten Kontrollphase eine starke Abstufung in der Nutzung 
der Areale vom Nutzungszentrum zu den Rändern hin ersichtlich wurde, lässt sich noch eine weitere Erklärung für die Unterschiede in der Arealnutzung finden. In der ersten Kontrollphase konnte die Familie das Semifreiland nach einer mehrmonatigen Winterphase neu erkunden und hielt sich anfangs noch nahe bei der ,Winterhütte“ in Areal 4 auf. Während der folgenden Phase dehnte die Familie die core-area immer weiter aus, bis sie sich während des zweiten Experiments wieder mehr auf die Umgebung der Winterhütte konzentrierte, da wegen der niedrigen Temperaturen (Anfang Oktober) vermehrt in der Hütte übernachtet wurde.

Im zweiten Beobachtungsjahr wurde eine Verschiebung des Nutzungsraumes der Familie vom Zentrum (Areal 4) in Richtung der Areale 1 und 2 deutlich. Insbesondere an Areal 1 wird deutlich, dass sich die Familie im Laufe der Experimente immer häufiger in Areal 1 (und 2) und damit weiter weg von der Nachbarfamilie aufhielt. Durchgehend wurden jedoch die Areale 3 und 4 immer noch am häufigsten genutzt. Die verringerte Aufenthaltswahrscheinlichkeit in den Arealen 5 und 6 während des dritten Experiments ging mit der vermehrten Nutzung der Areale 4 und 1 einher. Das Familienmitglied I hatte sich am Ende des zweiten Experiments häufig in Areal 1 versteckt und das Streifgebiet auch von hier aus verlassen. Mehrere Familienmitglieder suchten das Areal während und nach der Anwesenheit von I auf; möglicherweise um zu kontrollieren, ob I noch zur Familie gehörte bzw. wo er sich aufhielt.

In mehreren Studien wurde beobachtet, dass Jungtiere von den Grenzen des Streifgebiets ferngehalten wurden (König und Rothe 1991, Goldizen 1987 für S. fuscicollis, Wolters 1978 für S. o. oedipus). Es ist plausibel, dass diese Maßnahme dem Schutz der Jungtiere vor fremden Familien bzw. vor Auseinandersetzungen mit diesen Familien diente. Die Verschiebung der Aufenthaltswahrscheinlichkeit der Familie während des zweiten Beobachtungsjahres in die von der Nachbarfamilie abgewandte Richtung kann daher ebenfalls mit dem Schutz der Jungtiere in Zusammenhang gebracht werden.

\subsubsection{Arealnutzung der Familienmitglieder}

Die häufigsten Nutzungsräume der Alpha-Tiere und der adoleszenten und juvenilen Nachkommen waren während des ersten Beobachtungsjahres die Areale 2 und 3, welche von den Nachbarfamilien weiter entfernt lagen. Die adulten Nachkommen hielten sich seltener in Areal 2 und D und E seltener in Areal 3 auf, waren dafür aber häufiger in den Arealen 4 und 5 anzutreffen. Obwohl die Alpha-Tiere, als auch die adulten Nachkommen nah um das Zentrum herum ihre Aufenthaltsschwerpunkte hatten, waren die adulten Söhne stärker zu den Nachbarfamilien orientiert. Eine vergleichbare Verteilung der Gruppenmitglieder fand Radespiel 
(1990) bei Experimenten mit Weißbüschelaffen in Laborhaltung. Die größere Aufenthaltswahrscheinlichkeit von $\mathrm{G}$ in Areal 1 lässt sich vielleicht damit erklären, dass sein Aufenthaltsschwerpunkt als nicht-adulter Nachkomme zwar meist in der Nähe der Eltern lag (die vermehrt in Areal 2 und 3 waren), er aber häufiger Exkursionen nach Areal 1 unternahm, aber dennoch in akustischem Kontakt mit den Eltern stand, wenn diese sich in Areal 2 aufhielten.

Ähnlich wie im ersten Jahr hielten sich die adulten Nachkommen C und D im zweiten Beobachtungsjahr seltener in Areal 1 und $\mathrm{C}$ sich seltener in Areal 2 auf, als die übrigen Familienmitglieder. C war dafür häufiger als die nicht-adulten Nachkommen in Areal $5 \mathrm{zu}$ finden. Da er während des zweiten Jahres häufig die Jungtiere trug, vermied er die Randareale 1, 2, 6 und 7 und konnte sich am effektivsten in der dichten Vegetation der Areale 3 und 5 verbergen, um die Jungtiere vor Raubfeinden zu schützen. Da G sich häufiger als alle anderen Familienmitglieder in Areal 6 aufhielt, ist zu vermuten, dass er besonders stark zu den Nachbarfamilien bzw. Locktieren orientiert war, vergleichbar mit dem Verhalten von E im ersten Beobachtungsjahr. Offen bleibt, ob sein Interesse der Suche nach potentiellen Reproduktionspartnerinnen oder der Verteidigung des Streifgebietes galt. Darms (1987) erkennt für den Aufenthalt adulter Nachkommen an den Grenzen der Streifgebiete beide Erklärungsmöglichkeiten an. Solange G zur Natalfamilie gehörte, verteidigte er nach seiner Interpretation das Streifgebiet gegen Eindringlinge. Gleichzeitig hatte er am Rand des Streifgebietes die Möglichkeit, auf den besten Emigrationszeitpunkt zu warten.

\subsubsection{Peripheralisationen}

Die räumliche Assoziation der Mitglieder einer Weißbüschelaffenfamilie besteht aus den drei Hauptclustern Alpha-Tiere, Subgruppe der nicht-adulten Nachkommen und den adulten Nachkommen, die voneinander und von den Alpha-Tieren eine periphere Position einnehmen. Sie sind mehr oder weniger mit der Subgruppe der nicht-adulten Nachkommen assoziiert (Jünemann 1990, König und Rothe 1991, siehe auch Maier et al. 1982). Die stärkste Kohäsion besteht zwischen den Alpha-Tieren (Darms 1987), während die interindividuellen Distanzen (IID) der nicht-adulten Nachkommen mit den IID der Eltern vergleichbar sind. Nach einer Geburt schränken die Eltern die Zuwendungen zu den nicht-adulten Nachkommen ein und kümmern sich vermehrt um die Jungtiere. Die Nicht-adulten finden in ihrer Subgruppe (peergroup) weiterhin sozialen Anschluss. Die adulten Nachkommen befinden sich nach Dawson (1976 für S. oedipus geoffroyi) in ihrer peripheren Position möglicherweise in einer Art Übergangsstadium zwischen dem Verbleib im elterlichen Streifgebiet und dem Erkunden von 
Emigrationsmöglichkeiten an den Grenzen des Streifgebietes. Sie meiden alle potentiellen Konkurrenten (adulte Geschwister und die Eltern), halten sich an der Peripherie auf und können so nach zukünftigen Sexualpartnern Ausschau halten (Darms 1987). Der Aufenthalt an den Grenzen des Streifgebietes bedeutet für die Gruppe auch eine bessere Verteidigung des Streifgebietes, da die adulten Nachkommen so eher fremde Artgenossen wahrnehmen und das elterliche Streifgebiet gegen Eindringlinge verteidigen können (König und Rothe 1991).

Während der beiden Beobachtungsjahre der Studie war anhand der engen Assoziation der Alpha-Tiere zur Familie und ihren durchgehend überdurchschnittlich hohen Nahbereichshäufigkeiten zu Familienmitgliedern auch eine hohe Kohäsion zueinander zu vermuten. Die Assoziation der adoleszenten Tochter H zur Familie im ersten Beobachtungsjahr und der adoleszenten Töchter $\mathrm{K}$ und $\mathrm{M}$ im zweiten Beobachtungsjahr entsprechen den Untersuchungen von Darms (1987), dass die IID der nicht-adulten Nachkommen denen der Eltern ähnelt. Die räumliche Assoziation des adoleszenten Sohnes G im ersten Beobachtungsjahr muss hingegen anders bewertet werden. Seine geringe Kohäsion zur Familie entsprach nicht den Erwartungen für adoleszente Familienmitglieder. Da er sich bevorzugt in den Arealen aufhielt, die weit von nachbarlichen Streifgebieten entfernt lagen, ist es unwahrscheinlich, dass er sich zur Verteidigung des Streifgebietes oder zur Herstellung des Kontaktes mit fremden Artgenossen an der Peripherie der Familie aufhielt. Seine Nahbereichshäufigkeiten wichen nicht vom Erwartungsbereich ab. Daraus kann geschlossen werden, dass seine lockere räumliche Assoziation zur Familie nicht mit einer sozialen Isolation zusammenhing (Digby 1995b). Die fast durchgängig lockere Assoziation der adulten Nachkommen gegenüber den Eltern und nicht-adulten Nachkommen fügt sich in die Angaben von König und Rothe (1991) und Darms (1987) ein. Im ersten Beobachtungsjahr waren die Nahbereichhäufigkeiten aller adulten Nachkommen geringer als der Familiendurchschnitt, was ebenfalls mit der Erwartung übereinstimmt.

Im ersten Beobachtungsjahr gab es Hinweise darauf, dass die peripheren adulten Nachkommen vermehrt nicht-taktil mit fremden Artgenossen interagierten. Dies unterstützt die Annahme von Darms (1987), dass sich adulte Nachkommen an der Peripherie aufhalten, um Kontakte zu fremden Artgenossen herzustellen. Jedoch konnte für keinen der adulten Nachkommen im ersten Beobachtungsjahr eine Abhängigkeit der räumlichen Assoziation von den Experimenten festgestellt werden. Ebenso war kein Zusammenhang zwischen der räumlichen Assoziation und der nicht-taktilen Interaktion mit fremden Artgenossen zu erkennen. Die Anwesenheit der Locktiere war demnach nicht für die peripheren Positionen 
der adulten Nachkommen im ersten Beobachtungsjahr entscheidend.

Während der Abendstunden des ersten Beobachtungsjahres waren alle Familienmitglieder außer F enger mit der Familie assoziiert als im Tagesmittel. Die lockere Assoziation von F zur Familie hing möglicherweise mit seinem bevorstehenden Rauswurf zusammen. Freilandstudien definieren den Ausschluss eines Tieres aus seiner Sozietät zum Teil derart, dass die Gruppenmitglieder mit dem Tier dissoziativ interagierten, wenn es sich dem Gruppenschlafplatz näherte (z.B. Caine 1993). Eigene Beobachtungen im Semifreiland konnten die Angaben bestätigen. Infolge der dissoziativen Handlungen seines Bruders E suchte F häufig später als andere den gemeinsamen Schlafplatz auf, womit die weniger enge Assoziation von $\mathrm{F}$ in den Abendstunden zur Familie erklärt wird.

Während des zweiten Beobachtungsjahres hatte nur die älteste Tochter D im ersten Experiment einen erhöhten Anteil an nicht-taktiler Interaktion mit fremden Artgenossen. Bis auf die Morgenstunden waren bei ihr, wie auch bei den anderen adulten und subadulten Nachkommen, keine Abhängigkeiten der räumlichen Assoziation zur Familie von den Experimenten zu verzeichnen. Morgens hatte D im zweiten Experiment eine lockere Assoziation zur Familie, so dass dies vielleicht als Reaktion auf die Anwesenheit der beiden Lockmännchen gedeutet werden kann. Die morgendliche Peripheralisation von der Familie spricht für Erkundungen der Umgebung nach Reproduktionspartnern, da sexuelle Interaktionen nur in Abwesenheit von Gruppenmitgliedern gezeigt werden (Anzenberger 1983, Digby 1999, Geyer 1993). Sie hielt sich außerdem seltener als andere im Nahbereich von Familienmitgliedern auf. Da sie jedoch nicht häufiger als erwartet mit fremden Artgenossen kommunizierte, kann diese Vermutung nicht durch alle Verhaltenseinheiten/bzw. -bereiche belegt werden.

Die räumliche Assoziation zwischen den Eltern und adulten Nachkommen ändern sich, wenn Jungtiere geboren werden, so dass die ältesten und erfahrensten Helfer Zugang zu den Jungtieren bekommen (Jünemann 1990). Da es am Ende des ersten Experiments im zweiten Beobachtungsjahr eine Geburt gab kann ein Zusammenhang zwischen der Geburt und den verringerten Nahbereichshäufigkeiten der adulten Nachkommen vermutet werden. Während der ersten Woche nach der Geburt wurden die Jungtiere jedoch überwiegend von der Mutter getragen, so dass eine Jungtierübernahme noch nicht notwendig war. Trotzdem hielten sich die Söhne schon im ersten Experiment häufiger im Nahbereich von Familienmitgliedern auf. Die Anwesenheit der Jungtiere stellte für alle Familienmitglieder eine Attraktion dar, auch wenn anfangs nur wenige Geschwister die Jungtiere übernehmen durften. So würde eine 
Annäherung aller Familienmitglieder zu den Jungtieren gleichzeitig ihre Nahbereichshäufigkeiten erhöhen. Dies wird durch die Beobachtungen im ersten Experiment unterstützt, da in dieser Phase kaum Unterschiede in der Nahbereichshäufigkeit zwischen den Familienmitgliedern festzustellen waren. Da die Tochter D vor allem während des zweiten Experiments nicht in die Nähe der Jungtiere gelassen wurde, wäre zumindest in dieser Phasee auch hierin ein Begründung für die Verringerung ihrer Nahbereichshäufigkeiten zu finden.

Ein Zusammenhang zwischen NTA und der räumlichen Assoziation zur Familie konnte nur bei einem Tier nachgewiesen werden. Bei dem subadulten Nachkommen I verringerte sich mit dem zweiten Experiment die räumliche Assoziation zur Familie. Da er am Ende des zweiten Experiments aus der Familie vertrieben wurde, kann die Peripheralisation mit dem Rauswurf in Zusammenhang gebracht werden. Zeitgleich hatte sich bei I die Nahbereichshäufigkeit zu Familienmitgliedern vermindert. Der Anteil an taktiler Interaktion war schon während des ersten Experiments seltener als erwartet. Die Annahmen von Rothe und Darms (1993) und Digby (1995b), dass bei freilebenden Weißbüschelaffen Emigrationen bzw. Rauswürfen Peripheralisationen vorausgehen, konnte mit den Ergebnissen aus der Semifreilandhaltung in einem von zwei Fällen für Rauswürfe bestätigt werden. Die Vermutung von Rothe und Darms (1993), dass Peripheralisationen anstelle von Beißkämpfen vor Rauswürfen geschehen, wurde ebenfalls durch die Beobachtungen an dem Rauswurf von I bestätigt, da I von seinem Verfolger zwar angegriffen wurde, es jedoch zu keinen Beißkämpfen oder starken Verletzungen kam. Statt dessen distanzierte sich I räumlich von der Familie. Demnach konnten bei diesem Rauswurf die Beobachtungen von McGrew (1997) an Saguinus oedipus, dass Peripheralisationen infolge von Angriffen durch Gruppenmitglieder entstehen können, ebenfalls bestätigt werden.

Zwischen der Häufigkeit, mit der dissoziative Handlungen innerhalb der Familie auftraten und der räumlichen Assoziation zu Familienmitgliedern konnte allerdings bei keinem Familienmitglied eine Korrelation gefunden werden. Es gibt somit keine direkten Hinweise darauf, dass allgemein aggressive Interaktionen innerhalb der Familie die Verringerung von räumlichen Assoziationen bewirkten. Freiwillige Peripheralisationen von Subadulten ohne vorhergehende (elterliche) Aggression beschrieben schon Sutcliffe (1980) und Ingram (1977), wobei fraglich ist, ob diese Laborstudien mit Beobachtungen aus der Semifreilandhaltung vergleichbar sind.

Der Vergleich der räumlichen Assoziation der Familienmitglieder zur Familie verdeutlichte Unterschiede zwischen den Altersklassen bzw. Hauptclustern, wie sie von König und Rothe 
(1991) beschrieben wurde. Allerdings konnte bei diesem Vergleich eine Lockerung der räumlichen Assoziation von I zur Familie nicht ermittelt werden. Erst ein Vergleich der Assoziationskoeffizienten des Einzeltieres zwischen den Experimenten machte die Peripheralisation im zweiten Experiment gegenüber dem ersten Experiment deutlich. Die beinahe durchgängige Peripheralisation der adulten und z.T. subadulten Nachkommen von der Familie war in dieser Studie jedoch kein Hinweis auf eine bevorstehende Emigration bzw. einen Rauswurf aus der Familie. Nur ausgehend von einem dauerhaft gleichbleibenden Assoziationskoeffizienten können Lockerungen der räumlichen Assoziation, verbunden mit der Verminderung von Nahbereichshäufigkeiten und der taktilen Interaktion mit Familienmitgliedern nach dieser Studie als Indikator für bevorstehende Emigrationen bzw. Rauswürfe bewertet werden.

Da vor Rauswürfen weiblicher Nachkommen weder eine Lockerung ihrer räumlichen Assoziation zur Familie, noch der Nahbereichshäufigkeiten oder taktilen Interaktion zu verzeichnen war, bestehen möglicherweise Unterschiede im Verlauf der Rauswürfe von Männchen und Weibchen. Die Datenlage über Rauswürfe weiblicher Nachkommen war für eine Untersuchung dieses Aspekts allerdings zu dürftig. Inwiefern freiwilligen Emigrationen Peripheralisationen von der Familie vorausgehen, konnte in dieser Arbeit nicht geklärt werden, da keine freiwilligen Emigrationen beobachtet werden konnten. So bleibt z.B. unklar, ob die Verringerung der Nahbereichshäufigkeit und die morgendliche Peripheralisierung von D im zweiten Experiment als Emigrationsbereitschaft interpretiert werden kann.

\section{5 Überprüfung einzelner Modellkomponenten}

\subsubsection{Potentielle Reproduktionspartner}

Da die Mitglieder einer Weißbüschelaffenfamilie, mit Ausnahme der Eltern, zu 50\% miteinander verwandt sind, sollten sie daran interessiert sein, dass die Fitness ihrer Verwandten maximiert wird, weil dadurch ihre inklusive Fitness ebenfalls steigen würde. Es wäre damit die Frage berechtigt, ob Rauswürfe nur dann vollzogen werden, wenn das rausgeworfene Tier die besten Reproduktionschancen erhält. Da die nicht-taktile Interaktion mit fremden Artgenossen keine Aussage über die Emigrationsbereitschaft eines Nachkommen zuließ, wurde jedoch erwartet, dass Rauswürfe besonders häufig geschehen, wenn potentielle Paarungspartner, also Locktiere präsent sind. Die Untersuchungen konnten diese Vermutung aber nicht bestätigen. Von acht beobachteten Rauswürfen geschah nur einer in einer 
Experimentalphase, in welcher der Familie Locktiere geboten wurden (vgl. Tab. 3.3, S. 68). Da das Locktier und das ausgestoßene Tier männlich waren, kann nicht davon ausgegangen werden, dass der Rauswurf aufgrund optimaler Reproduktionsbedingungen vollzogen wurde.

\subsubsection{Mortalitätsrisiko}

Das Modell beinhaltet Mortalitätsrisiken in drei unterschiedlichen Umgebungen. Zunächst befindet sich ein Individuum innerhalb des elterlichen Streifgebietes und ist dort einem bestimmten Mortalitätsrisiko ausgesetzt. Wandert es von der Natalfamilie ab oder hat es ein neues Streifgebiet gefunden, wirkt jeweils ebenso ein bestimmtes Mortalitätsrisiko auf es ein. Das Mortalitätsrisiko setzt sich bei freilebenden Krallenaffen aus dem Risiko, nicht genügend Nahrung zu finden, Krankheiten zu erleiden und der Prädation durch Beutegreifer zu unterliegen, zusammen. In der vorliegenden Studie konnten nur Aussagen zum Prädationsrisiko auf dem Studiengelände, also im Streifgebiet der Natalfamilie, gemacht werden.

Obwohl die Familie J18 nicht in ihrer natürlichen Umgebung lebte, bestand für sie ein reales Prädationsrisiko, weil die Semifreianlage für Greifvögel der Umgebung (Milane/ Bussarde/ Habichte/ Sperber) frei zugänglich war und streunende Katzen, als auch Marder und Rotfüchse auf dem Gelände gesichtet wurden. Der Prädationsdruck der Affen in Sennickerode ließ sich aus der Zeitperiode der bisher im Semifreiland lebenden Gruppen (1995 - 2003) und der Anzahl an Tieren, die der Prädation in dieser Zeit zum Opfer fielen, abschätzen. Die Anzahl der Tiere pro Jahr auf dem Gelände, die jeweilige Aufenthaltsdauer der Gruppen, sowie die Anzahl der beobachteten Raubfeindangriffe sind in Tab. 3.41 dargestellt.

Es kam während der acht Jahre zu keinem Todesfall durch Beutegreifer, obwohl während der Sommermonate regelmäßig Greifvögel in der Nähe und über dem Gelände gesichtet wurden. Die Ursache für die effektive Raubfeindvermeidung liegt wahrscheinlich in dem Wächtersystem der Gruppen begründet.

Abschätzungen des Prädationsrisikos von Migranten in neuen Streifgebieten konnte für $C$. jacchus nicht gemacht werden. An dieser Stelle muss grundsätzlich in Frage gestellt werden, ob Studien zu Mortalitäts- und Prädationsrisiken im Semifreiland überhaupt aussagekräftig und damit sinnvoll sind, da die Habitat- und Umweltbedingungen des natürlichen Lebensraumes von $C$. jacchus selbst dann nicht erreicht werden, wenn die Studientiere naturnahen Bedingungen mitteleuropäischer Landschaften ausgesetzt werden. Da jedoch Beutegreifer für die Studientiere zumindest als potentielle Bedrohung existent waren, und das Wächtersystem 
„aus der Sicht der Affen“ notwendig war, scheint die Haltung im Semifreiland bisher die beste Alternative zu Freilandbeobachtungen zu sein, um die Auswirkungen von Umwelteinflüssen auf das Verhalten von $C$. jacchus zu erforschen. Insbesondere für die Analyse der Entscheidung über Philopatrie und Emigration ist sie eine wichtige Voraussetzung.

Tabelle 3.41 Gruppen und Familien auf dem Semifreigelände der Ethologischen Station Sennickerode der Universität Göttingen (1995-2003). Ebenfalls sind die Gruppengröße, Aufenthaltsdauer im Gelände und Prädationsbeobachtungen.

\begin{tabular}{|c|c|c|c|c|}
\hline \multirow{4}{*}{1995} & & & & \\
\hline & J16 & $8-10$ & 86 & 1 Habichtattacke \\
\hline & J18 & $6-8$ & 94 & \\
\hline & $1022 / 1023 / 1024$ & 3 & 58 & \\
\hline \multirow[t]{3}{*}{1996} & J16 & 10 & 121 & \\
\hline & J18 & 11 & 121 & \\
\hline & $\mathrm{J} 25$ & 3 & 112 & \\
\hline \multirow[t]{4}{*}{1997} & J18 & $12-14$ & 145 & \\
\hline & J25 & $4-6$ & 148 & \\
\hline & 991 & 1 & 119 & \\
\hline & $\mathrm{J} 23$ & $10-13$ & 89 & \\
\hline \multirow[t]{4}{*}{1998} & J18 & $11-14$ & 163 & \\
\hline & $J 23$ & $10-12$ & 163 & \\
\hline & $\mathrm{J} 25$ & $7-8$ & 80 & \\
\hline & $1049 / 1051$ & 2 & 81 & \\
\hline \multirow[t]{2}{*}{1999} & J18 & $7-10$ & 135 & \\
\hline & $\mathrm{J} 23$ & $7-10$ & 57 & \\
\hline \multirow[t]{2}{*}{2000} & J18 & $9-10$ & 128 & 1 Habichtattacke* \\
\hline & $\mathrm{J} 27 / 1$ & $6-7$ & 131 & \\
\hline \multirow[t]{3}{*}{2001} & $\mathrm{~J} 18$ & $9-12$ & 162 & \\
\hline & J29 & $8-10$ & 112 & Gruppe mobbt einen Marder \\
\hline & $\mathrm{J} 27 / 1$ & $1-4$ & & \\
\hline \multirow[t]{3}{*}{2002} & J18 & $9-10$ & 5 & \\
\hline & $\mathrm{J} 27 / 1$ & $8-10$ & 129 & \\
\hline & $\mathrm{J} 29$ & $8-10$ & 145 & \\
\hline \multirow[t]{2}{*}{2003} & $\mathrm{~J} 28$ & 11 & & Gruppe mobbt einen Greifvogel \\
\hline & $\mathrm{J} 29$ & 10 & & Gruppe mobbt einen Greifvogel \\
\hline
\end{tabular}

* aus Rothe und Thieß (2001a) 


\subsubsection{Geschlecht}

Im Entscheidungsmodell hat das Geschlecht des Individuums Auswirkungen auf die Entscheidung über Philopatrie oder Emigration. Einerseits wurde angenommen, dass die Entscheidung indirekt durch das Angebot potentieller Reproduktionspartner beeinflusst wird und andererseits durch die Anwesenheit von Emigrationspartnern innerhalb der Natalfamilie.

Bei der Berechnung der Rauswurfwahrscheinlichkeit wurden keine Unterschiede in Abhängigkeit vom Geschlecht der Nachkommen festgestellt. Es war damit für männliche als auch für weibliche Nachkommen gleich wahrscheinlich, aus der Familie geworfen zu werden. Von männlichen Nachkommen wurden bevorzugt Emigrationen bei der Anwesenheit von Lockweibchen erwartet, während die Emigration weiblicher Nachkommen in der Anwesenheit männlicher Locktiere provoziert werden sollte. Es wurde deutlich, dass weder die männlichen, noch die weiblichen Nachkommen aus der Fokusfamilie freiwillig abwanderten, wenn ihnen potentielle Reproduktionspartner präsentiert wurden. Rauswürfe ereigneten sich ebenfalls nicht in der Anwesenheit gegengeschlechtlicher fremder Artgenossen. Da es keine gemeinschaftlichen Emigrationen von Brüdern oder Schwestern gab, bleibt offen, inwiefern das Geschlecht eines Individuums bei Anwesenheit von Emigrationspartnern Auswirkungen auf die Entscheidung über Philopatrie und Emigration hat. Die Zusammenhänge sind im Teilmodell in Abb. 3.159 dargestellt.

Eine mögliche Interpretation der ausbleibenden Co-Emigrationen ist die, dass potentielle Emigranten keine abwanderungswilligen gleichgeschlechtlichen Geschwister bzw. Emigrationspartner innerhalb ihrer Natalfamilie zur Verfügung hatten.

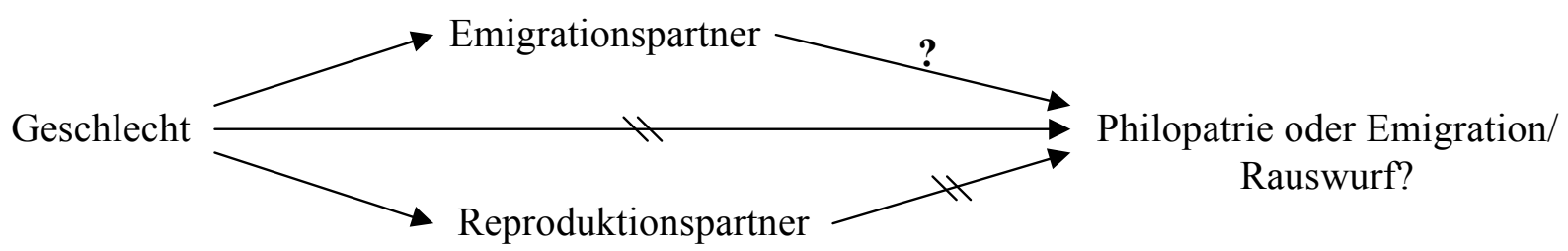

Abb. 3.159 Auswirkungen des Geschlechts eines Individuums auf die Wahrscheinlichkeit, die Natalgruppe zu verlassen. Ein direkter Einfluss auf die Entscheidung zwischen Philopatrie und Emigration konnte nicht gefunden werden. Indirekte Auswirkungen des Geschlechts auf die Entscheidung über die Anwesenheit von potentiellen Emigrationspartnern konnten nicht ermittelt werden und bleiben damit ungeklärt. Da während der Anwesenheit der Locktiere keine Emigrationen oder Rauswürfe beobachtet werden konnten, waren keine Hinweise auf einen Einfluss von potentiellen Reproduktionspartnern auf die Entscheidung über Philopatrie oder Emigration zu finden.

Im ersten Beobachtungsjahr gab es zwei adulte Brüder ( $\mathrm{C}$ und $\mathrm{E})$ und im zweiten Beobachtungsjahr vier adulte bzw. subadulte Brüder, die Emigrationsgemeinschaften hätten 
bilden können (C, G, I und J). Warum sie dennoch nicht gemeinsam emigrierten, wurde bereits in 3.3.4.2 diskutiert. Die älteste Tochter D hatte im ersten Beobachtungsjahr eine jüngere Schwester $(\mathrm{H})$ und im zweiten Beobachtungsjahr bei der Präsentation der Lockmännchen keine geschlechtsreifen Schwestern, so dass in diesem Falle auch keine CoEmigration möglich war. Anders interpretiert war die Anwesenheit geeigneter Emigrationspartner möglicherweise kein ausreichender Anreiz für eine gemeinschaftliche Emigration aus der Familie. Die Brüder C und E emigrierten auch in Anwesenheit der Lockweibchen nicht aus der Familie, so dass sogar die Kombination aus Anwesenheit von adulten Brüdern und potentiellen Reproduktionspartnern bei den männlichen Nachkommen keine Emigration auslöste.

Die Ergebnisse können das vorliegende Modell über direkte Auswirkungen des Geschlechts auf die Entscheidung über Philopatrie und Emigration nicht stützen. Die Anwesenheit von potentiellen Emigrations- und Reproduktionspartnern wirkten sich in der Studie immer zugunsten der Philopatrie, also entgegen der Annahmen des Modells aus.

\subsubsection{Alter}

Nach dem Modell hat das Alter über den Restreproduktionswert (RRV) einen direkten Einfluss auf die Entscheidung über Philopatrie und Emigration. Stearns (1992) berechnete die Restreproduktionswerte der Altersklasse $A$ wie folgt:

$$
R R V_{A}=p_{A} \mathrm{e}^{-r} v_{A+1}
$$

Erläuterungen der Formel:

$$
\begin{aligned}
& v_{A}=\frac{\mathrm{e}^{r(A-1)}}{l_{A}} \sum_{X=A}^{X=\omega} \mathrm{e}^{-r X} l_{X} m_{X} \\
& p_{X}=1-d_{X} \\
& p_{X}=\text { Wahrscheinlichkeit, von Alter } \mathrm{x} \text { bis Alter } \mathrm{x}+1 \text { zu überleben } \\
& d_{X}=\text { Wahrscheinlichkeit, zwischen } \mathrm{x} \text { und } \mathrm{x}+1 \text { zu sterben } \\
& l_{X}=\text { Wahrscheinlichkeit, bis zur Altersklasse } \mathrm{X} \text { zu überleben } \\
& m_{X}=\text { erwartete Anzahl an Nachkommen eines Weibchens in Altersklasse } \mathrm{X} \\
& r=\text { Populationswachstum } \\
& v_{X}=\text { Reproduktionswert der Altersklasse } \mathrm{X} \\
& \omega=\text { Alter bei der letzten Reproduktion }
\end{aligned}
$$


Die Reproduktions- und Restreproduktionswerte wurden für die Weißbüschelaffen der Kolonie des IfA berechnet. Für die Simulation der Restreproduktionswerte wurde angenommen, dass die Geburtenrate eines Weibchens ca. vier Nachkommen pro Jahr beträgt (Ross und Jones 1999). Dasselbe gilt für die Reproduktionsrate der strikt monogamen Männchen. Das Alter der letzten Reproduktion wird auf zehn Jahre gesetzt ${ }^{19}$. Die Population soll ihr maximales Wachstum $\left(r_{m}\right)$ erreicht haben. In Tab. 3.42 ist eine Lebenstafel über die Population der Universität Göttingen dargestellt. Die Lebenstafel beinhaltet die Anzahl der Überlebenden und Gestorbenen, die Sterbe- und Überlebensraten und die Wahrscheinlichkeit, bis zum Alter x zu überleben.

Tab. 3.43 bildet die Reproduktions- und Restreproduktionswerte und Geburtenrate für die einzelnen Altersklassen der weiblichen Weißbüschelaffen aus der Kolonie des IfA ab.

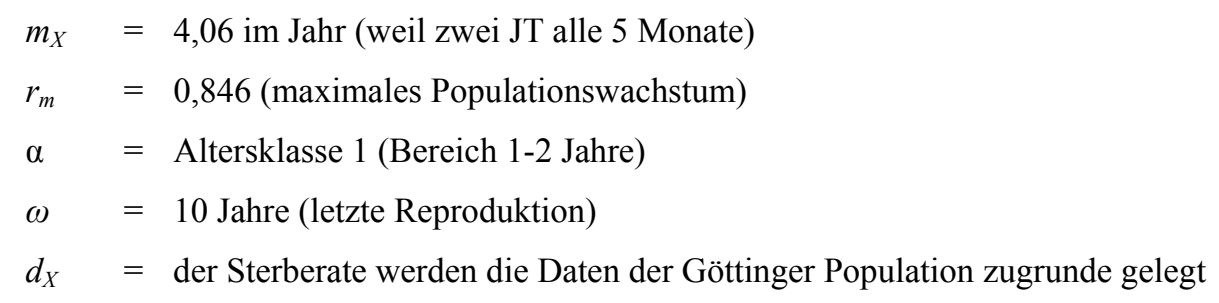

Tabelle 3.42 Lebenstafel für die Weibchen der Callithrix jacchus-Kolonie des IfA, die im Zeitraum von 19682003 auf natürliche Weise gestorben ist $(\mathrm{N}=252)$.

\begin{tabular}{cccccc}
\hline $\begin{array}{c}\text { Altersklasse } \\
(\mathrm{x})\end{array}$ & $\begin{array}{c}\text { Überlebende } \\
(\mathrm{Sx})\end{array}$ & $\begin{array}{c}\text { Gestorbene } \\
(\mathrm{Dx})\end{array}$ & $\begin{array}{c}\text { Sterberate } \\
(\mathrm{dx})\end{array}$ & $\begin{array}{c}\text { Überlebensrate } \\
(\mathrm{px})\end{array}$ & $\begin{array}{c}\text { Überlebt bis zum } \\
\text { Alter } \mathrm{x}(\mathrm{Ix})\end{array}$ \\
\hline 0 & 252 & 189 & 0,75 & 0,25 & 1,000 \\
1 & 63 & 0 & 0,00 & 1,00 & 0,250 \\
2 & 63 & 14 & 0,22 & 0,78 & 0,250 \\
3 & 49 & 11 & 0,22 & 0,78 & 0,195 \\
4 & 38 & 11 & 0,29 & 0,71 & 0,152 \\
5 & 27 & 7 & 0,26 & 0,74 & 0,108 \\
6 & 20 & 6 & 0,30 & 0,70 & 0,080 \\
7 & 14 & 6 & 0,43 & 0,57 & 0,056 \\
8 & 8 & 4 & 0,50 & 0,50 & 0,031 \\
9 & 4 & 3 & 0,75 & 0,25 & 0,016 \\
10 & 1 & 1 & 1,00 & 0,00 & 0,004 \\
\hline
\end{tabular}

\footnotetext{
${ }^{19}$ In der Ethologischen Station Sennickerode des IfA gab es auch ältere reproduktive Weibchen.
} 
Tabelle 3.43 Reproduktionswerte und Restreproduktionswerte verschiedener Altersklassen der Weibchen der $C$. jacchus-Kolonie des IfA unter der Annahme $\left(\mathrm{m}_{\mathrm{x}}=4,06, \mathrm{r}_{\mathrm{m}}=0,846, \alpha=1, \omega=10\right)$.

\begin{tabular}{cccc} 
Altersklasse & Restreproduktionswert & Geburtenrate & Reproduktionswert \\
\hline 0 & 0,307 & 0,00 & 0,000 \\
1 & 1,118 & 4,06 & 5,178 \\
2 & 0,864 & 4,06 & 4,924 \\
3 & 0,840 & 4,06 & 4,900 \\
4 & 0,765 & 4,06 & 4,825 \\
5 & 0,769 & 4,06 & 4,829 \\
6 & 0,678 & 4,06 & 4,738 \\
7 & 0,531 & 4,06 & 4,591 \\
8 & 0,414 & 4,06 & 4,474 \\
9 & 0,187 & 4,06 & 4,247 \\
10 & 0,000 & 4,06 & 4,060 \\
\hline
\end{tabular}

Wie im oben dargestellten Beispiel zu erkennen ist, nimmt der Reproduktionswert und damit auch der Restreproduktionswert mit zunehmendem Alter ab. Tardif und Ziegler (1986) konnten belegen, dass sich bei Tamarinweibchen ( $S$. oedipus und S. fuscicollis) die Ovulationsrate mit dem Alter verringert. Die Entscheidung, zu emigrieren führt also nur dann zu einer optimalen Fitnessauszahlung, wenn ein Tier relativ jung ist (bis ca. 5-6 Jahre). Ältere Tiere mit niedrigem RRV (ab ca. 7 Jahre) haben nur noch geringe Chancen, ihre direkte Fitness zu maximieren und sollten eher bemüht sein, in die Aufzucht ihrer Geschwister zu investieren, da der so erhaltene Nutzen höher ausfiele, als ein später eigener Reproduktionsversuch. So findet der Zeitpunkt der Emigration aus dem Natalgebiet bei den meisten Arten im subadulten Alter mit Beginn der Geschlechtsreife statt (Dawson 1976 für S. oedipus, Gaines und McClenaghan 1980 für Microtus, Harcourt 1978 für Gorilla). Goldizen und Terborgh (1989) schätzten das Alter migrierender S. fuscicollis allerdings auf 1,3 - 4,3 Jahre.

$\mathrm{Da}$ in der Studienfamilie weder subadulte, noch adulte Nachkommen freiwillig emigrierten können nur die Zeitpunkte der Rauswürfe in diesem Zusammenhang diskutiert werden. Die Rauswurfdaten der C. jacchus-Kolonie des IfA machen einen Einfluss des Alters eines Tieres auf die Wahrscheinlichkeit, rausgeworfen zu werden deutlich, da bevorzugt Männchen im Alter von 15 bis 25 und für Weibchen im Alter von 15 bis 30 Monaten rausgeworfen werden (Abb. 3.160). 


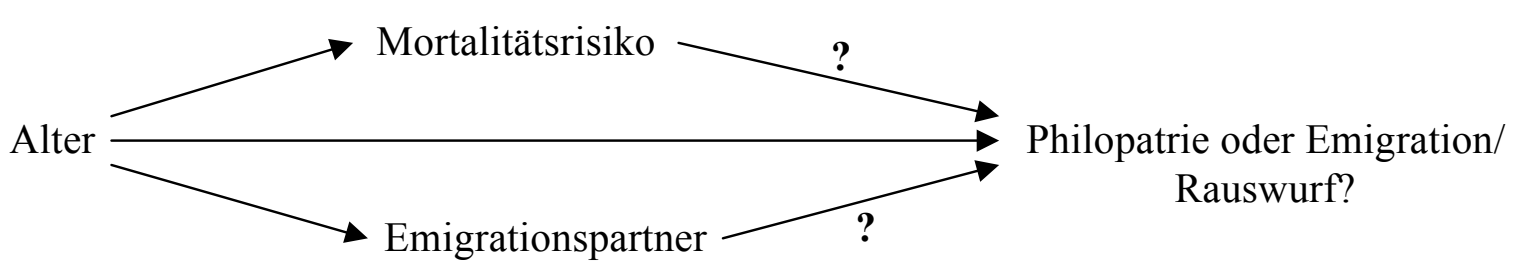

\begin{abstract}
Abb. 3.160 Auswirkungen des Alters eines Individuums auf die Wahrscheinlichkeit, die Natalgruppe zu verlassen. Es konnte ein direkter Einfluss auf die Entscheidung zwischen Philopatrie und Emigration/Rauswurf gefunden werden, da bevorzugt Tiere der Altersklassen 15-30 Monaten rausgeworfen wurden. Indirekte Auswirkungen des Alters auf die Entscheidung über unterschiedliche Mortalitätsrisiken und die Anwesenheit von potentiellen Emigrationspartnern konnten nicht ermittelt werden und bleiben damit ungeklärt.
\end{abstract}

Das Alter eines Individuums steht nach dem Modell in Wechselwirkung mit dem Mortalitätsrisiko im alten Streifgebiet der Natalgruppe, während einer Migration und in einem zukünftigen neuen Streifgebiet. Die Mortalitätsrisiken, denen Individuen während einer Emigration oder in einem neuen Streifgebiet ausgesetzt sind, konnten nicht analysiert werden, weil die Untersuchungen auf das 6,5ha große Semifreigelände beschränkt bleiben mussten. Da keine Co-Emigrationen stattfanden und die Brüder trotz der Anwesenheit potentieller Emigrationspartner in beiden Beobachtungsjahren philopatrisch blieben, bleibt unklar, inwiefern das Alter bei Anwesenheit von Emigrationspartnern auf die Entscheidung über Philopatrie und Emigration einwirkt.

\title{
3.5.5 Gruppengröße
}

Die Gruppengröße hat in dem Modell aus 1.2 Auswirkungen auf die Entscheidung über Philopatrie und Emigration. Indirekte Auswirkungen hat die Gruppengröße, da sie die Verfügbarkeit von Helferpositionen und das Mortalitätsrisiko im Streifgebiet der Natalgruppe beeinflusst. Es konnte nachgewiesen werden, dass das Rauswurfrisiko innerhalb eines Monats bei den Gruppengrößen 11, 13 und 17 größer als bei den anderen getesteten Gruppengrößen ist, so dass ein direkter Einfluss der Gruppengröße auf die Entscheidung zwischen Philopatrie und Emigration vorhanden ist. Inwiefern die Gruppengröße über die Verfügbarkeit von Helferpositionen auf die Entscheidung einwirkt, konnte nicht detailliert untersucht werden. Das Prädationsrisiko von Individuen in unterschiedlich großen Gruppen konnte nicht statistisch bewertet werden, weil keines der Gruppenmitglieder durch einen Raubfeindangriff ums Leben kam und Wahrscheinlichkeitsberechnungen daher unmöglich waren (Abb. 3.161). 


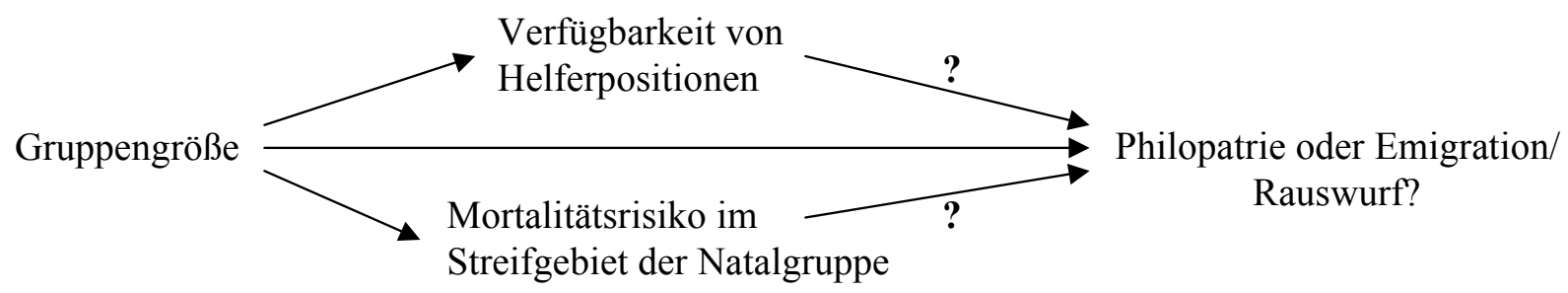

Abb. 3.161 Auswirkungen der Gruppengröße auf die Wahrscheinlichkeit, dass ein Nachkomme die Natalgruppe verlässt. Es konnte ein Einfluss auf die Entscheidung zwischen Philopatrie und Emigration gefunden werden, da das Rauswurfrisiko bei Gruppengrößen von 11, 13 und 17 größer als bei anderen Gruppengrößen ist. Indirekte Auswirkungen auf die Entscheidung hat die Gruppengröße über die Verfügbarkeit von Helferpositionen und über das Mortalitätsrisiko im Streifgebiet der Natalgruppe.

Rauswürfe ereigneten sich bei der Familie J18 bei Gruppengrößen von 10 und 11 und bewegen sich damit im unteren Bereich der größten Rauswurfwahrscheinlichkeit. Demnach lohnt es sich für die Familie nicht mehr, nicht-reproduktive Helfer zu tolerieren, wenn die Gruppengröße von 11 überstiegen wird.

\subsubsection{Geschwister-Konkurrenz}

Garber et al. (1993, 1997) legen dar, dass kooperatives Verhalten und Konkurrenz als Taktiken nicht-reproduktiver Nachkommen verstanden werden können, die den Zugang zu Ressourcen ermöglichen, die dazu verhelfen eine soziale Position zu erlangen, zu erhalten oder zu verbessern oder die reproduktive Möglichkeiten verbessern.

Bei Konkurrenz um gruppendienliche Aktivitäten zwischen den Geschwistern war eine Verringerung der affiliativen und eine Zunahme der dissoziativen Interaktionen zwischen den konkurrierenden Geschwistern nach der Geburt von Jungtieren zu erwarten. Es konnte in E1 und E2 aber für keine Dyade eine Reduktion der affiliativen Interaktionen verzeichnet werden. Aus ad libitum Beobachtungen wurde allerdings ersichtlich, dass alle Nachkommen Interesse an den Jungtieren hatten und versuchten, die Jungtiere zu übernehmen. Dissoziative Interaktionen als Folge der Konkurrenz um Jungtiere waren, von einer Ausnahme abgesehen, nicht zu beobachten. Nachdem das Alpha-Männchen die älteste Tochter D jedes Mal, wenn diese sich den Jungtieren annäherte, aggressiv abwehrte, begegneten auch alle übrigen Nachkommen ihrer Schwester dissoziativ, wenn sie den Jungtieren nahe kam. Es wäre möglich, dass das dissoziative Verhalten der Geschwister durch Konkurrenz um die Jungtierfürsorge motiviert war (Price 1991). Die höhere Wahrscheinlichkeit für Rauswürfe innerhalb der ersten zwanzig Tage nach Geburten lässt auf Konkurrenz um Helferpositionen in der Jungtierfürsorge schließen, da direkt nach der größten Rauswurfwahrscheinlichkeit das Tragen der Jungtiere für die Nachkommen relevant wird. Die Ankunft neuer Jungtiere 
stimuliert die Nachkommen demnach, Konkurrenten um Helferpositionen zu eliminieren. Der einzige Rauswurf, der direkt nach einer Geburt beobachtet werden konnte, ging jedoch nicht mit einer daraufhin gesteigerten Beteiligung an der Jungtierfürsorge einher. Es fiel soger auf, dass der Rauswerfer G nur wenig in der Nähe der Jungtiere gesichtet wurde. Inwiefern andere Hilfeleistungen (z.B. Verteigung des Streifgebietes) durch Konkurrenten geschmälert werden können, muss in dieser Studie offen bleiben, da Untersuchungen zur Besetzung von Helferpositionen und möglicherweise Rollen für den Rahmen dieser Studie zu umfangreich wären.

Falls Rauswürfe aufgrund von Konkurrenz um Sozialpartner zwischen Geschwistern entstehen, sollte sich die Häufigkeit und/oder Qualität der Interaktionen des Rauswerfers mit Sozialpartnern von der Zeit vor dem Rauswurf zu der Zeit nach dem Rauswurf verändern. Nach den Clique-Analysen und Vergleichen affiliativer Interaktionen ließen sich für die Rauswerfer E und G keine Hinweise für soziale Konkurrenz zu F bzw. E ermitteln. Da E und G während des ersten Beobachtungsjahres die häufigsten Grooming-Partner ihres Bruders C waren und G nach dem Rauswurf von E häufiger Grooming-Kontakt zu C hatte als alle übrigen männlichen Familienmitglieder, ist zu vermuten, dass G mit E um Kontakt zu dem ältesten Bruder C konkurrierte (siehe Tab. 7.1-4 im Anhang). Das zweite Beobachtungsjahr lieferte Hinweise für soziale Konkurrenz zwischen dem rausgeworfenen Tier I und seinem Bruder G, der ihn aus der Familie verjagt hatte. I war Mitglied aller Cliquen der jeweiligen Phasen unabhängig vom Geschlecht der übrigen Cliquenmitglieder, während $G$ nur an einer Grooming-Clique beteiligt war. I hatte zudem häufig Grooming-Interaktionen mit dem AlphaMännchen A. Nach dem Rauswurf von I konnten im vierten Experiment drei extreme Cliquen ermittelt werden, denen $\mathrm{G}$ angehörte, so dass dieser hiermit eine zentrale Grooming-Position innehatte (vgl. Tab. 3.18-19, S. 105). In zwei Cliquen von G war nun auch das AlphaMännchen A beteiligt. Es liegen damit Hinweise vor, dass G unter anderem mit I um Grooming-Interaktionen mit dem Alpha-Männchen konkurrierte.

Eine andere Erklärung für den Rauswurf von I durch G ergibt sich durch Beobachtungen von Ehardt und Bernstein (1992) an Macaca mulatta. Adulte Männchen zeigten bevorzugt gegenüber adulten oder adoleszenten Männchen Aggressionen, die mit Weibchen dissoziativ interagierten. Der adaptive Wert dieses Verhaltens wird in persönlichen Vorteilen für das intervenierende Männchen als auch in der Erhaltung der Gruppenstabilität vermutet. Da I vor seinem Rauswurf seiner Schwester D gegenüber stark dissoziatives Verhalten zeigte, muss auch hier eine Kausalität zwischen dem Rauswurf und den dissoziativen Interaktionen 
zwischen I und D als Ursache für den Rauswurf in Betracht gezogen werden.

Hinweise auf eine Konkurrenz um Sozialpartner zwischen den Schwestern D und H konnten aus den Beobachtungen zu affiliativem und Grooming-Verhalten im ersten Beobachtungsjahr nicht gefunden werden. In der Hüttenphase des zweiten Jahres (D1) war D in keine Clique involviert, während $\mathrm{H}$ mit $\mathrm{C}$, I und $\mathrm{G}$ eine 2-Clique bildete. D verjagte $\mathrm{H}$ am Ende dieser Phase aus der Familie und bildete daraufhin mit I und $\mathrm{M}$ eine 2-Clique. Diese Ergebnisse lassen sich als Hinweis verstehen, dass D ihre jüngere Schwester H aus der Familie verdrängte, um sozialen Anschluss an die Geschwister zu erreichen.

Eine alternative, möglicherweise validere Erklärung für den Rauswurf von $\mathrm{H}$ bietet allerdings die sexuelle Konkurrenz. Nach Garber (1994) und Goldizen et al. (1996) sichern weibliche Tamarin-Nachkommen ihre eigene Reproduktion, indem sie so lange in der Natalgruppe verweilen, bis die Mutter stirbt und sie die Position des reproduktiven Weibchens erben können. Strier (1999) weist jedoch darauf hin, dass nicht alle Töchter die mütterliche Position des dominanten reproduktiven Weibchens erben können. Halten sich mehr als eine Tochter innerhalb der Gruppe auf, die das Erbe antreten könnten, entsteht unter ihnen Konkurrenz um das Erbe. Der Rauswurf von $\mathrm{H}$ geschah spontan und unerwartet, nachdem $\mathrm{H}$ wegen einer vorangegangenen Operation geschwächt war. Dass D diese Situation ausgenutzt hatte, um die jüngere Schwester aus der Familie zu verdrängen, weist auf eine bereits bestehende oder zumindest zukünftige Konkurrenz zwischen den Töchtern hin (vgl. Snowdon und Pickhard 1999). Vermutlich wäre bei den Töchtern der Fokusfamilie spätestens im Falle des Todes der Mutter diese Konkurrenz im Verhalten deutlich geworden. Hubrecht (1989) fand, dass ovulierende Töchter gegenüber ihren jüngeren Schwestern besonders aggressives Verhalten zeigten. In 3.2.6.2. wurde angenommen, dass D möglicherweise als älteste Tochter ovuliert hatte. Ein Nachweis dafür hätte auf Zusammenhänge der Ovulationen mit der Aggression gegen $\mathrm{H}$ hingewiesen.

Der Rauswurf von M durch K kann ähnlich wie bei der eben beschriebenen Schwester-Dyade mit zukünftiger sexueller Konkurrenz interpretiert werden. Die Ergebnisse bieten keine Hinweise auf eine Konkurrenz um Sozialpartner. Der Rauswurf ereignete sich allerdings erst sechs Monate nach dem Ende des zweiten Beobachtungsjahres, so dass nicht ermittelt werden konnte, wie die Familie zu der Zeit strukturiert war. Es kann daher zu diesem Rauswurf keine weitere Eingrenzung möglicher Ursachen gemacht werden. 
Bei der Anwesenheit des Lockweibchens im ersten Experiment (C1) gab es zwischen allen Bruder-Dyaden seltener affiliative Kontakte als vor- und nachher, während sich die räumliche Assoziation aber nicht änderte. Der Rückgang der affiliativen Interaktionen weist darauf hin, dass die Brüder während der Präsentation des Lockweibchens ihre Beziehungen lockerten, anstelle sich enger aneinander zu binden, um beispielsweise gemeinsam abzuwandern. Da Konkurrenz in Krallenaffengruppen selten über dissoziative Interaktionen geäußert wird (Ferrari 1988b, Lazaro-Perea et al. 1997, Garber 1997), kann die Vermeidung affiliativer Interaktionen als Konkurrenz gedeutet werden - in diesem Falle als sexuelle Konkurrenz zwischen den Brüdern um eine potentielle Reproduktionspartnerin.

Ekman et al. (2002) erkennen in der Geschwister-Konkurrenz einen wesentlichen Bestandteil der Emigrations-Entscheidung. Dominante Geschwister könnten es sich leisten, auf den Moment zu warten, der die besten Möglichkeiten für eine Emigration bietet, da die Dominanz ihnen den Zugang zu Ressourcen im heimatlichen Territorium sichert. In ihrer Studie über den Sibirischen Jay (Perisoreus infaustus) mussten subordinierte Nachkommen immer zuerst gehen, während spätere Emigrationen ausschließlich bei dominanten Nachkommen beobachtet wurden. Die Dominanzverhältnisse zwischen den Rauswerfern und Rausgeworfenen der Familie J18 entsprechen nicht exakt diesem Modell, da E durch den jüngeren Bruder $\mathrm{G}$ rausgeworfen wurde, über den er im ersten Beobachtungsjahr dominant war. Dafür spricht allerdings die Beteiligung des Alpha-Männchens am Rauswurf, ohne dessen Hilfe G es möglicherweise nicht geschafft hätte, seinen älteren Bruder rauszuwerfen. Das Dominanzverhältnis von G und I konnte anhand der Hierarchie am Futterplatz nicht ermittelt werden. Der Seniorität zufolge war G über I dominant, ebenso wie über J. Letzteres wurde aus der Hierarchie am Futterplatz deutlich. D war über H dominant (ad libitum-Beobachtungen), während den Zwillingsschwestern K und M kein Rangverhältnis zugeordnet werden konnte. In den meisten Fällen wurden die Rauswürfe also von dominanteren gegenüber subordinierten Geschwistern durchgeführt, so dass das Modell von Ekman et al. (2002) mit Einschränkungen für die Familie J18 unterstützt werden kann. Da bei C. jacchus Zwillinge geboren werden und die Eltern sich an Rauswürfen beteiligen, kann das Modell jedoch nicht strikt angewendet werden und verdeutlicht die wesentlich komplexeren und individuengeprägten Sozialstrukturen von Primaten gegenüber Vögeln.

Überlegungen zu den Ursachen der Geschwister-Konkurrenz in einer Callithrix-Sozietät wurden in 1.3 dargestellt. Die Modelle von Creel (1990) und Lucas et al. (1996) weisen auf den Einfluss der Anzahl an Helfern hin, die mit der Gruppengröße in direktem Zusammen- 
hang steht. Mit zunehmender Helferanzahl kann die inklusive Fitness der nicht-reproduktiven Helfer negativ werden (siehe Abb. 3.162). Im Falle der Validität des Modells für C. jacchus kann angenommen werden, dass ab einer Gruppengröße von 11 die inklusive Fitness eines Helfers negativ wird und damit die Rauswurfwahrscheinlichkeit ansteigt. Die inklusive Fitness eines Helfers entspricht dem Anteil der Helfer in der Population $(p)$, subtrahiert von dem Verwandtschaftsgrad zu den reproduktiven Tieren $(r)$ multipliziert mit dem Effekt des Helfers auf den Aufzuchtserfolg der reproduktiven Tiere (b). Zur Berechnung der Formel $(r-p) \times b$ für die beobachtete $C$. jacchus-Familie waren die Variablen $r$ und $p$ zugänglich, wohingegen $b$ nicht ermittelt werden konnte, da nicht bekannt war, wie groß der Aufzuchtserfolg des reproduktiven Paares gewesen wäre, wenn keine Helfer anwesend gewesen wären.

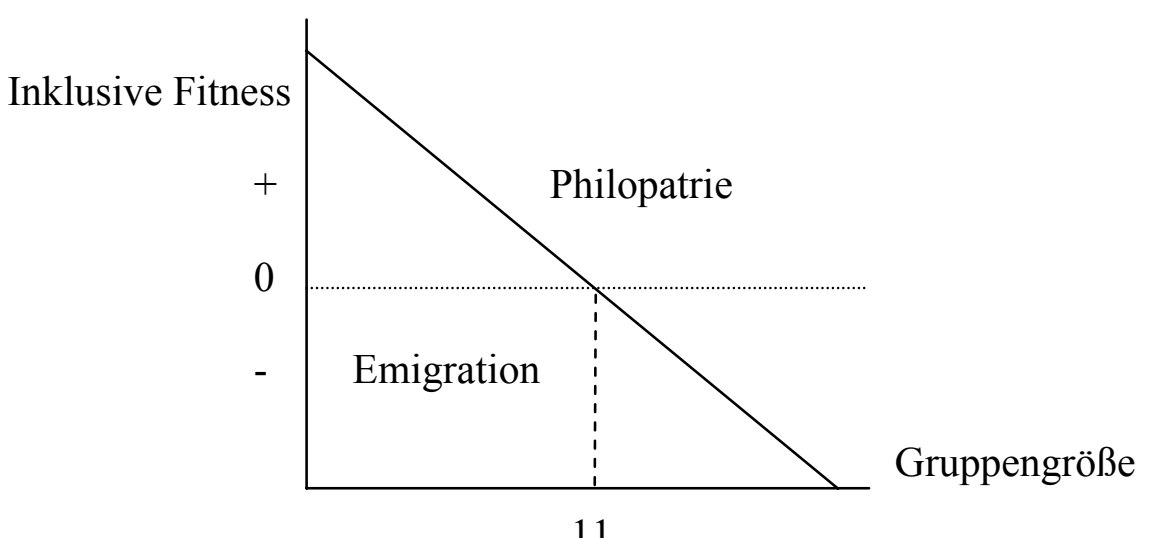

11

Abb. 3.162 Mit zunehmender Gruppengröße sinkt die inklusive Fitness der nicht-reproduktiven Helfer. Ab einem bestimmten Schwellenwert (kritische Gruppengröße) wird die inklusive Fitness negativ, so dass sich die philopatrische Lebensweise als nicht-reproduktiver Helfer nicht mehr lohnt und eine Emigration aus der Familie die inklusive Fitness steigern könnte. Die Ergebnisse liefern Hinweise darauf, dass ab einer Gruppengröße von 11 die inklusive Fitness negativ wird, so dass ab dieser Größe die meisten Rauswürfe geschehen.

Ein weiteres Problem für die Berechnung der inklusiven Fitness ist die Notwendigkeit zu beurteilen, ob das Verhalten eines Tieres als Hilfeleistung zu interpretieren ist bzw. zumindest der Jungtieraufzucht diente. Das Modell kann daher in dieser Studie noch nicht für C. jacchus angewendet werden. Anhand der Formel kann allerdings eingegrenzt werden, ab welcher Helferzahl bei einer gegebenen Gruppengröße die inklusive Fitness negativ würde. Bei einer Gruppengröße von 10 Tieren und einer Helferzahl von 4 (10 minus Alpha-Tiere, Juvenile und Infantes), wäre die inklusive Fitness eines Helfers positiv, vorausgesetzt der Effekt der Helfer auf den Aufzuchtserfolg $(b)$ ist auch positiv (Gleichung 1).

$(0,5-0,4) \times b=0,1 \times b$ 
Gibt es bei den gleichen Voraussetzungen einen Helfer mehr, so erreicht die inklusive Fitness den Nullwert (Gleichung 2).

$(0,5-0,5) \times b=0 \times b$

Gibt es bei einer Gruppengröße von 10 insgesamt 6 Helfer, beispielsweise weil alle Nachkommen außer den Jungtieren bereits mindestens subadult sind, so ergibt sich eine negative inklusive Fitness, vorausgesetzt der Effekt der Helfer auf den Aufzuchtserfolg $(b)$ ist positiv (Gleichung 3).

$(0,5-0,6) \times b=-0,1 \times b$

Bei einer Gruppengröße von 12 wäre bis zu einer Helferanzahl von 5 und bei einer Gruppengröße von 8 nur noch bei 3 Helfern die inklusive Fitness der Helfer positiv. Die Voraussetzung für Konkurrenz um Helferpositionen hängt damit eng mit der Gruppengröße und der Altersverteilung der Gruppenmitglieder zusammen, da sie bestimmt, welche Nachkommen theoretisch helfen könnten. Ob tatsächlich diejenigen Nachkommen, die zum qualifizierten Helfen bereits in der Lage wären, sich zur Hilfeleistung entscheiden, beeinflusst die Anzahl der Helfer in der Gruppe. Somit prägt die Entscheidung aller Nachkommen, Hilfe zu leisten oder nicht, die Entscheidung eines jeden Nachkommen über Philopatrie und Emigration. Es wurde damit deutlich, dass Entscheidungen nicht nur hierarchisch geordnet sind, sondern viele Entscheidungen auch wechselseitig voneinander abhängen, so dass von einem Entscheidungsnetzwerk gesprochen werden kann.

Die Studie liefert Hinweise, dass Hypothese 4, die besagt, dass Rauswürfe aufgrund von Geschwister-Konkurrenz entstehen, zurückgewiesen werden kann.

\subsubsection{Eltern-Nachkommen-Konflikt}

Die Ergebnisse über die Beteiligung der Eltern an Rauswürfen verdeutlichen, dass Geschwister-Konkurrenz überwiegend zwischen männlichen Nachkommen ausgeprägt ist, während der Eltern-Nachkommen-Konflikt vor allem zwischen weiblichen Gruppenmitgliedern stattfindet. Die Konsequenz aus dieser Schlussfolgerung zeigt, dass reproduktive Weibchen durch die Anwesenheit von potentiellen Rivalinnen mehr zu verlieren haben (vgl. Schaffner und French 1997) als reproduktive Männchen durch die Anwesenheit von potentiellen Rivalen. Es wird damit die Hypothese unterstützt, dass männliche Nachkommen als Helfer von den Eltern bevorzugt werden (Epple 1975a). Zusätzlich zur Annahme, dass der Aufzuchtserfolg durch Söhne mehr gesteigert wird als durch Töchter (König 1995), ist für das 
Alpha-Männchen die Kontrolle über das Alpha-Weibchen (mate-guarding) leichter als für das Alpha-Weibchen die Kontrolle über ovulierende Töchter (daughter-guarding). Ein reproduktives Weibchen ist meistens trächtig und zumindest zeitweilig mit dem Säugen und Tragen ihrer Jungtiere beschäftigt, so dass die Verhinderung sexueller Kontakte einer Tochter hohe Kosten verursacht. Die Kosten durch den Verlust einer helfenden Tochter (via Rauswurf) wären demnach geringer als die Kosten des daughter-guardings. Das KostenNutzen-Verhältnis, welches sich durch die Anwesenheit von helfenden Söhnen für das AlphaMännchen ergibt, ist nach der Annahme dieser Zusammenhänge günstiger. Nachweise dafür können in der vorliegenden Arbeit allerdings nicht erbracht werden, da weder Ovulationen bei Töchtern bestätigt werden konnten, noch ausgeschlossen werden konnte, dass einer der Söhne an der Vaterschaft der Jungtiere beteiligt war. In der C. jacchus-Kolonie des IfA wurden allerdings Ovulationen bei einigen Töchtern nachgewiesen (Rothe pers. Mtlg.). Zur Klärung der ultimaten Ursachen für das unterschiedliche Verhalten der Eltern sind in zukünftigen Untersuchungen derartige Nachweise deshalb dringend notwendig.

Ist das Verhalten der Eltern so ausgerichtet, dass die Nachkommen die bestmöglichen Reproduktionschancen erhalten und greift gleichzeitig ein ethologischer Polymorphismus, indem ein bestimmter Anteil an Nachkommen aus der Familie geworfen und zur eigenen Reproduktion gedrängt und ein anderer Teil der Nachkommen zur Mithilfe bei der Jungtieraufzucht in der Familie gehalten wird, so bleibt die Frage offen, nach welchen Kriterien Nachkommen für die eine oder andere ethologische Morphe ,ausgewählt“ wird. Da zwischen der Häufigkeit, mit der Eltern ihre Nachkommen aufsuchen, als auch ihren affiliativen Interaktionen gegenüber Nachkommen kein Zusammenhang mit gruppendienlichen Verhaltensweisen gefunden werden konnte, musste nach anderen Rauswurfkriterien seitens der Eltern gesucht werden. Der Rauswurf von E wird in dieser Studie auf sexuelle Konkurrenz zurückgeführt, so dass der Rauswurf als eine gesteigerte Form des mate-guarding aufgefasst werden kann. Da für den Rauswurf von $\mathrm{H}$ durch das Alpha-Weibchen keine Hinweise auf Konkurrenz um Sozialpartner etc. gefunden werden konnten, bleibt auch hier nur eine Erklärung auf der Ebene der sexuellen Konkurrenz. Indem die Eltern bedrohten Nachkommen (z.B. J) Schutz gewährten und anderen diesen Schutz verweigerten (z.B. E und M) nahmen sie Einfluss auf die Soziodynamik der Familie. Wie weitreichend dieser Einfluss generell ist, hängt von den situationsbegleitenden Umständen ab. Der Schutz, den die Eltern J gewährten, war, langfristig betrachtet, nicht ausreichend, um J vor dem Rauswurf von G zu bewahren. Ebenfalls war der Einsatz, den das Alpha-Männchen für den Schutz von H zeigte, 
wirkungslos. Die Alpha-Tiere können damit nicht als regulatorische „Überinstanz“ der Familie betrachtet werden, sondern eher als wichtige Teilnehmer an dynamischen Prozessen der Familie. Ein Hinweis auf die Bedeutung der Eltern für die Gruppenstabilität verdeutlicht sich in der hohen Rauswurfwahrscheinlichkeit während der ersten zwanzig Tage nach einer Geburt. Die Alpha-Tiere sind während dieser Phase verstärkt mit der Jungtierfürsorge beschäftigt und nur vermindert in der Lage, dissoziative Interaktionen zwischen Geschwistern zu kontrollieren, weshalb möglicherweise vorwiegend zu dieser Zeit Rauswürfe auftreten.

Da der Eltern-Nachkommen-Konflikt eine Ursache für Rauswürfe sein kann, muss Hypothese 6 zurückgewiesen werden. 


\section{SCHLUSSFOLGERUNG UND AUSBLICK}

Durch die Untersuchungen dieser Studie konnten einige Zusammenhänge zwischen den Faktoren, die auf die Entscheidung zwischen Philopatrie und Emigration bzw. Rauswurf einwirken, geklärt werden. Es wurde deutlich, dass keine der experimentellen Bedingungen zu einer freiwilligen Emigration eines nicht-reproduktiven Nachkommen führte und möglicherweise auch die familieninternen Voraussetzungen nicht erfüllt waren, dass ein Individuum bereit war, seine Taktik zur Optimierung seiner inklusiven Fitness durch eigene Reproduktion von sich aus zu ändern.

Mit der Analyse der räumlichen Assoziation der Nachkommen zur Familie sollten Emigrationsneigungen ermittelt werden, welche die Bereitschaft zur Abwanderung von der Familie anzeigen sollten. Da die adulten Nachkommen dauerhaft peripher waren und sich auch während der Anwesenheit von Locktieren keine stärkere räumliche Isolation von der Familie abzeichnete, gibt es keine Hinweise auf die Bereitschaft zur freiwilligen Emigration bestimmter Nachkommen durch Peripheralisationen. Für einige männliche Tiere, die von Familienmitgliedern rausgeworfen wurden, konnten vermehrt räumliche Distanzen zur Familie nachgewiesen werden. Es wurde allerdings auch deutlich, dass Peripheralisationen von der Familie nicht als alleinige Indikatoren für bevorstehende Rauswürfe bewertet werden können, und nicht allen Rauswürfen Peripheralisationen vorausgehen. Erstens gab es Hinweise auf einen Zusammenhang zwischen der Dauer einer Peripheralisation und der Voraussagbarkeit von Rauswürfen, da Nachkommen mit dauerhaft geringen Assoziationskoeffizienten nicht rausgeworfen wurden, währenddessen ein subadulter männlicher Nachkomme kurzfristig direkt vor seinem Rauswurf peripher wurde. Zweitens ging mit den Peripheralisationen vor Rauswürfen eine Verringerung der Nahbereichshäufigkeiten und der taktilen Interaktion mit Familienmitgliedern einher.

Nachkommen dieser Sozietät verließen das Natalgebiet also nur aufgrund von dissoziativen Aktionen anderer Familienmitglieder. Die Wahrscheinlichkeit für Rauswürfe ist bei einer Gruppengröße von 11, 13 und 17 Gruppenmitgliedern, innerhalb der ersten 20 Tage nach Geburten und für männliche Nachkommen im Alter von 15-25 Monaten und für Weibchen im Alter von 15-30 Monaten am größten. Unabhängig ist die Wahrscheinlichkeit für einen Rauswurf dagegen von der Anwesenheit potentieller Reproduktionspartner, vom Geschlecht des betroffenen Tieres, von der Beteiligung an der Jungtierfürsorge, der Ressourcensicherung und Raubfeindvermeidung, der nicht-taktilen Interaktionshäufigkeit mit fremden Artgenossen und der Häufigkeit taktiler Interaktion mit Familienmitgliedern der einzelnen Individuen. Es 
gibt Hinweise, dass sich bei C. jacchus individuelle Charakteristika, z.B. Ängstlichkeit ${ }^{20}$ und die Spielhäufigkeit mit Jungtieren auf die Rauswurfwahrscheinlichkeit für ein Tier auswirken. Unsicher bleibt die Auswirkung des Östrus des Alpha-Weibchens auf die Wahrscheinlichkeit für einen Rauswurf, da Ovulationen nicht eindeutig nachgewiesen werden konnten, als auch die Auswirkung der Jahreszeit auf Rauswurfwahrscheinlichkeiten, da die Stichprobengröße hierfür zu klein war.

Die räumliche Assoziation der Geschwister-Dyaden war im zweiten Beobachtungsjahr von den Experimenten abhängig, während sie im ersten Jahr im Verlauf der Experimente stabil blieb. Die affiliativen Interaktionen waren während beider Beobachtungsjahre bei den Schwester-Dyaden, den Bruder-Schwester-Dyaden und den meisten Bruder-Dyaden abhängig von den Experimenten und zeigten damit eine gewisse Instabilität. Es gibt somit Hinweise auf Auswirkungen fremder Artgenossen auf die freundschaftlichen Interaktionen zwischen den Geschwistern. Da im zweiten Beobachtungsjahr die Ereignisse Geburt und Postpartum-Östrus des Alpha-Weibchens mit der Präsentation der Kleinfamilie bzw. zweier Lockmännchen zusammenfielen und ein subadulter Bruder rausgeworfen wurde, lassen sich für das zweite Beobachtungsjahr keine zuverlässigen Rückschlüsse auf die Auslöser des Rauswurfs ziehen. Die Untersuchungen über Rauswurfwahrscheinlichkeiten unterstützen allerdings mehr die Annahme, dass die familieninternen Faktoren (Geburt und/oder Östrus) und damit einhergehende dissoziative Aktionen zwischen den Geschwistern zum Rauswurf führten. Möglicherweise war auch die Kombination der verschiedenen Umstände die proximate Ursache dieses speziellen Rauswurfs.

Rauswürfe von Schwestern ereigneten sich infolge kurzfristiger Abwesenheit einzelner Tiere von der Familie (kurze operative Eingriffe), wobei nicht ausgeschlossen werden kann, dass die später rausgeworfenen Tiere von der Narkose körperlich geschwächt waren. Dieser Umstand macht die Unabhängigkeit der Rauswürfe von und durch Weibchen von familienexternen Faktoren, wie z.B. der Präsenz von Locktieren, deutlich und unterstützt die Erbschafts-Hypothese, als deren Nebeneffekt die Konkurrenz zwischen Schwestern beschrieben wird.

Da dissoziatives Verhalten bei Callitrichiden vergleichsweise selten beobachtet wird, musste bei der Analyse der ultimaten Gründe für die Geschwister-Konkurrenz auf andere Verhaltens-

\footnotetext{
${ }^{20}$ gemeint ist Verhalten, welches am ehesten mit Ängstlichkeit beschrieben werden kann (Syndrom aus Büschelanlegen, Fellsträuben, Fliehen, „Angstgesicht“)
} 
einheiten zurückgegriffen werden. Die Ergebnisse über die Familienstruktur und den Einfluss der Locktiere auf die Beziehungen der Geschwister weisen auf Konkurrenz zwischen den nicht-reproduktiven Nachkommen hin, z.B. auf Konkurrenz um Sozialpartner (G und E um den Zugang zu C, G und I um den Zugang zu A und einer zentralen Position im GroomingNetzwerk) und auf Konkurrenz um potentielle Reproduktionspartner (Rückgang affiliativer Interaktionen zwischen den Brüdern bei der Präsentation der Lockweibchen). Die Erklärungsmodelle greifen jedoch nur, solange davon ausgegangen werden kann, dass das jeweilige Verhalten richtig interpretiert wurde, also dass z.B. der Rückgang der affiliativen Interaktionen zwischen den Brüdern tatsächlich ein Ausdruck von Konkurrenz war und keine anderen Ursachen hatte.

Die Ergebnisse dieser Studie liefern Hinweise, dass der Eltern-Nachkommen-Konflikt bei $C$. jacchus in sexueller Konkurrenz begründet liegt, wobei sich der Konflikt häufiger zwischen den Weibchen äußert. Interventionen bei dissoziativen Interaktionen zwischen den Geschwistern durch die Eltern werden vor allem auf die Erhaltung der Gruppenstabilität zurückgeführt.

Die Geschwister und die Eltern haben somit Einfluss auf die dynamischen Prozesse und möglicherweise familienregulatorische Funktionen. Inwiefern Individuen durch ihr Verhalten die Wahrscheinlichkeit für ihren eigenen Rauswurf beeinflussen, konnte nur bedingt festgestellt werden (siehe Spielhäufigkeit mit Jungtieren und Ängstlichkeit vor aggressiven Familienmitgliedern).

Da die Familienmitglieder eng miteinander verwandt sind (mit Ausnahme der Eltern) und Individuen von der Reproduktion ihrer Verwandten profitieren, wurde erwartet, dass Rauswürfe nur dann geschehen, wenn das rausgeworfene Tier gute Überlebens- und Reproduktionschancen vorfindet. Haben die Rausgeworfenen außerhalb der Familie gute Reproduktionschancen, so bleibt fraglich, warum nicht die Rauswerfer selber abgewandert sind, um diese Chancen zu nutzen (vorausgesetzt die Rauswerfer waren nicht-reproduktive Nachkommen). Plausibler erscheint eine Erklärung in Anlehnung an das Modell von Brown und Brown (1984), in dem es indifferent ist, welches Tier von der Familie ausgestoßen wird, da letzten Endes nur das Verhältnis von Bleibenden und Emigranten (Rausgeworfenen) für die Selektion entscheidend ist. Demnach hätten sich die Gene jener Individuen evolutiv durchgesetzt, deren Nachkommen in einem bestimmten Verhältnis philopatrisch waren bzw. emigrierten. Erreicht werden konnte dieses Verhältnis möglicherweise, indem die bevorzugte Strategie der Nachkommen die Philopatrie war und in Folge der geschwisterlichen oder 
Eltern-Nachkommen-Konkurrenz Gruppenmitglieder ausgestoßen wurden. Das elterliche Streifgebiet konnte so von einer Generation zur nächsten vererbt und die genetische Vermischung der Population durch Migrationen gewährleistet werden. Wie groß der Anteil der bleibenden bzw. emigrierenden Nachkommen sein muss, damit das Verhältnis adaptiv ist, hängt möglicherweise von diversen Umwelt- und Sozialfaktoren ab, die in ihrer Gesamtheit noch nicht abgeschätzt werden können.

Die Ergebnisse und Schlussfolgerungen der vorliegenden Arbeit ergaben folgende Vorschläge für weitere Untersuchungen zur Gruppendynamik bei C. jacchus:

- Untersuchung der Mortalitätsrisiken in verschiedenen Habitaten und dem Reproduktionserfolg von Emigranten im Freiland, z.B. durch den Einsatz von Radiotelemetrie.

- Körpertemperaturmessungen mit weiterentwickelten Geräten, kürzeren Messintervallen und Implantationen der Temperaturbuttons in jüngere Tiere (ca. 12 Monate alt), so dass der Zeitpunkt der Entscheidung über eine Emigration oder einen Rauswurf anhand physiologischer Parameter mitverfolgt werden kann.

- Untersuchung des Serotonin-Transportergens bei C. jacchus auf mögliche Zusammenhänge zwischen der Kettenlänge des Gens und der Emigrationsentscheidung.

- Langzeitforschungsprogramme zur Berechnung der inklusiven Fitness nicht-reproduktiver Helfer bei verschiedenen Gruppengrößen, Mortalitätsrisiken, Altersklassen, Geschlechterverhältnissen etc. Dazu zählt unter anderem die Entwicklung zuverlässiger Vaterschaftsanalysen bei C. jacchus.

- Experimente mit Lockmännchen zur Emigrationsbereitschaft der ältesten Töchter bei $C$. jacchus in Abhängigkeit von Ovulationszyklen.

- Untersuchung der Verfügbarkeit von Helferpositionen bzw. des Vorkommens von „Rollen“, inklusive der Fragen, ob die Anzahl der Rollen in einer Gruppe begrenzt ist, Rollen besetzt werden können und sie so zu einer begrenzten Ressource werden können.

Es wäre wünschenswert, wenn in zukünftigen Forschungsprojekten einigen der offengebliebenen oder aufgeworfenen Fragen dieser Studie nachgegangen würde, um die Lücken im Verständnis über die dynamischen Prozesse von Callithrix jacchus-Familien zu schließen. 


\section{ZUSAMMENFASSUNG}

In einer zweieinhalbjährigen Beobachtungsperiode wurde analysiert, welche Mechanismen zu Emigrationen und Rauswürfen nicht-reproduktiver Nachkommen einer großen semifreilebenden Weißbüschelaffenfamilie (Callithrix jacchus) führen. Dazu wurden familieninterne und familienexterne Faktoren auf Auswirkungen auf die Entscheidung über Philopatrie und Emigration überprüft. Emigrationsneigungen sollten anhand von Peripheralisationen deutlich werden.

Die Untersuchung wurde in der Ethologischen Station Sennickerode des Instituts für Zoologie und Anthropologie der Universität Göttingen durchgeführt. Die Fokusfamilie bestand während des gesamten Untersuchungszeitraumes aus 7-11 Mitgliedern und lebte seit 1995 in einem ca. 1,5 ha großen Streifgebiet in einem ca. 6,5 ha großen Semifreigelände und war allen Wetterbedingungen, als auch Prädationsrisiken durch Beutegreifer (insbesondere Greifvögel) ausgesetzt. In beheizten Blockhütten, Schlafkästen und einem sich über weite Teile des Streifgebietes erstreckenden Baum- und Strauchbestand konnte die Familie jederzeit Schutz finden.

Mit instantaneous sampling wurden das Verhalten, ggf. der Rezipient des Verhaltens, sowie der Nahbereich zu anderen Familienmitgliedern notiert. Als Ergänzung wurden ad libitum besondere und seltene Ereignisse festgehalten. Mit einer Transponderanlage wurden die Aufenthaltsorte der Familienmitglieder im Streifgebiet ermittelt. Nach einer Kontrollphase (B1) ohne und einer Kontrollphase (B2) mit Nachbarfamilie wurden der Fokusfamilie in darauf folgenden Experimenten (C1, C2, E1, E2, E3, E4) verschiedene Locktiere in einem Streifgebiet präsentiert, welches durch einen $30 \mathrm{~m}$ breiten Grasgürtel von dem Gebiet der Fokusfamilie getrennt war. Durch die Anwesenheit der Locktiere sollten die nichtreproduktiven Nachkommen zu einer Peripheralisation und/oder Emigration aus dem heimatlichen Streifgebiet provoziert werden. Die Erstellung von Zeitbudgets war die Grundlage zur Beantwortung verschiedener Hypothesen.

Während der gesamten Beobachtungszeit emigrierte kein Nachkomme freiwillig aus der Natalfamilie. Es kam allerdings zu acht Rauswürfen durch meist gleichgeschlechtliche Geschwister. Die Alpha-Tiere beteiligten sich an jeweils einem Rauswurf eines gleichgeschlechtlichen Nachkommen, was nach Prüfung der Gesamtergebnisse als Folgen sexueller Konkurrenz interpretiert wurde. 
Es konnten anhand der Daten der C. jacchus-Kolonie des Instituts für Anthropologie aus dem Zeitraum von 1972-2003 Wahrscheinlichkeiten für Rauswürfe in Abhängigkeit von verschiedenen Faktoren, wie Gruppengröße, Alter der Jungtiere, Geschlecht und Alter der rausgeworfenen Tiere berechnet werden, anhand derer die Ergebnisse der Studie bewertet werden konnten. Ebenfalls wurden Rauswurfwahrscheinlichkeiten für individuell gezeigte Verhaltenseinheiten oder - bereiche ermittelt.

Um familiendynamische Prozesse vor ihrer Manifestation im Verhalten der Tiere zu ermitteln, wurden die Körpertemperaturen einiger Fokustiere mit implantierten Temperaturbuttons gemessen. Aufgrund technischer und methodischer Probleme konnten keine stressbedingten Körpertemperaturerhöhungen bei den Fokustieren dokumentiert werden.

Bei allen adulten Nachkommen führte die Anwesenheit bestimmter Locktiere zur nichttaktilen Interaktion mit fremden Artgenossen. Untersuchungen zur Familienstruktur ergaben für männliche Nachkommen Hinweise auf Geschwister-Konkurrenz um Sozialpartner. Durch die experimentelle Präsentation von Lockweibchen konnte bei den männlichen Nachkommen ein Rückgang der affiliativen Interaktionen festgestellt werden, was als sexuelle Konkurrenz interpretiert werden kann. Die räumliche Assoziation blieb unbeeinflusst. Bei der Präsentation von zwei Lockmännchen verringerten sich die affiliativen Interaktionen zwischen den Schwestern und die räumliche Assoziation zwischen Bruder- und Bruder-Schwester-Dyaden. Die affiliativen Interaktionen zwischen Brüdern und der ältesten Schwester waren bei der Anwesenheit zweier Lockmännchen vermindert. Da während dieser Phase der Rauswurf eines Familienmitgliedes geschah und Jungtiere geboren worden waren, sind die Ergebnisse des zweiten Beobachtungsjahres jedoch nicht eindeutig interpretierbar.

Eine lockere räumliche Assoziation zur Familie war durchgängig bei adulten Nachkommen beiderlei Geschlechts zu verzeichnen, ohne dass es zu freiwilligen Emigrationen kam, so dass diese Peripheralisationen nicht mit einer Emigrationsneigung in Zusammenhang gebracht werden konnten. Plötzliche Lockerung der räumlichen Assoziation eines Tieres zur Familie, begleitet von geringeren Nahbereichshäufigkeiten und verringerten affiliativen Interaktionen konnten als Indikator für bevorstehende Rauswürfe gewertet werden.

Es wurde deutlich, dass die Geschwister und die Eltern an den dynamischen Prozessen der Familie beteiligt sind, wobei noch nicht vollständig erfasst werden konnte, inwiefern Individuen ihren eigenen Ausschluss aus der Familie durch ihr Verhalten fördern oder ihm entgegen wirken können. 


\section{ANHANG}

\subsection{Tier-Identifikation}

Abb. 6.1 Identifikation der Mitglieder von Familie J 18

\begin{tabular}{|c|c|c|c|c|}
\hline Tier $-\mathrm{Nr}$. & Name & Geschlecht & Fellschnitt & Chip-Code \\
\hline 877 & $\mathrm{~A}$ & $\hat{0}$ & - & 00-01D2-496E \\
\hline 850 & $\mathrm{~B}$ & q & - & $00-01 D 2-3675$ \\
\hline 1039 & $\mathrm{C}$ & $\widehat{0}$ & Doppel-Mitte & 00-012A-28AA \\
\hline 1115 & $\mathrm{D}$ & q & Mitte-Spitze & 00-01C6-6386 \\
\hline 1133 & $\mathrm{E}$ & $\pi$ & Wurzel-Spitze & 00-01C6-6DE9 \\
\hline 1150 & $\mathrm{~F}$ & $0^{\lambda}$ & Spitze & 00-01C6-0EC1 \\
\hline 1166 & G & $\hat{O}$ & Mitte & 00-01C6-76FA \\
\hline 1167 & $\mathrm{H}$ & q & Doppel-Spitze & 00-01C6-07A3 \\
\hline 1172 & I & 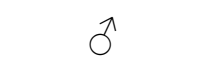 & Wurzel & 00-01C6-7636 \\
\hline 1173 & $\mathrm{~J}$ & $\hat{0}$ & Spitze & 00-01C6-7679 \\
\hline 1186 & $\mathrm{~K}$ & q & Wurzel-Spitze & 00-01C6-6Е9C \\
\hline 1187 & M & q & Wurzel-Mitte & 00-01C6-638D \\
\hline 1201 & $\mathrm{P}$ & $\hat{0}$ & Spitze & \\
\hline 1202 & $\mathrm{R}$ & $\lambda$ & Doppelspitze & \\
\hline 1218 & $\mathrm{~S}$ & $\hat{\sigma}$ & Wurzel-Mitte & \\
\hline 1219 & $\mathrm{~T}$ & 우 & Wurzel & \\
\hline
\end{tabular}

\subsection{Kürzellisten}

Abkürzung: Ind.=Individuum (Subjekt); FM=Familienmitglied

\begin{tabular}{lll}
\hline Verhaltenseinheit mit Kürzel & Beschreibung des Verhaltens & Verhaltensbereich \\
\hline aa - Aufforderung zum Grooming & nach Rothe (1979) & TIF \\
abe - Jungtierabstreifen, erfolgreich & nach Rothe (1979) & TIF \\
abv - Jungtierabstreifen, Versuch & nach Rothe (1979) & TIF \\
ae - Äugen & nach Rothe (1979) & NTF \\
ag - Angriff & nach Rothe (1979) & TIF \\
an - Annähern & Ind. bewegt sich auf ein FM zu & NTF \\
aw - Ausweichen & nach Rothe (1979) & NTF \\
ba - Aufblicken & Ind. blickt vorher nach untern und & KL \\
bb - beobachtet Beobachterin & dann plötzlich auf &
\end{tabular}




\begin{tabular}{|c|c|c|}
\hline be - Beobachten & $\begin{array}{l}\text { Ind. sitzt, liegt oder steht und } \\
\text { beobachtet ein Objekt oder FM }\end{array}$ & $\mathrm{RR}$ \\
\hline bh - Büschelheben & nach Rothe (1979) & NTF \\
\hline bg - Benagen (Gegenstände) & $\begin{array}{l}\text { Ind. nagt an einem Gegenstand (z.B. } \\
\text { Ast oder Grashalm) }\end{array}$ & $\mathrm{RR}$ \\
\hline bi - Blicken, Anblicken & Relativ kurzes Anblicken eines FM & NTF \\
\hline bk - Büschelklappen & nach Rothe (1979) & NTF \\
\hline bn - Büschelanlegen & nach Rothe (1979) & NTF \\
\hline bm - Bißmarkeanlegen & nach Rothe (1979) & $\mathrm{RR}$ \\
\hline bs - Beschnuppern & nach Rothe (1979) & TIF \\
\hline bss - beißen & nach Rothe (1979) & TIF \\
\hline bst - Büschelstellen & nach Rothe (1979) & NTF \\
\hline bw - Blickabwenden & nach Rothe (1979) & NTF \\
\hline ch - chatter & $\begin{array}{l}\text { Keckerlaute nach Winter \& Rothe } \\
\text { (1979) }\end{array}$ & NTF \\
\hline df - Doppelphi & Phiilaute nach Winter \& Rothe (1979) & NTA \\
\hline ec - Ecklaut & $\begin{array}{l}\text { Keckerlaute nach Winter \& Rothe } \\
\text { (1979) }\end{array}$ & NTF \\
\hline ex - Erkunden (explore) & $\begin{array}{l}\text { Manipulieren, Beschnuppern und/oder } \\
\text { Beobachten von Gegenständen }\end{array}$ & $\mathrm{RR}$ \\
\hline $\mathrm{fb}-$ Futterbetteln & nach Rothe (1979) & $\mathrm{RR}$ \\
\hline fc - Defäkieren & nach Rothe (1979) & SK \\
\hline fg - Folgen & nach Rothe (1979) & TIF \\
\hline fi - phi (laut) & Phiilaute nach Winter \& Rothe (1979) & NTA \\
\hline fil - phi (leise) & Phiilaute nach Winter \& Rothe (1979) & NTF \\
\hline $\mathrm{fl}$ - Fliehen & nach Rothe (1979) & NTF \\
\hline $\mathrm{fr}-$ fressen & nach Rothe (1979) & SK \\
\hline fs - Futterstehlen & nach Rothe (1979) & $\mathrm{RR}$ \\
\hline $\mathrm{ft}$ - Futtertransport & nach Rothe (1979) & $\mathrm{RR}$ \\
\hline fut - Futtertriller & nach Rothe (1979) & TIF \\
\hline$f x-$ Fixieren & nach Rothe (1979) & $\mathrm{RR}$ \\
\hline ga - Autogrooming & nach Rothe (1979) & SK \\
\hline gr - Grooming & nach Rothe (1979) & TIF \\
\hline $\mathrm{gk}$ - Genitalkontrolle & nach Rothe (1979) & TIF \\
\hline gn - Gähnen & nach Rothe (1979) & SK \\
\hline gp - Genitalpräsentieren & nach Rothe (1979) & NTF \\
\hline ha - Hängen & nach Rothe (1979) & KL \\
\hline ht - Hüten & nach Rothe (1979) & TIF \\
\hline jae - Jungtieraufnahme, erfolgreich & nach Rothe (1979) & TIF \\
\hline jaf - Jungtieraufsteigen & nach Rothe (1979) & TIF \\
\hline jas - Jungtierabsteigen & nach Rothe (1979) & TIF \\
\hline jav - Jungtieraufnahme, Versuch & nach Rothe (1979) & TIF \\
\hline jue - Jungtierübernahme, erfolgreich & nach Rothe (1979) & TIF \\
\hline
\end{tabular}




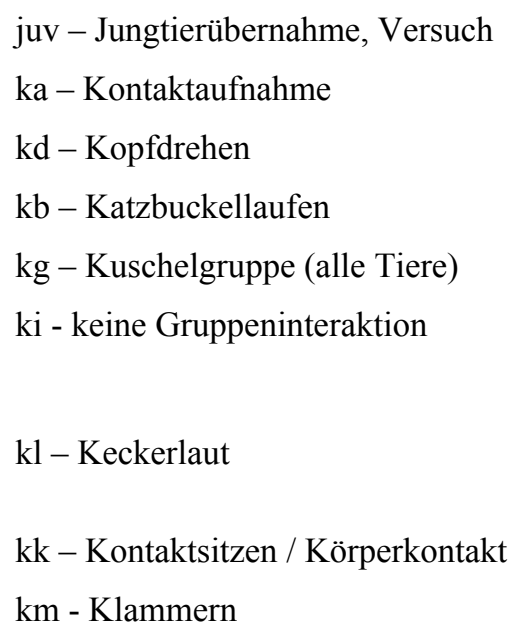

nach Rothe (1979)

TIF

nach Rothe (1979) TIF

nach Rothe (1979) KL

nach Rothe (1979) NTF

nach Rothe (1979) TIF

Aufenthaltsort des Ind. ist bekannt und KL es interagiert nicht mit FM; es kann jedoch nicht beobachtet werden

Keckerlaute nach Winter \& Rothe NTF (1979)

nach Rothe (1979)

TIF

Mit allen vier Extremitäten am Sub- $\quad$ KL strat festgekrallt hängt das Ind. senkrecht mit dem Kopf nach oben am Substrat nach Rothe (1979) TIF nach Rothe (1979) SK nach Rothe (1979) NTF nach Rothe (1979) TIF

Ind. holt mit Arm Richtung FM aus NTF wie bei Schlagen, berührt FM jedoch nicht

Ind. leckt FM oder an Gegenstand TIF

Ind. liegt allein auf dem Bauch oder SK auf der Seite

nach Rothe (1979) KL

Ind. fährt mit der Zunge über die $\quad$ SK Lippen; meist nach dem Fressen nach Rothe (1979) RR nach Rothe (1979) RR nach Rothe (1979) RR trillerartiger Laut, nur von Weibchen NTF nach Rothe (1979) NTF nach Rothe (1979) RR nach Rothe (1979) NTF Keckerlaute nach Winter \& Rothe NTF nach Rothe (1979) KL nach Rothe (1979) NTF nach Rothe (1979) NTF nach Rothe (1979) TIF nach Rothe (1979) NTF Keckerlaute nach Winter \& Rothe NTF nach Rothe (1979) SK nach Rothe (1979) SK nach Rothe (1979) NTF 


\begin{tabular}{|c|c|c|}
\hline sc-Scannen & nach Rothe (1979) & $\mathrm{RR}$ \\
\hline sg - Schlagen & nach Rothe (1979) & TIF \\
\hline sh - Schlafen & nach Rothe (1979) & SK \\
\hline si-Schleichen & nach Rothe (1979) & KL \\
\hline sl - sit and look & $\begin{array}{l}\text { Ind. sitzt ohne Körperkontakt und } \\
\text { blickt kein Objekt oder FM direkt an }\end{array}$ & KL \\
\hline sp - Schupsen & $\begin{array}{l}\text { Ind. schubst FM mit den Händen von } \\
\text { sich weg; meist auf Sitzbrett }\end{array}$ & TIF \\
\hline st - sich schütteln & nach Rothe (1979) & SK \\
\hline td - Drillingstragen & nach Rothe (1979) & TIF \\
\hline te - Einlingstragen & nach Rothe (1979) & TIF \\
\hline tl - Triller & nach Winter \& Rothe (1979) & NTF \\
\hline $\operatorname{tr}-$ Trinken & nach Rothe (1979) & SK \\
\hline ts - Tsiklaut & nach Rothe (1979) & NTF \\
\hline $\mathrm{tt}$ - Tragtierwechsel & nach Rothe (1979) & TIF \\
\hline tw - Twitter & nach Rothe (1979) & NTF \\
\hline tz - Zwillingstragen & nach Rothe (1979) & TIF \\
\hline ub - Umherblicken & $\begin{array}{l}\text { Ind. dreht Kopf gleichmäßig hin und } \\
\text { her, schneller als beim Scannen }\end{array}$ & $\mathrm{RR}$ \\
\hline uk - Unterkriechen & nach Rothe (1979) & TIF \\
\hline um - Umarmen & nach Rothe (1979) & TIF \\
\hline ur - Urinieren & nach Rothe (1979) & SK \\
\hline us - über die Schulter blicken & nach Rothe (1979) & NTF \\
\hline uw - umwenden & Ind. dreht sich am Sitzplatz um & $\mathrm{KL}$ \\
\hline ve - Verstecken & $\begin{array}{l}\text { Verbergen vor anderen FM, Greif- } \\
\text { vögeln oder in anderen erschreckenden } \\
\text { Situationen }\end{array}$ & RR \\
\hline vh - Verharren & $\begin{array}{l}\text { Ind. verhält in einer Bewegung inne } \\
\text { und erstarrt einen Moment }\end{array}$ & $\mathrm{KL}$ \\
\hline wd - wegdrücken & $\begin{array}{l}\text { Ind. drängt FM mit eigenem Gewicht } \\
\text { von sich weg am Sitzbrett }\end{array}$ & TIF \\
\hline wg - Getragenwerden & nach Rothe (1979) & TIF \\
\hline wh - Whisper & Leiser, kaum wahrnehmbarer Pfeiflaut & NTF \\
\hline wj - Wegjagen & nach Rothe (1979) & NTF \\
\hline wl - Warnlaut & Pfifflaute nach Winter \& Rothe (1979) & RR \\
\hline wn - Weggehen & nach Rothe (1979) & NTF \\
\hline zie - Zitzenkontakt, ein Jungtier & nach Rothe (1979) & TIF \\
\hline ziz - Zitzenkontakt, zwei Jungtiere & nach Rothe (1979) & TIF \\
\hline
\end{tabular}




\subsection{Protokolle der Rauswürfe}

Rauswürfe, die ausführlich beobachtet werden konnten, sind im Folgenden als Protokollauszüge dargestellt.

\subsubsection{Rauswurf von $\mathbf{F}$}

Datum: 06.07.00

Drei Wochen vor dem Rauswurf von F wurde dieser vermehrt von seinem älteren Bruder E und von dem Alpha-Weibchen B gejagt. In der Familie herrschte insgesamt Anspannung. Am 15.06.2000 sonderte F sich von der Familie kurzzeitig ab. Dann nahmen Aggressionen zwischen D und diversen Familienmitgliedern zu und F trug z.T. die Jungtiere. Die AlphaTiere beschützten F in Auseinandersetzungen. Am Morgen des 06.07.2000 wurde F von E attackiert und sprang ins Gras. Er wanderte zum südlich gelegenen Areal ab und wurde vom Pflegepersonal eingefangen.

\subsubsection{Rauswurf von $\mathrm{E}$}

Datum: 27.03.01

E saß im Außenkäfig und lief Gefahr zu erfrieren. Er wurde in die Schutzhütte gebracht. 9:30 Uhr: E saß in der Kuschelgruppe zwischen G und D.

9:50 Uhr: A und B markierten und liefen in der Hütte umher; A ging auf E zu und begann, ihn anzukeckern, G genitalpräsentierte; A, B, G mobbten E und jagten ihn; E versteckte sich hinter der Heizung; G griff E an, worauf E aufschrie, die Familie jagte E, bis er sich wieder hinter der Heizung versteckte. A, B, G und H mobbten, E röchelte und schrie tief und heiser; G keckerte am stärksten; A, G, B markierten, G griff E wieder an, H keckerte dazu. E, D und $\mathrm{C}$ hielten sich heraus, I und J liefen mit der Familie, formierten sich mit, griffen aber nicht an, keckerten auch nicht; dann genitalpräsentierte $\mathrm{C}$ gegen $\mathrm{E}, \mathrm{D}$ saß immer noch abseits; $\mathrm{H}$ keckerte viel, G immer an erster Stelle und griff als einziger an. G, H, A, B katzbuckelten durch die Hütte (in dieser Reihenfolge).

10:10A, B, G, J und die Babies sammelten sich im Schlafkasten; C kuschelte mit D im anderen Schlafkasten; H und I waren je allein in der Hütte, I lief zur Familie, G guckte nach E und markierte. Dann war alles ruhig.(G blickte immer wieder zu E und markierte danach, G piepste hoch in regelmäßigen Abständen; auch schon, als er in der Kuschelgruppe neben E $\mathrm{sa})$. 
10:28 G sprang zur Familie in den Schlafkasten.

10:35Bei dem Versuch, E einzufangen, geriet Familie in Panik; E setzte sich zu C und D; Familie hielt still, bewegte sich nur im Zeitlupentempo; E schlief fast ein, C und D tolerierten ihn, blickt ihn an, E groomte sein Fell und war aufgeplustert.

10:50 A ging wieder auf E zu, stand vor ihm, E quärrte, A blickte sich um, worauf G neben ihn sprang und zu E genitalpräsentierte, E floh in die Heizungsecke; die Familie inklusive C katzbuckelte viel, Verfolgung von E durch die ganze Familie mit Ausnahme von C und D. G griff E erneut als Einziger an; E wurde daraufhin aus der Familie herausgefangen.

\subsubsection{Rauswurf von $H$}

Datum: 21.05.01

Dem Rauswurf ging die Implantation von fünf Tieren der Affenfamilie, inklusive D und H, voraus. H war zuletzt operiert worden und hatte eine Anästhesie-Verlängerung bekommen, so dass sie zum Zeitpunkt des Einsetzens in den Hütten-Käfig-Komplex physisch noch relativ schwach war und Lokomotionsprobleme hatte. Sofort begann D, ihre jüngere Schwester H zu attackieren. Sie jagte H durch den gesamten Hütten-Käfig-Komplex und biss sie bei kurzen Angriffen. A, der Vater, versuchte D von den Angriffen abzuhalten und stellte sich ihr mehrfach in den Weg. Auch wenn D und H in unterschiedlichen Käfigen saßen, setzte er sich in den Durchgang zum angrenzenden Käfig, um ihn zu blockieren. B schloss sich D bei den Angriffen auf die jüngste Tochter an und keckerte laut, wenn sie $\mathrm{H}$ anblickte. A versuchte, $\mathrm{H}$ vor beiden Angreiferrinnen zu schützen, bis B anfing, sehr schrille Chatter- und Tsiklaute abzugeben. Von da an verschwand A in der Hütte und mischte sich nicht mehr in die Konflikte ein. Der Rest der Familie kam näher, beobachtete die Situation und sprang teils mit auf $\mathrm{H} \mathrm{zu}$, verlor jedoch bald das Interesse an der Jagd und blieb neutral. Als $\mathrm{H}$ Hautverletzungen hatte und nur noch am Boden des Käfigs saß, wurde sie als „von der Familie ausgestoßen“ betrachtet und aus dem Käfigkomplex entfernt.

\subsubsection{Rauswurf von I}

Datum: 29.06.01

Bevor der Konflikt zwischen I und seinem Aggressor G offensichtlich wurde, gerieten verschiedene Dyaden der Familie in spannungsreiche Konflikte. D begann. I anzugreifen, zu keckern und ihn zu jagen, woraufhin die Familie I verteidigte. D geriet darüber mit $\mathrm{C}$ in eine Auseinandersetzung und es herrschte extreme Spannung zwischen den beiden. I unterstützte 
C wiederum im Konflikt mit D. Die Familie schloss sich dem Streit gegen D an und C, G und I jagten und mobbten sie gemeinsam. I verfolgte $\mathrm{D}$ allein in die Areale 6 und 7, beobachtete sie, blickte zu den Lockmännchen herüber und gab Distanz-Phi-Laute ab. D versuchte, I zu verjagen, worauf $\mathrm{G}$ sich anschloss und $\mathrm{I}$ fortan aggressiv begegnete und attackierte. $\mathrm{G}$ jagte zwei Tage lang hinter I her, der sich nach einiger Zeit im Gras am äußersten Ende des Streifgebiets vor $G$ versteckte. $G$ patrouillierte regelmäßig ca. alle $30 \mathrm{~min}$ in das Randareal und suchte I auf, um ihn von Neuem anzukeckern und anzugreifen. D und J unterstützten G dabei. I übernachtete allein in einer Schützhütte in Areal 2 und wurde an nächsten Morgen von $\mathrm{G}$ wieder an den Rand getrieben, wo er sich am Boden im hohen Gras versteckte. Am Vormittag des 29.06.01 war die Familie, insbesondere D, sehr aufgeregt und blickte zum Zaun im Osten der Semifreianlage. I hatte den Grasgürtel zum Zaun passiert und war gen Süden am Zaun entlang gewandert. Er kletterte in das Gewächshaus und nahm Kontakt zu den dort lebenden Gruppen auf.

\subsubsection{Rauswurf von J}

Datum: 21.10.01

Eine Woche vor dem Rauswurf wurde J aus der Familie entfernt, da er krank war und intensiver Pflege bedurfte. Nachdem sein Gesundheitszustand verbessert war, wurde er wieder in die Familie gegeben, die ihn zuerst neutral behandelte, d.h. er wurde weder gemobbt, angegriffen oder in affiliative Interaktionen eingebunden. Als J begann, affilitiv mit Familienmitgliedern zu interagieren, versuchte $\mathrm{G}$, alle affiliativen Interaktionen zwischen J und den restlichen Familienmitgliedern zu unterbinden. Am darauffolgenden Morgen wurde J von $\mathrm{G}$ so hart attackiert, so dass er aus der Familie entfernt werden musste.

\subsubsection{Rauswurf von $M$}

Datum: 24.03.01

$\mathrm{K}$ und $\mathrm{M}$ wurden Temperaturbuttons implantiert. Beide Tiere mussten dafür kurzzeitig aus der Familie entfernt werden. Um einen Streit wegen eines geschwächten Tieres zu vermeiden, wurden beide Tiere erst wieder in die Familie gesetzt, nachdem sie mehrere Stunden aus der Narkose aufgewacht waren. Dennoch herrschte beim Einsetzen Aufregung in der Familie. Beim gemeinsamen Katzbuckellaufen in der Hütte sammelten sich die Familienmitglieder zunächst vor $\mathrm{K}$, die in einer Ecke saß. Die Familienmitglieder machten Schlitzaugen, Büschelklappen und Genitalpräsentieren. K wirkte verunsichert, sprang dann aber bei der 
nächsten Katzbuckelrunde in die Familie hinein und katzbuckelte selber hinter den anderen her. Danach machte hielten die Familienmitglieder vor $\mathrm{M}$ an mit den gleichen dissoziativen Handlungen wie zuvor bei K. M war jedoch verschreckt, schrie auf und floh vor den Familienmitgliedern in den Außenkäfig. Jedes mal, wenn sie die Hütte betrat, kam die Familie auf sie zu gesprungen und schüchterte sie ein. K zeigte sich dabei von mal zu mal aggressiver und entschiedener, bis sie anfing, $M$ ernsthaft $\mathrm{zu}$ jagen. Sie scheuchte $M$ von einer Heizungsecke (Versteck der Affen hinter dem Heizkörper) in die andere und keckerte sie an. Kam M nicht mehr aus der Ecke hervor, wagte sich $\mathrm{K}$ bis kurz vor ihre Schwester und keckerte, bis $M$ das Versteck verließ. Die Familie wollte neugierig zur Heizungsecke laufen, was A jedoch nicht zuließ. Er saß am vordersten Rand der Heizung und schirmte die übrigen Familienmitglieder von $\mathrm{K}$ und $\mathrm{M}$ ab, so dass niemand Partei ergreifen konnte. Er selber blieb beiden gegenüber neutral, ließ von $\mathrm{K}$ aber häufiger eine Annäherung zu. Zeitweise unterstützte D die Angreiferin bei der Jagd auf M. M wurde aus der Familie herausgefangen.

\subsubsection{Rauswurf von $P$}

Datum: 30.07 .02

Am 26.07.02 wurde B, das Alpha-Weibchen wegen Altersschwäche und Krankheit eingeschläfert. $\mathrm{G}$ war an $\mathrm{K}$ interessiert und keckerte $\mathrm{D}$ an. Außerdem sprang er mit aufgestellten Büscheln und gesträubtem Fell mehrmals neben P. D zeigte am 27.07.02 Aggressionen gegen K, woraufhin G einen Angriff auf D startete. Am 28.07.02 keckerte G P offen an; P versuchte mit Quärren und Anstupsen zu beschwichtigen. Viel Spannung in der Familie (zwischen D und K). P nagte viel und wurde weiter von $G$ angekeckert. G genitalpräsentierte häufig und die Adulten der Familie katzbuckelten. G näherte sich $\mathrm{P}$ immer häufiger an, während P G beobachtete. Am 30.07.02 wurde zum ersten Mal beobachtet, wie P von $\mathrm{G}$ gejagt wurde. Die Jagd dauerte den ganzen Tag an und führte soweit, dass P sich im Gras versteckt hielt. $\mathrm{K}$ und $\mathrm{A}$ schlossen sich der Jagd auf $\mathrm{P}$ am Ende des Tages an. Gleichzeitig griff A auch D an und warf sie aus dem Schlafkasten. D konnte sich jedoch wieder in die Familie eingliedern, während es P nicht gelang, in den Schlafkasten zu klettern. Er zog sich in das Gewächshaus zurück und wurde von der Familie getrennt. 


\subsection{Grooming-Tabellen}

Tabelle 6.1 Grooming in der ersten Kontrollphase (B1) des ersten Beobachtungsjahres Rezipient

\begin{tabular}{|c|c|c|c|c|c|c|c|c|c|c|}
\cline { 2 - 11 } \multicolumn{1}{c|}{ Aktor } & $\mathrm{A}$ & $\mathrm{B}$ & $\mathrm{C}$ & $\mathrm{D}$ & $\mathrm{E}$ & $\mathrm{F}$ & $\mathrm{G}$ & $\mathrm{H}$ & $\mathrm{I}$ & $\mathrm{J}$ \\
\hline $\mathrm{A}$ & & 10 & & & & & & 1 & 1 & \\
\hline $\mathrm{B}$ & & & 1 & & & & & 1 & 6 & 3 \\
\hline $\mathrm{C}$ & & 2 & & & 1 & & & 9 & 6 & 4 \\
\hline $\mathrm{D}$ & & & & & 1 & & 5 & & 1 & 1 \\
\hline E & & & 5 & 8 & & 1 & 2 & & & \\
\hline F & & & & & & & 4 & 9 & & 1 \\
\hline G & & 1 & 1 & 1 & & & & 2 & 1 & 1 \\
\hline H & & & 2 & & 3 & & 5 & & & 3 \\
\hline I & & & & & & & & & & \\
\hline J & & & & & & & & & & \\
\hline
\end{tabular}

Tabelle 6.2 Grooming in der zweiten Kontrollphase (B2) des ersten Beobachtungsjahres Rezipient

\begin{tabular}{|c|c|c|c|c|c|c|c|c|c|}
\cline { 2 - 10 } Aktor & $\mathrm{A}$ & $\mathrm{B}$ & $\mathrm{C}$ & $\mathrm{D}$ & $\mathrm{E}$ & $\mathrm{G}$ & $\mathrm{H}$ & $\mathrm{I}$ & $\mathrm{J}$ \\
\hline $\mathrm{A}$ & & 18 & & & & 2 & & & 2 \\
\hline $\mathrm{B}$ & 3 & & & & & & & & 2 \\
\hline $\mathrm{C}$ & & 1 & & & & & & & 3 \\
\hline $\mathrm{D}$ & & 1 & & & 2 & & 1 & & \\
\hline $\mathrm{E}$ & & & 4 & & & 3 & 1 & & \\
\hline $\mathrm{G}$ & & & 1 & & & & 2 & & \\
\hline $\mathrm{H}$ & & 1 & 3 & & & 12 & & 4 & 1 \\
\hline $\mathrm{I}$ & & & & & & & & & 1 \\
\hline $\mathrm{J}$ & & & & & & & & & \\
\hline
\end{tabular}

Tabelle 6.3 Grooming im ersten Experiment (C1) des ersten Beobachtungsjahres

Rezipient

\begin{tabular}{|c|c|c|c|c|c|c|c|c|c|}
\cline { 2 - 11 } Aktor & $\mathrm{A}$ & $\mathrm{B}$ & $\mathrm{C}$ & $\mathrm{D}$ & $\mathrm{E}$ & $\mathrm{G}$ & $\mathrm{H}$ & $\mathrm{I}$ & $\mathrm{J}$ \\
\hline $\mathrm{A}$ & & 14 & 1 & 8 & & & 1 & & 2 \\
\hline $\mathrm{B}$ & 2 & & 1 & & & & & 5 & 3 \\
\hline $\mathrm{C}$ & 1 & 4 & & 4 & 5 & & 3 & & 2 \\
\hline $\mathrm{D}$ & 3 & & 8 & & 1 & & & & 5 \\
\hline E & & & 5 & 2 & & & 4 & & \\
\hline $\mathrm{G}$ & & & 3 & & 7 & & 6 & 1 & 9 \\
\hline $\mathrm{H}$ & 3 & & 3 & & & 2 & & & 7 \\
\hline $\mathrm{I}$ & & & & & & & & & \\
\hline J & & & & & & & & & \\
\hline
\end{tabular}


Tabelle 6.4 Grooming im zweiten Experiment (C2) des ersten Beobachtungsjahres

Rezipient

\begin{tabular}{|c|c|c|c|c|c|c|c|c|c|}
\cline { 2 - 10 } \multicolumn{1}{c|}{ Aktor } & $\mathrm{A}$ & $\mathrm{B}$ & $\mathrm{C}$ & $\mathrm{D}$ & $\mathrm{E}$ & $\mathrm{G}$ & $\mathrm{H}$ & $\mathrm{I}$ & $\mathrm{J}$ \\
\hline $\mathrm{A}$ & & 4 & 4 & & & & & 5 & 1 \\
\hline $\mathrm{B}$ & 2 & & & & & & & 5 & 1 \\
\hline $\mathrm{C}$ & & & & 3 & 5 & & & & \\
\hline $\mathrm{D}$ & & & 2 & & & 1 & & 1 & 1 \\
\hline $\mathrm{E}$ & & & 12 & & & & & & 3 \\
\hline $\mathrm{G}$ & 10 & 1 & & 5 & & & 1 & & 3 \\
\hline $\mathrm{H}$ & 1 & & & & & 1 & & & 3 \\
\hline $\mathrm{I}$ & 1 & & & & & & & & \\
\hline $\mathrm{J}$ & & 1 & & & & & & & \\
\hline
\end{tabular}

Tabelle 6.5 Grooming im ersten Experiment (E1) des zweiten Beobachtungsjahres

Rezipient

\begin{tabular}{|c|c|c|c|c|c|c|c|c|c|}
\cline { 2 - 10 } \multicolumn{1}{c|}{ Aktor } & A & B & C & D & G & I & J & K & M \\
\hline A & & 11 & & & & 1 & 2 & 3 & \\
\hline B & & & & & & & & 4 & \\
\hline C & & 1 & & & 2 & & 4 & & 5 \\
\hline D & & & & & & & & & 2 \\
\hline G & 3 & & 7 & & & 1 & 3 & & 5 \\
\hline I & 3 & 1 & 4 & 1 & & & 3 & 2 & \\
\hline J & & & 2 & & & 1 & & 1 & \\
\hline K & & 4 & & 2 & & & & & \\
\hline M & & 2 & 1 & & & 1 & & & \\
\hline
\end{tabular}

Tabelle 6.6 Grooming im zweiten Experiment (E2) des zweiten Beobachtungsjahres

Rezipient

\begin{tabular}{|c|c|c|c|c|c|c|c|c|c|}
\multicolumn{1}{c|}{ Aktor } & A & B & C & D & G & I & J & K & M \\
\hline A & & 12 & & & & & & & \\
\hline B & & & & & & & & & \\
\hline C & & & & 1 & 5 & 2 & & & 4 \\
\hline D & & & 3 & & & & & 1 & \\
\hline G & & & 11 & & & & & & \\
\hline I & & & 2 & & & & 8 & & 2 \\
\hline J & & 3 & & & & 6 & & 4 & 6 \\
\hline K & & 1 & 2 & & & & & & \\
\hline M & & 1 & & & & & 1 & & \\
\hline
\end{tabular}


Tabelle 6.7 Grooming im dritten Experiment (E3) des zweiten Beobachtungsjahres

\begin{tabular}{|c|c|c|c|c|c|c|c|c|}
\hline \multirow[b]{2}{*}{ Aktor } & \multicolumn{8}{|c|}{ Rezipient } \\
\hline & A & B & C & D & G & $J$ & K & M \\
\hline A & & 3 & & & & & & 1 \\
\hline B & & & & & & & & \\
\hline C & 1 & & & & & 1 & 1 & \\
\hline D & 2 & & & & & & & 4 \\
\hline G & 10 & & 5 & & & & 3 & \\
\hline $\mathrm{J}$ & 1 & 3 & & & & & 2 & \\
\hline K & & 1 & & & & 3 & & \\
\hline M & 1 & & & & & & & \\
\hline
\end{tabular}

Tabelle 6.8 Grooming im vierten Experiment (E4) des zweiten Beobachtungsjahres Rezipient

\begin{tabular}{|c|c|c|c|c|c|c|c|c|}
\cline { 2 - 9 } \multicolumn{1}{c|}{ Aktor } & A & B & C & D & G & J & K & M \\
\hline A & & 4 & & & 4 & 2 & 2 & \\
\hline B & & & & & & & & \\
\hline C & & & & & 1 & & 7 & \\
\hline D & & & & & & & 1 & \\
\hline G & 4 & & & & & 11 & 1 & 1 \\
\hline J & 1 & 1 & 1 & & 4 & & 4 & \\
\hline K & 1 & 4 & 3 & & 2 & & & 1 \\
\hline M & 1 & 2 & & & 3 & & 1 & \\
\hline
\end{tabular}




\section{LITERATUR}

Abbott, D.H.(1984): Behavioral and physiological suppression of fertility in subordinate marmoset monkeys. Am. J. Primatol. 6: 169-186.

Abbott, D.H.(1986): Social suppression of reproduction in subordinate marmoset monkeys (Callithrix jacchus jacchus). In: de Mello, M.T. (ed.): A primatologia no Brasil - 2. Sociedade Brasileira de Primatologia, Brasilia. S.16-31.

Abbott, D.H. und Hearn, J.P. (1978): Physical, hormonal and behavioural aspects of sexual development in the marmoset monkey, Callithrix jacchus. J. Reprod. Fert. 53: 155-166.

Ahlborn, S. (1996): Untersuchungen zur Nahrungsökologie semifreilebender Weißbüschelaffen (Callithrix jacchus). Unveröffentlichte Diplomarbeit, Universität Göttingen, Göttingen.

Alexander, .R.D.(1979): The evolution of social behaviour. Ann. Rev. Ecol. Sys. 5: 325-383.

Altmann, J. (1974): Observational study of behavior: sampling methods. Behaviour 49: 227267.

Amann, E. (1999): Evolutionäre Spieltheorie, Grundlagen und neue Ansätze. Studies in Contemporary Economics, Physica Verlag, A Springer-Verlag Company.

Anzenberger, A.A. (1983): Bindungsmechanismen in Familiengruppen von Weißbüscheläffchen (Callithrix jacchus). Inaugural-Dissertation, Universität Zürich, Juris Druck + Verlag Zürich.

Baker, A.J. (1987): Emigration in wild groups of golden lion tamarins (Leontopithecus rosalia). Int. J. Primatol. 8: 500.

Baker, A.J. (1991): Evolution of the social system of the golden lion tamarin. Ph.D. dissertation, University of Maryland, College Park.

Barash, D.P. (1980): Soziobilogie und Verhalten. Verlag Paul Parey.

Bartecki, U. und Heymann, E.W. (1987): Field observation on snake-mobbing in a group of saddle-back tamarins, Saguinus fuscicollis nigrifrons. Folia primatol. 48: 199-202.

Bekoff, M. (1977): Mammalian dispersal and the ontogeny of individual behavioural phenotypes. Am. Nat. 111: 715-732.

Bortz, J., Lienert, G.A. und Boehneke, K.(1990): Verteilungsfreie Methoden in der Biostatistik. Springer-Verlag, Berlin.

Box, H.O. (1977): Quantitative data on the carrying of young captive monkeys (Callithrix jacchus) by other members of their family groups. Primates 18 (2): 475-484.

Brown, J.L. (1987): Helping and communal breeding in birds: ecology and evolution. Princeton, Princeton University Press.

Brown, J.L. und Brown, E.R. (1984): Parental facilitation: parent-offspring relations in communally breeding birds. Behav. Ecol. Sociobiol. 14: 203-209.

Buchanan-Smith, H. (1990): Polyspecific association of two tamarin species, Saguinus labiatus and Saguinus fuscicollis, in Bolivia. Am. J. Primatol. 22: 205-214.

Caine, N.G. (1984): Visual scanning by tamarins: a description of the behavior and test of two derived hypotheses. Folia Primatol. 43: 59-67. 
Caine, N.G. (1986): Visual monitoring of threatening objects by captive tamarins (Saguinus labiatus). Am. J. Primatol. 10:1-8.

Caine, N.G. (1993): Flexibility and cooperation as unifying themes in Saguinus social organization and behaviour: the role of predation pressure. In: Ryland, A.B.: Marmosets and tamarins. Oxford University Press. S. 200-219.

Caine, N.G. (1997): Cutting costs in response to predatory threat by Geoffroy's marmosets. Am. J. Primatol. 42: 98.

Carr, G.M. und Macdonald, D.W. (1986): The sociality of social foragers: a model based on resource dispersion. Anim. Behav. 34: 1540-1549.

Chaoui, N.J., Hasler-Gallusser, S. (1999): Incomplete sexual suppression in Leontopithecus chrysomelas: a behavioural and hormonal study in a semi-natural environment. Folia Primatol. 70: 47-54.

Charnov, E.L. (1976): Optimal foraging: attack of a mantid. Am. Nat. 110:141-151.

Chase, I.D. (1980): Cooperative and non-cooperative behaviour in animals. Am. Nat. 115: 827-857.

Cheney, D.L. und Seyfarth, R.M. (1983): Nonrandom dispersal in free-ranging vervet monkeys: social and genetic consequences. Am. Nat. 122: 392-412.

Clarke, F.M. (1987): The effects of carrying infants on the marmoset (Callithrix jacchus) caregivers. Int. J. Primatol. 8: 536.

Clutton-Brock, T.H., Gaynor, D., McIlrath, G.M, Maccoll, A.D.C., Kansky, R., Chadwick, P., Manser, M., Skinner, J.D. und Brotherton, P.N.M. (1999): Predation, group size and mortality in a cooperative mongoose, Suricata suricatta. J. Anim. Ecol. 68: 672-683.

Colvin, J. (1983): Influences of the social situation on male emigration. In: Hinde, R.A. (ed.): Primate social relationships: an integrated approach. S. 160-170. Blackwell Scientific Publications.

Creel, S.R. (1990): How to measure inclusive fitness. Proc. R. Soc. Lond. Ser. B, 241: 229231.

Darms, K. (1987): Analyse interindividueller Distanzen zwischen den Mitgliedern zweier Weißbüschelaffengruppen (Callithrix jacchus ERXLEBEN 1777). Dissertation, Universität Göttingen, Göttingen.

Darms, K. (1989): Dynamics of group size in the common marmoset, Callithrix jacchus: social conflicts, disintegration, and expulsion of familiy members. Paper präsentiert bei der Tagung der Tropical Ecology Group of the British Ecological Society, „Behavioural Ecology of the Neotropical Primates“, Linnaean Society, London, 16. Juni 1989.

Dawson, G.A. (1976): Behavioural ecology of the panamanian tamarin, Saguinus oedipus (Callitrichidae, Primates). Michigan State University, Ph.D. Zoology.

Dawson, G.A. (1978): Composition and stability of social groups of the tamarin, Saguinus oedipus geoffroyi, in Panama: Ecological and behavioural implications. In: Kleiman, D.G. (ed.) The biology and conservation of the callitrichidae - the symposia of the National Zoological Park, Smithsonian Institution. S.23-37.

Digby, L.J. (1995a): Infant care, infanticide, and female reproductive strategies in polygynous groups of common marmosets (Callithrix jacchus). Behav. Ecol. Sociobiol. 37: 51-61. 
Digby, L.J. (1995b): Social organization in a wild population of Callithrix jacchus: II. Intragroup social behaviour. Primates 36 (3): 361-375.

Digby, L.J. (1999): Sexual behaviour and extragroup copulations in a wild population of common marmosets (Callithrix jacchus). Folia Primatol. 70: 136-145.

Digby, L.J. und Barreto, C. E. (1993): Social organization in a wild population of Callithrix jacchus. I. Group composition and dynamics. Folia Primatol. 61: 123-134.

Digby, L.J. und Barreto, C. E. (1996): Activity and ranging patterns in common marmosets (Callithrix jacchus): Implications for reproductive strategies. In: Norconk, M.A., Rosenberger, A.L. und Garber, P.A. (eds.): Adaptive radiations of neotropical primates. Plenum Press, New York. S. 173-185.

Digby, L.J. und Ferrari, S.F. (1994): Multiple breeding females in free-ranging groups of Callithrix jacchus. Int. J. Primatol. 15 (3): 389-397.

Dreschler, A. P. (2002): Zur Markierung der Weißbüschelaffen (Callithrix jacchus) unter semi-freien Haltungsbedingungen. Unveröffentlichte Diplomarbeit, Universität Göttingen, Göttingen.

Dugatkin, L.A. und Reeve, H.K. (1998): Game theory and animal behaviour. Oxford University Press, New York, Oxford.

Dunbar, R.I.M. (1988): Primate social systems. Cornell University Press.

Dunbar, R.I.M. (1991): Functional significance of social grooming in primates. Folia Primatol. 57: 121-131.

Ehardt, C.L. und Bernstein, I.S. (1992): Conflict intervention behaviour by adult male macaques: structural and functional aspects. In: Harcourt, A. H. und de Waal, F.B.M. (eds.): Coalitions and alliances in humans and other animals. Oxford University Press, Oxford, New York, Tokyo. S. 83-111.

Emlen, S.T. (1982a): The evolution of helping. I. An ecological constraints model. Am. Nat. 119 (1): 29-39.

Emlen, S.T. (1982b): The evolution of helping. II. The role of behavioural conflict. Am. Nat. 119 (1): 40-53.

Emlen, S.T. und Wrege, P.H. (1992): Parent-offspring conflict and the recruitment of helpers among bee-eaters. Nature 356: 331-333.

Emlen, S.T. und Vehrenkamp, S.L. (1985): Cooperative breeding strategies among birds. In: Holldobbler, B. und Lindauer, M. (eds): Fortschritte der Zoologie 31: 359-374. G. Fischer, Stuttgart.

Ekman, J. und Rosander, B. (1992): Survival enhancement through food sharing: a means for parental control of natal dispersal. Theoret. Popul. Biol. 42, 117-129.

Ekman, J., Eggers, S. und Griesser, M. (2002): Fighting to stay: the role of sibling rivalry for delayed dispersal. Anim. Behav.64 (3): 453-459.

Epple, G. (1967): Vergleichende Untersuchungen über Sexual- und Sozialverhalten der Krallenaffen (Hapalidae). Folia Primatol. 7: 37-65.

Epple, G. (1975a): The behaviour of marmoset monkeys (Callitrichidae). In: Rosenblum, L.A. (ed.): Primate behaviour, Vol. 4, S.195-239. Academic Press, New York.

Epple, G., Belcher, A.M., Küderling, I., Zeller, U., Scolnick, L., Greenfield, K.L. und Smith, A.B. III (1993): Making sense out of scents: species differences in scent glands, scent- 
marking behaviour, and scent-mark composition in the Callitrichidae. In: Rylands, A.B.(ed.): Marmosets and tamarins - systematic, behaviour and ecology. Oxford University Press, Oxford. S.123-151.

Evans, S. und Poole, T.B. (1984): Long-term changes and maintenance of the pair bond in common marmosets (Callithrix jacchus jacchus). Folia Primatol. 42: 33-41.

Ferrari, S.F. (1987): Food transfer in a wild marmoset group. Folia Primatol. 48: 203-206.

Ferrari, S.F. (1988a): Social behaviour in Callithrix flaviceps: an ecological perspective. Paper präsentiert beim Workshop über Monogamie bei Krallenaffen, 26-28. Feb. Universität Göttingen, Göttingen.

Ferrari, S.F. (1988b): The behaviour and ecology of the buffy-headed marmoset, Callithrix flaviceps (O.Thomas, 1903). Unveröffentlichte Ph.D. Thesis, University College, London.

Ferrari, S.F. und Diego, V.H. (1992): Long-term changes in a wild marmoset group. Folia Primatol. 58: 215-218.

Ferrari, S.F. und Digby, L.J. (1996): Wild Callithrix groups: stable extended families? Am. J. Primatol. 38: 19-27.

Ferrari, S.F. und Lopes Ferrari, M.A. (1989): A re-evaluation of the social organisation of the callitrichidae, with reference to the ecological differences between the genera. Folia Primatol. 52: 132-147.

Ferrari, S.F. und Lopes Ferrari, M.A. (1990): Predator avoidance behaviour in the buffyheaded marmoset, Callithrix flaviceps. Primates 31(3): 323-338.

Fröhlich, W. (2000): Wörterbuch Psychologie. 23., aktualisierte, überarbeitete und erweiterte Auflage September 2000. Deutscher Taschbuch Verlag GmbH \& Co. KG, München.

Gaston, A.J. (1978): The evolution of group territorial behaviour and coopreative breeding. Am. Nat. 112: 1091-1100.

Gaines, M.S. und McClenaghan, L.R. (1980): Dispersal in small mammals. Ann. Rev. Ecol. Syst. 11: 163-196.

Garber, P.A. (1994): Phylogenetic approach to the study of tamarin and marmoset social systems. Am. J. Primatol. 34: 199-219.

Garber, P.A. (1997): One for all and breeding for one: cooperation and competition as a tamarin reproductive strategy. Evol. Anthropol. 5: 187-199.

Garber, P.A., Moya, L. und Malaga, C. (1984): A preliminary field study of the moustached tamarin monkey (Saguinus mystax) in northeastern Peru: Questions concerned with the evolution of a communal breeding system. Folia Primatol. 42: 17-32.

Garber, P.A., Encarnacion. F., Moya, L. and Pruetz, J. D. (1993): Demographic and reproductive patterns in moustached tamarin monkeys (Saguinus mystax): Implications for reconstructing platyrrhine mating systems. Am. J. Primatol. 29: 235-254.

Garber, P.A. und Teaford, M.F. (1986): Body weights in mixed species troops of Saguinus mystax mystax and Saguinus fuscicollis nigrifrons in Amazonian Peru. Am. J. Phys. Anthropol. 71: 331-336.

Geyer, U. (1993): Peripheralisation adulter Krallenaffenmännchen (Callithrix jacchus). Untersuchung zweier Einflussfaktoren: Optische Kontrolle durch die Restgruppe und Konfrontation mit gruppenfremden Artgenossen. Unveröffentlichte Examensarbeit, Universität Göttingen, Göttingen. 
Giraldeau, L.A. und Livoreil, B. (1998): Game-theory and social foraging. In: Dugatkin, L.A. und Reeve, H.K. (ed.): Game theory and animal behaviour. Oxford University Press, Oxford, New York. S. 16-37.

Goldizen, A.W. (1987): Facultative polyandry and the role of infant-carrying in wild saddleback tamarins (Saguinus fuscicollis). Behav. Ecol. Sociobiol. 20: 99-109.

Goldizen, A.W., Terborgh, J., Cornejo, F., Porras, D.T. und Evans, R. (1988): Seasonal food shortage, weight loss, and timing of births in saddle-back tamarins (Saguinus fuscicollis). J. Anim. Ecol. 57: 893-901.

Goldizen, A.W. und Terborgh, J. (1989): Demography and dispersal patterns of tamarin population: possible causes of delayed breeding. Am. Naturalist 134 (2): 208-224.

Goldizen, A.W., Mendelson, J., van Vlaardingen, M. und Terborgh, J. (1996): Saddle-back tamarin (Saguinus fuscicollis) reproductive strategies: evidence from a thirteen-year study of a marked population. Am. J. Primatol. 38: 57-83.

Goodman, D. (1982): Optimal life histories, optimal notation, and the value of reproductive value. Am. Nat. 119: 803-823.

Hamilton, W.D. (1964): The genetical evolution of social behaviour. I, II. J. Theoret. Biol. 7: $1-52$.

Harcourt, A.H. (1978): Strategies of emigration and transfer by primates, with particular reference to gorillas. Z. Tierpsychol. 48: 401-420.

Hearn, J.P. (1983): The common marmoset (Callithrix jacchus). In: Hearn, J.P. (ed.): Reproduction in new world primates: new models in medical science. MTP Press Ltd., Lancaster. S. 181-215.

Henzi, S.P. und Lucas, J.W. (1980): Observations on the intertroop movement of adult vervet monkeys (Cercopithecus aethiops). Folia Primatol. 33: 220-235.

Heymann, E.W. (1987): A field observation of predation on a moustached tamarin (Saguinus mystax) by an anaconda. Int. J. Primatol. Vol. 8 (2): 193-194.

Heymann, E.W. (1990b): Social behaviour and infant carrying in a group of moustached tamarins, Saguinus mystax (Primates: Platyrrhini: Callithrichidae), on Padre Isla, peruvian Amazonia. Primates 32: 183-196.

Hinde, R.A. (1983): Primate social relationships. Blackwell Scientific Publications.

Hinde, R.A. und Stevenson-Hinde, J. (1976): Towards understanding relationships: dynamic stability. In: Bateson, P.P.G. und Hinde, R.A. (eds.): Growing points in ethology. Cambridge University Press, Cambridge.

Holler, M.J. und Illing, G. (2000): Einführung in die Spieltheorie. Springer Verlag Berlin Heidelberg.

Hubrecht, R.C. (1984): Field observations on group size and composition of the common marmoset (Callithrix jacchus jacchus), at Tapacura, Brazil. Primates 25: 13-21.

Hubrecht, R.C. (1985): Home-range size and use and territorial behaviour in the common marmoset, Callithrix jacchus jacchus, at the Tapacura Field Station, Recife, Brazil. Int. J. Primatol. Vol. 6 (5): 533-550.

Hubrecht, R.C. (1989): The fertility of daughters in common marmosets (Callithrix jacchus jacchus) familiy groups. Primates 30: 423-432.

Hrdy, S.B. (1977): The langurs of Abu. Cambridge: Harvard University Press. 
Ingram, J.C. (1977): Interactions between parents and infants, and the development of independence in the common marmoset (Callithrix jacchus). Anim. Behav. 25: 811-827.

Isbell, L.A. (1994): Predation on primates: ecological patterns and evolutionary consequences. Evol. Anthropol. 4: 61-71.

Jünemann, B. (1990): Analyse der räumlichen Struktur einer Großfamilie des Weißbüschelaffen (Callithrix jacchus Erxleben 1777) unter besonderer Berücksichtigung möglicher Regulationsmechanismen. Unveröffentlichte Diplomarbeit, Universität Göttingen, Göttingen.

Keller, L. und Reeve, H.K. (1994): Partioning of reproduction in animal societies. Trends Ecol. Evol. 9: 98-102.

Kerl, J. (2000): Der Einfluß abiotischer Umweltparameter auf die Lebensäußerungen von Weißbüschelaffen (C. jacchus). Cuvillier Verlag Göttingen.

Kleiman, D.G. (1979): Parent-offspring conflict and sibling competition in a monogamous primate. Am. Nat. 114: 753-760.

Kleiman, D.G. (1981): Correlations among life history characteristics of mammalian species exhibiting two extreme forms of monogamy. In: Alexander, R.D. und Tinkle, D.W. (eds.): Natural selection and social behaviour. S. 332-344. Chiron Press, Oxford.

Knox, K.L. und Sade, D.S. (1991): Social behaviour of the emperor tamarin in captivity. Components of agonistic display and the agonistic network. Int. J. Primatol. 12: 439-480.

Kokko, H. und Johnstone, R.A. (1999): Social queing in animal societies: a dynamic model of reproductiv skew. Proceedings of the Royal Society of London, Series B, 266: 571-578.

Komdeur, J. (1992): Importance of habitat saturation and territory quality for evolution of cooperative breeding in the seychelles warbler. Nature: 358: 493-495.

König, A. (1992): Untersuchungen zum Scanning-Verhalten beim Weißbüschelaffen (Callithrix jacchus ERXLEBEN 1777). Dissertation, Universität Göttingen, Verlag Dr. Kovač, Hamburg.

König, A. (1995): Group size, composition and reproductive success in wild common marmosets (Callithrix jacchus). Am. J. Primatol. 35: 311-317.

König, A., Rothe, H., Siess, M. Darms, K., Gröger, D., Radespiel, U. und Rock, J. (1988): Reproductive reorganization in incomplete groups of the common marmoset (Callithrix jacchus) under laboratory conditions. Z. Säugetierkunde. 53: 1-6.

Koenig, W. und Pitelka, F. (1981): Relatedness and inbreeding: counterploys in the communally nesting acorn woodpecker. Science 206: 1103-1105.

König, A., Radespiel, U., Siess, M., Rothe, H. und Darms, K. (1990): Analysis of pairingparturition- and interbirth-intervals ina colony of common marmosets (Callithrix jacchus). Z. Säugetierkunde 55: 308-314.

König, A. und Rothe, H. (1991): Social relationships and individual contribution to cooperative behaviour in captive common marmosets (Callithrix jacchus). Primates 32: 183-195.

Krebs, J.R. und Davies, N.B. (1996): Einführung in die Verhaltensökologie. 3. neubearbeitete und erweiterte Auflage, Blackwell Wissenschafts-Verlag Berlin, Wien. 
Kretzer, N. (1996): Experimentelle Untersuchung zur fraternalen kooperativen Polyandrie bei Weißbüschelaffen (Callithrix jacchus) unter Semifreilandbedingungen, Unveröffentlichte Diplomarbeit, Universität Göttingen.

Lazaro-Perea, C., Snowdon, C.T. und Santee, D.P. (1997): Competition and chemical communication in wild groups of common marmosets (Callithrix jacchus). The Integrative Neurobiology of Affiliation 807: 534-537.

Lazaro-Perea, C., Arruda, M.F. und Snowdon, C.T. (2004): Grooming as a reward? Social function in cooperatively breeding marmosets. Anim. Behav. 67: 627-636.

Lee, P.C. (1983): Play as a means for developing relationships. In: Hindes, R.A. (ed.): Primate social relationships: an integrated approach. Blackwell Scientific Publications. S. 82-89.

Lehner, P.N. (1996): Hanbook of ethological methods. Second edition. Cambridge University Press, Cambridge.

Leutenegger, W. (1980): Monogamy in callitrichids: A consequence of phyletic dwarfism? Int. J. Primatol. 1 (1): 95-98.

Lidicker, W.Z.JR. (1975): The role of dispersal in the demography of small mammals. In: Golley, F.B., Petrusewicz, K. und Ryszkowski, L. (eds.): Small mammals: their reproduction and population dynamics. Cambridge University Press, Cambridge. S. 103128.

Lucas, J.R., Creel, S.R. und Waser, P.M. (1996a): How to measure inclusive fitness, revisited. Anim. Behav. 51: 225-228.

Lucas, J.R., Creel, S.R. und Waser, P.M. (1997): Optimization and cooperative breeding: future fitness. In: Solomon, N.G. und French, J.A.(eds.): Cooperative breeding in mammals. Cambridge University Press. S. 171-198.

Lucas, J.R., Howard, R.D. und Palmer, J.G. (1996b): Callers and satellites: chorus behaviour in anurans as a stochastic dynamic game. Anim. Behav. 51: 501-518.

Maier, J.G. (1988): Untersuchungen zur Körpertemperatur von Weißbüscheläffchen (Callithrix jacchus) mit Hilfe eines neu entwickelten Telemetriesystems. InauguralDissertation, Universität München, München.

Maier, W., Alonso, C. und Langguth, A. (1982): Field observations on Callithrix jacchus jacchus L. Z. Säugetierkunde 47 (6): 334-346.

Malcolm, J. und Marten, K. (1982): Natural selection and the communal rearing of pups in african wild dogs (Lycaon pictus). Behav. Ecol. Sociobiol. 10: 1-13.

Manson, J.H., Rose, L.M., Perry, S. und Gros-Luis, J. (1999): Dynamics of female-female relationships in wild Cebus capucinus: data from two Costa Rican sites. Int. J. Primatol. 20: 679-706.

Martin, P. und Bateson, P. (1993): Measuring behaviour. Second Edition. Cambridge University Press.

Maynard-Smith, J. (1964): Group selection and kin selection. Nature 201: 1145-1147.

Maynard-Smith, J. (1982): Evolution and the theory of games. Cambridge University Press.

Maynard-Smith, J. und Price, G.R. (1973): The logic of animal conflict. Nature 246: 15-18.

MacArthur, R.H. und Pianka, E.R. (1966): On the optimal use of a patchy environment. Am. Nat. 100: 603-609. 
Macdonald, D.W. und Moehlmann, P.D. (1983): Cooperation, altruism, and restraint in the reproduction of carnivores. In Bateson, P. und Klopfer, P. (eds.): Perspectives in ethology, S. 433-467. New York: Plenum Press.

McGrew, W.C. (1986): Kinship terms and callitrichid mating patterns: a discussion note. Primate Eye 30: 25-26.

McGrew, W.C. (1997): Sex differences in the familiy life of cotton-top tamarins: socioecological validity in the laboratory? In: Kinzey, W.G.(ed.): New world primates ecology, evolution, and behaviour. Aldine de Gruyter - New York. S. 95-107.

McGrew, W.C. und McLuckie, E.C. (1986): Philopatry and dispersion in the cotton-top tamarin, Saguinus (o.) oedipus: an attempted laboratory simulation. Int. J. Primatol. 7: 401422.

Mendes Pontes, A.R. und Monteiro da Cruz, M.A.O. (1995): Home range, intergroup transfers, and reproductive status of common marmosets Callithrix jacchus in a forest fragment in north-eastern Brazil. Primates 36: 336-347.

Missler, M., Wolff, J.R., Rothe, H., Heger, W., Merker, H.; Treiber, A., Scheid, R. und Crook, G.A. (1992): Developmental biology of the common marmoset: Proposal for a "postnatal staging". J. Med. Primatol. 21: 285-298.

Mock, D.W., Parker, G.A. und Schwagmeyer, P.L. (1998): Game theory, sibling rivalry, and parent-offspring conflict. In: Dugatkin, L.A. und Reeve, H.K.: Game theory and animal behaviour. Oxford University Press, New York, Oxford. S. 146-167.

Moura, A.C. de A. (2003): Sibling age and intragroup aggression in captive Saguinus midas midas. Int. J. Primatol. 24 (3):639-652.

Neymann, P. (1978): Aspects of the ecology and social organization of free-ranging cottontop tamarins (Saguinus oedipus) and the conservation status of the species. In: Kleiman, D.G. (ed.) The biology and conservation of the callitrichidae - the symposia of the National Zoological Park, Smithsonian Institution. S. 39-71.

Nievergelt, C.M., Digby, L.J., Ramakrishnan, U. and Woodruff, D.S.(2000): Genetic analysis of group composition and breeding system in a wild common marmoset (Callithrix jacchus) population. Int. J. Primatol. 21 (1): 1-20.

Oliveira, M.S., Lopes, F.A., Alonso, C. und Yamamoto, M.E. (1999): The mothers's participation in infant carrying in captive groups of Leontopithecus chrysomelas and Callithrix jacchus. Folia Primatol. 70: 146-153.

Packard, J.M., Seal, U.S., Mech, L.D. und Plotka, E.D. (1985): Causes of reproductive failure in two family groups of wolves (Canis lupus). Z. Tierpsychol. 68: 24-40.

Petry, H. und Maier, J. (1990): Radiotelemetrische Untersuchungen der Körpertemperatur von Weißbüscheläffchen (Callithirx jacchus). Z. Ernährungswiss. 29: 197-207.

Price, E.C. (1990): Infant-carrying as a courtship strategy of breeding male cotton-top tamarins. Animal Behav. 40: 784-786.

Price, E.C. (1991): Competition to carry infants in captive families of cotton-top tamarins (Saguinus oedipus). Behaviour 118 (1-2): 66-88.

Price, E.C. (1992): Changes in the activity of captive cotton-top tamarins (Saguinus oedipus) over the breeding cycle. Primates 33: 99-106.

Price, E.C. und Evans, S. (1991): Terminology in the study of callitrichid reproductive strategies. Anim. Behav. 42: 1025-1027. 
Price, E.C. und McGrew (1991): Departures from monogamy in colonies of captive cottontop tamarins. Folia Primatol. 57: 16-27.

Pryce, C.R. (1988): Individual and group effects on early caregiver-infant relationships in redbellied tamarin monkeys. Anim. Behav. 36: 1455-1464.

Pulliam, H.R. (1974): On the theory of optimal diets. Am. Nat. 108: 59-74.

Pusey, A.E. und Packer, C. (1987): Dispersal and philopatry. In: Smuts, B., Cheney, R., Wrangham, R. und Struhsaker, T. T. (ed.): Primate societies, University of Chicago Press, Chicago. S. 250-266.

Radespiel, U. (1990): Die räumlichen und sozialen Strukturen innerhalb einer Familie von Weißbüschelaffen (Callithrix jacchus ERXLEBEN 1777) unter dem experimentellen Einfluß der zeitweiligen Abwesenheit der Eltern. Unveröffentlichte Diplomarbeit, Universität Göttingen, Göttingen.

Ralls, K. und Ballou, J. (1982): Effects on inbreeding on infant mortality in captive primates. Int. J. Primatol. 3: 491-505.

Ralls, K., Harvey, P.H. und Lyles, A.M. (1986): Inbreeding in natural populations of birds and mammals. In Soulé, M.E.: Conservation biology: the science of scarcity and diversity. Sinauer Associates, Inc, Sunderland, Massechusetts. S. 35-56.

Rasa, O.A.E. (1989): Helping in dwarf mongoose societies: An alternative reproductive strategy. In: Rasa, O.A.E. und Vogel, C. (eds.): The sociobiology of sexual and reproductive strategies. Croom Helm, Beckenham.

Rasa, O.A.E. (1984): Dwarf mongoose and hornbill mutualism in the Taru desert, Kenya. Behav. Ecol. Sociobiol. 12: 181-190.

Reyer, H.-U. (1980): Flexible helper structure as an ecological adaptation in the pied kingfisher, Ceryle rudis L. Behav. Ecol. Sociobiol. 6: 219-227.

Reyer, H.-U. (1984): Investment and relatedness: a cost/benefit analysis of breeding and helping in the pied kingfisher. Anim. Behav. 32: 1163-1178.

Ross, C. und Jones, K.E. (1999): Socioecology and the evolution of primate reproductive rates. In: Lee, P.C.(ed.) Comparative primate socioecology. Cambridge University Press. S. 73-110.

Rothe, H. (1974): Allogrooming by adult Callithrix jacchus in relation to postpartum oestrus. J. of Human Evol. 3: 535-540.

Rothe, H. (1975): Some aspects of sexuality and reproduction in groups of captive marmosets (Callithrix jacchus). Z. Tierpsychol. 37: 255-273.

Rothe, H. (1978): Sub-grouping behaviour in captive Callithrix jacchus families: a preliminary investigation. In: Rothe, H.; Wolters, H.J. und Hearn, J.P.(eds.) Biology and behaviour of marmosets; proceeding of the marmoset workshop, Eigenverlag H. Rothe, Göttingen. S. 233-258.

Rothe, H. (1979): Das Ethogramm von Callithrix jacchus ERXLEBEN, 1777 (Primates, Ceboidea, Callithrichidae). Eine morphaktische Analyse des Verhaltens mit besonderer Berücksichtigung des sozialen Umfeldes. Unveröffentlichte Habilitation, Universität Göttingen.

Rothe, H. und Darms, K. (1993): The social organization of marmosets: a critical evaluation of recent concepts. In: Rylands, A.B.: Marmosets and tamarins - systematic, behaviour and ecology. Oxford University Press, Oxford. S. 176-199. 
Rothe, H., Darms, K. und König, A. (1992): Sex ratio and mortality in a laboratory colony of the common marmoset (Callithrix jacchus). Lab. Anim. 26: 88-99.

Rothe, H., Darms, K., König, A., Radespiel, U. und Jünemann, B. (1993a): Long-term study of infant-carrying behavior in captive common marmosets (Callithrix jacchus): effect of nonreproductive helpers on the parent's carrying performance. Int. J. Primatol. 14 (1): 7993.

Rothe, H., Darms, K., König, A., Siess, M. und Brusek, P. (1986): Dynamics of group size in the common marmoset (Callithrix jacchus) under laboratory conditions. Primate Report 14: 74-75.

Rothe, H. und König, A. (1987): Polygynes Verhalten einer Weißbüschelaffen-Gruppe (Callithrix jacchus). Zool. Garten N.F. 57: 368-369.

Rothe, H. und König, A. (1991): Variability of social organization in captive common marmosets (Callithrix jacchus). Folia Primatol. 57: 28-33.

Rothe, H., König, A. und Darms, K. (1993b): Infant survival and number of helpers in captive groups of common marmosets (Callithrix jacchus). Am. J. Primatol. 30: 131-137.

Rothe, H. und Radespiel, U. (1988): Relationships of expelled common marmosets to their native families and unrelated conspecifics. Int. J. Primatol. 8: 499. Abstract.

Rothe, H. und Thieß, A. (2001a): Bemerkenswerte Beobachtungen an einer Gruppe semifreilebender Weißbüschelaffen (Callithrix jacchus). Zool. Garten N.F. 71: 69.

Rothe, H. und Thieß, A. (2001b): Temporary cooperative polyandry in semifree common marmosets (Callithrix jacchus). In: Apfelbach, R. (ed.): Advances in Ethology 36, Supplements to Ethology, Contributions to the XXVIIth International Ethological Conference, Blackwell Wissenschafts-Verlag Berlin Vienna 2001. S. 255.

Rothe, H., Thieß, A. und Wiesemüller, B. (2003): Does infant-carrying influence the selection of feeding sites in semifree common marmosets (Callithrix jacchus). Crook, G. (ed.) International Primatological Congress, Selbstverlag Adelaide, CD-Rom.

Rothe, H.; Westermann, H. und Kerl, J. (1997): Freigehege für Krallenaffen am Institut für Anthropologie der Universität Göttingen. Zool. Garten N.F. 67 (3): 85-91.

Ruiz, J.C. und Colillas, O.J. (1962): Ciclo ovarico del marmoset comun. Data. Physiol. Lat. Amer. 32 (1): 59-61.

Rylands, A.B. (1982): The behaviour and ecology of the three species of marmosets and tamarins (Callitrichidae, Primates) in Brazil. Dissertation, University of Cambridge.

Rylands, A.B. (1986): Infant-carrying in a wild marmoset group, Callithrix humeralifer: evidence for a polyandrous mating system. In de Mello, M.T. (ed.): A primatologia no Brasil-2: 131-144. Sociedade Brasileira de Primatologia, Brasilia.

Rylands, A.B. (1990): Scent marking behaviour of wild marmosets, Callithrix humeralifer (Callitrichidae, Primates). In: Macdonald, D.W., Müller-Schwarze, D. und Natynczuk, S.E.(Hrsg.): Chemical signals in vertebrates 5: 415-429. Oxford University Press, Oxford.

Rylands, A.B. (1996): Habitat and the evolution of social and reproductive behavior in Callitrichidae. Am. J. Primatol. 38: 5-18.

Sade, D.S. (1972): Sociometrics of Macaca mulatta. I. Linkages and cliques in grooming matrices. Folia Primatol. 18: 196-223. 
Saltzman, W., Schultz-Darken, N.J. und Abbott, D.H. (1997a): Familial influences on ovulatory function in common marmosets (Callithrix jacchus). Am. J. Primatol. 41: 159177.

Saltzman, W., Schultz-Darken, N.J., Severin, J.M. und Abbott, D.H. (1997b): Escape from social suppression of sexual behavior and of ovulation in female common marmosets. The Integrative Neurobiology of Affiliation 807: 567-570.

Sanchez, S., Kaumanns, W. und Pelaez, F. (1996): Costs of cooperation in cotton-top tamarins (Saguinus oedipus) in captivity. Antiquity 70: 145.

Sanchez, S. Pelaez, F. Gil-Bürmann, C. und Kaumann, W. (1999): Costs of infant-carrying in the cotton-top tamarin (Saguinus oedipus). Am. J. Primatol. 48: 99-111.

Sanchez-Viagra, M.R., Pope, T.R. und Salas, V. (1998): Relation of intergroup variation in allogrooming to group social structure and ectoparasite loads in red howlers (Alouatta seniculus). Int. J. Primatol. 19: 473-491.

Savage, A., Giraldo, L.H., Soto, L.H. und Snowdon, C.T. (1996): Demography, group composition, and dispersal in wild cotton-top tamarin (Saguinus oedipus) groups. Am. J. Primatol. 38 (1): 85-100.

Scanlon, C.E., Chalmers, N.R. und Monteiro da Cruz, M.A.O. (1988): Changes in the size, composition, and reproductive condition of wild marmoset groups (Callithrix jacchus jacchus) in north east Brazil. Primates 29 (3): 295-305.

Schäfers, B. (2000): Grundbegriffe der Soziobiologie. 6.Auflage, UTB für Wissenschaft, Leske +Budrich, Opladen.

Schaffner, C.M. und Caine; N. (2000): The peacefulness of cooperative breeding primates. In: Aureli, F. und de Waal, F.B.M.: Natural conflict resolution. University California Press, Berkeley. S. 155-169.

Schmidtke, J., Nürnberg, P. und Krawczak, M. (2003): Der Rhesusaffe und die Vermehrungsstrategie. Das Magazin der Deutschen Forschungsgemeinschaft 4: 20-22.

Schnell, C. (1993): Radiotelemetrische Untersuchungen von Blutdruck, Herzfrequenz, Elektrokardiogramm, Körpertemperatur und Spontanaktivität an Weißbüschelaffen (Callithrix jacchus). Z. Säugetierkunde 58 (Sonderheft): 63-64.

Schnell, C. (1997): Haemodynamic measurements by telemetry in concious unrestrained marmostes: Responses to social and non social stress events. In: Pryce, C., Scott, L. und Schnell, C. (eds.): Marmosets and tamarins in biological and biomedical Research, DSSD Imagery, Salisbury, S. 170-180.

Schradin, C. und Anzenberger, A.A. (2001): Infant-carrying in family groups of Goeldi's monkeys (Callimico goeldii). Am. J. Primatol. 53: 57-67.

Seyfarth, R.M. (1983): Grooming and social competition in primates. In: Hinde, R.A. (ed.): Primate social relationships: an integrated approach. S. 182-189. Blackwell Scientific Publications.

Shahuano Tello, N., Huck, M. und Heymann, E.W. (2002): Boa constrictor attack and successful group defense. Folia Primatol. 73 (2-3): 146-148.

Sherman, P.W. (1985): Alarm calls of Belding's ground squirrels to aerial predators: nepotism or selfpreservation? Behav. Ecol. Sociobiol. 17: 313-323.

Sherman, P.W., Lacey, E.A., Reeve, H.K. und Keller, L. (1995): The eusociality continuum. Behav. Ecol. 6: 102-108. 
Siess, M. (1988): Experimente zur koopertiven Polyandrie beim Weißbüscheläffchen (Callithrix jacchus ERXLEBEN 1777). Unveröffentlichte Diplomarbeit, Universität Göttingen, Göttingen.

Siegel, S. (1987): Nichtparametrische statistische Methoden. 3. unveränderte Auflage Fachbuchhandlung für Psychologie, Verlagsabteilung Eschborn bei Frankfurt am Main.

Smith, A.T. (1987): Population structure of pikas: dispersal versus philopatry. In: ChepkoSade, B.D. und Halpin, Z.T. (eds.): Mammalian dispersal patterns: The effects of social structure on population genetics. The University of Chicogo Press, Chicago, London. S. 128-142.

Smith, T.E., Schaffner, C.M. und French, J.A. (1997): Social and developmental influences on reproductive function in female Wied's black tufted-ear marmosets (Callithrix kuhli). Hormones and Behaviour 31: 159-168.

Snowdon, C.T. und Pickhard, J.J. (1999): Family feuds: severe aggression among cooperatively breeding cotton-top tamarins. Int. J. Primatol. 20(5): 651-663.

Snowdon, C.T. und Soini, P. (1988): The tamarins, genus Saguinus. In: Mittermeier, R., Coimbra-Filho, A.F. und da Fonseca, G.A.B (eds.): Ecology and behavior of neotropical primates, Vol. 2, Academica Brasiliera de Sciencias, Rio de Janeiro. S. 223-98.

Snowdon, C.T., Ziegler, T.E. und Widowski, T.M. (1993): Further hormonal suppression of eldest daughter cotton-top tamarins following birth of infants. Am. J. Primatol. 31:11-21.

Soini, P. (1982): Ecology and population dynamics of the pygmy marmoset, Cebuella pygmaea. Folia Primatol. 39: 1-21.

Soini, P. (1987): Sociosexual behaviour of a free-ranging Cebuella pygmaea (Callithrichidae, Platyrrhini) troop during postpartum estrus of its reproductive female. Am. J. Primatol. 13: 223-230.

Soini, P. (1988): The pygmy marmoset, genus Cebuella. In: Mittermeier, R.A., Rylands, A.B., Coimbra-Filho, A.F. und da Fonseca, G.A.B. (eds.): Ecology and behaviour of neotropical primates. Academica Brasiliera de Sciencias, Rio de Janeiro,Vol. 2, S.79-129.

Spichiger-Carlsson, P. (1982): Beziehungen unter Familienmitgliedern bei Weißbüscheläffchen. Lizentiatsarbeit, Universiät Zürich, Zürich.

Stacey, P.B. und Ligon, J.D. (1991): The benefits-of-philopatry hypothesis for the evolution of kooperative breeding: variation in territory quality and group size effects. Am Nat. 137: 831-846.

Stearns, S.C. (1992): The evolution of life histories. Oxford University Press.

Stevenson, M. und Rylands, A.B. (1988): The marmoset monkeys, genus Callithrix. In Mittermeier, R., und Coimbra-Filho, A.F. und da Fonseca, G.A.B (eds.): Ecology and behavior of neotropical primates, Vol. 2, Academica Brasiliera de Sciencias, Rio de Janeiro. S. 131-222.

Strier, K. (1999): Why is female kin bonding so rare? Comparative sociality of neotropical primates. In: Lee, P.C.(ed.) Comparative primate socioecology. Cambridge University Press. S. 300-319.

Suchi, S. (1996): Der Einfluß abiotischer Faktoren auf die Aktivitätsperiode und das TimeBudget einer Gruppe von Weißbüschelaffen (Callithrix jacchus). Unveröffentlichte Diplomarbeit, Universität Göttingen. 
Sugiyama, Y. (1976): Life history of male Japanese macaques. Adv. Study Behav. 7: 255284.

Sussman, R.W. und Garber, P.A. (1987): A new interpretation of the social organisation and mating system of the callitrichidae. Int. J. Primatol. 8 (1): 73-92.

Sutcliffe, A.G. (1980): Agonistic and related social behaviours in the common marmoset (Callithrix jacchus jacchus). Ph.D. Thesis, University College of Wales, Aberystwyth.

Sutcliffe, A.G. und Poole, T.B. (1984): Intragroup agonistic behaviour in captive groups of the common marmoset Callithrix jacchus jacchus. Int. J. Primatol. 5: 473-489.

Tardif, S.D. (1994): Relative energetic cost of infant care in small-bodied neotropical primates and ist relation to infant-care patterns. Am. J. Primatol. 3: 133-143.

Tardif, S.D. (1997): The bioenergetics of parental behaviour and the evolution of alloparental care in marmosets and tamarins. In: Solomon, N.G. und French, J.A. (eds.): Cooperative breeding in mammals. Cambridge University Press, Cambrigde. S. 11-33.

Tardif, S.D. und Carson, R.L. (1987): Relation of intra-group aggression to reproductive strategies. Int. J. Primatol. 8 (5): 157.

Tardif, S.D. und Harrison, M.L. (1990): Estimates of the energetic cost of infant transport in tamarins. Am. J. Phys. Anthrop. 81: 306.

Tardif, S.D., Harrison, M.L. und Simek, M.A. (1993): Communal infant care in marmosets and tamarins: relation to energetics, ecology, and social organization. In: Rylands, A.B. (ed.) Marmosets and tamarins: systematics, behaviour and ecology. Oxford University Press, Oxford, New York, Tokyo. S. 220-234.

Tardif, S.D., Richter, C.B. und Carson, R.L. (1984): Effects of sibling rearing experience on future: reproductive success in two species of callitrichidae. Am. J. Primatol. 6: 377-380.

Tardif, S.D. und Ziegler, T.E. (1986): Age-related effects on ovarian function in tamarins (Saguinus). Primate Report 14: 146.

Terborgh, J. und Goldizen, A.W. (1985): On the mating system of the cooperatively breeding saddle-backed tamarin (Saguinus fuscicollis). Behav. Ecol. Sociobiol. 16: 293-299.

Terborgh, J. und Janson, C.H. (1986): The socioecology of primate groups. Annual Review of Ecology and Systematics 17: 111-135.

Terborgh, J. und Stern, M. (1987): The surrepitious life of the saddle-back tamarin. Am. Scientist 75: 260-269.

Thieß, A. und Rothe, H. (2002): Usability of temperature buttons in common marmosets (Callithrix jacchus). In: Dehnhard, M. und Hofer, H. (ed.): Advances in Ethology 37, Supplements to Ethology, S. 77.

Trivers, R.L. (1972): Parental investment and sexual selection. In: Campbell, B. (ed): Sexual selection and the descent of man 1871-1971. Chicago, Aldine. S. 136-179.

Trivers, R.L. (1985): Social evolution. Menlo Park, Benjamin/Cummings.

Vasquez, und Heymann, E.W. (2001): Crested eagle predation on infant tamarins. Folia Primatol. 72 (5): 301-303.

Voland, E. (2000): Grundriss der Soziobiologie. 2. überarbeitete Auflage. Spektrum Akademischer Verlag, Gustav Fischer.

von Neumann, J. und Morgenstern, O. (1944): Theory of games and economic behaviour. Princeton University Press, Princeton. 
von Werthern, C. Frhr. (1990): Untersuchungen zu Körpertemperatur und Spontanaktivität von Braunrückentamarinen (Saguinus fuscicollis) mittels Radiotelemetrie bzw. Videometrie. Inaugural-Dissertation, Tierärztliche Fakultät der Universität München, München.

Waser, P.M. (1988): Resources, philopatry, and social interactions among mammals. In: Slobodchikoff, C.N.(ed): The ecology of social behaviour, Academic Press. S. 109-130.

Waser, P.M. und Jones, W.T. (1983): Natal philopatry among solitary mammals. The Quarterly Review of Biology 58: 355-390.

Westermann, H. und Rothe, H. (1996): Using Newton Message Pad 120/130 for ethological data collection on semi-free ranging common marmosets (Callithrix jacchus). Folia Primatol. 69: 240.

Williams, G.C. (1966): Natural selection, the cost of reproduction, and a refinement of Lack's principle. Am. Nat. 100: 687-690.

Wilson, O.E (1994): Naturalist. Island Press, Washington, D.C. S. 380.

Winter, M. und Rothe, H. (1979): Darstellung des Lautrepertoires handaufgezogener Weißbüschelaffen (Callithrix jacchus jacchus) unter besonderer Berücksichtigung der fließenden Übergänge zwischen den einzelnen Lautgruppen. Primates 20: 259-276.

Wolters, H.J. (1978): Some aspects of role taking behaviour in captive family groups of the cotton-top tamarin Saguinus oedipus oedipus. In: Rothe, H.; Wolters, H.J. und Hearn, J.P.(eds.) Biology and behaviour of marmosets; proceeding of the marmoset workshop, Eigenverlag H. Rothe, Göttingen. S. 233-258.

Woolfenden, G.E. und Fitzpatrick, J.W. (1984): The Florida scrub jay. Princeton University Press, Princeton.

Wright, P.C. (1984): Biparental care in Aotus trivirgatus and Callicebus moloch. In: Small, M.R. (ed.): Female primates: Studies by women primatologists. Alan R. Liss. New York. S. 59-75.

Yamamoto, M.E. (1993): From dependence to sexual maturity: the behavioural ontogeny of Callithrichidae. In: Rylands, A.B.(ed.) Marmosets and tamarins. Oxford University Press, Oxford. S. 235-254.

Yamamoto, M.E.; Alencar, A.; Araujo, A.; Albuquerque, F. und Sousa, M.B. (2001): Breeding strategies in captive and wild Callithrix jacchus. In: Apfelbach, R. (ed.): Advances in Ethology 36, Supplements to Ethology, Contributions to the XXVIIth International Ethological Conference, Blackwell Wissenschafts-Verlag Berlin Vienna 2001. S. 290.

Zahavi, A. (1990): Arabian babblers: the quest for social status in a cooperative breeder. In: Stacey, P.B. und Koenig, W.D.(eds.): Cooperative breeding in birds. Long term studies of ecology and behaviour. Cambridge University Press, Cambridge. S.103-130. 


\section{DANKSAGUNG}

Mein herzlicher Dank gilt allen Personen, die mich finanziell, fachlich und ideell in dieser Studie unterstützt haben:

Deutsche Forschungsgemeinschaft (DFG): Finanzierung der Studie von 2000-2002, AZ: GRK 289/2-00

Meine Familie: finanzielle und ideelle Unterstützung

Andreas Friedrichs: Unterstützung bei computer-technischen Problemen

Anja Friedrichs: Korrektur des Manuskriptes und ideelle Unterstützung

Andrea Karadura: Pflege der Studientiere

Michael Kroll: Durchsicht und Korrektur des Manuskriptes, anregende Diskussionen, computer-technische und ideelle Unterstützung

Prof. Dr. Stefan Steinlechner (Tierärztliche Hochschule Hannover, Institut für Zoologie): Wissenschaftliche Beratung bei Temperaturmessungen

Stefan Suchi: Statistische Beratung

Prof. Dr. Jürgen Zimmermann (Universität Clausthal-Zellerfeld, Wirtschaftswissenschaften): Wissenschaftliche Beratung 


\section{LEBENSLAUF}

\section{Persönliche Daten}

Dipl. Biol. Ariane Thieß

geboren am 13.03.1972 in Hamburg

deutsche Staatsangehörigkeit

\section{Schulbildung}

1978 - $1982 \quad$ Grundschule Schimmelmannstraße in Hamburg

1982 - 1991 Gymnasium Marienthal in Hamburg, Abitur mit der Note 1,6

\section{Hochschulausbildung}

$\begin{array}{ll}1991-1998 & \text { Universität Hamburg, Biologie-Studium, Diplom am 20.11.1998 } \\ & \text { Hauptfach: Zoologie, Nebenfächer: Naturschutz und Hydrobiologie } \\ & \text { Diplomarbeit: Fluchtverhalten und Kooperation weiblicher } \\ & \text { Riesenkänguruhs (Macropodidae) und ihrer Folgejungen } \\ & \text { Universität Göttingen, Promotion im Institut für Zoologie und } \\ & \text { Anthropologie }\end{array}$

Publikationen

Thieß A. \& Croft D. B. (1997): Flight behaviour and cooperation of female kangaroos and their young-at-foot. (Veröffentlichung im Internet unter http://www.bios.unsw.edu.au/fgap/res1997.htm\#thiess)

Rothe H. \& Thieß A. (2001): Bemerkenswerte Beobachtungen an einer Gruppe semifreilebender Weißbüschelaffen (Callithrix jacchus). Zool. Garten N.F. 71, (1) S. 69.

Rothe H. \& Thieß A. (2001): Temporary cooperative polyandry in semifree common marmosets (Callithrix jacchus). In: Apfelbach, R. (ed.): Advances in Ethology 36, Supplements to Ethology, Contributions to the XXVIIth International Ethological Conference, Blackwell Wissenschafts-Verlag Berlin Vienna 2001. S. 255.

Thieß A. \& Rothe H. (2001): Analysis of sociodynamic processes in large families of semifree living common marmosets (Callithrix jacchus). Folia Primatol 72 (3) S. 186.

Thieß A. \& Rothe H. (2002):Usability of temperature buttons in common marmosets (Callithrix jacchus). In: Dehnhard, M. \& Hofer, H. (ed.): Advances in Ethology 37, Supplements to Ethology, Contributions to the 4th International Symposium on Physiology and Behaviour of Wild and Zoo Animals, Blackwell Wissenschafts-Verlag, Berlin Vienna 2002. S. 77.

Rothe H., Thieß A. \& Wiesemüller B. (2003): Does infant-carrying influence the selection of feeding sites in semifree common marmosets (Callithrix jacchus)? In: Crook G (ed.): Primates in the New Millenium, Selbstverlag Adelaide, CD-ROM. 